Florida International University FIU Digital Commons

3-7-2016

\title{
Hybrid Energy Storage Implementation in DC and AC Power System for Efficiency, Power Quality and Reliability Improvements
}

Mustafa Farhadi

mfarh003@fiu.edu

DOI: $10.25148 /$ etd.FIDC000253

Follow this and additional works at: https://digitalcommons.fiu.edu/etd

Part of the Electrical and Electronics Commons, and the Power and Energy Commons

\section{Recommended Citation}

Farhadi, Mustafa, "Hybrid Energy Storage Implementation in DC and AC Power System for Efficiency, Power Quality and Reliability Improvements" (2016). FIU Electronic Theses and Dissertations. 2471.

https://digitalcommons.fiu.edu/etd/2471 


\title{
FLORIDA INTERNATIONAL UNIVERSITY
}

Miami, Florida

\section{HYBRID ENERGY STORAGE IMPLEMENTATION IN DC AND AC POWER SYSTEM FOR EFFICIENCY, POWER QUALITY AND RELIABILITY IMPROVEMENTS}

\author{
A dissertation submitted in partial fulfillment of \\ the requirements for the degree of \\ DOCTOR OF PHILOSOPHY \\ in \\ ELECTRICAL ENGINEERING \\ by
}

Mustafa Farhadi 
To: $\quad$ Interim Dean Ranu Jung

College of Engineering and Computing

This dissertation, written by Mustafa Farhadi, and entitled Hybrid Energy Storage Implementation in DC and AC Power System for Efficiency, Power Quality and Reliability Improvements, having been approved in respect to style and intellectual content, is referred to you for judgment.

We have read this dissertation and recommend that it be approved.

$\begin{array}{r}\hline \text { Arif Sarwat } \\ \hline \text { Kinzy Jones } \\ \hline \text { Sakhrat Khizroev } \\ \hline \text { Mark J. Roberts } \\ \hline \text { Osama Mohammed, Major Professor }\end{array}$

Date of Defense: March 7, 2016

The dissertation of Mustafa Farhadi is approved.

Interim Dean Ranu Jung College of Engineering and Computing

Dean Andrés G. Gil Vice President for Research and Economic Development and Dean of the university Graduate School

Florida International University, 2016 
(C) Copyright 2016 by Mustafa Farhadi All rights reserved. 


\section{DEDICATION}

This dissertation is dedicated to my devoted and loving wife, Samaneh, and to my

darling parents, Alie and Anoshiravan. Without their support, encouragement, and most importantly their everlasting love, the completion of this work would never have been possible. 


\section{ACKNOWLEDGMENTS}

I would like to express my sincere gratitude to my principal advisor, Professor Osama Mohammed, for his guidance, support, encouragement, and thought-provoking discussions during my doctoral research. This dissertation would not have been possible without his help, support, resolute dedication, and patience. His passion for success has inspired me to come up with new ideas. His expert advice and unsurpassed knowledge of the various fields of electrical energy systems has always provided me an endless supply of idea to move to the next step and complete this dissertation. I am also grateful for the chance he gave me to work under his supervision at his excellent research group at the Energy Systems Research Laboratory. In addition, this atmosphere enabled me to develop

professionally in an environment where research work and engineering ethics are highly respected.

I highly acknowledge the support I received as a research assistant under my major professor research grants received from the Office of Naval Research and the US Department of Energy throughout my research at the energy systems research laboratory. I thank the university graduate school for awarding me the Dissertation Year Fellowship (DYF) which significantly helped me complete this dissertation.

I would like to also thank my dissertation committee members for their insightful comments and constructive suggestions in the review of my dissertation.

Also, I am thankful to all my colleagues in the Energy Systems Research Laboratory. Many graduate and undergraduate student scholars have helped me with discussions, contributions, and assistance contributing to achieving my research goals. I 
also want to mention the support of our departmental staff, especially Ms. Pat Brammer and Mr. Oscar Silveira, who gave me great help and support.

Finally, I would like to give a special thanks to my wife for her everlasting love and to my mother and father for their unconditional support and love. I also extend my gratitude to my dearest brother and sister, Mosa and Somaye, for their encouragement and assistance. I would also like to express my gratitude to my father and mother in law, Jamal and Zahra, thank you for all the support and encouragement. 
ABSTRACT OF THE DISSERTATION

HYBRID ENERGY STORAGE IMPLEMENTATION IN DC AND AC POWER

SYSTEM FOR EFFICIENCY, POWER QUALITY AND RELIABILITY

IMPROVEMENTS

by

Mustafa Farhadi

Florida International University, 2016

Miami, Florida

Professor Osama Mohammed, Major Professor

Battery storage devices have been widely utilized for different applications. However, for high power applications, battery storage systems come with several challenges, such as the thermal issue, low power density, low life span and high cost. Compared with batteries, supercapacitors have a lower energy density but their power density is very high, and they offer higher cyclic life and efficiency even during fast charge and discharge processes. In this dissertation, new techniques for the control and energy management of the hybrid battery-supercapacitor storage system are developed to improve the performance of the system in terms of efficiency, power quality and reliability.

To evaluate the findings of this dissertation, a laboratory-scale DC microgrid system is designed and implemented. The developed microgrid utilizes a hybrid lead-acid battery and supercapacitor energy storage system and is loaded under various grid conditions. The developed microgrid has also real-time monitoring, control and energy management capabilities. 
A new control scheme and real-time energy management algorithm for an actively controlled hybrid DC microgrid is developed to reduce the adverse impacts of pulsed power loads. The developed control scheme is an adaptive current-voltage controller that is based on the moving average measurement technique and an adaptive proportional compensator. Unlike conventional energy control methods, the developed controller has the advantages of controlling both current and voltage of the system. This development is experimentally tested and verified. The results show significant improvements achieved in terms of enhancing the system efficiency, reducing the AC grid voltage drop and mitigating frequency fluctuation.

Moreover, a novel event-based protection scheme for a multi-terminal DC power system has been developed and evaluated. In this technique, fault identification and classifications are performed based on the current derivative method and employing an artificial inductive line impedance. The developed scheme does not require high speed communication and synchronization and it transfers much less data when compared with the traditional method such as the differential protection approach. Moreover, this scheme utilizes less measurement equipment since only the DC bus data is required. 


\section{TABLE OF CONTENTS}

CHAPTER

PAGE

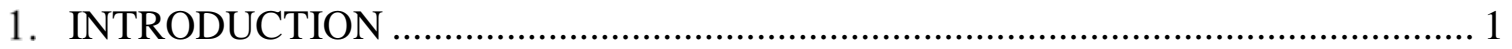

1.1. Introduction to Energy Storage Systems.......................................................... 1

1.2. Energy Storage Technology Overview ......................................................... 2

1.2.1. Pumped Hydroelectric Energy Storage ............................................................

1.2.2. Compressed Air Energy Storage................................................................4

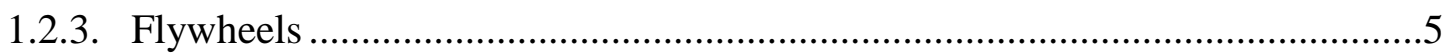

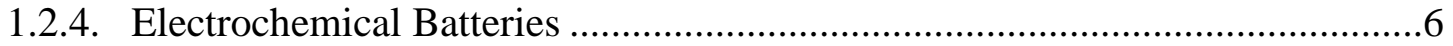

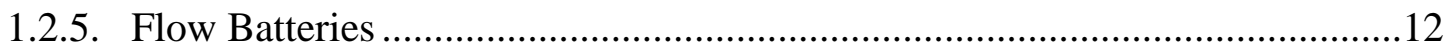

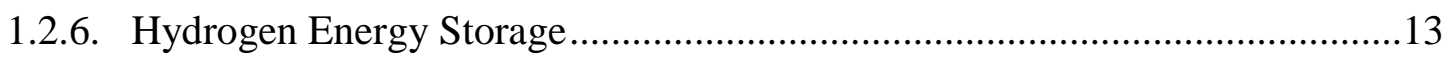

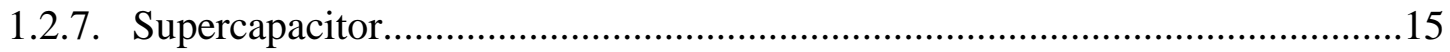

1.2.8. Superconductive Magnetic Energy Storage (SMES)....................................19

1.2.9. Comparison of energy storage technologies ..............................................20

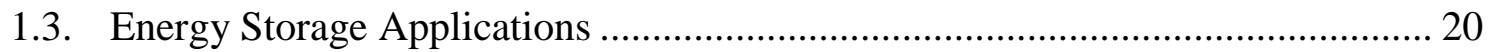

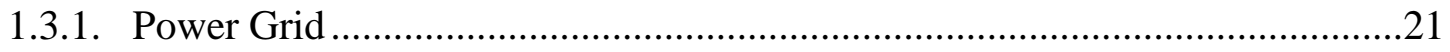

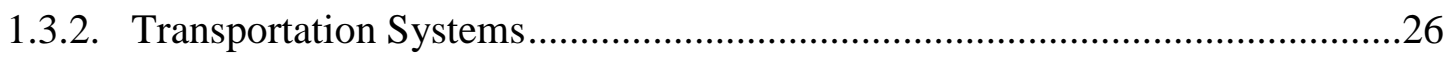

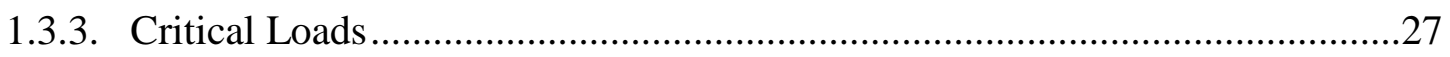

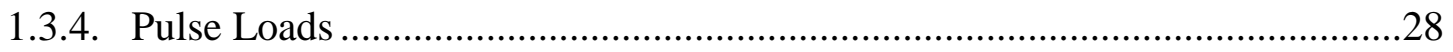

1.4. Hybrid Energy Power System.................................................................... 29

1.4.1. Hybrid Power System Configuration .............................................................30

1.4.2. Real-time Control of Hybrid Power System ..................................................34

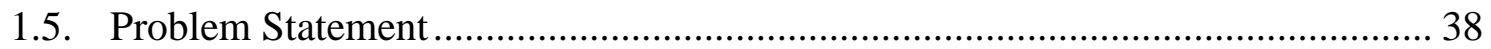

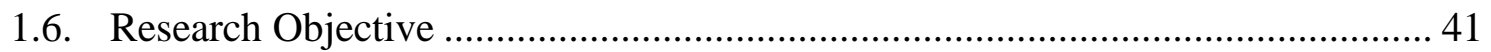

1.7. Original Contributions of the Dissertation..................................................... 44

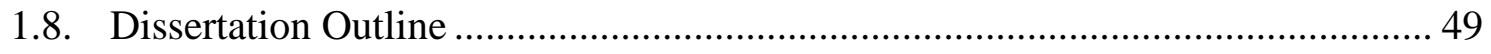

2. SUPERCAPACITOR-BATTERY ENERGY STORAGE INTEGRATION............ 53

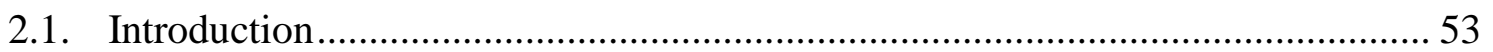

2.2. Power and Energy Requirements of the Pulse Loads ....................................... 55 
2.3. Battery-Supercapacitor Hybrid Configurations ............................................ 57

2.3.1. Passive Hybrid Configuration......................................................................58

2.3.2. Active Supercapacitor-Battery Configuration .............................................59

2.3.3. Active Battery-Supercapacitor Configuration ............................................60

2.3.4. Series Active Hybrid Configuration ...........................................................61

2.3.5. Parallel Active Hybrid Configuration ..........................................................61

2.4. Performance Analysis of Hybrid Storage System for Pulse Load Applications .. 62

2.4.1. Passive Battery-Supercapacitor Storage for Pulsed Load Application............63

2.4.2. Active Battery-Supercapacitor Storage for Pulsed Load Application .............69

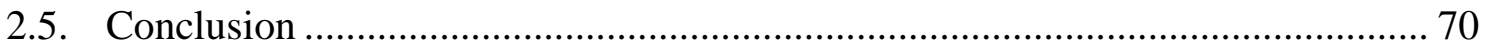

3. DESIGN AND IMPLEMENTATION OF HYBRID DC POWER SYSTEM........... 72

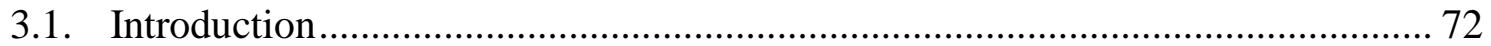

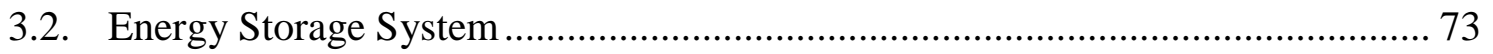

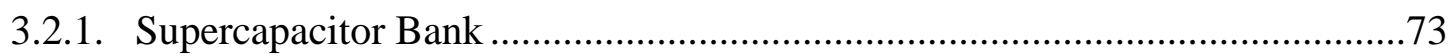

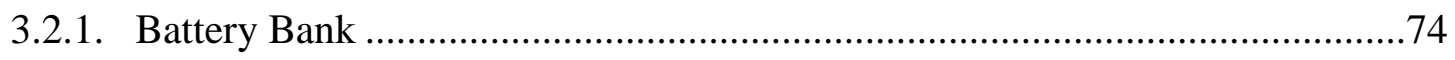

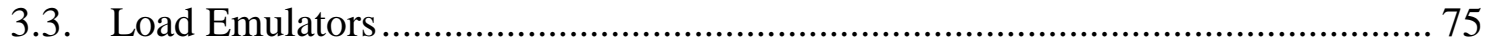

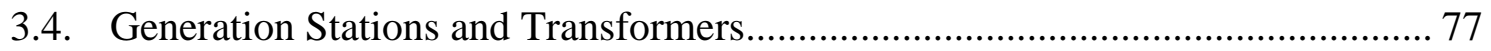

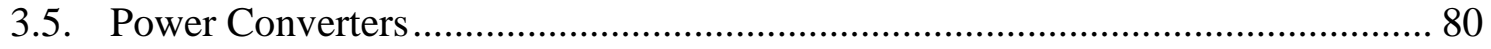

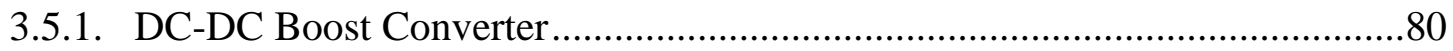

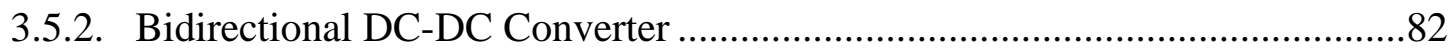

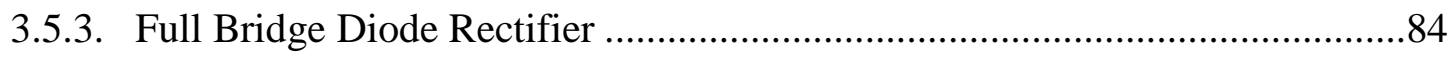

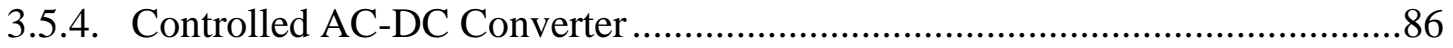

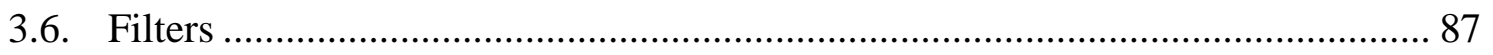

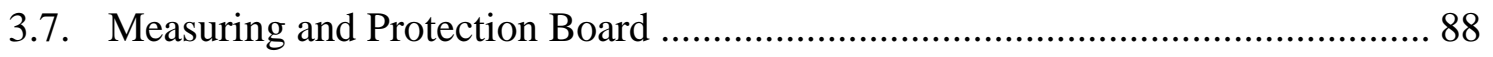

3.7.1. DC Voltage and Current Measurement Board..............................................8

3.7.2. Voltage Measurement with Common-Mode Rejection ..................................90

3.7.3. Isolated switching signal board ...............................................................91

3.7.4. AC Voltage and Current Measurement Box ..................................................93

3.7.5. Analog Hysteresis Voltage Protection ..........................................................94

3.8. AC Grid Test-Bed System Description............................................................ 98 


\section{REAL-TIME CONTROL AND ENERGY MANAGEMENT OF HYBRID DC} POWER SYSTEM

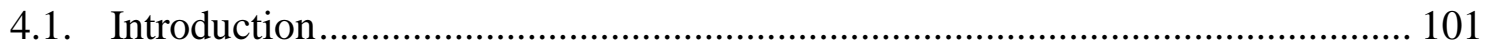

4.2. Bidirectional Energy Transfer in DC Microgrid............................................... 102

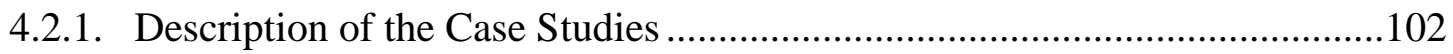

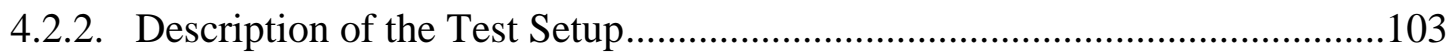

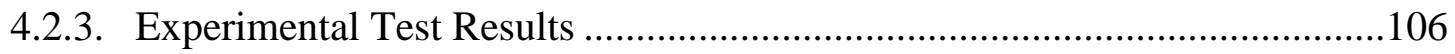

4.2.4. Summary of the Bidirectional Energy Transfer Results ..............................111

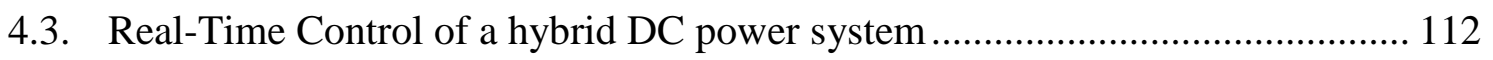

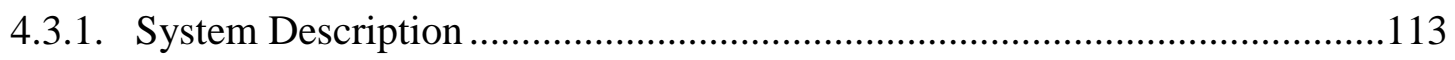

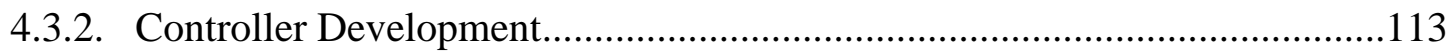

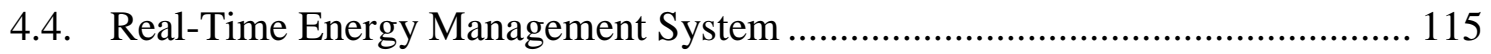

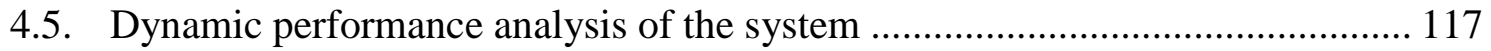

4.6. Operation and Harmonic Analysis of Isolated and Non-Isolated Grid............... 120

4.6.1. Isolated hybrid DC microgrid operation ................................................120

4.6.2. Non-isolated Hybrid DC Microgrid Operation ............................................126

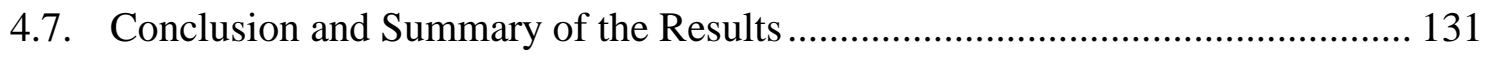

\section{DEVELOPMENT OF AN ADAPTIVE ENERGY MANAGEMENT SYSTEM}

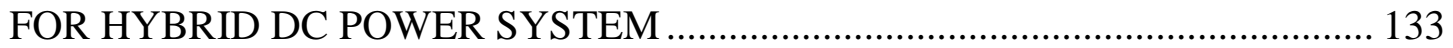

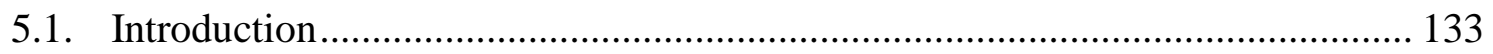

5.2. Actively Controlled Redundant Hybrid DC Power System .............................. 134

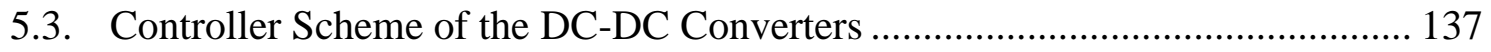

5.4. Development of an Adaptive Energy Management System ............................. 139

5.4.1. Errors and Their Effect on the Energy Management ....................................139

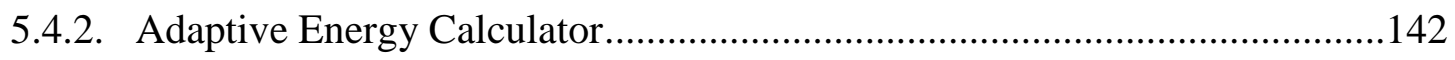

5.4.3. The Real-Time Energy Management System .............................................144

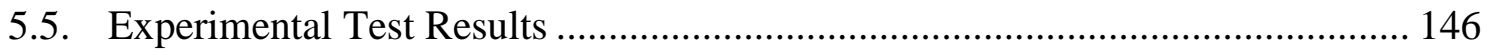

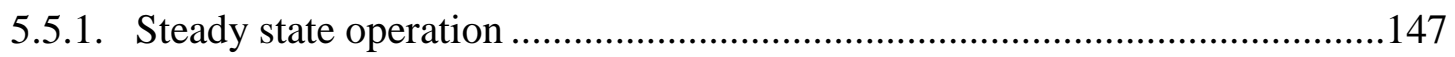

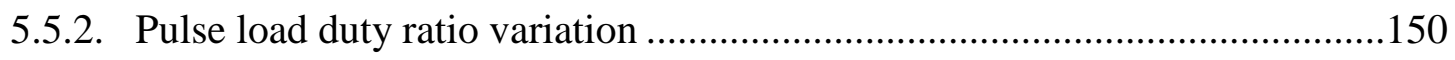

5.5.3. Operation with inaccurate measurement...................................................151 
5.5.4. Grid support during transient time.

5.5.5. Converter or supercapacitor outage

5.6. Conclusion and Summary of the Results

6. PERFORMANCE ANALYSIS OF GRID CONNECTED HYBRID DC MICROGRID INCORPORATING PULSE LOAD.............................................. 158

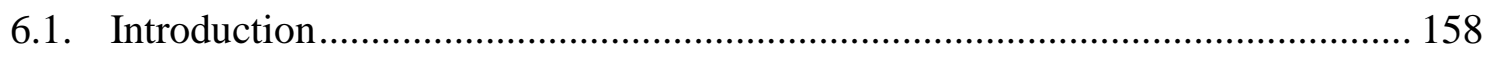

6.2. Energy Control for Grid Connected Hybrid DC Power System........................ 159

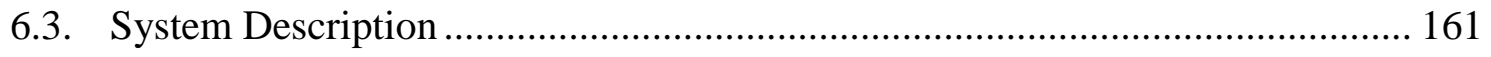

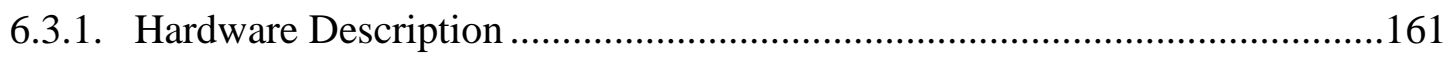

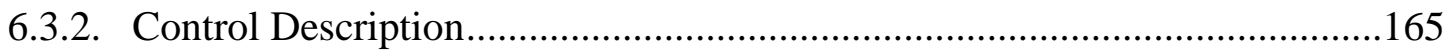

6.4. Energy Control Schemes for Grid Connected Hybrid DC Microgrid ................ 166

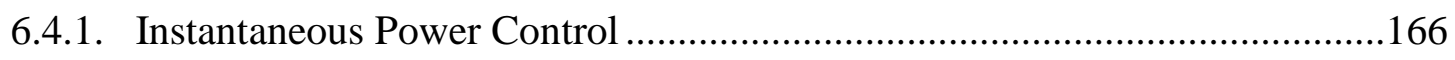

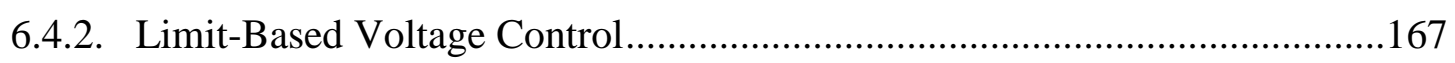

6.4.3. Continuous Average Current Control ........................................................168

6.5. Developed Adaptive Current-Voltage Control .............................................. 169

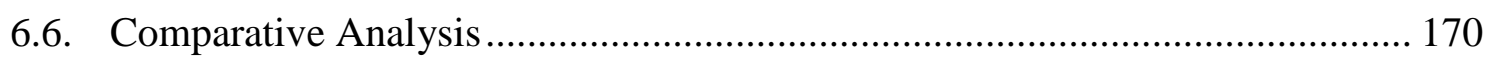

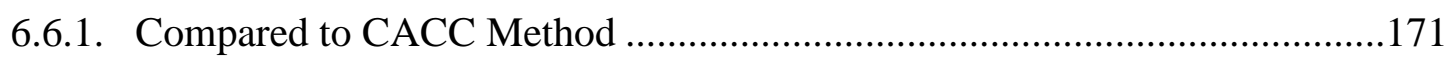

6.6.2. Compared to IPC and LBVC Methods .....................................................179

6.7. Summary of the Results and Conclusion .................................................... 189

7. VIRTUAL TETS-BED MODELING FOR DYNAMIC AND TRANSIENT STUDY OF HYBRID POWER SYSTEM ........................................................ 191

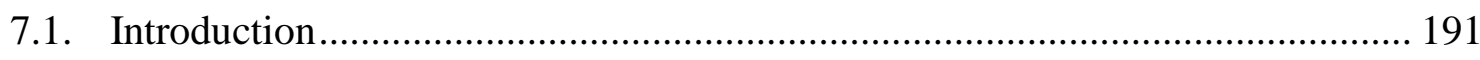

7.2. Modeling of the Energy Storage System ....................................................... 192

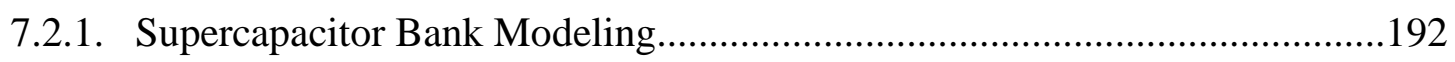

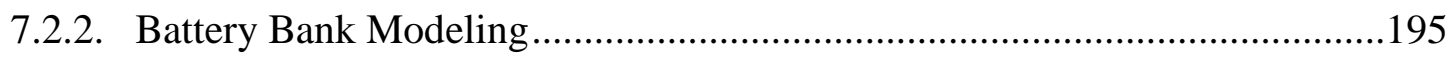

7.3. Virtual Test-Bed Model Implementation....................................................... 196

7.4. Evaluation of the Virtual Test-Bed Platform ................................................. 201

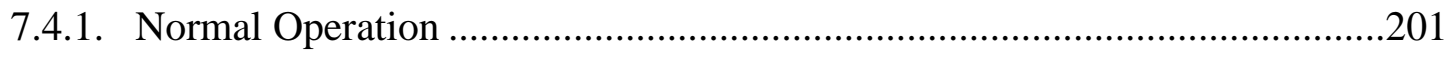

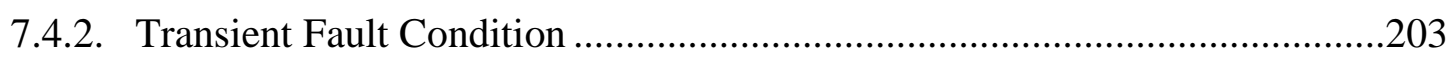

8. DESIGN OF DC POWER SYSTEM WITH HIGH RELIABILITY ...................... 205 


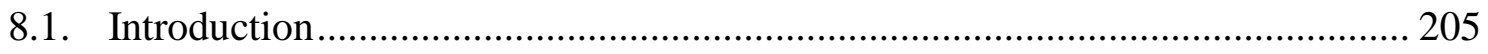

8.2. DC Power System Protection Requirements ..................................................... 206

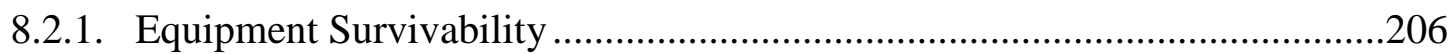

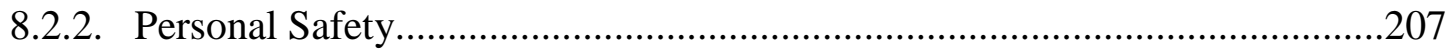

8.2.3. Fault Detection and Location Capabilities.......................................................207

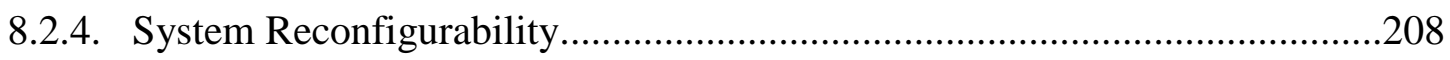

8.2.5. Ride-Through Capability ...........................................................................209

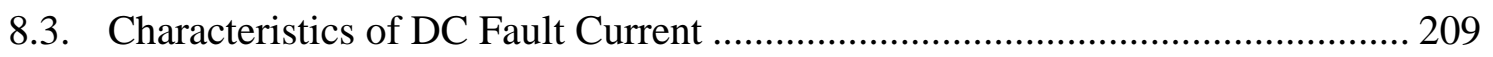

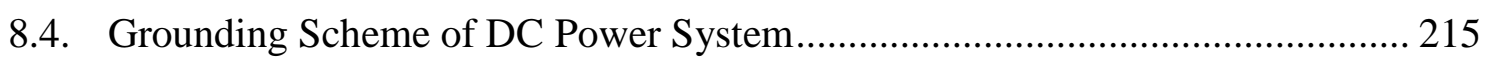

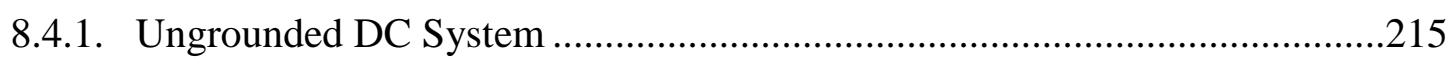

8.4.2. Resistance Grounded System..................................................................216

8.4.3. Solidly Grounded System ...........................................................................218

8.5. Circuit Breakers and Disconnecting Switches .................................................. 220

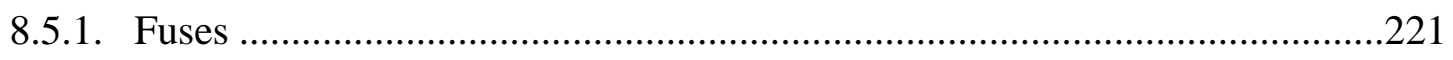

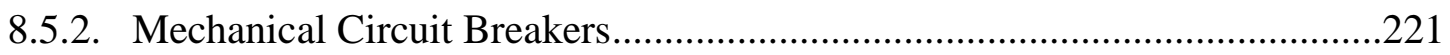

8.5.3. Solid-State Circuit Breakers ........................................................................222

8.5.4. Hybrid Solid-State Circuit Breakers ..............................................................224

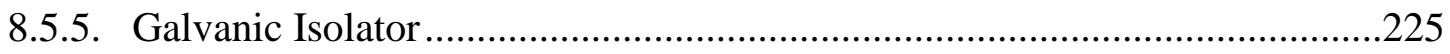

8.5.6. Fault-Tolerant Converters ............................................................................226

8.5.7. Superconductive Fault Current Limiter ……………………………..............226

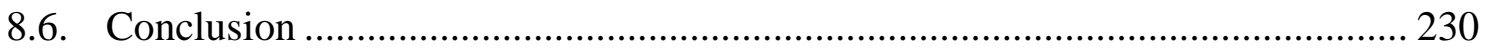

9. FAULT IDENTIFICATION AND LOCATION TECHNIQUES IN DC SYSTEM 233

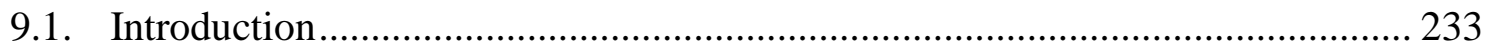

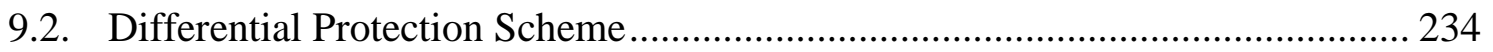

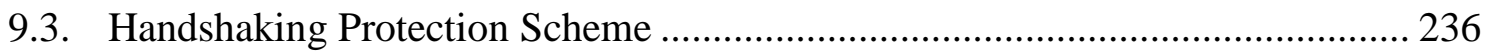

9.4. Traveling Wave Protection Techniques.............................................................. 240

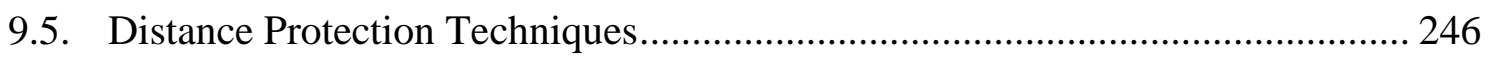

9.6. Artificial Intelligence Protection Techniques …………………......................... 248

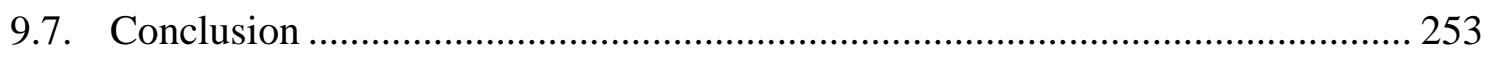


10. DEVELOPMENT OF AN EVENT-BASED PROTECTION SCHEME FOR HYBRID DC POWER SYSTEM …………………..................................... 254

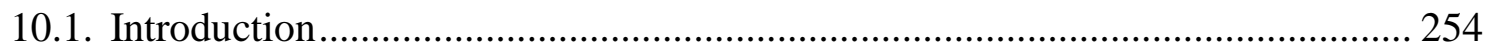

10.2. Proposed Approach for Protection of Hybrid DC Microgrid .............................. 254

10.3. Developed Event-based Protection Scheme ……................................................ 256

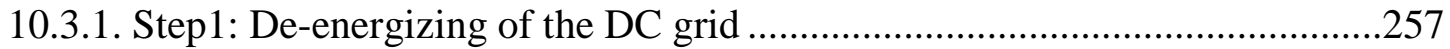

10.3.2. Step 2: High Level Data Communication ....................................................258

10.3.3. Step 3: Identification and Isolation of the Faulted Area ...................................258

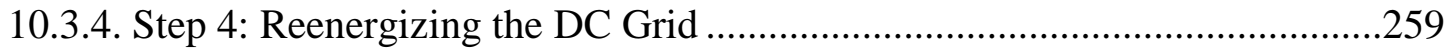

10.4. Hybrid DC microgrid Power System Design for Advanced Protection Scheme 260

10.5. Short Circuit Current Calculations.................................................................. 262

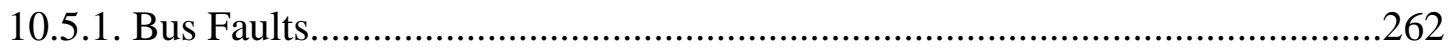

10.5.2. Feeder Fault and Remote Bus Fault..............................................................267

10.5.3. Low Pass Filter Effects …………………………….....................................269

10.6. Fault Event Classification Approach …………………………..................... 271

10.6.1. Artificial Inductive Line Impedance Technique .............................................272

10.6.2. Fault Current Derivative Method....................................................................274

10.6.3. Analysis of the Effects of the Cable Length .................................................277

10.7. Performance Evaluation of the proposed Protection Scheme............................... 279

11. CONCLUSIONS AND RECOMMENDATIONS FOR FUTURE WORK.............. 283

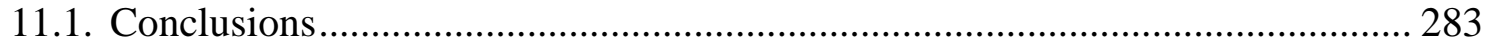

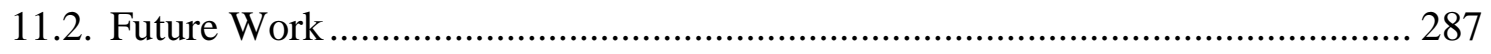

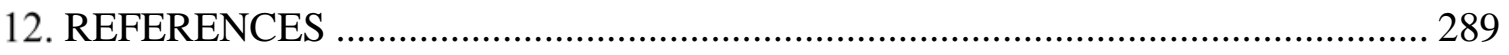

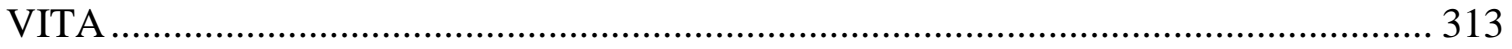




\section{LIST OF TABLES}

TABLE

PAGE

Table 1.1: Power and energy comparison of the storage technologies [55]-[59]............ 20

Table 1.2: Performance comparison of the storage technologies [55]-[59]..................... 21

Table 3.1: Specification of the supercapacitor bank ................................................... 73

Table 3.2: Specification of the battery bank. ............................................................ 74

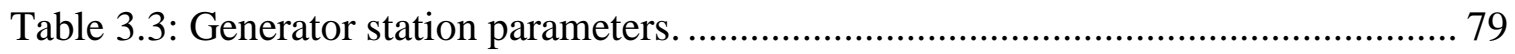

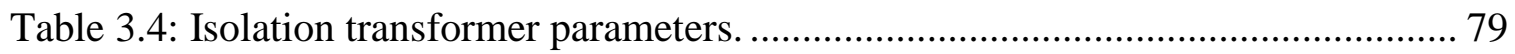

Table 5.1: Test results summary for AEC and DVC control methods. ......................... 149

Table 6.1: Hybrid DC Microgrid System Parameters.................................................. 163

Table 6.2: Energy Storage and Bidirectional Converter Parameters. ............................. 164

Table 7.1: Supercapacitor bank specifications......................................................... 194

Table 7.2: Sixth order model parameters of the 2.9-f supercapacitor bank................... 195

Table 7.3: Battery model parameters. ................................................................... 196

Table 7.4: AC side components’ parameters. .......................................................... 198

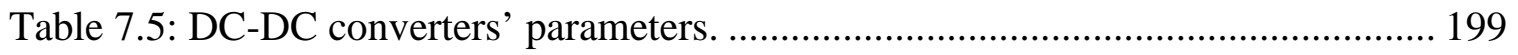

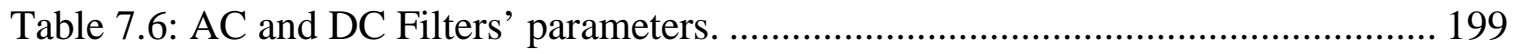

Table 7.7: DC microgrid power line Parameters. ........................................................ 201

Table 8.1: Comparison of different grounding schemes for DC power system applications. .................................................................................. 219

Table 8.2: Comparison of different circuit breaker technologies for DC power system

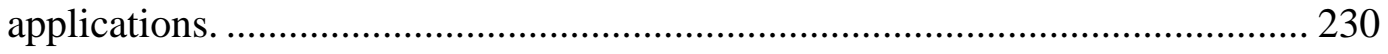

Table 10.1: Artificial inductive line impedance parameters ...................................... 273 


\section{LIST OF FIGURES}

FIGURE

PAGE

Figure 1.1: Classification of energy storage technologies.......................................... 3

Figure 1.2: Comparison of the different battery technologies in terms of volumetric and gravimetric energy density [16]-[18] ..................................................... 7

Figure 1.3: Comparison of different battery technologies in terms of capital cost per unit power and capital cost per energy [16]-[18].............................................. 8

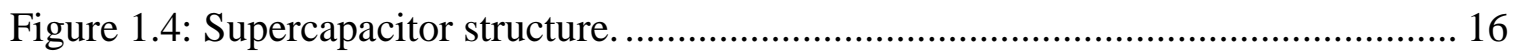

Figure 1.5: Effective capacitance of supercapacitor as a function of frequency [40]....... 18

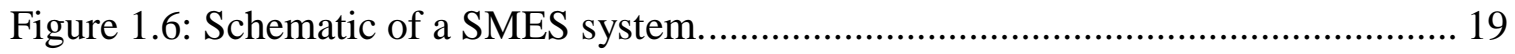

Figure 1.7: Utilization of energy storage devices for load leveling............................... 23

Figure 1.8: Schematic diagram of AC-coupled hybrid power system............................ 31

Figure 1.9: Schematic diagram of DC-coupled hybrid power system............................ 32

Figure 1.10: Schematic diagram of hybrid AC/DC power system................................. 33

Figure 1.11: Schematic diagram of high-frequency AC hybrid power system. .............. 34

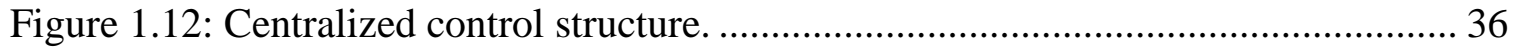

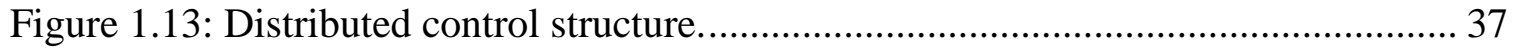

Figure 1.14: Distributed-centralized hybrid control structure. ................................... 38

Figure 2.1: Consumption power profile of a pulse load. ............................................ 57

Figure 2.2: Power density vs. energy density for various energy-storing devices. .......... 58

Figure 2.3: Passive battery-supercapacitor hybrid configuration. ................................. 59

Figure 2.4: Active supercapacitor-battery hybrid configuration................................... 60

Figure 2.5: Active battery-supercapacitor hybrid configuration................................... 60

Figure 2.6: Series active battery-supercapacitor hybrid configuration. .......................... 61

Figure 2.7: Parallel active battery-supercapacitor hybrid configuration. ....................... 62 
Figure 2.8: Simplified equivalent circuit of a passive hybrid configuration. (a):Time domain, (b): Frequency domain.

Figure 2.9: Normalized load, battery and supercapacitor currents for 1 per unit pulse load with 10 -s period and 0.1 duty ratio. 66

Figure 2.10: Current contribution of the battery bank for different pulse periods and duty ratios 68

Figure 2.11: Current contribution of the battery bank for different supercapacitor sizes.

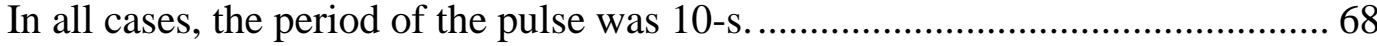

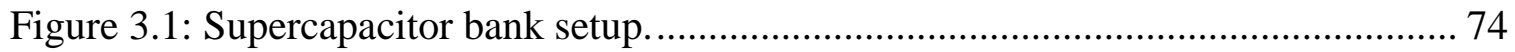

Figure 3.2: Experimental test equipment: (a) battery bank; (b) load emulator................. 75

Figure 3.3: A dynamic load consumption profile.................................................... 76

Figure 3.4: A pulse load consumption profile. ........................................................... 77

Figure 3.5: Schematic diagram of the generator stations' configuration and their

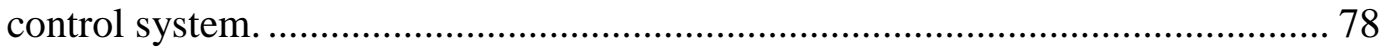

Figure 3.6: Variable frequency drive topology of the generator stations. ...................... 79

Figure 3.7: Experimental test setup components. (a): AC generator; (b) Galvanic

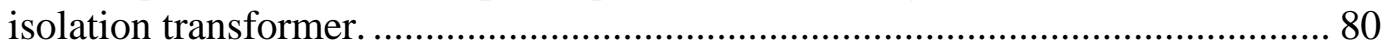

Figure 3.8: Power converter test setup. (a): DC-DC boost converter; (b): DC-DC

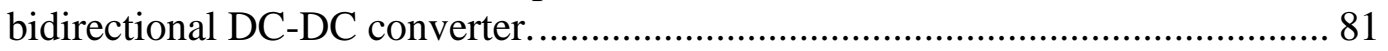

Figure 3.9: Configuration of the boost converter..................................................... 82

Figure 3.10: Block diagram of the boost converter controller..................................... 82

Figure 3.11: Configuration of the bidirectional DC-DC converter................................ 83

Figure 3.12: Block diagram of the bidirectional DC-DC converter controller................. 84

Figure 3.13: AC-DC power converter test setup. (a): Full bridge diode rectifier; (b): Three-phase controlled rectifier................................................................ 85

Figure 3.14: Three-phase full wave uncontrolled rectifier configuration........................ 85

Figure 3.15: Input AC voltage and the output DC voltage of an uncontrolled full bridge rectifier without capacitive filter.................................................................. 86

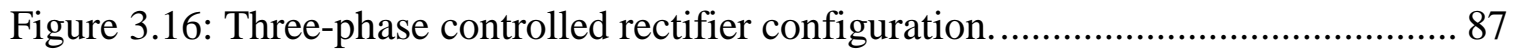


Figure 3.17: DC measurement board.

Figure 3.18: DC measurement circuit configuration. (a): Voltage measurement. (b) Current measurement.

Figure 3.19: Voltage measurement with common-mode rejection................................. 90

Figure 3.20: Measurement board with Common-Mode Rejection................................ 91

Figure 3.21: Experimental test results for two measurement modes. ........................... 92

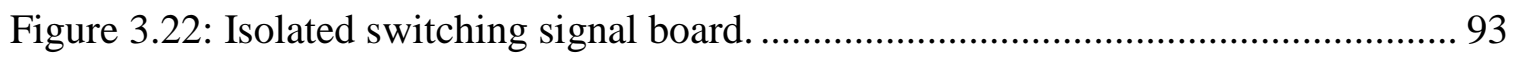

Figure 3.23: Schematic diagram of the analog hysteresis voltage protection.................. 95

Figure 3.24: Analog hysteresis voltage protection circuit board................................. 96

Figure 3.25: Relative error of the $V_{A r H}$ for the ten supercapacitor arrays....................... 97

Figure 3.26: Relative error of the $V_{A r L}$ for the ten supercapacitor arrays. ...................... 97

Figure 3.27: Schematic diagram of the smart grid test-bed....................................... 98

Figure 3.28: Monitoring and control infrastructure of the hybrid DC microgrid............ 99

Figure 3.29: Control desk and monitoring system of the hybrid DC microgrid............. 100

Figure 4.1: A schematic diagram for the bidirectional energy transfer study: two DC buses linked with a DC/DC converter. ......................................................... 102

Figure 4.2: Output voltage of the three-phase full wave uncontrolled rectifier: 6.0 V/div, $2.5 \mathrm{~ms} / \mathrm{div}$.

Figure 4.3: Backward current to the bidirectional DC-DC converter when the converter

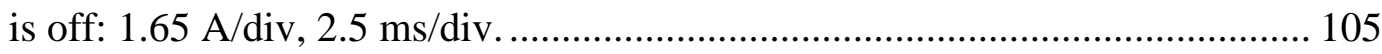

Figure 4.4: Drawn current from bus $1\left(\mathrm{I}_{\mathrm{LV}}\right)$ and injected current into the bus $2\left(\mathrm{I}_{\mathrm{HV}}\right)$ when the reference of the $\mathrm{I}_{\mathrm{HV}}$ is changed from 0 to $4 \mathrm{~A}$. $\mathrm{I}_{\mathrm{LV}}: 5.0 \mathrm{~A} / \mathrm{div}, 25$ ms/div; I $\mathrm{HV}_{\mathrm{V}}$ : $1.65 \mathrm{~A} / \mathrm{div}, 25 \mathrm{~ms} / \mathrm{div}$.

Figure 4.5: $\mathrm{I}_{\mathrm{LV}}$ and $\mathrm{I}_{\mathrm{HV}}$ when the reference of $\mathrm{I}_{\mathrm{HV}}$ is changed from 4 to $-3 \mathrm{~A}$. $\mathrm{I}_{\mathrm{LV}}: 5.0$ A/div, 25 ms/div; $\mathrm{I}_{\mathrm{HV}}$ : $1.65 \mathrm{~A} / \mathrm{div}, 25 \mathrm{~ms} / \mathrm{div}$.....

Figure 4.6: $\mathrm{I}_{\mathrm{LV}}$ and $\mathrm{I}_{\mathrm{HV}}$ when the reference of $\mathrm{I}_{\mathrm{LV}}$ is changed from -4 to $2 \mathrm{~A}$. $\mathrm{I}_{\mathrm{LV}}: 5.0$ A/div, 25 ms/div; I $\mathrm{HV}_{\mathrm{HV}} 1.65 \mathrm{~A} / \mathrm{div}, 25 \mathrm{~ms} / \mathrm{div}$ 108

Figure 4.7: $\mathrm{I}_{\mathrm{LV}}$ and $\mathrm{I}_{\mathrm{HV}}$ when the reference of $\mathrm{I}_{\mathrm{LV}}$ is changed from 2 to $6 \mathrm{~A}$. $\mathrm{I}_{\mathrm{LV}}: 5.0$ $\mathrm{A} /$ div, 25 ms/div; $\mathrm{I}_{\mathrm{HV}}$ : $1.65 \mathrm{~A} / \mathrm{div}, 25 \mathrm{~ms} /$ div. 
Figure 4.8: $\mathrm{I}_{\mathrm{LV}}$ and $\mathrm{I}_{\mathrm{HV}}$ when the three-phase full wave uncontrolled rectifier is connected to bus 2 and the reference of $\mathrm{I}_{\mathrm{LV}}$ is changed from -4 to $5 \mathrm{~A}$. $\mathrm{I}_{\mathrm{LV}}: 5.0$ A/div; I $\mathrm{HV}: 1.65 \mathrm{~A} / \mathrm{div}, 25 \mathrm{~ms} / \mathrm{div}$

Figure 4.9: A zoom-in on the Figure 4.8 with $5 \mathrm{~ms} / \mathrm{div}$.

Figure 4.10: $\mathrm{I}_{\mathrm{LV}}$ and $\mathrm{I}_{\mathrm{HV}}$ when the three-phase full wave uncontrolled rectifier is connected to bus 2 and the reference of $\mathrm{I}_{\mathrm{HV}}$ is changed from -3 to $5 \mathrm{~A}$. $\mathrm{I}_{\mathrm{LV}}: 5.0$ A/div, 25 ms/div; I $\mathrm{HV}_{\mathrm{HV}} 1.65 \mathrm{~A} / \mathrm{div}, 25 \mathrm{~ms} / \mathrm{div}$.

Figure 4.11: Schematic diagram of the system for the real-time control and energy management study.

Figure 4.12: Block diagram of the first boost converter controller with current control and digital hysteresis voltage protection.

Figure 4.13: Energy management of hybrid DC microgrid...................................... 116

Figure 4.14: Dynamic performance of the microgrid test results. .............................. 117

Figure 4.15: Current-voltage control method test result............................................ 118

Figure 4.16: Instantaneous power control method test results................................... 119

Figure 4.17: First mode of operation test results for the isolated DC microgrid............ 121

Figure 4.18: Output current of the transformer in the first mode of operation............... 122

Figure 4.19: Harmonic analysis of the output current of the transformer in the first

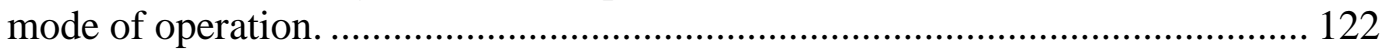

Figure 4.20: Second mode of operation test results for the isolated DC microgrid........ 123

Figure 4.21: Output current of the transformer in the second mode of operation. ......... 124

Figure 4.22: Harmonic analysis of the output current of the transformer in the second mode of operation.

Figure 4.23: Third mode of operation test results for the isolated DC microgrid. ......... 125

Figure 4.24: Output current of the transformer when the third mode of operation was selected.

Figure 4.25: Harmonic analysis of the Output current of the transformer in the third mode of operation.

Figure 4.26: Input current of the AC filter in the non-isolated grid when first mode of operation is selected. 
Figure 4.27: Harmonic analysis of the Input current of the AC filter in the non-isolated grid when first mode of operation is selected.

Figure 4.28: Second mode of operation test results for the non-isolated DC microgrid.

Figure 4.29: Input current of the AC filter in the non-isolated grid when the second mode of operation was selected.

Figure 4.30: Harmonic analysis of the input current of the AC filter in the non-isolated grid when the second mode of operation was selected.

Figure 4.31: Input current of the AC filter in the non-isolated grid when the third mode of operation was selected.

Figure 4.32: Harmonic analysis of the Input current of the AC filter in the non-isolated grid when the third mode of operation was selected.

Figure 5.1: A schematic diagram of a redundant hybrid DC power system.................. 135

Figure 5.2: Block diagram of the first boost converter controller. .............................. 138

Figure 5.3: Block diagram of the bidirectional DC-DC converter controller................. 138

Figure 5.4: Block diagram of the Adaptive energy calculator..................................... 142

Figure 5.5: Compensation factor $k_{\text {com }}$ profile with respect to the normalized supercapacitor voltage.

Figure 5.6: A flow chart of the real-time energy management algorithm.

Figure 5.7: DC microgrid performance in the case of the AEC and the DVC control methods for pulse load $=3.25-\mathrm{kW}$ (1.0 p.u.) and load $1=500-\mathrm{W}(0.25$ p.u.) and load $2=1.35-\mathrm{kW}$ (0.675 p.u.). The dashed lines in the voltage graph show the $5 \%$ thresholds.

Figure 5.8: Generator performance comparison of the AEC and the DVC control methods.

Figure 5.9: Operation test results of the microgrid with time averaging current measurement method for load $1=500-\mathrm{W}(0.25$ p.u.) and load $2=2-\mathrm{kW}(1.0$ p.u.) while the duty ratio of the $3.25-\mathrm{kW}$ (1.0 p.u.) pulse load is changed from $20 \%$ to $10 \%$ at $\mathrm{t}=30$-s.

Figure 5.10: Operation test result of the microgrid with AEC method for load $1=500$ $\mathrm{W}(0.25$ p.u.) and load $2=2-\mathrm{kW}$ (1.0 p.u.) while the duty ratio of the $3.25-\mathrm{kW}$ (1.0 p.u.) pulse load is changed from $20 \%$ to $10 \%$ at $t=10$-s. 
Figure 5.11: Operation test results of the microgrid without adaptive gain compensation for pulse load $=3.25-\mathrm{kW}(1.0$ p.u. $)$ and load $2=2-\mathrm{kW}$ (1.0 p.u.) while the load 1 is changed from 0 to $1500-\mathrm{W}(0.75$ p.u. $)$ at $\mathrm{t}=8$-s with $10 \%$ over-measurement.

Figure 5.12: Operation test results of the microgrid with AEC for pulse load $=3.25$ $\mathrm{kW}$ (1.0 p.u.) and load $2=2-\mathrm{kW}$ (1.0 p.u.) while the load 1 is changed from 0 to $1500-\mathrm{W}(0.75$ p.u. $)$ at $\mathrm{t}=8$-s with $10 \%$ over-measurement.

Figure 5.13: Operation test results of the microgrid with the proposed controller for pulse load $=3.25-\mathrm{kW}$ (1.0 p.u.) and load $2=2-\mathrm{kW}$ (1.0 p.u.) while the load 1 is changed from 0 to $2-\mathrm{kW}(1.0$ p.u. $)$ at $\mathrm{t}=6$-s with three times overshoot and damping factor of $1-\mathrm{s}$.

Figure 5.14: Test results for the grid with pulse load $=3.25 \mathrm{~kW}(1.0 \mathrm{p} . \mathrm{u}$.$) , load 1=$ $500-\mathrm{W}$ ( 0.25 p.u.) and load $2=2-\mathrm{kW}$ (1.0 p.u.) while outage of the converter 1 is occurred at $\mathrm{t}=10$-s.

Figure 5.15: Test results for the grid with pulse load $=3.25-\mathrm{kW}(1.0$ p.u. $)$, load $1=$ 1500-W (0.75 p.u.) and load $2=2 \mathrm{~kW}$ (1.0 p.u.) while the supercapacitor and pulse load are disconnected at $\mathrm{t}=10$-s.

Figure 6.1 Schematic diagram of the grid connected hybrid dc microgrid under study.

Figure 6.2: Block diagram of the DC microgrid three layer control system. ................ 165

Figure 6.3: Block diagram of the instantaneous power control................................... 166

Figure 6.4: Block diagram of the limit-base voltage control..................................... 167

Figure 6.5: Block diagram of continues average current control................................ 168

Figure 6.6: Block diagram of adaptive current-voltage control................................ 170

Figure 6.7: Experimental test results of ACVC and CACC technique during constant pulse load operation.............................................................................. 172

Figure 6.8: Experimental test results of CACC method and ACVC technique during test case 1.

Figure 6.9: Experimental test results of CACC method and ACVC technique when pulse load frequency changes from $0.1-\mathrm{Hz}$ to $0.2-\mathrm{Hz}$ and its duty ratio increased from $20 \%$ to $40 \%$. 
Figure 6.10: Variation of the normalized average DC bus voltage and the $\mathrm{kv}$ in the proposed ACVC technique when pulse load frequency changes from $0.1-\mathrm{Hz}$ to $0.2-\mathrm{Hz}$ and its duty ratio increased from $20 \%$ to $40 \%$.

Figure 6.11: Experimental test results of CACC method and ACVC technique when pulse load duty ratio changes from $20 \%$ to $10 \%$.

Figure 6.12: Variation of the $k_{v}$ in the proposed ACVC technique when pulse load duty ratio changes from $20 \%$ to $10 \%$

Figure 6.13: Experimental test results of CACC method and ACVC technique when pulse load changed from 2-kW to 3-kW.

Figure 6.14: Hybrid DC microgrid performance comparison when ACVC, LBVC and IPC methods were utilized.

Figure 6.15: AC grid performance comparison when ACVC, LBVC and IPC methods are utilized

Figure 6.16: Hybrid DC microgrid performance comparison during the operation of high duty ratio pulsed power load.

Figure 6.17: AC grid performance comparison during the operation of low duty ratio pulsed power load.

Figure 6.18: Normalized DC bus voltage variation comparison between the developed ACVC technique and the LBVC and IPC methods.

Figure 6.19: AC bus voltage drop comparison between the developed ACVC technique and the LBVC and IPC methods.

Figure 6.20: AC grid frequency variation performance comparison between the developed ACVC technique and the LBVC and IPC methods.....

Figure 6.21: System efficiency comparison between the developed ACVC technique and the LBVC and IPC methods.

Figure 7.1: Electrical equivalent circuit models for supercapacitor: (a) first order model, (b) third order model with voltage dependent branch.....

Figure 7.2: Sixth order equivalent circuit models for supercapacitor bank.

Figure 7.3: Schematic diagram of the virtual test-bed power system....

Figure 7.4: Performance of the hybrid DC microgrid during normal operation.

Figure 7.5: Performance of the hybrid DC microgrid during transient condition when the boost converter at bus 2 fails at $\mathrm{t}=10 \mathrm{~s}$. 
Figure 8.1: Schematic diagram of a notional three-bus MVDC.

Figure 8.2: Equivalent circuit of the grid shown in Figure 8.2 during short circuit conditions: (a) seen from AC source at bus 1, (b) seen from battery bank at bus 2

Figure 8.3: Grounding scheme of DC power system. (a), (b) and (c) are the resistance grounded DC power system. (d) is a solidly grounded system in middle point. 218

Figure 8.4: Configuration of the passive MCB..................................................... 222

Figure 8.5: Schematic of a Unidirectional SSCB system........................................ 223

Figure 8.6: Schematic of a Bidirectional SSCB system. .......................................... 223

Figure 8.7: Hybrid circuit breaker for high-voltage applications. ................................ 225

Figure 8.8: Circuit breaker with galvanic isolator. ................................................. 226

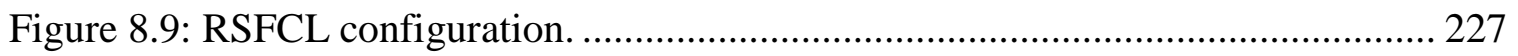

Figure 8.10: Passive ISFCL configuration......................................................... 228

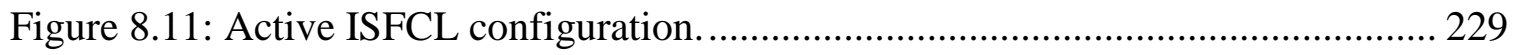

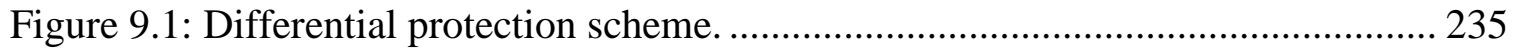

Figure 9.2: Illustration of the potential faulted line Selection in the handshaking

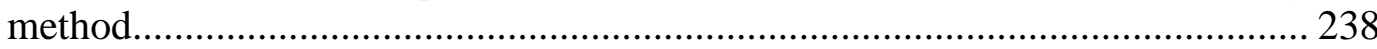

Figure 9.3: Illustration of the potential faulted line opening in the handshaking method.

Figure 9.4: Lattice diagram corresponding to fault F1 to illustrate the travelling wave flow along the HVDC transmission line........................................................ 242

Figure 9.5: Schematic diagram WT-based MRA decomposition................................. 251

Figure 9.6: WT-based MRA protection flowchart................................................... 252

Figure 10.1: Schematic diagram of a three-bus MVDC. ........................................... 257

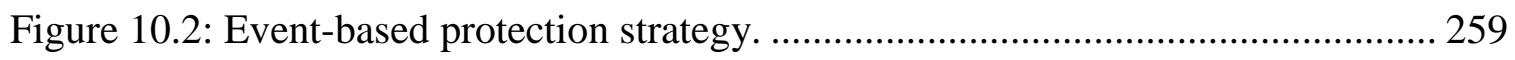

Figure 10.3: Load and its hold-up capacitor configuration........................................ 260

Figure 10.4: Schematic diagram of the hybrid dc microgrid under study. .................... 261 
Figure 10.5: Equivalent circuit of the grid in the short circuit conditions seen from uncontrolled rectifier at bus 1 .

Figure 10.6: Equivalent circuit of the grid in the short circuit conditions seen from battery bank at bus 2 .

Figure 10.7: Simulation result during fault $\mathrm{F} 1$, showing $i_{C G C}, i_{C D F}$ and $i_{U R}$ contributions to the $i_{b 1}$.

Figure 10.8: Sub-transient short circuit current $i_{b 1}$ during faults F1, F5 (a) when occurs $5 \mathrm{~m}$ from bus 1, F5 (b) when occurs $5 \mathrm{~m}$ from bus 2, and fault F7 when occurs $5 \mathrm{~m}$ from bus 2 .

Figure 10.9: Sub-transient short circuit current $i_{b 1}$ during faults F1, F5 (a) when AILI $=0.02-\mathrm{mH}$ were employed, F5 (b) when AILI $=0.1-\mathrm{mH}$ were employed and F5 (c) when AILI = 0.5-mH were employed.

Figure 10.10: Derivative of short circuit current $i_{b 1}$ during fault F1 and fault F5 $5 \mathrm{~m}$ from bus 1 with AILI $=0.1 \mathrm{mH}$.

Figure 10.11: Sub-transient short circuit current $i_{b 1}$ with AILI $=0.1 \mathrm{mH}$ during faults F5 $5 \mathrm{~m}$ from bus 2, F6 middle of the feeder, F7 middle of the feeder, F8 middle of the feeder and F9 $5 \mathrm{~m}$ from bus 4 .

Figure 10.12: Derivative of short circuit current $i_{b 1}$ with AILI $=0.1 \mathrm{mH}$ during faults F5 $5 \mathrm{~m}$ from bus 2, F6 middle of the feeder, F7 middle of the feeder, F8 middle of the feeder and F9 $5 \mathrm{~m}$ from bus 4 .

Figure 10.13: Sub-transient short circuit current $i_{b 1}$ with AILI $=0.1 \mathrm{mH}$ during faults F5 $5 \mathrm{~m}$ from bus 2 and F6 middle of the feeder. Case 1: length of the cable 12 was $40 \mathrm{~m}$. Case 2: length of the cable 1-2 was $150 \mathrm{~m}$.

Figure 10.14: Derivative of short circuit current $i_{b 1}$ with AILI $=0.1 \mathrm{mH}$ during faults F5 $5 \mathrm{~m}$ from bus 2 and F6 middle of the feeder. Case 1: length of the cable 12 was $40 \mathrm{~m}$. Case 2: length of the cable 1-2 was $150 \mathrm{~m}$.

Figure 10.15: Performance of the event-based protection method during fault F5........ 280

Figure 10.16: Performance of the event-based protection scheme during fault F1........ 281 


\section{LIST OF ACRONYMS}

\begin{tabular}{|c|c|}
\hline CHS & Pumped Hydro Storage \\
\hline CAES & Compressed Air Energy Storage \\
\hline PHES & Pumped Hydroelectric Energy Storage \\
\hline Li-ion & Lithium-Ion \\
\hline $\mathrm{Ni}-\mathrm{Cd}$ & Nickel-Cadmium \\
\hline $\mathrm{NaS}$ & Sodium Sulfur \\
\hline $\mathrm{NaS}$ & Sodium Sulphur \\
\hline VR & Vanadium Redox \\
\hline PSB & Polysulphide Bromide \\
\hline $\mathrm{ZnBr}$ & Zinc Bromine \\
\hline HES & Hydrogen Energy Storage Systems \\
\hline ICE & Internal Combustion Engine \\
\hline FC & Fuel Cell \\
\hline SMES & Superconductive Magnetic Energy Storage \\
\hline NbTi & Niobium-Titanium \\
\hline VRT & Voltage Ride Through \\
\hline UPS & Uninterruptible Power Supply \\
\hline HFAC & High-Frequency AC \\
\hline $\mathrm{EV}$ & Electric Vehicle \\
\hline $\mathrm{HEV}$ & Hybrid Electric Vehicle \\
\hline PHEV & Plug-In Hybrid Electric Vehicle \\
\hline ESR & Internal Series Resistance \\
\hline
\end{tabular}




\begin{tabular}{|c|c|}
\hline AVR & Automatic Voltage Regulator \\
\hline IGBT & Isolated Gate Bipolar Transistor \\
\hline PI & Proportional-Integral \\
\hline THD & Total Harmonic Distortion \\
\hline $\mathrm{CT}$ & Current Transformer \\
\hline PT & Potential Transformer \\
\hline DAQ & Data Acquisition System \\
\hline LV & Low Voltage \\
\hline HV & High Voltage \\
\hline DG & Distributed Generator \\
\hline EMS & Energy Management System \\
\hline AEC & Adaptive Energy Calculator \\
\hline DVC & Direct Voltage Control \\
\hline CCA & Continuous Current Averaging \\
\hline CCA & Continuous Current Averaging \\
\hline ACVC & Adaptive Current-Voltage Control \\
\hline PWM & Pulse Width Modulation \\
\hline IPC & Instantaneous Power Control \\
\hline LBVC & Limit-Based Voltage Control \\
\hline CACC & Continuous Average Current Control \\
\hline BPDC & Bipolar DC \\
\hline NPCI & Neutral-Point-Clamped Inverter \\
\hline MPDC & Monopole DC \\
\hline
\end{tabular}




\begin{tabular}{|c|c|}
\hline МСB & Mechanical Circuit Breaker \\
\hline MACCB & Mechanical AC Circuit Breaker \\
\hline SSCB & Solid-State Circuit Breaker \\
\hline IGCT & Integrated Gate-Commutated Thyristor \\
\hline HHVCB & High Voltage Circuit Breaker \\
\hline SFCL & Superconductive Fault Current Limiter \\
\hline RSFCL & Resistive Superconductive Fault Current Limiter \\
\hline ISFCL & Inductive Superconductive Fault Current Limiter \\
\hline $\mathrm{SiC}$ & Silicon-Carbide \\
\hline $\mathrm{GaN}$ & Gallium-Nitride \\
\hline MVDC & Medium Voltage DC \\
\hline LVDC & Low Voltage DC \\
\hline HVDC & High voltage DC \\
\hline FFT & Fast Fourier Transform \\
\hline ANN & Artificial Neural Network \\
\hline CWT & Continuous Wavelet Transform \\
\hline DWT & Discrete Wavelet Transform \\
\hline $\mathrm{HPF}$ & High Pass Filter \\
\hline LPF & Low Pass Filters \\
\hline MRA & Multiresolution Analysis \\
\hline AILI & Artificial Inductive Line Impedance \\
\hline $\mathrm{ADC}$ & Analog to Digital Converters \\
\hline
\end{tabular}




\section{INTRODUCTION}

\subsection{Introduction to Energy Storage Systems}

In order to deal with the projected energy requirements, and address the environmental concerns through the integration of more renewable energy sources, the current electric power grid is required to undergo essential modernization. Advances to the electric grid must also increase the efficiency of non-renewable energy sources while improving the performance of the electricity delivery system. In order to help meet these challenges, energy storage systems can play a significant role by enhancing the operating capabilities of the power system, ensuring its reliability, and lowering the cost while reducing future infrastructure investments. Moreover, energy storage systems, as backup power, can be essential for system stabilization services and emergency preparedness [1][2].

Pursuit of a renewable energy future is inspiring significantly increased efforts for global energy storage installations. As of December 2014, the United State has about 21.4GW of operational grid storage, in which more than 200-MW is electro-chemical storage [3]. Energy storage systems are an instrumental tool for improving the power grid reliability by regulating variable generations and improving smart grid and microgrid functionality. In smart grid and microgrid power systems, storage devices provide redundancy in a system with limited transmission capacity, and for a system with variable and changing load profiles.

Different energy storage technologies have been developed for various smart grid and microgrid applications. These technologies include, but are not limited to, pumped 
hydro storage (CHS), compressed air energy storage (CAES), electrochemical capacitors, different types of batteries, and flywheels. However, various challenges are related to the widespread deployment of these technologies: manufacturing and grid integration cost, reliability and safety of the system, and industrial performance acceptance. Depending on the application and the performance required, an energy storage system or hybrid combination of these technologies should be employed. In this chapter, the latest technology developments of energy storage devices are presented. This chapter also discusses the detailed application of these storage technologies in a power grid and in transportation system, in addition to critical and pulse loads.

\subsection{Energy Storage Technology Overview}

Various energy storage technologies have been developed for different grid services. The storage system capabilities in meeting different grid requirements also makes the storage system proper for multiple services and gives the storage system a greater degree of operational flexibility than the traditional grid energy resources, such as a diesel generator or combustion turbines. Moreover, the multi-functionality of the storage system makes these technologies more flexible in terms of design and investment. However, depending on the performance characteristics of an energy storage technology, it can be ideal for certain power system services and less suitable for other applications.

Figure 1.1 groups energy storage systems based on their technologies. Some of these technologies, such as pumped hydro and CEAS, are widely employed and mature in technology, while some others, such as hydrogen and thermal energy storage, are in their early stages of development. Moreover, these technologies are different in term of cost and 
performance. As a result, not every technology is suitable for every kind of application. In this section, the main energy storage technologies developed for various power system applications are described and their advantages and drawbacks are presented.

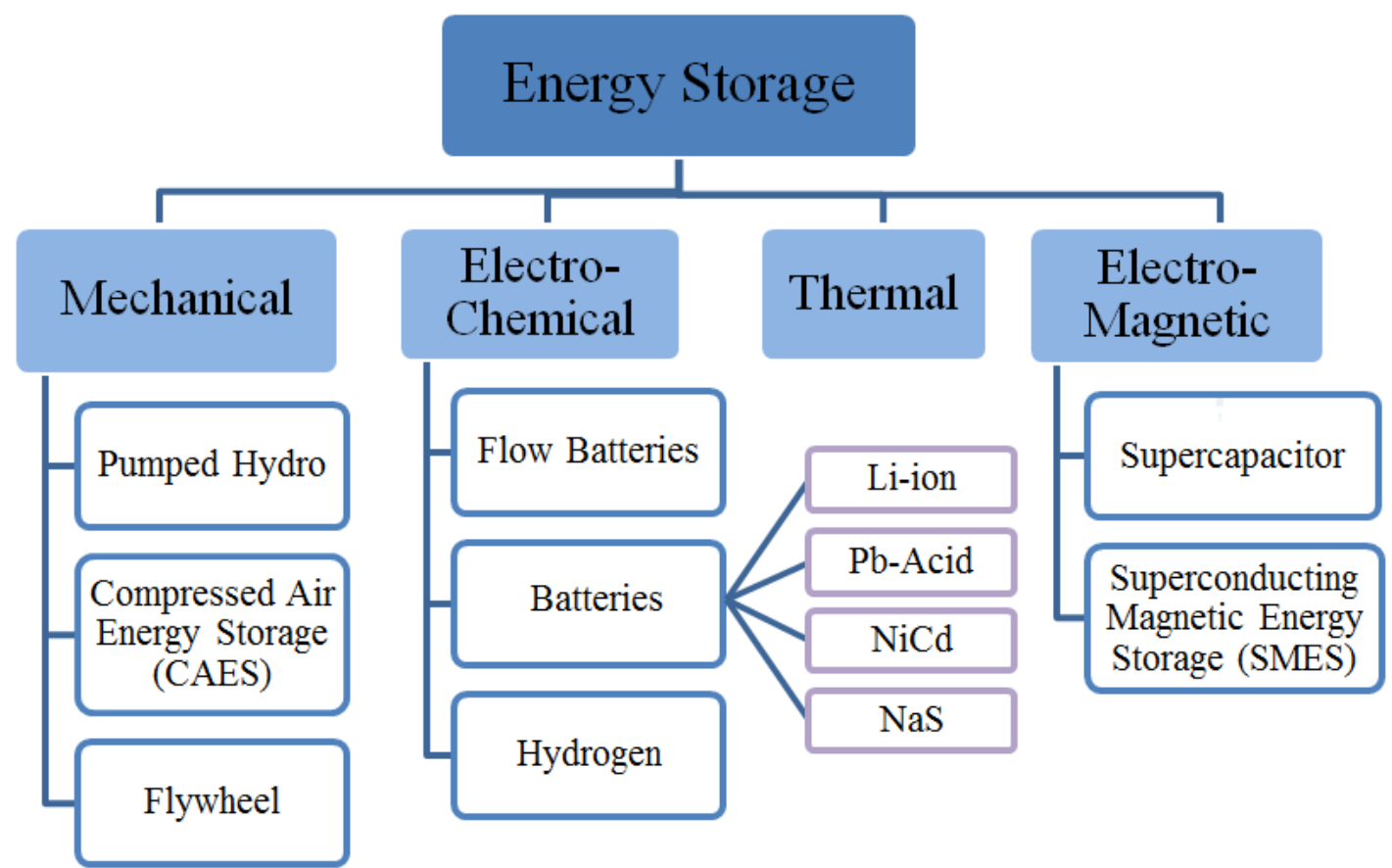

Figure 1.1: Classification of energy storage technologies.

\subsubsection{Pumped Hydroelectric Energy Storage}

Pumped hydroelectric energy storage (PHES) is the most mature and widespread large-scale energy storage system among the available technologies and is currently used at many locations in the United States and around the world. At present, the United States has about 20.4-GW operational PHES capacity, which is $95 \%$ of the total grid scale available storage systems [3]. A pumped hydro system is typically comprised of two water reservoirs at different heights which are connected by a waterway system including a number of pump and turbine units. During off-peak electricity demand, PHES pump water from a reservoir up to another reservoir at a higher elevation. When the demand is high, 
water from the upper reservoir is released through a hydroelectric turbine into the lower reservoir to support the power grid.

The typical efficiency of the PHES is about $76 \%-85 \%$, while the system can be practically sized up to 4-GW [4]-[5]. Recently, the variable speed pumping technology commenced new capabilities to the PHES to assist in the integration of variable generation sources. The PHES system can respond in a range of a few minutes up to hours. Therefore, it is suitable for power quality improvement of the grid and in applications such as energy management, load leveling and frequency regulation [6]-[8]. As a result, the PHES system can enhance the grid to allow successful integration of large amounts of renewable resources.

However, the PHES has very specific geographic requirements, making its installation site-dependent. Generally, a reservoir one kilometer in diameter, 25 meters deep and 200 meters head is required to store enough water for a 10-GWh PHES system [9]. Although the cost per kWh of the PHES system is relatively low in comparison to the other storage technologies, the high initial installation cost of the PHES plants makes these systems less economically attractive for low-power applications [6]-[8].

\subsubsection{Compressed Air Energy Storage}

Compressed air energy storage (CAES) systems have a similar concept as pumped hydro while they store the energy in the form of pressurized air. However, they are not as largely utilized as PHES systems. The CAES systems store compressed air in underground caverns in geological formations such as naturally occurring aquifers and constructed rock caverns [5]-[9]. 
The energy requirement of the CAES systems is absorbed during off-peak hours from renewable energy sources or the grid and can be discharged for various services. Large-scale CAES systems are capable of discharge times in tens of hours and with high module sizes that can reach 1-GW. These capabilities are highly effective in increasing the penetration level of renewable energy resources while enhancing the grid stability during intermittent operations. Also, the CAES systems have high flexibility that enables the power plants to be a reliable power supply and react within a few minutes. As a result, the CAES are very suitable for use in providing ancillary services such as reactive power control for voltage support and capacity reserve to maintain and improve the grid reliability[6]-[8].

The current total capacity of all CAES plants in the United States, including the recently announced projects, is 420-MW [9]. Although the CAES technology is highly effective in providing grid service that can assist in achieving the full potential of renewables, there are main disadvantages related to its high installation cost and its dependence on geographical location [6]-[8].

\subsubsection{Flywheels}

The flywheel energy storage system is a mechanical energy storage technology that absorbs energy from the grid and converts it into a spinning disc. The disc is connected to a central shaft and rotates on two magnetic bearings that are placed in a vacuum to reduce windage losses occurring due to air resistance against moving parts. The flywheel can be sped up to take in electricity from the grid or slowed down to rapidly utilize the energy. The faster a flywheel rotates, the more energy it can store. The amount of kinetic energy, E, stored in a spinning object is a function of its mass and its velocity as (1.1). 


$$
E=\frac{1}{2} I \omega^{2}
$$

where, $\mathrm{I}$ is the moment of inertia and $\omega$ is the rotational velocity.

In a case of a flywheel system with a solid disc, the stored kinetic energy can be expressed by:

$$
E=\frac{1}{4} M r^{2} \omega^{2} \approx \frac{1}{4} M v^{2}
$$

where $M$ is the mass of the rotating disc, $v$ is the linear velocity of the outer rim of the cylinder and $r$ is its radius. Equation (1.2) shows that increasing the rim speed is more effective than increasing the mass of the disc for increasing the energy capacity of a flywheel system. However, a flywheel system is limited by the strength of the disc material to withstand the stresses caused by rotation [10]- [13].

Although the power density of the flywheel is very high, its energy density is relatively low. Thus, in grid applications, they are only suitable for frequency regulation and short-time power quality services. Moreover, the flywheel energy storage can be utilized in a hybrid configuration with another electric energy storage system such as batteries or fuel cells. Examples include aircraft powertrains and shipboard power systems where the systems require very high power for short periods of time in order of a few seconds and even milliseconds [10]-[15].

\subsubsection{Electrochemical Batteries}

In contrast to the pumped hydro and CAES technologies that require specific geographical features at their installation site, various electrochemical batteries can be utilized without this limitation. However, the energy capacity of the battery technology 
storages are lower and their discharge times are shorter, ranging from a few seconds to ten hours [16]-[18].

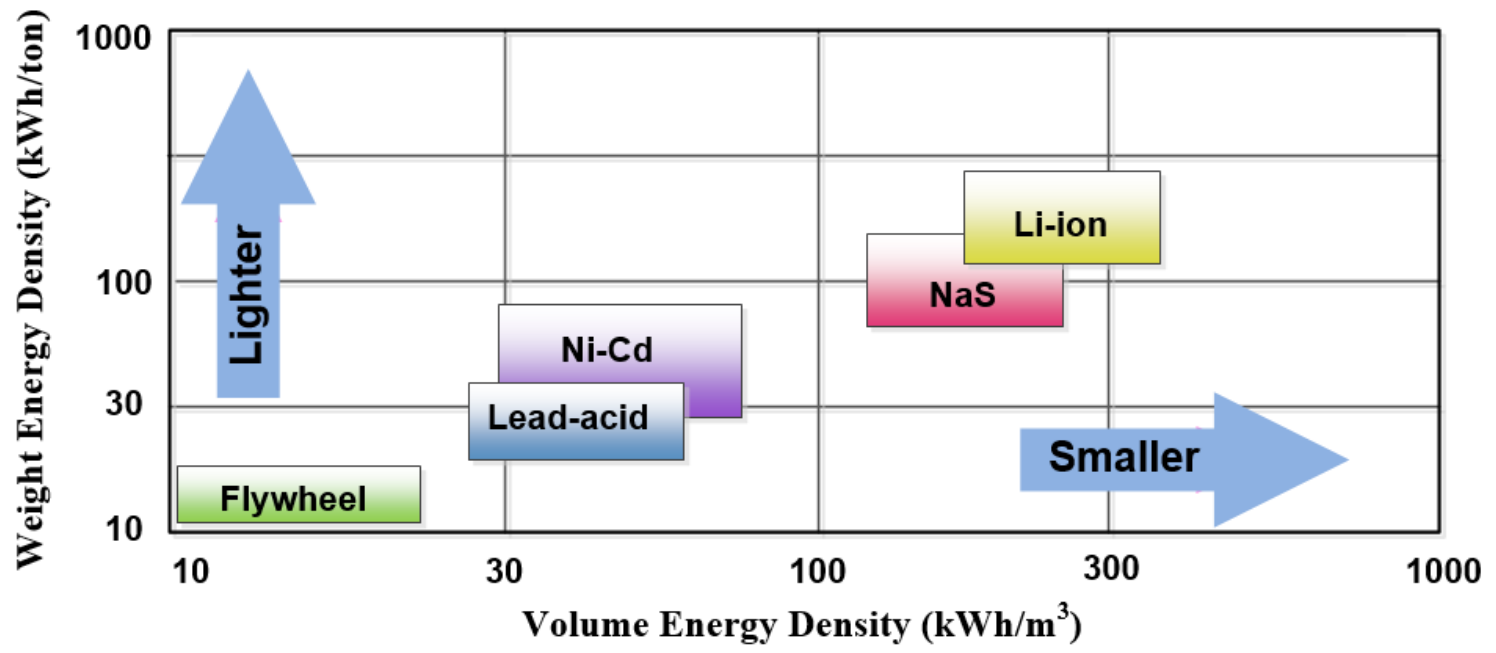

Figure 1.2: Comparison of the different battery technologies in terms of volumetric and gravimetric energy density [16]-[18].

There are several battery technologies that are commercially available and have been successfully utilized in both distributed and centralized power applications. However, there are several challenges for their widespread deployment, such as lifetime, safety, system cost, charging capabilities, and power management. The most mature and modern battery technologies that are currently available include lithium-ion (Li-ion), nickelcadmium (Ni-Cd), sodium sulfur ( $\mathrm{NaS})$, and lead acid batteries.

Volumetric and gravimetric energy density comparison of different battery technologies and flywheel energy storage is shown in Figure 1.2 . As can be seen, the battery technologies have much higher volumetric and gravimetric energy density which results in a lighter and more compact system. Among the different battery technologies, the lithium-ion storage system has the highest energy and is very suitable for applications where system weight and volume are concerns. Figure 1.3 also shows the comparison of 
the different battery technologies and the flywheel in terms of cost per unit power output and cost per energy. Lead acid battery storage, as a mature technology with good battery life, is relatively cheap and is suitable for power quality and energy management applications. Also, lithium-ion, as a modern battery technology, is more expensive. It can be seen that the flywheel energy storage for high power application is feasible while it is not economic for high-energy applications. In this section, the most mature and modern battery technologies, namely Li-ion, Ni-Cd, NaS, and lead acid batteries, are explained here with their technical advantages and limitations.

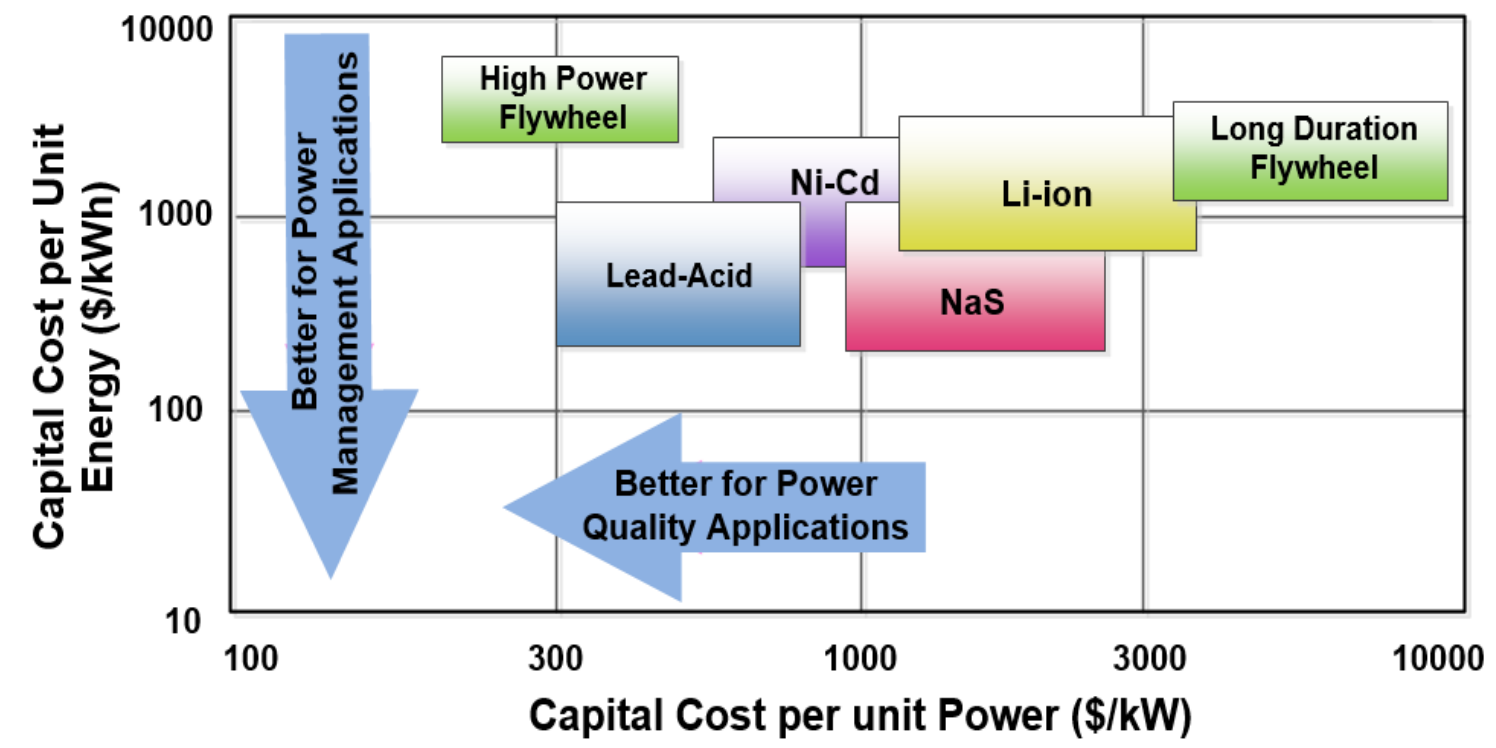

Figure 1.3: Comparison of different battery technologies in terms of capital cost per unit power and capital cost per energy [16]-[18].

\subsubsection{Lithium-ion batteries}

Li-ion energy storage has been utilized in a wide range of applications, ranging from a few kilowatt hours in residential applications, such as rooftop photovoltaic arrays, to multi-megawatts for grid ancillary services, such as frequency regulation and spinning reserve. The anode in Li-ion batteries is made of graphitic carbon while different lithiated metal oxides, such as LiCoO2 and LiMO2, can be used as a cathode. When the battery is 
charged, the cathode is turned into lithium ions and moved through the lithium salts electrolyte towards the anode where they combine with external electrons.

Li-ion batteries have a very high energy density, fast response and long cyclic lifetime. Also, the efficiency of Li-ion batteries is very high, reaching above 95\%. These advantages led Li-ion batteries to be widely used in portable electronic devices and considered as promising devices in electric vehicles and hybrid vehicles. Currently, Li-ion batteries took over $50 \%$ of the small portable application market. However, the main challenges for large-scale Li-ion battery utilization are the high cost due to the requirements of special packaging and internal overcharge protection circuits. These needs are essential to ensure the chemical and mechanical long-term reliability of lithium ion battery technologies. Moreover, the limited sources for lithium are an important issue for the viability of Li-ion batteries [19]-[23].

\subsubsection{Lead-Acid Battery}

Lead-acid batteries are the most developed and mature battery storage technology. Lead-acid batteries consist of lead plates, each with an anode and cathode which are immersed in a mixture of sulphuric acid and water as an electrolyte. During the charging process, the electrodes sit in electrolyte and store the energy. When the battery is discharged, the electrodes turn into lead sulphate while the sulphuric acid dissolves and becomes mainly water.

The energy and power capacities of lead-acid batteries depend on the size and geometry of the electrodes. The power capacity of lead-acid batteries depends on the surface area for each electrode. On the contrary, in order to improve the energy capacity of 
the battery, the mass of each electrode should be increased. Thus, depending on the application, a compromise must be made.

The success of lead-acid batteries is due to their relatively low cost, fast response, and low self-discharge rate. Also, the average efficiency of a storage system is around $85 \%$. These attributes made the lead-acid batteries widely utilized for both short-term and longterm applications, ranging from seconds up to 8 hours. For instance, in grid applications, they are deployed for grid services such as frequency regulation and energy management. However, compared with other modern battery technologies, the lead-acid batteries suffer from low power density. Thus, hybrid utilization of the batteries with high power density storage, such as a supercapacitor, for high-power applications is necessary. Moreover, the limitation of the lead-acid batteries in fast-charging processes and high sensitivity in temperature changes are the other drawbacks of this storage system [24]-[25].

\subsubsection{Nickel Cadmium Batteries}

NiCd batteries are a type of electro-chemical battery using nickel oxide hydroxide and metallic cadmium as electrodes. During discharge, the nickel oxide hydroxide mixes with water and produces a hydroxide ion and nickel hydroxide. Also, cadmium hydroxide is generated at the negative electrode. During the charging process, the reaction is reversed, while oxygen and hydrogen are produced at the positive electrode and negative electrode, respectively. As a result, some venting and water addition is required [26]-[28].

Compared to lead-acid batteries, the NiCd battery technologies have higher energy density and a wider temperature operating range. Also, the life of these batteries is relatively high and they have a charge and discharge cycle durability of up to 2500 cycles. At lower depth of discharge rates, NiCd batteries have a much longer cycle life that can 
reach 50,000 cycles. The NiCd batteries have very fast response times and can operate at full power within milliseconds. As a result, they are widely deployed in grid application for providing standby power in severe conditions and protecting against voltage sags. Moreover, high temperature durability of the NiCd batteries makes them a suitable storage system for photovoltaic generation.

One of the disadvantages of NiCd battery technologies is that the life of the batteries can be significantly reduced during rapid charge/discharge cycles when depth of discharge is considerable. Moreover their efficiency is relatively low and varies from $60 \%$ to $70 \%$. Another disadvantage is that the NiCd battery technologies suffer from memory effect and also self-discharge in standby. Consequently, they are not likely to be deployed for largescale systems and they are not a suitable candidate for load management and peak shaving applications [26]-[28].

\subsubsection{Sodium Sulphur batteries}

Sodium Sulphur (NaS) batteries consist of liquid sulfur as the positive electrode and liquid sodium as the negative electrode. The electrodes are separated by a solid beta alumina ceramic electrolyte that allows only the positive sodium ions to be combined with the sulfur and form sodium polysulfides. During discharge, positive Na ions pass through the electrolyte and the electrons flow in the external circuit. During charging, this process is reversed and the charging causes sodium polysulfides to deliver the positive sodium ions back through the electrolyte [29]-[32].

One of the main advantages of the NaS battery technologies is their high efficiency, which is approximately $89 \%$. Also, the NaS batteries are able to provide power up to five times greater than the continuous rating when they are discharged in short large pulses [31]. 
These characteristics enable the NaS battery to be economically deployed in combined power quality and peak shaving applications. The major disadvantage of NaS batteries is keeping the system at a high temperature above $500-^{\circ} \mathrm{F}$. This is required to retain the sodium and the sulphur molten and also to keep the conductivity of the electrolyte. This constraint brings several challenges, such as extra energy consumption, thermal management and safety issues [15], [29]-[32].

\subsubsection{Flow Batteries}

Flow battery energy storage was deployed specifically to provide storage in the MW-scale. The three primary types of flow battery technologies are vanadium redox (VR), polysulphide bromide (PSB) and zinc bromine ( $\mathrm{ZnBr}$ ). The operation principle of these technologies is similar; two charged electrolytes are pumped to a cell stack to form a chemical reaction. This allows the current to be obtained from the device when required.

Compared to the traditional batteries, flow batteries are able to allow more charge utilization with a higher number of discharge cycles and longer unit life. These advantages are due to the separation of the chemical components in the flow batteries. The main difficulties related to flow batteries are their low energy density and integrated design requirements, which has led to low commercial traction especially at sub-MW scale. However, recently announced flow battery energy storage projects have potential energy capacities of up to 20-MW [3].

PSB battery is a fast-response flow battery technology that can react within 20 milliseconds. Also, the average efficiency of this battery technology is about $75 \%$ [16], [18]. These attributes enable the PSB battery to be used for many grid energy storage applications, including peak shaving, load leveling, and renewable resources integration. 
One of the disadvantages of this technology is the maintenance required to remove sodiumsulphate produced during the chemical reactions [16], [18], [33].

The VR batteries have the lowest power density compared with other flow battery technologies and require more cells to achieve the same power output. However, the net efficiency of this battery is relatively high and reaches $85 \%$. Also, this technology has more design flexibility in large power applications, specifically in MW power range [16], [18].

Compared with all flow battery technologies, the $\mathrm{ZnBr}$ battery has the highest energy density. Although the electrolyte in this technology is not discharged and has indefinite life, the membrane degrades slightly during the chemical reaction. Thus, the membrane must be replaced at the end of the battery's life, which is approximately 2,000 cycles [16], [18], [34].

\subsubsection{Hydrogen Energy Storage}

Hydrogen energy storage systems (HES) are considered as one of the most promising storage techniques; however they are still technologically immature and require significant advancement to become more economically and technically attractable. Hydrogen is the most important component in this technology. The three essential stages for hydrogen energy utilization are hydrogen production, hydrogen storage and energy generation from the hydrogen.

Various methods have been studied and developed for hydrogen production. Currently, the dominant technologies for hydrogen production are methane steam reformation and coal gasification. However, hydrogen production from electrolysis through renewable electricity is considered much more clean and efficient [35]. In this 
method, the water is broken down into hydrogen and oxygen using different electrolyte solutions.

The two common methods for hydrogen storage are compressed gas storage and liquid hydrogen storage. In the compressed gas storage method, the hydrogen is compressed via cylinders to use large-capacity tanks. In the second method, the hydrogen is cooled and liquefied to increase the density of the storage system. However, the liquefaction process requires considerable energy consumption, which increases the system cost. Recent advancements have led to a new technique that utilizes metal alloys for these hydrogen storage systems [36].

There are two methods to generate power from stored hydrogen. Deploying an internal combustion engine (ICE) is the simplest method to take mechanical power from the hydrogen. On the other hand, a fuel cell (FC) directly converts the stored chemical energy into electricity. Compared to ICEs, the FCs are more efficient and reliable as well as offer higher overall power density.

A fuel cell consists of two electrodes that are separated by an electrolyte. At the anode electrode, hydrogen is supplied to be oxidized and yield electrons, which passes to an external circuit. At the cathode, the electrons are absorbed from the external circuit to reduce the oxidant. In addition, the system includes subsystems to manage water, air, thermal energy and power.

Fuel cell systems can be deployed in a wide range of applications, such as large buildings, transportation, and the distributed power grid. In large building, the FCs provide both power and heat with an efficiency of up to $80 \%$. In transportation systems, the fuel cells are utilized as a zero emission high efficiency storage system. The main disadvantage 
of this technology is the high initial costs of the system since they are still under development [16], [18], [37].

\subsubsection{Supercapacitor}

Supercapacitor technology stores the electrical energy directly between two electrodes rather than converting the charge to another form, such as mechanical energy in flywheel or chemical energy in batteries. The capacitance and energy density of the supercapacitors are thousands of times larger than conventional electrolytic capacitors.

Also known as double layer capacitors, supercapacitors store energy across the double layer formed at the interface between an electronically conducting carbon and the electrolyte. In ordered to increase the energy density of the supercapacitors, high surfacearea materials, such as activated carbon, are used, as shown in Figure 1.4. The supercapacitor design and modeling are described in [38]-[40]. In order to make the analysis traceable, the governing equations presented here take into account a non-faradic double layer capacitor and a rough aspect of the electrode/electrolyte interface. Also, it is assumed that the capacitor parameters are constant over the voltage and temperature window of operation.

In the case of uniform electrolyte concentration, the current per cross-sectional area in the electrode, denoted by $i_{d}$, and in the ideal electrolyte, denoted by $i_{y}$, can be expressed by (1.3) and (1.4) using Ohm’s law.

$$
\begin{aligned}
& i_{d}=-\sigma \frac{\partial \varphi_{d}}{\partial x} \\
& i_{y}=-\kappa \frac{\partial \varphi_{y}}{\partial x}
\end{aligned}
$$


where, $\varphi_{d}$ and $\varphi_{y}$ are the potential in the electrode and in the electrolyte, respectively. Also, $\sigma$ and $\kappa$ are the conductivities of the electrode and the electrolyte, respectively.

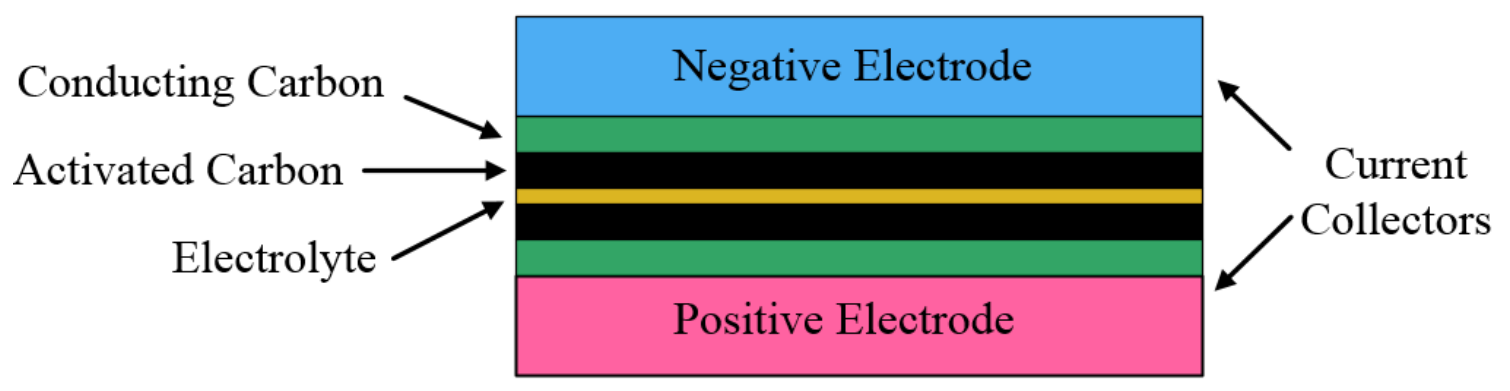

Figure 1.4: Supercapacitor structure.

Based on the charge conservation principle, the current density of the cell, $I$ and the relation between $i_{d}$ and $i_{y}$ can be expressed as:

$$
\begin{gathered}
I=i_{d}+i_{y} \\
\frac{\partial i_{d}}{\partial x}=-\frac{\partial i_{y}}{\partial x}=a i_{n}
\end{gathered}
$$

where $a$ is the interface area of the electrode and electrolyte and $i_{n}$ is the current per interfacial area.

Also, the relationship between $i_{n}, \varphi_{d}$ and $\varphi_{y}$ can be found as:

$$
i_{n}=-C \frac{\partial\left(\varphi_{d}-\varphi_{y}\right)}{\partial t}
$$

In [40], it is shown that the dimensionless imaginary impedance of a supercapacitor, $L m^{*}$ can be found as:

$$
\begin{aligned}
L m^{*} & =\frac{\left(1+\gamma^{2}\right)}{(1+\gamma)^{2} \omega^{*}}\left\{\frac{\sinh \left(\omega^{*}\right) \cosh \left(\omega^{*}\right)+\sin \left(\omega^{*}\right) \cos \left(\omega^{*}\right)}{\cosh ^{2}\left(\omega^{*}\right)-\cos ^{2}\left(\omega^{*}\right)}\right\}+ \\
& 2 \frac{\gamma}{(1+\gamma)^{2} \omega^{*}}\left\{\frac{\sinh \left(\omega^{*}\right) \cos \left(\omega^{*}\right)+\cosh \left(\omega^{*}\right) \sin \left(\omega^{*}\right)}{\cosh ^{2}\left(\omega^{*}\right)-\cos ^{2}\left(\omega^{*}\right)}\right\}
\end{aligned}
$$


where $\gamma$ is the ratio of the electrolyte and the electrode conductivity $(\kappa / \sigma)$ and $\omega^{*}$ is the dimensionless angular frequency that can be found using (1.9).

$$
\omega^{*}=\sqrt{\omega a C L^{2}(\kappa+\sigma) / 2 \kappa \sigma}
$$

Based on (1.9), it can be found that the imaginary impedance of the supercapacitor highly depends on the dimensionless angular frequency and the ratio of the electrolyte and the electrode conductivity. For a pure capacitor, the cell capacitance is equal to the inverse of the frequency times the imaginary component of the frequency response. Thus, the cell capacitance can be found as a function of $\mathrm{Lm}^{*}$ as:

$$
C=\frac{\kappa \sigma}{L m^{*} \omega(\kappa+\sigma) L}
$$

The supercapacitor model can be used to plot the effective capacitance of the supercapacitor cell under different working frequencies and different electrode thicknesses. The result is plotted in Figure 1.5. As the result shows, at low frequencies, the capacitance increases as the thickness increases. On the contrary, at high frequencies, the thinner electrodes result in a higher capacitance. Also, increasing $\gamma($ decreasing $\sigma$ ) increases the effective capacitance of the cell since the current distribution will be more uniform. 


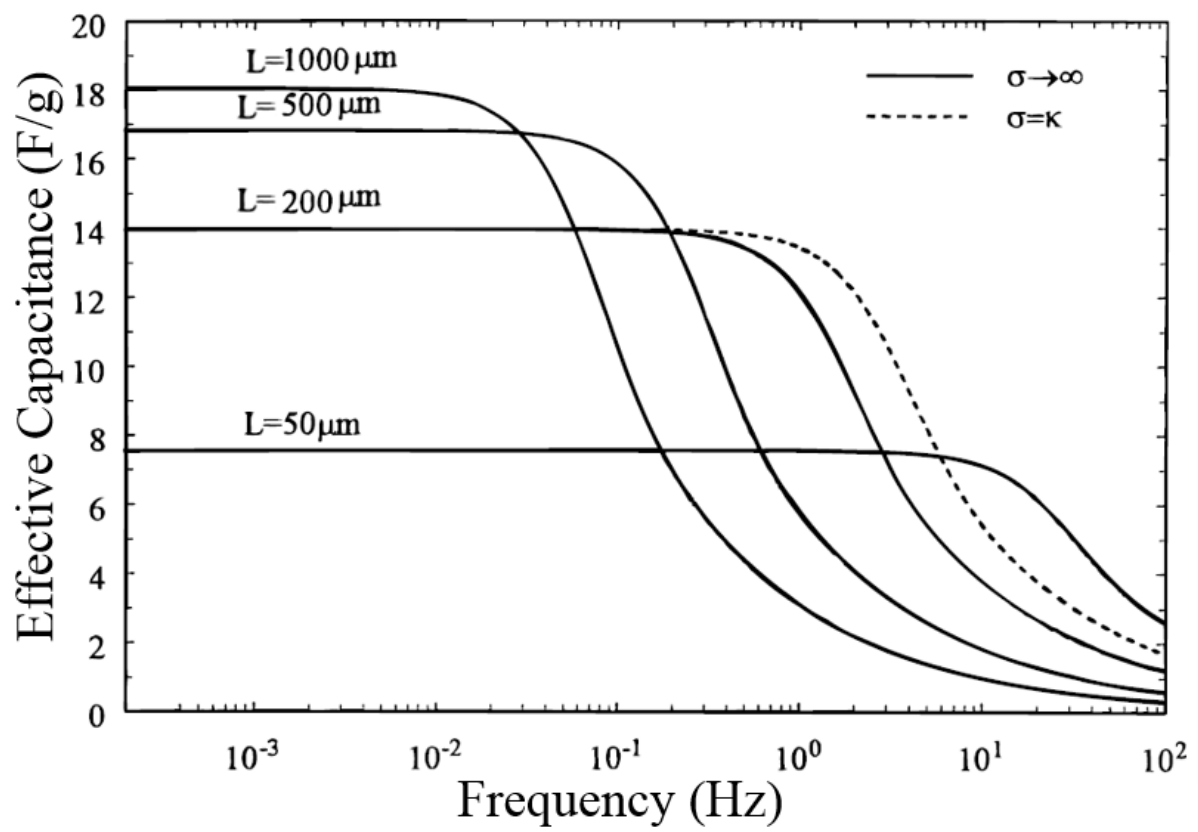

Figure 1.5: Effective capacitance of supercapacitor as a function of frequency [40].

There are two types of electrolytes utilized for supercapacitor technologies: organic and aqueous. Aqueous electrolytes are stable to $0.9-\mathrm{V}$, while the organic electrolytes are stable to 2.7-V. As a result, organic electrolyte capacitors offer a much higher energy density than the aqueous capacitors due to a higher cell voltage. However, they are more expensive.

Since the supercapacitor technology directly stores electrical energy, the charging and discharging process is very fast and efficient. Typical efficiency of supercapacitor systems is around $95 \%$ and they also have a long lifetime that can reach 200,000 cycles. On the contrary, the energy density of the supercapacitors is very low. As a result, a hybrid combination of supercapacitors with a higher energy density storage device, such as battery, offers a very useful solution for numerous applications, from large-scale grid applications to electric vehicles and portable devices [41]-[46]. This is discussed thoroughly in section 2 . 


\subsubsection{Superconductive Magnetic Energy Storage (SMES)}

One of the new storage technologies in the demonstration/applied research phase is the SMES. This system stores electric energy in the form of a magnetic field in a superconducting coil. The superconducting coil stores the energy by the flow of a direct current based on (1.11).

$$
E=\frac{1}{2} L i^{2}
$$

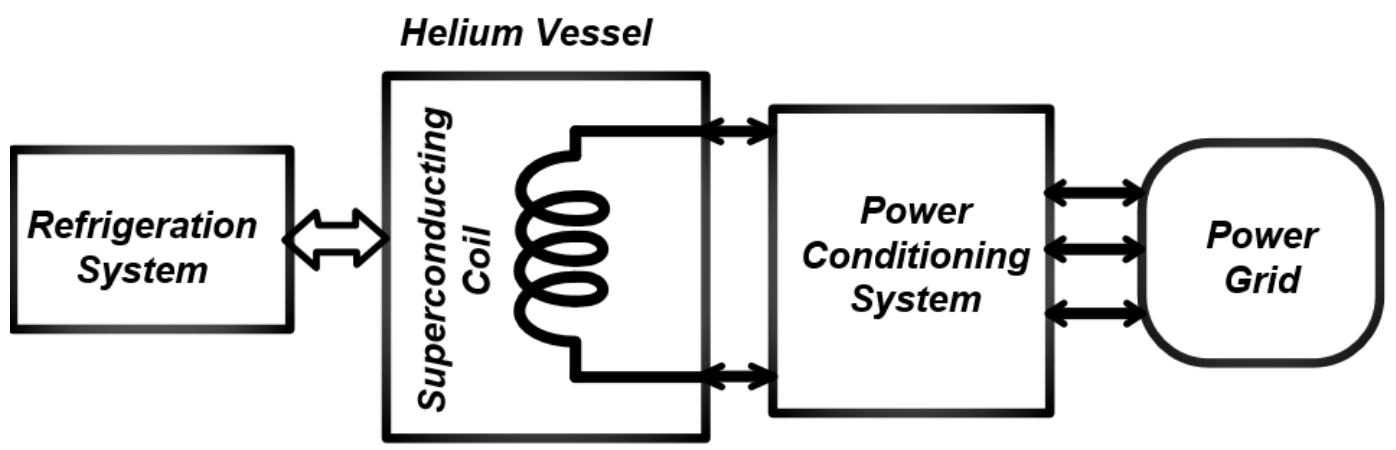

Figure 1.6: Schematic of a SMES system.

where $\mathrm{L}$ is the coil inductance which depends on the size and geometry of the coil as well as the cross-sectional area of the wire.

A typical SMES system structure is shown in Figure 1.6. As can be seen, the system includes a superconducting coil, a refrigerator and a power conditioning system. The superconducting material utilized in a SMES system enhances the storage capacity since currents pass with almost no resistance. However, in order to maintain the coil in the superconducting state, it needs to be cryogenically cooled at a very low temperature. The low-temperature superconductor, niobium-titanium (NbTi), typically is utilized in SMES system due to its ductile nature, which makes it possible to produce the fine filamentary structure. This characteristic enables the conductor to endure the alternating stress 
conditions produced in a pulsed magnet winding [47]-[49]. In the case of the NbTi conducting wire, the coolant can be liquid helium at $4.2 \mathrm{~K}$, or super fluid helium at $1.8 \mathrm{~K}$.

Recent advances in superconducting materials and cryogenic systems have made the SMES a feasible and attractive solution for many applications. Depending on the superconducting coil and the power conditioning system, the SMES can respond very quickly (within a millisecond) and for power ratings up to several megawatts. The SMES technologies also have a high power density and long cyclic life. However, the energy density of the system is relatively low and its cost is high. Currently, the SMES system is utilized in shipboard power systems, industrial power quality applications and for supplying short bursts of electricity in power grids [50]-[54].

\subsubsection{Comparison of energy storage technologies}

The energy storage technologies explained in this section are compared in Table 1.1 in terms of power, energy and cost. Also, Table 1.2 summarizes the overall performances of these storage technologies in terms of discharge time, self-discharge, efficiency, cycle life and life time.

\subsection{Energy Storage Applications}

Energy storage devises are widely utilized for various applications to improve the performance of the system. This section presents the application of these storage devices in the power grid, transportation systems, critical loads as well as pulse loads.

Table 1.1: Power and energy comparison of the storage technologies [55]-[59].

\begin{tabular}{|l|c|c|c|c|}
\hline & $\begin{array}{c}\text { Energy } \\
\text { density } \\
(\mathrm{Wh} / \mathrm{kg})\end{array}$ & $\begin{array}{c}\text { Energy } \\
\text { capital cost } \\
(\$ / \mathrm{kWh})\end{array}$ & $\begin{array}{c}\text { Power } \\
\text { capital cost } \\
(\$ / \mathrm{kW})\end{array}$ & $\begin{array}{c}\text { Power } \\
\text { rating }\end{array}$ \\
\hline PHES & $0.5-1.5$ & $5-100$ & $600-2000$ & $\begin{array}{c}100- \\
5000 \mathrm{MW}\end{array}$ \\
\hline
\end{tabular}




\begin{tabular}{|l|c|c|c|c|}
\hline CAES & $30-60$ & $2-50$ & $400-800$ & $5-300 \mathrm{MW}$ \\
\hline Flywheel & $10-30$ & $1000-5000$ & $250-350$ & $<250 \mathrm{~kW}$ \\
\hline $\mathrm{Li}-$ ion & $100-150$ & $600-2500$ & $1200-4000$ & $<100 \mathrm{~kW}$ \\
\hline Lead - acid & $30-50$ & $200-400$ & $300-600$ & $<20 \mathrm{MW}$ \\
\hline NiCd & $50-75$ & $800-1500$ & $500-1500$ & $<40 \mathrm{MW}$ \\
\hline NaS & $150-240$ & $300-500$ & $1000-3000$ & $<10 \mathrm{MW}$ \\
\hline VR & $25-45$ & $150-1000$ & 1000 & $<10 \mathrm{MW}$ \\
\hline PSB & $75-85$ & 750 & 2000 & $1-15 \mathrm{MW}$ \\
\hline ZnBr & $0.5-5$ & 500 & 2000 & $<2 \mathrm{MW}$ \\
\hline HES & $1000+$ & $1000+$ & $10-50$ & $<8 \mathrm{MW}$ \\
\hline Supercapacitor & $0.05-5$ & $300-2000$ & $100-300$ & $<200 \mathrm{~kW}$ \\
\hline SMES & $0.5-5$ & $\begin{array}{c}1000- \\
10,000\end{array}$ & $200-400$ & $\begin{array}{c}100 \mathrm{~kW}- \\
10 \mathrm{MW}\end{array}$ \\
\hline
\end{tabular}

\subsubsection{Power Grid}

The aim of this section is to explain the most significant applications of power storage technologies for a power grid with a considerable penetration of renewable energy.

\subsubsection{Load Management}

Energy storage systems can be utilized for grid load management in two different aspects: load leveling and load following.

Table 1.2: Performance comparison of the storage technologies [55]-[59].

\begin{tabular}{|l|c|c|c|c|c|}
\hline & $\begin{array}{c}\text { Discharge } \\
\text { time }\end{array}$ & $\begin{array}{c}\text { Self- } \\
\text { discharge } \\
\text { (\% per day) }\end{array}$ & $\begin{array}{c}\text { Efficiency } \\
(\%)\end{array}$ & $\begin{array}{c}\text { Cycle life } \\
\text { (cycles) }\end{array}$ & $\begin{array}{c}\text { Life } \\
\text { time } \\
\text { (years) }\end{array}$ \\
\hline PHES & $1-24 \mathrm{~h}+$ & very small & $70-85$ & $\begin{array}{c}12,000- \\
35,000\end{array}$ & $40-60$ \\
\hline
\end{tabular}




\begin{tabular}{|l|c|c|c|c|c|}
\hline CAES & $1-24 \mathrm{~h}+$ & small & $70-80$ & $\begin{array}{c}9000- \\
20,000\end{array}$ & $20-40$ \\
\hline Flywheel & $<15$ minutes & $100 \%$ & 85 & $20000+$ & $10-15$ \\
\hline Li-ion & seconds-hours & $0.1-0.3 \%$ & $90-100$ & $\begin{array}{c}1000- \\
10,000\end{array}$ & $5-15$ \\
\hline Lead-acid & minutes-hours & $0.1-0.3 \%$ & 85 & $500-1500$ & $5-15$ \\
\hline NiCd & minutes-hours & $0.2-0.6$ & $60-70$ & $2000-2500$ & $10-20$ \\
\hline NaS & minutes-hours & $20 \%$ & $75-90$ & 2500 & $10-15$ \\
\hline VR & minutes-hours & small & $70-90$ & 10,000 & $5-10$ \\
\hline PSB & minutes-hours & small & 75 & 2000 & $10-15$ \\
\hline ZnBr & minutes-hours & small & $75-80$ & 2000 & $5-10$ \\
\hline HES & seconds-24h + & very small & $35-40$ & $1000+$ & $10-15$ \\
\hline Supercapacitor & $\begin{array}{c}\text { seconds- } \\
\text { minutes }\end{array}$ & $20-40 \%$ & $90-98$ & $100,000+$ & $10-20$ \\
\hline SMES & seconds & $10-15 \%$ & $95-98$ & $100,000+$ & $20+$ \\
\hline
\end{tabular}

Load leveling is an operation scheme that stores cheap energy during off-peak hours and subsequently allows it to supply it back to the grid during times of high electricity demand. Thus, the overall power generation requirements become flatter and utilization of efficient and cheaper base load generation can be effectively maximized. Figure 1.7 shows the utilization of energy storage devices for load leveling applications. Load leveling is a long-term power application and requires storage systems with the power capabilities ranging from $1 \mathrm{MW}$ to $100+\mathrm{MW}$ to operate within the time frame of 1-10 h. As a result, PHES, CAES, HES, flow batteries and other high-capacity electrochemical battery storages, such as lead-acid and NaS, can be utilized for this application [6], [60]-[61]. 


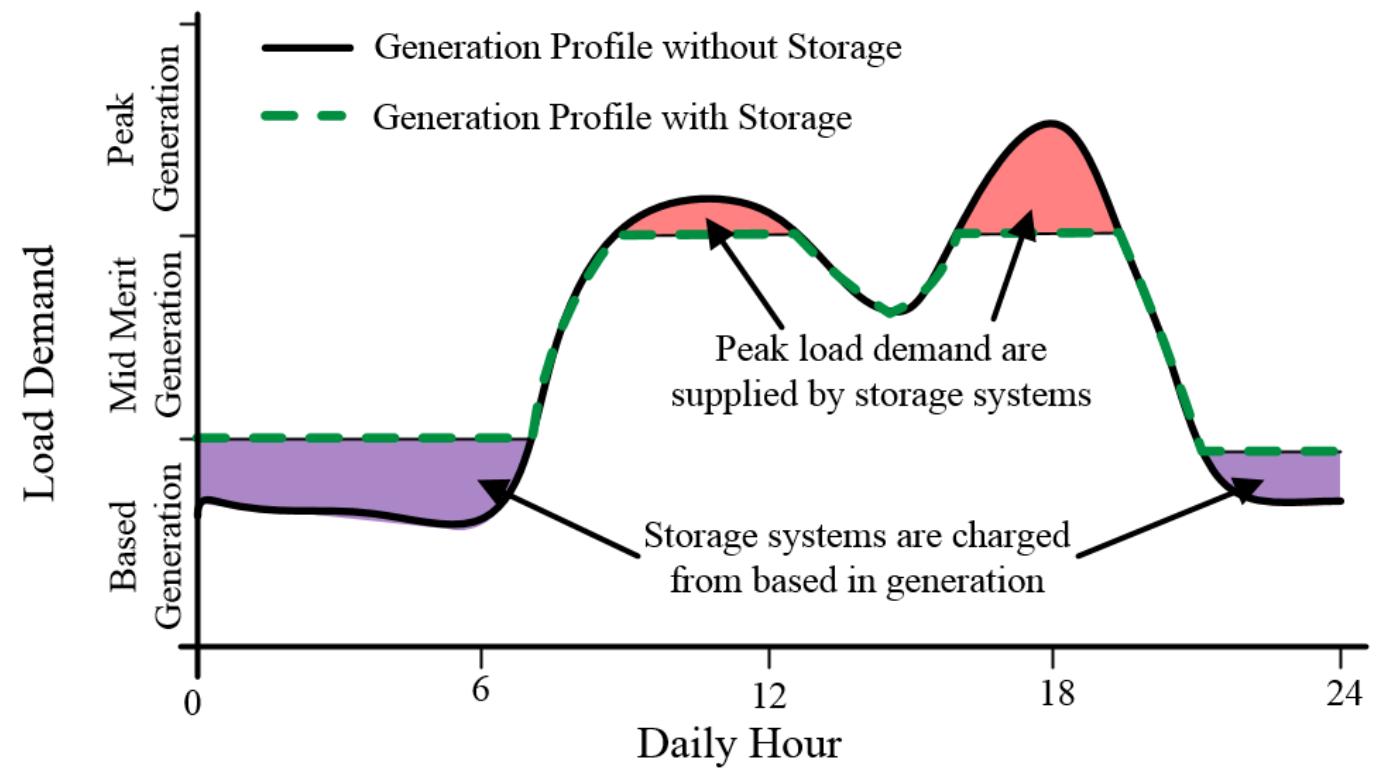

Figure 1.7: Utilization of energy storage devices for load leveling.

Also, the energy storage system can be utilized to provide load following energy service so that the generation plant output does not change with the load variation. With this service, the storages act as a source when power required is more than production levels and perform as a sink when there is power surplus. As a result, the efficiency of the power system can be improved and the greenhouse gas emissions can be considerably reduced. To provide the load following service, the storage devices should deliver electrical power in a time frame of several minutes to an hour. As a result, HES, electrochemical and flow battery storage devices are suitable for this application [56], [62].

In addition to the load leveling and the load following services, load management can be performed for financial purposes. Energy arbitrage is a bulk load management service that involves the purchase of inexpensive electricity when its cost is low, to charge the storage plant, so that the energy can be sold when the price and the demand are high. Similar to load leveling service, PHES, CAES, HES, flow batteries and high capacity electrochemical battery storages are suitable for this application [16], [18], [31], [56]. 


\subsubsection{Frequency Regulation}

Frequency regulation is one of the important grid ancillary services that balances between generation and load to maintain a $60 \mathrm{~Hz}$ frequency. High-power energy storage systems are a very suitable alternative to provide spinning reserve for frequency regulation services since their response times are very fast and they have a very high efficiency when compared to traditional storage devices, such as PHES and CAES systems. New policies on the adoption of energy storage systems made them already near commercial viability in enhancing frequency regulation techniques [9]. For instance, combined large flywheel installations and power monitoring software are very useful for this service [10], [11], [63]. Moreover, the studies on the utilization of other high-power energy storage technologies have shown that most batteries and other short-time scale storage technologies, such as the supercapacitor and SMES, are well suited for the frequency control, especially the primary control [19],[20], [64]-[66].

\subsubsection{Voltage Control}

Voltage control is the use of grid equipment to maintain voltages on the transmission system within required limits by injecting or absorbing reactive power [67][68]. Energy storage devices with the appropriate power converter systems can perform as a dynamic reactive power supply for voltage support. In this application, batteries and other high-power energy storage technologies are well suited as they can respond immediately to the voltage variations [12], [50], [69]-[72]. For this purpose, the four quadrant power converters enable the storage devices to continuously provide reactive power at grid frequency, with minimum impact on the real energy being stored [73]-[74]. 


\subsubsection{Oscillation Damping}

In stable grids without disturbances, the relative rotor angular positions of synchronous machines remain constant. However, power variations in renewable resources or loss of a major transmission line can cause considerable power oscillation in an interconnected grid system. This can result in a rotor angular instability and an undamped electromechanical oscillations of the synchronous generator. Such oscillations across a weak transmission link may separate a number of generators and can result in partial or total power interruption if not properly mitigated [75]-[77].

This kind of disturbance can be effectively mitigated by injecting or absorbing real oscillatory power at frequencies of 0.5 to $1 \mathrm{~Hz}$, opposing the disturbance. Using generation plants with fast relief valves is a typical solution to damp such an oscillation. Advances in both high-power energy storage devices and power electronic interfaces have made them into viable technologies for this application. The storage system must detect the disturbance and respond within 20 milliseconds at full power and inject or absorb oscillatory power for up to 20 cycles. As a result, SMES and other high-power storage technologies, including supercapacitors, flywheels and high power batteries, are suitable for this application [51][54], [78]-[80].

\subsubsection{Voltage Ride Through}

With the increasing penetration of renewable power plants, many countries have enforced stringent technical requirements for plants to maintain the grid stability during grid disturbances. Modern grid codes that have been recently developed require the large wind farm to not only withstand various grid faults, but also contribute to the network stability and support the grid similarly to conventional generation units. Such requirements 
are referred to as voltage ride through (VRT) capability [81]-[84]. During a major fault in the AC grid, the voltage collapses at the point of common coupling of the plant and the grid. As a result, the power that can be transferred from the DC link of the plant to the AC side will be highly limited. In this case, the trapped energy in the DC side can cause severe overvoltage in the bus capacitor and voltage stress on the converter. In order to bypass the excess power and maintain the voltage of the DC link, rapid response energy storage devices, such as flywheel and supercapacitor, are utilized. This also enables the power plant to ride through the fault uninterrupted and support the grid by injecting reactive power [14], [15], [85]-[87].

\subsubsection{Transportation Systems}

Transportation systems typically utilize high-energy storage devices with a long discharge time to maximize the overall system efficiency and to minimize the system cost, weight and volume simultaneously. The most commonly utilized battery technologies for transportation systems include lithium-ion and nickel-based batteries [21]-[23]. Other technologies being explored include the internal combustion hydrogen engine, fuel cell and other battery-type energy storage systems [23], [88], [89]. For this application, high-power storage devices, such as supercapacitors and flywheels, are of significant interest to provide short, rapid discharges during starting and acceleration periods. The high-power storage technologies are deployed as a rapid response buffer between the electric drive system and the primary storage device to improve the performance of the system in terms of cost, system life and overall efficiency [90]-[95]. Besides the transportation system, there are many industrial and railway vehicles and aircraft applications where the concept of high- 
power storage hybridization can be utilized with similar improvements and advantages being discussed [96]-[98].

\subsubsection{Critical Loads}

Many commercial, public and even residential end-users require a highly reliable power supply to reduce the effects associated with outages and poor power quality. In general, this requirement is fulfilled by uninterruptible power supplies (UPSs). The UPS system usually utilizes an energy storage device shunt connected to an intermediate DClink. For these applications, the lead acid battery has been the predominant storage system. However, most power failures and disturbances are very short (less than one second); therefore, the use of battery banks in these applications requires oversized banks and paying an extra cost for unusable energy reserve. Also, telecommunication centers and many safety and public health facilities are required to have standby gensets. For this application, short-duration energy storage can be adapted to mitigate brief power disturbances and provide a bridge to startup and synchronize standby gensets for long-term supply.

As a short-time, high-power UPS, flywheel energy storage systems can be utilized for immediate power availability to a critical load during power disturbances, such as an outage, dips or surges in voltage and/or current. Compared to batteries, the flywheel UPSs have lower operating costs, due to lower maintenance and replacement costs, more space savings, due to a high-power density of the storage, and higher yearly energy efficiency improvement. Moreover, the flywheel technology is environmentally friendly and has a broad operating temperature range up to $100^{\circ} \mathrm{F}$ [99]-[102]. 
Since supercapacitors have a high power and low energy density, it is well suited for a system that suffers from short-duration grid faults. Also, it can be employed as a bridging power supply to a more permanent backup energy source, such as a genset or fuel cell. Typical supercapacitor UPS sizes can range from 20KW to MW with modular solution systems. In addition to having a lower cost, the supercapacitor UPS offers very low maintenance and standby power costs [103]-[106].

\subsubsection{Pulse Loads}

Utilization of high-power energy storage is significantly important in telecommunications, shipboard and spacecraft power systems. In these applications, there are various types of loads with a common profile that have a high instantaneous power requirement but a relatively low average power requirement. Such a current behavior is collectively referred to as a pulsed load. The duration of such a load typically ranges from hundreds of milliseconds to seconds, with power levels that depend on the applications [45], [46], [107], [108].

In the case of a single energy source, the high instantaneous power requirements of a pulse load can cause considerable power disturbances and thermal issues. The possibility of introducing a high-power storage system, such as a supercapacitor and flywheel, with appropriate power electronic infrastructure and control strategies, will highly improve the performance of the system. In this case, the long-time primary energy source only supplies the average pulse load requirements while a high-power energy storage supplies the dynamic power requirements. As a result, the system configuration will have several advantages, including less weight and volume of the system and elimination of thermal issues, frequency fluctuation and voltage disturbances [109]-[114]. 


\subsection{Hybrid Energy Power System}

As discussed in section 1.3, most of the renewable energy resources have intermittent characteristics, and their output fluctuates on a daily and seasonal basis. Therefore, storage facilities play an important role in safeguarding against disruptions in delivery and supply. Moreover, each energy storage technology has a different energypower characteristic. For instance, PHES and hydrogen storage have a high energy capacity, but their energy density and response time is relatively low and cannot be utilized for fast load transients or power quality applications. As a result, hybrid combinations of two or more power generation technologies, along with storage devices, can significantly improve the system's performances. For example, a fuel cell combined with a microturbine system can better utilize the stored energy in the fuel to achieve a higher overall system efficiency than either source can possibly achieve [115]-[118].

In a hybrid power system, all energy resources are converted into electricity to supply different types of loads while the energy can be stored into mechanical, chemical, thermal or electromagnetic form. In order to achieve full benefit from the system, appropriate coordination and output power control of various sources in a hybrid system are essential. Also, for proper power dispatch of the energy sources, each energy source should be appropriately sized and controlled. This will allow the system to be sustainable to the maximum extent possible and minimize the energy production cost, while reducing greenhouse gas emissions.

In this section, the possible hybrid configurations of the power system for connection of two or more energy sources are discussed. Then, the energy management and control schemes for a hybrid power system are presented. 


\subsubsection{Hybrid Power System Configuration}

Since energy sources in a hybrid power system have different characteristics, it is essential to properly standardize the hybridization framework and procedure. The hybrid power system can be connected in a microgrid, where local and distributed sources and loads are connected together with autonomous operating capabilities. In order to improve the robustness of the microgrid, the plug-and-play operation capability also can be added to the system. The concept of the plug-and-play capability is to add or disconnect any grid components, namely generating power or load, into an existing system without requiring system reconfiguration [119]-[122].

There are many methods to connect energy sources to form a hybrid power system. These methods differ in their configuration scheme, and the types and the number of power converters. The system configuration can be generally classified into AC-coupled, DCcoupled, hybrid AC/DC, and high-frequency AC hybrid configurations [45], [111], [118], [123]-[128]. The general review of these configurations is presented here.

\subsubsection{AC-Coupled Hybrid Power System}

Figure 1.8 shows an AC-coupled hybrid power system, which is the most commonly used hybrid configuration. In this scheme, the AC sources and the power grid are directly connected to a common AC bus. Also, the DC sources and energy storage system are connected to the common coupling AC bus through a DC/AC converter. In the

case of a storage system, the converter should have the bidirectional power flow capability to enable the charging and discharging of the storage device. 


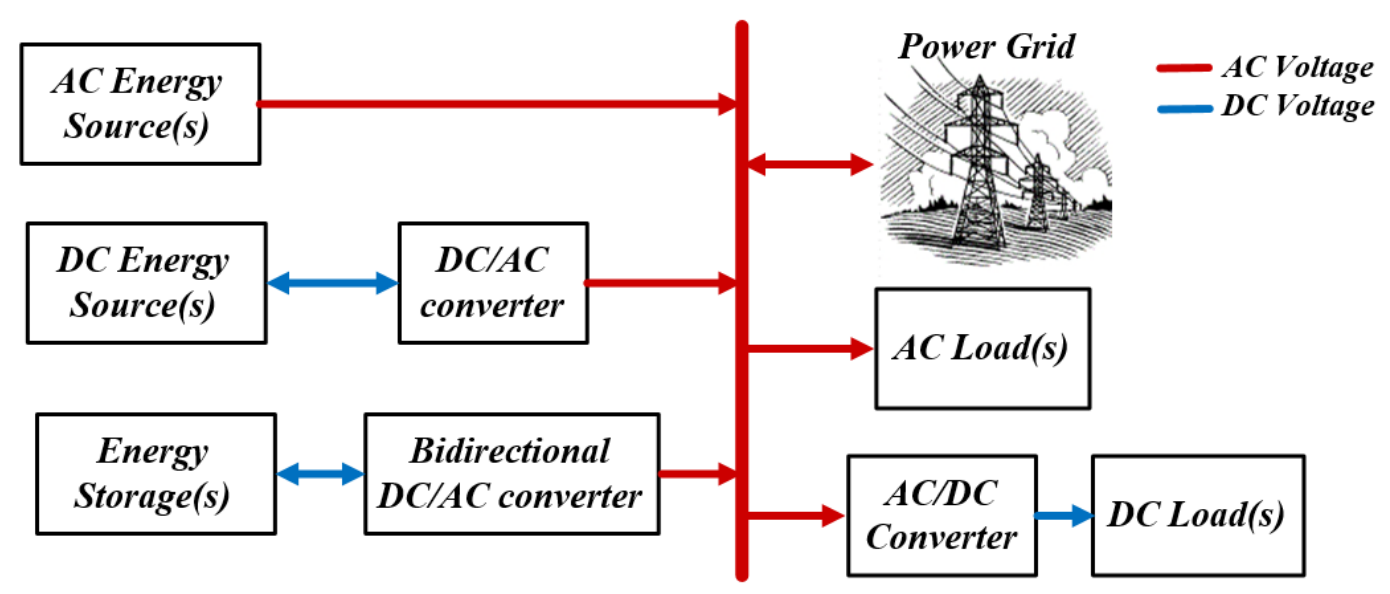

Figure 1.8: Schematic diagram of AC-coupled hybrid power system.

\subsubsection{DC-Coupled Hybrid Power System}

The schematic diagram of a DC-coupled hybrid power system is shown in Figure 1.9. In this configuration, different energy sources are integrated to a common DC bus through power electronic interfaces. In this schematic, it is assumed that the DC energy source(s) have proper power regulation capabilities. Otherwise, installation of a DC/DC converter is necessary. The DC load can be directly connected to the common coupling DC bus or it can be interfaced through a DC/DC power converter if the operating voltage of the load differs from the microgrid voltage. The AC load is also connected to the DC bus via a DC/AC converter, which is commonly regulated via a voltage control scheme. In order to enable power flow between the DC bus and the AC power grid, a bidirectional DC/AC power converter is required. This converter can be regulated based on a DQ control scheme to achieve independent active and reactive power flow control [45], [111], [125]. 


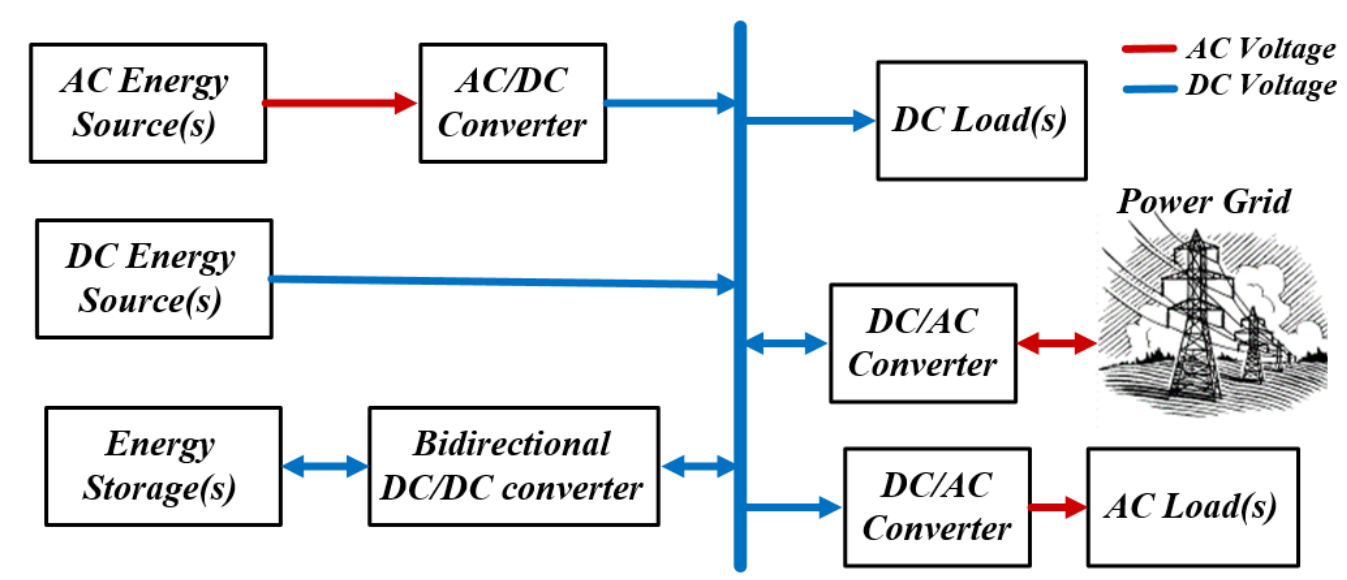

Figure 1.9: Schematic diagram of DC-coupled hybrid power system.

The DC-coupled hybrid power system has several advantages. This configuration is simple since different energy sources can be connected to the system without synchronization. Moreover, since most renewable energy sources and energy storage devices, as well as many loads are operating with DC power, their integration to the microgrid can be achieved with fewer power electronic converters. As a result, the overall efficiency of the system is improved, and the total cost of the system will be reduced. However, failure of the grid connected DC/AC converter is problematic, since the whole system will not be able to receive power from the AC grid or supply it. To solve this issue, multiple inverters can be connected in parallel with a lower power rating. This requires a power sharing scheme between the converters and power converter synchronization, which increase the cost and complexity of the system [118], [124].

\subsubsection{Hybrid AC/DC Power System}

Figure 1.10 shows a hybrid AC/DC power system. As can be seen, in this configuration, the energy resources and loads are connected to either the DC or AC bus of the hybrid system rather than a single DC or AC bus. The hybrid AC/DC configuration will enable the DC energy sources to be integrated directly to the common DC bus, without 
extra power electronic interfaces. The energy sources mainly supply the DC loads, and the extra power is transferred to the AC bus and the power grid. Similarly, the AC sources and the AC loads are connected to the AC bus with a minimum number of converters. As a result, the system's efficiency is improved and its cost is minimized. However, control and energy management of this subsystem might be more complicated than for the DC-coupled or the AC-coupled hybrid power system [125]-[126].

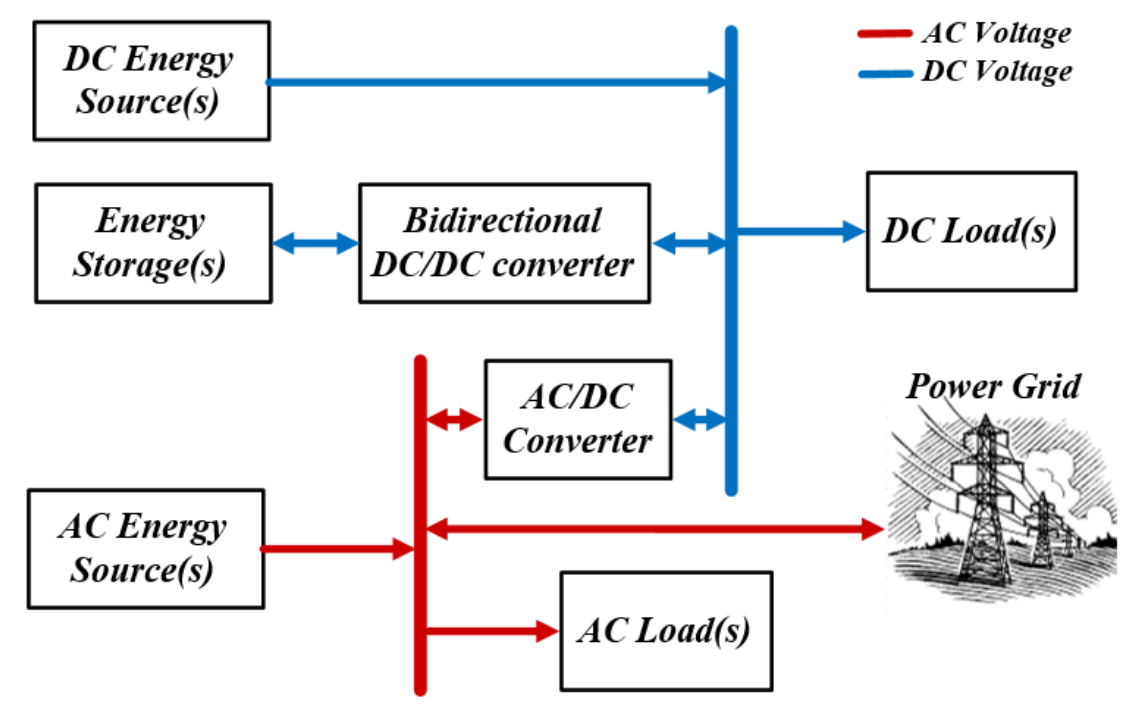

Figure 1.10: Schematic diagram of hybrid AC/DC power system.

\subsubsection{High-Frequency AC Hybrid Power System}

Figure 1.11 shows the schematic diagram of a high-frequency AC (HFAC) hybrid power system. In this configuration, both HFAC power and DC power are provided through a number of power electronic converters. The HFAC power systems deliver electrical power at frequencies greater than $60 \mathrm{~Hz}$. Research on the HFAC system has shown many potential benefits, compared with the grid frequency AC system, in terms of ease of electrical isolation using compact, high-frequency transformers and flexibility to meet loads demand at different voltage levels. Moreover, high-frequency operation can improve the dynamic response of the system, and reduce or eliminate current harmonics 
that are at higher frequency ranges. The HFAC can also result in considerable savings in the number of converter components and the system cost.

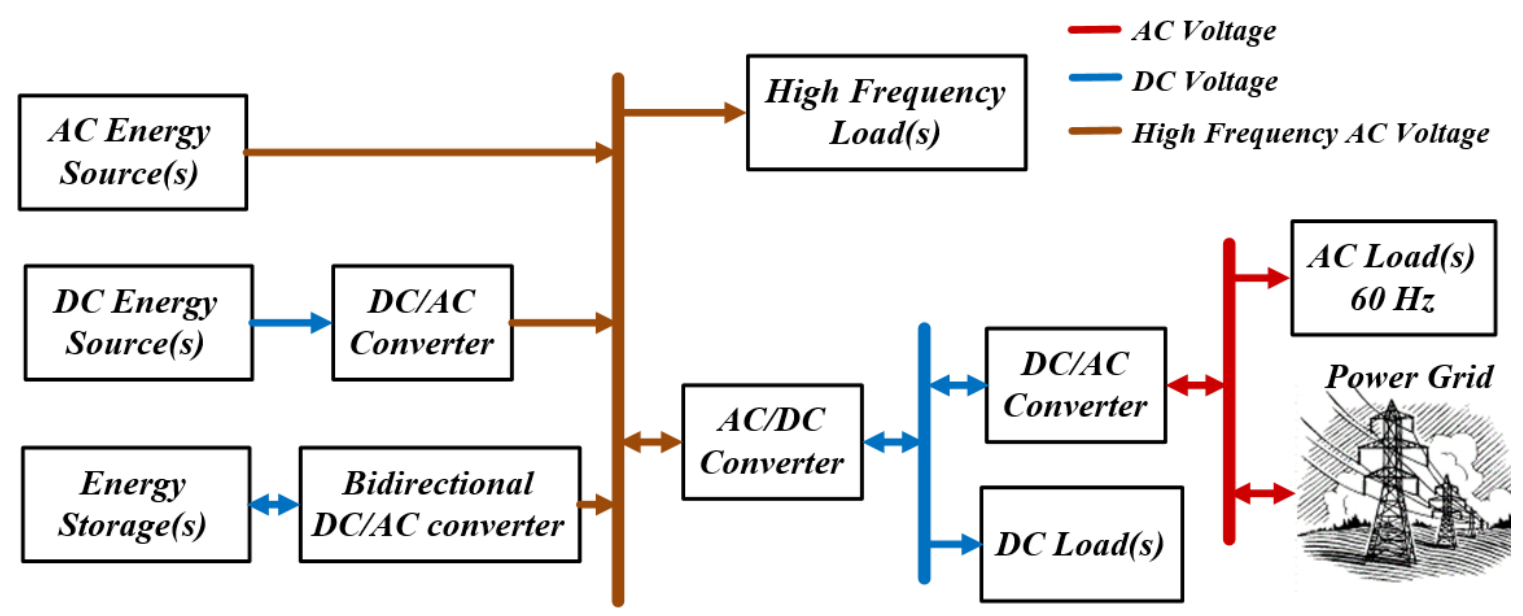

Figure 1.11: Schematic diagram of high-frequency AC hybrid power system.

Significant studies have been done for the grid-connected HFAC hybrid power system to improve the performance of this system and make this configuration more economically attractive. Currently, the standalone HFAC hybrid power system has been utilized in applications, such as ship power systems, airplanes and space station applications [127]-[130].

\subsubsection{Real-time Control of Hybrid Power System}

Real-time control and energy management of a hybrid power system are very important since the number of power components is usually high and there are multiple parameters that need to be satisfied. Generally, the real-time control system should keep the voltage level and frequency of the grid within a predetermined limit while it realizes the desired active and reactive power of each power unit. Control structure of such a system based on the coordination method of the power unit can be classified to centralized, distributed, and hybrid control schemes. In this section, these methods are explained, and their advantages and limitations are discussed. 


\subsubsection{Centralized Control Scheme}

The centralized control scheme is a hierarchical control wherein the information becomes more centralized the more the control system proceeds up the command levels. Figure 1.12 shows the general structure of a centralized control scheme. As can be seen, the measurement signals and equipment status of all power units are sent to a centralized controller to make decisions.

The centralized controller, as a supervisory controller, uses all measured signals, a set of power unit constraints, and objectives to make decisions based on a predefined energy management algorithm and power units prioritizes. The centralized controller may also need to use state estimation to estimate network power flow and voltage profiles before power dispatching. In addition to power and voltage control, the centralized controller may have other objectives, such as maximizing system efficiency, minimizing system operation and maintenance costs, as well as reducing the environmental impact. In such a system, multi-objective optimization is required, however, it may not lead to a single solution. As a result, a complete non-dominated or Pareto set solution should be found to represent potential compromise of the alternative solutions among the objectives [118], [131]-[132]. 


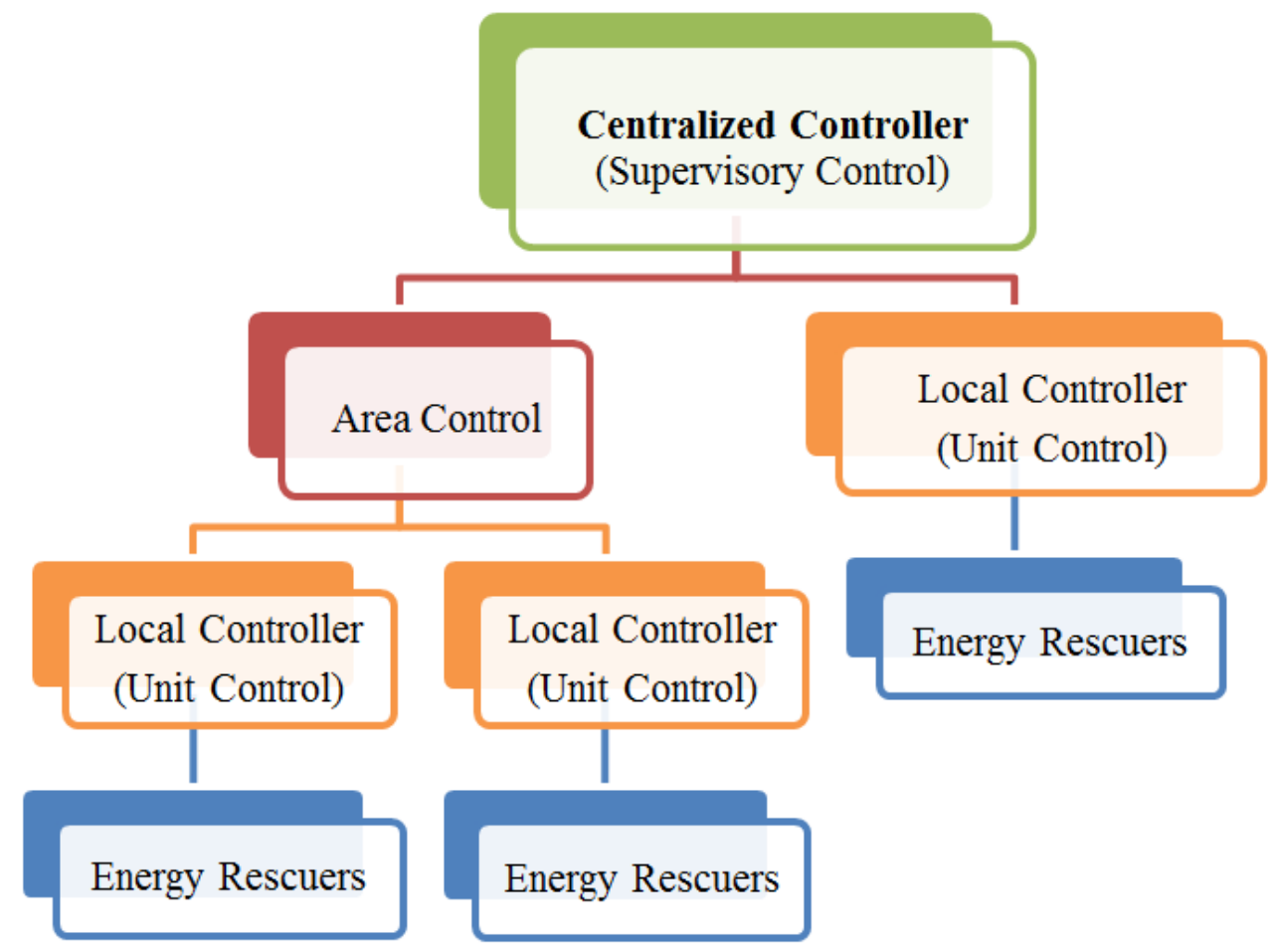

Figure 1.12: Centralized control structure.

The centralized control approach is a typical solution for many automated industrial plants. The main advantage of this control structure is that the system can more accurately reach its optimum operation since the decision is made by only the supervisory control. However, the transferred data is complex, and the computational burden of the centralized controller is very heavy. As a result, the system requires significant investment in its communication network and the controller processor, especially for large hybrid power systems. Moreover, in a case that the centralized controller is out-of-service, the whole system will fail.

\subsubsection{Distributed Control}

Recent advances in communication technology and distributed generation have created opportunities for distributed control schemes and the development of intelligent agents. This control scheme is a flat control architecture that distributes the data within the 
units rather than sending all data to just a single supervisory controller. Figure 1.13 shows the schematic diagram of a fully distributed control system. As can be seen, the measurement signals of each energy source is sent to their corresponding local controller, while each control unit communicates with one another to make compromised operating decisions [132]-[134].

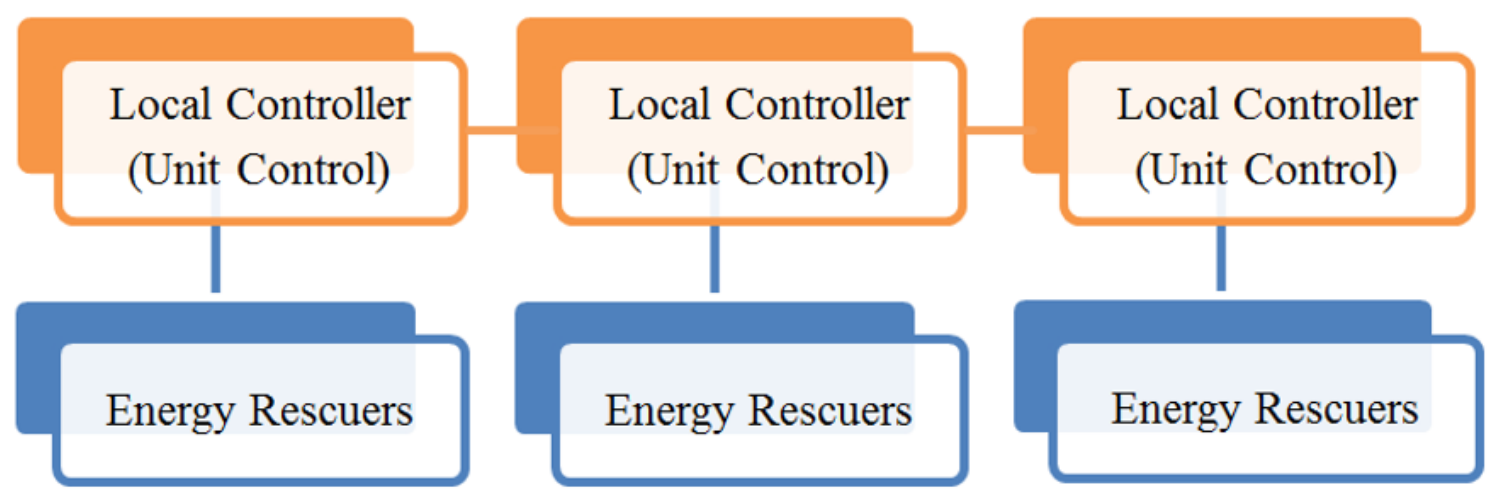

Figure 1.13: Distributed control structure.

In a distributed control structure, the computational burden across the system's controller is significantly reduced since there is no central controller. Also, this control scheme has a plug-and-play capability, which makes the system easily reconfigurable in cases when a control unit fails. Significant research has been done towards the applications of distributed control in power system. The main issue for utilization of this controller is the intelligent operation of the local controller; the controller needs to make a decision based on the exchanged information with their neighbors to reach a stable condition very fast, while the operation point is optimized. This requires an intelligent operation, and accurate design of local controllers and the data networks [118], [131]-[132]. 


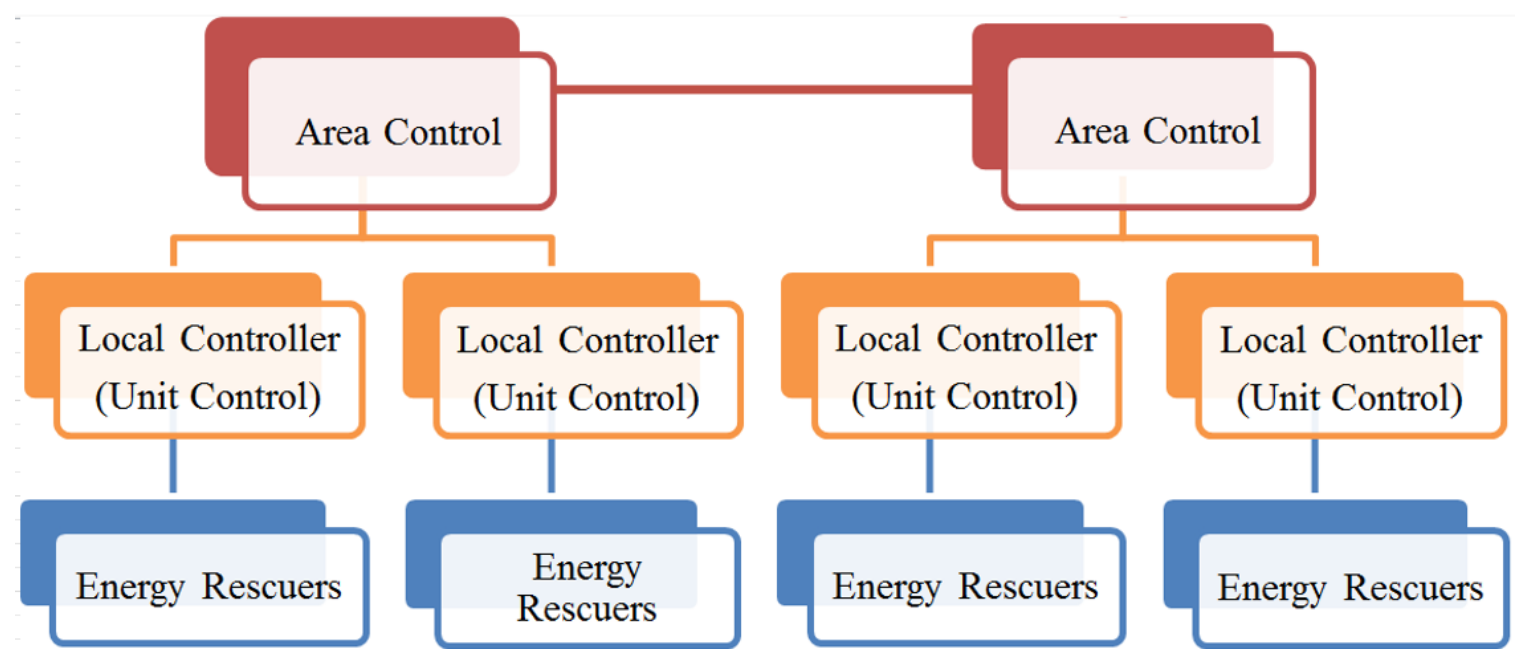

Figure 1.14: Distributed-centralized hybrid control structure.

\subsubsection{Distributed-Centralized Hybrid Control}

The distributed-centralized hybrid control scheme is a more practical structure, which utilizes both the hierarchical and flat control architecture. Figure 1.14 shows an example of this control structure. In this approach, a centralized controller is applied for the control of the distributed energy resources within a microgrid while distributed control is utilized to a set of centralized controllers. This method reduces the computational burden on the centralized controller and minimizes the risk of problems that may occur due to failure of the centralized controller. With the distributed-centralized hybrid structure, the centralized controller is utilized to optimize the operation of the energy resources, while global coordination among the different distributed generation resources is achieved through distributed control [118], [131]-[132].

\subsection{Problem Statement}

As discussed in previous sections, energy storage systems are of significant importance in advanced power systems for the purpose of meeting peak demands and for the improvement of power reliability and efficiency. Battery energy storages have been 
utilized for many years for different applications. However, the battery-based energy storage systems come with several challenges. Batteries are not able to handle the dynamic peak power demands efficiently and may result in a reduced life span. A typical solution to this problem is to increase the size of the battery bank. However, this could cause an increase in total cost, thermal issues and imbalances across battery cells. Compared to batteries, ultracapacitors have a lower energy density, but their power density is very high. Also, the transient response of ultracapacitors is very fast, their charging cycles are over $100 \mathrm{~K}$ cycles, and they offer very high efficiency even during very fast charge and discharge situations. This dissertation proposes utilization of a battery-ultracapacitor hybrid storage system, as a storage device with high power and energy capability, to improve the overall performance of the system.

Hybrid energy storage can provide viable solutions for increased efficiency, improved power quality and reliability issues in AC/DC power systems, including power grids with considerable penetration of renewable energy. It is also very practical for shipboard power systems, aircraft powertrains, electric vehicles, and hybrid electric vehicles. However, there are many issues with the hybrid energy storage implementation. A power system with hybrid energy storage should be designed and controlled properly to maintain the balance between the state of charge of the battery storage and ultracapacitor, while minimizing the system's power losses. Moreover, depending on the application and the required power of the system, the storage devices should be sized so that the total cost and the energy consumption of the system are reduced. Currently, issues related to the hybrid DC power system, such as system configuration, energy management and real-time 
control, as well as the system efficiency for different loading schemes, are still an open problem.

The importance of the DC power system is not only because of the fact that most of the renewable energy sources, such as solar energy and fuel cells, have a DC output, but also because implementations of energy storage systems are easier and more efficient in DC power systems. Moreover, the recent advancement of power electronic converters has made power regulation in DC architectures more efficient and effective. This dissertation is aimed at developing and verifying new techniques for hybrid DC power system design and its real-time control to provide a power system with self-diagnosing, self-healing, and self-reconfiguring capabilities.

A power system with self-diagnosing and self-healing capabilities is able to prevent unplanned outages and abnormalities through better prediction, analysis and control. Such a system should have an accurate fault detection capability to prevent spreading of the disturbances to other healthy parts of the power system. The self-reconfiguring capability of the system also requires a proper design and coordination of the switching devices to enable fast transition from fault condition to a new safe operation.

One of the important aspects of dynamic and transient study in power system is the modeling of components, such as power resources, energy storage devices, power converters, cables and loads. Since testing various techniques in an actual power system is cost prohibitive, utilization of a virtual test-bed with a high level of precision is very useful. The virtual test-bed model can be used for various studies, such as power system design, control and energy management, system optimization and protection scheme evaluation. 


\subsection{Research Objective}

The hybrid energy storage system is an effective solution to provide a reliable power with high efficiency in various power systems, such as those in transportation, shipboard and spacecraft, as well as distribution systems involving sustainable energy sources. For these applications, the supercapacitor as a high-power buffer device, in combination with conventional electrochemical batteries, can play a significant role in improvements of the power system efficiency, power quality and reliability. This dissertation involves the development and experimental validation of various techniques for hybrid energy storage utilization and its real-time control and energy management to enhance the performance of the system.

The main research objectives and contributions of this dissertation can be summarized as follows:

- To accurately design, model and implement a hybrid DC power system incorporating different energy storage technologies and various loading scheme at the Energy Systems Research Laboratory, Florida International University. The hybrid DC power system is a hardware/software-based setup with power generation and storage capability in a laboratory scale of up to $10-\mathrm{kW}$. This platform is actively connected to a previously implemented 72-kW smart grid testbed [135]-[136]. The developed system includes implementations of control strategies with monitoring capability for all parameters of the interconnected components. Also, implementation of a real-time data analysis system, as a powerful tool, enables the investigation of many challenging aspects of a hybrid power system, which includes system efficiency, voltage profile, current 
harmonics, power fluctuation and frequency variation, as well as system reliability.

- To develop and experimentally evaluate an advanced energy control technique to reduce the adverse effects of pulsed power loads on the power system. The developed method should be able to properly coordinate power sharing between different energy sources and the available energy storage devices based on their inherent characteristics. Characteristics that should be considered are powerenergy capabilities, maximum current limitation, operating voltage threshold, power loss, thermal limitation and cyclic life time, as well as energy cost of the energy devices. Also, the adverse effects of the pulse load that need to be fully mitigated are voltage drop of the DC bus, power pulsation of the energy devices, frequency fluctuation of the interconnected AC bus and instability of the hybrid power system.

- To develop and experimentally evaluate an intelligent energy management system to ensure continuous operation of the hybrid power system under different grid and loading conditions. The energy management algorithm should set the current or the voltage reference of each energy device based on the availability of the energy devices and the required power of the grid. The energy management system should properly add self-reconfiguration capabilities to the system so that disconnection or failure of a grid component does not cause disconnection of the other healthy parts of the power system. Moreover, the energy management system, in coordination with the energy control scheme, should be able to 
effectively supply a dynamic load with a large startup current while the energy capacity and the cost of the system are minimized.

- To design and implement different AC and DC filters in the system to improve the power quality of the AC and DC power system so that the current harmonics of the AC system are minimized and the performance of the DC power system, in terms of control capability and voltage ripple, is improved. The power quality improvement of the system not only needs well-designed AC and DC filters, but also requires appropriate implementation of the galvanic transformers and utilization of proper power converter switching patterns.

- To accurately model different components of the hybrid power system and implement a virtual test-bed model of this system for dynamic and transient study. The developed virtual test-bed should accurately model the implemented energy storage devices, i.e. batteries and supercapacitor, for very fast charge and discharge processes. Also, power electronic converters, involved in the actual hybrid power system, should be accurately implemented in the virtual test bed system. The power converters include boost and bi-directional converters needed for DC power regulation, and the controlled and uncontrolled rectifiers needed to link the DC microgrid to the main AC grid. The virtual test-bed should also accurately model the switching devices, such as circuit breakers and disconnecting switches, while their switching time must be set based on their technology and speed of the contactors. The developed model needs to be evaluated using the experimental test results under both normal operation and transient fault conditions. The virtual test-bed system can be effectively utilized for hybrid power 
system optimization purposes, such as system performance enhancement, optimization of the controller parameters and the size and cost minimization of the storage devices.

- To develop a protection scheme for a hybrid DC power system that is fast, accurate and reliable. The developed protection scheme should be able to identify the type of fault using a fast fault current signal processing technique so that the maximum fault current is effectively limited and the transition time to a new safe operation is minimized. For this purpose, the protection relays need to be accurately set and well-coordinated to prevent malfunction. Proper design of the hybrid DC power system, including the implementation of the disconnecting switches and circuit breakers, is also very important to add self- reconfiguration and self-healing capabilities to the grid. Another design aspect of the protection system is accurate setting of the protection relay that requires a detailed fault current analysis based on analytical calculation. These analyses can be used as a powerful tool for protection relays setting calculation, and to identify the maximum and minimum of a fault current when it occurs in different zones of the hybrid power system.

\subsection{Original Contributions of the Dissertation}

In order to verify the contributions of this dissertation, a laboratory-scale hardware/software-based hybrid DC power system is developed. This research platform has a power generation and storage capability that is able to supply the loads with power up to $10-\mathrm{kW}$ in a steady state condition and $18-\mathrm{kW}$ in transient conditions. The implemented hybrid power system utilizes a 5.8-F supercapacitor, which adds a very fast 
response and short time storage capability to the system. All the power electronic converters, energy storage devices, programmable loads and main energy resources are completely reconfigurable for different research scenarios. The developed platform has a real-time control and monitoring system to fully analyze the power grid and its components for various studies, such as efficiency, voltage profile, current harmonics, power fluctuation and frequency variation, as well as system reliability. Also, this power system is actively connected to the previously developed $72-\mathrm{kW}$ smart grid test-bed to realize the interaction of the hybrid DC power system and the AC grid in various grid configurations and loading conditions.

In the past decade, researchers were reconsidering hybrid DC microgrids and DC distribution power systems due to their multiple advantages. In this dissertation, different aspects involved in the design, control and operation of a hybrid power system that incorporates a supercapacitor and pulse loads are investigated. An analog voltage measurement system with a common mode rejection capability is developed to monitor the supercapacitor bank with a high level of accuracy and noise rejection capability. Also, the design requirements of the hybrid DC power system used to prevent DC current circulation to the interconnected AC grid are fully examined and experimentally evaluated. This study also included the challenges of the filter design and switching patterns of the converters to reduce both the even and the odd-order harmonics.

In this dissertation, two protection systems for safe operation of the supercapacitor bank are developed; digital voltage protection and analog hysteresis voltage protection. The digital protection is embedded to the converter controller and monitors the entire supercapacitor bank. Also, the analog hysteresis protection is designed to monitor the 
supercapacitor arrays. If the voltage of any of the supercapacitor arrays exceeds the preset limit, the analog control circuit will open the charging path of the supercapacitor bank. This will ensure that uneven charge distribution, especially during very fast charges, does not cause the supercapacitor to exceed its maximum voltage. Both protection systems are experimentally evaluated and their high accuracy is verified.

Extensive research has been done on an actively controlled hybrid DC system in which the energy delivered to the supercapacitor and the pulse load is controlled via power electronic converters. In most of these studies, the supercapacitor and the pulse load are connected to a grid through a single DC/AC or DC/DC converter. The advantage of these configurations is the ease of converter control and energy management. However, the system redundancy of these configurations is low, i.e. a fault in the bus connecting converter will cause the outage of the supercapacitor and the pulse load. In this dissertation, a new configuration for a direct connection of supercapacitor and pulse load to a DC bus has been proposed. The developed configuration not only improves the redundancy of the system, but also reduces the system cost and the power losses. Moreover, in addition to supplying the pulse load, the proposed configuration enables the supercapacitor to support the grid during the transient startup of the dynamic loads with large startup current.

In the case of heavy pulse loads or loads with a large startup current, energy management of the hybrid power systems is a fundamental concern. Such a high, shorttime current behavior not only requires a higher rating of the power components, but also can potentially cause the system voltage and frequency to drop in the entire microgrid since there is no infinite bus in the system. To reduce the adverse effects of the pulse load, a novel, real-time energy management system is developed in this dissertation. The 
developed energy management system utilizes an adaptive energy calculator based on the moving average measurement technique. The performance of this development is experimentally compared with other approaches suggested in the literature. The results proved that the developed energy management system with adaptive energy calculation capability properly shares the power, and controls the bus voltage while the adverse effects of the pulse load, such as power pulsation, frequency fluctuation and AC voltage drop, are highly reduced.

Grid connected DC microgrid power systems with hybrid energy resources are of significant interest in many applications. Multiple sources and reconfiguration characteristics of the hybrid DC microgrid highly improve the efficiency and reliability of the system. However, existence of the pulsed loads not only causes thermal and stability issues on the DC microgrid, but also leads to additional disruptive impacts in the interconnected AC grid. In this dissertation, a novel, three-layer control scheme is developed to actively control a hybrid DC microgrid that is connected to a main AC grid. The developed control structure utilizes an adaptive current-voltage energy control system. Unlike conventional energy control methods, the developed adaptive current-voltage energy control approach has the advantage of controlling both the voltage and the current of the system while keeping the output current of the power converter at a relatively constant value. Using experimental test results, it is shown that the developed strategy highly improves the dynamic performance of the hybrid DC microgrid. Although the adaptive current-voltage energy control technique causes slightly more bus voltage variation, it effectively eliminates the high current and power pulsation of the power 
converters. The experimental test results for different pulse duty ratios are analyzed in terms of system efficiency, AC grid voltage drop and the frequency fluctuations.

A reliable hybrid DC power system with a self-diagnosing and self-reconfiguring capability is in high demand for many applications. However, protection of such a system against short circuit faults, especially in multi-source distribution systems and multiterminal DC lines, is problematic. In this dissertation, a new protection scheme for a multiterminal DC power system is developed. This protection scheme is an event-based protection strategy, which transfers less data when compared with commonly used databased protection methods, and does not require high speed communication and synchronization. Each protection relay is able to autonomously identify the type of event using the current derivative fault identification method and employing novel, artificial inductive line impedance techniques. In order to accurately set the protection relays, detailed fault current analysis considering low pass RC filter effects are investigated in detail. The performance of the developed protection scheme is evaluated under different DC feeder and bus faults. The results show that the developed protection scheme is fast and accurate, and the grid can ride-through the fault uninterrupted. The detailed analytical analysis given in this dissertation provided essential guidelines to set the protection relays for an event-based protection scheme. This can be utilized in multi-terminal DC microgrids and for various applications, such as telecommunication systems, shipboards and spacecraft, and distribution systems involving a large number of electronic loads and data centers. 


\subsection{Dissertation Outline}

This dissertation is organized in ten chapters, including the current chapter, which contains a brief introduction to the dissertation and statement of the problem. Chapter 2 is focused on the battery-supercapacitor hybrid system and its application for pulse load mitigation. The effects of pulsed power loads on the AC and DC power system are discussed. Also, the power and the energy requirements of the pulse load in terms of dynamic and average power and energy are analyzed. This chapter also presents passive battery-supercapacitor hybrid configuration and different active hybrid configurations. The advantages and limitations of each configuration are discussed in terms of power and energy capability, power converter requirement, complexity of the control system and the implementation cost.

Chapter 3 presents a description of the hybrid DC power system setup developed as a verification tool for this dissertation. The components of this system that include energy storage devices, load emulators, generator stations and transformer are described. Also, hardware test setups, the configuration and the main control strategy of various implemented power converters are included in this chapter. Moreover, the hybrid power system test setup contains various measurement circuits, protection boards and a drive system. These boards are detailed and their performances are evaluated. Finally, this chapter presents the technique for the main control and monitoring strategy of the developed hybrid DC microgrid.

Chapter 4 investigates the connectivity issues related to linking two DC buses featuring different voltage characteristics. The focus of the study is to achieve effective bidirectional energy transfer between two DC buses, whose voltages contain a significantly 
different content of ripple. Also, the real-time operation and current harmonic analysis of isolated and non-isolated DC microgrids incorporating hybrid energy sources with various loading schemes are investigated. In order to properly manage the energy and prevent AC grid power pulsation, a new current-voltage control technique based on a master-slave control concept is developed. Various experimental tests based on different power sharing patterns are performed and the results are analyzed.

Chapter 5 explains the development of a hybrid power system with high redundancy that improves the performance of the system and reduces the total power losses. The concept of the actively controlled hybrid DC power system with a high redundancy is described. Then, the system configuration of the proposed microgrid and the developed energy control concept are explained. This chapter also includes the detailed scheme of the developed energy management system with an adaptive energy calculator. The source of errors and their effects on the performance of the system are illustrated. Experimental tests of the proposed microgrid, its controllers and the new energy management system under different loading schemes and grid conditions are presented and the results are compared with other methods suggested in the literature.

Chapter 6 focuses on the grid connected hybrid DC microgrid power systems that incorporate a pulsed load. This chapter includes a detailed description and performance evaluation of a new three layer control structure that utilizes an adaptive current-voltage control scheme. Using experimental test results, the performance of the developed control scheme for different pulse rates and duty ratios is evaluated. The results are compared with other control techniques in terms of normalized DC bus voltage variation, normalized AC grid voltage drop, frequency fluctuations and system efficiency. 
Section 7 presents implementation and evaluation of a virtual test-bed platform as an accurate model of the hybrid power system. Different techniques for modeling of supercapacitor and battery energy storage devices are explained. This is followed by a description of a four bus hybrid DC microgrid and its simulation model. In order to evaluate the accuracy of the developed model, the normal and transient performances of the virtual test-bed are analyzed and are compared with the experimental results.

Chapter 8 is focused on the protection of DC power systems that incorporate hybrid energy storage devices. Various grounding schemes for DC power systems including their advantages and limitations are discussed. Also, different DC power system protection devices, which are commercially available or in the research stages, are presented.

Chapter 9 reviews different protection techniques suggested in the literature for radial, distributed and multi-terminal DC power systems. Various fault identification and location techniques are presented, and the requirements and applications of these techniques are compared.

Chapter 10 presents a new event-based protection scheme for a multi-terminal hybrid DC microgrid. In order to fully illustrate and evaluate the proposed protection scheme, a three-bus medium-voltage DC system and a four-bus hybrid DC power system are considered. Also, this section presents the detailed fault current analysis using analytical calculation and virtual test-bed model. The developed current derivative event classification method and artificial inductive line impedance are described. Finally, the performance of the proposed event-based fault identification method is evaluated and verified in terms of fault isolation, load support during a fault, and system restoration. 
Chapter 11 provides general outcomes of the results and the conclusion of this dissertation. Also, some suggestions and recommendations for further work related to this topic are given. 


\section{SUPERCAPACITOR-BATTERY ENERGY STORAGE INTEGRATION}

\subsection{Introduction}

With the global environmental pollution concerns, there has been a push to utilize more electric vehicles (EVs) and hybrid electric vehicles (HEVs) in transportation systems. Many studies have been done in the control and integration of these vehicles in power systems to accommodate higher penetration of EVs and HEVs [70], [88]. A group of plugin hybrid electric vehicles (PHEVs) during their charging process draw very high current for a short duration of time, which can create a transient imbalance between power production and the power consumption of the grid. Such a current behavior is collectively referred to as pulsed loads and cause considerable voltage and frequency fluctuation in the power system [137].

However, pulsed load behaviors are not limited to the AC grid power network. Such a load profile is a major concern in hybrid AC/DC power systems in shipboard, spacecraft and telecommunication applications [107], [138]-[139]. Due to the absence of an infinite bus in these systems, the existence of heavy pulsed loads can potentially create several issues. They not only cause voltage drops in the common coupling DC bus, but also lead to additional disruptive impacts in the main AC grid and the slack bus generator [107], [138] -[139].

One method to reduce the disruptive impact of the pulsed loads is through load coordination. In this approach, the load coordination is achieved through load shedding so that the total system load remains constant [46]. For this approach there are two necessary requirements. First, there should be enough based load connected on the system and, 
second, those loads should be noncritical so that their disconnections do not cause problems. Clearly, since these requirements are not always met, the load coordination approach is not an effective solution for many applications.

One effective method to reduce the adverse impacts of the pulsed loads is the utilization of supplementary energy storage devices. Although the energy densities of the battery energy storage are high and their terminal voltage variation are limited, their power density is low and they have significant difficulty providing very high power over a short time period. In order to solve this problem and increase the power capacity of a battery energy storage system, multiple cells need to be put in parallel and series. However, this will increase the system cost considerably. Moreover, unequal current sharing between battery cells are problematic and cause thermal issues within the system [140]-[142].

Recently, supercapacitor technology has been introduced that has the potential to improve the performance of a power system with heavy pulse loads. Also known as double layer capacitors, supercapacitors have a high power density, low internal resistance and high cycle life [46], [108]-[113]. Compact in size, supercapacitors can store an incomparably higher amount of energy than conventional capacitors and they can release that energy at either a high or a low rate with capacitance ranges up to 2700 Farads. Also, they can deliver up to 10-20 times more power than batteries. However, the relative energy densities of the supercapacitors are low and, as a result, a hybrid combination of the supercapacitor with other energy resources is necessary.

Hybrid battery-supercapacitor storage system can be classified in two categories; passive hybrid and active hybrid systems. The passive hybrid configuration is the simplest configuration in which the supercapacitor is directly connected to the battery energy 
storage with no power management circuitry [108]-[109], [142]. The active hybrid configuration utilizes power converter/converters to more effectively regulate the power sharing between the battery, supercapacitor and the pulse load [109]-[111].

This chapter discusses the power and the energy requirements of the pulsed load in terms of average and dynamic energy and, also average and dynamic power. The most simple hybrid configuration of the battery-supercapacitor, which is the passive configuration, is explained. Moreover, different possible battery-supercapacitor active hybrid topologies are presented and their advantages and limitations are discussed. Finally, the steady state operations of the passive hybrid and the active battery-supercapacitor hybrid systems, which are the most effective configurations for pulsed load applications, are analytically analyzed.

\subsection{Power and Energy Requirements of the Pulse Loads}

In order to understand the requirements of a pulse load, consider a consumption profile plotted in Figure 2.1, which defines the load power versus time $P_{L}(t)$ (solid line). The load profile used here is simplified for the sake of making the analysis tractable. The load is considered as a constant power changing between $P_{\text {Min }}$ and $P_{\text {Max }}$ with a period $T$ (the frequency $f=1 / T$ ) and duty cycle $D$. Based on Figure 2.1, the instantaneous load power, $P_{L}(t)$ for the first $N$ pulses can be expressed as:

$$
P_{L}(t)=P_{\text {Min }}+\sum_{k=0}^{N-1}\left(P_{\text {Max }}-P_{\text {Min }}\right)[\phi(t-k T)-\phi(t-(k+D) T)]
$$

where $\phi$ is a unit step function at $t=0$. 
In order to describe the dynamic behavior of the pulse load, the instantaneous load power can be decoupled into an average power profile, $P_{L a v}$ and a dynamic power profile, $P_{L d y}$. Figure 2.1 also shows $P_{L a v}$ as a dotted line and $P_{L d y}$ as a dashed line. The $P_{L a v}$ and $P_{L d y}$ can be found as (2.2) and (2.3), respectively.

$$
\begin{gathered}
P_{\text {Lav }}=\frac{1}{T} \int_{0}^{T} P_{L}(t) d t=D\left(P_{\text {Max }}-P_{\text {Min }}\right)+P_{\text {Min }} \\
P_{\text {Ldy }}=P_{L}(t)-P_{\text {Lav }}=-D\left(P_{\text {Max }}-P_{\text {Min }}\right) \\
+\sum_{k=0}^{N-1}\left(P_{\text {Max }}-P_{\text {Min }}\right)[\phi(t-k T)-\phi(t-(k+D) T)]
\end{gathered}
$$

Note that the average of the $P_{L d y}$ over a period $T$ is zero. In case of a single source system, the source must supply the instantaneous power requirements, i.e. both the average and the dynamic power components. While, in a hybrid energy system, the dynamic power and the average power can be shared between different energy devices.

The energy system for the pulsed power load not only should be able to satisfy the load power requirements, but also it needs to provide enough energy to operate in a long period of time. The energy demand of the pulse load can be given by:

$$
\begin{gathered}
E_{L}=E_{L a v}=\int_{0}^{N T} P_{L}(t) d t=\left[D\left(P_{\text {Max }}-P_{\text {Min }}\right)+P_{\text {Min }}\right] N T \\
E_{L d y}=\int_{0}^{N T} P_{L d y}(t) d t=0
\end{gathered}
$$

where, the $E_{L}, E_{L a v}$ and $E_{L d y}$ are the total energy, the average energy and the dynamic energy demands of the load, respectively. Equation (2.5) implies that the dynamic energy demand of a pulse load is zero. However, the dynamic energy demands can be decomposed into two nonzero components as a consumed energy $E_{L d y}^{C}$ and an accumulated energy $E_{L d y}^{A}$ (shown in Figure 2.1): 


$$
\begin{aligned}
E_{L d y}^{C} & =E_{L d y}^{A}=\int_{0}^{D T} P_{L d y}(t) d t \\
& =\int_{D T}^{T} P_{L d y}(t) d t=D(1-D)\left(P_{M a x}-P_{M i n}\right)
\end{aligned}
$$

The $E_{L d y}^{C}$ is the maximum dynamic energy that is temporarily required by the pulse load, which is also equal to $E_{L d y}^{A}$.

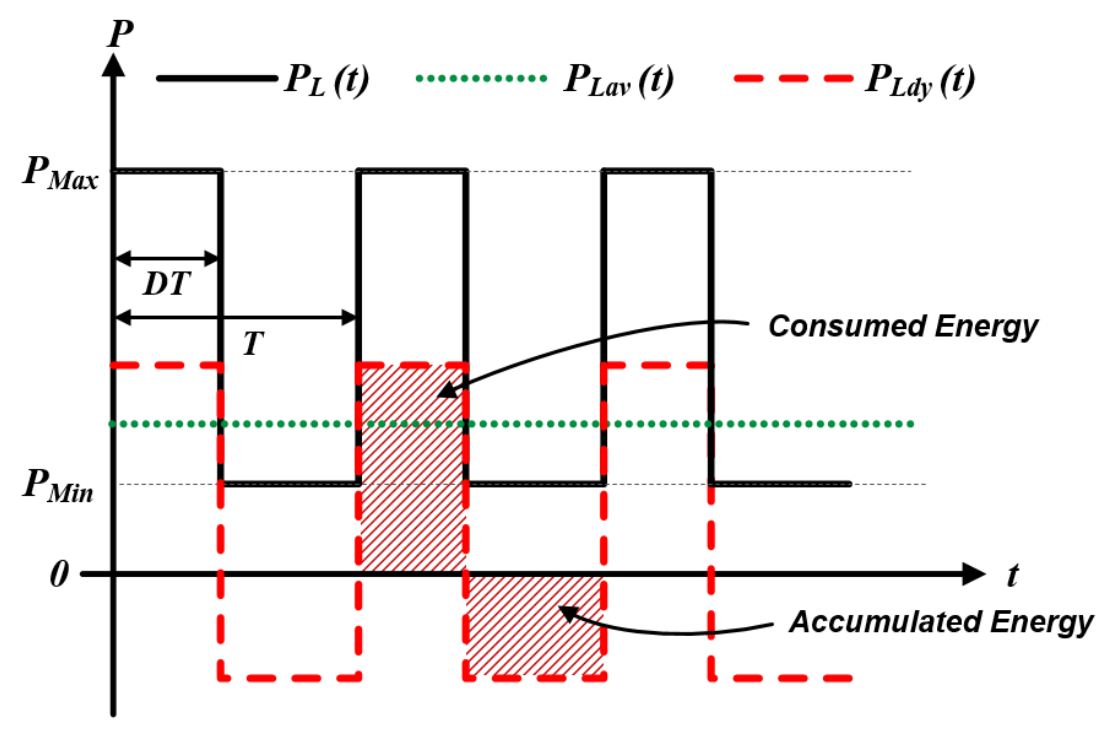

Figure 2.1: Consumption power profile of a pulse load.

\subsection{Battery-Supercapacitor Hybrid Configurations}

For the design of a hybrid energy storage system and classification of storage devices, Ragone Plot is usually utilized [141]. Figure 2.2 shows the Ragone Plot, the power density versus energy density of different energy storage systems. Lithium battery energy storages have the power density of $100-500 \mathrm{~W} / \mathrm{kg}$, which is the highest energy density of all modern batteries. On the other hand, the power density of the supercapacitors is around ten times greater than all batteries. Also, the internal series resistance (ESR) of the supercapacitors is much lower than that of the batteries. Therefore, supercapacitors have much higher charging/discharging efficiency [46], [108]-[113]. However, the power 
density of the supercapacitors is relatively low. Thus, in order to retain the benefits of high specific power and energy, a hybrid battery/supercapacitor storage system can be utilized.

Various topologies for the hybrid battery-supercapacitor system have been studied and utilized over the past few years. In this section, a review of the most commonly used hybrid battery-supercapacitor configurations is given.

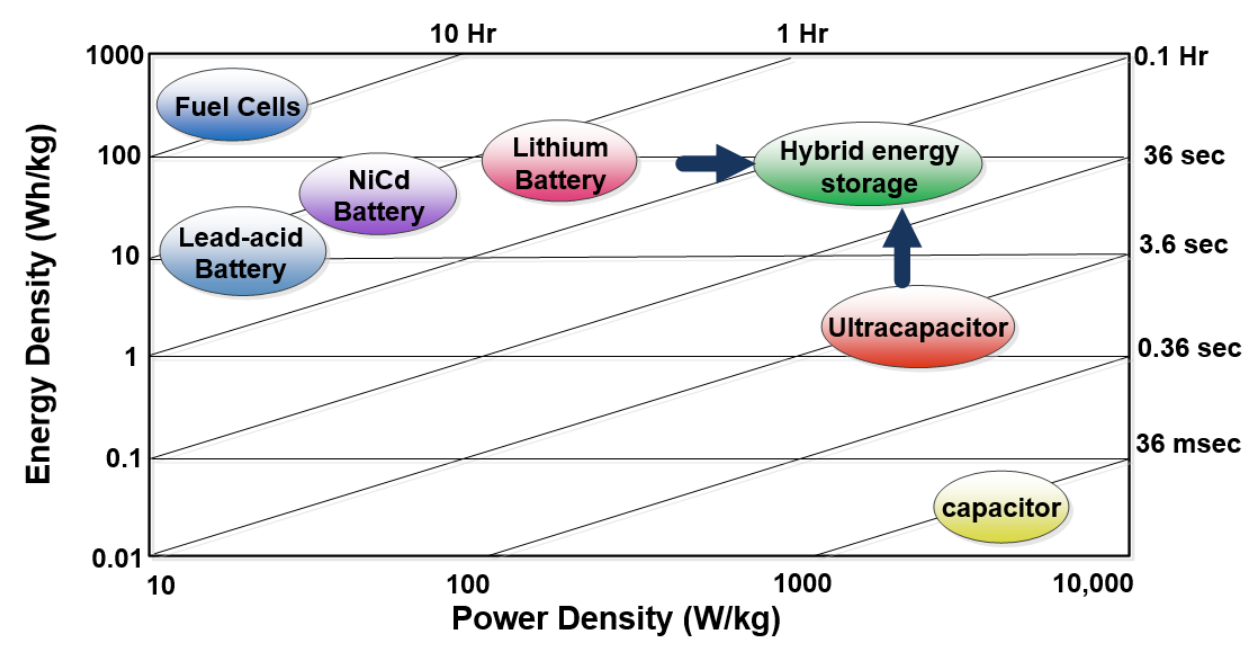

Figure 2.2: Power density vs. energy density for various energy-storing devices.

\subsubsection{Passive Hybrid Configuration}

Figure 2.3 is the direct connection of the battery and the supercapacitor in parallel, which is known as passive configuration. In this configuration, the terminal voltage of the battery and the supercapacitor are not regulated. As a result, the power sharing between the storage devices is mainly determined by their voltage-current characteristics and their respective internal resistances. Therefore, the usable energy from the supercapacitor is very limited and it acts as a low pass filter for the hybrid storage system. In the next section, the performance of this configuration for pulsed load applications will be analytically analyzed. 


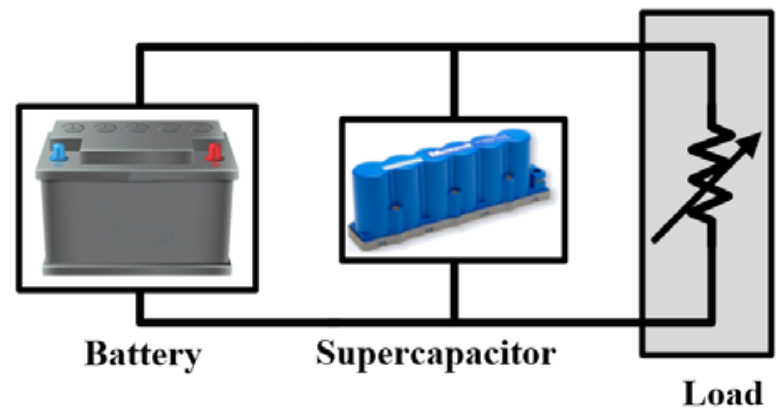

Figure 2.3: Passive battery-supercapacitor hybrid configuration.

\subsubsection{Active Supercapacitor-Battery Configuration}

In an active supercapacitor-battery hybrid configuration, a bidirectional DC-DC converter is implemented between the supercapacitor and the load, while the battery bank is directly connected to the load. Figure 2.4 shows the circuit topology of this system. Implementation of the DC/DC converter allows the supercapacitor voltage to be decoupled from the battery bank. As a result, the supercapacitor voltage can change in a wide range and its maximum energy utilization is highly improved. Moreover, the converter, acting as a power regulator, can effectively decompose the dynamic and the average power of the load, as explained in Figure 2.1.

The main disadvantage of this topology is that in this configuration the converter size needs to be relatively large in order to handle the power of the supercapacitor, especially in the case of a deep discharge. Moreover, since the battery bank is directly connected to the load, there is less flexibility for the design of the bank.

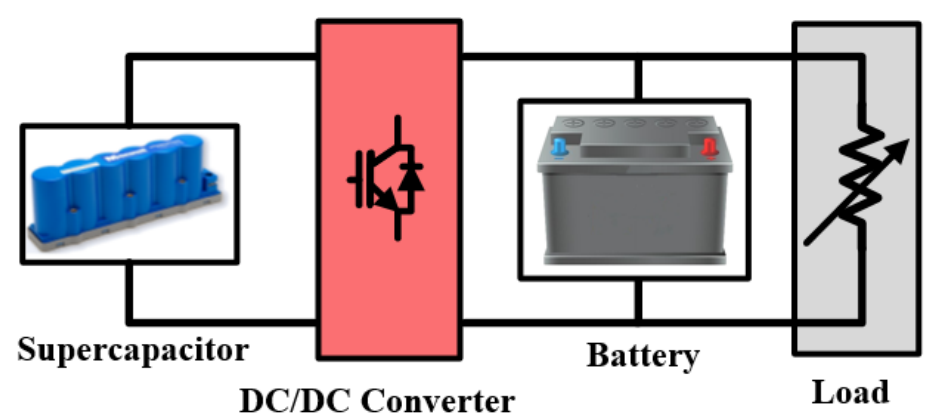


Figure 2.4: Active supercapacitor-battery hybrid configuration.

\subsubsection{Active Battery-Supercapacitor Configuration}

Figure 2.5 is an active battery-supercapacitor hybrid configuration. In this configuration, a DC/DC converter is utilized between the two energy storage devices. The converter continuously controls the power that flows from the battery to the supercapacitor and it is able to accurately regulate the output voltage in a broad voltage range. As a result, the active hybrid configuration effectively enhances the power sharing between the energy storage devices.

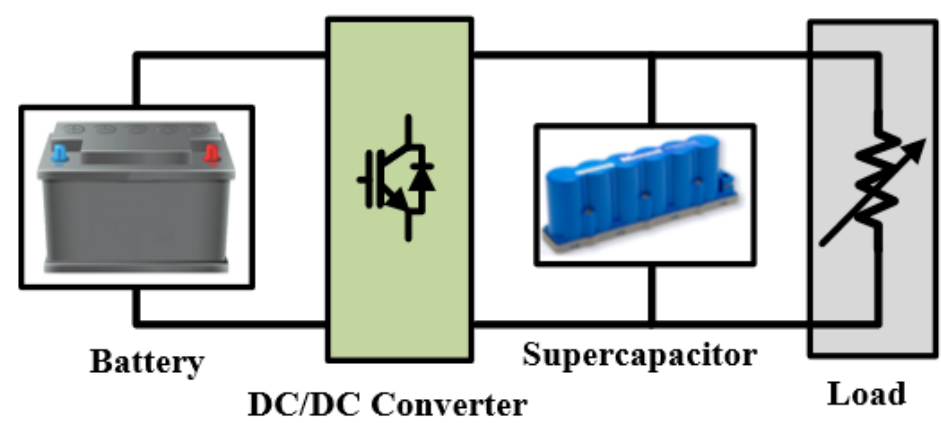

Figure 2.5: Active battery-supercapacitor hybrid configuration.

This configuration is the most studied and researched hybrid configuration for pulse load applications. In [109], it is shown that for a pulse load with $0.2-\mathrm{Hz}$ frequency and a $10 \%$ duty ratio, the power capability of an active hybrid configuration is three times greater than that of a passive hybrid. Furthermore, the active hybrid configuration results in a smaller battery current ripple, and less weight and volume than that of the passive configuration. In the next section, the performance of the active hybrid configuration for pulsed load applications will be analytically analyzed. 


\subsubsection{Series Active Hybrid Configuration}

In a series active hybrid configuration, two DC-DC converters are employed to interface the battery and the supercapacitor bank to the load. Figure 2.6 shows the topology of this system. Utilization of the converters makes a better working range of the battery and the supercapacitor bank. As a result, the controllability and performance of the system is improved when compared with the active battery-supercapacitor or the active supercapacitor-battery hybrid configurations. It should be noted that, as an alternative configuration, the placement of the battery and the battery converter can be changed with the supercapacitor and its converter [22].

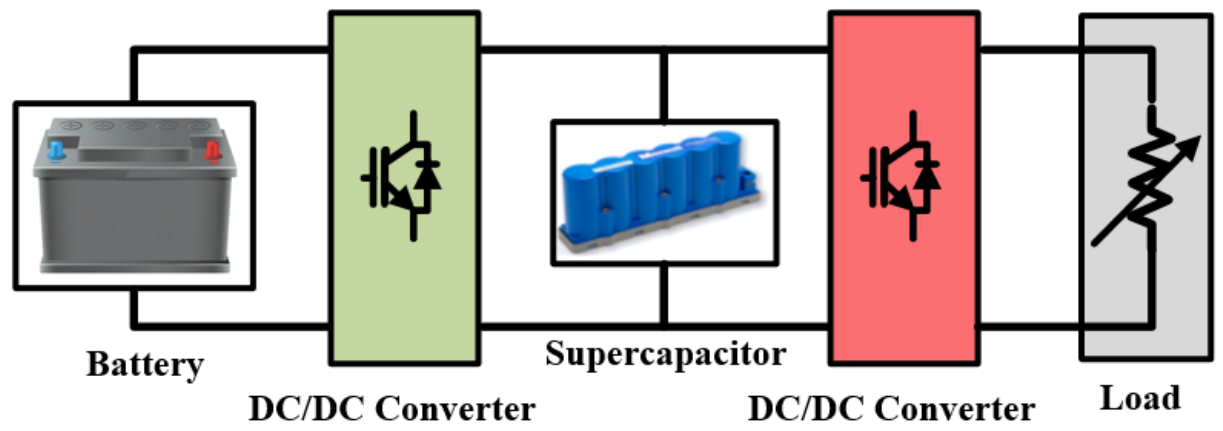

Figure 2.6: Series active battery-supercapacitor hybrid configuration.

The disadvantages of this configuration are the extra cost and complexity of the system due to utilization of two converters. Moreover, utilization of the two conversion stages between the storage devices and the load considerably reduces the overall efficiency of the system.

\subsubsection{Parallel Active Hybrid Configuration}

The parallel active hybrid system utilizes two converters for power regulation of the battery bank and the supercapacitor, which are connected in parallel. The topology of this hybrid system is shown in Figure 2.7. Similar to the series active hybrid configuration, 
utilization of two converters will improve the performance of the system. However, the cost and the complexity of the system are increased and its overall efficiency is reduced.

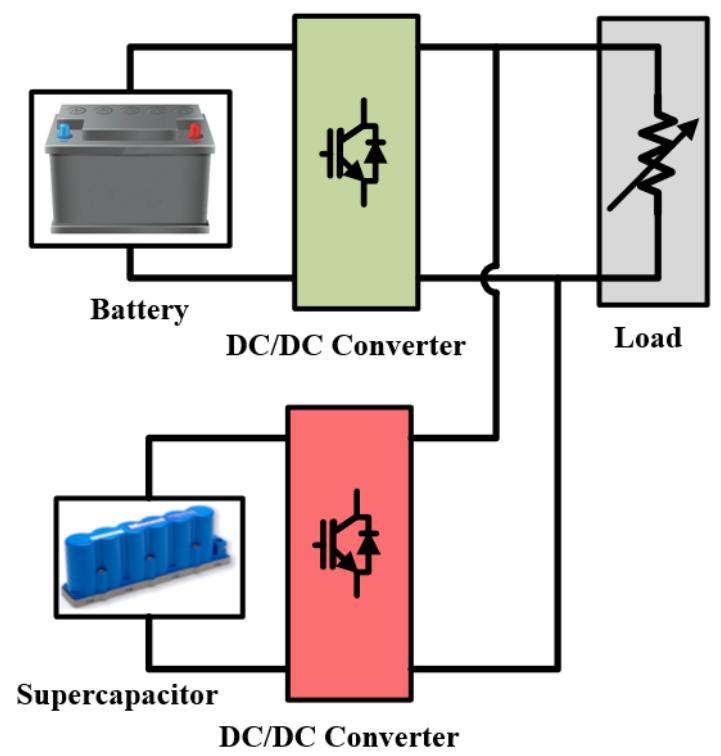

Figure 2.7: Parallel active battery-supercapacitor hybrid configuration.

\subsection{Performance Analysis of Hybrid Storage System for Pulse Load Applications}

According to (2.2) and (2.6), a hybrid energy system for pulse load application should be designed so that the $P_{L a v}$ and the $E_{L d y}^{C}$ is provided. Assuming that the maximum voltage limit and the minimum voltage limit of the pulse load are $V_{\max }$ and $V_{\text {Min }}$, respectively, the total usable energy from a supercapacitor bank can be expressed as:

$$
E_{C}=\frac{N_{p} C_{\text {cell }}\left(V_{\text {Max }}^{2}-V_{\text {Min }}^{2}\right)}{2 N_{s}}
$$

where $C_{c e l l}$ is the capacitance of a supercapacitor cell, $N s$ is the number of supercapacitor cells connected in series in each array to reach the rated DC bus voltage and $N_{p}$ is the number of parallel supercapacitor arrays to meet the required storage energy. Also, $V_{L}$ is the minimum voltage of the pulse load after discharging the supercapacitor. Based on (2.7), 
there is a tradeoff between the maximum $E_{L d y}^{C}$ that can be supplied by a supercapacitor bank and the $V_{\max }$ and the $V_{\text {Min }}$ limit of a pulse load.

As discussed in the previous section, the passive hybrid and active batterysupercapacitor hybrid storage systems are the most effective configurations for the pulsed load applications. In this section, the steady state operations of these topologies under pulsed mode are analytically analyzed.

\subsubsection{Passive Battery-Supercapacitor Storage for Pulsed Load Application}

Supercapacitor is a highly nonlinear component that is usually modeled by distributed capacitor-resistor network. However, for the study presented here, a simplified model of a supercapacitor is used to make the analysis more tractable. Also, the battery bank model is simplified as an ideal voltage source in series with an internal resistor. The circuit model of a passive hybrid configuration considered for this study is shown in Figure 2.8 (a). Figure 2.8 (b) also shows its equivalent model in S-domain using Laplace transform. The $C_{S C}$ is the nominal capacitance of the supercapacitor bank, $V c_{0}$, is its initial voltage and $R_{C}$ is its ESR. Also, $V_{b}$ is the internal voltage of the battery bank and $R_{b}$ is its equivalent resistance.

Based on the circuit model of the hybrid system shown in Figure 2.8 (b), Thevenin's equivalent voltage and impedance of the system can be found as:

$$
\begin{aligned}
& V_{T h}(S)=\frac{R_{C}}{R_{b}+R_{C}} V_{b} \frac{S+\alpha}{S(S+\beta)}+\frac{R_{b}}{R_{b}+R_{C}} V_{C 0} \frac{1}{S+\beta} \\
& Z_{T h}(S)=\frac{R_{b} R_{C}(S+\alpha)}{\left(R_{b}+R_{C}\right)(S+\beta)}
\end{aligned}
$$

where $\alpha$ and $\beta$ are: 


$$
\begin{aligned}
& \alpha=\frac{1}{R_{C} C_{C}} \\
& \beta=\frac{1}{\left(R_{b}+R_{C}\right) C_{C}}
\end{aligned}
$$

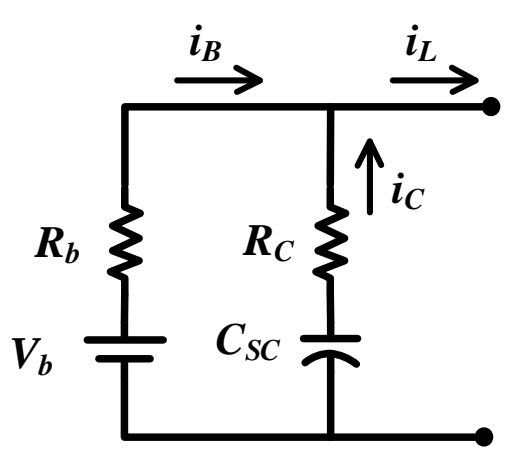

(a)

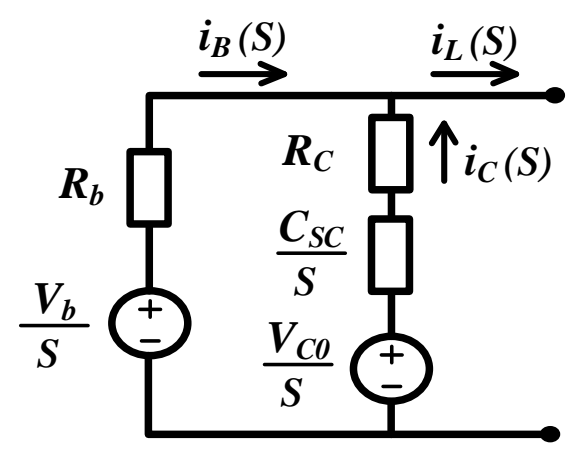

(b)

Figure 2.8: Simplified equivalent circuit of a passive hybrid configuration. (a):Time domain, (b): Frequency domain.

Using inverse Laplace transform, Thevenin’s equivalent voltage in the time domain can be found as:

$$
V_{T h}(t)=V_{b}+\frac{R_{b}}{R_{b}+R_{C}}\left(V_{C 0}-V_{b}\right) e^{-\beta t}
$$

With a same assumption made for (2.1) and assuming that the minimum current of the pulse load is zero, the instantaneous load current, $i_{L}(t)$ for the first $N$ pulses can be expressed as:

$$
i_{L}(t)=I_{m} \sum_{k=0}^{N-1}[\phi(t-k T)-\phi(t-(k+D) T)]
$$

where $I_{m}$ is the amplitude of the pulse load current.

Also, using (2.13), the load current in the S-domain can be expressed as:

$$
i_{L}(S)=I_{m} \sum_{k=0}^{N-1}\left[\frac{e^{-k T S}}{S}-\frac{e^{-(k+D) T S}}{S}\right]
$$


Based on the equivalent Thevenin's model of the circuit shown in Figure 2.8 (b), the load voltage at the output terminal in the S-domain can be expressed as:

$$
\begin{aligned}
V_{L}(S) & =V_{T h}(S)-Z_{T h}(S) I_{L}(S) \\
& =V_{T h}(S)-\frac{R_{b} R_{C}}{R_{b}+R_{C}} I_{L} \sum_{k=0}^{N-1}\left[\frac{1}{\beta}\left(\frac{\alpha}{S}+\frac{\beta-\alpha}{S+\beta}\right) \times\left(e^{-k T S}-e^{-(k+D) T S}\right)\right]
\end{aligned}
$$

In order to find the $V_{L}$ in the time domain, linear property of the Laplace transform can be applied to (2.15) [142]:

$$
\begin{aligned}
V_{L}(t)=V_{b}(t)+\frac{R_{b}}{R_{b}+R_{C}}\left(V_{C 0}-V_{b}\right) e^{-\beta t} \\
-R_{b} I_{L} \sum_{k=0}^{N-1}\left[\left(1-\frac{R_{b}}{R_{b}+R_{C}} e^{-\beta(t-k T)}\right) \phi(t-k T)\right. \\
\left.-\left(1-\frac{R_{b}}{R_{b}+R_{C}} e^{-\beta(t-(k+D) T)}\right) \phi(t-(k+D) T)\right]
\end{aligned}
$$

Also, the current drawn from the battery bank and the supercapacitor can be found based on the $V_{L}$ as:

$$
i_{b}(t)=\frac{1}{R_{b}}\left[V_{b}(t)-V_{L}(t)\right]
$$

Using KCL law, the current drawn from the supercapacitor is:

$$
i_{C}(t)=i_{L}(t)-i_{b}(t)
$$

Using (2.13), (2.17) and (2.18), the normalized load current, battery current and supercapacitor current for the case that $C_{S C}=20-F, R_{C}=0.025-\Omega$ and $R_{b}=0.15-\Omega$ are plotted in Figure 2.9. Also, for this case study, the pulse period and its duty ration were 10s and 0.1, respectively. During the pulse load on-state, most of the current is provided by the supercapacitor, while during the load off-state, the supercapacitor is smoothly charged 
by the battery bank. As a result, hybridization of the battery bank and the supercapacitor effectively reduced the maximum current that is drawn from the battery bank.

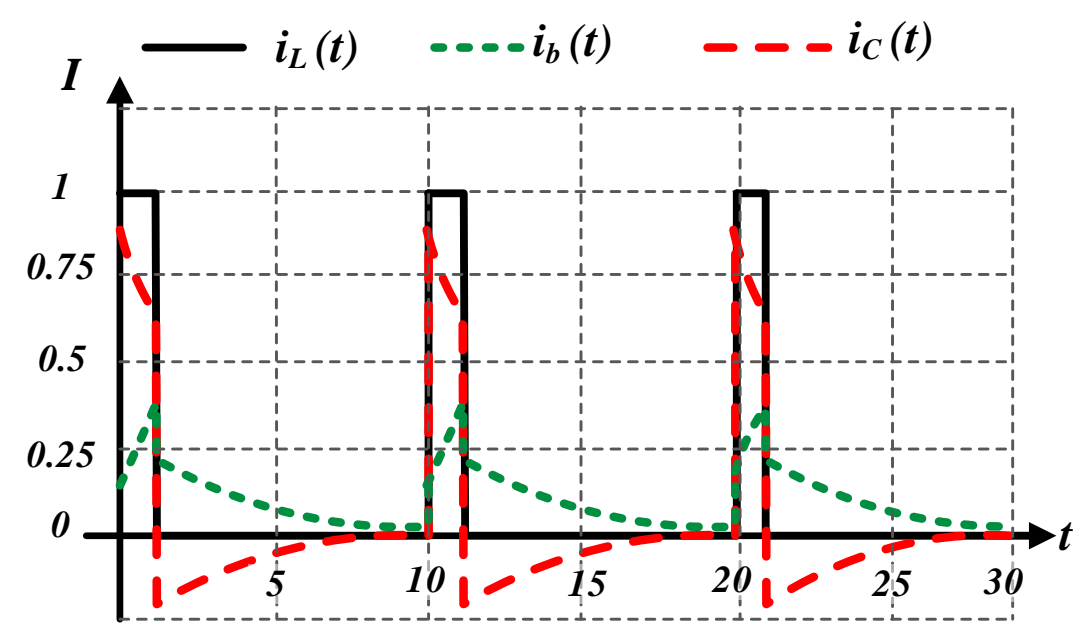

Figure 2.9: Normalized load, battery and supercapacitor currents for 1 per unit pulse load with 10 -s period and 0.1 duty ratio.

The supercapacitor assistance in reducing the peak current of the battery bank depends on both design aspects of the system, such as the supercapacitor size and its configuration, and also on the operating conditions, such as the pulse period, its amplitude and its duty ratio. In order to investigate the supercapacitor assistance in peak current reduction, the battery current is expressed based on (2.16) and (2.17) as:

$$
\begin{aligned}
i_{b}=\frac{1}{R_{b}}\left(V_{b}-V_{L}\right)= & -\frac{1}{R_{b}+R_{C}}\left(V_{C 0}-V_{b}\right) e^{-\beta t} \\
+I_{L} \sum_{k=0}^{N-1}[ & {\left[\left(1-\frac{R_{b}}{R_{b}+R_{C}} e^{-\beta(t-k T)}\right) \phi(t-k T)\right.} \\
& \left.\quad-\left(1-\frac{R_{b}}{R_{b}+R_{C}} e^{-\beta(t-(k+D) T)}\right) \phi(t-(k+D) T)\right]
\end{aligned}
$$

The loading profile shown in Figure 2.9 reveals that the maximum battery current occurs at the end of the pulse on-state, i.e. $t=(k+D) T$. Assuming that the supercapacitor's 
initial voltage in steady state is equal to the battery voltage, the maximum battery current can be found as [142]:

$$
i_{b}^{\max }=I_{L}\left(1-\frac{R_{b}}{R_{b}+R_{C}} \times \frac{e^{-\beta D T}-e^{-\beta T}}{1-e^{-\beta T}}\right)
$$

In order to show the effectiveness of the hybrid battery-supercapacitor system in reducing the maximum battery current, the $I_{L} / i_{b}^{\max }$ for different pulse ratios and periods are depicted in Figure 2.10. In this case, $C_{S C}=20-F, R_{C}=0.025$ and $R_{b}=0.15$. The results show that the hybrid battery-supercapacitor system effectively decreases the maximum current requirement of the battery bank. As the period of the pulse decreases, the $I_{L} / i_{b}^{\max }$ increases, which indicates more contribution of the supercapacitor in supplying the pulse load. Moreover, the $I_{L} / i_{b}^{\max }$ in all pulse periods increases when the pulse due ratio decreased. Maximum $I_{L} / i_{b}^{\max }$ is achieved when the pulse due ratio goes to zero and based on (2.20), it is equal to:

$$
\left(\frac{I_{L}}{i_{b}^{\max }}\right)^{\max }=\frac{R_{b}+R_{C}}{R_{C}}
$$

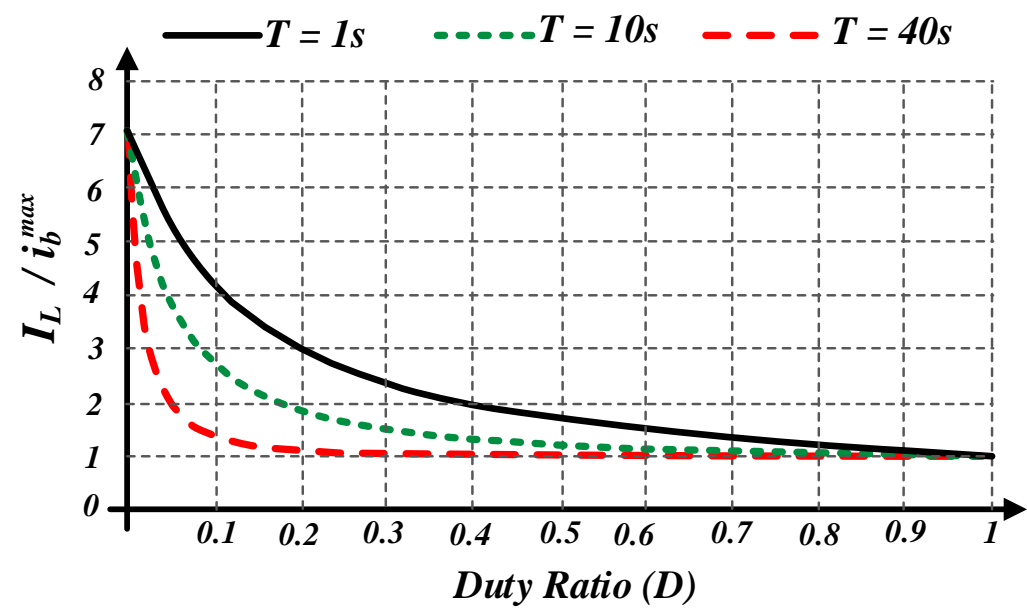


Figure 2.10: Current contribution of the battery bank for different pulse periods and duty ratios.

Figure 2.11 shows the effect of the supercapacitor size in reducing the battery bank current. As can be seen, increasing the size of the supercapacitor bank increases the $I_{L} / i_{b}^{\max }$ , which means more contribution of the supercapacitor in supplying the puled load and less current requirement from the battery bank. However, increasing the size of the supercapacitor increases the cost of the system and it may not be economically feasible, especially for high pulse duty ratio.

It can be concluded that the hybrid battery-supercapacitor system effectively decrease the maximum current requirement of the battery bank while, for a battery alone system, the $I_{L} / \mathrm{i}_{b}^{\max }$ is always equal to unity. Due to maximum current reduction, the internal voltage drop and power losses of the battery bank will be decreased. As a result, the hybridization of the battery and supercapacitor not only improves the system efficiency, but also it effectively increases the life time of the battery bank.

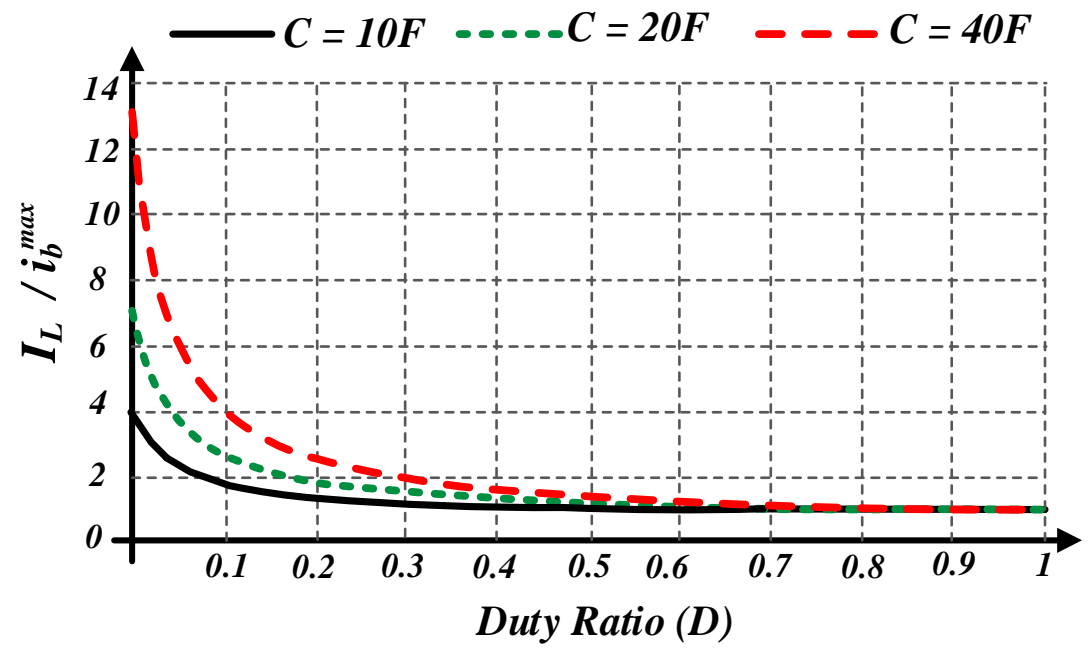

Figure 2.11: Current contribution of the battery bank for different supercapacitor sizes. In all cases, the period of the pulse was 10 -s. 


\subsubsection{Active Battery-Supercapacitor Storage for Pulsed Load Application}

A major characteristic of the active hybrid energy storage system, described in the previous section, is the utilization of a DC-DC converter that controls the power flow from the battery bank to the supercapacitor and the load. In this configuration, the supercapacitor voltage and current can be found as:

$$
\begin{aligned}
& V_{S C}=V_{L} \\
& i_{S C}=I_{L}-i_{b}=I_{L}-\kappa_{\text {Con }}(t) \frac{D I_{L \max }}{\eta_{\text {Con }}}
\end{aligned}
$$

Also, the battery bank voltage and its current can be expressed as:

$$
\begin{aligned}
& V_{b}=\frac{V_{L}}{\kappa_{\text {Con }}(t)} \\
& i_{b}=\kappa_{\text {Con }}(t) \frac{D I_{L \max }}{\eta_{\text {Con }}}
\end{aligned}
$$

where $\kappa_{C o n}$ and $\eta_{\text {Con }}$ are the converter voltage conversion ratio and its efficiency, respectively.

Utilization of the converter decouples the battery voltage from the supercapacitor and allows full control of the energy injected to the supercapacitor and the pulse load. As a result, the power capability of an active hybrid configuration can be effectively improved by decomposing the dynamic and the average power of the load as explained in Figure 2.1. In the case of grid connected pulse load, different performances can be achieved depending on the DC-DC converter control method. In this dissertation, various energy control techniques and power control schemes are developed for the active hybrid power system for both grid connected and standalone microgrid systems. In the following sections, the 
performances of the developed techniques are experimentally evaluated and are compared with traditional approaches suggested in the literature.

\subsection{Conclusion}

In this chapter, the issues related to the battery-supercapacitor hybrid systems and their applications for pulse load were studied. The effects of pulsed loads on the AC and DC power system were discussed. The power and the energy requirements of the pulsed load in terms of dynamic and average power, and also dynamic and average energy, were analyzed. Moreover, different possible passive battery-supercapacitor hybrid configuration and active hybrid configurations were explained in this section. The advantages and limitations of each configuration were discussed in terms of power and energy capability, complexity of the control system, power converter requirement and the implementation cost.

This chapter also presented a detailed analytical study of the passive hybrid and active battery-supercapacitor hybrid storage systems for pulsed load applications. The performance of the passive hybrid for different design aspects and the operating conditions, such as the supercapacitor size, the pulse period and pulse duty ratio, were studied. Moreover, different advantages of the active battery-supercapacitor hybrid over the passive configuration were discussed and their limitations were compared. 


\section{DESIGN AND IMPLEMENTATION OF HYBRID DC POWER SYSTEM}

\subsection{Introduction}

In this chapter, the design and implementation of a hybrid DC power system is presented. The developed test setup was implemented at the Energy Systems Research Laboratory, Florida International University. Depending on the application under study, the developed hybrid DC power system can be connected in a radial or a ring configuration. The power system can also be configured to be in a stand-alone operation mode, in which the grid is only supplied through the internal energy sources. Moreover, the system can be connected in a microgrid mode and be supplied through internal energy sources and by an interconnected AC network.

In the following section, the system components of the hybrid power system and the control schemes of the converters are described. Section 3.2 presents the energy storage devices implemented in the power system. This section is followed by an explanation on the design and implementation of the load emulator systems, which are the steady state load, the dynamic load and the pulse load. This is followed by a detailed specification of the generator station models and the isolation transformer are presented. Different power

converters are implemented in the developed power system. Hardware test setups, the configuration and the main control strategy of these converters are included in section 3.5.

The developed test setup contains various measurement circuits, protection boards and a drive system. These boards are detailed and their performances are evaluated in section 3.7. The hybrid DC power system is able to be connected in our smart grid testbed. Section 3.8 describes the system configuration and the control strategy of the AC grid 
test-bed. Finally, the real-time control and monitoring of the developed hybrid DC power system is presented in section 3.9.

\subsection{Energy Storage System}

The energy storage devices of the implemented hybrid DC power system include a supercapacitor bank and a battery bank. This subsection includes the detailed specifications and configuration of these systems.

\subsubsection{Supercapacitor Bank}

The supercapacitor bank is built using 350-F, 2.7-V cells of Maxwell Technologies supercapacitors. Six supercapacitor cells connected in series form a 58-F, 16-V module. Twenty supercapacitor modules were connected in series to configure a 2.9-F, 320-V supercapacitor bank. This bank is protected via an analog hysteresis voltage protection board, which is explained in section 3.7.5.

Table 3.1: Specification of the supercapacitor bank.

\begin{tabular}{|c|c|}
\hline Parameter & Specification \\
\hline Type & Maxwell (BMOD0058) \\
\hline Number of Cells & 20 \\
\hline Rated Capacity & $2.9 \mathrm{~F}$ \\
\hline Rated Voltage & $320 \mathrm{~V}$ \\
\hline Maximum Voltage & $340 \mathrm{~V}$ \\
\hline Maximum Continuous Current & $12 \mathrm{~A}\left(\Delta T=15^{\circ} \mathrm{C}\right)$ \\
\hline
\end{tabular}

Figure 3.1 shows the supercapacitor bank implemented in the test setup. Two 2.9F supercapacitor banks are available in our experimental test setup, which can be connected in parallel to form a 5.8-F, 320-V bank. Alternatively, they may be connected in series to 
form a 1.45-F, 650-V bank. The detailed parameters of the $2.9 \mathrm{~F}$ supercapacitor bank are summarized in Table 3.1.

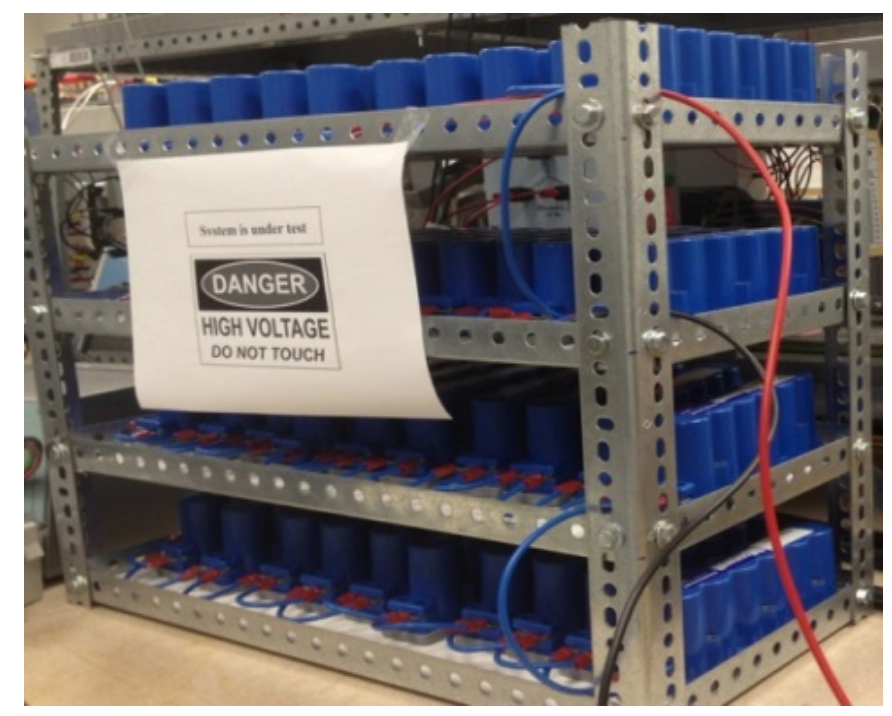

Figure 3.1: Supercapacitor bank setup.

\subsubsection{Battery Bank}

The battery energy storage systems are essential to meet the peak loads economically and to improve the power quality and stability of the grid. Figure 3.2 (b) shows the battery bank implemented in the test setup. The battery bank is composed of twelve lead-acid battery cells rated $120-\mathrm{V}, 110$-Ah. The specifications of this bank are summarized in Table 3.2.

Table 3.2: Specification of the battery bank.

\begin{tabular}{|c|c|}
\hline Parameter & Specification \\
\hline Type & $\begin{array}{c}\text { Universal (UB121100) } \\
\text { Lead Acid }\end{array}$ \\
\hline Number of Cells & 12 \\
\hline Rated Capacity & $110 \mathrm{Ah}$ \\
\hline Bank nominal Voltage & $120 \mathrm{~V}$ \\
\hline
\end{tabular}




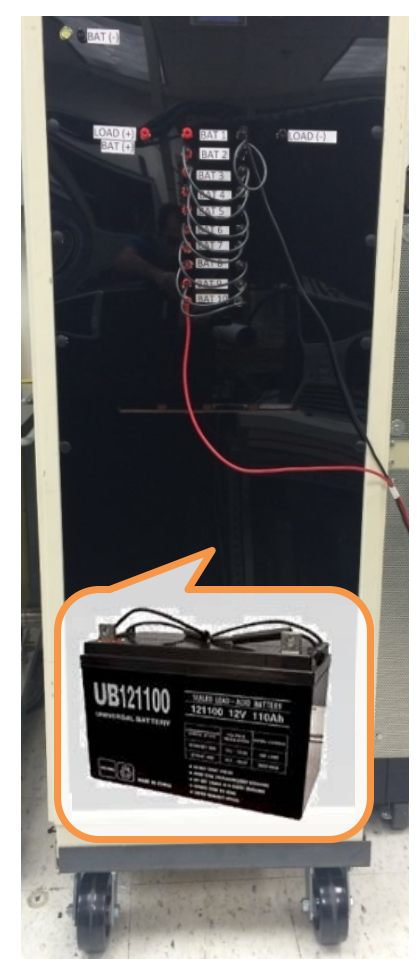

(a)

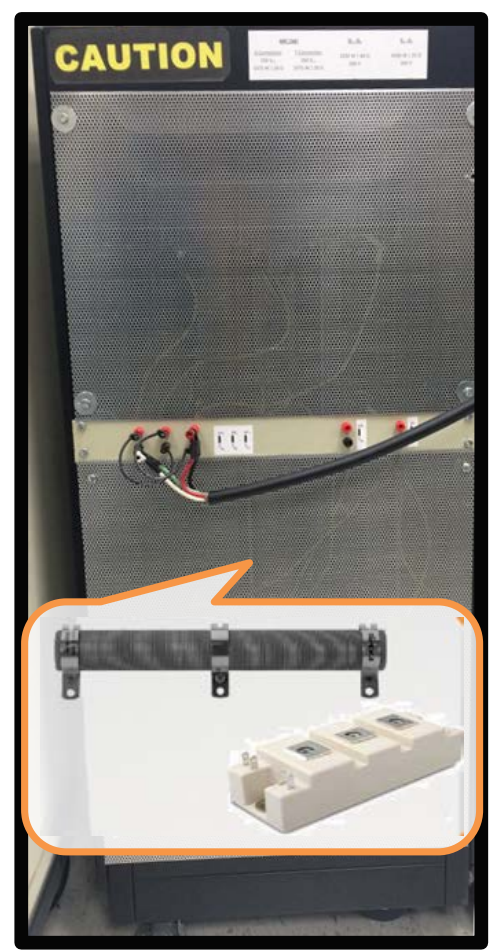

(b)

Figure 3.2: Experimental test equipment: (a) battery bank; (b) load emulator.

\subsection{Load Emulators}

The implemented hybrid DC microgrid includes three different types of loads. These include the steady state load, the dynamic load and the pulse load. Figure 3.2 (b) shows the load module that is built using the resistor bank and the buck converters. The rated power of the steady state load is $2-\mathrm{kW}$ at the rated $320-\mathrm{V}$ DC bus voltage while the dynamic load and the pulse load are fully programmable.

The dynamic load is a $15-\Omega$ resistor that is connected to the low voltage side of a buck converter, with a switching frequency of $2-\mathrm{kHz}$. This load is able to fully emulate the dynamic behavior of a load up to 6-kW. For instance, the consumption profile shown in Figure 3.3 is a load with high starting power based on the equation (3.1). 


$$
P_{D L}=P_{N}\left(1+k e^{\frac{-(t-T)}{\tau}}\right) u(t-T)
$$

where $P_{N}$ is the steady state power of the load, $k$ is the transient load coefficient and $\tau$ is its time constant. Also, $u$ is a unit step function and $\mathrm{T}$ is the load starting time. As Figure 3.3 shows, the maximum of the load at the starting point is $(1+k) P_{N}$ and it settles to $1.05 P_{N}$ after $3 \tau$ seconds. By adjusting the duty ratio of the buck converter, the implemented dynamic load is able to fully emulate the load profile such as the one shown in Figure 3.3.

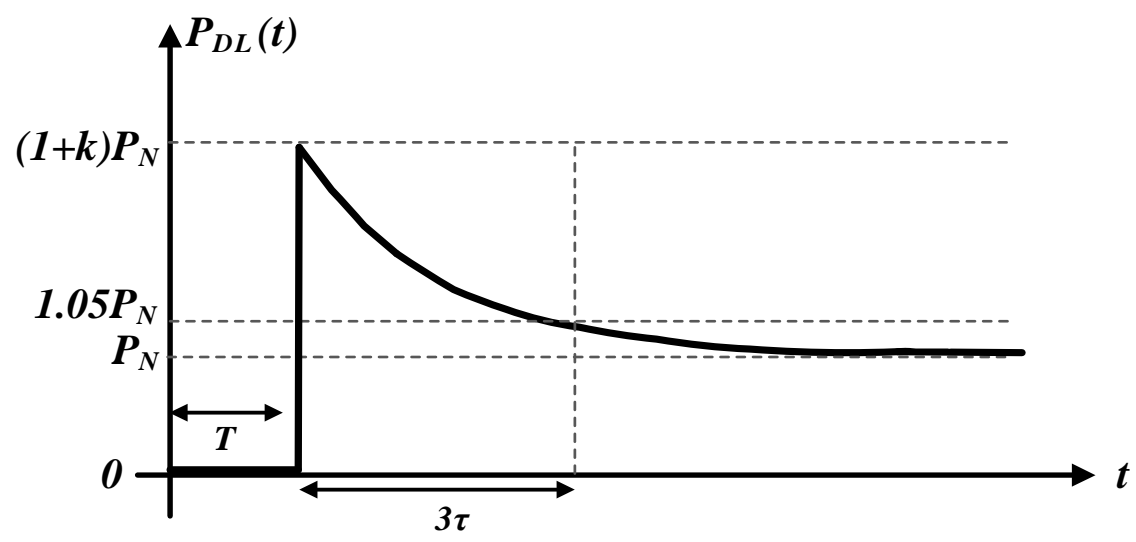

Figure 3.3: A dynamic load consumption profile.

Also, in order to emulate the pulse load behavior, a $20-\Omega$ resistor is connected to another buck converter. The switching frequency of this converter is 2-kHz. The pulse load is completely programmable up to $4-\mathrm{kW}$ at different pulse frequencies and duty ratios. 


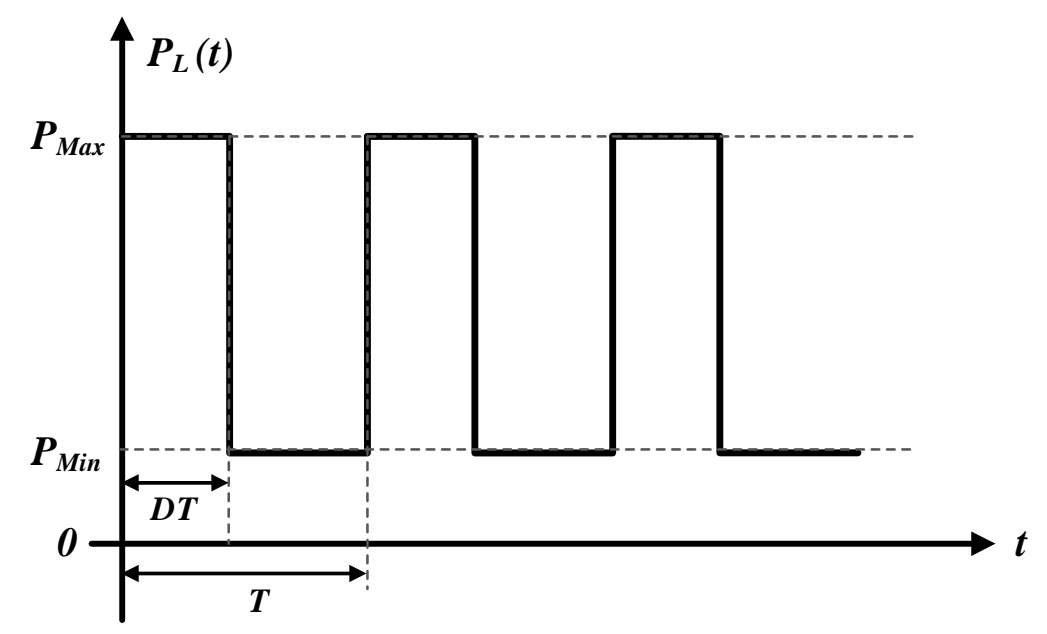

Figure 3.4: A pulse load consumption profile.

In order to understand the pulse load characteristics, consider a consumption profile

plotted in Figure 3.4, which defines the load power versus time $P_{L}(t)$. The load is considered to have constant power changing between $P_{\text {Min }}$ and $P_{\text {Max }}$ with a period $T$ (the frequency $f=1 / T$ ) and duty cycle $D$. Based on Figure 3.4, the instantaneous load power, $P_{L}(t)$ for the first $N$ pulses can be expressed as:

$$
P_{L}(t)=P_{M i n}+\sum_{k=0}^{N-1}\left(P_{M a x}-P_{M i n}\right)[u(t-k T)-u(t-(k+D) T)]
$$

where $u$ is a unit step function at $t=0$. By adjusting the duty ratio of the buck converter, the developed pulse power load emulator is able to completely control the pulse load parameters: the duty cycle $D$, the period $T$, the maximum power $P_{M a x}$ and the minimum power $P_{\text {Min }}$.

\subsection{Generation Stations and Transformers}

Four generating stations are implemented in an experimental test-setup. Figure 3.5 shows the schematic of the emulated generator stations and their measurements and 
controller components. As can be seen, the generation stations are 3-phase synchronous generators coupled to induction machines as prime movers. All stations are equipped with an automatic voltage regulator (AVR), which utilizes the feedback signal of the generator's output voltage to regulate the bus voltage.

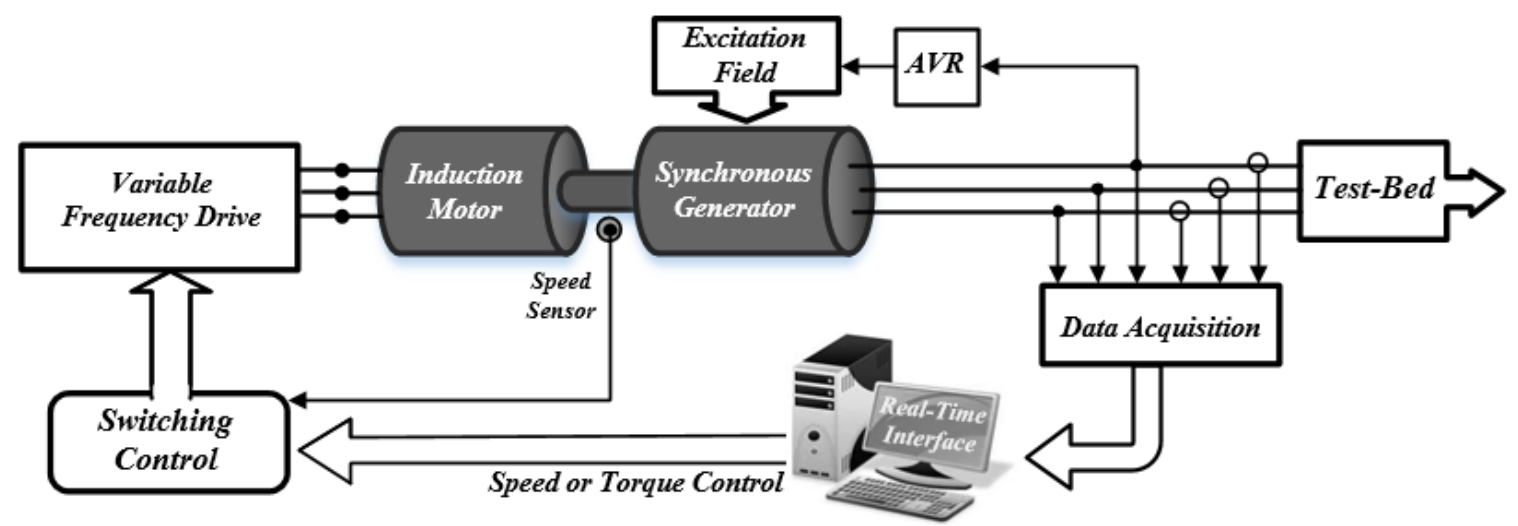

Figure 3.5: Schematic diagram of the generator stations' configuration and their control system.

Figure 3.6 shows the variable frequency drive system of the generator stations. The drive system is supplied through the AC grid. The input voltage is first converted to DC using an uncontrolled rectifier and it is connected to a full bridge inverter through an LC filter. The inverter controls the induction motor of the generator station as the prime mover controller. In the case of the slack bus generator, the prime mover is run through a constant frequency drive to maintain the grid frequency at $60-\mathrm{Hz}$. The frequency drive follows "vector speed control” that serves to keep the frequency constant. In order to emulate the PV buses, the prime mover of the generator is operated by a torque-controlled frequency drive, which regulates the output active power of the generation station. The generator station experimental test setup is shown in Figure 3.7 (a). The detailed parameters of the generator stations are given in Table 3.3. 


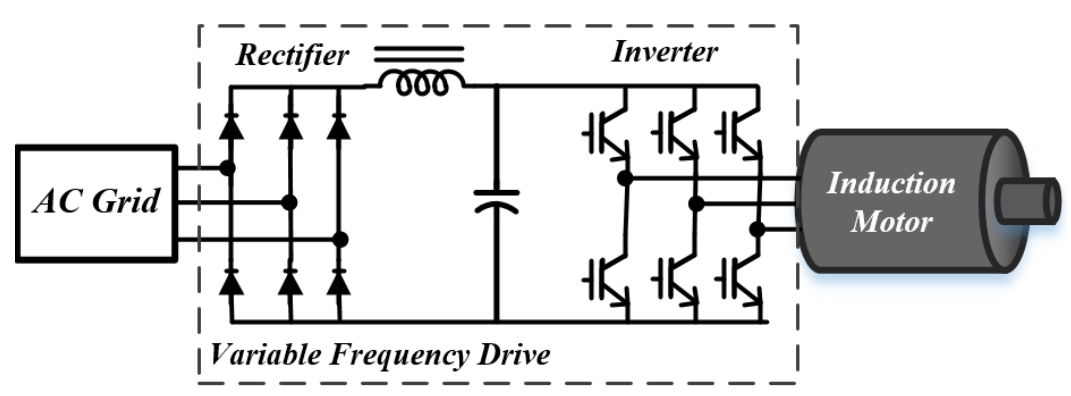

Figure 3.6: Variable frequency drive topology of the generator stations.

Table 3.3: Generator station parameters.

\begin{tabular}{|c|c|c|}
\hline Component & Parameter & Specification \\
\hline \multirow{3}{*}{ Generator } & $S_{N}$ & $3 \mathrm{kVA}$ \\
\cline { 2 - 3 } & $V_{N}$ & $208 \mathrm{~V}$ \\
\cline { 2 - 3 } & $F_{N}$ & $60 \mathrm{~Hz}$ \\
\cline { 2 - 3 } & $X_{L}, R s$ & $6.87 \mathrm{mH}, 1.45 \Omega$ \\
\hline
\end{tabular}

In order to galvanically isolate the AC generator from the grid, an isolation transformer was connected between the generator and the DC grid. The isolation transformers have a unity turn ratio and are intended for use as a part of an isolated power system. The implementation of the isolation transformer ensures no DC current flows to the generator through the neutral impedance. The implemented isolation transformers are connected in $\mathrm{Y} / \Delta$ configuration and their nominal power is $3-\mathrm{kVA}$. The general view of this transformer is shown in Figure 3.7 (b). The detailed parameters of the transformer are given in Table 3.4.

Table 3.4: Isolation transformer parameters.

\begin{tabular}{|c|c|c|}
\hline Component & Parameter & Specification \\
\hline \multirow{3}{*}{ Transformer } & Connection & $Y D$ \\
\cline { 2 - 3 } & $S_{N}$ & $3 \mathrm{kVA}$ \\
\cline { 2 - 3 } & $V_{N}$ & $208 \mathrm{~V}$ \\
\cline { 2 - 3 } & $R_{e q}, X_{e q}$ & $0.72 \Omega, 2.29 \mathrm{mH}$ \\
\hline
\end{tabular}




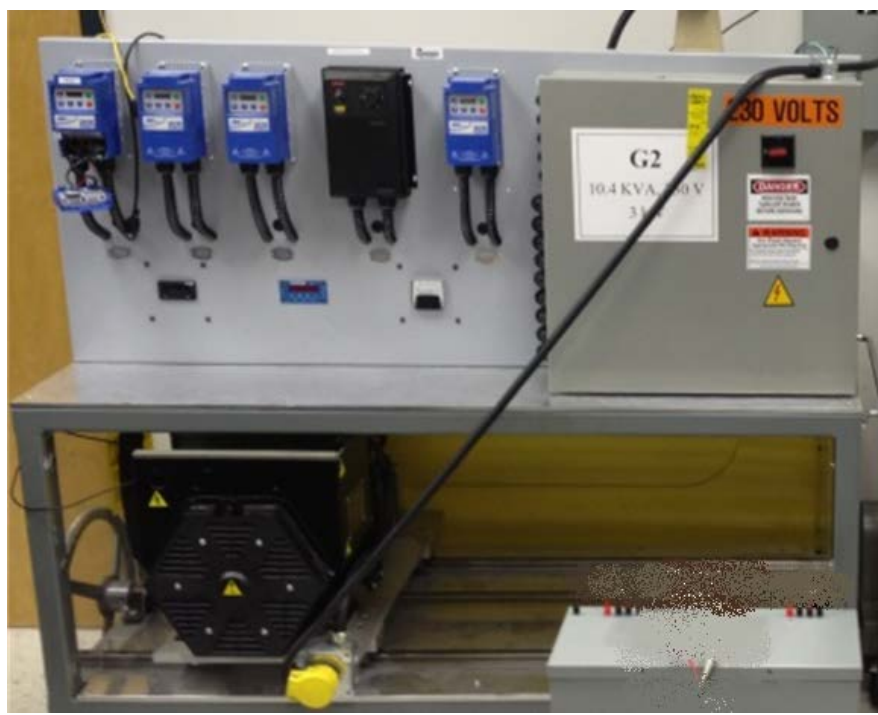

(a)

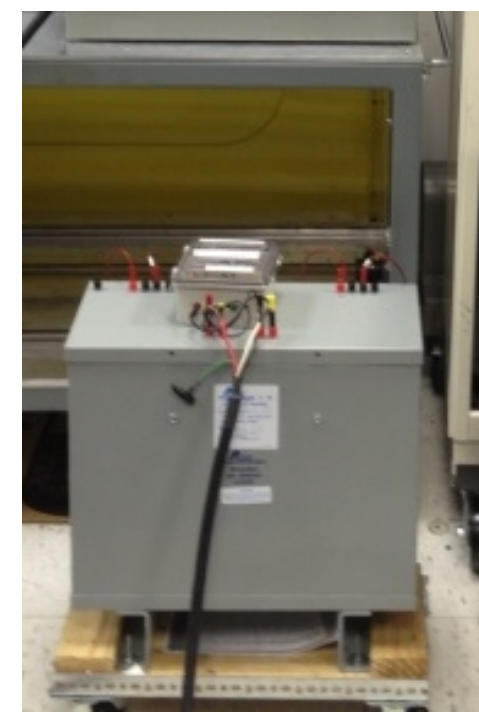

(b)

Figure 3.7: Experimental test setup components. (a): AC generator; (b) Galvanic isolation transformer.

\subsection{Power Converters}

Different power converters are utilized in the developed hybrid DC microgrid system. Hardware test setups, the configuration and the main control strategy of these converters are explained here.

\subsubsection{DC-DC Boost Converter}

The boost converters are mainly implemented to increase the input voltage level and to regulate the output power. Figure 3.8 (a) shows the DC-DC boost converter test setup. Also, the topology of this converter is shown in Figure 3.9. The main components of the converter are an isolated gate bipolar transistor (IGBT), a capacitor C, an inductor $\mathrm{L}$, and the freewheeling diodes $\mathrm{D}_{1}$ and $\mathrm{D}_{2}$. When the IGBT switch is turned on, the inductor is charged through the source connected in the input LV terminal. When the IGBT is switched off, the current flows through the freewheeling diode $\mathrm{D}_{2}$ and discharges the 
inductor and charges the capacitor. Thus, the load at the HV terminal is continuously supplied through the stored energy in the capacitor. It can be shown that the steady state output voltage and current $V_{H V}$ and $I_{H V}$ are [143]:

$$
\begin{gathered}
V_{H V}=\frac{V_{L V}}{1-k} \\
I_{H V}=\frac{V_{S L V} /(1-k)-V_{S H V}}{R_{H V}+R_{L V} /(1-k)^{2}}
\end{gathered}
$$

where, $V_{L V}, V_{S L V}$ and $V_{S H V}$ are the $L V$ terminal voltage, the Thevenin equivalent voltage of the source connected to the LV side and the equivalent voltage of the source connected to the $\mathrm{HV}$ side, respectively. In addition, the $k, R_{H V}$ and $R_{L V}$ are the duty cycle, the Thevenin equivalent resistor of the HV source and the equivalent resistor of the LV source, respectively.

Based on (3.4), the necessary condition to allow energy transfer is that the numerator of the fraction of (3.4) should be greater than zero; that is $\mathrm{V}_{\text {SLV }}>\mathrm{V}_{\text {SHV }}(1-\mathrm{k})$.

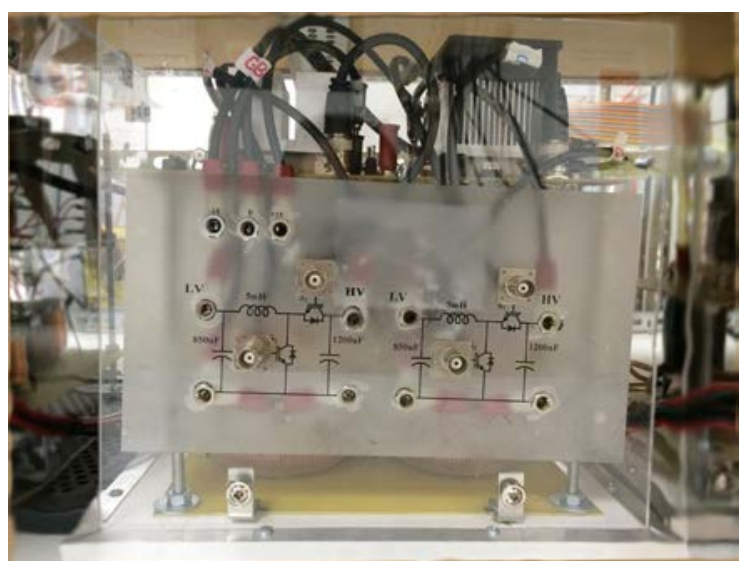

(a)

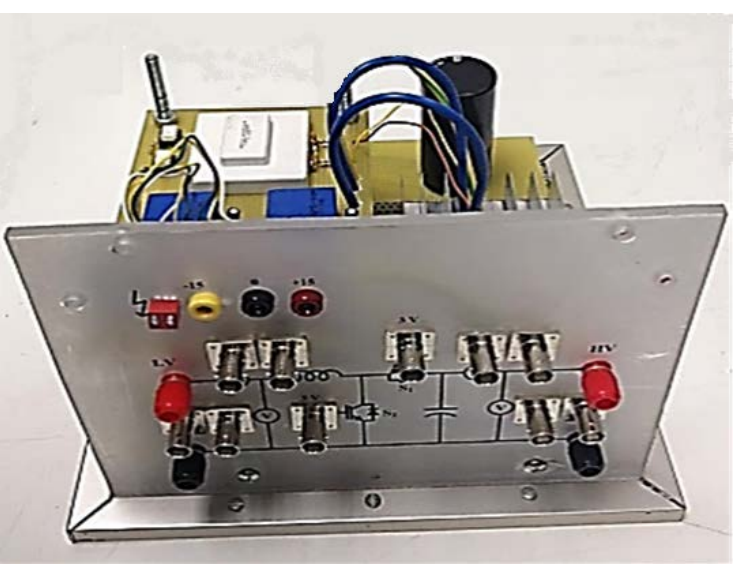

(b)

Figure 3.8: Power converter test setup. (a): DC-DC boost converter; (b): DC-DC bidirectional DC-DC converter. 


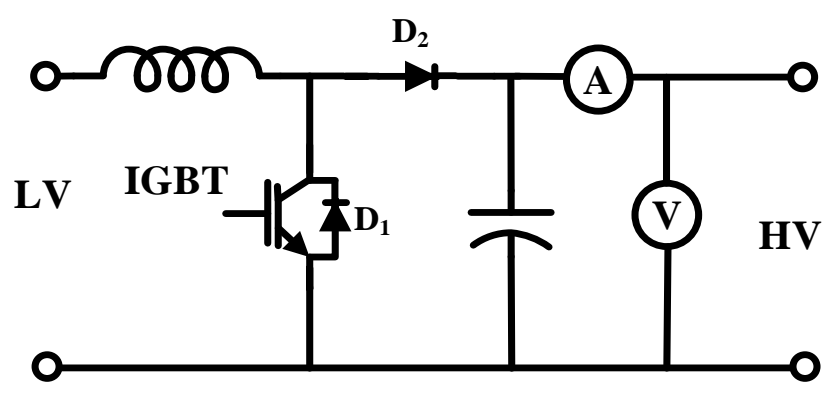

Figure 3.9: Configuration of the boost converter.

Figure 3.10 shows the control algorithm of the boost converter, which is controlled by a proportional-integral (PI) controller. As can be seen, the controller has two inputs: the current reference and the converter current as a feedback signal. The error signal is calculated and then is fed to a PI controller. The output is limited by a saturator limiter and a newly calculated duty ratio is then compared with a $5 \mathrm{kHz}$ sawtooth signal to generate the PWM pulses.

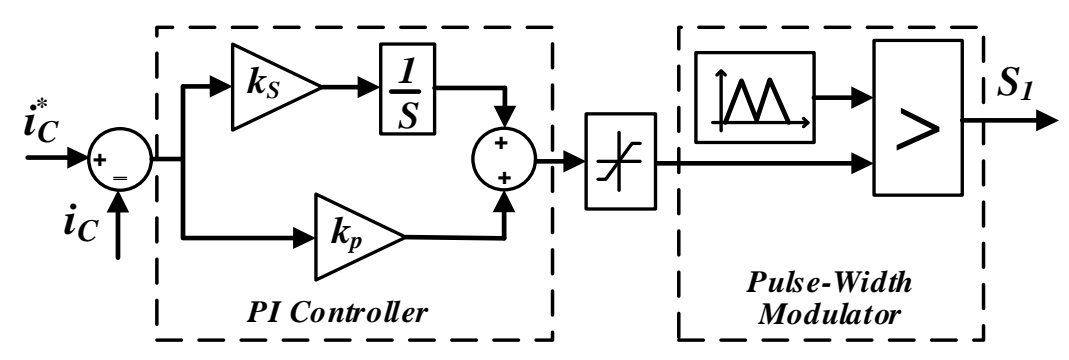

Figure 3.10: Block diagram of the boost converter controller.

\subsubsection{Bidirectional DC-DC Converter}

Figure 3.8 (b) and Figure 3.11 show the hardware setup and the configuration of the bidirectional buck-boost converter implemented in the hybrid DC microgrid system, respectively. The main components of the converter are the inductor $\mathrm{L}$, the capacitor $\mathrm{C}$, the IGBTs $S_{1}$ and $S_{2}$ and the freewheeling diodes $D_{1}$ and $D_{2}$. A fixed frequency PWM technique was used to control the IGBTs.

This converter has two modes of operation: boost and buck. The boost mode of operation allows energy transfer from the LV terminal to HV terminal by triggering IGBT 
$\mathrm{S}_{2}$. When $\mathrm{S}_{2}$ is switched on, the inductor $\mathrm{L}$ is charged and the capacitor $\mathrm{C}$ is discharged through the $\mathrm{HV}$ terminal. When $\mathrm{S}_{2}$ is switched off, the current that was flowing though IGBT $S_{2}$ would then flow through $\mathrm{L}$ and the freewheeling diode $\mathrm{D}_{1}$, charging the capacitor C and transferring energy to the HV terminal.

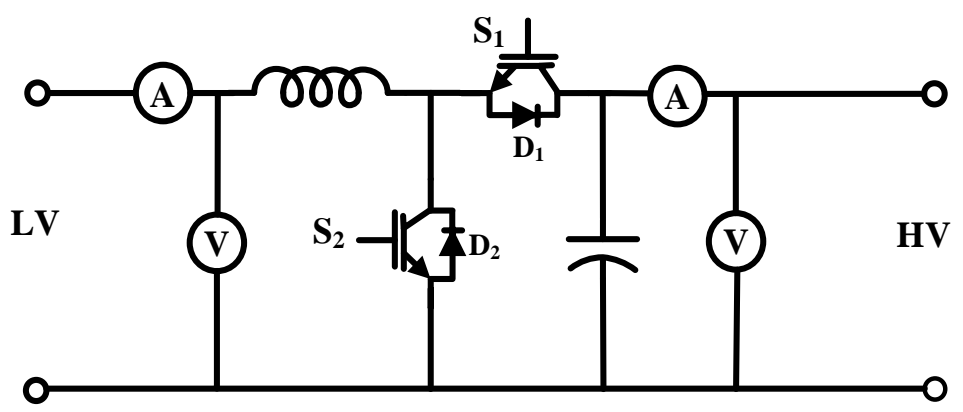

Figure 3.11: Configuration of the bidirectional DC-DC converter.

In the buck mode of operation, by switching on and off the IGBT $S_{1}$ at a controlled duty cycle, the energy transfers from the HV terminal to the LV terminal. When the IGBT $\mathrm{S}_{1}$ is switched on, the input current from the $\mathrm{HV}$ terminal flows through the filter inductor $\mathrm{L}$, and transfers the energy to the LV terminal. When IGBT $\mathrm{S}_{1}$ is switched off, the freewheeling diode $\mathrm{D}_{2}$ conducts due to the energy stored in the inductor. Therefore, the inductor current continues to flow through $\mathrm{L}$ and the diode $\mathrm{D}_{2}$. The inductor's current exponentially falls until IGBT $\mathrm{S}_{1}$ is switched on again in the next cycle.

During forward energy transfer, the converter is operating in boost mode and the steady state output voltage and current $V_{H V}$ and $I_{H V}$ can be similarly found using (3.3) and (3.4). During backward energy transfer, the converter is operating in buck mode and the output voltage and current $V_{L V}$ and $I_{L V}$ can be described by (3.5) and (3.6), respectively. The necessary condition of this operation is that $V_{S L V}$ should be smaller than $k V_{S H V}$.

$$
V_{L V}=k V_{H V}
$$




$$
I_{L V}=-\frac{k V_{S H V}-V_{S L V}}{k^{2} R_{H V}+R_{L V}}
$$

The bidirectional converter is controlled by a PI current controller, which adjusts the duty ratio of the IGBT switching at a fixed 5-kHz. Figure 3.12 shows the control diagram of the converter. Generally, the switching losses of the IGBTs are high compared to the diodes; however, these can be reduced by eliminating unnecessary switching. Depending on the mode of operation, only one IGBT is needed to be switched at a predefined frequency. In order to select the proper IGBT, the reference current is compared to zero. If the reference current is negative, the controlled PWM signal is sent to $S_{1}$, activating the buck mode of operation. On the other hand, if the current reference is positive, the signal is sent to $S_{2}$ to transfer energy from the LV side to the HV side, activating the boost mode of operation.

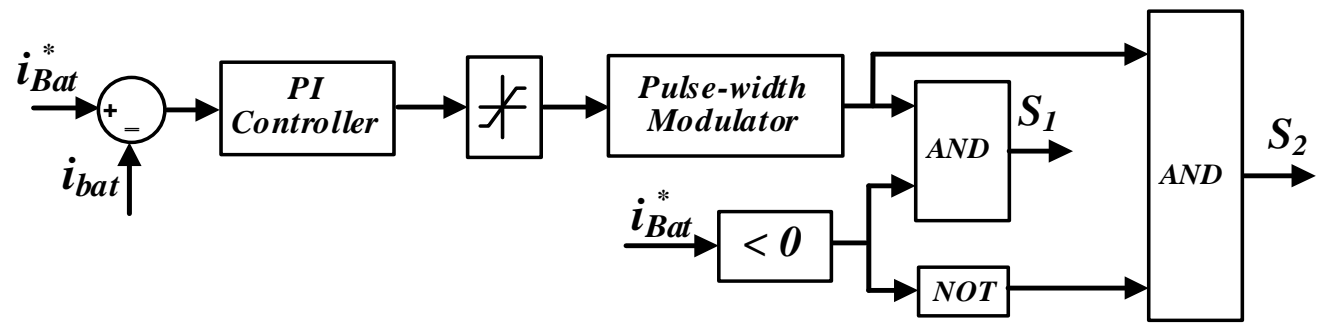

Figure 3.12: Block diagram of the bidirectional DC-DC converter controller.

\subsubsection{Full Bridge Diode Rectifier}

Diode rectifiers are very common in high-power applications. Figure 3.13 (a) shows the full bridge diode rectifier implemented in our hybrid DC power system test setup. Figure 3.14 shows a circuit diagram of this converter. The three-phase diode rectifier is also called an uncontrolled rectifier since there is no control over the switching of the diodes. The pair of diodes encountering the highest amount of instantaneous line-to-line voltage will conduct. As a result, each diode conducts for 120 degrees and the output 
contains six different ripple intervals per supply voltage time period. A capacitive filter was used at the output of the rectifier to reduce this voltage ripple.

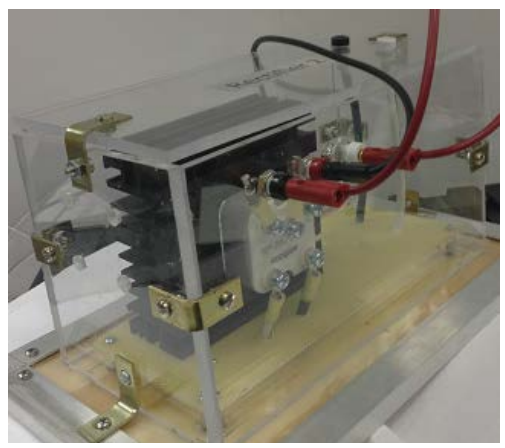

(a)

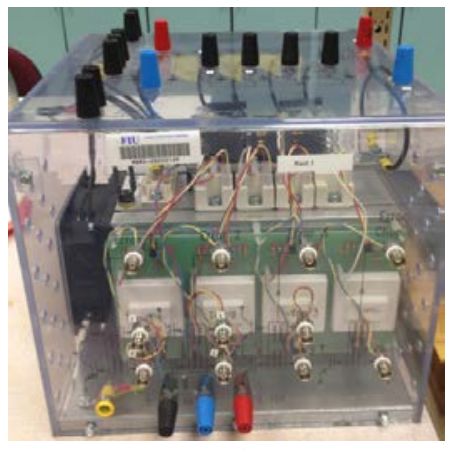

(b)

Figure 3.13: AC-DC power converter test setup. (a): Full bridge diode rectifier; (b): Three-phase controlled rectifier.

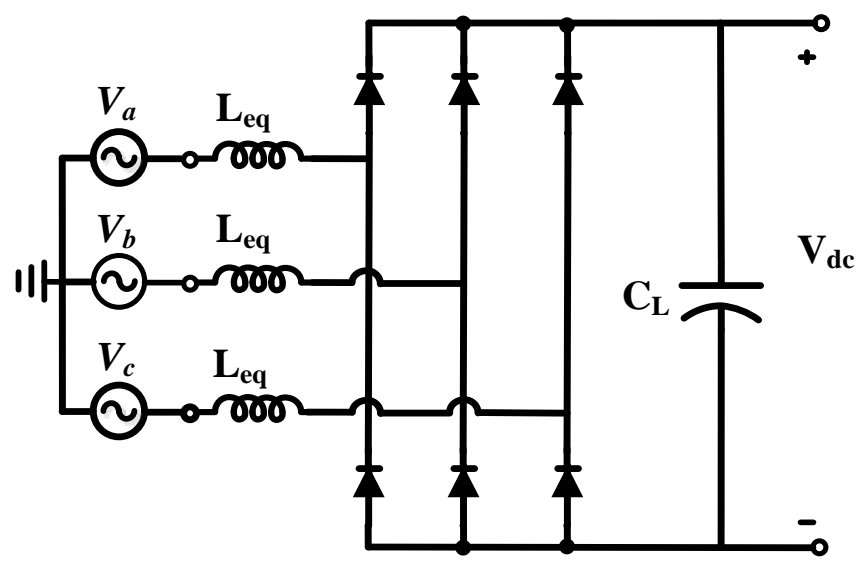

Figure 3.14: Three-phase full wave uncontrolled rectifier configuration.

Figure 3.15 shows the voltage waveform of the AC source and the DC output without a capacitive filter. The average output DC voltage $V_{d c}$, can be found as:

$$
V_{d c}=\frac{3}{\pi} \int_{\pi / 3}^{2 \pi / 3} V_{m} \sin (\omega t) d \omega t=\frac{\sqrt{3}}{\pi} V_{m}=1.3505 V_{L L}
$$




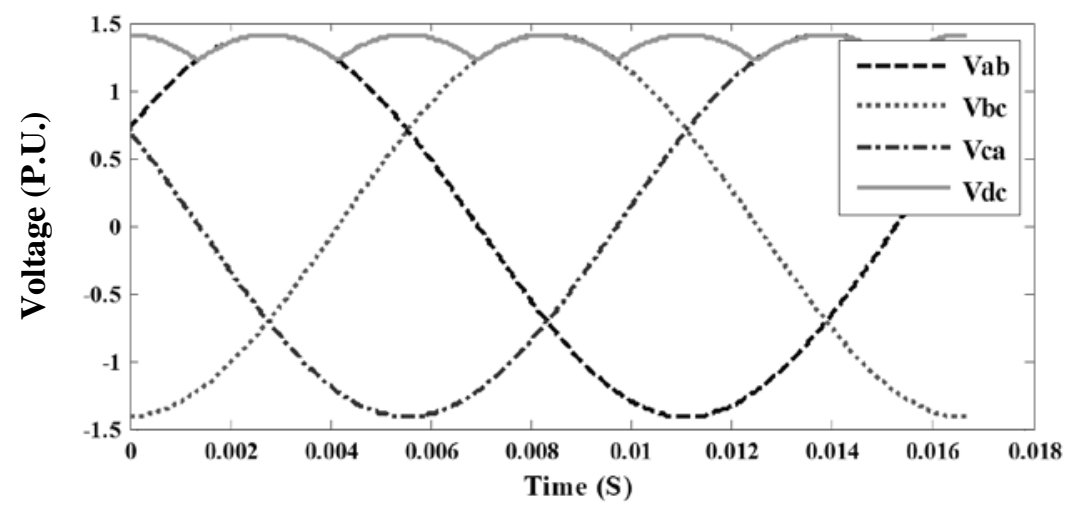

Figure 3.15: Input AC voltage and the output DC voltage of an uncontrolled full bridge rectifier without capacitive filter.

where $V_{m}$ and $V_{L L}$ are the maximum and RMS line-to-line AC voltages, respectively. Also, the peak-to-peak voltage ripple $V_{\text {ripple }}$ in this case can be found as:

$$
V_{\text {ripple }}=\left(1-\frac{\sqrt{3}}{2}\right) V_{m}=0.1809 V_{d c}
$$

Thus, in order to reduce the output voltage ripple, implementation of the capacitive filter is essential.

\subsubsection{Controlled AC-DC Converter}

A fully controlled three-phase rectifier has been designed and implemented for coupling the DC microgrid with the AC power system. This converter is built using Semikron technology SKM50GB063D, IGBTs (Figure 3.13 (b)). The topology of this converter is shown in Figure 3.16. The implemented converter has the capability of controlling both the active and reactive power drawn from the grid independently. This is achieved by using a vector decoupling PWM control technique. Vector decoupling PWM control of three-phase rectifiers requires coordinated transformation to the d-q frame of reference in order to obtain the desired controllability. Moreover, the controlled rectifier utilizes a PI controller to regulate the output power. Compared with a diode rectifier, the 
controlled rectifier operates within acceptable limits of the total harmonic distortion (THD) for the current drawn from the AC source. It can also yield very low voltage ripple on the DC output. More details about the operation principle of this converter and its controller can be found in [125] and [135].

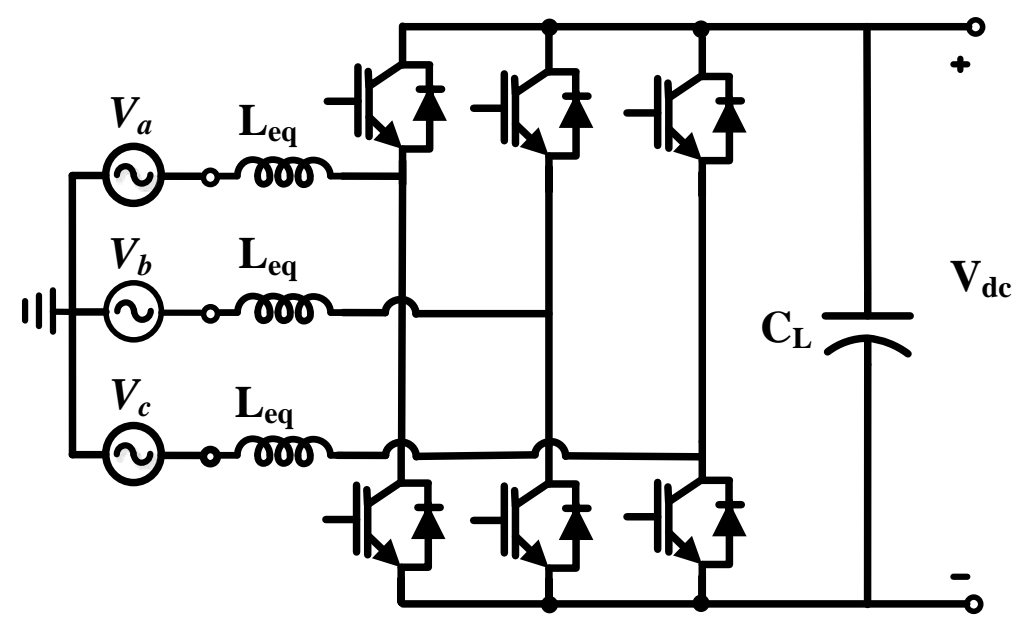

Figure 3.16: Three-phase controlled rectifier configuration.

\section{6. $\quad$ Filters}

The implemented filters in the hybrid DC power system test-setup include the AC filter and the DC filter. The AC filter is a three-phase line inductor that is implemented to reduce AC harmonics. Since the inductor impedance increases with frequency, it creates a larger impedance to the flow of higher order harmonic currents As a result, the current waveform is less discontinuous, resulting in a lower THD. The values of the inductors were chosen based on the value of effective impedance that forces THD to be less than 5\%.

Inspecting the output voltage of an uncontrolled rectifier shown in Figure 3.15, it can be seen that the output DC voltage pulsates at a frequency of six times that of the fundamental frequency of the AC source voltage. Therefore, an LC filter was used to reduce the voltage ripple. The LC filter is utilized to improve the performance of the DC- 
DC converters. Moreover, the capacitive filters were used at the output terminal of the DCDC converters to stabilize the DC bus voltage during the converters switching.

\subsection{Measuring and Protection Board}

Different circuit boards are implemented for DC and AC variable measurements. These boards are designed using Altium software and are implemented on printed circuit boards (PCBs). Also, an analog hysteresis protection board is designed for the protection of the supercapacitor bank against dangerous overvoltage. Moreover, the implemented hybrid DC power system includes an isolated switching board to boost the switching voltage signal and to protect the real-time control desk from short circuit faults that may occur in the grid. In this subsection, these boards are detailed and their performances are evaluated.

\subsubsection{Voltage and Current Measurement Board}

The design of printed circuit boards is necessary when highly reliable electrical circuits are required. Figure 3.17 shows the DC voltage and current boards implemented in PCB. In order to accurately measure the DC voltage and to isolate the measurement circuit from the power system, LV 25-P voltage transducers were utilized. Figure 3.18 (a) shows the configuration of the voltage measurement circuit board. The primary resistor $R_{T 1}$ is selected to adjust the input current of the transducer to its nominal current, which is 10$\mathrm{mA}$. Since the nominal DC bus voltage of the hybrid DC microgrid is $320-\mathrm{V}$, two $1.6-\mathrm{k} \Omega$ resistors are connected in series to form a 3-W, 3.2-k $\Omega R_{T 1}$. Also, the measurement resistor $R_{M}$ is $150-\Omega$. This will ensure very high accuracy voltage measurements with a 2500:1000 
current ratio. Moreover, in order to filter out high-frequency noises, $0.1 \mu \mathrm{F}$ ceramic capacitor $C_{T}$ is paralleled with the measurement resistor.

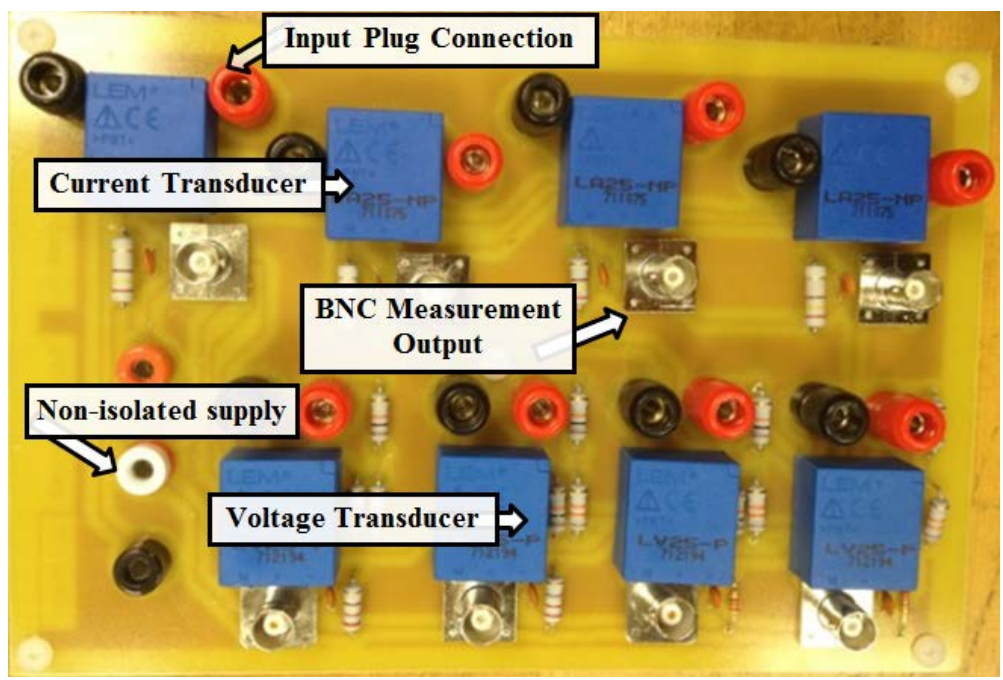

Figure 3.17: DC measurement board.

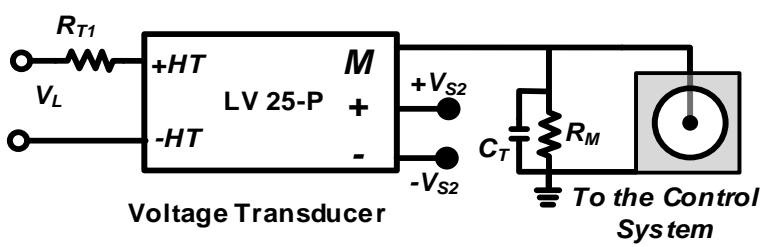

(a)

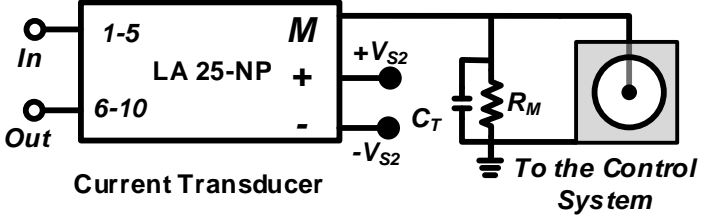

(b)

Figure 3.18: DC measurement circuit configuration. (a): Voltage measurement. (b) Current measurement.

Two types of current transducers were implemented for DC current measurements. The LA 25-NP transducers were utilized for current measurements up to 25-A. The LA 55 P was used for the higher current values up to 50-A. Figure 3.18 shows the configuration of the current measurement circuit board when LA 25-NP transducers were utilized. The measurement resistor $R_{M}$ was selected so that the nominal secondary current of the transducer became 25-mA to achieve the highest accuracy. A $0.1 \mu \mathrm{F}$ capacitor is in parallel to the output signal of the transducer to filter out any high-frequency noises. It should be 
noted that experimental results show that larger capacitors are not suitable for this application, as it may reduce the response speed of the measurement.

\subsubsection{Voltage Measurement with Common-Mode Rejection}

In applications such as battery and supercapacitor monitoring systems, a small differential voltage must be sensed in the presence of a high common voltage. In such a system, it is desired to remove the common voltage, which contains no useful information about the measurement, and could in fact reduce the measurement accuracy. Figure 3.19 shows the voltage measurement circuit with common-mode rejection. The voltage output of this circuit $V_{o}$ can be written as (3.9).

$$
V_{O}=\left(\frac{R_{2}+R_{1}}{R_{4}+R_{3}}\right)\left(\frac{R_{4}}{R_{1}}\right) V_{2}-\left(\frac{R_{2}}{R_{1}}\right) V_{1}
$$

In the case that the $R_{1}=R_{3}$ and $R_{2}=R_{4}$, (3.9) can be rewritten as:

$$
V_{O}=\left(\frac{R_{2}}{R_{1}}\right)\left(V_{2}-V_{1}\right)
$$

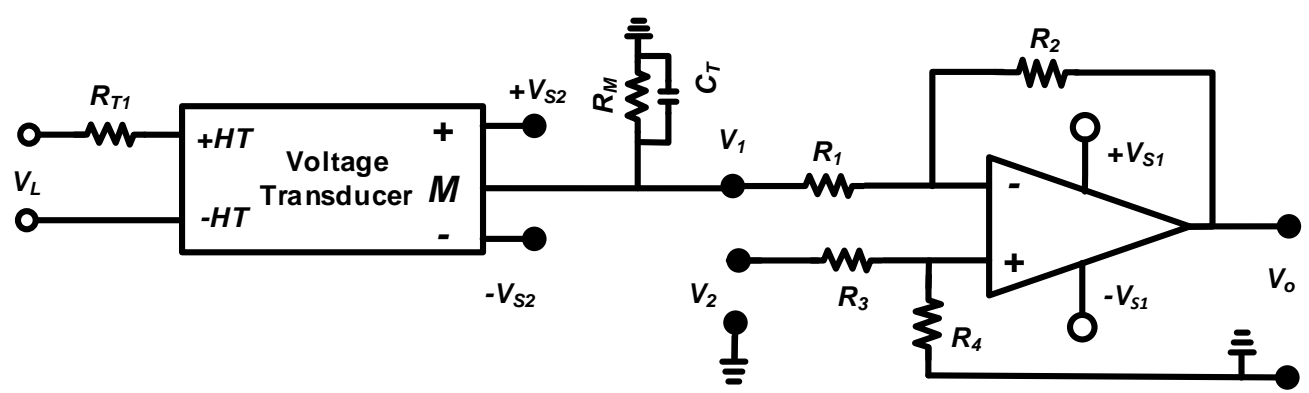

Figure 3.19: Voltage measurement with common-mode rejection.

For accurate measurement, the mirror resistors ( $R_{1}$ with $R_{3}$ and $R_{2}$ with $R_{4}$ ) should be very similar. The measurement system with common-mode rejection is designed based on the proposed configuration and is implemented on a printed circuit board (Figure 3.20). The measured voltage is compared with a $288-\mathrm{V}$ reference voltage and the difference is 
boosted 10 times. Due to a maximum voltage input limitation of the analog to digital input of dSPACE, the maximum voltage that is measurable is $332-\mathrm{V}$. This provides a very high accuracy in the voltage monitoring of the supercapacitor bank.

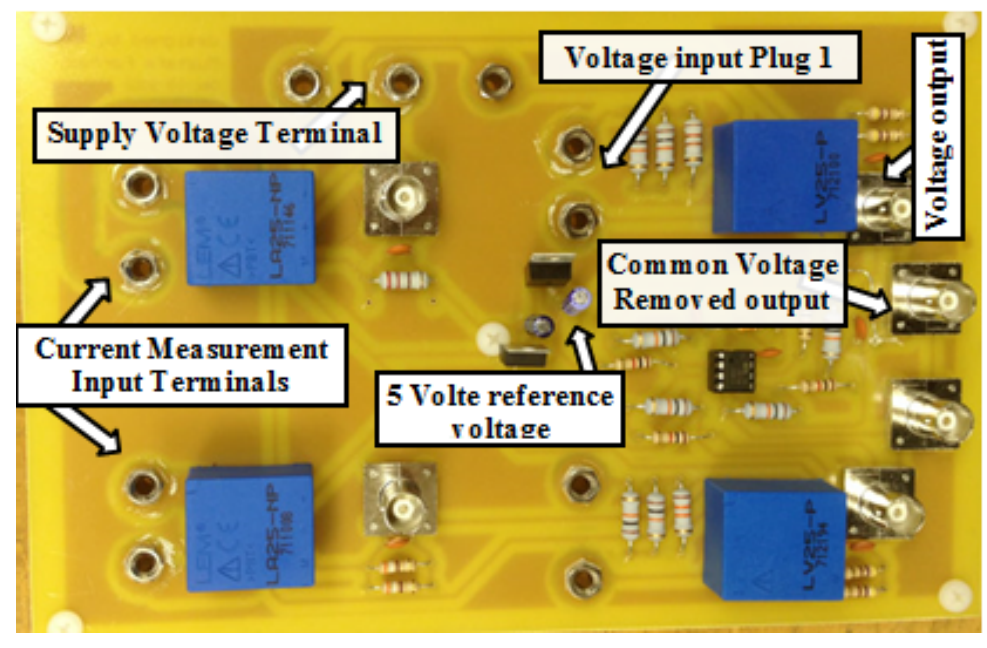

Figure 3.20: Measurement board with Common-Mode Rejection.

To show the effectiveness of the common-mode voltage rejection board, the voltage of a similar DC bus was measured via the common-mode rejection board and with the common voltage measurement circuit. Figure 3.21 shows the test results. As can be seen, in the case that the common voltage was not removed, the measurement was highly polluted with noises. On the other hand, the measurement of the common-mode rejection board was not affected by the noise. Hence, with this measurement technique, the voltage variation can be tracked properly and the control algorithm can work more accurately.

\subsubsection{Isolated switching signal board}

There are some problems and safety issues when using the real-time control boards for switching of the IGBTs. First, the maximum output signals from control boards are usually in the range of 3-10 V that is not sufficient to turn on the IGBTs. Also, the control board may be damaged due to excessive current drawn from IGBTs if a short circuit fault 
occurs in the microgrid or if the IGBTs burn. Moreover, in many applications, a floating switching signal is required, while the switching signals from the control board are usually grounded. Thus, in order to isolate the switching signal from the control board, as well as boost the signal, isolation of the switching in the PCB was designed.
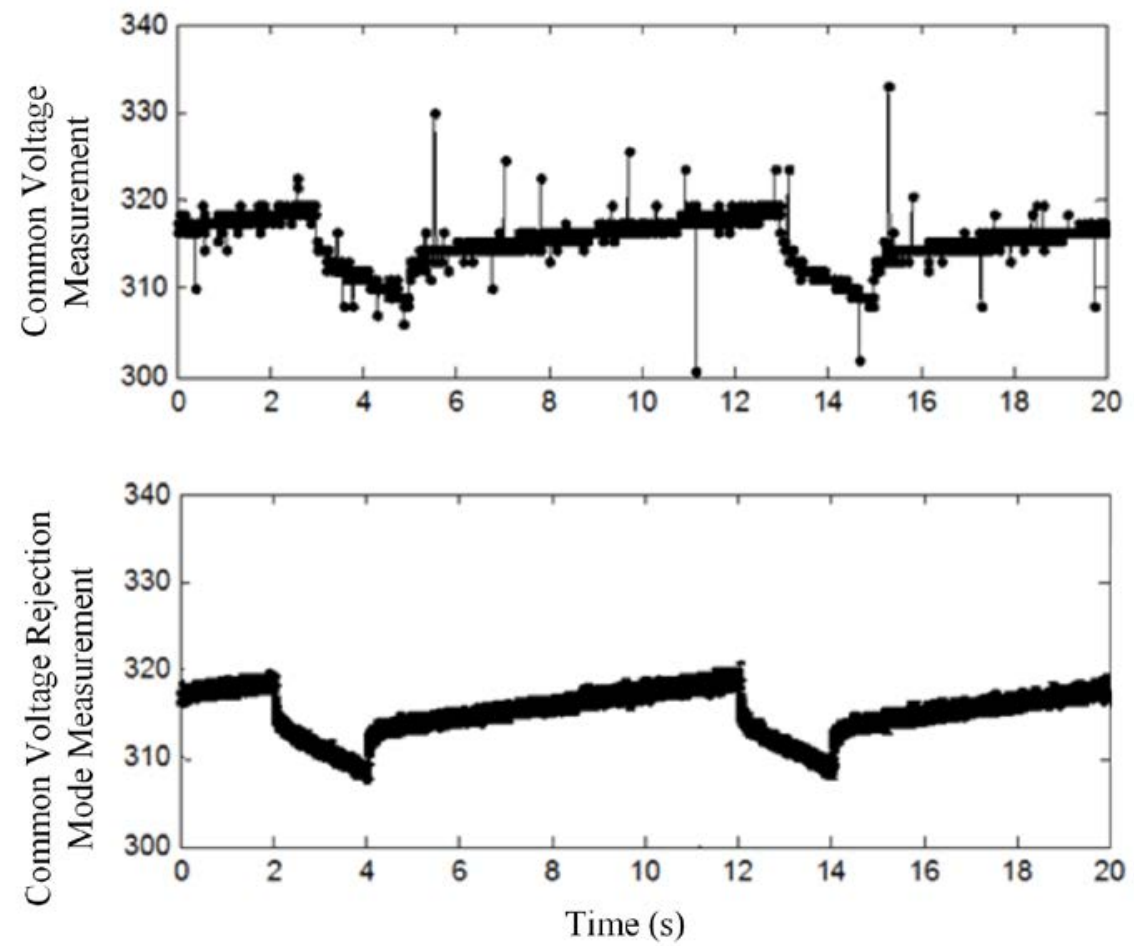

Figure 3.21: Experimental test results for two measurement modes.

Figure 3.22 shows the PCB for isolating the switching signal that is composed of an isolated DC-DC converter and optocoupler. The board provides four channels of a 15 V isolated signal with flotation reference voltage at maximum $100 \mathrm{kHZ}$. This PCB is used for the switching of the IGBTs of different DC-DC converters and load emulators. 


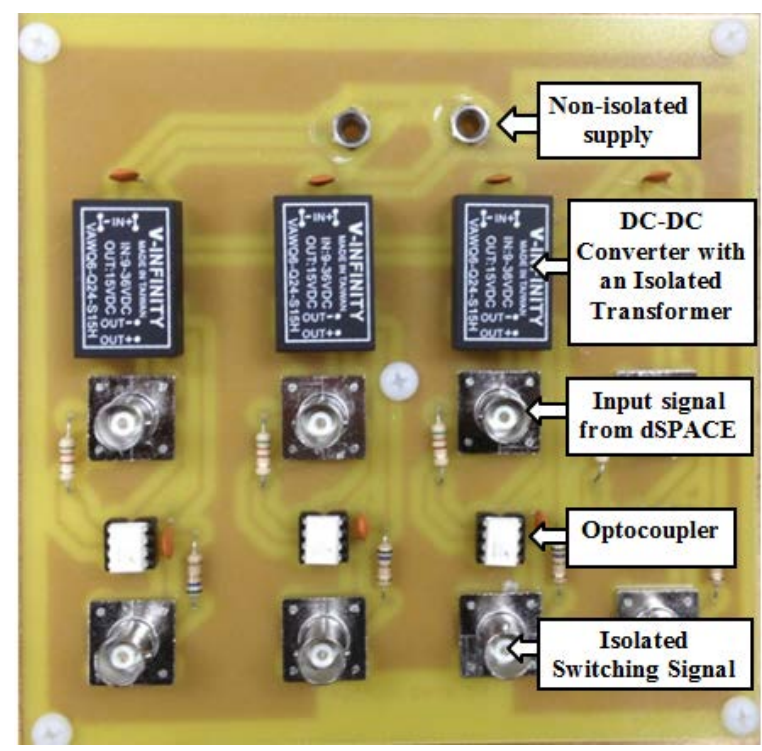

Figure 3.22: Isolated switching signal board.

\subsubsection{AC Voltage and Current Measurement Box}

Special boxes were built for measurement and filtering purposes. These boxes also have switching and protection functions. The current and voltage of each line is measured via current transformer (CT) and potential transformer (PT). The PTs are connected between phases and ground, so they can accurately measure the line-to-ground voltage. For control and monitoring purpose, the secondary sides of these CTs and PTs are connected to the real-time control boards. The current and voltage ratios for the PT and CT are 3:1and 20:1, respectively. Also, these boxes include an AC filter consisting of 24-mH inductors that are connected in series in each line. The inductor can also be reduced to $12-\mathrm{mH}$. The AC box components are designed for $208-\mathrm{V}$ line-to-line nominal voltage (120-V line-toground) and 15-A current per phase. As a result, the power transferring capacity for the three-phase system with nominal voltage is $5.4-\mathrm{kVA}$.

The AC measurement boxes are all equipped with a CRYDOM D53TP25D solidstate relay, whose switching can be controlled by a 3-32 V DC voltage via a real-time 
control board. The real-time control system can switch any branch according to the desired configuration and the real-time monitored voltage and current data. Also, in order to prevent damaging hardware components in the test-bed setup, all lines are protected by fuses.

\subsubsection{Analog Hysteresis Voltage Protection}

One of the important issues in the high voltage applications of the supercapacitor bank is the uneven charge distribution between the supercapacitor cells during fast charge. Uneven charge distribution can cause dangerous overvoltage in a cell while the overall bank voltage is under the high voltage threshold.

To solve the problem of uneven charge distribution, each 58-F supercapacitor module is protected by a passive balancing circuit. This balancing circuit consists of a resistor across each cell with the same resistance. This allows all cells to have the same voltage once the balancing process is completed. The passive balancing of the 58-F module draws 25-mA when the module is completely charged to $16-\mathrm{V}$.

Additionally, the analog hysteresis protection is designed to ensure that uneven charge distribution, especially during very fast charges, does not cause the supercapacitor to exceed its maximum voltage.

Figure 3.23 shows a simplified block diagram of the analog hysteresis voltage protection circuit. The hysteresis characteristics of the protection circuit prevent system oscillation. Every array of four 58-F supercapacitor modules is monitored. The voltage transducer measures the array voltage $V_{A r}$, while it isolates the protection circuit from the supercapacitor bank. 


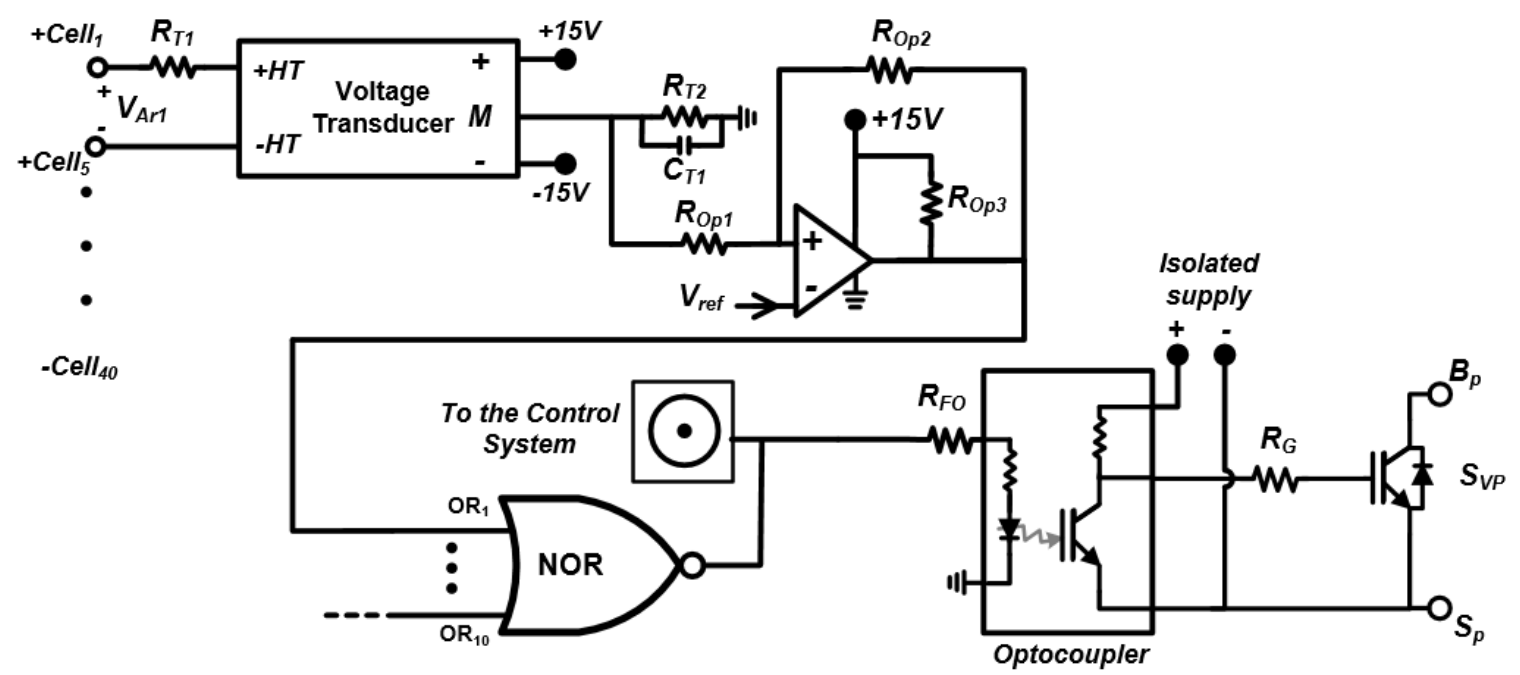

Figure 3.23: Schematic diagram of the analog hysteresis voltage protection.

Suppose that the high voltage level for the protection circuit activation is $V_{A r H}$, and its low limit is $V_{\operatorname{ArL}}$, while the reference voltage for the comparator is $V_{\text {ref }}$. It can be shown that the $V_{A r H}$ and $V_{A r L}$ can be expressed as:

$$
\begin{gathered}
V_{\text {ArH }}=\left[\left(R_{O p 1}+R_{O p 2}\right) V_{r e f}-\left(R_{O p 1} V_{O L}\right)\right] \frac{R_{T 1}}{R_{T 2} R_{O p 2} K_{n}} \\
V_{\text {ArL }}=\left[\left(R_{O p 1}+R_{O p 2}\right) V_{r e f}-\left(R_{O p 1} V_{O H}\right)\right] \frac{R_{T 1}}{R_{T 2} R_{O p 2} K_{n}}
\end{gathered}
$$

where, $V_{O L}$ and $V_{O H}$ are the low voltage level of the op-amp when it is off and high voltage level when it is on, respectively. Here, $K_{n}$ is the transducer conversion ratio (the ratio between the secondary current to the primary current of the voltage transducer). The other parameters can be found in Figure 3.23.

In an ideal circuit, it can be assumed that $V_{O L}$ is zero and $V_{O H}$ is equal to the opamp supply voltage $V_{S}$. Thus, equations (3.11) and (3.12) can be rewritten as:

$$
V_{A r H}=\frac{\left(R_{O p 1}+R_{O p 2}\right) R_{T 1} V_{r e f}}{R_{T 2} R_{O p 2} K_{n}}
$$




$$
V_{A r L}=\left[\left(R_{O p 1}+R_{O p 2}\right) V_{r e f}-\left(R_{O p 1} V_{S}\right)\right] \frac{R_{T 1}}{R_{T 2} R_{O p 2} K_{n}}
$$

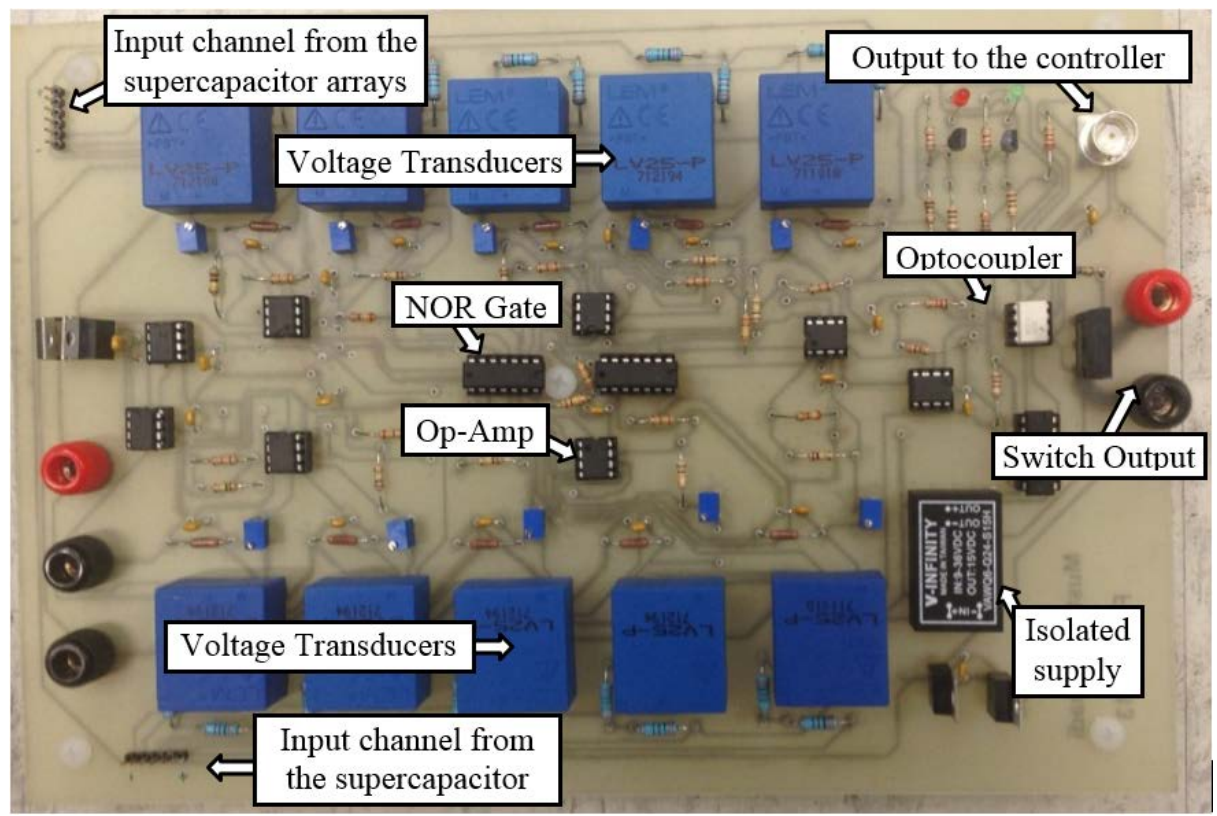

Figure 3.24: Analog hysteresis voltage protection circuit board.

For the proposed supercapacitor voltage protection circuit, based on the primary and secondary nominal current of the voltage transducer, $6.6-\mathrm{k} \Omega$ and $100-\Omega$ resistor for the $R_{T 1}$ and $R_{T 2}$ were selected. Also, since the rated voltage of the supercapacitor array is 64$\mathrm{V}, 1-\mathrm{k} \Omega$ and $100-\mathrm{k} \Omega$ resistors were selected for $R_{O p 1}$ and $R_{O p 2}$. This is done so that the $V_{\text {ArH }}$ and $V_{\text {ArL }}$ adjust to $66.67-\mathrm{V}$ and $62.68-\mathrm{V}$, respectively. If the voltage on any of the ten supercapacitor arrays exceeds the $V_{A r H}$, the output of the OR gate will be low and the IGBT switch $S_{V P}$, connected in series with supercapacitor bank, blocks the charging path. The charging path remains open until the supercapacitor is discharged through the bypass diode to the point that its voltage is less than $V_{\text {ArL }}$. Figure 3.24 shows the circuit board of the supercapacitor analog hysteresis voltage protection. 


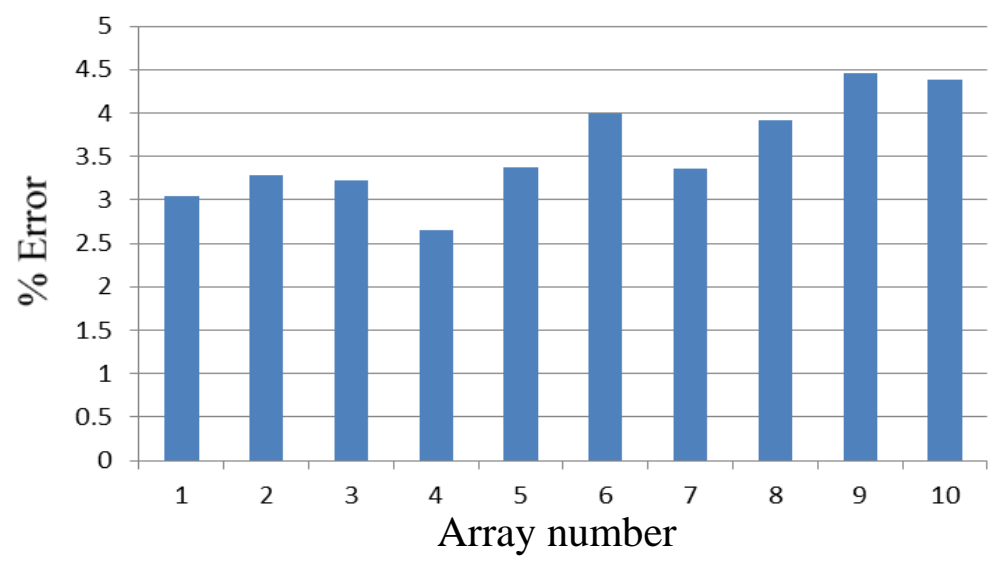

Figure 3.25: Relative error of the $V_{\mathrm{ArH}}$ for the ten supercapacitor arrays.

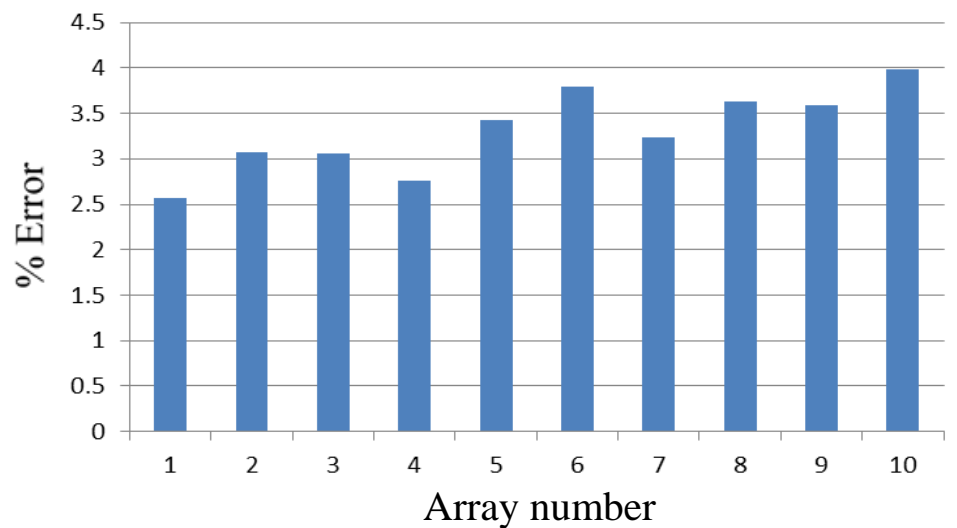

Figure 3.26: Relative error of the $V_{A r L}$ for the ten supercapacitor arrays.

The analog hysteresis voltage protection board was tested to verify its accuracy.

Figure 3.25 shows the relative error of the $V_{A r H}$ with respect to its nominal 66.67-V. As can be seen, the maximum error was only $4.49 \%$ and the average error was 3.57\%. Figure 3.26 shows the relative error of the $V_{\text {ArL }}$ for the ten arrays with respect to the designed threshold of $62.68-\mathrm{V}$. The experimental results show that the relative errors of the arrays were less than $4 \%$ and the average was only 3.31\%. The accuracy of the analog hysteresis protection circuit and the passive balancing capability of the supercapacitor module ensure the reliable operation of the supercapacitor bank. 


\section{8. $\quad$ AC Grid Test-Bed System Description}

The AC power system under study is a $72-\mathrm{kW}$ smart grid test-bed, which is developed in the Energy System Research Laboratory at Florida International University [135]-[136]. Figure 3.27 shows the schematic diagram of the AC grid test-bed. This system includes four generating stations, which are connected in a ring configuration through the transmission lines. The power lines are designed based on the $\pi$-model, which emulates power lines with different lengths through a series inductor and two parallel capacitors.

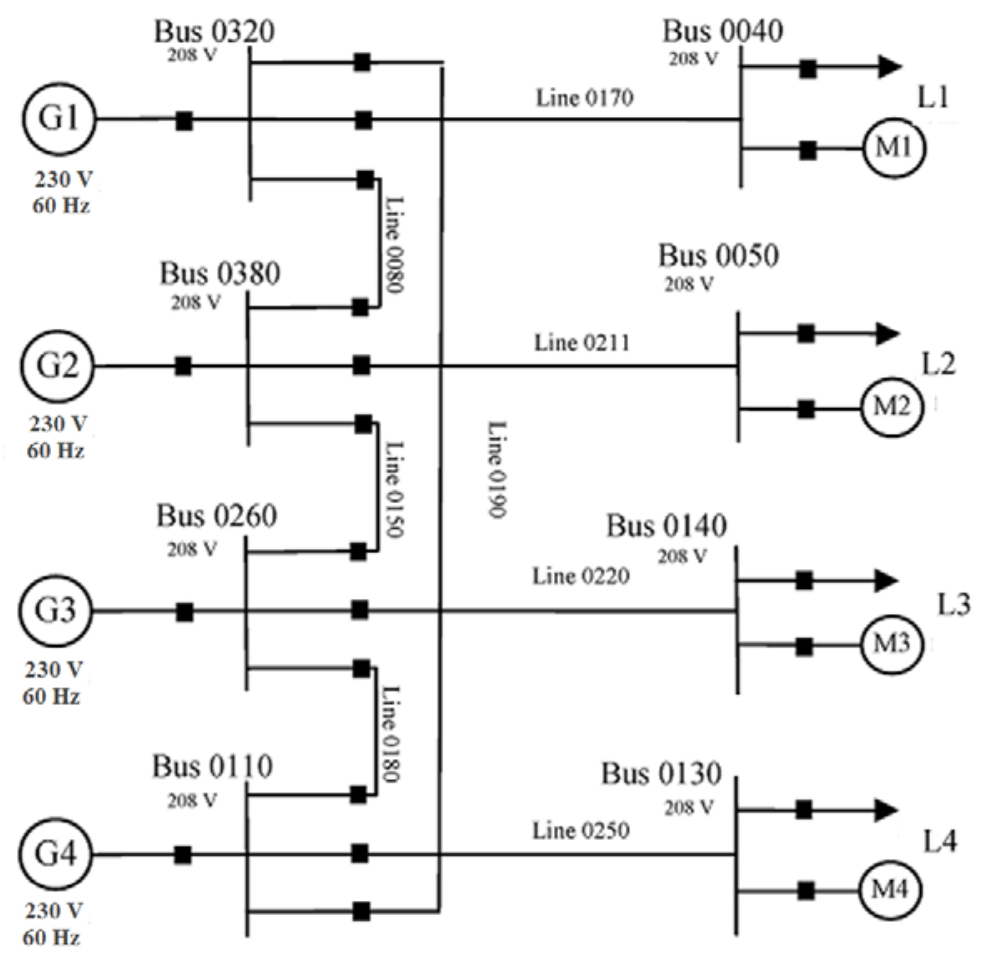

Figure 3.27: Schematic diagram of the smart grid test-bed.

The current and the voltage of the generator stations and transmission lines are measured via CTs and PTs, respectively. The secondary sides of these CTs and PTs are connected to the data acquisition systems (DAQs) through the communication terminals. Also, the LabVIEW software is utilized for real-time monitoring and control of the AC grid. The real-time control system can switch any branch according to the overall system 
strategy and the monitored voltage and current data. Further details on the AC grid testbed specifications and configuration can be found in [135]-[136].

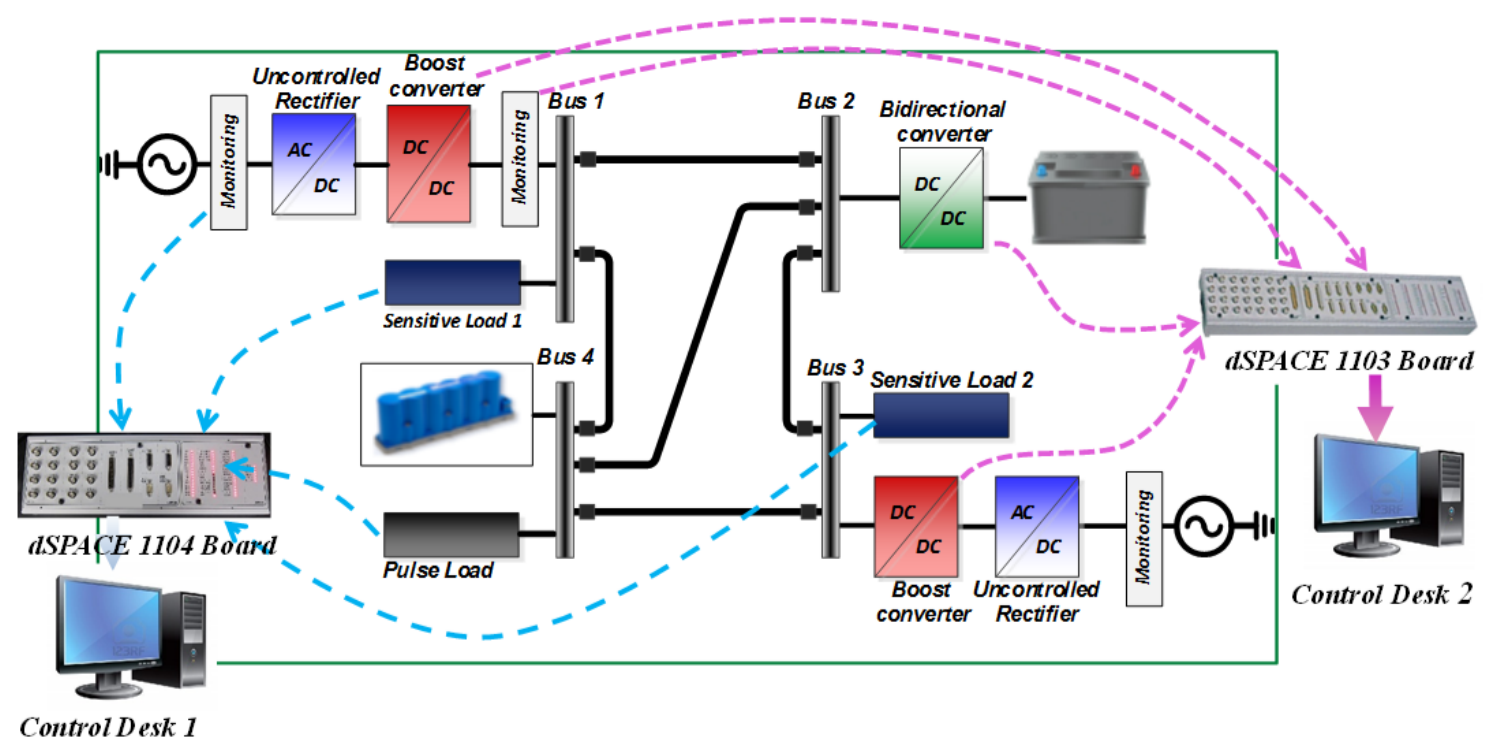

Figure 3.28: Monitoring and control infrastructure of the hybrid DC microgrid.

\subsection{Real-Time Control and Monitoring of the Hybrid DC power System}

Figure 3.28 shows an example of the hybrid DC microgrid that is connected in a ring configuration. This microgrid is composed of four DC buses with three different types of sources that supply a steady state load, a dynamic load and a heavy pulse load. The two AC generators are mainly supplying the microgrid while the battery bank provides extra power when the grid is highly loaded and/or any of the generators is out of service. Also, the supercapacitor bank, as an energy buffer, delivers high instantaneous power to the pulse load. The supercapacitor bank of the designed redundant microgrid is able to support the grid during the transient time and startup of the dynamic load.

Figure 3.28 also shows the strategy for the main control and monitoring system of the hybrid DC microgrid. As can be seen, the dSPACE 1103 real-time control module is utilized for the control of the power converters implemented in the hybrid DC microgrid. 
In the notional DC microgrid shown in Figure 3.28, dSPACE 1103 board is utilized for the energy management and control of the DC-DC boost converter implemented in bus 1 and 3 , and also for the control of the bidirectional converter implemented in bus 2. Moreover, this control board is utilized for the monitoring of the hybrid DC microgrid system.

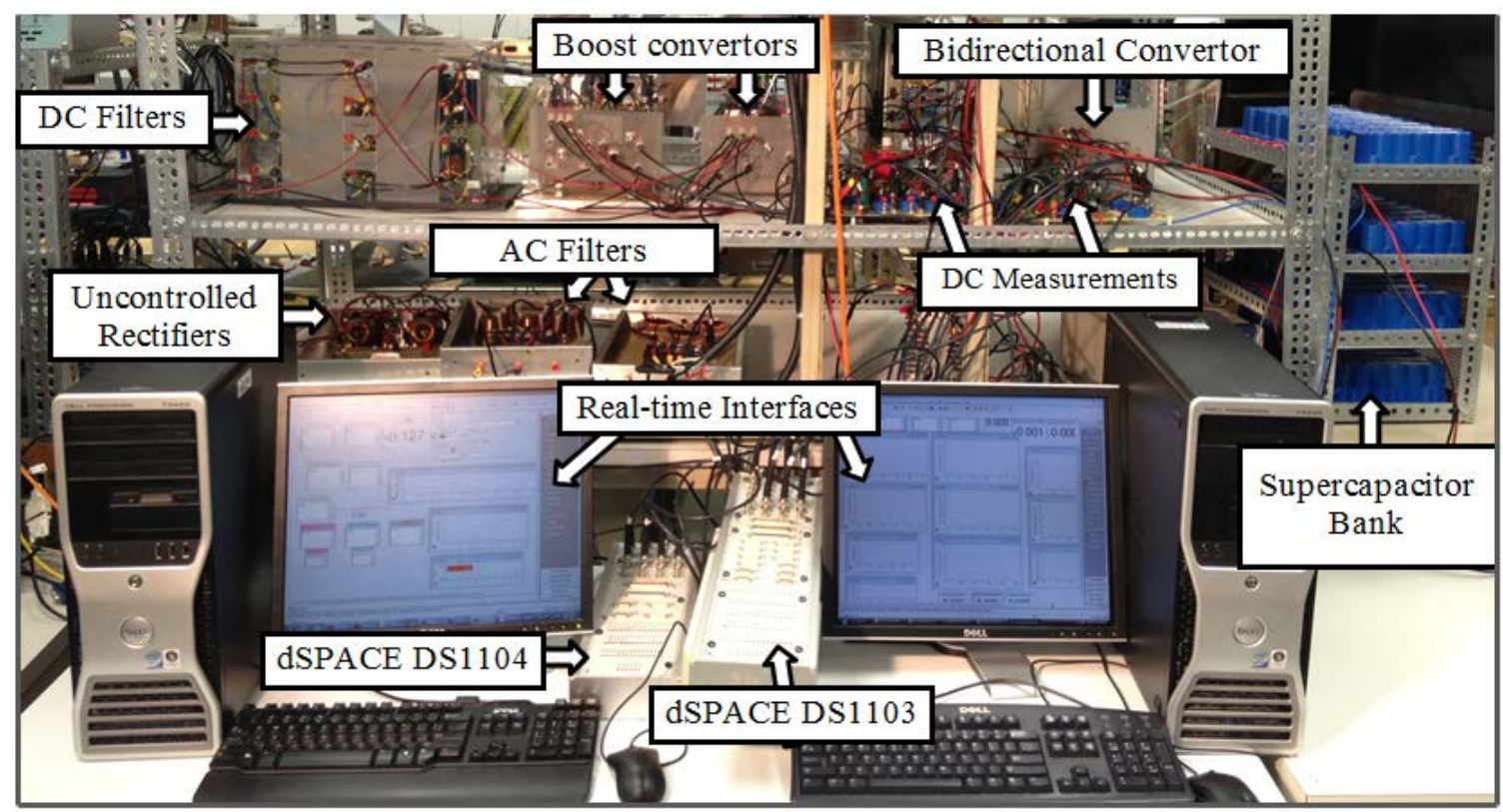

Figure 3.29: Control desk and monitoring system of the hybrid DC microgrid.

As shown in Figure 3.28, the dSPACE 1104 board was utilized to control and adjust the dynamic load, the pulse load and the steady state load. Also, this board is utilized for the control and monitoring of the power transferred to the AC generators. The experimental test setup of the hybrid DC microgrid including the control desk and monitoring system is shown in Figure 3.29. 


\section{REAL-TIME CONTROL AND ENERGY MANAGEMENT OF HYBRID DC POWER SYSTEM}

\subsection{Introduction}

In this chapter, the connectivity issues related to linking two DC buses featuring different voltage characteristics is investigated. The focus is to achieve effective bidirectional energy transfer between two DC buses whose voltages contain significantly different content of ripple. Various experiments are performed for bidirectional power flow under different system configurations and control modes.

Also, in this section, the real-time operation and harmonic analysis of isolated and non-isolated DC microgrid featuring hybrid energy sources with various loading schemes are investigated. In order to properly manage the energy and prevent the AC grid power pulsation, a current-voltage control technique based on the master-slave control concept is proposed. In this technique, the supercapacitor bank operates as the master and controls the DC bus voltage. The converters are working in current control mode and are equipped with hysteresis voltage control, which monitors the entire supercapacitor bank. Various operating modes based on different power sharing patterns were defined. The experimental test is carried out for both galvanically isolated and non-isolated DC grid systems. The results showed that the proposed energy management algorithm properly shares power and controls the voltage. Also, depending on the power sharing pattern, the isolation of the DC microgrid significantly affects the harmonic content of the current. 


\subsection{Bidirectional Energy Transfer in DC Microgrid}

In this section, the connectivity issues related to linking two DC buses featuring different voltage characteristics, in terms of voltage level and ripple, are identified, analyzed and discussed. A controlled DC-DC converter was used as the main bidirectional energy transfer enabler between the two buses. A three-phase full wave diode rectifier was used to achieve the high voltage ripple bus, whereas a three-phase controllable IGBT based rectifier was used as a pure DC bus. The focus of this section is on how to achieve the best controllability and performance levels of the bidirectional converter when it is linking a DC bus with a relatively high voltage ripple to another with a small ripple. An experimental test setup was devised to investigate and compare the performance of each configuration.

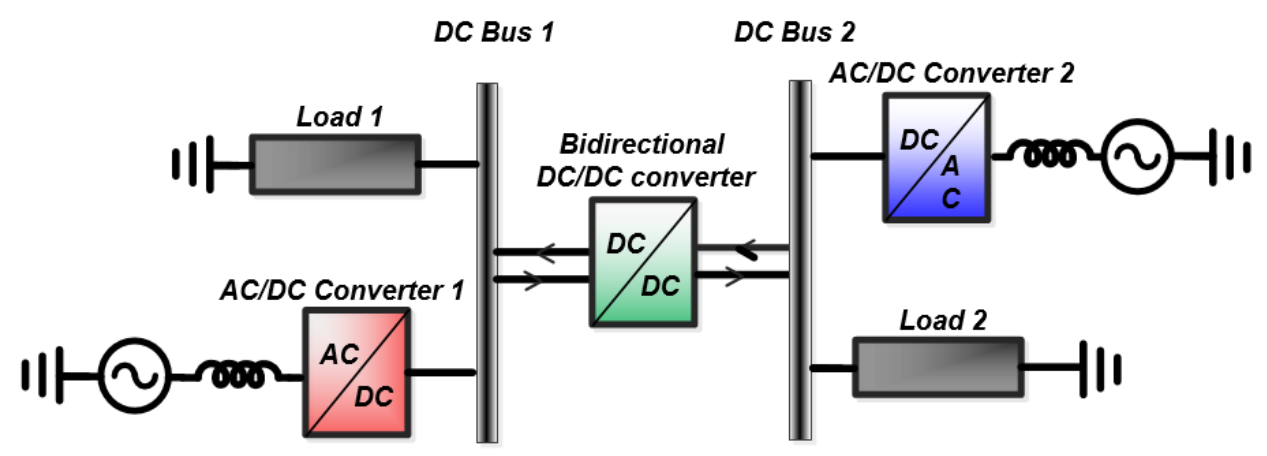

Figure 4.1: A schematic diagram for the bidirectional energy transfer study: two DC buses linked with a DC/DC converter.

\subsubsection{Description of the Case Studies}

Figure 4.1 shows the configuration of the system under study. The main components of this system include two DC buses, two AC-DC converters, two DC loads and a bidirectional DC-DC converter. A three-phase full wave diode rectifier was implemented as a high voltage ripple source, and a three-phase controllable IGBT based rectifier was used as a pure DC source. The bidirectional converter is a buck-boost 
converter that is operating in the current controlled mode. The low voltage (LV) side of the converter is connected to $24-\mathrm{V}$ DC bus, whereas the high voltage (HV) side is connected to 32-V DC bus. The voltage and current measurement transducers were placed on both sides of the bidirectional converter. Hence, the current feedback signal can be received either from the measurement at bus 1 or bus 2. Depending on which bus has high voltage ripple and whether the reference current was taken from the terminal with high voltage ripple or low voltage ripple, four case studies were defined as follows:

Case I: The uncontrolled rectifier, resulting in high voltage ripple, is connected to the LV 24-V DC bus 1, whereas the controlled rectifier was connected to the HV 32-V DC bus 2, and the current feedback was taken from the HV terminal.

Case II: The uncontrolled and controlled rectifier connection is similar to Case I; however, the current feedback was taken from the LV terminal.

Case III: The uncontrolled rectifier was connected to the HV 32-V DC bus 2, whereas the controlled rectifier was connected to the LV 24-V DC bus 1, and the current feedback was taken from the LV side.

Case IV: The uncontrolled and controlled rectifier connection is similar to Case III; however, the current feedback was taken from the HV terminal.

\subsubsection{Description of the Test Setup}

The bidirectional DC-DC converter with the topology shown in Figure 3.11 was implemented in hardware. The inductor and capacitor used in the converter are 2.7-mH and $1200-\mu \mathrm{F}$, respectively. The maximum input current into the LV side was limited to 12-A. For control and monitoring purposes, two voltmeters and two ammeters were connected to measure the current and voltage at the two terminals. In practice, for bidirectional energy 
flow, the DC-DC converters are usually operated in the current control mode. In this case, one of the ammeters may be enough for the current feedback of the controller. However, the other meters can still be placed for monitoring purposes.

A scaled-down setup of the system shown in Figure 4.1 was constructed to study the effect of high voltage ripple on the control of the energy flow between two DC buses. The nominal voltage of bus 1 and bus 2 are 24-V and 32-V, respectively. A 145-W and a 250-W constant loads were connected to bus 1 and bus 2, respectively. Two rectifiers were used. One rectifier is the three-phase full wave uncontrolled rectifier with an output voltage that can be adjusted by an autotransformer to be either $32 \mathrm{~V}$ or $24-\mathrm{V}$. In addition, a controlled AC-DC converter with very low voltage ripple, which operates in a voltage control mode, is used as a second source.

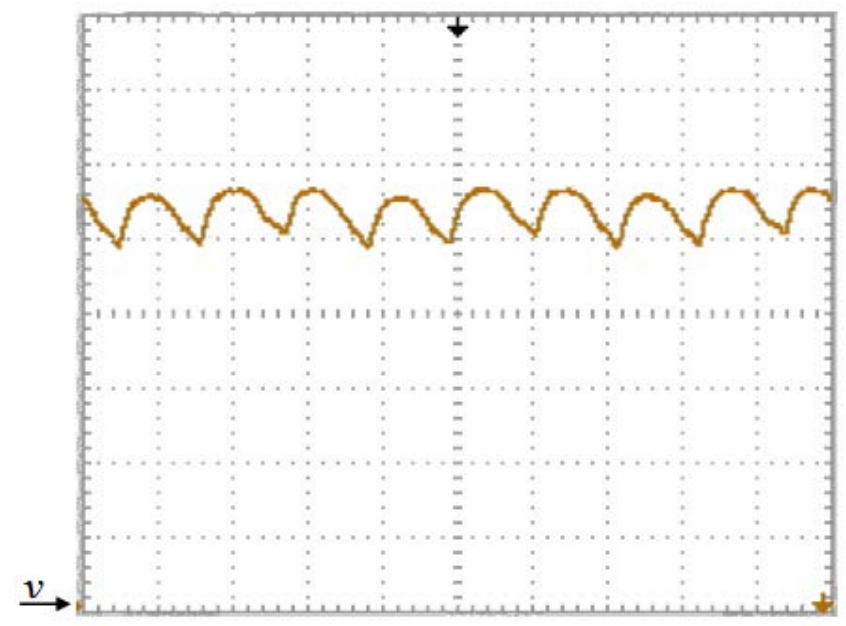

Figure 4.2: Output voltage of the three-phase full wave uncontrolled rectifier: $6.0 \mathrm{~V} / \mathrm{div}$, $2.5 \mathrm{~ms} / \mathrm{div}$.

\subsubsection{DC bus with High Voltage Ripple}

Figure 4.2 shows the output voltage of the three-phase full wave rectifier when it is adjusted to supply a $250-\mathrm{W}, 32-\mathrm{V}$ load. As can be seen, the peak to peak variation of the output voltage is $4.83-\mathrm{V}$, and as a result, the regulation factor, which is the ratio between 
the peak to peak ripple to the average DC voltage, is more than $15 \%$. Also, the fundamental frequency of the ripple voltage is $360-\mathrm{HZ}$ that is six times the AC supply frequency.

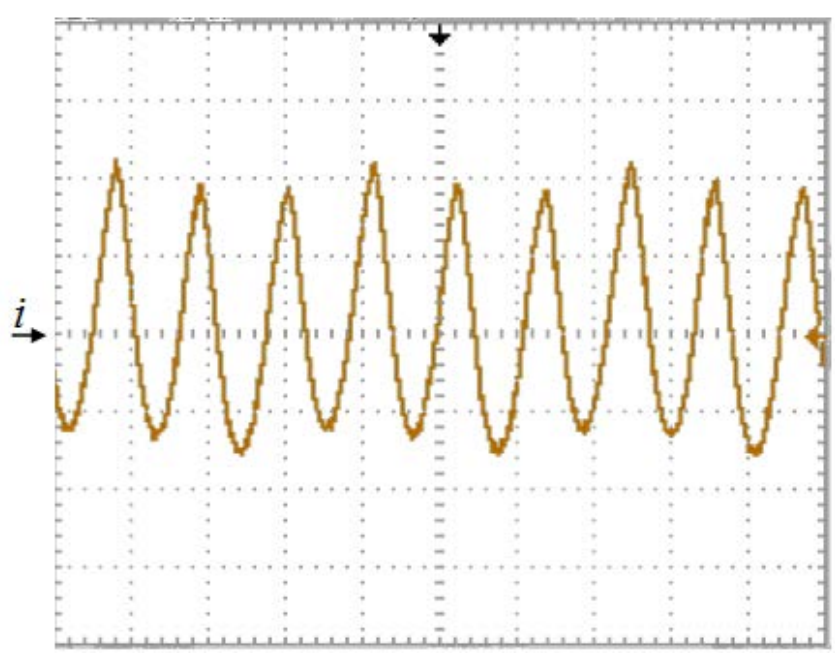

Figure 4.3: Backward current to the bidirectional DC-DC converter when the converter is off: $1.65 \mathrm{~A} / \mathrm{div}, 2.5 \mathrm{~ms} / \mathrm{div}$.

\subsubsection{Effect of Voltage Ripple}

Suppose that the three-phase full wave rectifier and its load are connected to bus 2 where the capacitor of the bidirectional DC- DC converter is also located. Furthermore, we also suppose that the bidirectional converter does not transfer any energy between the two busses. As a result of the voltage ripple of the DC bus, the capacitor will charge and discharge periodically. In this case, the capacitor acting as a filter will reduce the regulation factor to $9.8 \%$. The drawback of this phenomenon is that the ammeter at the HV terminal will see some current while the converter is off and, consequently, there will be more issues for controlling the bidirectional power flow. In other words, the difficulty of controlling the power flow is not only the voltage variation, but also the high current ripple. Figure 4.3 shows the backward current from bus 2 to the bidirectional converter. As can be seen, the peak to peak current varies between -2.30 to 3.63-A. 


\subsubsection{Experimental Test Results}

In order to study the bidirectional power transfer in a DC power system, four case studies were defined. In this subsection, the experimental tests were performed and the results are analyzed.

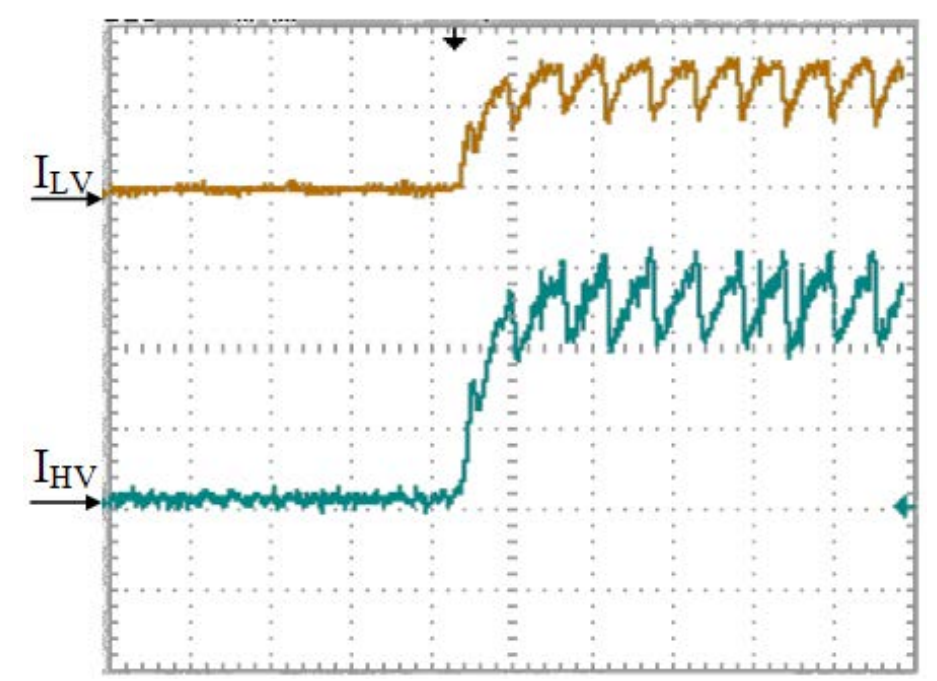

Figure 4.4: Drawn current from bus $1\left(\mathrm{I}_{\mathrm{LV}}\right)$ and injected current into the bus $2\left(\mathrm{I}_{\mathrm{HV}}\right)$ when the reference of the $\mathrm{I}_{\mathrm{HV}}$ is changed from 0 to $4 \mathrm{~A}$. $\mathrm{I}_{\mathrm{LV}}: 5.0 \mathrm{~A} / \mathrm{div}, 25 \mathrm{~ms} / \mathrm{div}$; $\mathrm{I}_{\mathrm{HV}}: 1.65$ A/div, 25 ms/div.

\subsubsection{Case I}

In the first case study, the three-phase full wave uncontrolled rectifier was connected to bus $1(\mathrm{LV})$ and the current control is based on $\mathrm{I}_{\mathrm{HV}}$. Figure 4.4 shows the experimental test results when the reference of $\mathrm{I}_{\mathrm{HV}}$ is changed from 0 to $4-\mathrm{A}$. As can be seen, the actual current reaches its steady state value in less than 25-ms with zero overshoot and zero steady state error. Figure 4.5 shows $I_{L V}$ and $I_{H V}$, when the reference of $I_{H V}$ is changed from 4 to -3-A. Similarly, in this case, the steady state error is zero and the transient time is less than 25-ms. However, there is about 0.5-A overshoot in $\mathrm{I}_{\mathrm{HV}}$. 


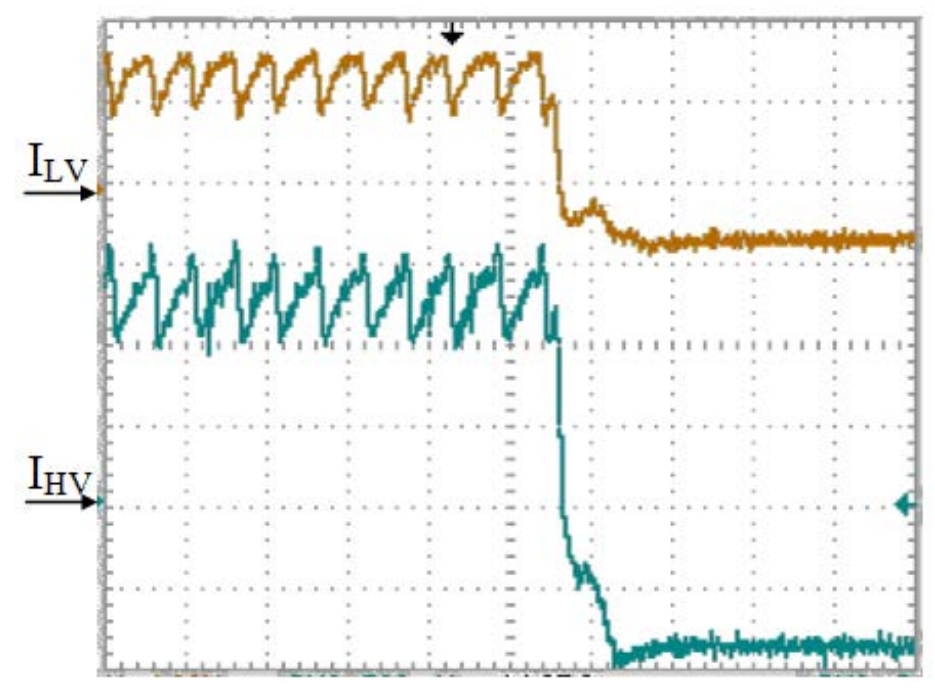

Figure 4.5: $\mathrm{I}_{\mathrm{LV}}$ and $\mathrm{I}_{\mathrm{HV}}$ when the reference of $\mathrm{I}_{\mathrm{HV}}$ is changed from 4 to $-3 \mathrm{~A}$. $\mathrm{I}_{\mathrm{LV}}$ : 5.0 $\mathrm{A} / \mathrm{div}, 25 \mathrm{~ms} / \mathrm{div} ; \mathrm{I}_{\mathrm{Hv}}$ : $1.65 \mathrm{~A} / \mathrm{div}, 25 \mathrm{~ms} / \mathrm{div}$.

Based on the experimental test results shown in Figure 4.4 and Figure 4.5, when the energy flow is negative (i.e. power is flowing from bus 2 to bus 1 ), the injected and drown current ripple is very small. However, in the case of positive power flow, there is a considerable current ripple in both $\mathrm{I}_{\mathrm{HV}}$ and $\mathrm{I}_{\mathrm{LV}}$. When $\mathrm{I}_{\mathrm{HV}}$ is 4-A, the peak to peak variation of the current is about 2-A. Also, in this case, the average current of $\mathrm{I}_{\mathrm{LV}}$ is about 5.5-A and its peak to peak ripple is 3-A. It can be noticed that current ripple frequency is about five times slower than the voltage ripple of the uncontrolled rectifier. This shows that the controller is also affected by the voltage ripple. This current ripple can be reduced by increasing the speed response of the controller; however, the experimental test results has shown that with a faster controller, the converter will be unstable for high current transfer.

\subsubsection{Case II}

In the second case study, the three-phase full wave uncontrolled rectifier is connected to bus 1 , whereas $I_{L V}$ is the reference current for the controller of the bidirectional DC-DC converter. Figure 4.6 shows $\mathrm{I}_{\mathrm{LV}}$ and $\mathrm{I}_{\mathrm{HV}}$ when the reference $\mathrm{I}_{\mathrm{LV}}$ is 
changed from -4 to 2-A. Also, Figure 4.7 shows $\mathrm{I}_{\mathrm{LV}}$ and $\mathrm{I}_{\mathrm{HV}}$ when the reference current is increased from 2 to 6-A. As can be seen, in both step changes of the reference, the controller responded in less than 25 -ms and the steady state error is zero. However, when the reference $\mathrm{I}_{\mathrm{LV}}$ is changed from 2 to 6 - $\mathrm{A}$, there are $8 \%$ and $19 \%$ overshoots in $\mathrm{I}_{\mathrm{LV}}$ and $\mathrm{I}_{\mathrm{HV}}$, respectively.

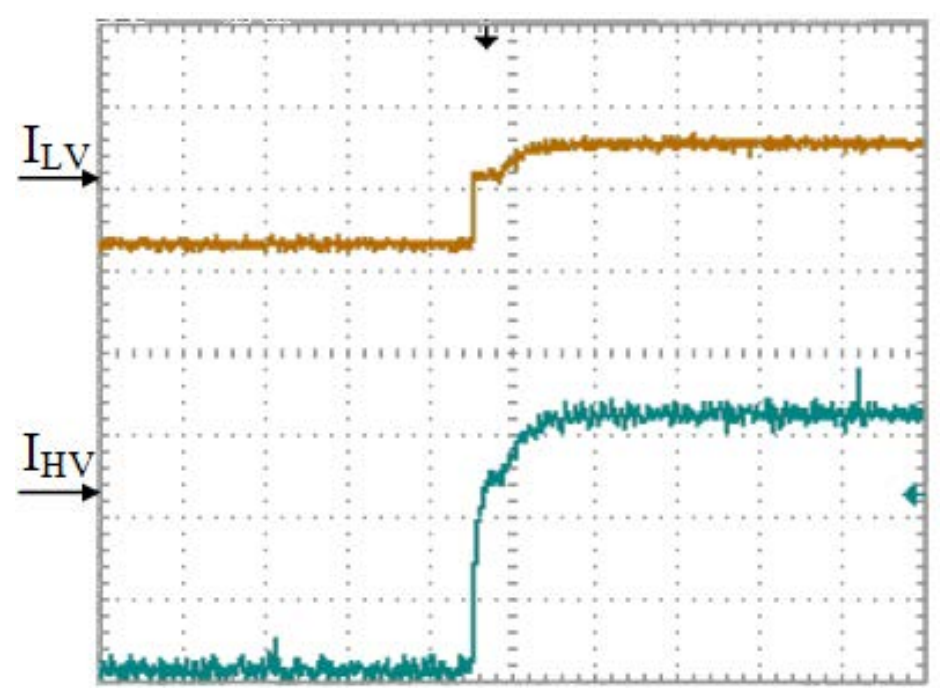

Figure 4.6: $\mathrm{I}_{\mathrm{LV}}$ and $\mathrm{I}_{\mathrm{HV}}$ when the reference of $\mathrm{I}_{\mathrm{LV}}$ is changed from -4 to $2 \mathrm{~A}$. $\mathrm{I}_{\mathrm{LV}}$ : 5.0 A/div, 25 ms/div; IHv: $1.65 \mathrm{~A} / \mathrm{div}, 25 \mathrm{~ms} / \mathrm{div}$.

In contrast to the first case study, when $\mathrm{I}_{\mathrm{LV}}$ is the reference of the controller, there is a small current ripple for both directions of the power flow. A more detailed study revealed that for the positive power flow, the frequency of the current ripple is the same as the voltage ripple of $360-\mathrm{Hz}$. The successive control of this scheme can be because of the fact that the controller measures the actual current at the terminal that the uncontrolled rectifier is connected to. As a result, it can be adapted by the voltage variation faster. 


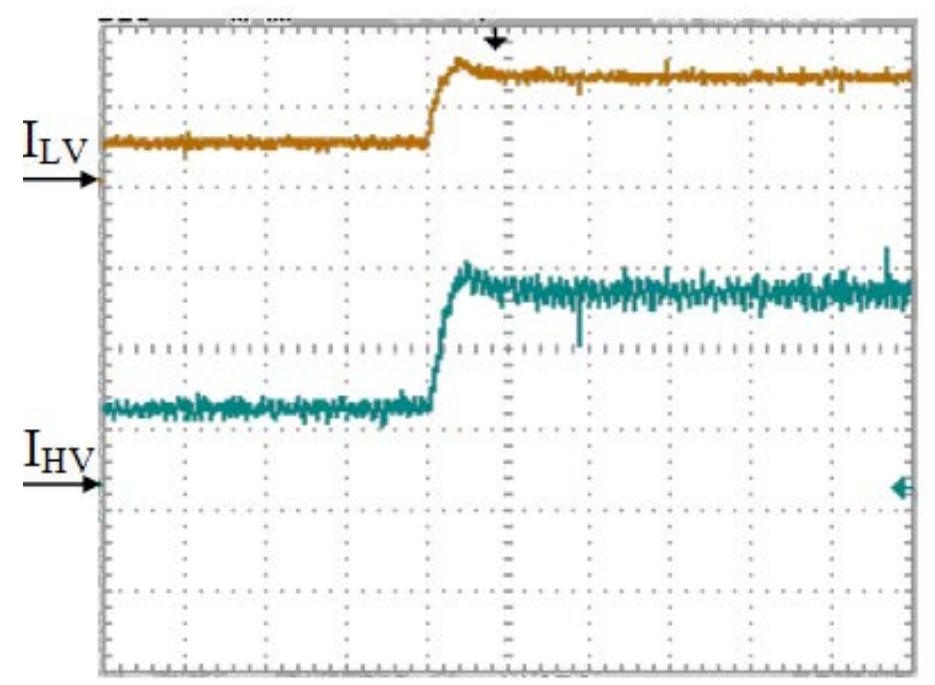

Figure 4.7: $\mathrm{I}_{\mathrm{LV}}$ and $\mathrm{I}_{\mathrm{HV}}$ when the reference of $\mathrm{I}_{\mathrm{LV}}$ is changed from 2 to 6 A. $\mathrm{I}_{\mathrm{LV}}$ : 5.0 $\mathrm{A} / \mathrm{div}, 25 \mathrm{~ms} / \mathrm{div} ; \mathrm{I}_{\mathrm{Hv}}$ : $1.65 \mathrm{~A} / \mathrm{div}, 25 \mathrm{~ms} / \mathrm{div}$.

\subsubsection{Case III}

As was explained in the subsection 4.2.2.2, when the HV terminal of the bidirectional converter is connected to a DC bus with a high voltage ripple, the capacitor at the $\mathrm{HV}$ terminal of the bidirectional converter will be charged and discharged periodically. In the third case study, the full wave uncontrolled rectifier is connected to the HV terminal (bus 2) and the current control is based on the $\mathrm{I}_{\mathrm{LV}}$. The $\mathrm{I}_{\mathrm{LV}}$ and $\mathrm{I}_{\mathrm{HV}}$ for this case when the reference is changed from -4 to 5-A are shown in Figure 4.8. As can be seen, there is a 1.8-A overshoot in the $\mathrm{I}_{\mathrm{Lv}}$ and the current reaches its steady state in less than 50ms. Also, there is a small current ripple in $\mathrm{I}_{\mathrm{LV}}$, which is within an acceptable range. The current ripple in $\mathrm{I}_{\mathrm{HV}}$ is more serious and it is about 3-A. This high ripple is basically because of the charging and discharging of the capacitor at the HV terminal. Figure 4.9 shows the result of the same case when the time per division of the graph is decreased for more clarification. As can be seen, the ripple is independent from the direction of the power flow and its fundamental frequency is $360-\mathrm{Hz}$, which is the same as the bus 2 voltage 
ripple. The interesting point of this control scheme is that bus 1 is not affected by the high current ripple at the HV terminal of the bidirectional converter.

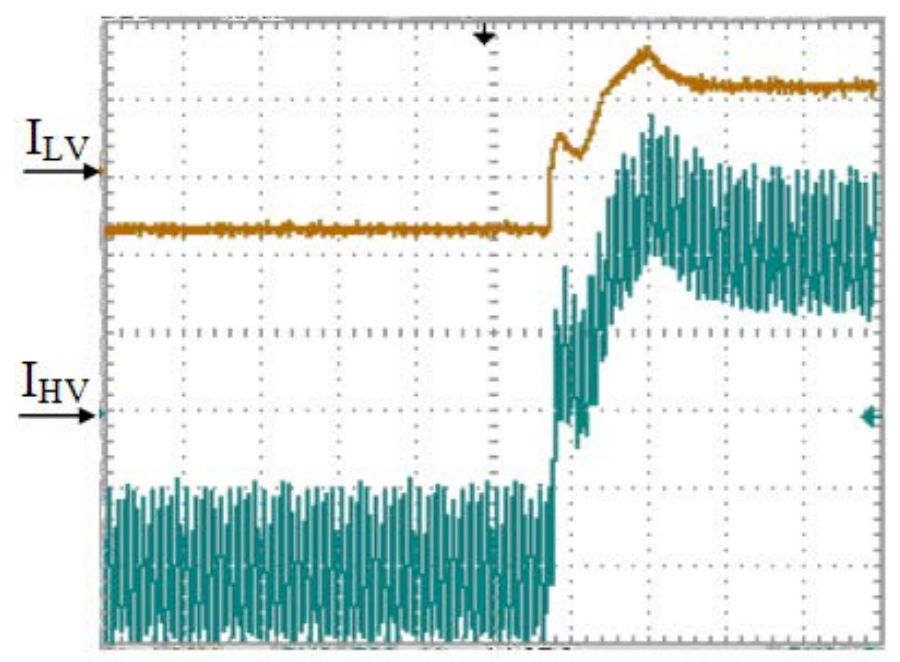

Figure 4.8: $\mathrm{I}_{\mathrm{LV}}$ and $\mathrm{I}_{\mathrm{HV}}$ when the three-phase full wave uncontrolled rectifier is connected to bus 2 and the reference of $\mathrm{I}_{\mathrm{LV}}$ is changed from -4 to $5 \mathrm{~A}$. $\mathrm{I}_{\mathrm{LV}}$ : $5.0 \mathrm{~A} / \mathrm{div}$; $\mathrm{I}_{\mathrm{HV}}$ : 1.65 A/div, $25 \mathrm{~ms} / \mathrm{div}$.

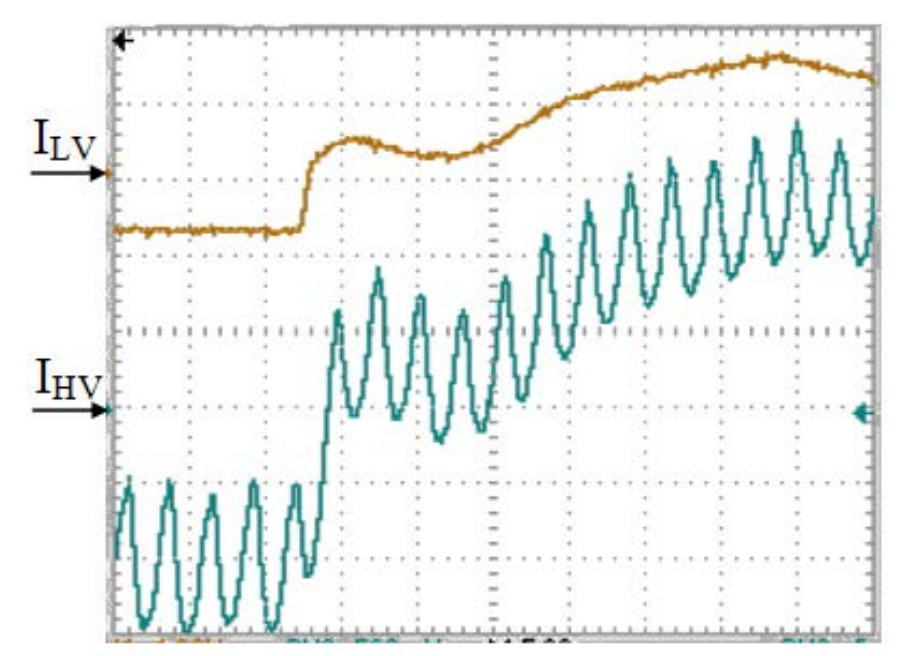

Figure 4.9: A zoom-in on the Figure 4.8 with 5 ms/div.

\subsubsection{Case IV}

In the last case study, the current control is based on $\mathrm{I}_{\mathrm{HV}}$ while the three-phase full wave uncontrolled rectifier is connected to bus 2 . Figure 4.10 shows $\mathrm{I}_{\mathrm{LV}}$ and $\mathrm{I}_{\mathrm{HV}}$ when the reference of $\mathrm{I}_{\mathrm{HV}}$ is changed from -3 to 5-A. As can be seen, $\mathrm{I}_{\mathrm{HV}}$ has about 2-A overshoot 
and it reaches its steady state in less than 60 -ms while its error is around zero. Similar to Case III, IHV has a high current ripple in both the steady state and transient conditions. This is because of the charging and discharging of the capacitor at the HV terminal. However, the current ripple of $\mathrm{I}_{\mathrm{LV}}$ is considerably higher than the previous case. This high current ripple is mainly because of the periodic change of the feedback $\mathrm{I}_{\mathrm{HV}}$.

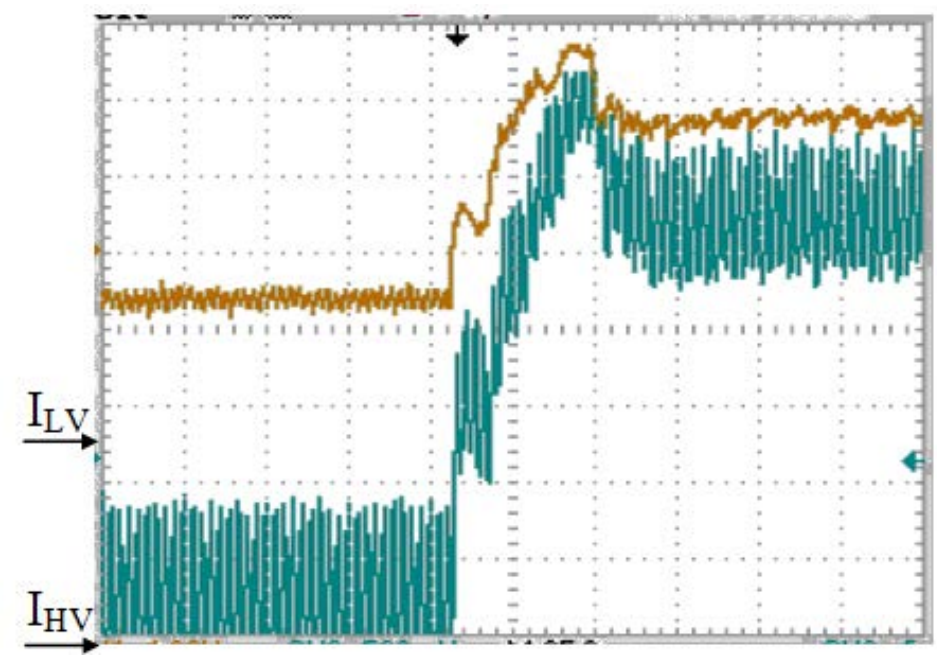

Figure 4.10: $\mathrm{I}_{\mathrm{LV}}$ and $\mathrm{I}_{\mathrm{HV}}$ when the three-phase full wave uncontrolled rectifier is connected to bus 2 and the reference of $\mathrm{I}_{\mathrm{HV}}$ is changed from -3 to $5 \mathrm{~A}$. $\mathrm{I}_{\mathrm{Lv}}$ : $5.0 \mathrm{~A} / \mathrm{div}, 25$ $\mathrm{ms} / \mathrm{div} ; \mathrm{I}_{\mathrm{HV}}: 1.65 \mathrm{~A} / \mathrm{div}, 25 \mathrm{~ms} / \mathrm{div}$.

\subsubsection{Summary of the Bidirectional Energy Transfer Results}

In this study, depending on the placement of the source with high voltage ripple and its connection with respect to the bidirectional DC-DC converter and whether the reference current is from the terminal with high voltage ripple or not, four case studies were defined. The comparative experimental test results showed that in the case that the diode rectifier is connected to the LV terminal of the bidirectional converter, the current ripple will be less if the reference current is taken from the LV terminal. This is due to the fact that, with this configuration, the controller can be adapted faster to the voltage variations. 
Also, better controllability of the bidirectional converter can be achieved with this configuration for high power flow.

On the other hand, experimental results for the two cases when the full wave diode rectifier was connected to the HV terminal of the bidirectional converter showed that when the voltage ripple at the $\mathrm{HV}$ terminal is considerable, better control can be achieved by choosing the low voltage terminal current as the reference. This is because of the fact that the high voltage variations cause considerable current ripple due to the charging and discharging process of the capacitor at the HV link. Hence, selecting the low voltage terminal current as the reference will prevent the controller from following periodic fast unnecessary variations. As a result, there will be less current ripple in the grid on the LV side.

\subsection{Real-Time Control of a hybrid DC power system}

The voltage control of a hybrid DC power system can be categorized as the masterslave control and the droop control techniques. In the master-slave method, the master controls the DC bus voltage while the slave communicates with the master to share the energy based on predefined limits and loading scenarios. In the droop control technique, the communication between the converters is not required. However, this method suffers from the stationary error as a result of the load variation [144].

In this section, the real-time operation and energy management for a notional DC microgrid that incorporates a pulsed load is presented. As discussed in section 2, the pulsed loads draw high currents during a short amount of time, which can cause considerable voltage and frequency fluctuation. These disturbances can trip other normal loads offline, 
causing a serious outage. To overcome these issues, a current-voltage control technique based on the master-slave control concept is proposed. In this technique, the supercapacitor bank, acting as the master, controls the DC bus voltage while the slave works in current control to share the required power of the load.

\subsubsection{System Description}

A notional hybrid DC microgrid proposed for this study is shown in Figure 4.11. This microgrid supplies two steady state loads and one pulsed load. The energy of the system is mainly supplied by an AC generator inside the grid (acting as a distributed generator) and through the main AC power grid. A 2.9-F supercapacitor bank functions as a power buffer device and provides the instantaneous power required. The AC generator is a 3-kVA, synchronous machine, which is explained in section 3.4. This generator is connected to the AC filter via a three-phase $3-\mathrm{kA} \mathrm{Y} / \Delta$ transformer to be galvanically isolated from the DC grid and the main AC network. This will ensure that no DC current flows to the generator through the neutral impedance. The AC filters are a 24-mH inductor to filter out harmonics to the AC generator and the AC power grid.

The LC type DC filter is connected between the uncontrolled rectifier and the boost converter to smooth out the DC voltage and current. The inductor and the capacitor of the DC filter are $2.7 \mathrm{mH}$ and $1200 \mu \mathrm{F}$, respectively.

\subsubsection{Controller Development}

Figure 4.12 shows the control diagram of the dc-dc converter. The converters are controlled by a PI current controller that adjusts the duty ratio of the IGBT switching at fixed 5-kHz. It should be noted that the uncertainty of the measurement can cause error accumulation. If the injected power to the DC bus exceeds the consumed power, the extra 
energy will be stored in the supercapacitor bank and, depending on the supercapacitor's time constant, an overvoltage can result. To solve this issue, the dc-dc converter is equipped with a hysteresis voltage control. The hysteresis voltage protection system monitors the entire supercapacitor bank. Once the voltage of the DC bus exceeds the preset limit, the converter will be turned off and it will stop sending power until the bus voltage drops to $95 \%$ of the nominal voltage.

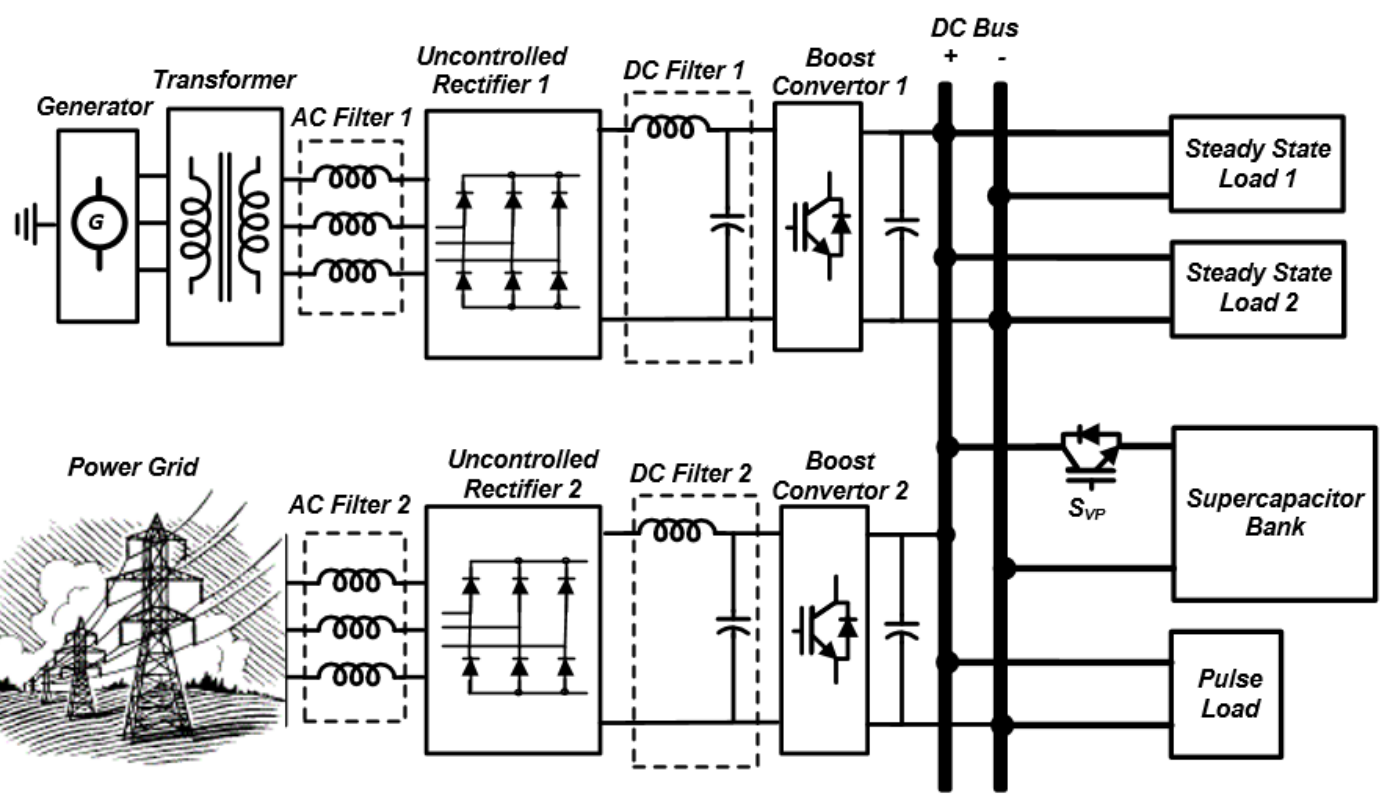

Figure 4.11: Schematic diagram of the system for the real-time control and energy management study.

Uneven charge distribution can cause dangerous overvoltage in a cell while the overall bank voltage is under the high voltage threshold. Thus, voltage control of the dcdc converter alone is not enough for supercapacitor cell overvoltage protection. The design of the analog hysteresis voltage protection circuit for the supercapacitor bank protection was described in section 3.7.5. This board was designed to protect the supercapacitor cell from dangerous overvoltage that can occur due to the uneven charge distribution among supercapacitor cells. This is an important issue for fast charging of the supercapacitor bank 
in high voltage applications. The output of the protection module is connected to an IGBT with an anti-parallel diode, which is indicated as $\mathrm{S}_{\mathrm{VP}}$ in Figure 4.11. If the voltage of any set of supercapacitors exceeds the maximum threshold voltage, the protection module will be activated and the charging path will be opened by turning off the $S_{\mathrm{vp}}$. During this time, the anti-parallel diode only allows the discharge of the supercapacitor bank until the voltage of the entire set drops to a safe threshold.

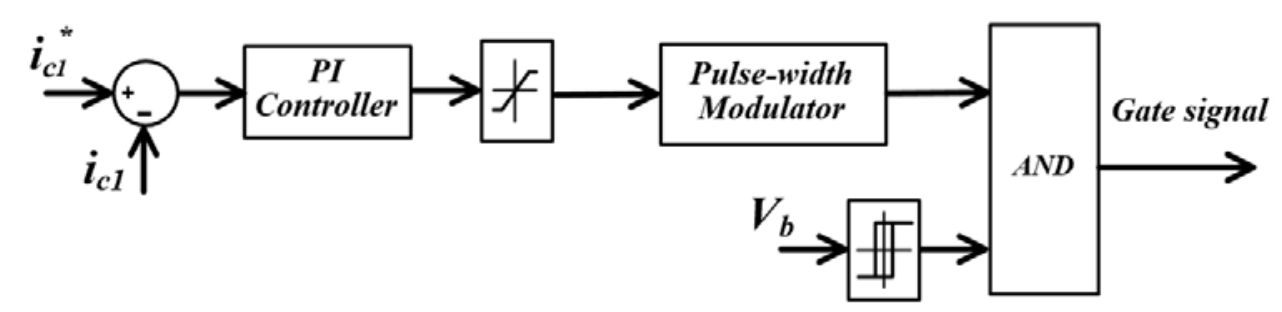

Figure 4.12: Block diagram of the first boost converter controller with current control and digital hysteresis voltage protection.

\subsection{Real-Time Energy Management System}

In order to properly supply the two normal steady state loads and the pulsed load, the injected power to the grid is controlled by the two dc-dc converters. In a DC microgrid, one of the power units should control the DC bus voltage while the other injects power via current control. In the proposed hybrid DC microgrid, the supercapacitor bank is precharged and serves to keep the bus voltage within the preset limits (i.e. variation of less than 5\%), while the two dc-dc boost converters are working in current control mode.

Figure 4.13 shows the energy management algorithm of the hybrid DC microgrid for three different modes of operation. The total instantaneous current of the two normal steady state loads and the average current of the pulsed load are calculated in the energy management system. In the first mode of operation, the required current is equally shared between the two boost converters. This will ensure that the input energy and the consumed 
energy of the DC microgrid are equal and, as a result, the supercapacitor's charge at the end of each pulse cycle is constant and the voltage of the DC bus will be within the limit. In the second mode of operation, the reference current of the second boost converter was set manually and the first converter injects the rest of the required power. On contrary, in the third mode of operation, the reference of the first boost converter is set manually by the operator and the second boost converter injects the rest of the power from the AC grid.

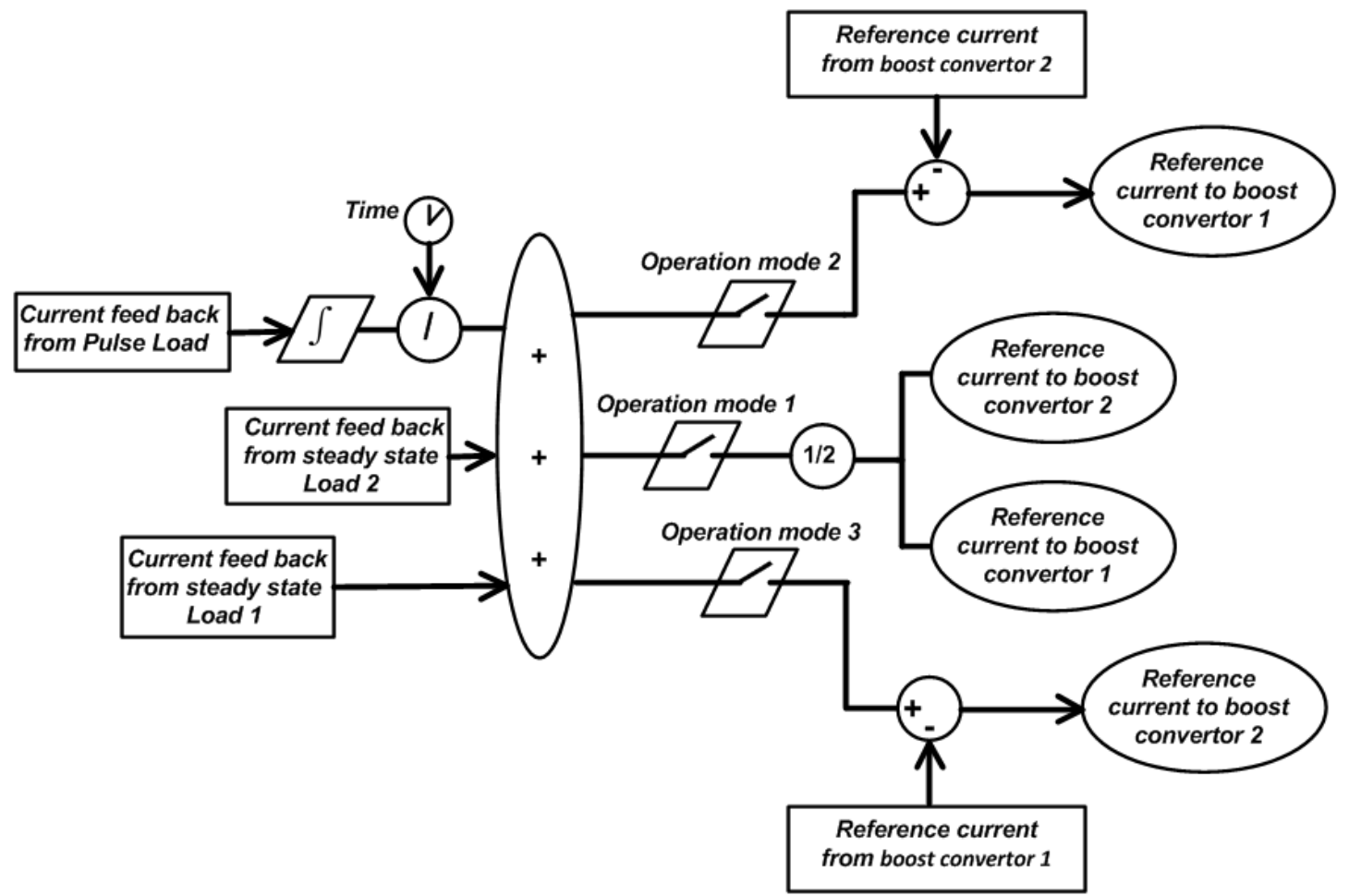

Figure 4.13: Energy management of hybrid DC microgrid.

Each mode of operation can be selected based on the required load power, availability of the AC sources and the power limitation of the converters. For instance, during the AC network failure, the second mode of operation can be selected and, by setting zero current reference for the second converter, the DC grid can operate in islanded conditions. Furthermore, smart techniques, including fuzzy logic concepts, can be applied 
to set the reference of the power converter for peak shaving purposes and to minimize the energy costs during long term operation [139]. Depending on the required power and whether the generator is isolated or not, the mode of operation highly affects the current harmonics of the system. This will be discussed in the next section.
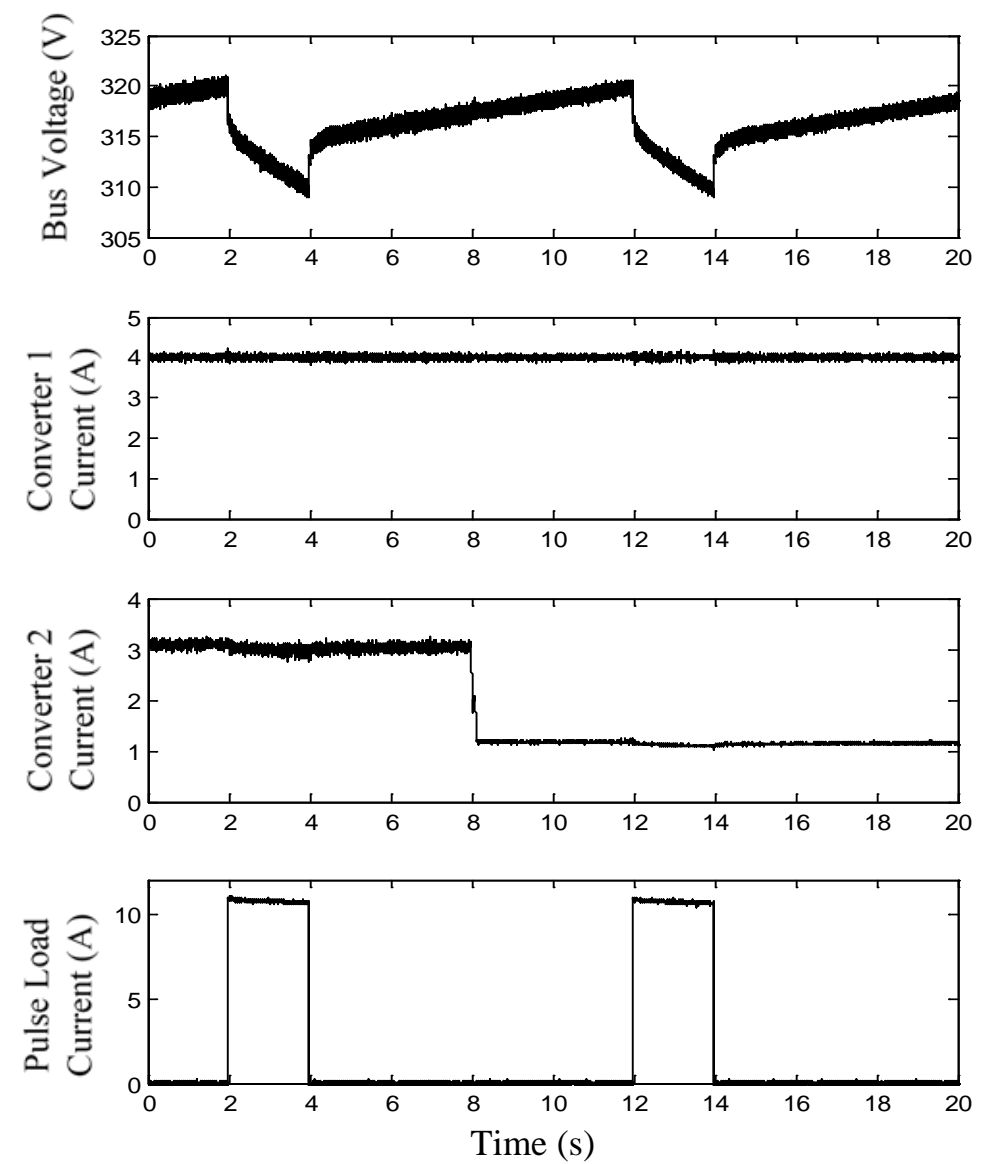

Figure 4.14: Dynamic performance of the microgrid test results.

\subsection{Dynamic performance analysis of the system}

In order to evaluate the real-time operation of the converter controller and the energy management system, the hybrid DC microgrid was tested under a step change of the steady state load. In this case, the second steady state load was 250-W and the pulsed load was $3.5-\mathrm{kW}$ at a frequency of $0.1-\mathrm{Hz}$ with a duty ratio of $20 \%$. Figure 4.14 shows the 
experimental test results when the third mode of operation was selected and the reference of the first converter was set to 4-A. The first steady state load was decreased from 1100$\mathrm{W}$ to $470-\mathrm{W}$ at $\mathrm{t}=8 \mathrm{~s}$. As can be seen, the second boost convertor tracked the load change very fast and decreased its output from 3.15-A to 1.25-A. Also, it can be seen that there was no voltage fluctuation for the step load change. This proves the effectiveness of the energy management algorithm and the developed converter controller.
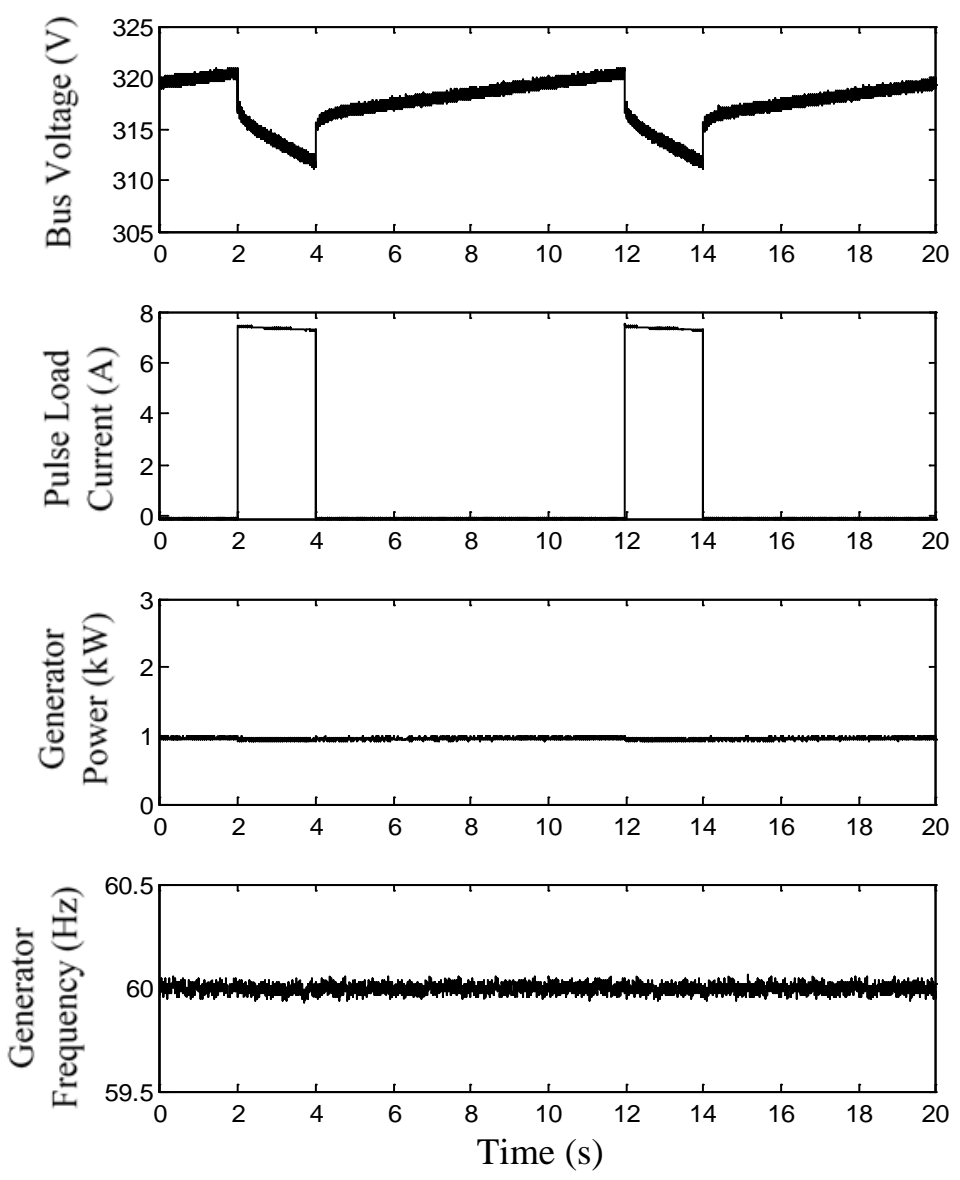

Figure 4.15: Current-voltage control method test result.

In order to demonstrate the effectiveness of the developed control technique, the performance of the internal generator during pulsed load operation was evaluated. Figure 4.15 shows the experimental test results while the first mode of operation was selected and the DC grid was supplying the $2.5-\mathrm{kW}$ pulsed load at a frequency of $0.1-\mathrm{HZ}$ with a duty 
ratio of $20 \%$. Also, the first and second steady state loads were $250-\mathrm{W}$ and $1100-\mathrm{W}$, respectively. As can be seen, with the current-voltage control technique, the supercapacitor properly supported the pulsed load and, as a result, the AC generator only supplied the average of the pulsed load. Moreover, since there was no power fluctuation on the generator, its frequency was almost constant at $60-\mathrm{Hz}$.
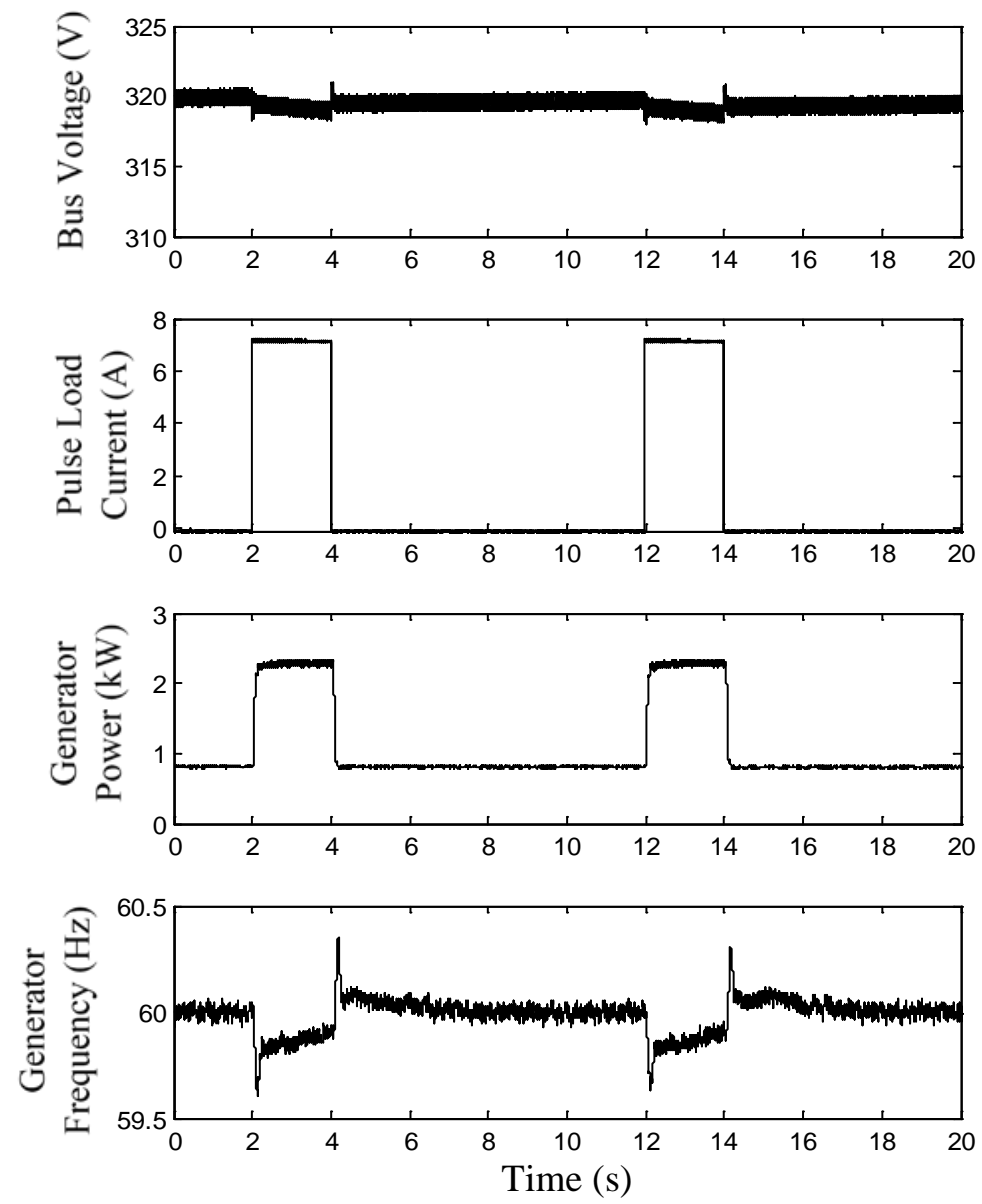

Figure 4.16: Instantaneous power control method test results.

Figure 4.16 shows the same experimental test results, while the instantaneous power control was applied and the required power was equally shared between the two converters. The test results show that the generator supplied around $0.8 \mathrm{~kW}$ during the pulse off-time and it raised up to 2.18-kW when the pulsed load occurred. Compared to the 
current voltage control technique, the DC bus voltage variation was reduced. However, the analysis of the generator frequency shows the power pulsation of the generator caused considerable frequency fluctuation, i.e. once the pulsed load occurred, the generator frequency dropped to $59.60-\mathrm{Hz}$ and it raised to $60.35-\mathrm{Hz}$ after the pulse ended. Such a power pulsation not only requires higher capacity of the converter and generator, but also decreases the system efficiency.

\subsection{Operation and Harmonic Analysis of Isolated and Non-Isolated Grid}

In the DC microgrid, the distributed generator (DG) and the DC loads are either connected directly or through a converter to the DC bus. Those that do not have a transformer are not galvanically isolated from the DC bus. Thus, the topology and the control of each converter should be designed such that no DC current flows to the machines via the neutral impedance. In this section, the operation of the hybrid DC microgrid with a galvanically isolated AC generator is studied. Also, the performance of the system without galvanic isolation under different loading and operating schemes are tested and the results are analyzed in this section.

\subsubsection{Isolated hybrid DC microgrid operation}

The proposed hybrid DC microgrid was tested while the generator and the AC grid were galvanically isolated by the 3-kW transformers. The first steady state load was 1-kW and the second load was 250-W. Also, the pulsed load was 3.5-kW at a frequency of 0.1HZ and duty ratio of $20 \%$. Figure 4.17 shows the experimental test results while the first mode of operation was selected. As can be observed, the two converters equally shared the power and each converter injected 3.1-A. Also, the experimental results show that the bus 
voltage variation was less than the preset limit of $5 \%$. During the pulse on-time, the supercapacitor bank was discharged and, since the generated and consumed power were almost equal, the supercapacitor bank was charged during the pulse off-time and the voltage returned to its origin.
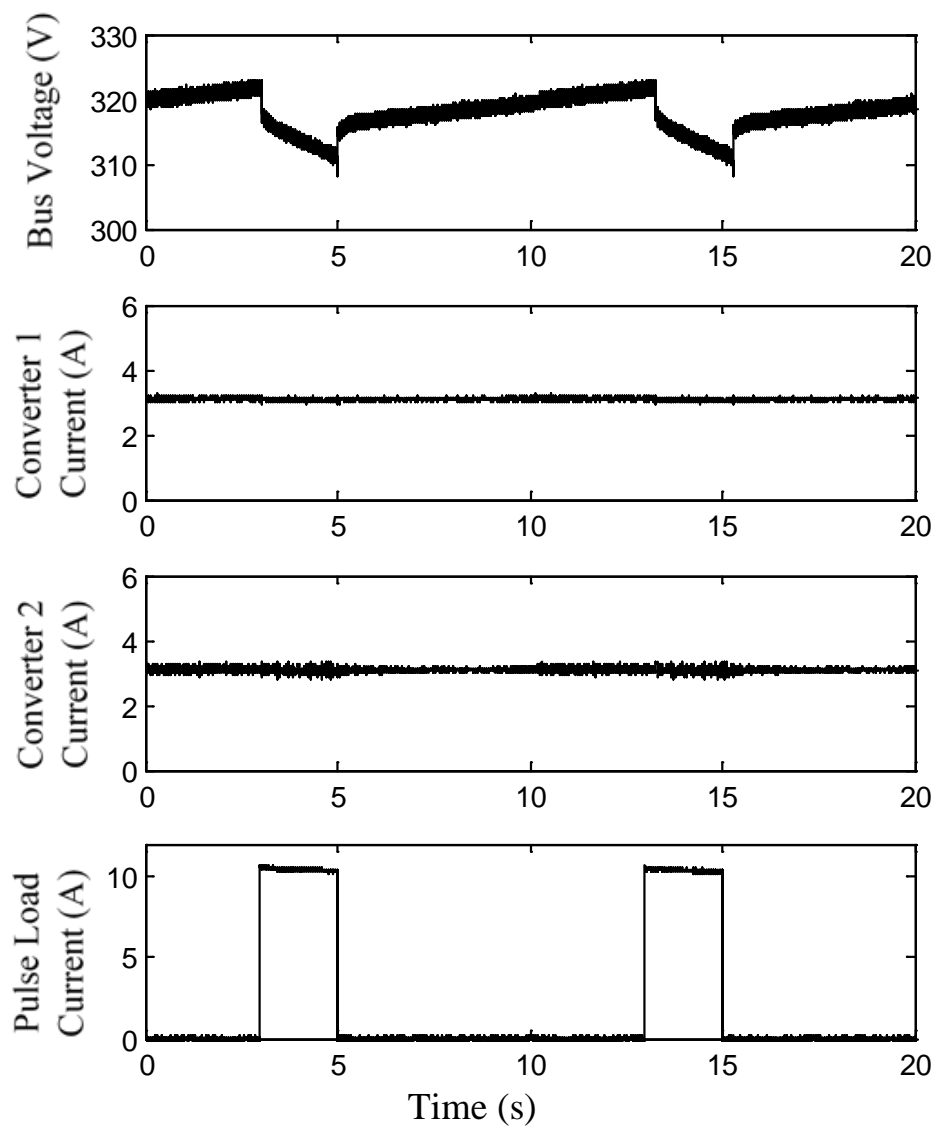

Figure 4.17: First mode of operation test results for the isolated DC microgrid.

Figure 4.18 shows the three-phase output current of the isolation transformer. As can be seen, the current was symmetric and its maximum value was 5-A. Figure 4.19 shows its harmonic components normalized with respect to the fundamental frequency at $60-\mathrm{Hz}$. The results show that the fifth harmonic had the highest amplitude; the fifth harmonic amounted to $8.2 \%$ of the fundamental harmonic. Also, the THD of the current was $11.86 \%$. 


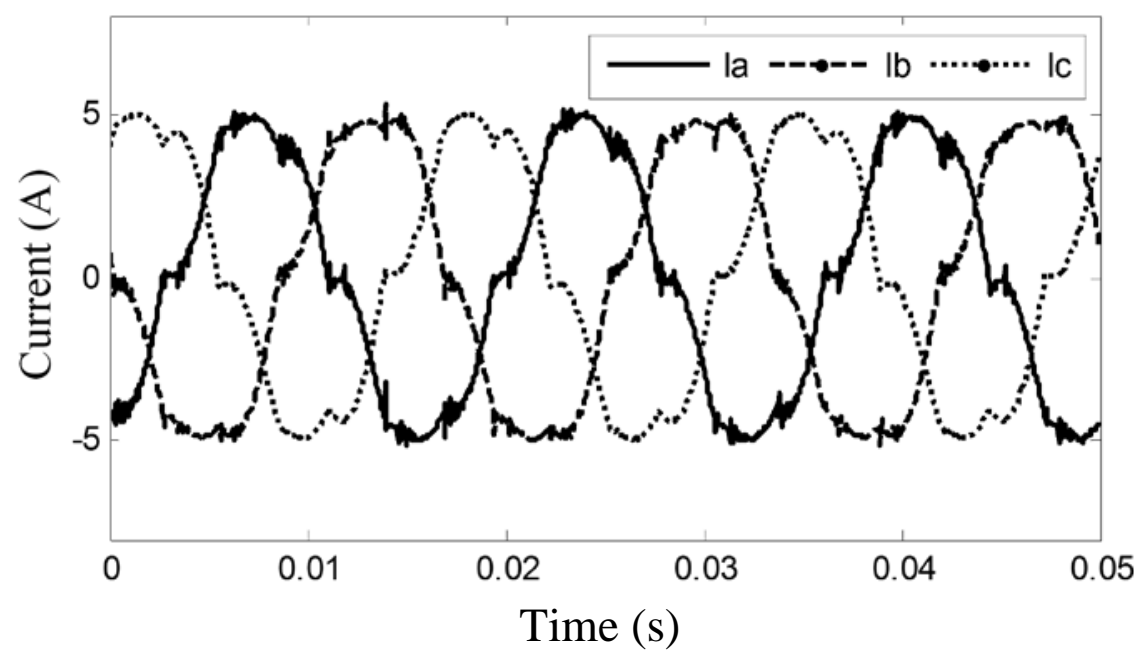

Figure 4.18: Output current of the transformer in the first mode of operation.

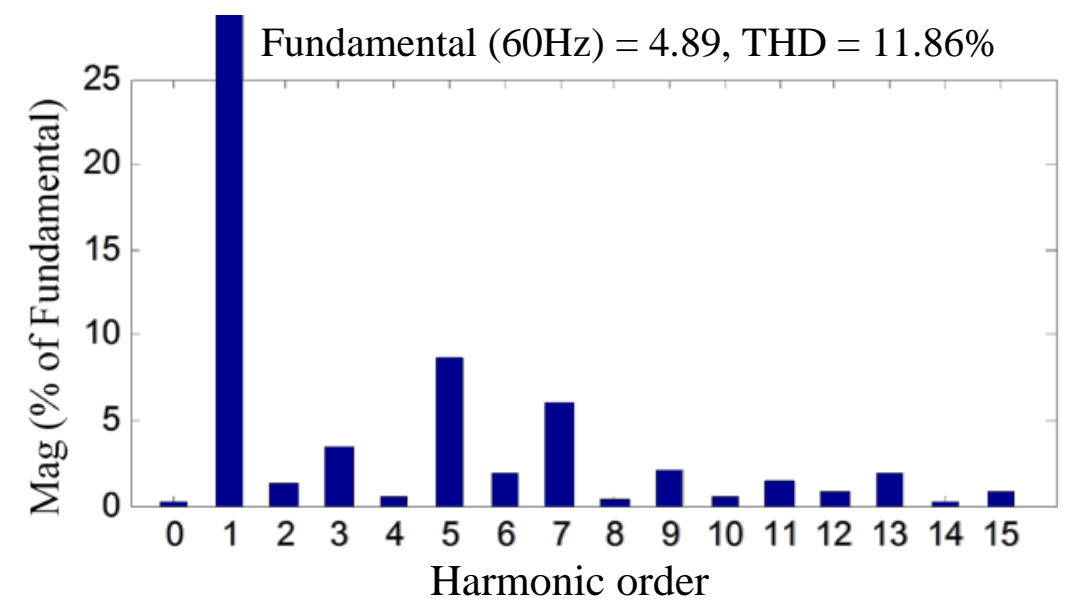

Figure 4.19: Harmonic analysis of the output current of the transformer in the first mode of operation.

Figure 4.20 shows the experimental results of the second operation mode when the reference current of the second converter was manually set to 1-A. In this case, the normal steady state and pulsed loads were similar to the previous case. As can be seen, the second converter tracked its reference with zero steady state error. Also, the first converter provided the rest of the required energy by calculating its reference current based on the algorithm explained in Figure 4.13. The test results show that the first converter injected 5.2-A to the DC bus. 

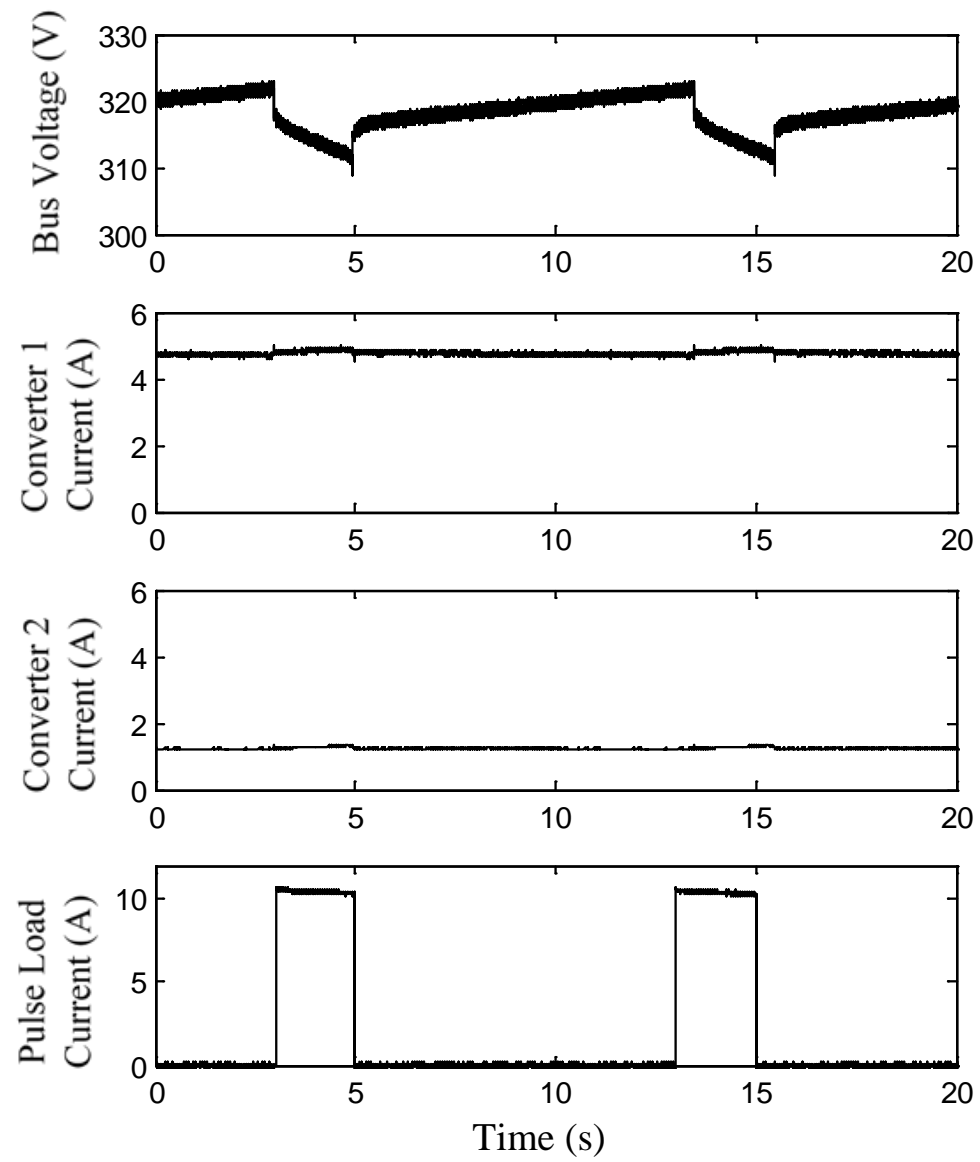

Figure 4.20: Second mode of operation test results for the isolated DC microgrid.

Figure 4.21 and Figure 4.22 show the output current of the transformer and its harmonic components up to fifteenth order. The maximum current in this case reached 10A, which was due to the high current injection to the DC bus and considerable voltage drop over the AC filter. As the harmonic analysis shows, the THD of the current was $4.05 \%$, while the fifth and seventh order harmonic had the highest values, which were $3.1 \%$ and $1.4 \%$, respectively. 


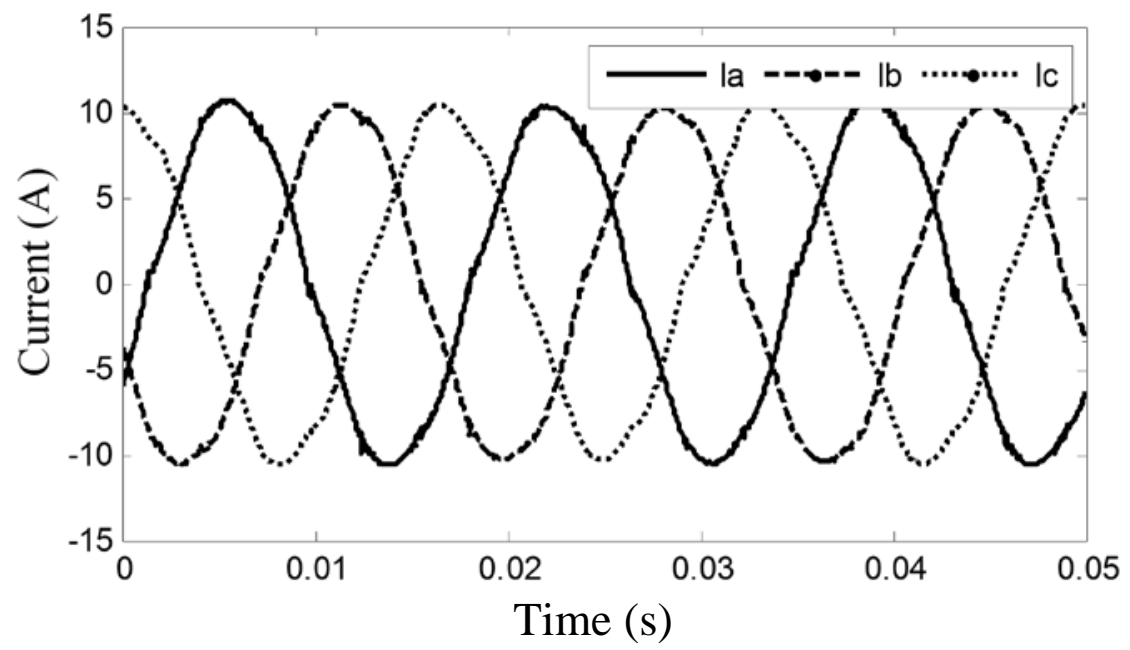

Figure 4.21: Output current of the transformer in the second mode of operation.

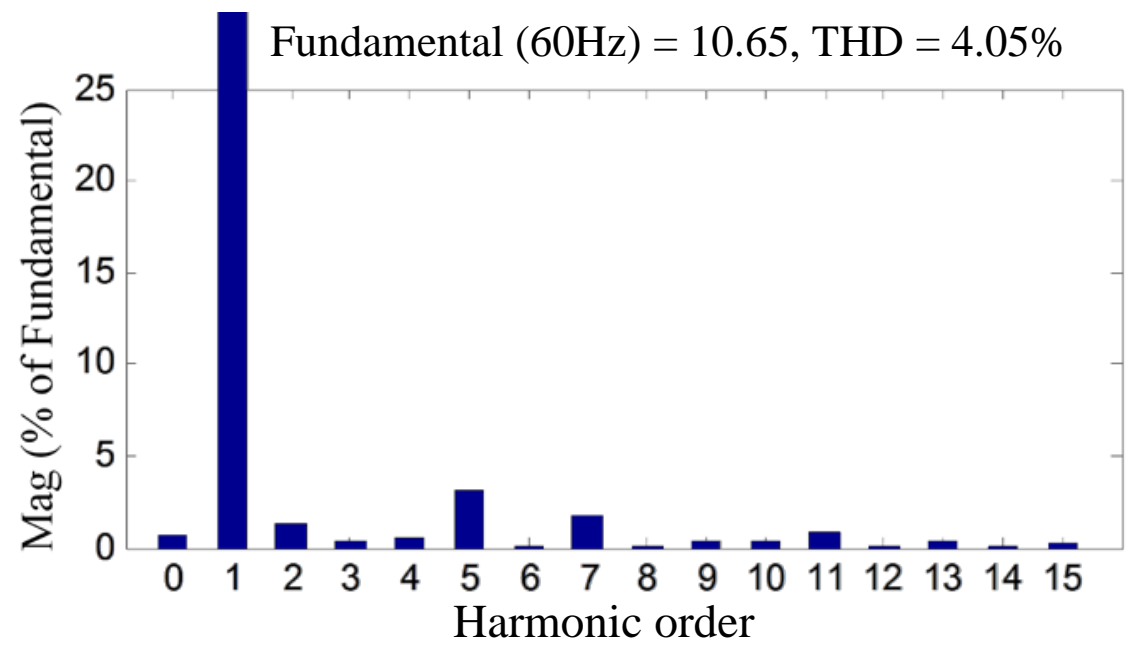

Figure 4.22: Harmonic analysis of the output current of the transformer in the second mode of operation.

The experimental results of the third mode of operation while reference of the first converter was set to 1-A is shown in Figure 4.23. As can be seen, the voltage variation of the bus was less than $4 \%$, i.e. the voltage decreased to $310-\mathrm{V}$ when the pulse ended and increased to 324-V before the next pulse occurred. Also, the steady state error of the first converter was almost zero and the second converter injected 5.2-A to the DC bus based on the energy management algorithm. 

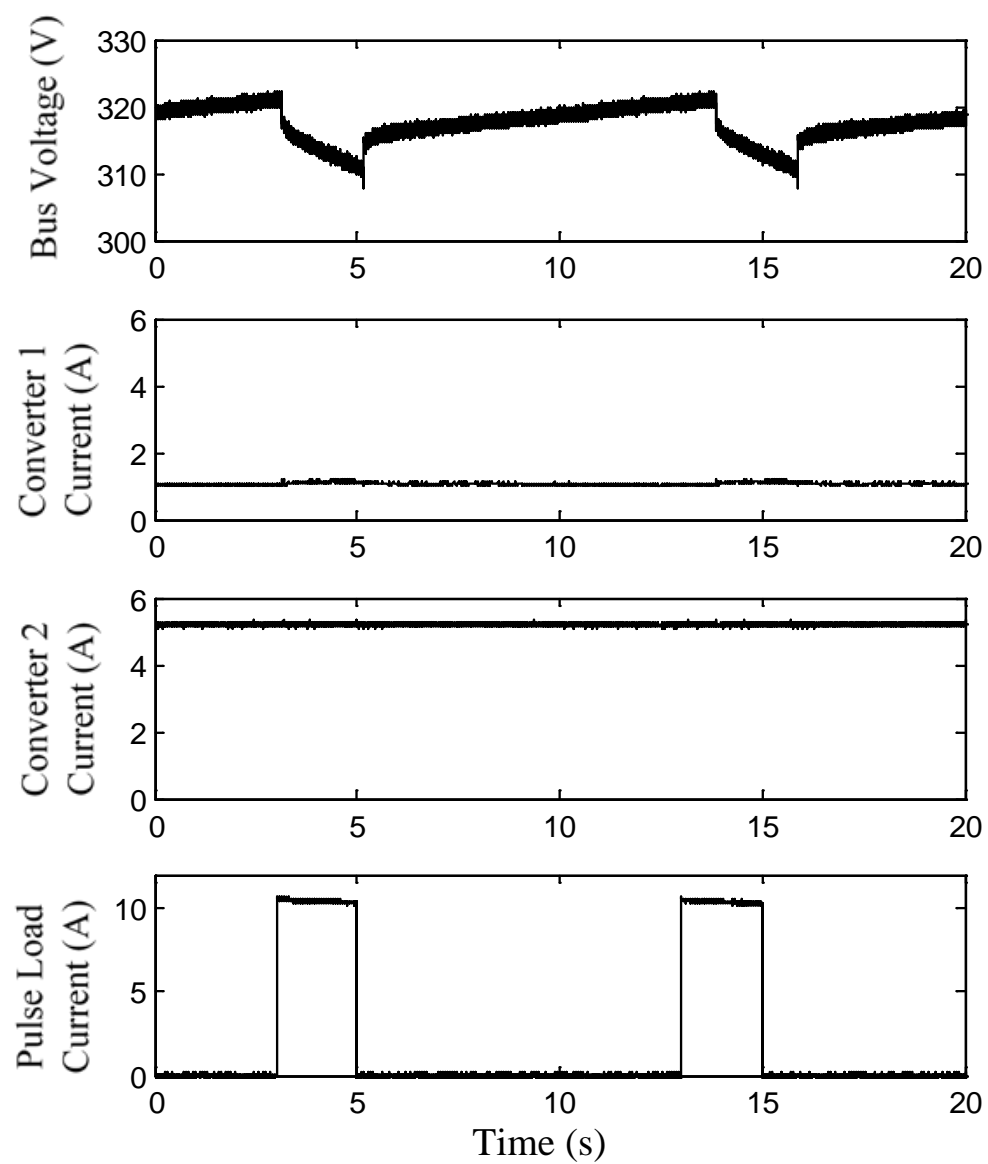

Figure 4.23: Third mode of operation test results for the isolated DC microgrid.

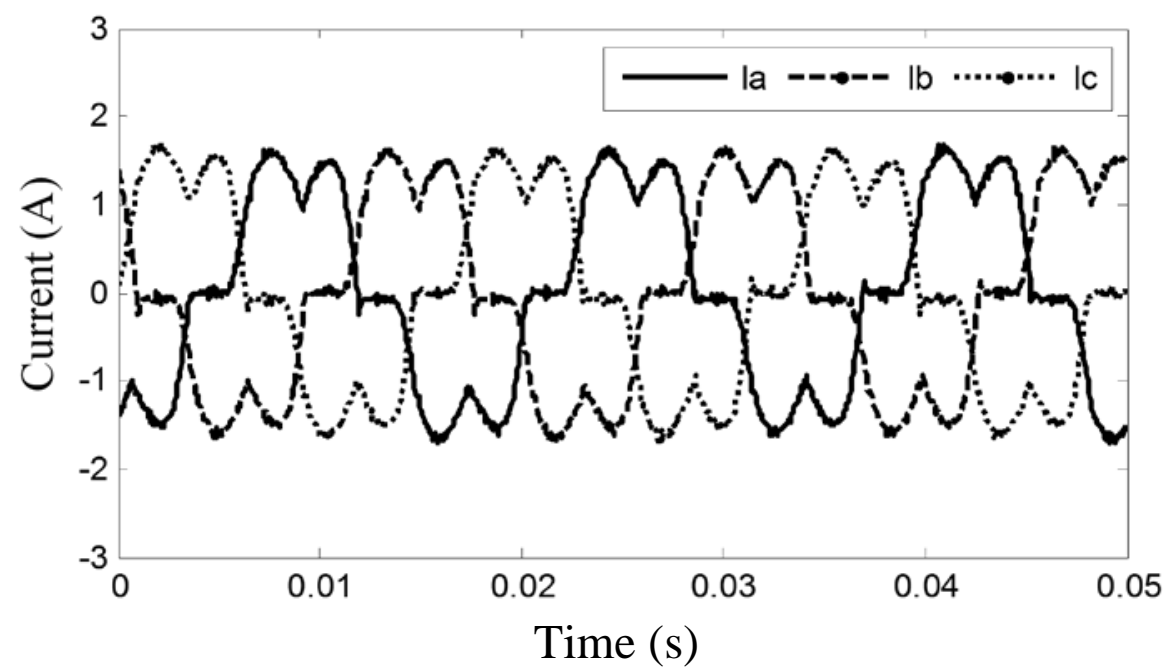

Figure 4.24: Output current of the transformer when the third mode of operation was selected. 
Figure 4.24 shows the waveform of the output current of the transformer. The maximum current was 1.82-A and it was highly distorted. Figure 4.25 shows the frequency spectrum of the current. The THD was $24.93 \%$ and the amplitude of the fifth harmonic was 23.92\%, which was relatively high. The high harmonics of the transformer output current were due to the small current that passes through the inductive filter. The results show that the THD of the current was close to the THD of a three-phase full wave uncontrolled rectifier without current filter, which is $\sqrt{\pi^{2} / 9-1}=31.08 \%$.

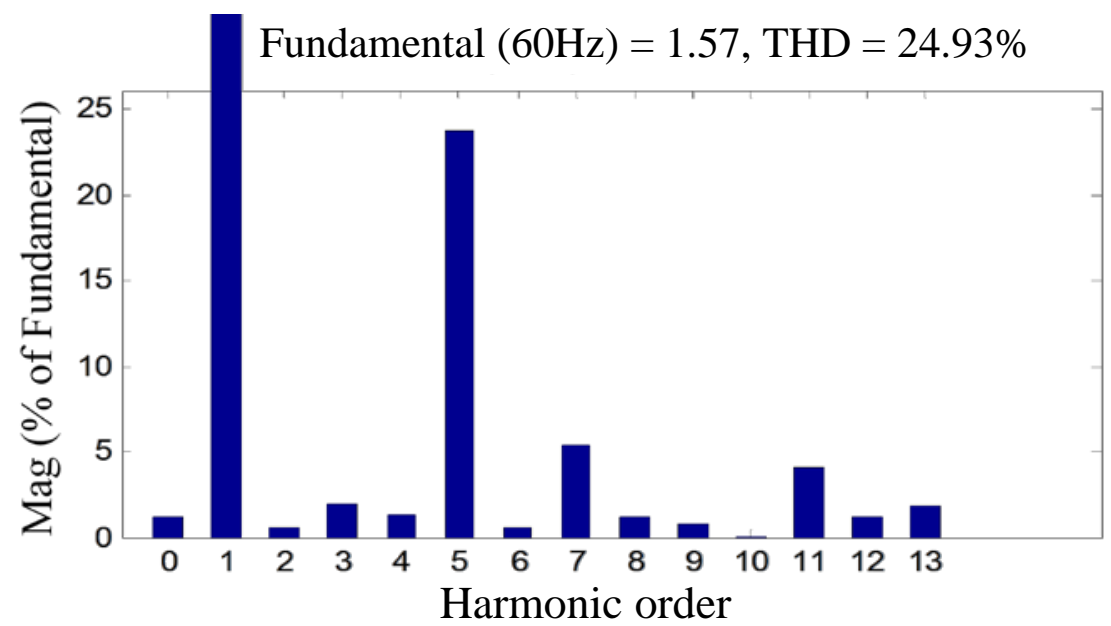

Figure 4.25: Harmonic analysis of the Output current of the transformer in the third mode of operation.

\subsubsection{Non-isolated Hybrid DC Microgrid Operation}

In this section, the hybrid DC microgrid shown in Figure 4.11 is tested while the generator was directly connected to the AC filter without any galvanic isolation. The DC bus voltage and DC currents when the first mode of operation is selected was very similar to test results for the case that the generator was isolated (Figure 4.17) and it is not presented. However, the generator current in the case of a non-isolated grid is much distorted. Figure 4.26 shows the input current to the AC filter and Figure 4.27 shows its harmonic spectrum. The current THD is $23.66 \%$ which is more than two times of the case 
that the generator was isolated. Interesting point of harmonic analysis is the high magnitude of second order harmonic, which is $7.82 \%$ of the fundamental harmonic. Moreover, the third harmonic has the highest amplitude while for isolated system the fifth harmonic was dominant.

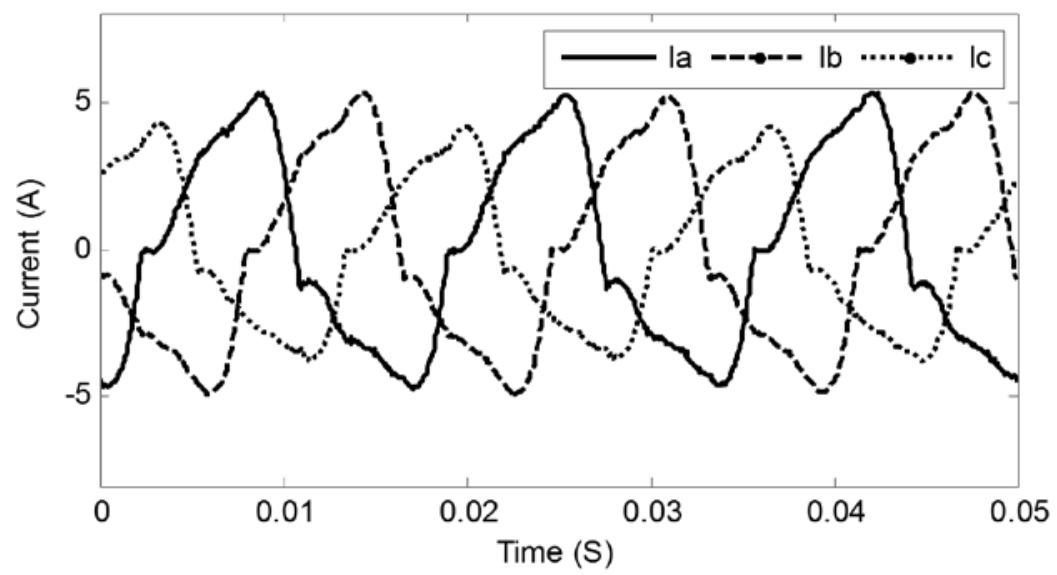

Figure 4.26: Input current of the AC filter in the non-isolated grid when first mode of operation is selected.

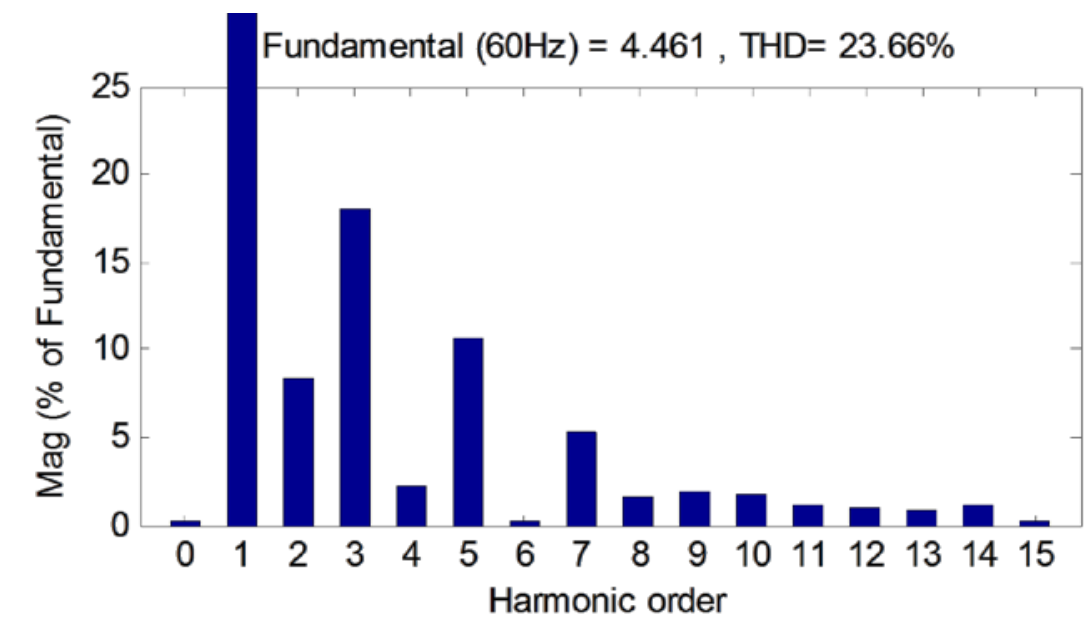

Figure 4.27: Harmonic analysis of the Input current of the AC filter in the non-isolated grid when first mode of operation is selected.

Figure 4.28 shows the DC bus voltage, the two converter's current and the pulse load current for non-isolated grid when the second mode of operation is selected. As can 
be seen, the first converter tracks its reference 5.2 A accurately with zero error while there is a small deviation in the second converter current, which is less than $6 \%$.
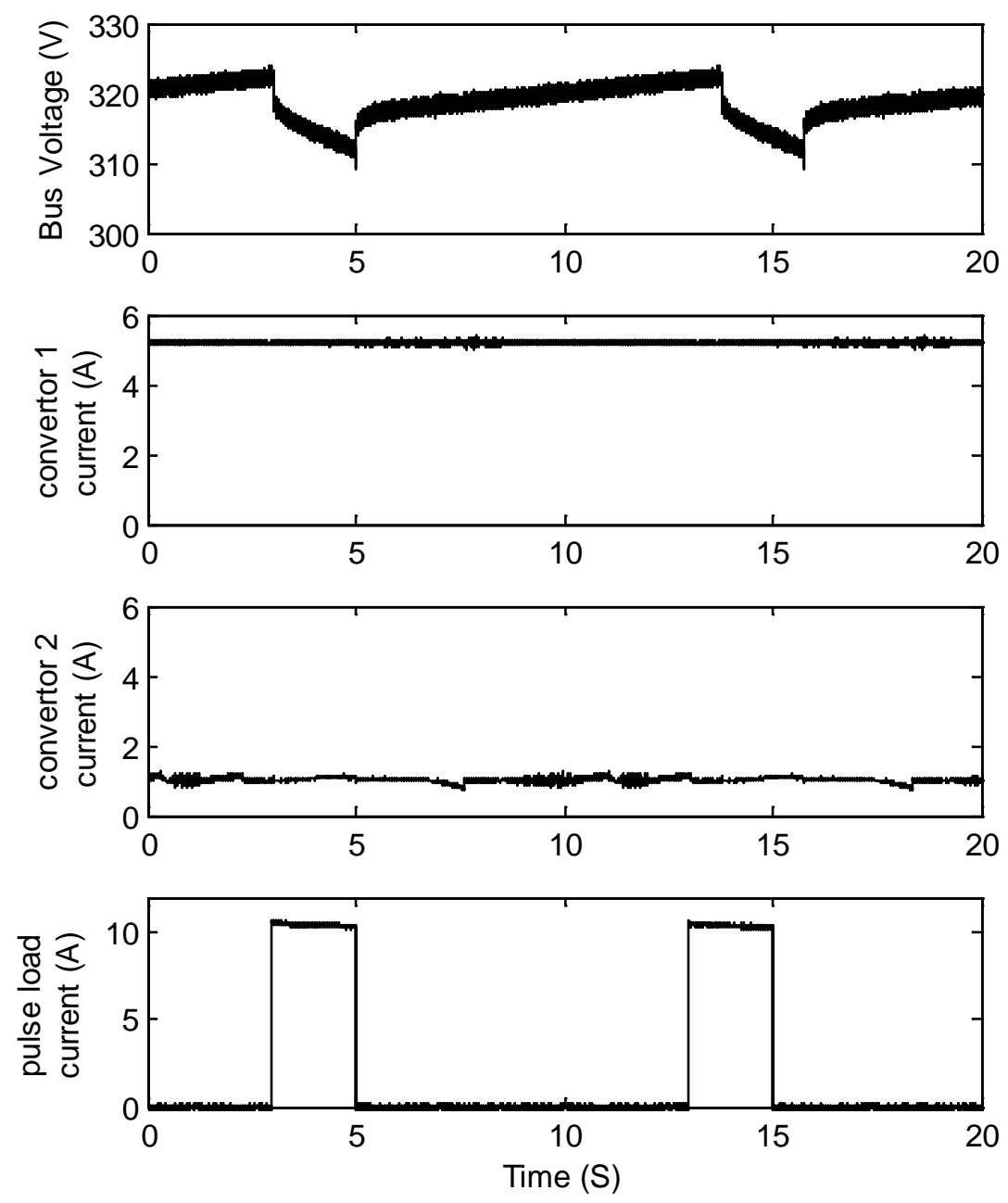

Figure 4.28: Second mode of operation test results for the non-isolated DC microgrid.

Figure 4.29 shows the input current to the AC filter in the second mode of operation for non-isolated grid. The results show that the AC current is asymmetric: the maximum positive current is $7.6 \mathrm{~A}$ and maximum negative is $5 \mathrm{~A}$. Clearly for the wave with asymmetries between positive and negative half wave even harmonic exist. Figure 4.30 shows the result of harmonic analysis of the current. It is known that the inductive AC filter can more effectively smooth the harmonics when the line current increases, which was seen 
in the isolated hybrid DC grid (Figure 4.19 and Figure 4.22). However, in the case of nonisolated grid, the THD of the current in the second mode of operation is more than the first case. This can be justified because the switching of the two converters are not similar since their current references are different. This results in circulating current between the generator and the AC network. As can be seen in Figure 4.30, the highest absolute amplitude of all the harmonics was the second harmonic, amounting to $20.83 \%$ of the amplitude of the fundamental.

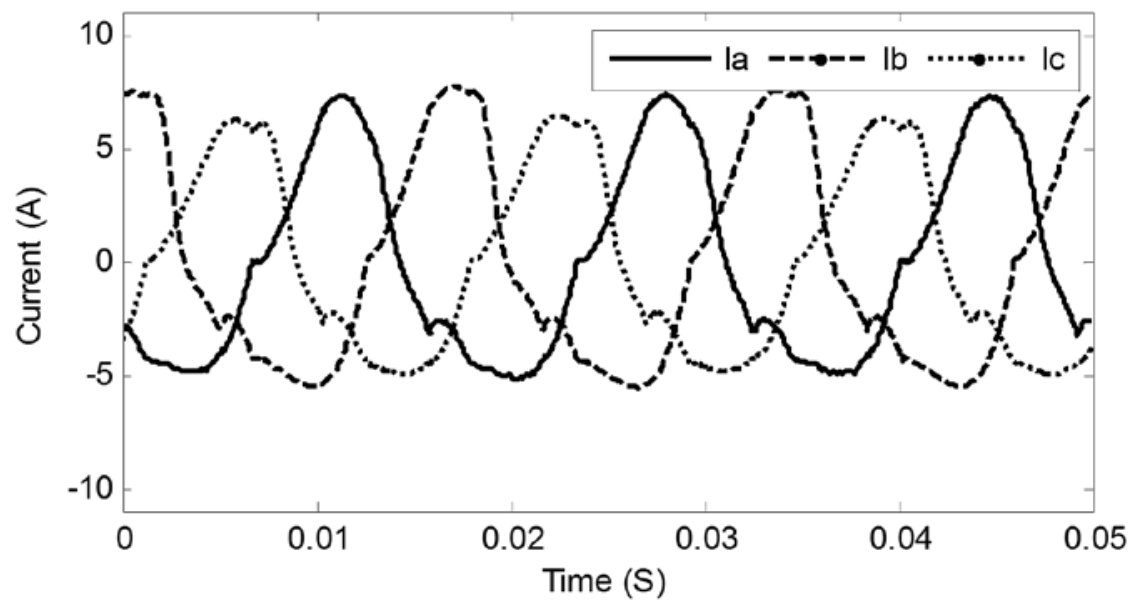

Figure 4.29: Input current of the AC filter in the non-isolated grid when the second mode of operation was selected.

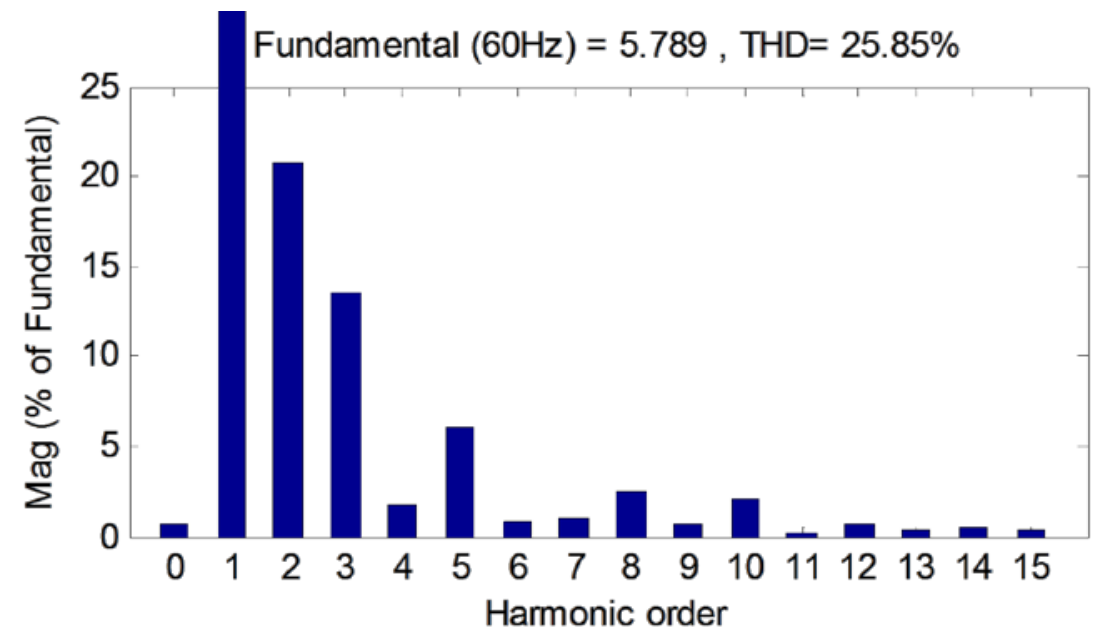

Figure 4.30: Harmonic analysis of the input current of the AC filter in the non-isolated grid when the second mode of operation was selected. 
Figure 4.31 shows the input current to the AC filter for the case that the third mode of operation was selected and the generator was not isolated. Similar to the previous case, asymmetries exist between the negative and positive half-wave. Figure 4.32 shows the harmonic spectrum of the current. The THD of the current is $37.55 \%$ and the even-order harmonics are dominant. The high amplitude of harmonics can be explained due to the low level of line current and the circulating current between the generator and AC network since the generator were not galvanically isolated.

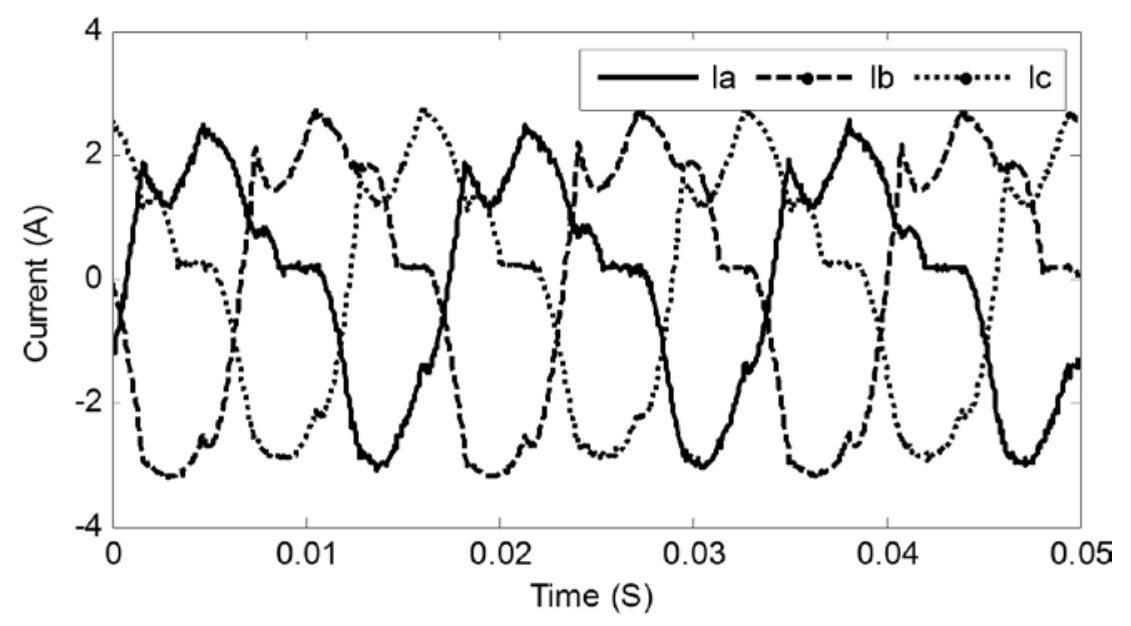

Figure 4.31: Input current of the AC filter in the non-isolated grid when the third mode of operation was selected.

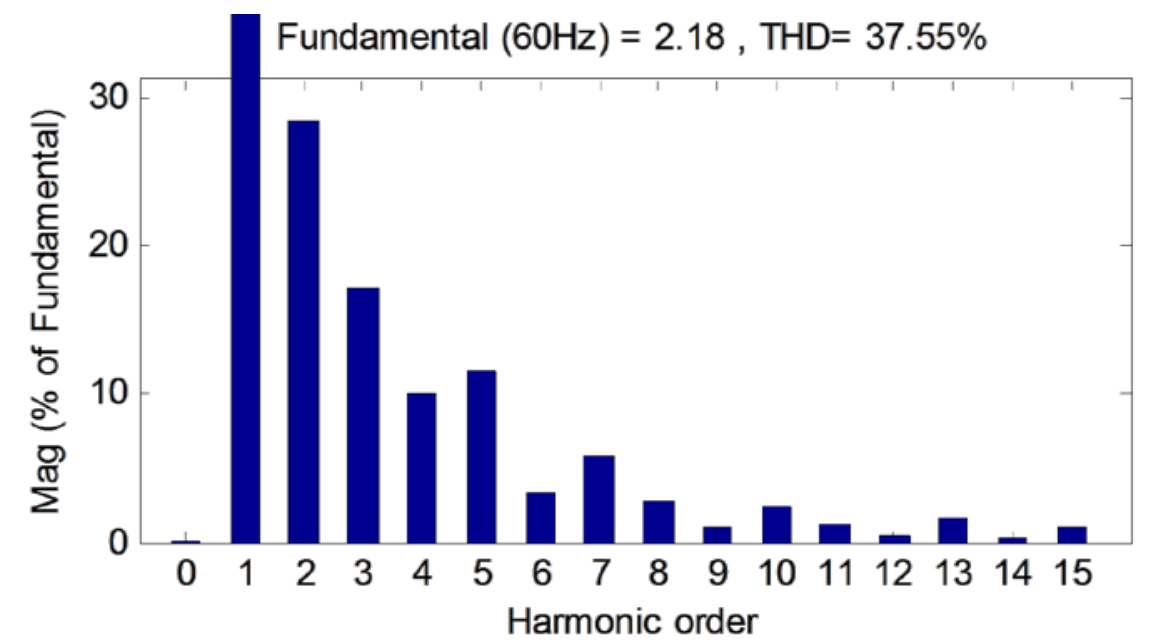

Figure 4.32: Harmonic analysis of the Input current of the AC filter in the non-isolated grid when the third mode of operation was selected. 
The result of the non-isolated grid shows that, although the DC bus voltage and convertor control were performed properly, the generator current for all modes of operation, especially in the second and third modes, were highly distorted. Thus, the implementation of transformers is necessary to avoid AC current distortion, particularly the even-order harmonics. Nevertheless, for harmonic suppression of the isolated grid, passive filters consisting of a bank of tuned LC filter and/or a high pass filter can be implemented as a low cost and high efficiency solution. Also, to have a better performance, and particularly to prevent parallel and series resonance, active filters and hybrid passiveactive filters are also applicable.

\subsection{Conclusion and Summary of the Results}

In this chapter, the issues of energy linking in a hybrid DC power system were studied. The goal was to achieve efficient bidirectional energy transfer between two DC buses whose voltages were at different levels and containing significantly different content of ripple. The results of the various experiments run for bidirectional power flow show that, depending on the configuration of the system, better controllability and lower current ripple can be achieved by the proper selection of current feedback.

Also, in this chapter, the real-time operation and energy management of the notional hybrid DC microgrid supplied by a three-phase DG and a supercapacitor bank on the microgrid was proposed. The converter controllers were designed with hysteresis voltage protection capability that was considered to ensure that uneven charge distribution of the supercapacitor bank, due to fast charge, does not cause the supercapacitor to exceed its maximum voltage. The experimental results showed that the voltage protection 
capability of the convertor and the accuracy of the analog hysteresis protection board ensure the reliable operation of the supercapacitor bank.

Moreover, the energy management algorithm based on master-slave control for three modes of operation was developed. The dynamic performance of the DC microgrid was experimentally tested and the proposed control method was compared with the instantaneous power control. The results showed that the power pulsation of the generator properly mitigated and there was no frequency fluctuations. However, it is very important to properly select the supercapacitor bank capacity to keep the DC bus voltage variation within an acceptable limit. This study also included the importance of the galvanic isolation on the performance of the system. A Y/D transformer was implemented between the AC supplies and the AC filter to isolate them from the DC microgrid. The test performed for both isolated and non-isolated hybrid DC grid showed that the energy management system properly shares power between the converters and the loads. As a result, the bus voltage variation was within the limit. However, the harmonic analysis showed that in the case of a non-isolated DC grid, the generator current was unacceptably distorted. The even-order harmonics existed due to circulating current between the non-isolated generator and the AC grid. 


\section{DEVELOPMENT OF AN ADAPTIVE ENERGY MANAGEMENT SYSTEM FOR HYBRID DC POWER SYSTEM}

\subsection{Introduction}

Significant enhancements in the performance of DC microgrids and DC distribution systems can be achieved with utilization of advanced power electronic converters and storage devices. A DC shipboard power system is an example of a DC microgrid concept where the system is capable of self-diagnosing, self-healing, and self-reconfiguring [46], [139]. In the case of heavy pulse loads or loads with a large startup current, energy management and power control of these systems are a fundamental concern. Such a high short-time current behavior not only requires higher rating of the power components, but also can potentially cause the system voltage and frequency to drop in the entire microgrid since there is no infinite bus in the system [46], [107], [139].

For pulse load mitigation, a supercapacitor offers an effective solution as a storage device with very high power density, long cyclic life, low power loss and relatively high energy density [109], [113], [146]. In this section, we investigate the real-time control and energy management of a DC microgrid incorporating hybrid energy sources with various loading schemes. In the proposed DC microgrid, a supercapacitor bank, working as a power buffer, and a pulse load are directly connected to a DC bus. The proposed system configuration highly improves the grid redundancy and reduces the total power losses. Moreover, the supercapacitor bank not only supplies the pulse load, but also supports the grid during transient periods when it is highly loaded. However, the energy management and control of such a system is more complex since the heavy pulse load can cause high 
power pulsation and voltage drop. To reduce the adverse effects of the pulse load, a new real-time energy management system (EMS) with an adaptive energy calculator (AEC) based on the moving average measurement technique is developed. The proposed microgrid was implemented in hardware and experimentally tested. The results were compared with other methods such as direct voltage control (DVC) and continuous current averaging (CCA) approaches. The results prove that the developed EMS with AEC technique properly share the power and control the bus voltage under different loading conditions while the adverse effects of the pulse load are highly reduced.

In the following section, the concept of the actively controlled hybrid DC power system with a high redundancy is explained. Then, the system configuration of the proposed microgrid and the developed energy control concept are described. Section 5.4 presents the source of errors and their effects on the performance of the system. This section also includes the detailed scheme of the AEC and the real-time EMS. Experimental tests of the proposed microgrid, its controllers and EMS under different loading schemes and grid conditions are presented in section 5.5. This is followed by the conclusions in section 5.6.

\subsection{Actively Controlled Redundant Hybrid DC Power System}

Several studies were performed on an actively controlled hybrid DC system in which the energy delivered to the supercapacitor and the pulse load was controlled via power electronic converters. Some of these studies dealt with power and efficiency improvement of the system [109], [113] and [145], and the others were conducted for stability enhancement of the AC grid [46], [107] and [139]. In most of these studies, the 
supercapacitor and the pulse load are connected to a grid through a single DC/DC or $\mathrm{DC} / \mathrm{AC}$ converter. The advantage of these configurations is the ease of energy management and converter control. However, the system redundancy of these configurations is low, i.e. a fault in the bus connecting converter will cause the outage of the supercapacitor and the pulse load. In this chapter, the real-time control and energy management of a redundant hybrid DC microgrid system, in which the supercapacitor and pulse load are directly connected to a DC bus, is studied.

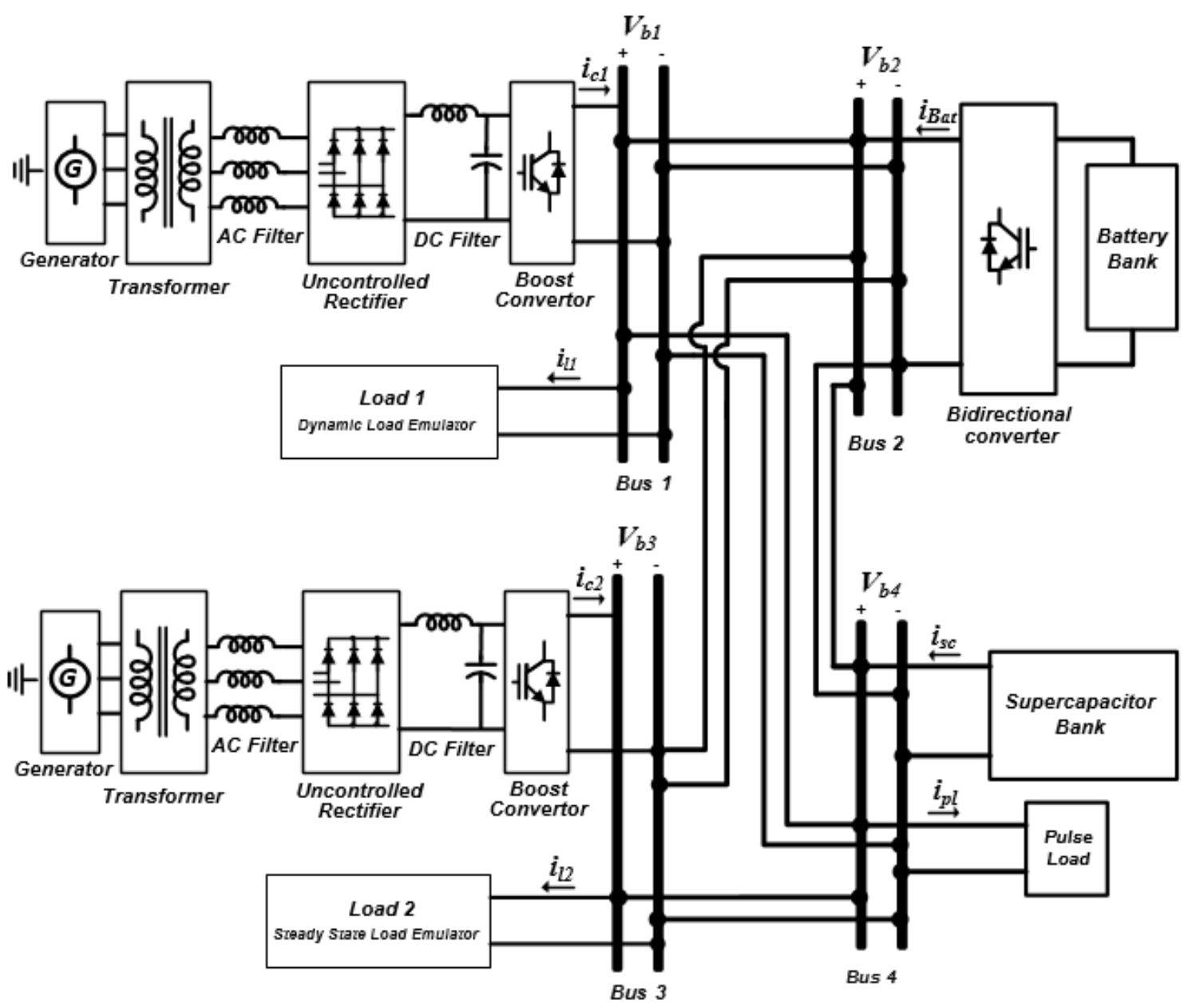

Figure 5.1: A schematic diagram of a redundant hybrid DC power system.

Figure 5.1 shows the schematic diagram of a notional hybrid DC microgrid under study. With the proposed configuration, not only the redundancy of the system is increased, 
but also the system cost and power losses are reduced since the extra power converter is eliminated. Moreover, with the proposed configuration, the supercapacitor is able to support the grid during the transient startup of the dynamic load in addition to supplying the pulse load.

Different energy control methods are suggested for actively controlled hybrid DC power systems that include energy storage and pulse load. The direct voltage control (DVC) is the most straightforward method [46], [139]. In the DVC method, one of the converters controls the DC bus voltage directly while it is usually working at its maximum power limit during the pulse load on-time. Although the DVC strategy is effective and simple, it is disruptive to the system and requires more complicated energy management and load coordination [46], [107], [139].

As an alternative, the average current control method can be implemented to reduce the disruptive effects of the pulse load. In this method, the input power to the grid and the consumed power are set to be equal during a pulse period. As a result, the bus voltage variation can be kept within a preset limit. In [109], the continuous current averaging (CCA) technique is suggested to calculate the average of the pulse load power. It is shown that the CCA strategy yields a higher specific power and also much lower main source current with very small ripples [109], [113]. However, it is shown in this study that the CCA control technique is not able to properly track the pulse load variation. Moreover, the CCA method suffers from error accumulation in the control of a grid with high redundancy, which can cause severe overvoltage or undervoltage. To solve all the aforementioned issues, a new adaptive energy calculator (AEC) is proposed. This method is based on the moving average current and voltage measurement technique in addition to an adaptive gain 
compensation. To show the effectiveness of the developed AEC technique, its performances are compared with the DVC and CCA methods under pulse load variation and an artificial measurement error. Moreover, the developed AEC method and the energy management system (EMS) is tested under different grid and loading conditions.

\subsection{Controller Scheme of the DC-DC Converters}

In order to regulate the injection of power to the grid, the uncontrolled rectifiers are connected to the DC buses through boost converters with the configuration that is shown in Figure 3.9. The input inductive filter and the output capacitive filter of each converter are 5.6-mH and $1200-\mu \mathrm{F}$, respectively. Figure 5.2 shows the controller of the converter that has voltage and current control modes set by the EMS. In each mode, a proportionalintegral (PI) controller with anti-windup is employed to further improve control loop responses by preventing the controller output saturation during transients. The controller adjusts the duty ratio of the IGBT switching at a 5-kHz fixed frequency.

It should be noted that error accumulation in the EMS may cause overvoltage, which will be discussed in the next section. In order to protect the system from dangerous overvoltage, the DC-DC converters are equipped with hysteresis voltage protection. If the voltage of the DC bus exceeds its high limit, the converter will be turned off until the supercapacitor is discharged to the low voltage limit. 


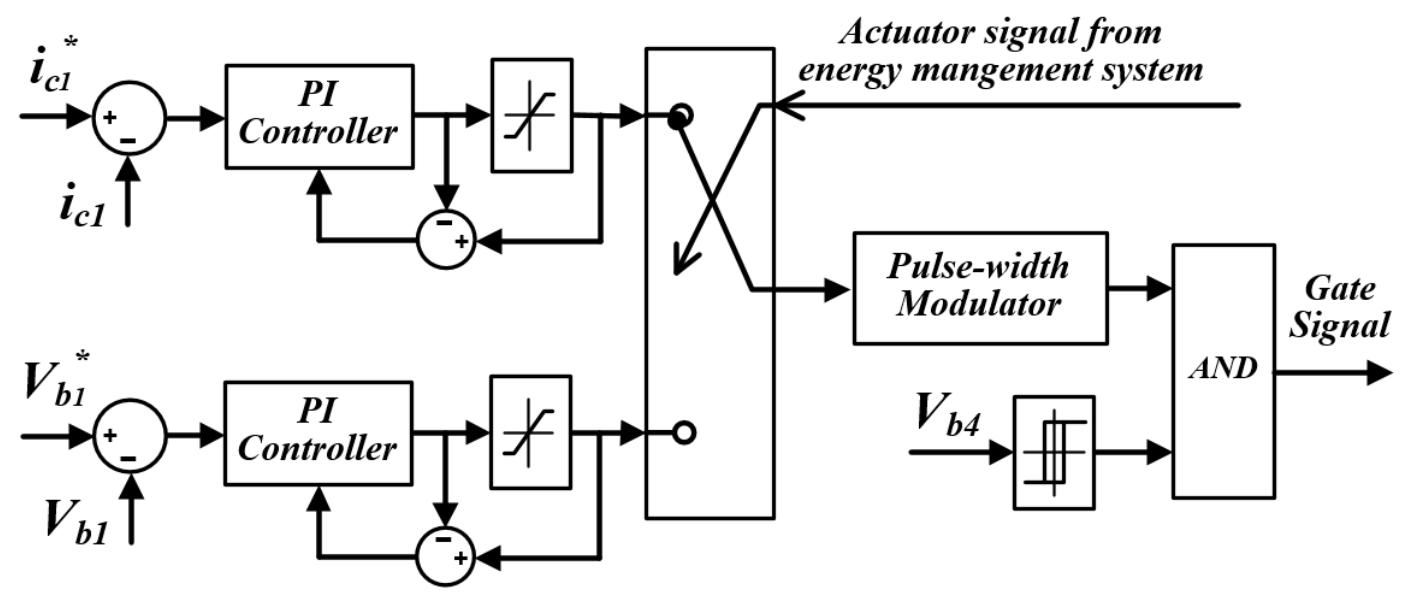

Figure 5.2: Block diagram of the first boost converter controller.

Also, the battery bank is connected to the grid through a bidirectional buck-boost converter with the topology that was shown in Figure 3.11. Figure 5.3 shows the controller of the converter. During the charging process, the converter is in buck mode and the duty ratio of the switching output S1 is controlled by the PI controller at a fixed frequency of 5$\mathrm{kHz}$ and the output S2 is turned off. Similarly, during grid support, the converter is in boost mode and the output S1 is turned off and S2 is switched on while the voltage limit of the grid is verified. The capacitance and inductance of the input filter of the bidirectional converter are $1200-\mu \mathrm{F}$ and 6-mH, respectively. This converter is connected to the DC bus through a $1200-\mu \mathrm{F}$ capacitive filter.

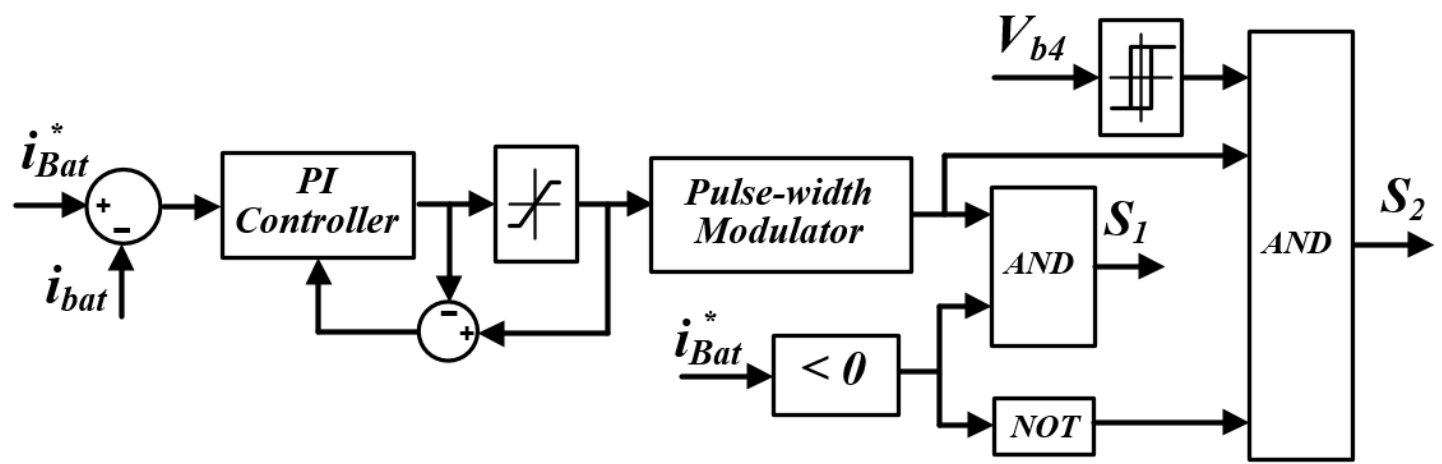

Figure 5.3: Block diagram of the bidirectional DC-DC converter controller. 


\subsection{Development of an Adaptive Energy Management System}

In this section, the sources of errors in the hybrid microgrid are studied and its effects on the EMS are analyzed. The proposed AEC and EMS are explained here in detail.

\subsubsection{Errors and Their Effect on the Energy Management}

For the real-time energy management of a redundant hybrid DC microgrid, one should ensure that the injected energy to the grid and the consumed energy are equal. However, the EMS that employs only current control is associated with three major sources of error, which are explained below:

i) Converter controller error: Any controller, depending on the application and whether it is optimized or not, suffers from the steady state and transient errors. The errors, especially the steady state error, highly affect the EMS performance.

ii) Measurement uncertainty: Measurements in the hybrid DC microgrid are incorporated with biased and unbiased noise [147]. Erroneous data in the current measurements for energy estimation can be removed by applying a low pass filter. More sophisticated techniques using statistical methods and random-fuzzy approaches are also applicable. However, it is often times problematic and may even be impossible to filter out all data corrupted with noise and bias errors.

iii) Power losses: The power consumption monitoring of a hybrid DC microgrid for proper energy management is highly important. However, the power losses of the DC buses and the cable cannot be easily measured. Ignoring the power losses, especially in a grid with a long cable, can cause considerable power consumption estimation error. 
For energy utilization of a supercapacitor bank, its terminal voltage should vary with time. The total energy that can be delivered by the supercapacitor can be expressed by (5.1):

$$
E=\frac{1}{2} C\left(V_{\max }^{2}-V_{\min }^{2}\right)
$$

where $V_{\max }$ is the maximum voltage of a supercapacitor bank during a pulse load and $V_{\min }$ is its minimum voltage. In the proposed hybrid DC microgrid, the maximum acceptable variation of the DC bus voltage is limited to $5 \%$.

To show the effect of the error on current control of a DC microgrid, assume that the losses of the cable and the DC bus during a short time interval are negligible. Then, equation (5.1) can be written based on the generated power of the converters and the consumed power of loads as (5.2):

$$
\sum_{i=0}^{N} \int_{0}^{t} V_{b i} i_{c i}-\sum_{j=0}^{M} \int_{0}^{t} V_{b j} i_{L i}=\frac{1}{2} C\left(V_{S C}^{2}-V_{S C 0}^{2}\right)
$$

where, $V_{S C O}$ and $V_{S C}$ are the supercapacitor voltage at $t=0$ and $t=t$, and $i_{L j}$ and $i_{c i}$ are the actual load current and converter output current, respectively. Also, $N$ and $M$ are the number of the converters and the loads connected to the DC buses. Furthermore, assuming that the converter and the load measurement have a constant error during the short time interval, $i_{c i}$ and $i_{L j}$ can be written as:

$$
\begin{gathered}
i_{c i}=i_{c i}^{*}\left(1+\alpha_{c i}\right) \\
i_{L j}=i_{L j}^{M}\left(1+\alpha_{M j}\right)
\end{gathered}
$$


where, $i_{c i}^{*}$ and $i_{L j}^{M}$ are the reference current of the $i^{\text {th }}$ converter and measured current of the $j^{t h}$ load. Also, the $\alpha_{c i}$ and $\alpha_{L j}$ are the error of the $i_{c i}$ and $i_{L j}$ with respect to the $i_{c i}^{*}$ and $i_{L j}^{M}$, respectively.

For the sake of simplicity, suppose that the converter and load bus voltage variation with respect to the converter and load power are negligible and they are equal to $V_{S C O}$.Thus, equation (5.2) can be written based on equations (5.3) and (5.4) as:

$$
\sum_{i=0}^{N} V_{S C 0} i_{c i}^{*}\left(1+\alpha_{c i}\right) t-\sum_{j=0}^{M} V_{S C 0} i_{L j}^{M}\left(1+\alpha_{M j}\right) t=\frac{1}{2} C V_{S C 0}^{2}\left(\beta(t)^{2}-1\right)
$$

where, $\beta(t)$ is the $V_{S C}$ to $V_{S C O}$ ratio.

Generally, in a real-time EMS, the current reference of each converter is set so that equation (5.6) is satisfied.

$$
\sum_{i=0}^{N}\left(V_{S C 0} i_{c i}^{*}\right) t=\sum_{j=0}^{M}\left(V_{S C 0} i_{L j}^{M}\right) t
$$

Thus, $\beta(t)$ can be found from equations (5.5) based on (5.6) as:

$$
\beta(t)=\sqrt{1+\frac{\sum_{i=0}^{N} \alpha_{c i} i_{c i}^{*} t-\sum_{j=0}^{M} \alpha_{M j} i_{L j}^{M} t}{V_{S C 0}}}
$$

It follows from equation (5.7) that with a current control, even if the generated and consumed power are set to be equal, the supercapacitor voltage highly depends on the error of the current measurements and it continually may change with time and cause error accumulation. 


\subsubsection{Adaptive Energy Calculator}

It is shown that the current control technique is not sufficient for the control of a grid with high redundancy. In order to improve the dynamic performance of the grid and to compensate the effect of error accumulation, a new AEC, based on the moving average measurement technique and an adaptive compensation gain, shown in Figure 5.4, is suggested.

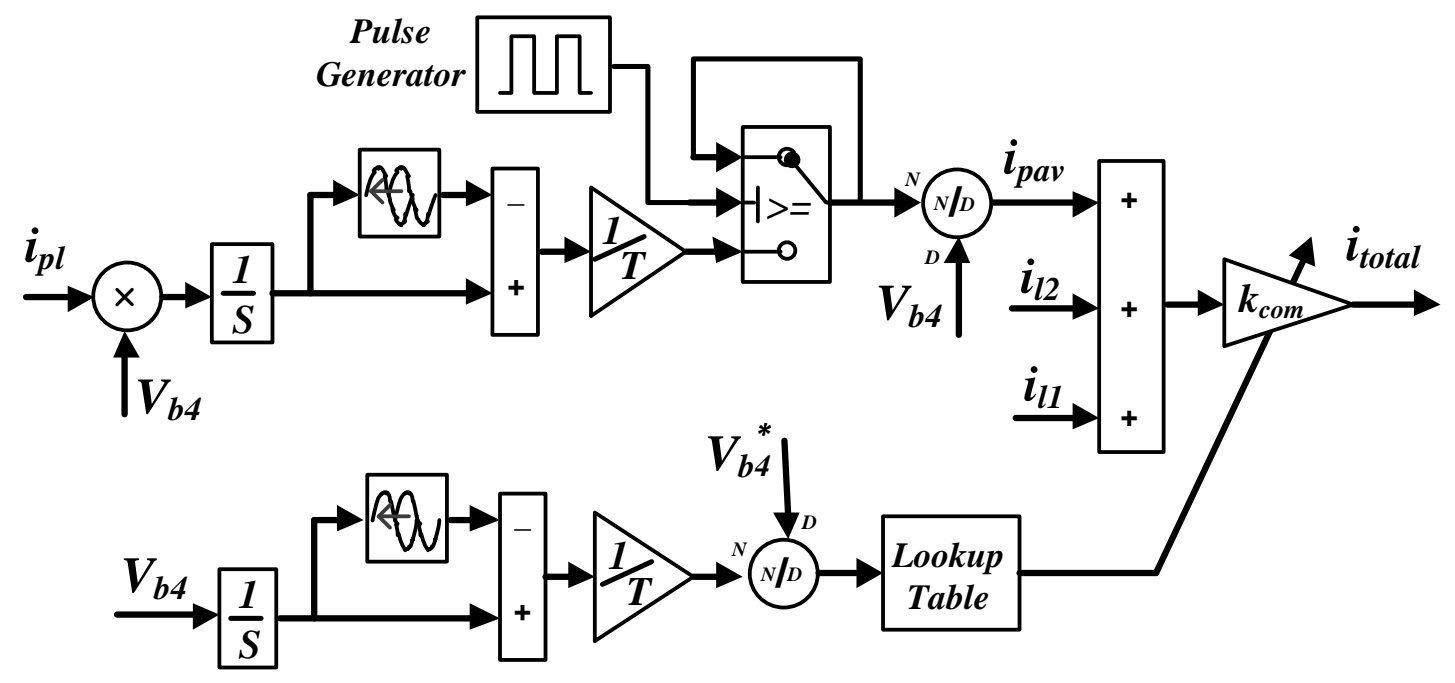

Figure 5.4: Block diagram of the Adaptive energy calculator.

The AEC unit calculates the total current that needs to be injected by the converters based on the loads power consumption and the supercapacitor bus voltage. In order to improve the dynamic performance of the AEC, the average current of the pulse load $i_{p a v}$, per each pulse duration $\mathrm{T}$, is calculated via the moving average measurement method and it is updated each $2 \mathrm{~T}$ second, while the average of the supercapacitor voltage is calculated continuously. As a result, the current measurement and voltage control will respond to the long-term and transient time variation of the grid, respectively. 
The voltage control is an adaptive proportional controller that continually changes the compensation factor $k_{c o m}$ to regulate the $i_{\text {total }}$ based on the normalized average bus voltage.

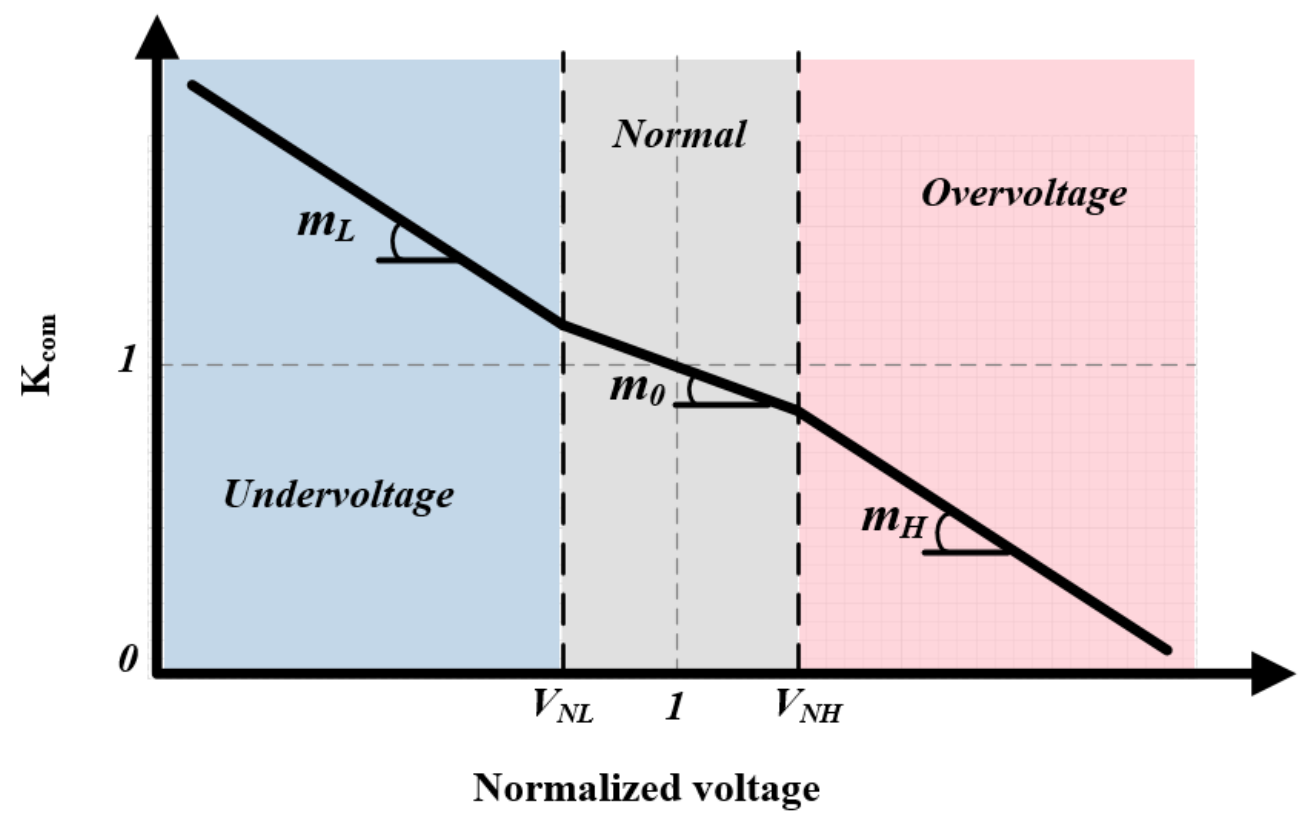

Figure 5.5: Compensation factor $k_{c o m}$ profile with respect to the normalized supercapacitor voltage.

Figure 5.5 shows the proposed scheme for $k_{c o m}$ variation with respect to the normalized supercapacitor voltage. In this figure, the $V_{N L}$ and $V_{N H}$ are the normal low voltage and normal high voltage thresholds of the supercapacitor, respectively. Three zones are defined in this profile: normal, overvoltage and undervoltage conditions in which the $k_{\text {com }}$ changes with $m_{0}, m_{L}$ and $m_{H}$ angle with respect to the normalized voltage. For better dynamic performance of the grid, the $m_{L}$ and the $m_{H}$ should be larger than $m_{0}$ to respond quickly to severe conditions. In this study, the nominal bus voltage $V_{b 4}, V_{N L}$ and $V_{N H}$ are $320 \mathrm{~V}, 0.995$ and 1.005, respectively. Also the $m_{0}, m_{L}$ and $m_{H}$ are 20, 86 and 46.7, respectively. 


\subsubsection{The Real-Time Energy Management System}

The main objective of the EMS unit is to set the current or the voltage reference of each converter based on the required power of the grid, availability of the converters and the battery state of charge. The EMS is composed of two parts: control mode selection and current reference assignment.

If the supercapacitor bank is connected to the grid, the EMS sets all of the converters to the current control mode. Otherwise, the EMS will set one of the converters to voltage control mode. In this case, the priority is given to the first converter.

Figure 5.6 shows how the EMS assigns the current reference of each unit. Initially, the EMS imports the $i_{\text {total }}$ from the AEC and then, based on the charge of the battery, the reference of the total required current $i_{\text {total }}^{*}$ is set. Following the verification of the availability of the converter, if both boost converters are connected, mode 11, the required current will be equally shared. Otherwise, if one of the converters is not available, mode 01 or mode 10 , the reference of the available converter will be set to the minimum of its maximum current limit $i_{c i}^{\max }$ and $i_{\text {total }}^{*}$, respectively.

The last step in the energy management system is to set the discharging or charging current reference of the bidirectional converter that is implemented in bus 2 . This will be determined based on the energy requirement of the grid and the battery bank state of charge. If the boost and bidirectional converters could not satisfy the power requirement, load shedding is necessary. However, load shedding is not covered in this study. 


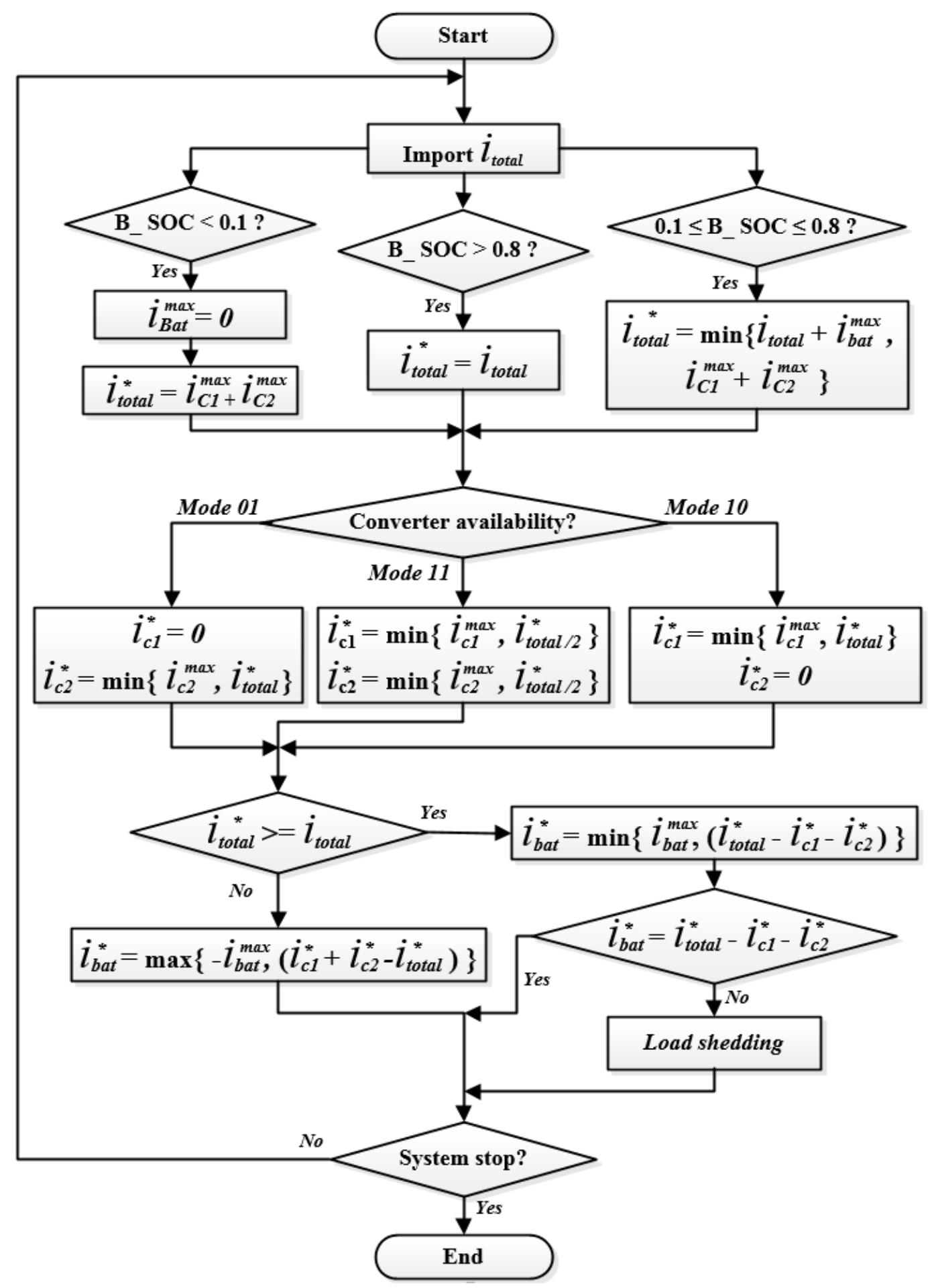

Figure 5.6: A flow chart of the real-time energy management algorithm. 


\subsection{Experimental Test Results}

The redundant hybrid DC microgrid, shown in Figure 5.1, has been implemented in our hybrid AC-DC power system test-bed to validate the feasibility of the proposed method. The developed EMS with AEC and the converters controller is built within Matlab/Simulink environment and executed with the dSPACE 1103 real-time interface. Also, for the control of the dynamic load 1 and the pulse load, the dSPACE 1104 board was used.

The nominal power of the AC generators are 3-kVA and are working at rated 60$\mathrm{Hz}$, frequency and 208- $\mathrm{V}$, nominal voltage. The AC filters are $12-\mathrm{mH}$ inductors that filter out harmonics to the AC generators. Also, the DC filters are connected between the uncontrolled rectifiers and the boost converters. The inductor and the capacitor of the DC filter are 6-mH and 850- $\mu \mathrm{F}$, respectively.

Three different types of loads were emulated in this microgrid. These include the steady state load, the dynamic load and the pulse load, which are connected to bus 3 , bus 1 and bus 4 , respectively. The rated power of the steady state load, load 1, is $2-\mathrm{kW}$ at the rated bus voltage. Also, in order to study the dynamic behavior of the microgrid, a $15-\Omega$ resistor was connected to the low voltage side of a buck converter, with a switching frequency of 2-kHz. This load, load 2, is able to fully emulate the dynamic behavior of a load up to 6-kW. Also, the nominal power of the pulsed load is $3.25-\mathrm{kW}$ and its pulse frequency is $0.1-\mathrm{Hz}$.

In this section, the performance of the developed EMS with the AEC is experimentally tested under various loading schemes and grid conditions. The results are compared with other methods such as DVC and CCA approaches. 


\subsubsection{Steady state operation}

In order to show the effectiveness of the current averaging techniques (AEC or CCA) in reducing the pulsed load disruptive effects, the performance of the DVC and the developed AEC method during steady state operation were experimentally evaluated. Figure 5.7 shows the tests results.
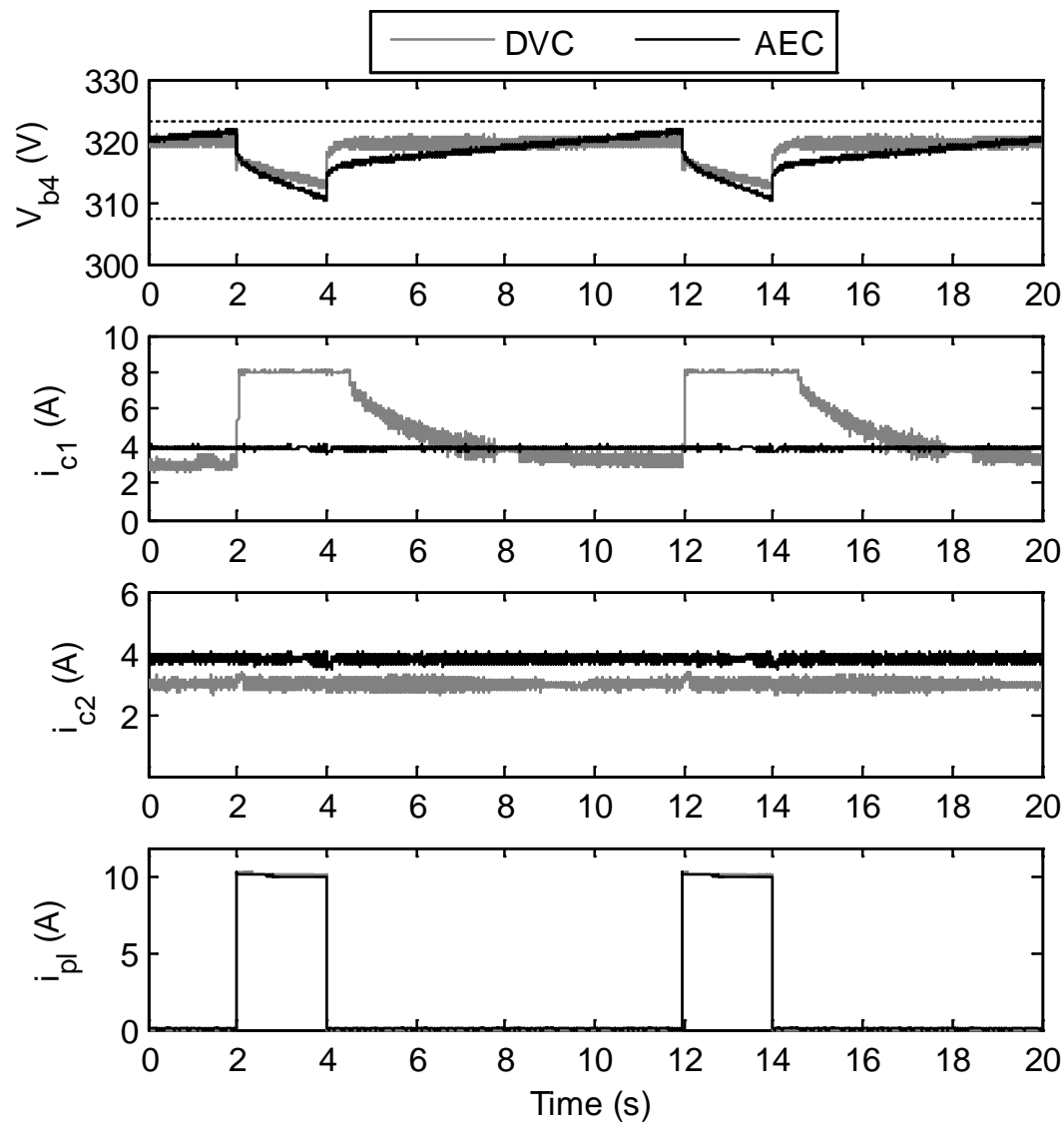

Figure 5.7: DC microgrid performance in the case of the AEC and the DVC control methods for pulse load $=3.25-\mathrm{kW}(1.0$ p.u. $)$ and load $1=500-\mathrm{W}(0.25$ p.u. $)$ and load $2=$

$1.35-\mathrm{kW}$ ( 0.675 p.u.). The dashed lines in the voltage graph show the $5 \%$ thresholds.

In the case of the DVC method, the first converter utilized the voltage-mode control and the second converter used the current control mode and supplied 3-A. As the results show, the DVC caused considerable current and power pulsation in the first converter. During the pulse on-time, the first converter reached its maximum power limit (2.5-kW) 
and injected 8-A to support the DC bus voltage. Within a short time following the pulse load being turned off, at t = 4.4-s, the bus voltage reached its reference. Consequently, upon the completion of supercapacitor bank charge redistribution, the converter's current reduced to 3-A asymptotically.

In the case of AEC technique, the power pulsation of the converter was prevented. It can be observed that the EMS equally shared the average required energy between the two boost converters and each converter injected 4-A to the grid continuously.
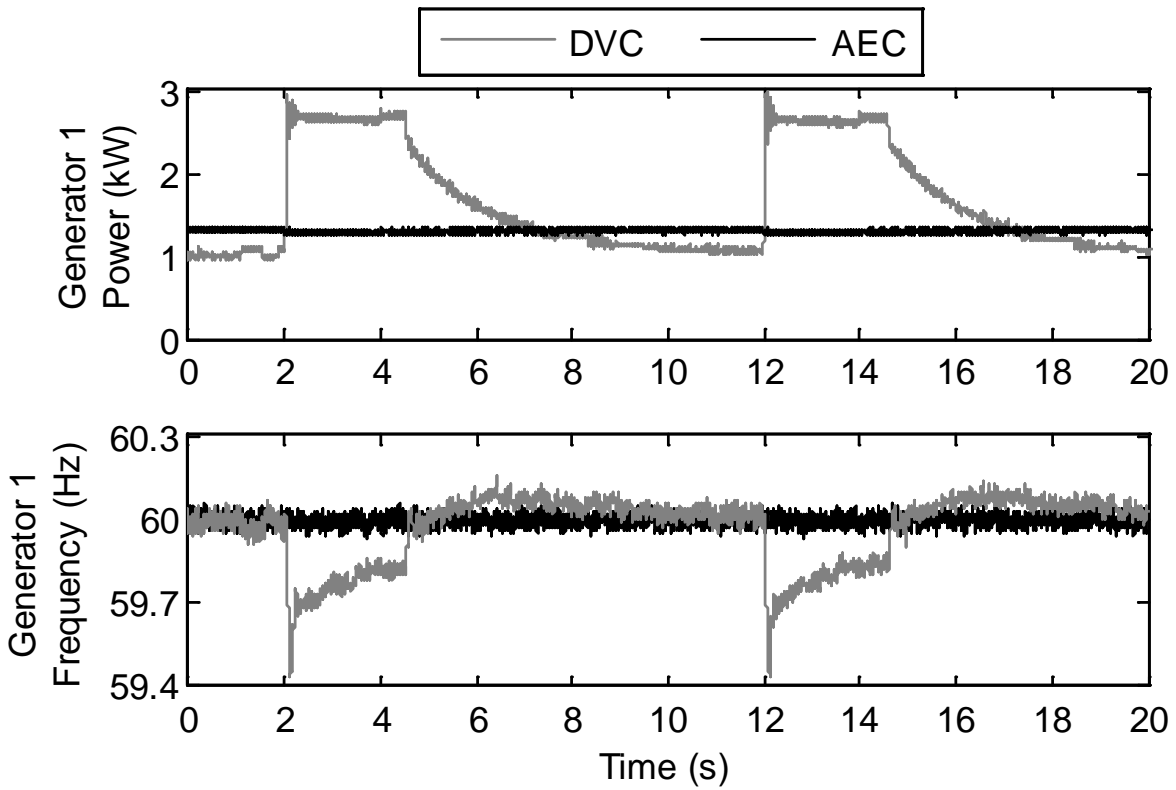

Figure 5.8: Generator performance comparison of the AEC and the DVC control methods.

To further show the superiority of the AEC method and the consequence of converter current pulsation, the generator performance during the two experiments are demonstrated in Figure 5.8. Also, the comparative analyses of the two methods are summarized in Table 5.1. It can be concluded that, although the AEC technique caused 0.84\% more bus voltage variation than the DVC, it effectively eliminated the high current and power pulsation of the converter and generator. Using the AEC method, the generator 
continuously supplied $1.3-\mathrm{kW}$ and its frequency was $60-\mathrm{Hz}$ with only $0.11-\mathrm{Hz}$ variation. However, the DVC method caused considerable power pulsation and frequency fluctuation, i.e. when the pulse occurred, the generator frequency dropped to $59.42-\mathrm{Hz}$ and returned to $60-\mathrm{Hz}$ with a small overshoot.

Table 5.1: Test results summary for AEC and DVC control methods.

\begin{tabular}{|c|c|c|c|c|}
\hline $\begin{array}{c}\text { Implemented } \\
\text { Method }\end{array}$ & $\frac{\Delta V_{b 4}}{V_{b 4}^{\text {nom }}}$ & $\frac{\boldsymbol{I}_{c 1}^{\max }}{\boldsymbol{I}_{c 1}^{\text {nom }}}$ & $\frac{\Delta P_{G 1}}{P_{G 1}^{\text {nom }}}$ & $\Delta f_{a c}$ \\
\hline DVC & 0.0272 & 1.0 & 0.611 & $0.69 \mathrm{~Hz}$ \\
\hline AEC & 0.0356 & 0.49 & 0.074 & $0.11 \mathrm{~Hz}$ \\
\hline
\end{tabular}
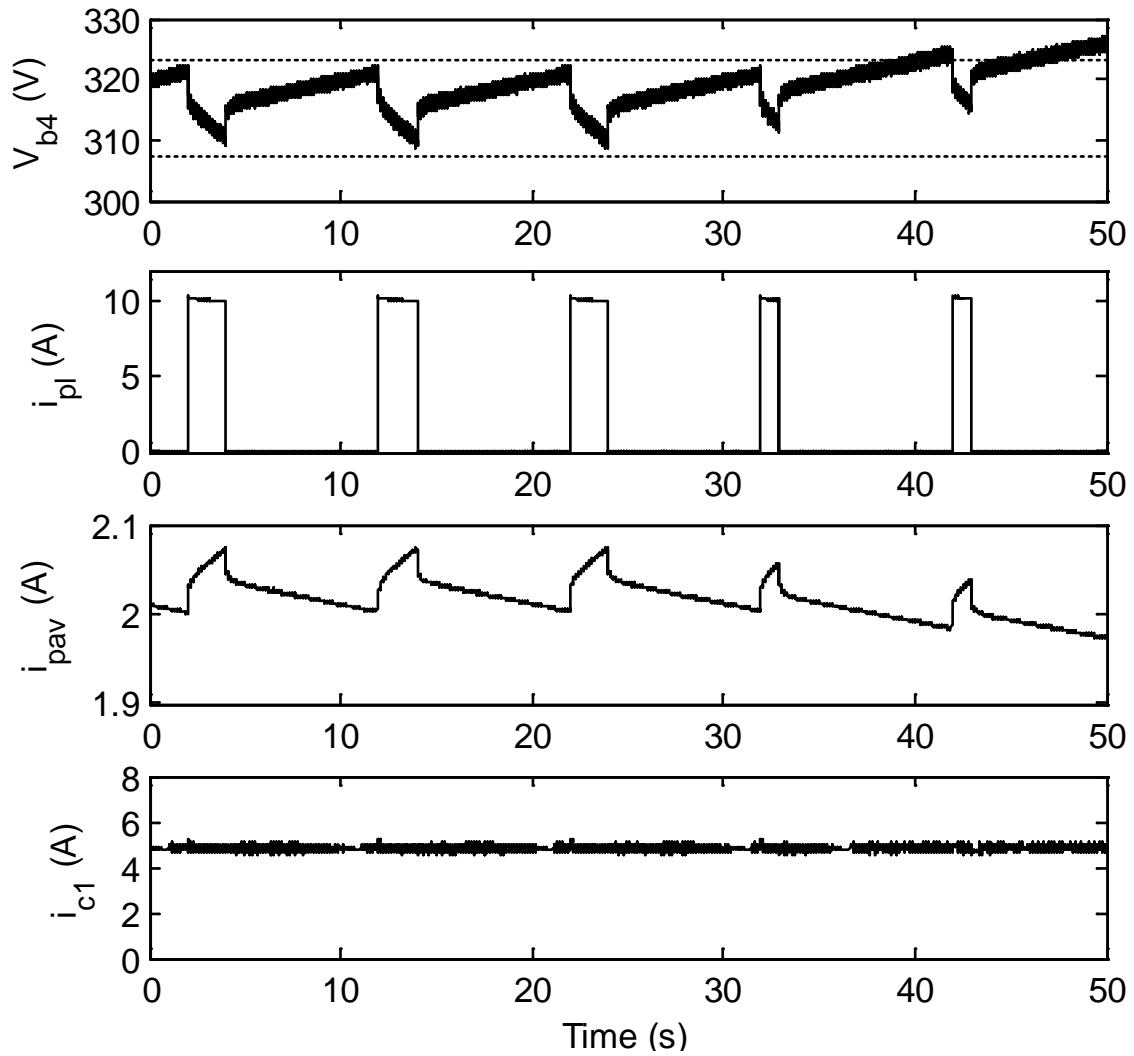

Figure 5.9: Operation test results of the microgrid with time averaging current measurement method for load $1=500-\mathrm{W}(0.25$ p.u.) and load $2=2-\mathrm{kW}$ (1.0 p.u.) while the duty ratio of the $3.25-\mathrm{kW}$ (1.0 p.u.) pulse load is changed from $20 \%$ to $10 \%$ at $\mathrm{t}=30$ -

S. 

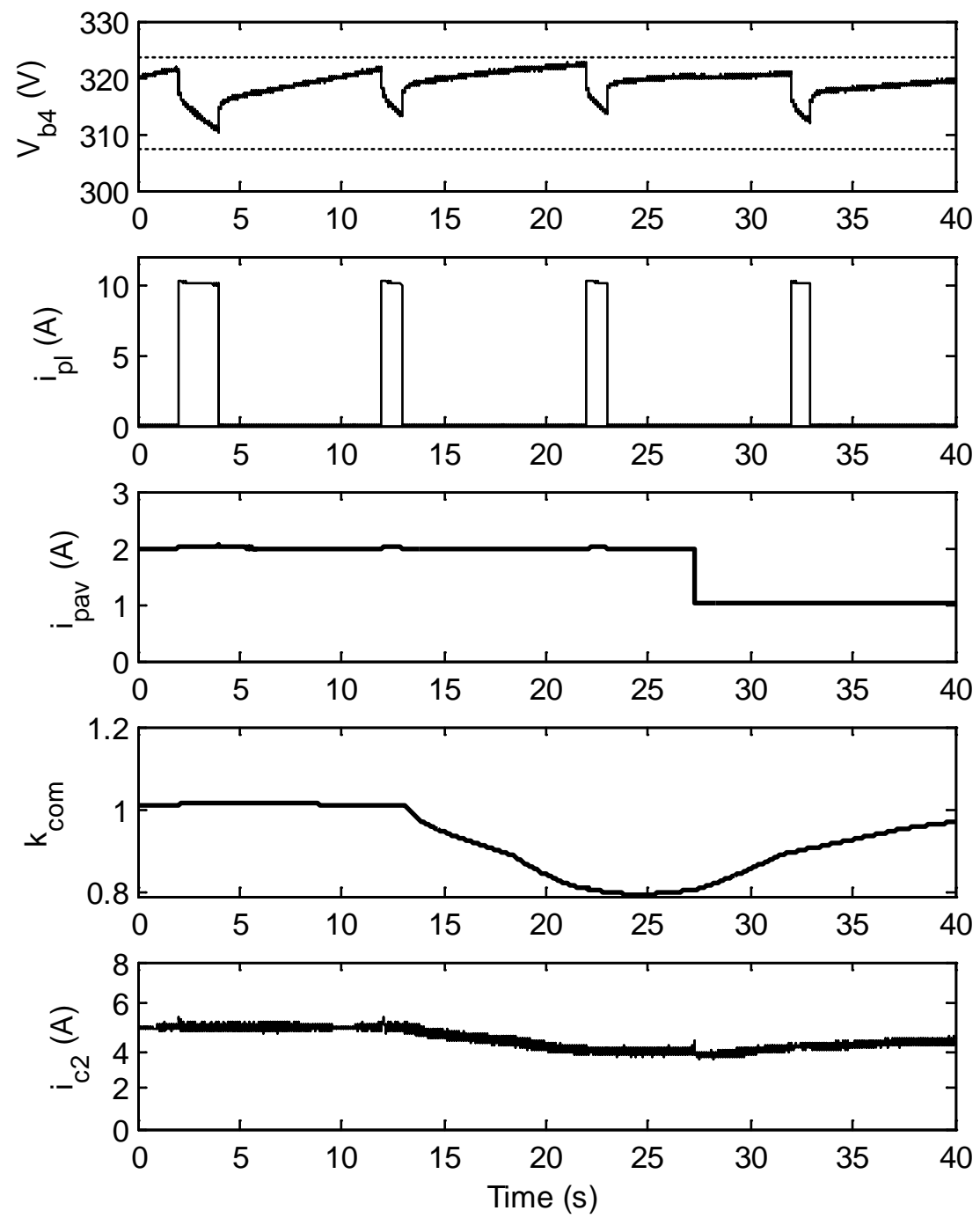

Figure 5.10: Operation test result of the microgrid with AEC method for load $1=500-\mathrm{W}$ (0.25 p.u.) and load $2=2-\mathrm{kW}$ (1.0 p.u.) while the duty ratio of the $3.25-\mathrm{kW}$ (1.0 p.u.) pulse load is changed from $20 \%$ to $10 \%$ at $\mathrm{t}=10$-s.

\subsubsection{Pulse load duty ratio variation}

In this section, the transient performance of the AEC is compared with the traditional CCA technique during the pulse load duty ratio variation. Figure 5.9 shows the experimental test results for the case when the CCA method was used and the pulsed load duty ratio was decreased from $20 \%$ to $10 \%$ at $t=30$-s. As observed, the $i_{p a v}$ decreased very slowly with time and it was not able to track the pulse load variation properly. This occurred as a result of the long memory of the CCA method, which caused an overpower 
injection to the grid and considerable voltage increase of the supercapacitor. The average of the $V_{b 4}$ increased from $320-\mathrm{V}$ to $324-\mathrm{V}$ in $20 \mathrm{~s}$.

Figure 5.10 shows the test results for a similar case when the AEC technique was implemented and the pulse duty ratio was changed at $t=10$ s. As observed, the $i_{p a v}$ was able to track the pulse duty ratio change in 20s. The transient overestimated energy was compensated by automatic reduction of the $K_{\text {com }}$ due to the small increase in the average bus voltage. Once $i_{p a v}$ tracked the pulse load variation and system error was reduced, the $K_{\text {com }}$ returned to 1 . Consequently, the AEC decreased the converters current with no overvoltage on the supercapacitor.

\subsubsection{Operation with inaccurate measurement}

In this section, the performance of the EMS without adaptive voltage gain compensation and the EMS with AEC technique under artificial measurement inaccuracy is analyzed. In order to have a considerable effect of the error in a short time, exaggerated $10 \%$ artificial over-measurement was applied on load 1 while it was changed from $0-\mathrm{W}$ to $1500-\mathrm{W}$ at $\mathrm{t}=8 \mathrm{~s}$. Figure 5.11 shows the experimental test results for the case that EMS without adaptive gain compensation was implemented. As the experimental results show, based on (5.7), the overestimation of the required energy caused considerable overvoltage during a short time, i.e. overcharging of the supercapacitor increased the bus voltage from $320-\mathrm{V}$ to $326-\mathrm{V}$ in $32 \mathrm{~s}$.

The experimental test results for a similar case while the EMS with AEC was utilized is depicted in Figure 5.12. The results show that the AEC properly tracked the load variation and changed the current reference of each converter from 4.05-A to 6.85-A at $\mathrm{t}=$ 8-S. Also, the AEC using the voltage feedback reduced the $K_{\text {com }}$ from 1.004 to 0.971 after 
25s and compensated the over-measurement. Therefore, the supercapacitor did not suffer any overvoltage.
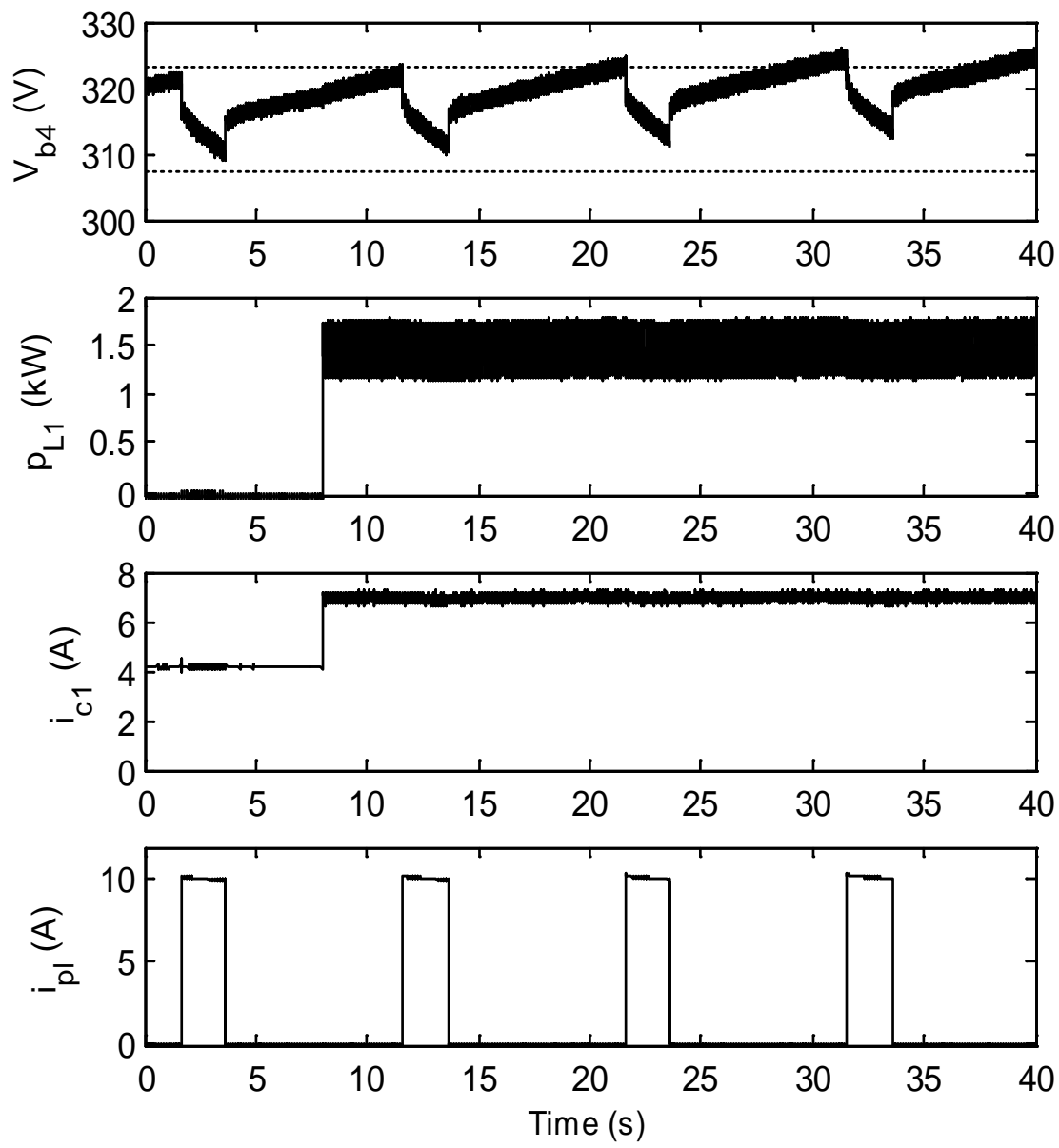

Figure 5.11: Operation test results of the microgrid without adaptive gain compensation for pulse load $=3.25-\mathrm{kW}(1.0$ p.u. $)$ and load $2=2-\mathrm{kW}(1.0$ p.u. $)$ while the load 1 is changed from 0 to $1500-\mathrm{W}$ ( 0.75 p.u.) at $\mathrm{t}=8$-s with $10 \%$ over-measurement.

\subsubsection{Grid support during transient time}

One of the advantages of the direct connection of the supercapacitor to a redundant DC microgrid is its capability to support the grid during the transient time when it is highly loaded. In order to evaluate the performance of the AEC and the EMS, the load 2 is emulated as a load with high starting power, based on equation (3.1). 

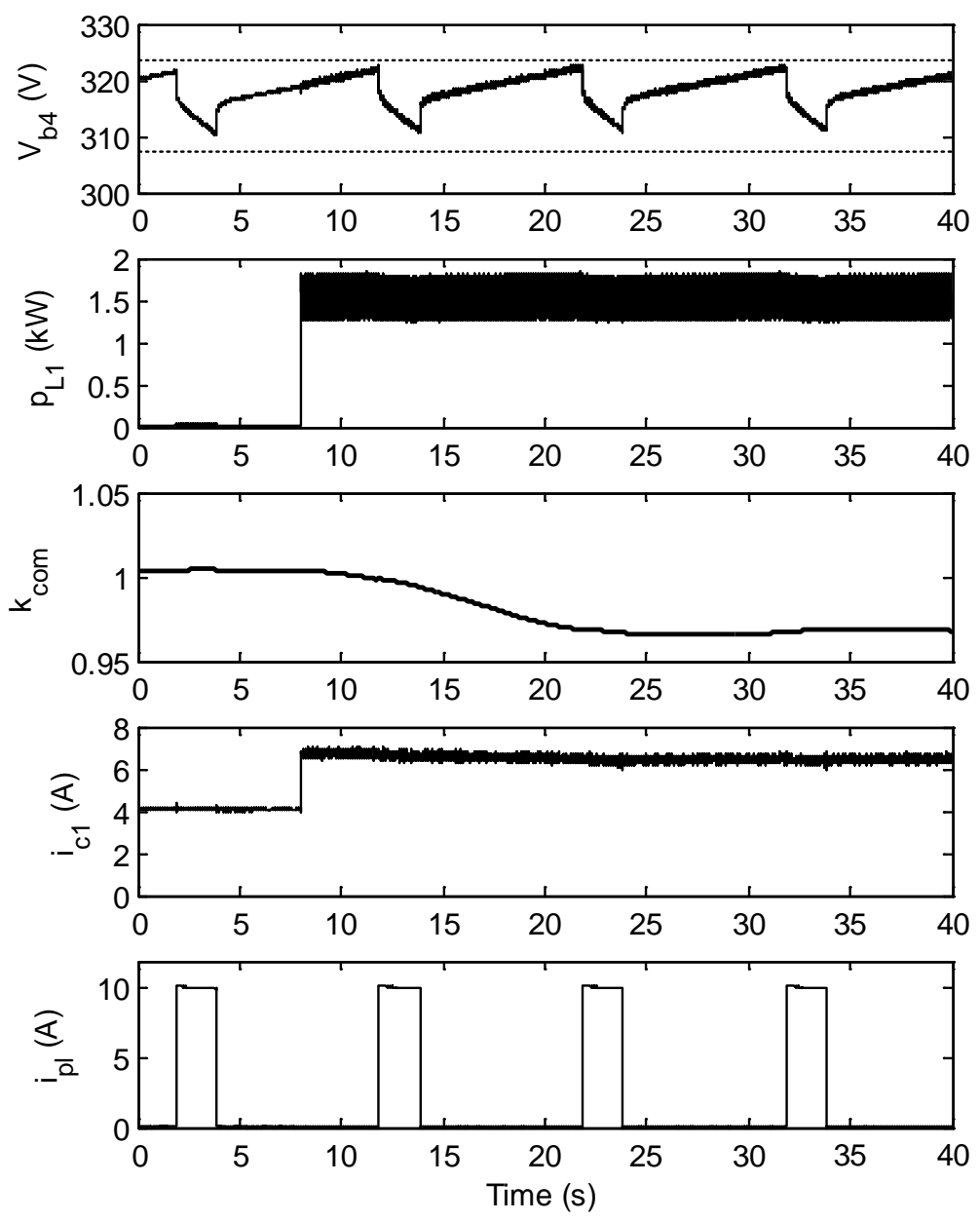

Figure 5.12: Operation test results of the microgrid with AEC for pulse load $=3.25-\mathrm{kW}$ (1.0 p.u.) and load $2=2-\mathrm{kW}$ (1.0 p.u.) while the load 1 is changed from 0 to $1500-\mathrm{W}$

(0.75 p.u.) at $\mathrm{t}=8$-s with $10 \%$ over-measurement.

Figure 5.13 shows the performance of the grid for the case when the battery bank was disconnected from the grid. The values of these parameters in equation (3.1) were; $P_{N}$ $=2000-\mathrm{W}, k=2$ and $\tau=1 \mathrm{~s}^{-1}$. As observed, load 1 increased to $6000-\mathrm{W}$ at $\mathrm{t}=6$-s and then settled at 2000-W after 3.36s. Since the total power requirement of the loads during the startup was higher than the two converters power capacity, the converters were saturated while the supercapacitor bank was discharged and supported the grid. Consequently, the average bus voltage was decreased and, as a result, the AEC increased the $K_{\text {com }}$ based on the control method explained in Figure 5.4 and Figure 5.5. The experimental results prove 
that the proposed AEC and EMS are able to properly manage the energy and prevent any considerable overvoltage or undervoltage even when the grid is highly overloaded.
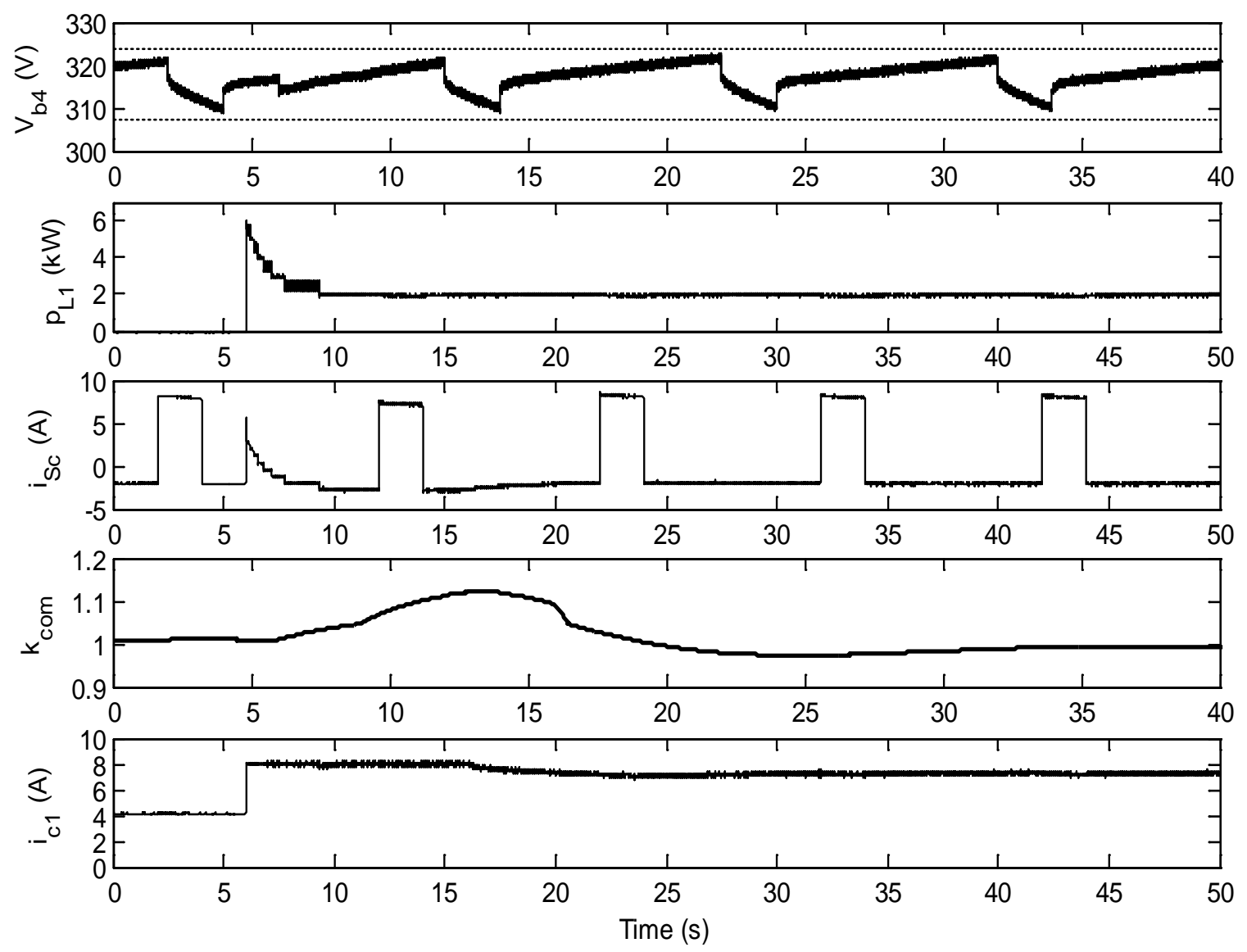

Figure 5.13: Operation test results of the microgrid with the proposed controller for pulse load $=3.25-\mathrm{kW}$ (1.0 p.u.) and load $2=2-\mathrm{kW}(1.0$ p.u. $)$ while the load 1 is changed from

0 to $2-\mathrm{kW}(1.0$ p.u. $)$ at $\mathrm{t}=6$-s with three times overshoot and damping factor of 1 -s.

\subsubsection{Converter or supercapacitor outage}

One of the important characteristics of a reliable hybrid DC microgrid is its selfhealing and self-reconfiguring capability. Figure 5.14 shows the test results for the case that an outage occurred in the first converter at $\mathrm{t}=10 \mathrm{~s}$. Based on the algorithm explained in Figure 5.6, the EMS set the second converter to its maximum power $\left(i_{c 2}^{*}=8 A\right)$ and the rest of the power was injected by the bidirectional converter. Also, it can be seen that there 
is no voltage fluctuation for the step load change, which proves the effectiveness of the energy management algorithm.
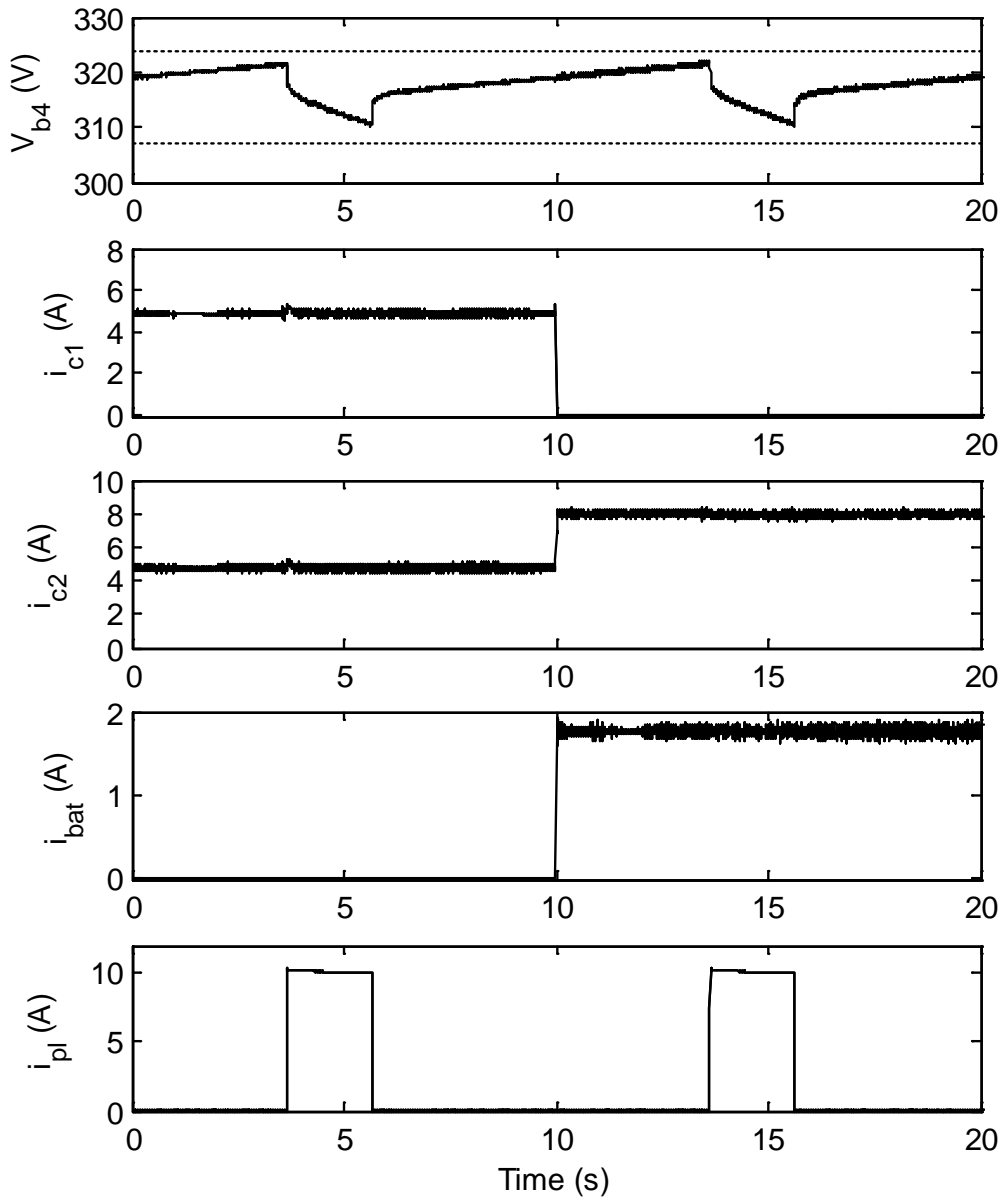

Figure 5.14: Test results for the grid with pulse load $=3.25 \mathrm{~kW}$ (1.0 p.u.), load $1=500$ $\mathrm{W}(0.25$ p.u. $)$ and load $2=2-\mathrm{kW}(1.0$ p.u.) while outage of the converter 1 is occurred at $\mathrm{t}$ $=10$-s.

The experimental test results for the case that the supercapacitor and pulse load at $\mathrm{t}=10 \mathrm{~s}$ were disconnected from the microgrid are shown in Figure 5.15. Based on the EMS priority list, the first converter was set to the voltage control mode. During this time, the second converter was in current control mode and continuously supplied 50\% of the load requirements. Thus, both converters equally shared the power even when the first converter was in voltage control mode. 

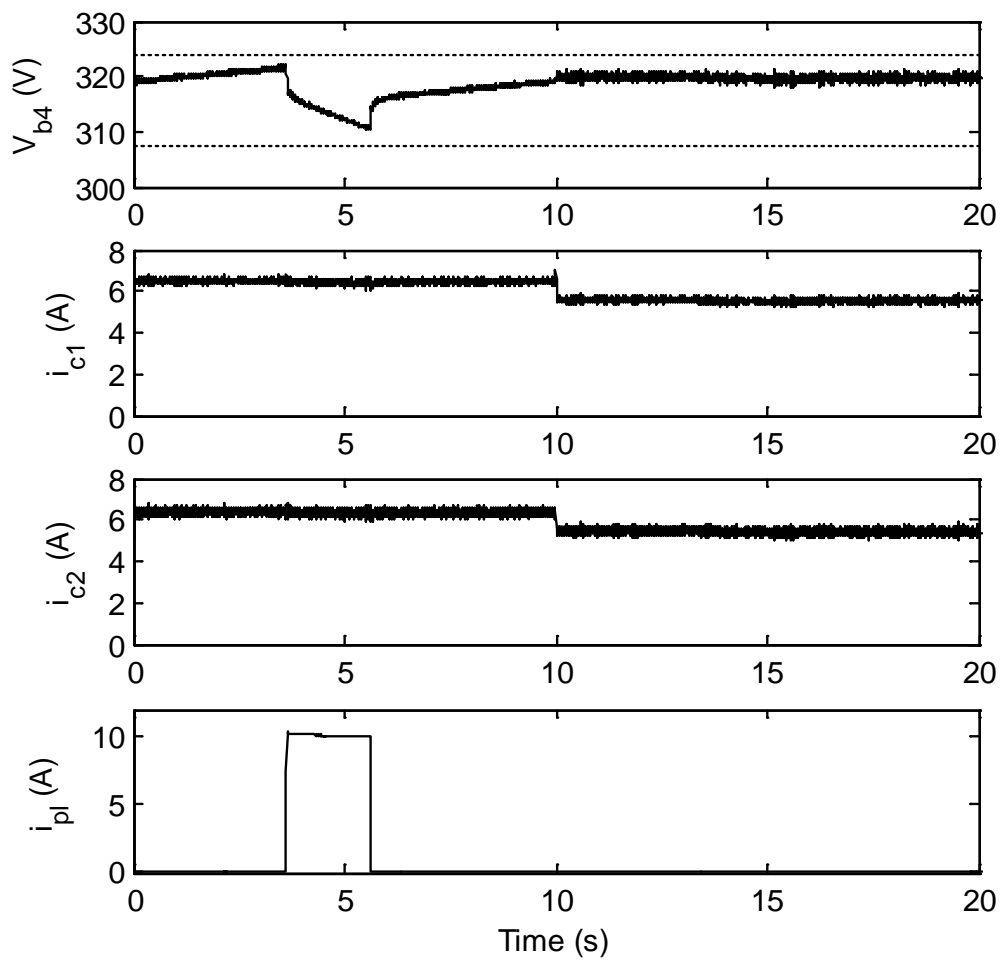

Figure 5.15: Test results for the grid with pulse load $=3.25-\mathrm{kW}$ (1.0 p.u.), load $1=1500$ W (0.75 p.u.) and load $2=2 \mathrm{~kW}(1.0$ p.u.) while the supercapacitor and pulse load are disconnected at $\mathrm{t}=10$-s.

\subsection{Conclusion and Summary of the Results}

A real-time control and energy management technique of a notional DC microgrid with high redundancy and heavy pulse load was studied. An adaptive energy calculator (AEC) based on a moving average measurement technique and a proportional voltage compensation was developed. Also, an energy management system (EMS) was designed to set the current or the voltage reference of each converter based on the AEC reference current, the battery state of charge and availability of the converters.

The proposed hybrid DC microgrid and its real-time controller was implemented in hardware and experimentally tested under various loading schemes and grid conditions. The results showed that the EMS with AEC technique caused 0.84\% more bus voltage variation than the direct voltage control (DVC). However, it effectively eliminated the high 
current pulsation of the converter, as well as prevented the power and frequency fluctuation of the generator. Also, the performance of the proposed method during transient pulse duty ratio variations was compared with continuous current averaging (CCA) method. The results showed that the EMS with AEC technique adaptively adjusts the total reference current during transient time. The bus voltage was maintained within the limit even if the measurement had a considerable error.

Moreover, the grid performance under transient high loading was evaluated and the results showed that using the proposed system configuration, the supercapacitor bank can support the grid and control the bus voltage. Finally, the reliability of the hybrid DC microgrid was tested and its self-reconfiguring capability was validated. 


\section{PERFORMANCE ANALYSIS OF GRID CONNECTED HYBRID DC MICROGRID INCORPORATING PULSE LOAD}

\subsection{Introduction}

Grid connected DC microgrid power systems with hybrid energy resources are of significant interest in many applications. Multiple sources and reconfiguration characteristics of the DC microgrid on these systems highly improve the efficiency and reliability of the system [148]-[150]. In the hybrid DC power system, there are some types of loads with a common profile that require relatively high pulse power, but with relatively low average power requirement. As discussed in chapter 2, such a high level of short time current behavior requires a higher power rating of the components and could potentially cause a significant disturbance to the entire grid [45], [107], [139].

Pulsed loads not only reduce the efficiency of the DC microgrid and cause voltage drop in the common coupling DC bus, but there are additional disruptive impacts in the interconnected AC grid. Since in such a system there is no infinite bus in the AC grid, the existence of large pulsed loads cause considerable voltage drop and frequency fluctuation [45]-[46], [139].

In this chapter, performance evaluation of the new developed energy control scheme for actively controlled hybrid DC microgrid that is connected to a main AC grid is proposed. The developed energy control is an adaptive current-voltage control (ACVC) scheme based on the moving average measurement technique and an adaptive proportional compensator. Unlike conventional energy control methods, the proposed ACVC approach has the advantages of controlling both the voltage and current of the system while keeping 
the output current of the power converter at a relatively constant value. For this study, a laboratory scale hybrid DC microgrid is developed to evaluate the performance of the ACVC strategy and to compare its performance with the other conventional energy control methods. Using experimental test results, it is shown that the proposed strategy highly improves the dynamic performance of the hybrid DC microgrid. Although the ACVC technique causes slightly more bus voltage variation, it effectively eliminates the high current and power pulsation of the power converters. The experimental test results for different pulse duty ratios demonstrate a significant improvement achieved by the developed ACVC scheme in enhancing the system efficiency, reducing the AC grid voltage drop and the frequency fluctuations.

\subsection{Energy Control for Grid Connected Hybrid DC Power System}

Different hybrid configurations are possible for pulse load applications. Passive configuration is the direct parallel connection of the supercapacitor with other energy resources and is considered the simplest hybrid configuration. The advantages of this method include ease of implementation, lower power losses and low cost due to the absence of additional power electronic converters. However, since the sources are always paralleled, the power sharing between them is highly limited and it is determined by their respective resistances and voltage-current characteristics [142]. On the contrary, in an active hybrid configuration, the battery and/or other energy resources are connected to the supercapacitor and pulse load through a power electronic converter to completely regulate the power injection. Previous studies have shown that the power capability of an actively controlled hybrid is much greater than that of a passive hybrid. Moreover, the active hybrid 
combinations have advantages of lower weight and volume, low current ripple of the main source and better output voltage regulation [109], [113], [146].

Several studies were performed on the control of an active hybrid DC power system. Some of these studies dealt with power and efficiency improvement of the system [109], [113], and others were conducted for stability enhancement of the AC grid [45], [107], [139]. For pulse load mitigation, the energy control scheme is very important as it largely governs the interaction of the pulse load with the AC/DC power system. In this study, a new energy control technique based on ACVC for hybrid DC microgrid is developed and its performances are compared with the other energy control schemes suggested in the literature. Unlike conventional methods, the proposed approach has the advantage of controlling both voltage and current of the DC microgrid. In order to improve the dynamic operation of the grid, the moving average current and voltage measurement is utilized for this method. The developed ACVC strategy is able to properly buffer the converters from the high pulse currents while maintaining the bus voltage variations within an acceptable range.

In order to test the performance of the proposed ACVC strategy and to compare it with the other conventional energy control methods, a laboratory scale hybrid DC microgrid was developed for this study. The comparative analysis is done both under steady state operation of the pulse load and under pulse load transients, such as duty ratio and frequency changes. The developed technique is compared with the other conventional methods in terms of the DC and AC power system performances. 
In the following sections, the hardware test setup of the system and its control scheme are described first. Section 6.4 describes conventional energy control techniques modified and adapted for our hybrid DC microgrid. Also, the detailed description of the developed ACVC strategy is presented in section 6.5. Section 6.6 discusses the performances of the ACVC strategy in comparison to other energy control methods. This is followed by the summary of the results and conclusions in section 6.7.

\subsection{System Description}

The notional hybrid DC microgrid considered for this study is depicted in Figure 6.1. This microgrid consists of several types of loads and hybrid energy sources that are connected to a common DC bus. The hardware setup and the control structure for this system and the interconnected AC grid are explained in this section.

\subsubsection{Hardware Description}

The energy sources of the DC microgrid are the AC grid, the battery bank and the supercapacitor bank. The AC grid is mainly supplying the microgrid while the battery bank provides extra power when the grid is highly loaded or if the DC microgrid is islanded. Also, the supercapacitor bank functions as a power buffer device and provides the instantaneous power required.

The interconnected AC grid is a hardware/software-based AC grid test-bed power system. This system includes generating stations, and programmable loads in a laboratory scale of up to $35 \mathrm{~kW}$. More details about the AC grid test-bed configuration and control can be found in section 3.8 . 


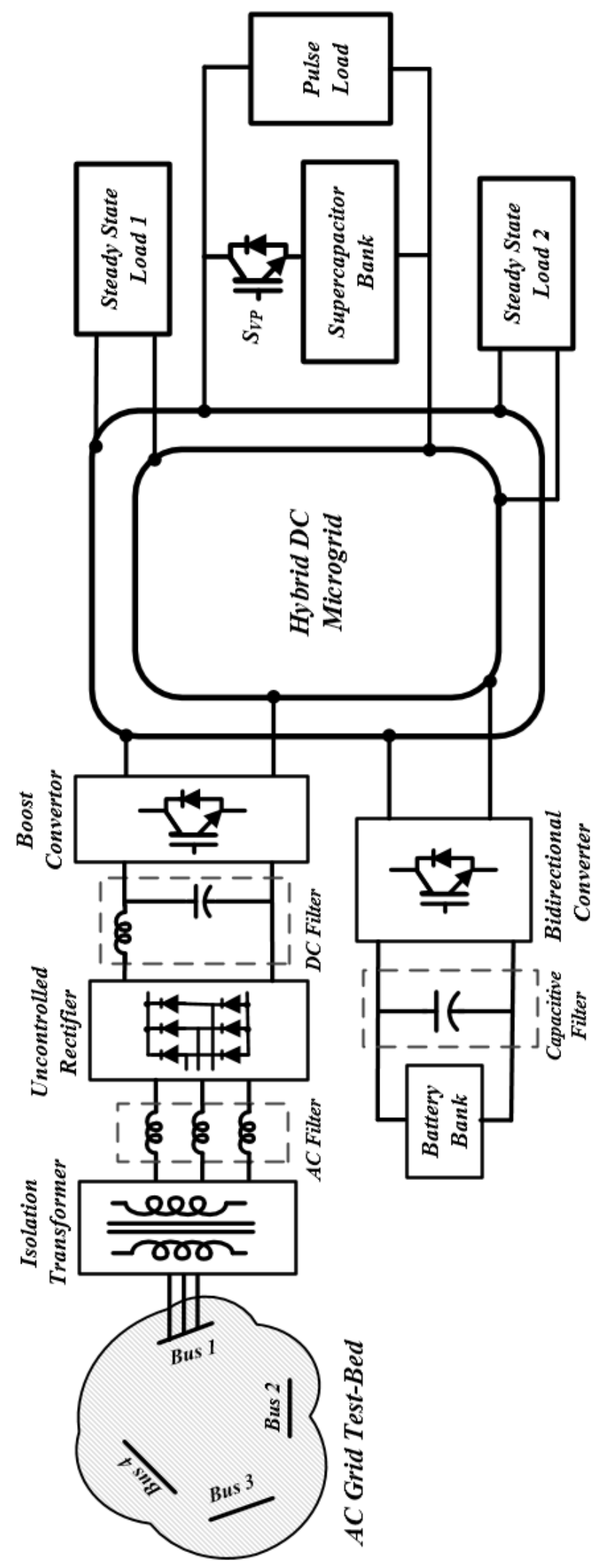

ठ્. 
The hybrid DC microgrid under study is connected to the AC grid through the uncontrolled rectifier and boost converter for power conversion and regulation. A threephase $\mathrm{Y} / \Delta$ transformer was implemented to galvanically isolate the AC grid from the DC microgrid. Also, an inductive AC filter is located between the transformer and the uncontrolled rectifier to filter out the harmonics to the AC grid. Moreover, an inductivecapacitive DC filter is connected between the boost converter and the uncontrolled rectifier to improve the performance of the converter and reduce the AC grid harmonics. The detailed parameters of the components are summarized in Table 6.1.

Table 6.1: Hybrid DC Microgrid System Parameters.

\begin{tabular}{|c|c|c|}
\hline Component & Parameter & Specification \\
\hline \multirow{5}{*}{ Transformer } & Connection & $Y D$ \\
\hline & $S_{N}$ & 3 kVA (1 p.u.) \\
\hline & $V_{N}$ & $208 V(1$ p.u. $)$ \\
\hline & $R_{e q}, X_{e q}$ & $\begin{array}{c}0.72 \Omega(0.05 \text { p.u. }) \\
0.86 \Omega(0.06 \text { р.и. })\end{array}$ \\
\hline & $R_{M}, X_{M}$ & $\begin{array}{l}4820 \Omega(334 \text { p.u.), } \\
16.45 \Omega(430 \text { p.u.) }\end{array}$ \\
\hline \multirow{4}{*}{ Boost Converter } & power rating & $2500 \mathrm{~W}$ \\
\hline & IGBT module & SKM100GAL12T4 \\
\hline & switching frequency & $5 \mathrm{kHz}$ \\
\hline & $L_{B C}$ & $6 \mathrm{mH}$ \\
\hline AC Filter & $L_{A F}\left(X_{L A C}\right)$ & $12 \mathrm{mH}(4.52 \Omega, 0.31 p . u)$ \\
\hline \multirow{2}{*}{ DC Filter } & $\overline{L_{D F}}$ & $2.7 \mathrm{mH}$ \\
\hline & $C_{D F}$ & $680 \mu F$ \\
\hline
\end{tabular}

As shown in Figure 6.1, the battery bank is connected through the bidirectional converter to the common coupling DC bus. The battery bank is rated 120-V, 110-Ah. Also, the supercapacitor bank is 2.9-F, and as an energy buffer, delivers high instantaneous power to the pulse load. The analog hysteresis protection explained in section 3.7.5, is 
implemented to ensure that uneven charge distribution, especially during very fast charges, does not cause the supercapacitor to exceed its maximum voltage limit. This protection unit also provides a backup for the energy control system. If the voltage on any of the supercapacitor arrays exceeds the preset limit, the analog control circuit will open the output of the IGBT switch $S_{v p}$, shown in Figure 6.1. The charging path remains open until the supercapacitor is discharged through the bypass diode to the point that its voltage is reduced to the predefined safe threshold. The detailed parameters of the storage system and the bidirectional converter are summarized in Table 6.2.

Table 6.2: Energy Storage and Bidirectional Converter Parameters.

\begin{tabular}{|c|c|c|}
\hline Component & Parameter & Specification \\
\hline \multirow{5}{*}{ Battery Bank } & Type & $\begin{array}{c}\text { Universal (UB121100) } \\
\text { Lead Acid }\end{array}$ \\
\hline & Number of Cells & 12 \\
\hline & Rated Capacity & $110 \mathrm{Ah}$ \\
\hline & Bank nominal Voltage & $120 \mathrm{~V}$ \\
\hline & Internal Resistance & $4 m \Omega$ \\
\hline \multirow{7}{*}{$\begin{array}{c}\text { Supercapacitor } \\
\text { Bank }\end{array}$} & Type & Maxwell (BMOD0058) \\
\hline & Number of Cells & 20 \\
\hline & Rated Capacity & $2.9 \mathrm{~F}$ \\
\hline & Rated Voltage & $320 \mathrm{~V}$ \\
\hline & Maximum Voltage & $340 \mathrm{~V}$ \\
\hline & Maximum Continuous Current & $12 A\left(\Delta T=15^{\circ} \mathrm{C}\right)$ \\
\hline & Leakage Current & $\begin{array}{c}25 \mathrm{~mA} \\
\text { (Passive Balancing) }\end{array}$ \\
\hline \multirow{4}{*}{$\begin{array}{l}\text { Bidirectional } \\
\text { Converter }\end{array}$} & power rating & $1800 \mathrm{~W}$ \\
\hline & IGBT module & SK45GB063 \\
\hline & switching frequency & $5 \mathrm{kHz}$ \\
\hline & $L_{B D}$ & $6 \mathrm{mH}$ \\
\hline
\end{tabular}




\subsubsection{Control Description}

Figure 6.2 shows the control of the hybrid DC microgrid, which consists of three layers. The first layer is the energy control system. This control layer utilizes the DC grid bus voltage and the load current to set the total current command, $i_{c}^{*}$. The next layer is the formulation of the reference current of the boost converter, $i_{c 1}^{*}$ and the reference current of the bidirectional converter, $i_{c 2}^{*}$ based on the converters availability and their power limitation. If both converters are available, the priority is given to the boost converter to supply the microgrid through the AC grid. The bidirectional converter is utilized in the case that an outage occurs in the AC grid or if the power requirement of the DC microgrid is higher than the boost converter power limitation.

The third layer is the converter controllers that regulate the output current of the converters. The converter controllers are PI with anti-windup that improves the control loop responses during transients and saturation. The controller adjusts the duty ratio of the IGBT switching at 5-kHz fixed frequency using pulse width modulation (PWM) technique. The switching signals PWM1 and PWM2 shown in Figure 6.2 are assigned to the boost converter and the bidirectional converter, respectively.

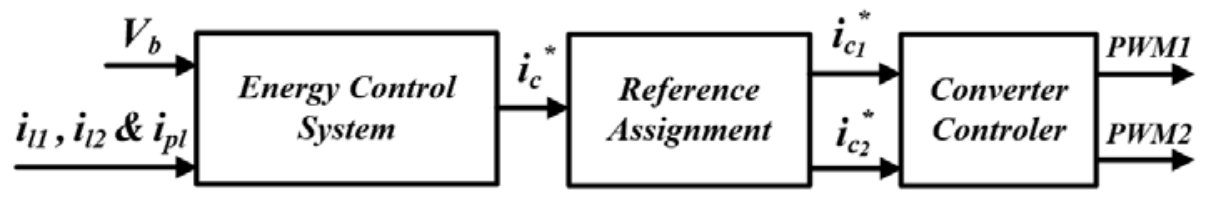

Figure 6.2: Block diagram of the DC microgrid three layer control system.

The energy control system largely governs the interaction of the pulse load with the DC microgrid and the AC power system. In the following sections, different energy control schemes, including the developed ACVC technique, are described. 


\subsection{Energy Control Schemes for Grid Connected Hybrid DC Microgrid}

Various energy control methods for pulse load mitigation suggested in the literature are adapted for our hybrid DC microgrid and are explained here. These methods differ from each other for the measurement data required and the performance level achieved.

\subsubsection{Instantaneous Power Control}

Instantaneous power control (IPC), as discussed in [45] and [139], is the simplest control technique of a hybrid DC power system. Figure 6.3 shows the schematic diagram of this controller, which is modified and adapted for our hybrid DC microgrid. As can be seen, this control method utilizes the instantaneous consumed power of the loads and the common coupling bus voltage $V_{b}$ to set $i_{c}^{*}$. The $\tau_{v m}$ and $\tau_{c r}$ are the time constants of the low pass filters for the measured $V_{b}$ and reference command $i_{c}^{*}$, respectively.

This controller is equipped with hysteresis voltage protection that monitors the entire supercapacitor bank. Once the voltage of the DC bus exceeds the preset maximum limit $V_{b \max }$, the controller will set $i_{c}^{*}$ to zero until the bus voltage drops to the preset minimum bus voltage threshold $\mathrm{V}_{\mathrm{bmin}}$.

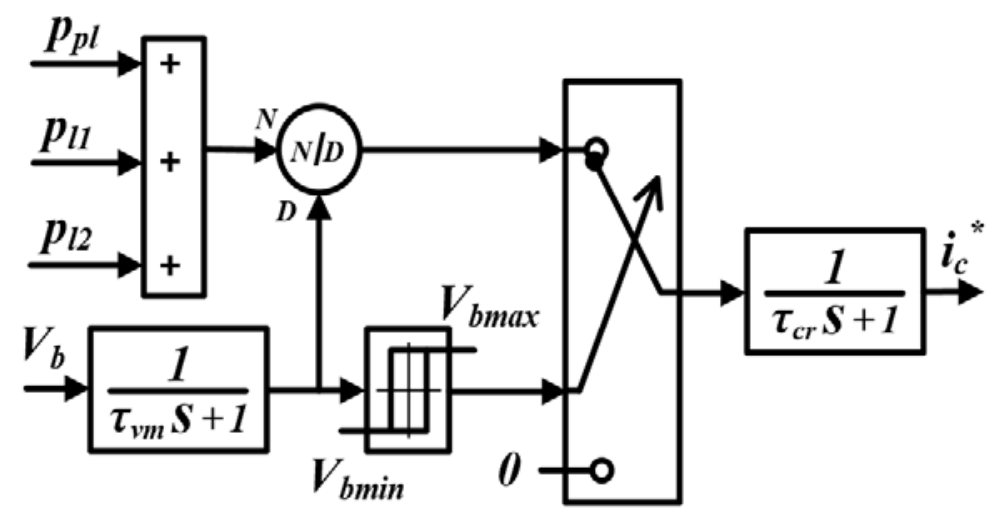

Figure 6.3: Block diagram of the instantaneous power control. 


\subsubsection{Limit-Based Voltage Control}

The basic concept of the limit-based voltage control (LBVC) is to charge the supercapacitor bank and control the DC bus voltage as rapidly as possible subject to the available power of the sources and the converter power limit [46], [139].

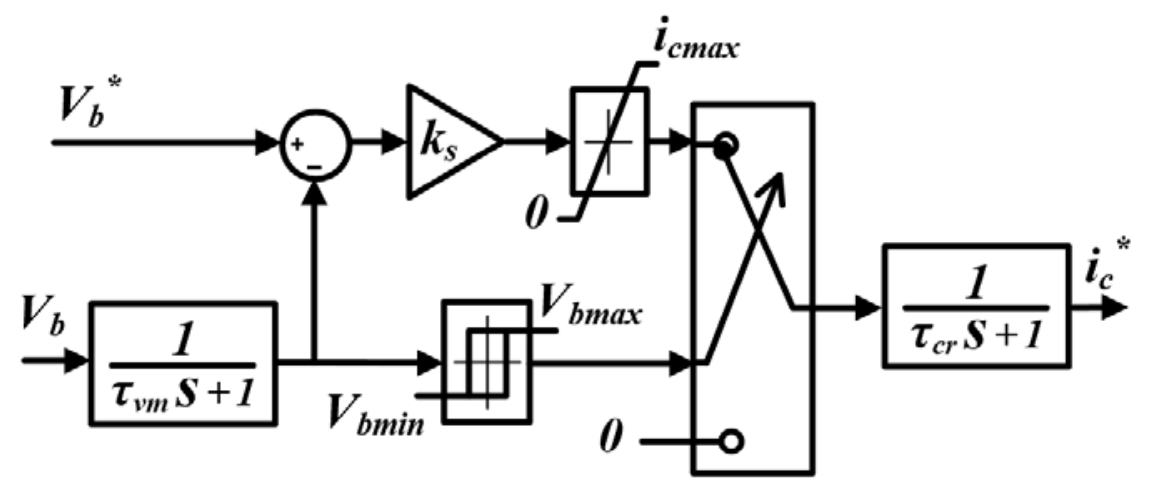

Figure 6.4: Block diagram of the limit-base voltage control.

The concept of this controller is shown in Figure 6.4. The two input ports are the target bus voltage $V_{b}^{*}$ and the measured bus voltage $V_{b}$ and the output port is the reference current command $i_{c}^{*}$. As can be seen, the measured bus voltage is first filtered by a low pass filter and then subtracted from $V_{b}^{*}$. The voltage error is then multiplied by a proportional gain $k_{s}$ and limited to the maximum current limit of the system $i_{\text {cmax }}$. The gain $k_{s}$ needs to be large enough so that the limit $i_{c \max }$ be in effect until the point where the bus voltage becomes very close to $V_{b}^{*}$ and then approaches it asymptotically. It should be noted that the $V_{b}^{*}$ is larger than the $V_{b \min }$ and smaller than the $V_{b \max }$. If the bus voltage is less than the $V_{b \max }$, the output of the current limiter goes through an output filter to form $i_{c}^{*}$. Otherwise, $i_{c}^{*}$ will be set to zero until the supercapacitor is discharged and the bus voltage is decreased to $\mathrm{V}_{\mathrm{bmin}}$. 


\subsubsection{Continuous Average Current Control}

Heavy pulse loads lead to significant disturbance in a power system due to their power profile characteristics. To reduce the power pulsation of the converters and sources, the continuous average current control (CACC) technique is suggested [45], [109], [113].

Figure 6.5 shows the schematic diagram of the CACC method adapted for our hybrid DC microgrid. Inputs to this controller are the bus voltage $V_{b}$ and the load currents $i_{p l}, i_{l 1}$ and $i_{l 2}$, where the output is the reference current command $i_{c}^{*}$. The goal for the CACC method is to keep the output current of the power converter at a relatively constant value equal to the sum of the steady state load current and pulse load time-averaged current. This buffers the converters from high pulse currents while maintaining the bus voltage. As can be seen in Figure 6.5, the pulse load current is first integrated and averaged according to the operation-time. Then, the calculated continuous averaged current is added to the steady state load's currents. If the safe operation of the system is ensured, the calculated current is then fed to the output filter to form $i_{c}^{*}$.

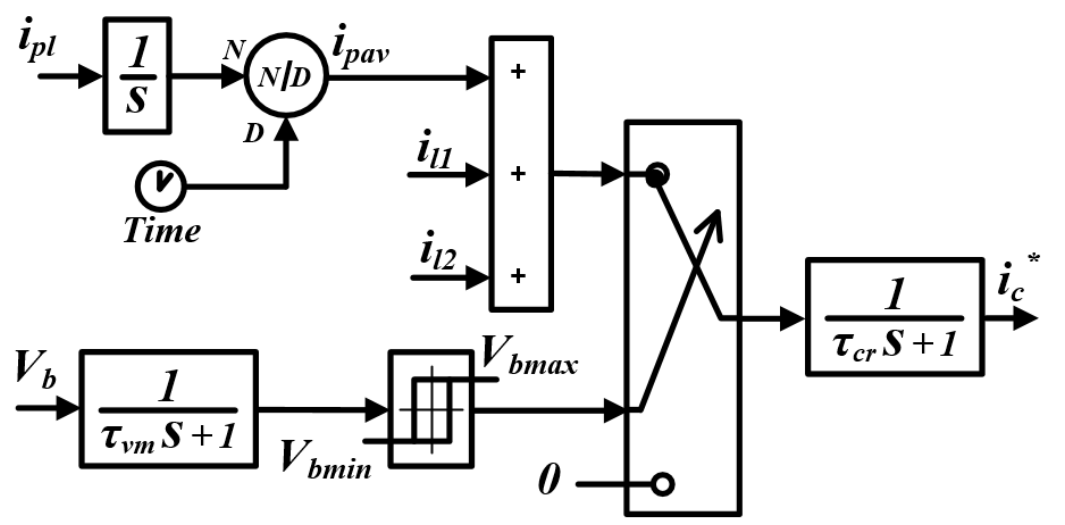

Figure 6.5: Block diagram of continues average current control. 


\subsection{Developed Adaptive Current-Voltage Control}

An effective energy control scheme should be able to keep the converters at a relatively constant value to prevent converter power pulsation. Although the CACC method effectively reduces the system disturbance during a pulse load with constant duty ratio and constant amplitude, it fails during pulse load changes and transient grid operation. In order to improve the dynamic performance of the grid and to buffer the battery bank and AC grid from high pulse currents, a new ACVC technique is developed in this study.

The ACVC is designed based on the moving average current and voltage measurement and an adaptive gain compensator. Figure 6.6 shows the schematic diagram of this controller. The input ports of the controller are the pulse load power, the steady state load currents and the bus voltage, while the output port is the reference current command $i_{c}^{*}$. As can be seen, the measured pulse load power is first integrated and passed through a delay block with a time delay of $\mathrm{T}$ seconds, where $\mathrm{T}$ is the averaging period. Then, the integrated power is subtracted from the delayed value to calculate the accumulated power. To calculate the average power during the last T period, $P_{p a v}$ the accumulated power is divided by the time period $\mathrm{T}$.

Similarly, the average of the bus voltage, $V_{b a v}$ is calculated and then normalized with respect to the desired voltage. The proposed ACVC is designed so that the current control and the voltage control respond to the long-term and transient variation of the grid, respectively. Thus, the $p_{p a v}$ is calculated per $\mathrm{T}$ period and is updated every $\mathrm{T}$ cycle, while the $V_{b a v}$ is continually updated. 


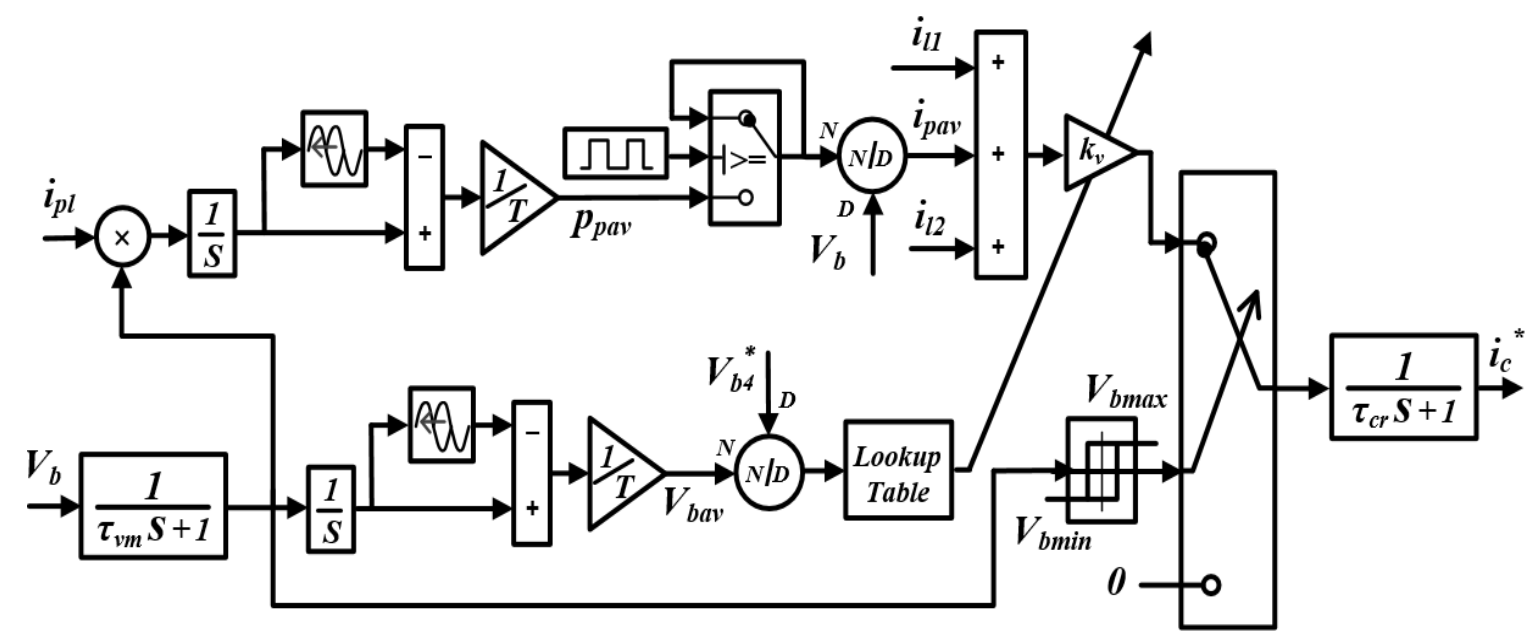

Figure 6.6: Block diagram of adaptive current-voltage control.

To set the reference current $i_{c}^{*}$, the updated $p_{p a v}$ is divided by the $V_{b}$ to form the $i_{\text {pav }}$ and then is added to the steady state loads currents. This current is fed to the gain $k_{v}$, which is an adaptive proportional voltage controller. The $k_{v}$ is continually updated using a lookup table and the normalized average bus voltage. The lookup table is composed of three zones: normal, overvoltage and undervoltage conditions. These zones are defined based on the voltage thresholds $V_{N L}$ and $V_{N H}$, which are the normalized low voltage and normalized high voltage thresholds of the DC bus. The $k_{v}$ changes with $m_{0}, m_{L}$ and $m_{H}$ angle in normal, overvoltage and undervoltage conditions, respectively. For better dynamic performance of the grid, the $m_{L}$ and the $m_{H}$ should be larger than $m_{0}$ to respond quickly to severe undervoltage and overvoltage conditions. In this study, the $m_{0}, m_{L}$ and $m_{H}$ are 20, 86 and 46.7, respectively, where $V_{b}, V_{N L}$ and $V_{N H}$ are $320 \mathrm{~V}, 0.995$ and 1.005, respectively.

\subsection{Comparative Analysis}

In this section, the performance of the developed ACVC technique is experimentally validated and it is compared to the other energy control methods. The three 
layer control system, which includes the energy control, the reference current formulation and converter control, are built within Matlab/Simulink environment and executed with the dSPACE 1103 real-time interface. In order to obtain the optimal values of the PI controllers, the Simulink Response Optimization toolbox was employed [151]. Also, the dSPACE 1104 board was utilized to control and adjust the pulse load and the steady state loads. In the following experimental test, the steady state load 1 and load 2 had power consumptions of 700-W and 300-W, respectively.

\subsubsection{Compared to CACC Method}

The developed ACVC technique and the CACC method are aimed at keeping the power converter at a relatively constant value to buffer the converter and the AC grid from power pulsation. Figure 6.7 shows the comparative analysis of the hybrid DC microgrid performance when the ACVC technique and CACC method are employed. In this experiment, the pulsed load was $2-\mathrm{kW}$ at a frequency of $0.1-\mathrm{HZ}$ and duty ratio of $20 \%$. As can be seen, the ACVC and CACC methods had a similar performance; the energy control system adjusted the boost converter to a relatively constant 4.5-A, which is equal to the sum of the steady state load current and the pulse load time-averaged current. This resulted in a slight variation of common coupling DC bus voltage and allowed the supercapacitor to supply the pulsed load. During the pulse on-time, the supercapacitor bank was discharged to $314.7-\mathrm{V}$ and was charged during the pulse off-time and the voltage increased to 322.6-V. Since the generated and consumed power were equal, the average voltage was always $320-\mathrm{V}$ and the loads were continually supplied. 

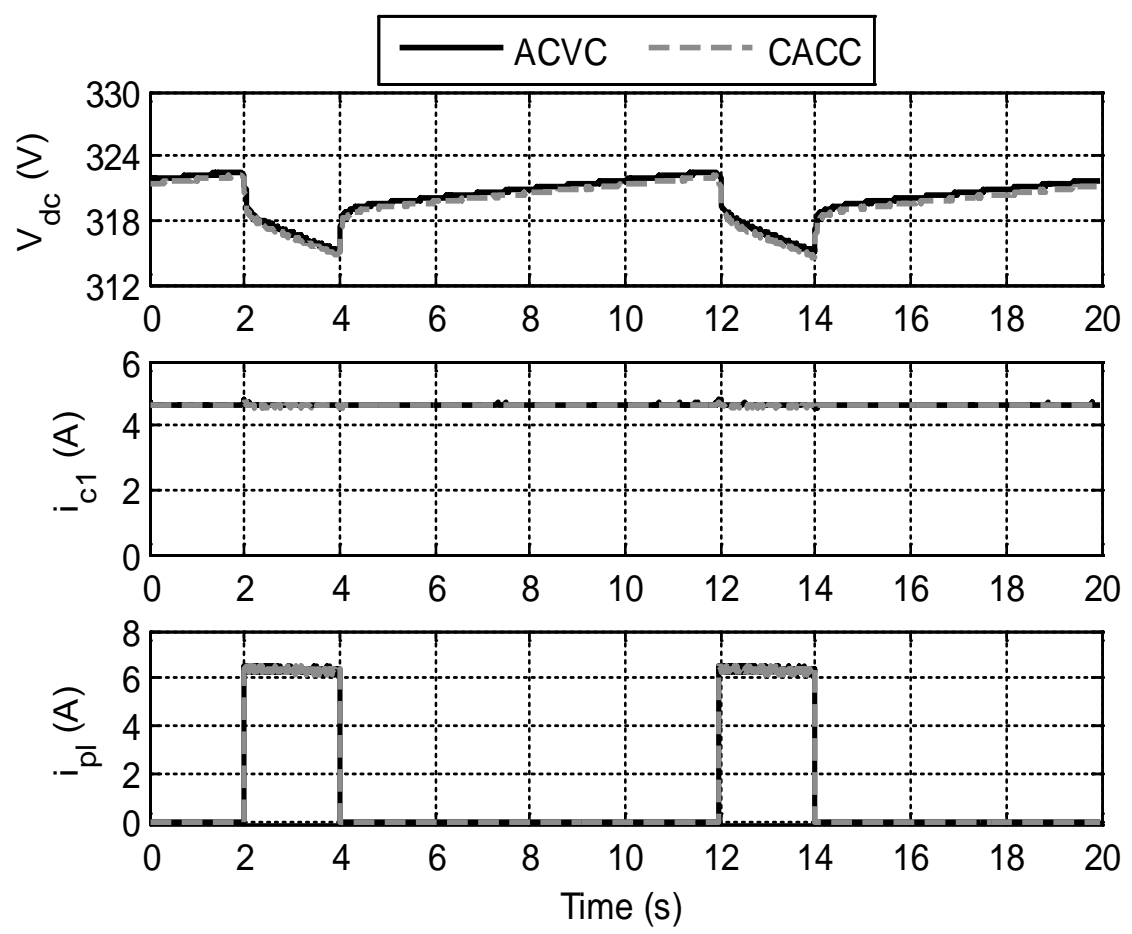

Figure 6.7: Experimental test results of ACVC and CACC technique during constant pulse load operation.

In order to evaluate the transient and dynamic performance of the CACC method and the developed ACVC technique, various experimental tests are performed when the pulse load characteristics were changed. In the first case study, the frequency of the pulse load was changed while its duty ratio was constant. In this situation, the average of the pulse load will not change. Figure 6.8 shows the performance of the developed ACVC technique and the conventional CACC method when the pulse frequency changed from 0.1 $\mathrm{HZ}$ to $0.2 \mathrm{~Hz}$ at $\mathrm{t}=30$-s. As can be seen, the average of the current is constant and both methods are able to perform accurately during pulse load variations. 

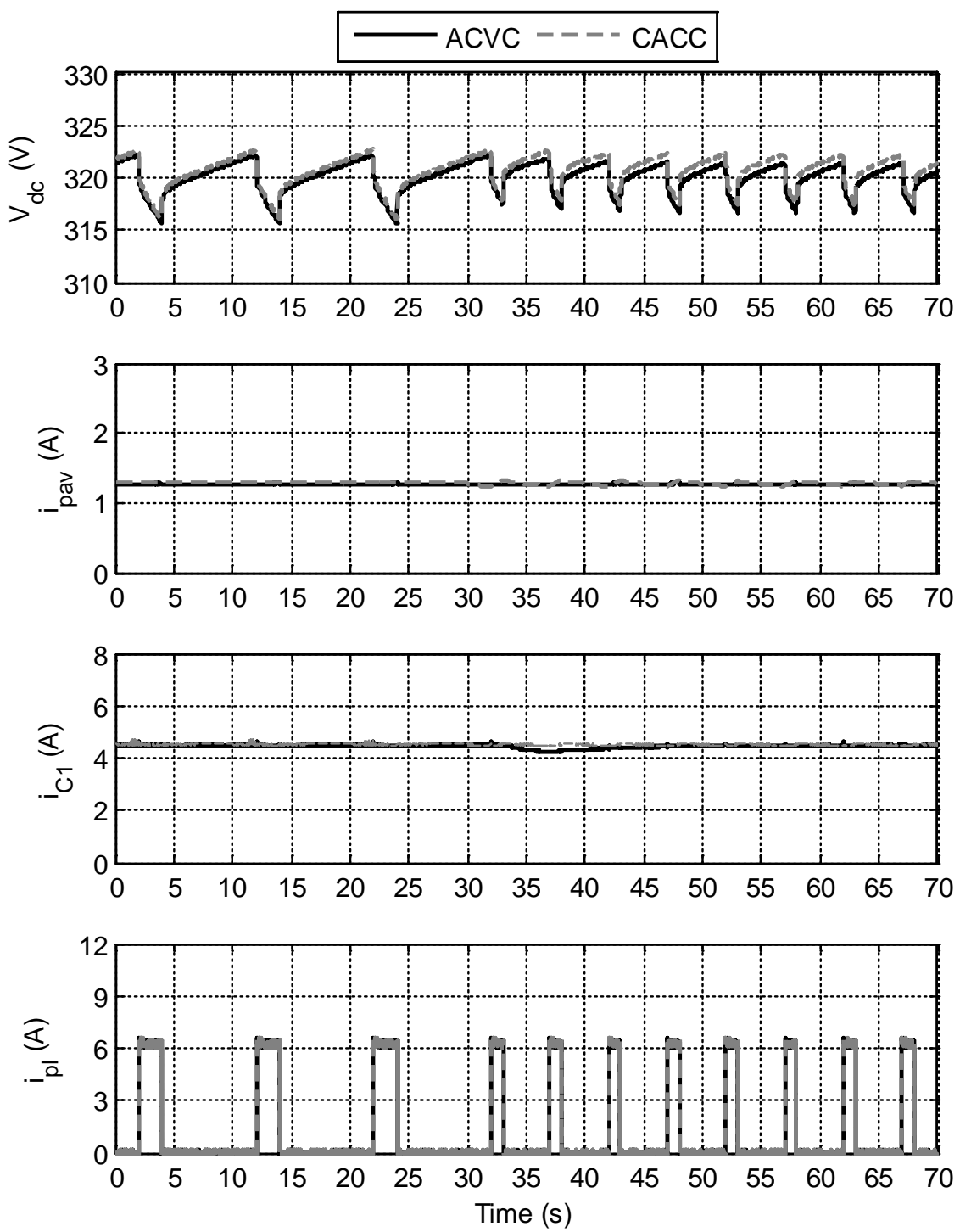

Figure 6.8: Experimental test results of CACC method and ACVC technique during test case 1.

Figure 6.9 shows the experimental test results when the pulse frequency was decreased from $0.1-\mathrm{Hz}$ to $0.2-\mathrm{Hz}$ and its duty ratio was increased from $20 \%$ to $40 \%$ at $\mathrm{t}=$ $30 \mathrm{~s}$. As can be seen, in the case of the CACC method, 1.25 A pulse load average current was calculated initially and then it decreased slowly after pulse change happened. The slow response of the CACC method caused an imbalance between the injected power and the consumed power. As a result, considerable undervoltage occurred in the common coupling 
DC bus, i.e. the average voltage dropped to $209.8-\mathrm{V}$ in 40 -s. The CACC method not only suffers from undervoltage during transient pulse load changes, but also a dangerous overvoltage may happen due to overpower estimation and slow response of the CACC method. It should be noted that the longer the CACC operates in a pulse load condition, the slower it will respond to pulse load variation.
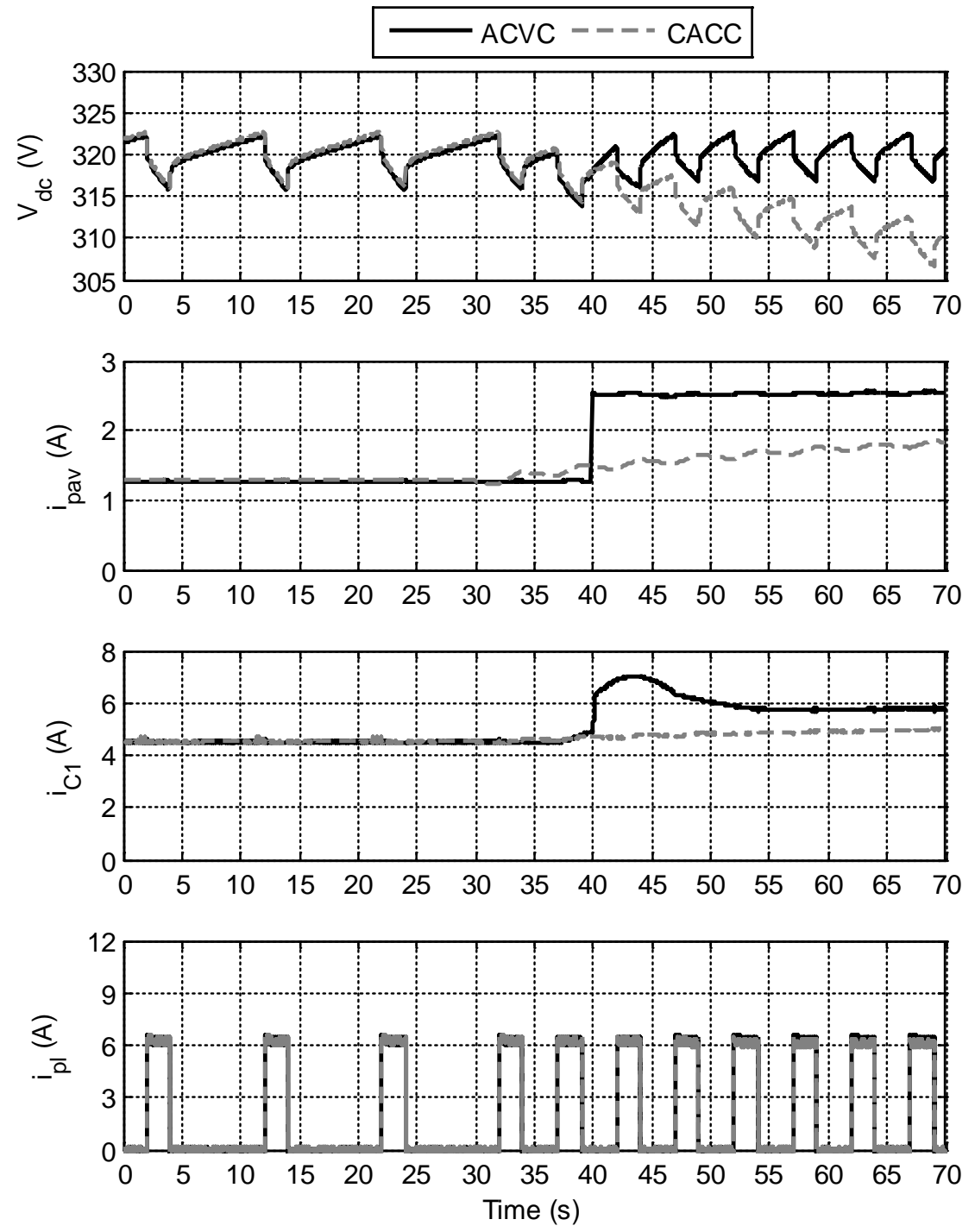

Figure 6.9: Experimental test results of CACC method and ACVC technique when pulse load frequency changes from $0.1-\mathrm{Hz}$ to $0.2-\mathrm{Hz}$ and its duty ratio increased from $20 \%$ to $40 \%$. 

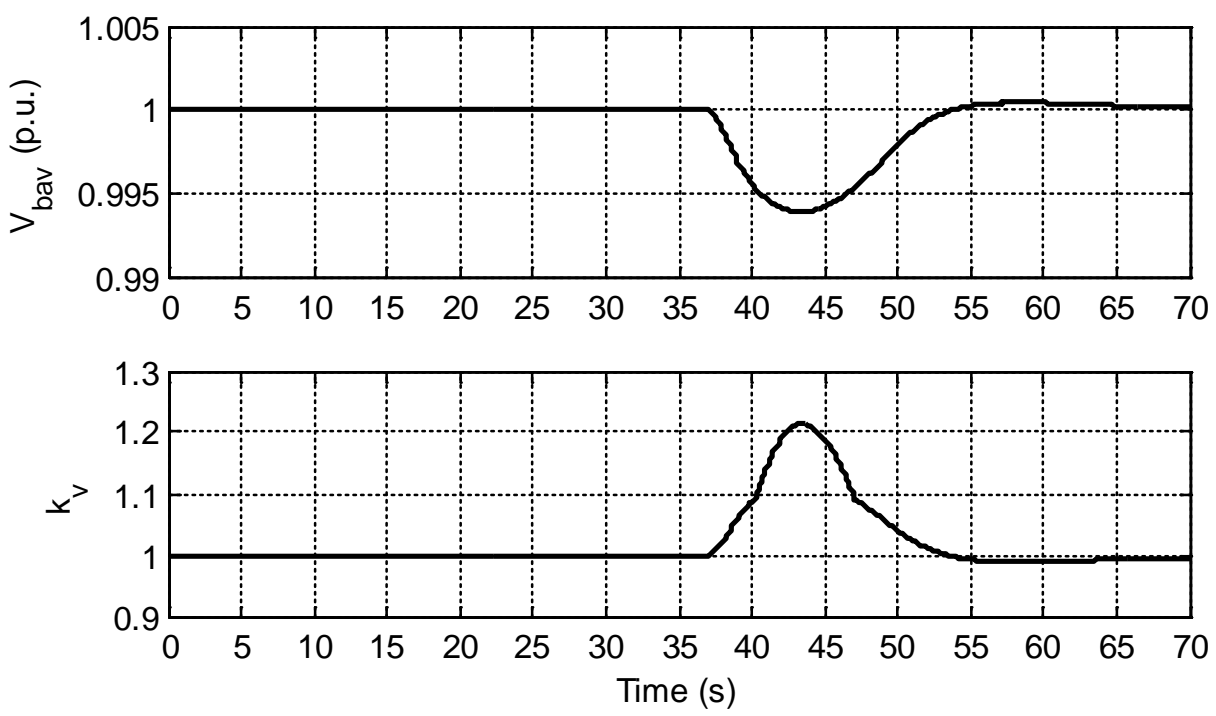

Figure 6.10: Variation of the normalized average DC bus voltage and the $\mathrm{kv}$ in the proposed ACVC technique when pulse load frequency changes from $0.1-\mathrm{Hz}$ to $0.2-\mathrm{Hz}$ and its duty ratio increased from $20 \%$ to $40 \%$.

Figure 6.9 also shows the results for a similar test when the ACVC technique was employed. As can be seen, the developed ACVC technique is able to track the pulse load variation very quickly and reduce the calculated average pulse load current from 1.25-A to 2.5-A in 10-s. By employing the adaptive compensation gain $k_{v}$, the ACVC method was able to effectively regulate the DC bus voltage and the injected power to the microgrid. Figure 6.10 shows the normalized average DC bus voltage and the gain $k_{v}$ variation during the test. As observed, during time interval $\mathrm{t}=37-\mathrm{s}$ and $\mathrm{t}=40-\mathrm{s}$, the $k_{v}$ increased slowly, since the average voltage was less than 0.995 and the system was in normal operation zone. Then, the $k_{v}$ increased with a higher slope to a maximum of 1.21 when the system moved to the undervoltage zone. Once the voltage was completely compensated, the $k_{v}$ returned to 1 and the system worked in a new operation state.

Figure 6.11 shows the experimental test results when the duty ratio was decreased from $20 \%$ to $10 \%$ at $t=30 \mathrm{~s}$. As can be seen, in the case of the CACC method, $1.25 \mathrm{~A}$ 
pulse load average current was calculated initially and, then, after pulse change happened, it decreased gradually. The overpower injection of the CACC method due to slow response caused considerable voltage increase of the DC bus. The result shows that the maximum of the DC bus voltage increased from $322.4-\mathrm{V}$ to $324.2-\mathrm{V}$ in $30-\mathrm{s}$.
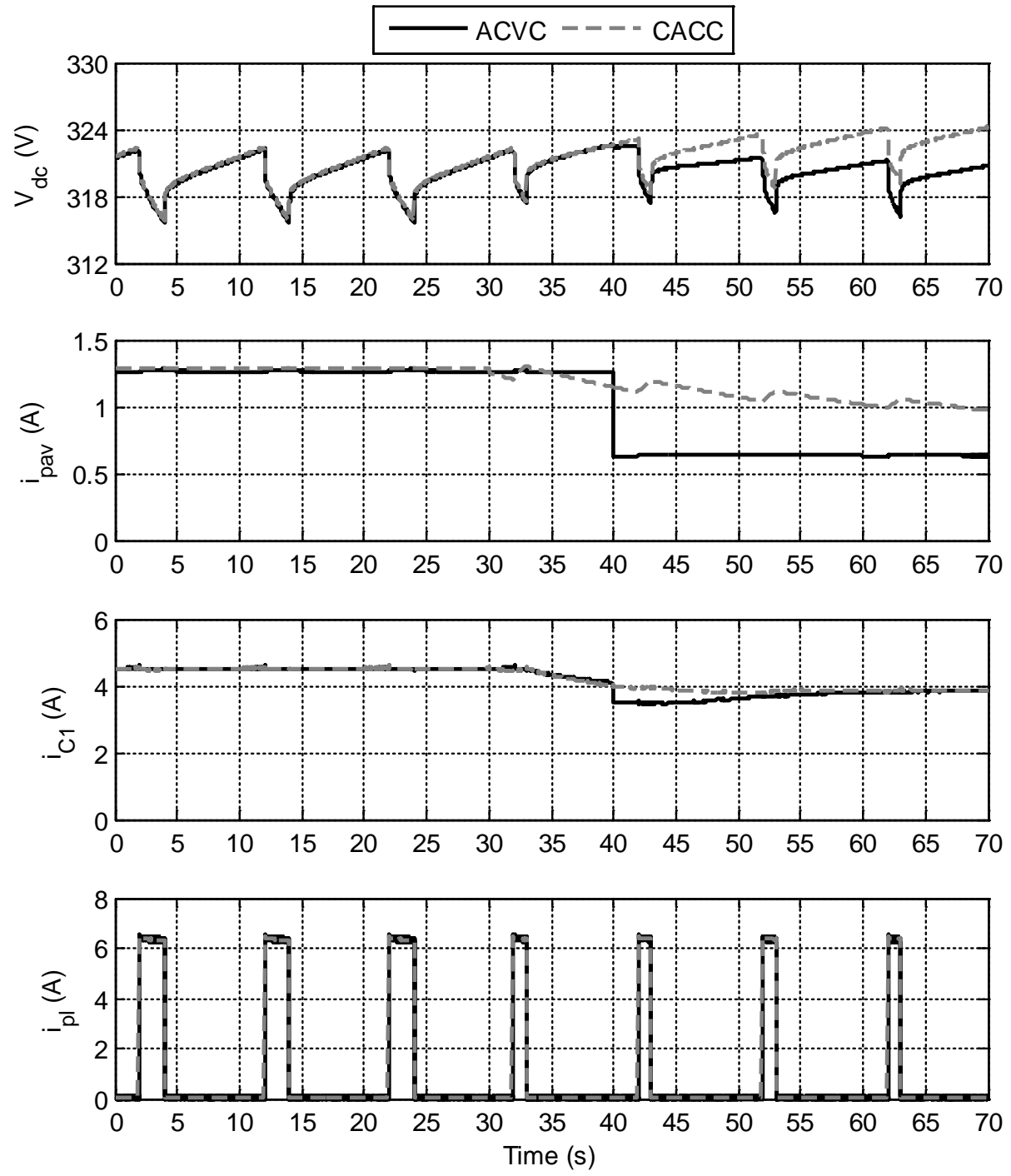

Figure 6.11: Experimental test results of CACC method and ACVC technique when pulse load duty ratio changes from $20 \%$ to $10 \%$.

Figure 6.11 also shows the ACVC technique test result when the same experiment was performed. As can be observed, the developed ACVC technique is able to track the 
pulse load variation very fast and reduce the average pulse load current from 1.25-A to 0.625-A in 10 seconds. By employing the adaptive compensation gain $k_{v}$, the ACVC method was able to effectively regulate the DC bus voltage and the injected power to the microgrid. Figure 6.12 shows the $k_{v}$ variation during the test. The overestimated energy during transient time caused a small increase of the average bus voltage. Consequently, the $k_{v}$ is decreased to 0.9 based on the continuous average bus voltage measurements and the lookup table. Once the pulse load variation was tracked and the calculated energy was reduced, the $k_{v}$ returned to 1 and the system will work in a new operation state. Thus, the transient response of the system is highly improved and any steady state error or dangerous over voltage is prevented.

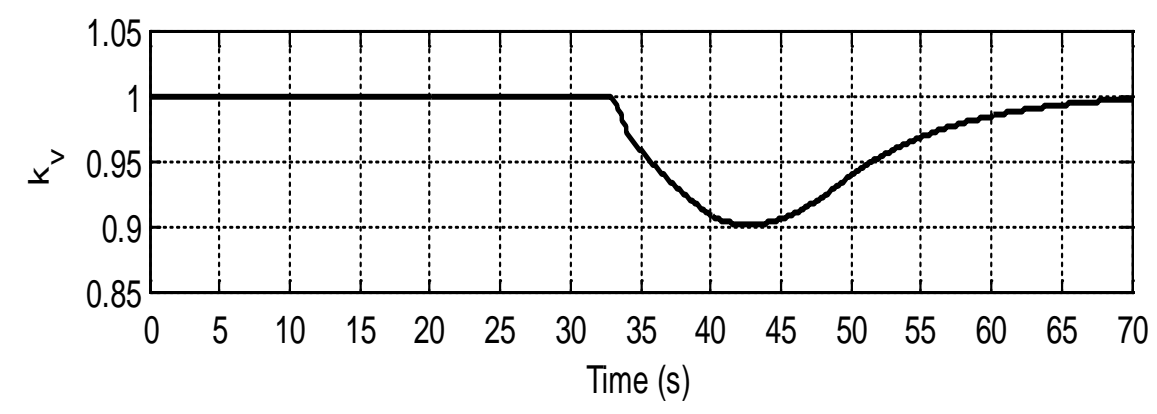

Figure 6.12: Variation of the $k_{v}$ in the proposed ACVC technique when pulse load duty ratio changes from $20 \%$ to $10 \%$.

From the three test cases that performed for transient studies, it can be concluded that the developed ACVC technique is able to properly handle pulse load variation and it only requires the maximum pulse load period, which is considered as a known parameter in this study. However, in the case that the maximum frequency of the pulse load is unknown or if the load frequency is varying in a very wide range, a pulse frequency calculator function could be added to the controller. 

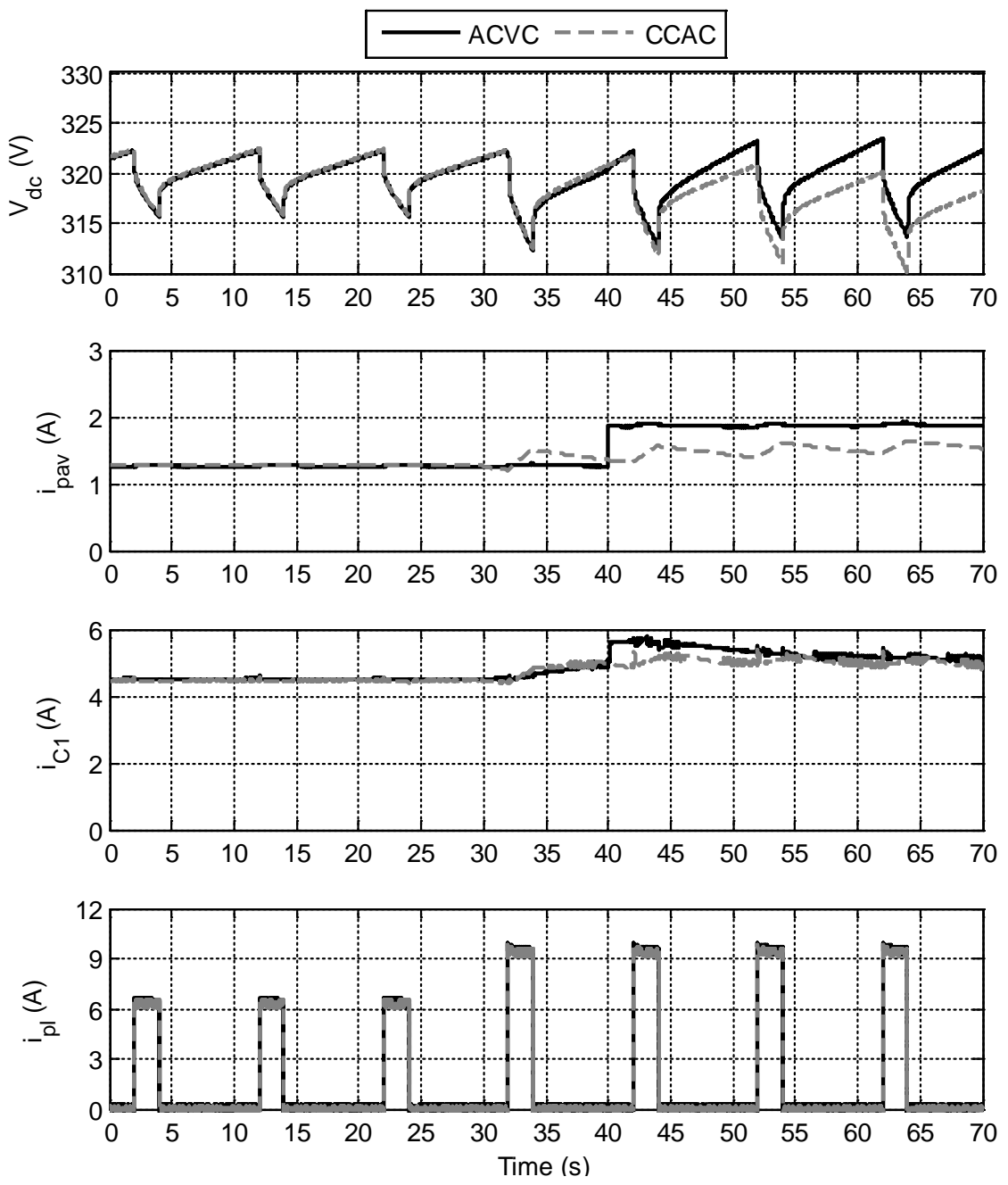

Figure 6.13: Experimental test results of CACC method and ACVC technique when pulse load changed from 2-kW to 3-kW.

In order to further investigate the effectiveness of the developed ACVC technique, its performance was tested while the pulse load power was changed from 2-kW to 3-kW. Figure 6.13 shows the comparative performance of the ACVC technique and the CACC method. As observed, the ACVC technique was able to accurately calculate the $i_{p a v}$, which was changed from 1.25-A to 1.875-A. The transient underestimated power was compensated with the adaptive variation of the $k_{v}$. The system reached a new steady state 
condition in which the DC bus voltage varied from 323.4-V to 313.6-V to support the 3kW pulsed power load while the average voltage was 320-V. In contrast, the CCAC method failed to properly track the pulse load variation and the $i_{p a v}$ was slowly increased from 1.25-A. The slow response of the CACC method is due to the large memory of the integrator and the continuous averaging characteristic of the CACC method. As a result, the average DC bus voltage was decreased from $320-\mathrm{V}$ to $316.8-\mathrm{V}$ in $40-\mathrm{s}$.

The experimental test results show that the presented ACVC technique prevented any steady state voltage error or dangerous over voltage during transient and dynamic operation and its performance was effectively improved compared with the CACC method.

\subsubsection{Compared to IPC and LBVC Methods}

In order to prove the effectiveness of the developed ACVC technique in reducing the pulsed load disruptive effects, the performance of this scheme is experimentally compared to the LBVC and IPC methods. In the following experiments, the pulsed load was a constant $2-\mathrm{kW}$ at a frequency of $0.1-\mathrm{HZ}$ and duty ratio of $20 \%$, while the steady state load 1 and load 2 were 700-W and 300-W, respectively. Comparative analysis of the hybrid DC microgrid and AC grid performances are shown in Figure 6.14 and Figure 6.15, respectively.

Figure 6.14 shows the common coupling DC bus voltage, the boost converter current, the bidirectional converter current and the pulse load current for the three different energy control methods. As can be observed, unlike to the ACVC technique, the IPC and LBVC methods caused considerable current and power pulsation of the converters. For the given case study, the ACVC technique caused 2.47\% maximum DC bus voltage variation, which was $1.28 \%$ and $1.59 \%$ more than the IPC and LBVC methods, respectively. 

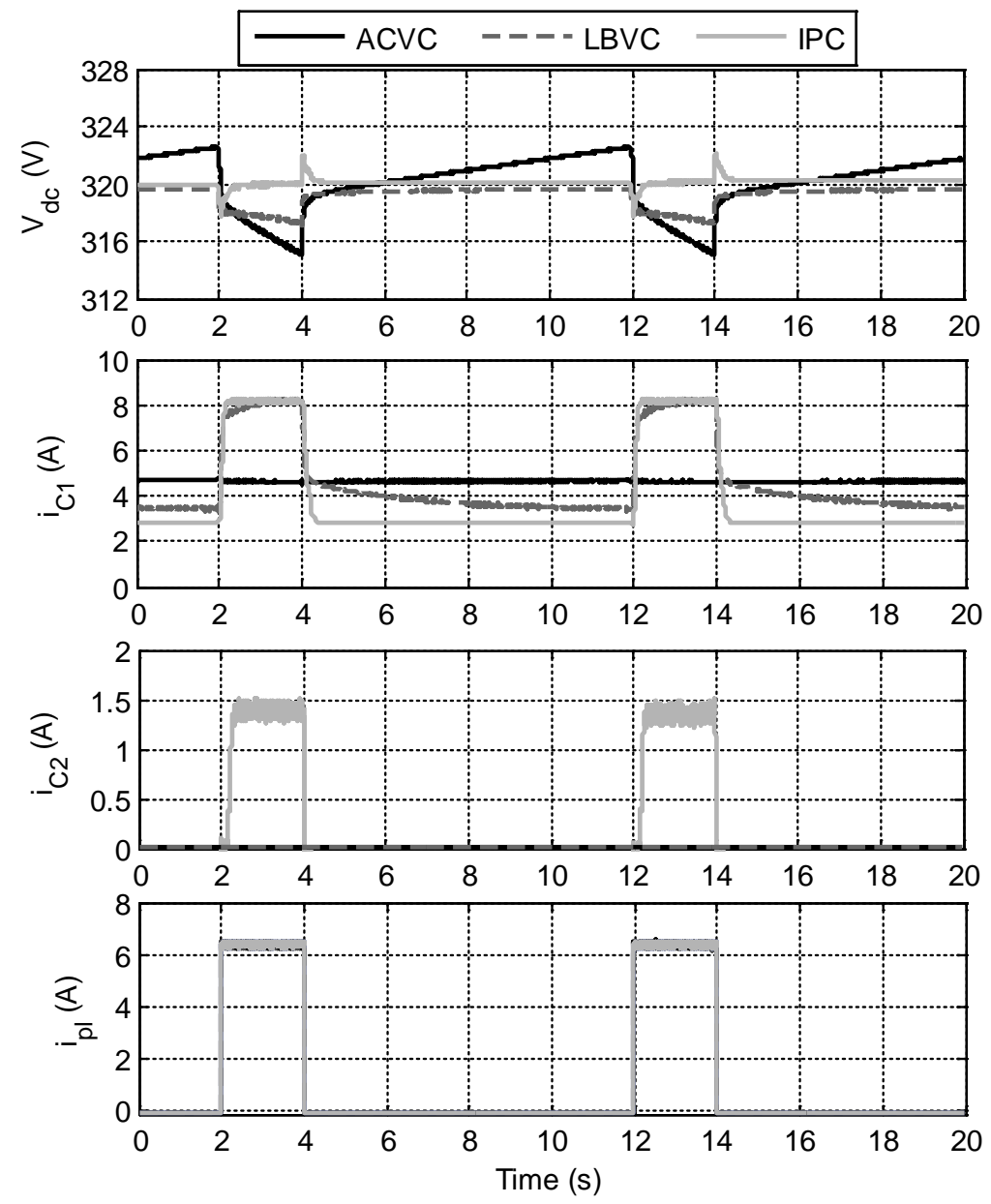

Figure 6.14: Hybrid DC microgrid performance comparison when ACVC, LBVC and IPC methods were utilized.

The test results depicted in Figure 6.14 show that, although the ACVC technique made slightly more bus voltage variation than the LBVC and the IPC method, it effectively eliminated the high current pulsation of the converter. The IPC method utilized the boost converter and the bidirectional converter to supply the grid during the pulse on-time, while during the pulse off-time, only the boost converter supplied the DC microgrid, i.e. when the pulse load turned on, the boost converter operated at its maximum power limit at 8-A and the rest of the power was provided by the bidirectional converter and battery bank. When the pulse load turned off, the boost converter was only required to supply the steady 
state loads. Also, Figure 6.14 shows that the LBVC method only utilized the boost converter to supply the DC microgrid. Once the pulse load turned on, the converter reached its maximum power limit with a slow-front impulse and then asymptotically decreased to 3.5-A when the pulse turned off.

To further show the effectiveness of the ACVC technique and the consequence of converter current pulsation, the AC grid performance during the DC microgrid operation is shown in Figure 6.15. In the case of the ACVC scheme, the slack bus generator at bus 1 continuously supplied $1.56-\mathrm{kW}$ and its frequency was almost constant at $60-\mathrm{Hz}$. Also, the ACVC caused only a $2.9 \%$ voltage drop on the interconnected AC bus.

On the contrary, the IPC and LBVC methods caused considerable power, voltage and frequency fluctuation in the AC grid. In the case of the LBVC method, the slack bus generator power raised from $1.1-\mathrm{kW}$ to $2.87-\mathrm{kW}$. As a result, the AC grid frequency dropped to $59.44-\mathrm{Hz}$ when the pulse was turned on and it increased to $60.24-\mathrm{Hz}$ after the pulse ended. This power pulsation also caused a 9.10\% maximum voltage drop in the interconnected AC bus. Similarly, the IPC method caused 2.02-kW power pulsation and considerable frequency fluctuation. When the pulse occurred, the generator frequency dropped to $59.28-\mathrm{Hz}$ and returned to $60-\mathrm{Hz}$ with a $0.31-\mathrm{Hz}$ overshoot. Also, the maximum AC bus voltage drop in the case of IPC was $7.08 \%$. These results show the improvement provided by the proposed ACVC scheme over the IPC and LBVC methods in reducing the impact of pulsed power loads on the AC grid. 

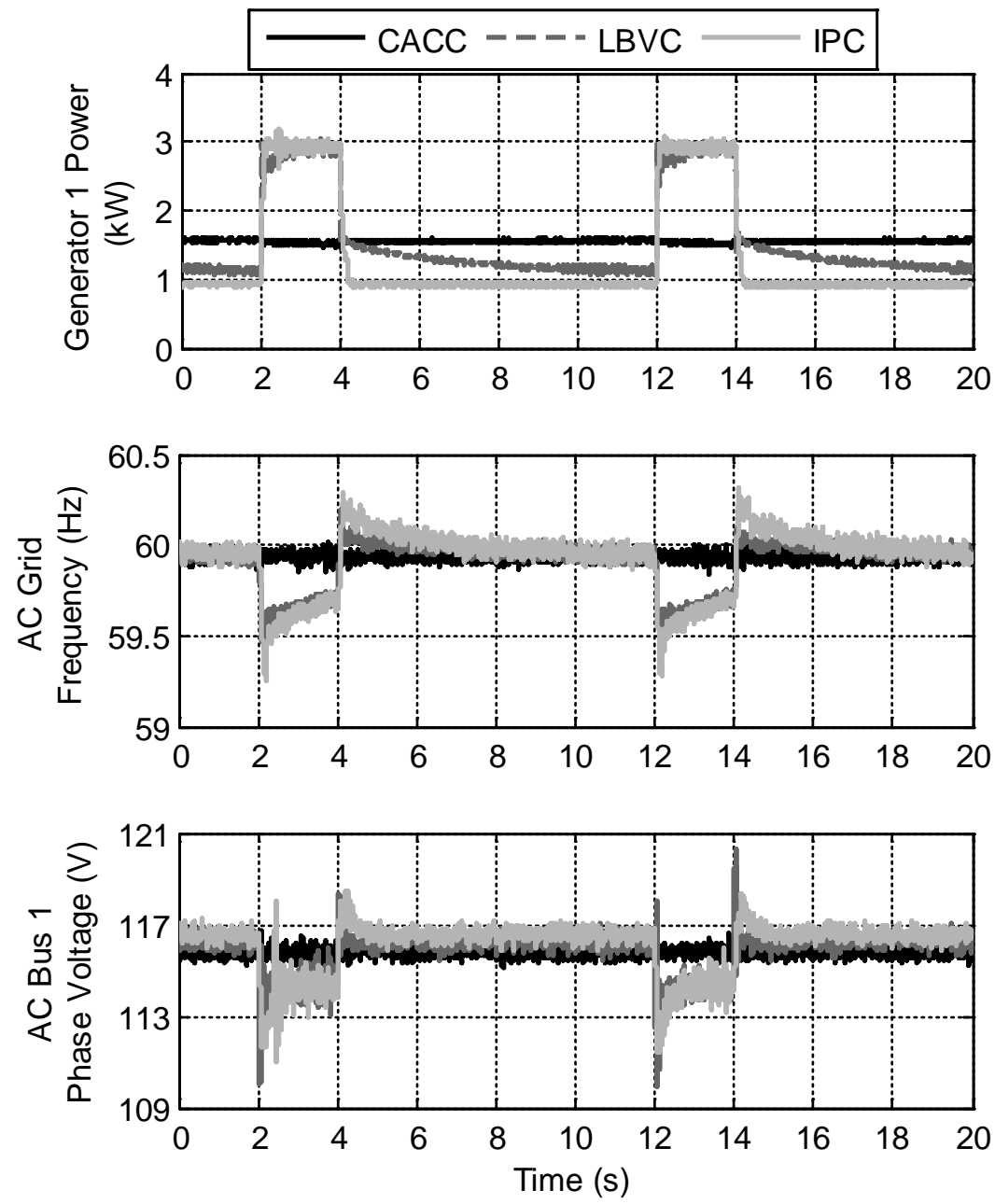

Figure 6.15: AC grid performance comparison when ACVC, LBVC and IPC methods are utilized.

In order to do more study on the effectiveness of the developed energy control scheme, experimental tests were performed under a high duty ratio pulse load. In the following experiments, the loads were similar to the previous case study while the duty ratio of the pulse load was increased to $40 \%$. Figure 6.16 and Figure 6.17 show the performances of the DC microgrid and AC grid for the three energy control methods. As can be seen in Figure 6.16, the LBVC have the lowest maximum voltage fluctuation, which was only $0.47 \%$. Also, the maximum DC bus voltage variation of the IPC method and ACVC scheme were $0.75 \%$ and $1.47 \%$, respectively. 

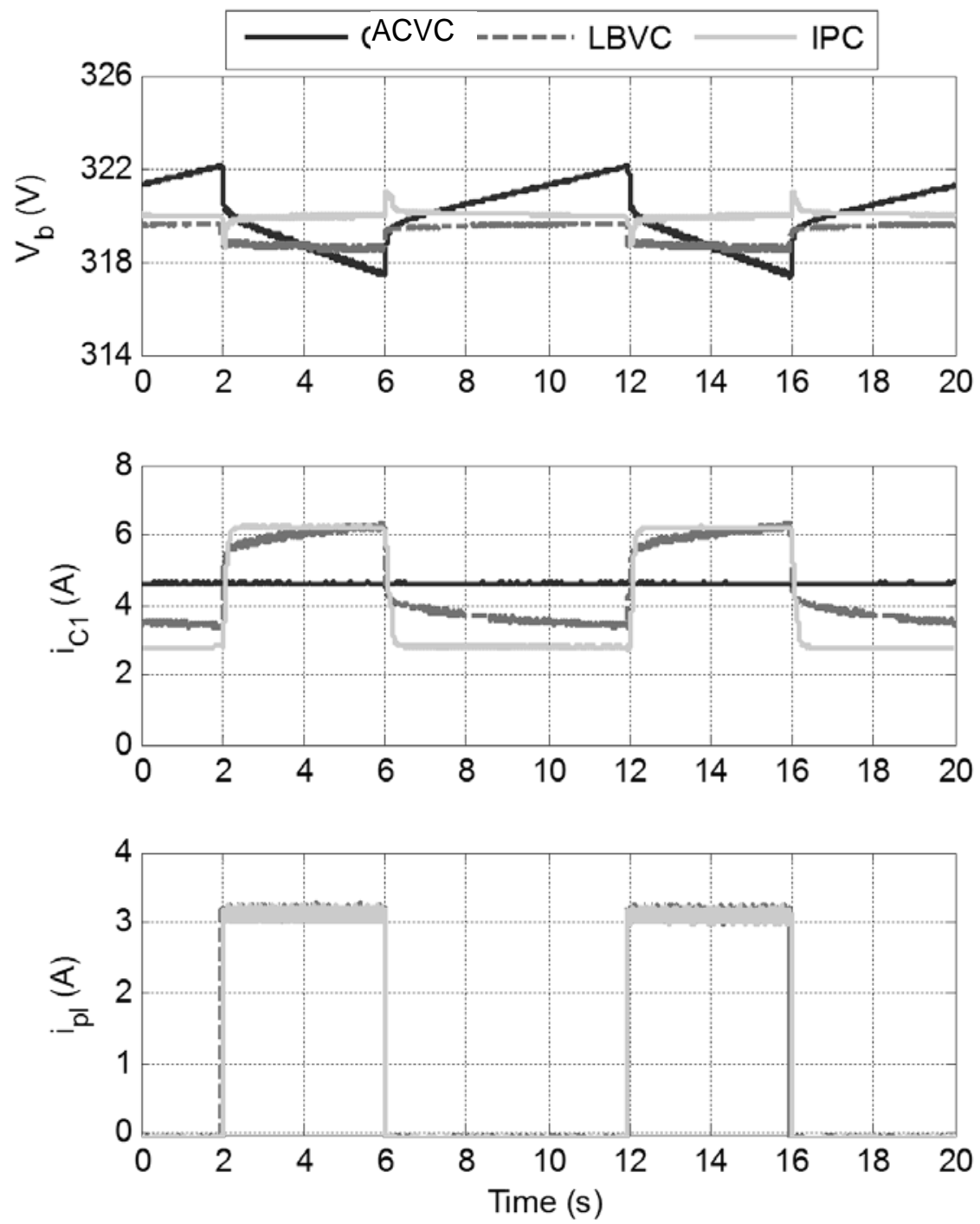

Figure 6.16: Hybrid DC microgrid performance comparison during the operation of high duty ratio pulsed power load.

The test results show that, in this case study, all the energy control schemes required only the boost converter to support the DC grid through AC grid. The developed ACVC method adjusted the boost converter output current to a relatively constant 4.5-A. In contrast, the IPC caused power pulsation of the boost converter from 2.79-A to 6.27-A. Also, the LBVC set the output current of the boost converter to a maximum of 6.27-A and then decreased to 3.3-A before the next pulse occurred. The analysis of the DC microgrid 
performance shows that similar to the previous case study, the developed ACVC method effectively eliminated the high current pulsation of the boost converter. However, the ACVC method causes slightly more DC bus voltage variation than the LBVC and the IPC methods.

The AC grid performance during the DC microgrid operation for the three energy control methods is shown in Figure 6.17. In the case of the ACVC technique, the generator 1 supplied $1.56-\mathrm{kW}$ and its frequency was almost constant at $60-\mathrm{Hz}$. Similar to the previous case study, the voltage drop of the interconnected AC bus was $2.9 \%$. In the case of the LBVC method, the slack bus generator power increased from 1.17-kW to 2.18-kW. As a result, the AC grid frequency dropped to $59.63-\mathrm{Hz}$ when the pulse turned on and it increased to $60.25-\mathrm{Hz}$ after the pulse ended. Also, the results show that the IPC technique caused $1.23-\mathrm{kW}$ power pulsation and $0.72-\mathrm{Hz}$ frequency fluctuation. Moreover, the maximum AC bus voltage drop in the case of LBVC and IPC was $6.16 \%$ and $5.71 \%$, respectively.

The performance of the AC grid shows that, similar to the previous case study, the developed ACVC method successfully prevents the negative impact of pulsed power loads on the AC grid. In contrast, the power pulsation and frequency fluctuation of the AC grid in the case of LBVC and IPC were considerable. The results show that the IPC control caused a little more frequency fluctuation than the LBVC in the case of a high duty ratio pulse load. This occurs due to the higher power pulsation of the slack bus generator and non-utilization of the battery bank. 

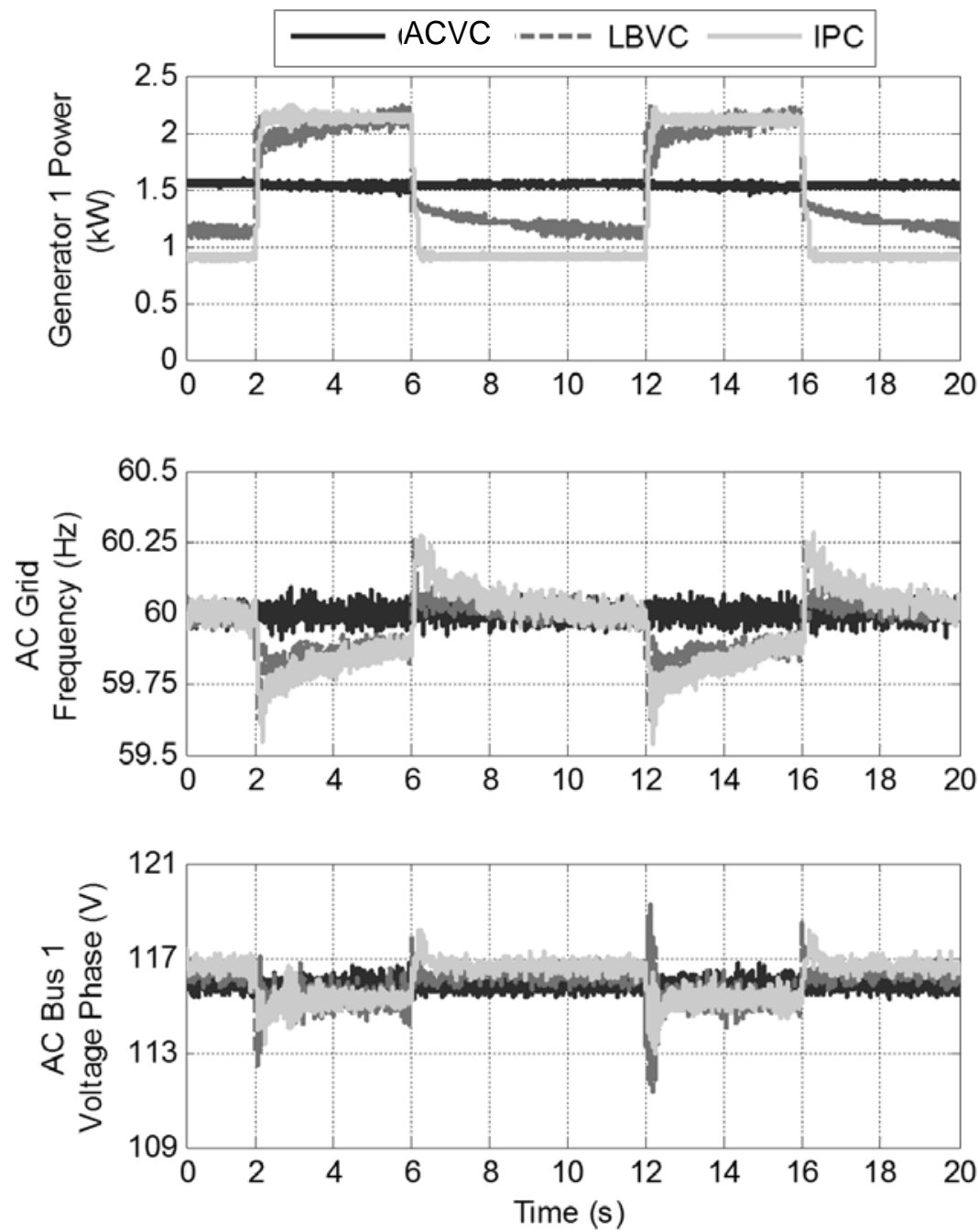

Figure 6.17: AC grid performance comparison during the operation of low duty ratio pulsed power load.

The performances of the ACVC strategy in comparison to the LBVC and IPC methods were studied for $2 \mathrm{~kW}$ and $1 \mathrm{~kW}$ pulse load. In the following, two more cases for $4 \mathrm{~kW}$ and $1.3 \mathrm{~kW}$ pulse load were performed. In these experiments, the pulse load was 4$\mathrm{kJ}$ at a frequency of $0.1-\mathrm{HZ}$ while the steady state load 1 and load 2 were $700-\mathrm{W}$ and 300$\mathrm{W}$, respectively. It should be noted that the IPC method was not able to supply the DC microgrid in the case of 0.1 duty ratio pulse load since the maximum power requirements for this energy control scheme was higher than the DC microgrid power limitation. 
Comparison of the normalized DC bus voltage variation for different energy control methods is shown in Figure 6.18. It can be seen that, as the duty ratio increased, the common coupling DC bus voltage variation in all energy control methods decreased due to the decrease in pulse rate. The highest voltage variation occurred in the case of the ACVC method, while the LBVC method had the lowest voltage variation. The lower voltage variation in the case of the LBVC and IPC methods is a result of the higher power that is drawn from the AC grid.

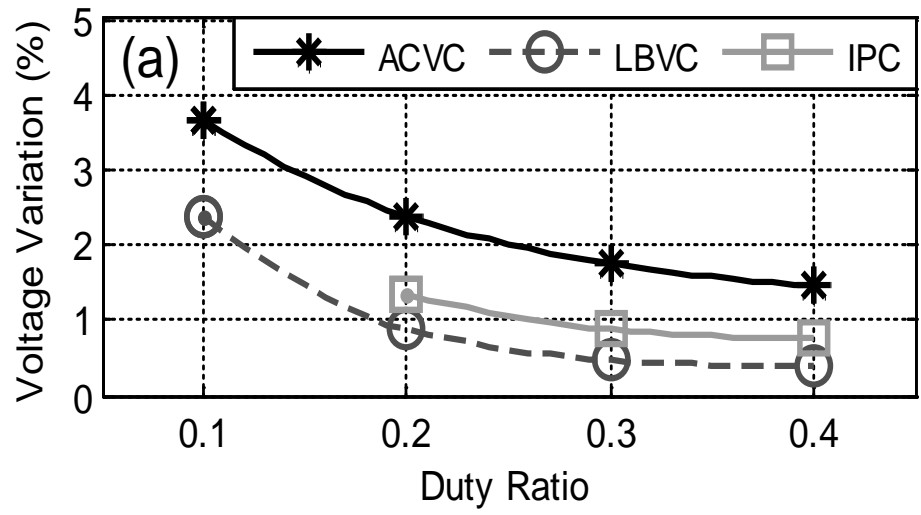

Figure 6.18: Normalized DC bus voltage variation comparison between the developed ACVC technique and the LBVC and IPC methods.

Figure 6.19 shows the effect of pulse load characteristics on the maximum voltage drop of AC bus 1 for different energy control techniques. As can be seen, the developed ACVC technique successfully reduced the maximum voltage drop, which was less than 4.8-V and it was nearly constant for different duty ratios. However, the LBVC and the IPC methods caused considerable voltage drop. Reducing the duty ratio of the pulse load increased the AC bus voltage drop since the absorbed power from the AC grid was increased. Maximum voltage drop in the case of LBVC was $12.5-\mathrm{V}$, which occurred when the duty ratio was 0.1 . 


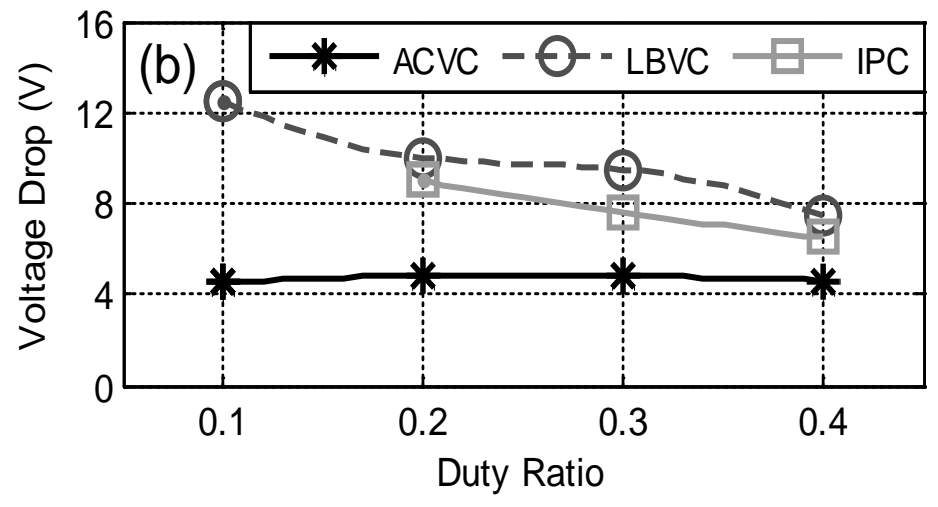

Figure 6.19: AC bus voltage drop comparison between the developed ACVC technique and the LBVC and IPC methods.

Also, the AC grid frequency fluctuations for different energy control methods are shown in Figure 6.20. The frequency fluctuation in the case of the ACVC method was effectively limited to less than $0.14-\mathrm{Hz}$. In contrast, the LBVC and IPC caused considerable frequency variation and it was inversely related to the pulse duty ratio. However, in the case of LBVC, the frequency variation was decreased when the duty ratio was reduced to 0.1. This was due to the fact that the voltage drop of the DC bus was so high that the LBVC reduced the converter reference current very slowly when the pulse turned off. As a result, the frequency overshoot of the AC grid was avoided.

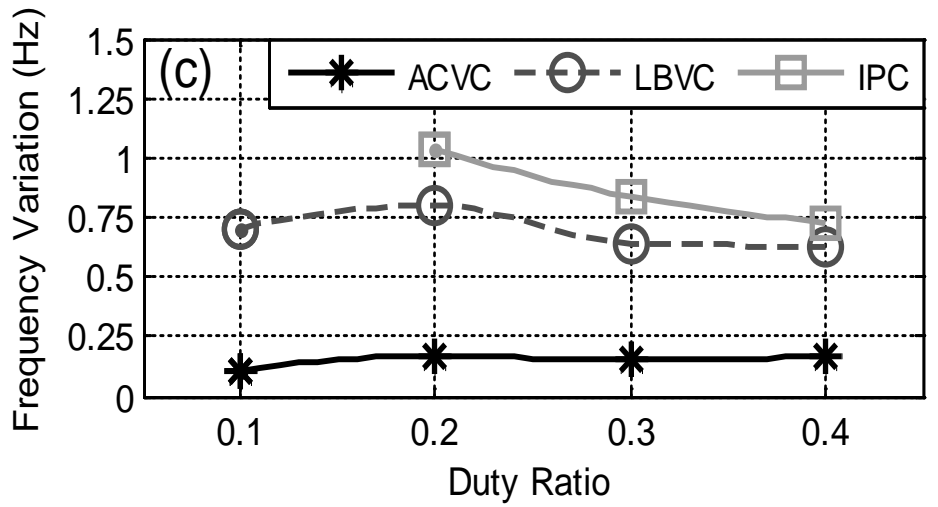

Figure 6.20: AC grid frequency variation performance comparison between the developed ACVC technique and the LBVC and IPC methods. 
Figure 6.21 shows the comparison of the system efficiency for different energy control techniques and pulse load duty ratios. It can be seen that the proposed ACVC method yielded higher efficiency than the LBVC and IPC methods. This is because of the fact that the power losses of the system are proportional to the square of the current and the ACVC technique reduced the maximum current of the converter. For the higher duty ratios, the system efficiency for IPC and LBVC methods approached the ACVC technique since the converter power pulsation was reduced and they were approaching the average power. In the case of the IPC method, the efficiency of the system was slightly increased when the duty ratio was decreased from 0.3 to 0.2 . The higher efficiency in the case of 0.2 duty ratio was because of the fact that $450-\mathrm{W}$ of the pulse load was supplied through the highly efficient bidirectional converter and the battery bank.

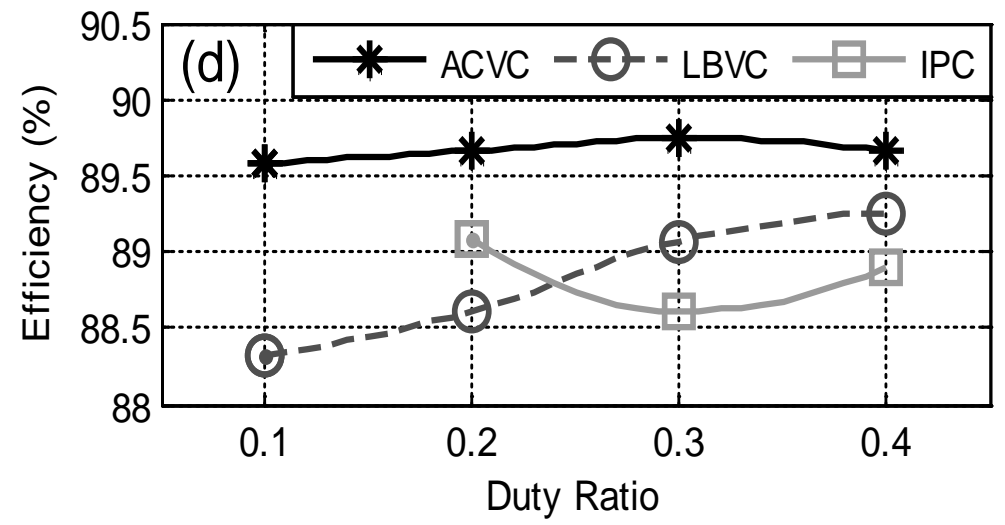

Figure 6.21: System efficiency comparison between the developed ACVC technique and the LBVC and IPC methods.

The experimental test results for different pulse duty ratios further demonstrated the significant improvement achieved by the developed ACVC scheme in enhancing the system efficiency, reducing the AC grid voltage drop and frequency fluctuations. The DC bus voltage variation in the case of the ACVC scheme was slightly higher than the IPC and LBVC methods; however, it was always less than $4 \%$, which was in an acceptable range. 


\subsection{Summary of the Results and Conclusion}

In this chapter, a new energy control scheme was developed to reduce the adverse impact of pulsed power loads for the grid connected hybrid DC power system. The proposed energy control was an adaptive current-voltage control (ACVC) scheme based on the moving average current and voltage measurement and a proportional voltage compensator. The performance of the developed ACVC technique was experimentally evaluated and it was compared to the other common energy control methods.

The test results showed that the ACVC scheme has a similar performance with the continuous average current control (CACC) method during a constant pulsed power load operation. However, the transient response of the ACVC technique during pulse load variation was effectively improved and it prevented any steady state voltage error or dangerous over voltage.

Also, the performance of the developed ACVC technique was compared with the limit-based voltage control (LBVC) and instantaneous power control (IPC) methods for different pulse rates and duty ratios. The comparative analysis showed that, although the maximum DC bus voltage variation in the case of the ACVC scheme was higher than the IPC and LBVC methods, the proposed ACVC technique required smaller power capacity of the converter and energy resources. Moreover, the developed ACVC method effectively eliminated the power pulsation of the slack bus generator and frequency fluctuation of the interconnected AC grid while the AC bus voltage drop was well reduced. Additionally, the efficiency analysis for different pulse duty ratios showed that the developed ACVC method considerably improved the efficiency of the system since the maximum current of the converter was reduced and the converter was operating at a relatively constant value. 


\section{VIRTUAL TETS-BED MODELING FOR DYNAMIC AND TRANSIENT STUDY OF HYBRID POWER SYSTEM}

\subsection{Introduction}

Hybrid DC microgrid power systems are becoming increasingly common in a host of applications. The design, energy management and power control of such systems are a fundamental concern. Moreover, protection of the system against short circuit faults needs to be fully studied. This section presents implementation and evaluation of virtual test-bed platform as an accurate model for hybrid power systems.

The virtual test-bed models can be used to develop protection systems and test system-level performance. Also, the virtual test-bed model can be parameterized to optimize the design of the system and to improve the performance of the system in terms of efficiency, power quality and reliability.

Also, the virtual test-bed provides an environment that is effective for design training and assistance. With predictive simulation, not only can an engineer perform analysis using real-time system parameters, but he can also simulate "what if” scenarios simply by taking action using the system model. For instance, using real-time data, the designer can simulate the impact of starting a large motor without actually starting the motor.

In order to set the stage for the virtual test-bed modeling, this section begins with a description of the energy storage modeling. This is followed by an explanation of the four bus hybrid DC microgrid specification and modeling. Following the virtual test-bed 
modeling description, the normal and transient performance of the microgrid is evaluated using both simulations and laboratory results.

\subsection{Modeling of the Energy Storage System}

A precise model of a hybrid power system for pulse load study shall provide an accurate model of the energy storage systems for a wide range of frequencies. In this section, the detailed models of the energy storage systems utilized in our virtual test-bed platform are presented.

\subsubsection{Supercapacitor Bank Modeling}

Several models of supercapacitors have been suggested in the literature for a variety of applications [39], [152]-[153]. Supercapacitors are usually modeled with resistivecapacitive networks, which can be obtained through analyses in the time domain or frequency domain. Figure 7.1 and Figure 7.2 show the most common electrical equivalent circuits of a supercapacitor for pulsed power load applications. Figure 7.1 (a) is the supercapacitor's classical first order approximation model. The model is comprised of three components: the capacitance $\mathrm{C}_{\text {sc }}$, which simulates the supercapacitor's capacitance due to the double layer effect; the equivalent parallel resistance $R_{E P R}$, which represents self-discharging or the effect of leakage current; and the equivalent series resistance $\mathrm{R}_{\mathrm{ESR}}$

, which models the supercapacitor's ohmic loss and voltage drop during the charging and discharging process. 


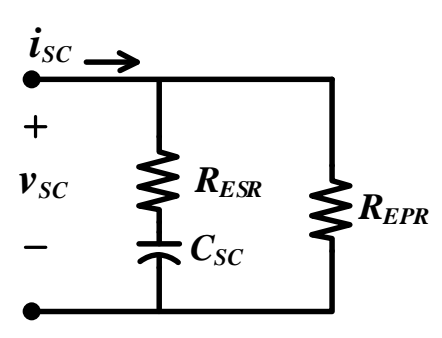

(a)

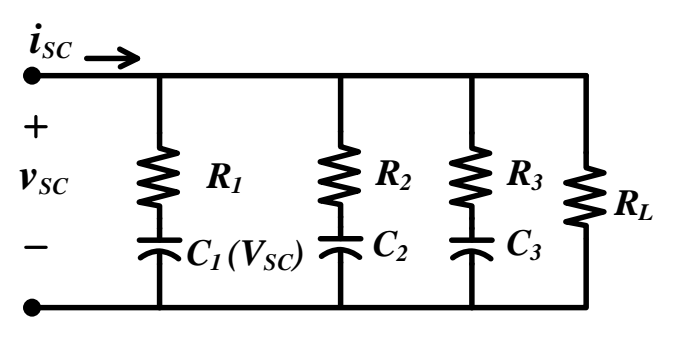

(b)

Figure 7.1: Electrical equivalent circuit models for supercapacitor: (a) first order model, (b) third order model with voltage dependent branch.

Although the first order model is simple and its parameters can be easily extracted based on the manufacturer datasheet, this model is not able to capture the nonlinear rise and fall of the supercapacitor voltage, especially during fast charge and discharge processes. Therefore, for better accuracy, more detailed models are required. Figure 7.1 (b) is a third order supercapacitor model with a voltage-dependent differential capacitor in its first branch. Each of the three branches has a distinct time constant to reflect the internal charge distribution process and will result in easily measurable parameters. The first branch, with the voltage-dependent capacitor, dominates the immediate behavior of the supercapacitor in the time range of seconds while the second and third branches determine terminal behavior in the range of minutes and for times longer than 10 minutes, respectively. Also, a leakage resistor parallel to the terminals models the self-discharge property. Although this model is proper for low frequencies and for voltages above $40 \%$ of the rated terminal voltage, it may not have enough accuracy at high frequencies and lower voltage ranges.

Figure 7.2 is a sixth order model of a supercapacitor using impedance spectroscopy and nonlinear least-squares fitting method. The electrochemical impedance spectroscopy is a method that allows the investigation of the complex impedance of electrochemical cells 
in a wide frequency range from millihertz to megahertz. It should be noted that in the sixth order model, the circuit topology for each order is not unique; however, by proper spreading of the time constant of each branch, this model will have high accuracy in many applications, accounting for a frequency range of up to tens of $\mathrm{kHz}$ [152]. In order to prevent numerical oscillation, the simulation time step should be smaller than two times the time constant of the fastest branch.

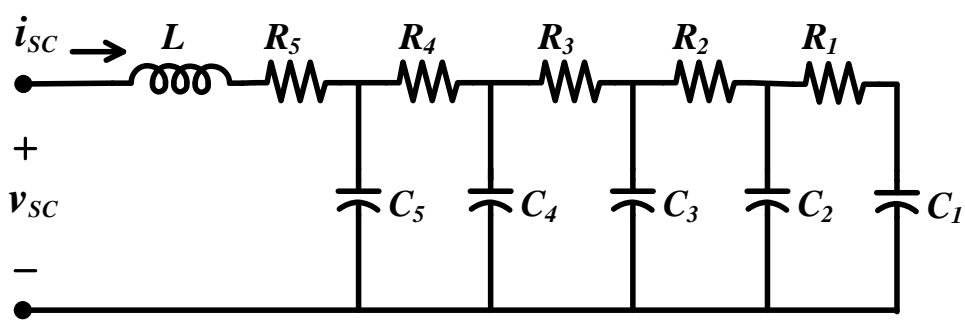

Figure 7.2: Sixth order equivalent circuit models for supercapacitor bank.

The virtual test-bed hybrid DC microgrid includes a 2.9-F supercapacitor bank and, as an energy buffer, delivers high instantaneous power to the pulsed load. The supercapacitor bank is composed of twenty Maxwell's 58-F, 16-V modules and rated 320V. The supercapacitor bank specifications are listed in Table 7.1.

Table 7.1: Supercapacitor bank specifications.

\begin{tabular}{|c|c|}
\hline Type & Maxwell (BMOD0058) \\
\hline Number of Cells & 20 \\
\hline Rated Capacity & $2.9 \mathrm{~F}$ \\
\hline Rated Voltage & $320 \mathrm{~V}$ \\
\hline Maximum Voltage & $340 \mathrm{~V}$ \\
\hline Maximum Continuous Current & $12 \mathrm{~A}\left(\Delta \mathrm{T}=15^{\circ} \mathrm{C}\right)$ \\
\hline
\end{tabular}

Since the transient and the dynamic characteristics of the hybrid DC power system are a concern, the sixth order ladder model of the supercapacitor bank is employed in our 
virtual test-bed platform for this study. The detailed model parameters of a 2.9-F supercapacitor can be found in Table 7.2.

Table 7.2: Sixth order model parameters of the 2.9-f supercapacitor bank.

\begin{tabular}{|c|c|c|c|}
\hline $\mathrm{R}_{1}$ & $1.02069 \Omega$ & $\mathrm{C}_{1}$ & $0.9193 \mathrm{~F}$ \\
\hline $\mathrm{R}_{2}$ & $0.17034 \Omega$ & $\mathrm{C}_{2}$ & $1.5428 \mathrm{~F}$ \\
\hline $\mathrm{R}_{3}$ & $0.05069 \Omega$ & $\mathrm{C}_{3}$ & $0.5481 \mathrm{~F}$ \\
\hline $\mathrm{R}_{4}$ & $0.05862 \Omega$ & $\mathrm{C}_{4}$ & $0.0594 \mathrm{~F}$ \\
\hline $\mathrm{R}_{5}$ & $0.22828 \Omega$ & $\mathrm{C}_{5}$ & $0.0008 \mathrm{~F}$ \\
\hline $\mathrm{L}$ & $2.2413 \mathrm{E}-3 \mathrm{mH}$ & & \\
\hline
\end{tabular}

\subsubsection{Battery Bank Modeling}

The lead-acid battery, which is commonly utilized in pulsed load DC power systems, is a complex and nonlinear device. Modeling of the lead-acid batteries for transient and dynamic operation analysis must account for the dependence of battery parameters on the battery state of charge, battery storage capacity and other internal phenomenon, such as self-discharge and internal heating losses. The analytical-electrical battery model suggested in [154]-[155] provides sufficient accuracy for an actively controlled battery energy storage system. In this method, the battery terminal voltage is a function of the battery internal voltage and its charge depletion based on equations (7.1) and (7.2):

$$
\begin{gathered}
U(q)=U_{0}-K\left(\frac{Q}{Q-q}\right)+A e^{- \text {Bq }} \\
V_{\text {Batt }}=U(q)-R_{\text {batt }} \frac{d q}{d t}
\end{gathered}
$$

Where $K$ is the polarization constant, $U_{0}$ is the internal voltage of the battery, $Q$ is the battery capacity, $A$ is the exponential zone voltage amplitude, $B$ is the exponential zone 
time constant inverse and $R_{\text {batt }}$ is the internal resistance of the battery. The detailed parameters of the $120-\mathrm{V}$ lead-acid battery bank implemented in our simulation platform can be found in Table 7.3.

Table 7.3: Battery model parameters.

\begin{tabular}{|c|c|}
\hline Parameter & Specification \\
\hline Internal Voltage $\left(U_{0}\right)$ & $130.65-\mathrm{V}$ \\
\hline Battery Capacity $(Q)$ & $110-\mathrm{Ah}$ \\
\hline Internal Resistance $\left(R_{\text {batt }}\right)$ & $0.011-\Omega$ \\
\hline Exponential Zone Voltage $(A)$ & $122.17-\mathrm{V}$ \\
\hline Exponential Zone Time Constant Inverse $(B)$ & $0.367-(\mathrm{Ah})^{-1}$ \\
\hline
\end{tabular}

\subsection{Virtual Test-Bed Model Implementation}

The hybrid energy storage power system and its interconnected test-bed power grid has been developed for power system studies, such as real-time control and operation, energy management, efficiency, power quality and reliability analysis of hybrid microgrids. The performance analysis of a four bus hybrid DC microgrid was presented in section 5. In this section, for transient and dynamic operation study, a virtual test-bed platform of this microgrid is developed on Matlab/Simulink environment. Figure 7.3 shows the schematic diagram of the hybrid DC microgrid virtual test bed. This simulation model and the components’ parameters are presented in detail in the following.

The AC generators, which mainly supply the DC microgrid, are 3-kVA, 60-Hz, 208-V, 1800-RPM synchronous machines coupled to individual induction motors as prime 
movers. These generators are modeled via a synchronous machine model. Stator windings are connected in wye and its middle point is connected to an internal neutral point.

In order to regulate the mechanical input power of the generator, the mechanical power $P_{m}$ is set. The $P_{m}$ and the reference voltage $V_{r}$ is set by the generator controller. Thus, the machine speed is determined by the machine inertia and by the difference between the mechanical torque resulting from the applied mechanical power $P_{m}$ and the internal electromagnetic torque $T_{e}$. The detailed generator specifications utilized for the virtual test-bed model can be found in Table 7.4.

As discussed in section 3, the generation stations are equipped with an automatic voltage regulator (AVR), which utilizes the feedback signal of the generators output voltage to regulate the bus voltage. In order to simulate the AVR function, the excitation system block is utilized. The excitation system is formed by the voltage regulator and it can be represented by the following transfer function between the exciter voltage $V_{f d}$ and the regulator's output $e_{f}$ :

$$
\frac{V_{f d}}{e_{f}}=\frac{1}{K_{e}+s T_{e}}
$$

where the $K_{e}$ and $T_{e}$ are the first-order exciter system gain and time constant, respectively. The $K_{e}$ and $T_{e}$ are 1 and zero to set proportional relation between the exciter voltage and the regulator's output.

In order to represent the stator terminal voltage transducer, a first-order low pass filter with a 0.02-s time constant is utilized. Also, the gain $K_{a}$ and time constant $T_{a}$ of the first-order system representing the main regulator are 300 and 0.001-s, respectively. 
AS shown in Figure 7.3, the AC generators are connected to $3-\mathrm{kVA} \mathrm{Y} / \Delta$ transformers to isolate the AC generators from the DC microgrid. This will prevent the even-order harmonics and the DC circulating current into the AC generators [45]. The specification of the galvanic isolation transformer can be found in Table 7.4.

Table 7.4: AC side components’ parameters.

\begin{tabular}{|c|c|c|}
\hline Component & Parameter & Specification \\
\hline \multirow{4}{*}{ Generator } & $S_{N}$ & $3 \mathrm{kVA}$ \\
\cline { 2 - 3 } & $V_{N}$ & $208 \mathrm{~V}$ \\
\cline { 2 - 3 } & $F_{N}$ & $60 \mathrm{~Hz}$ \\
\cline { 2 - 3 } Transformer & $L_{S}, R_{S}$ & $6.87 \mathrm{mH}, 1.45 \Omega$ \\
\cline { 2 - 3 } & Connection & $Y \mathrm{D}$ \\
\cline { 2 - 3 } & $S_{N}$ & $208 \mathrm{VVA}$ \\
\cline { 2 - 3 } & $V_{N}$ & $0.72 \Omega, 2.29 \mathrm{mH}$ \\
\hline
\end{tabular}

The full diode bridge implemented in the virtual test-bed consists of six diodes connected in a bridge configuration. The snubber resistance and the snubber capacitance of the diodes are $100-\mathrm{k} \Omega$ and $0.001-\mu \mathrm{F}$, respectively. This will also avoid the numerical oscillations of the model during the simulation. In order to consider the internal loss of the diodes during conduction mode, a $0.001-\Omega$ resistor is set as an internal resistance of the diode.

In order to boost the voltage and to regulate the injected power of the battery bank to the grid, a buck-boost converter is implemented at bus 2. Also, two boost converters regulate the output power of the uncontrolled rectifiers to the DC buses. The detailed parameters of the converters are given in Table 7.5. The configurations of the converters and their control strategy can be found in section 5 . 
Table 7.5: DC-DC converters' parameters.

\begin{tabular}{|c|c|c|}
\hline Component & Parameter & Specification \\
\hline \multirow{3}{*}{ Boost Converter } & power rating & $2500 \mathrm{~W}$ \\
\cline { 2 - 3 } & IGBT module & SKM100GAL12T4 \\
\cline { 2 - 3 } & switching frequency & $5 \mathrm{kHz}$ \\
\cline { 2 - 3 } & $\mathrm{LBC}_{\mathrm{BC}}, \mathrm{R}_{\mathrm{LBC}}$ & $6 \mathrm{mH}, 0.21 \Omega$ \\
\hline \multirow{3}{*}{ Bidirectional Converter } & power rating & $1800 \mathrm{~W}$ \\
\cline { 2 - 3 } & IGBT module & SK45GB063 \\
\cline { 2 - 3 } & Switching frequency & $5 \mathrm{kHz}$ \\
\cline { 2 - 3 } & $\mathrm{L}_{\mathrm{BD}}, \mathrm{R}_{\mathrm{LBD}}$ & $6 \mathrm{mH}, 0.20 \Omega$ \\
\hline
\end{tabular}

Figure 7.3 also shows the location and configuration of the AC and DC filters. These filters are implemented to filter out the harmonics to the AC generators and to smooth the output current of the boost converters. The specifications of each filter are listed in Table 7.6.

Table 7.6: AC and DC Filters’ parameters.

\begin{tabular}{|c|c|c|}
\hline Component & Parameter & Specification \\
\hline AC Filter & $L_{A F}, R_{A F}$ & $12 \mathrm{mH}, 0.31 \Omega$ \\
\hline \multirow{2}{*}{ DC Filter } & $L_{D F}, R_{L D F}$ & $2.7 \mathrm{mH}, 0.08 \Omega$ \\
\cline { 2 - 3 } & $C_{D F}, R_{C D F}$ & $680 \mu \mathrm{F}, 0.161 \Omega$ \\
\hline Bus Capacitor & $C_{G C}, R_{C G C}$ & $330 \mu F, 0.249 \Omega$ \\
\hline
\end{tabular}

The power lines for high voltage application are usually formed based on $\pi$-model, which emulates the power line through a series inductor and two parallel capacitors. However, since the medium and low voltage hybrid power system is considered in this study, the power lines are modeled via RL series impedance. The detailed specification of the power cable emulated in our virtual test-bed platform can be found in Table 7.7 . 


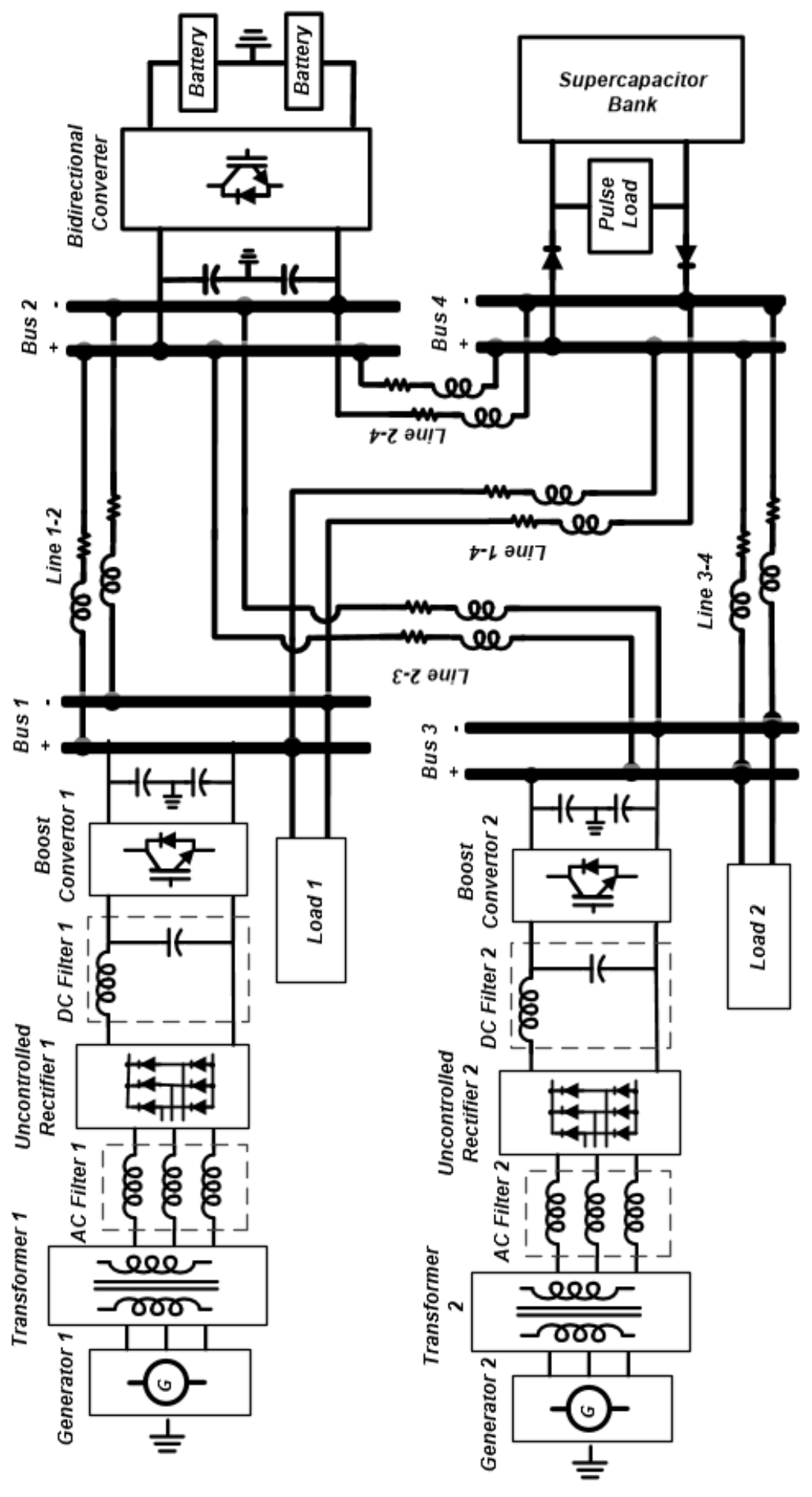

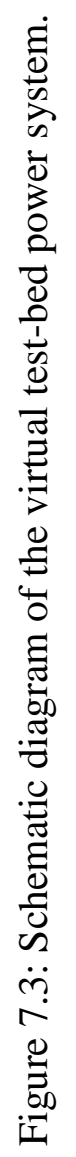


Table 7.7: DC microgrid power line Parameters.

\begin{tabular}{|c|c|c|c|c|c|}
\hline Cable & Length & $\mathrm{R}(\Omega / \mathrm{km})$ & $\mathrm{L}(\mathrm{mH} / \mathrm{km})$ & $\mathrm{R}(\Omega)$ & $\mathrm{L}(\mathrm{mH})$ \\
\hline Line1-2 & $70 \mathrm{~m}$ & 1.41 & 0.357 & 0.0987 & 0.02499 \\
\hline Line2-3 & $70 \mathrm{~m}$ & 1.41 & 0.357 & 0.0987 & 0.02499 \\
\hline Line3-4 & $50 \mathrm{~m}$ & 2.24 & 0.430 & 0.112 & 0.0215 \\
\hline Line1-4 & $40 \mathrm{~m}$ & 2.24 & 0.430 & 0.0896 & 0.0172 \\
\hline Line2-4 & $100 \mathrm{~m}$ & 1.41 & 0.357 & 0.141 & 0.0357 \\
\hline
\end{tabular}

\subsection{Evaluation of the Virtual Test-Bed Platform}

The notional hybrid DC microgrid, shown in Figure 7.3, has been implemented in our hybrid AC-DC power system test-bed. The three level control systems are built within the Matlab/Simulink environment and executed with the dSPACE 1103 board. Also, the dSPACE 1103 interface was utilized for the real-time monitoring and control of the system.

The detailed model of the notional hybrid DC microgrid, explained in section 5, was also implemented in our virtual test-bed platform using the Matlab/Simulink software. The fixed step Euler solver was utilized. The fundamental sample time of the simulation model was adjusted to $10-\mu$ s. The performance of the hybrid DC microgrid during normal operation and transient fault condition were experimentally tested and the results are compared with the simulation model.

\subsubsection{Normal Operation}

Figure 7.4 shows the hybrid DC microgrid test results under normal operation condition. During this test, the power of the pulse load was 3250-W and its frequency was $0.2-\mathrm{Hz}$, while its duty ratio was adjusted to $20 \%$. Also, the rated power of the dynamic load and the steady state load were 2250-W and 250-W, respectively. As can be seen, since 
both boost converters were available, the required current of the grid was equally shared between the two converters, i.e. each converter injected 4.9-A to the grid. Figure 7.4 also shows the bus voltage variation. When the pulsed load ended, the voltage of the DC bus dropped to $310.6-\mathrm{V}$ and it charged to $322-\mathrm{V}$ before the next pulse occurred. Since the generated and consumed power levels were equal, the average supercapacitor bank voltage was always $320-\mathrm{V}$ and its variation was $3.56 \%$, which was less than the preset $5 \%$ limit. The simulation results of this case study are also shown in Figure 7.4. As the results show, the supercapacitor voltage variations, and the converters and the load current accurately followed the experimental test results, which validated the accuracy of the simulation model.
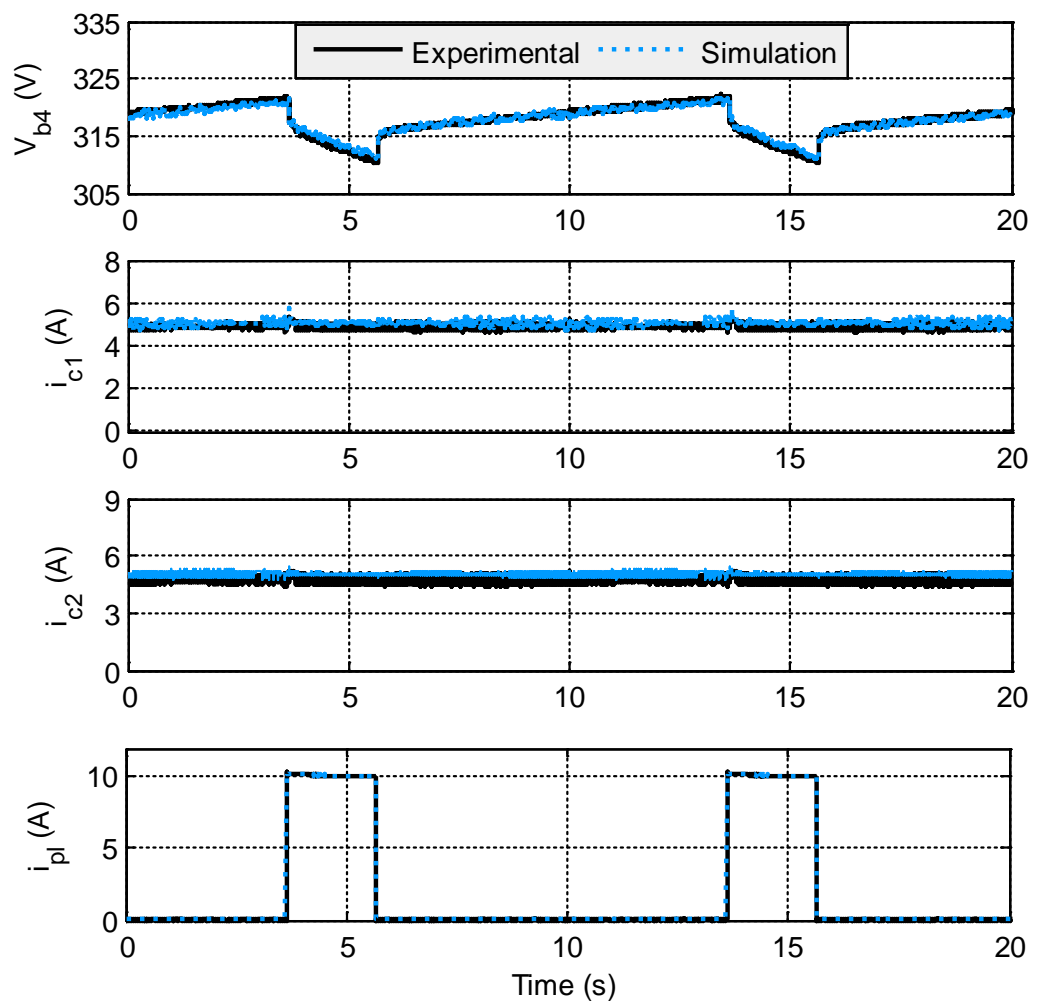

Figure 7.4: Performance of the hybrid DC microgrid during normal operation. 

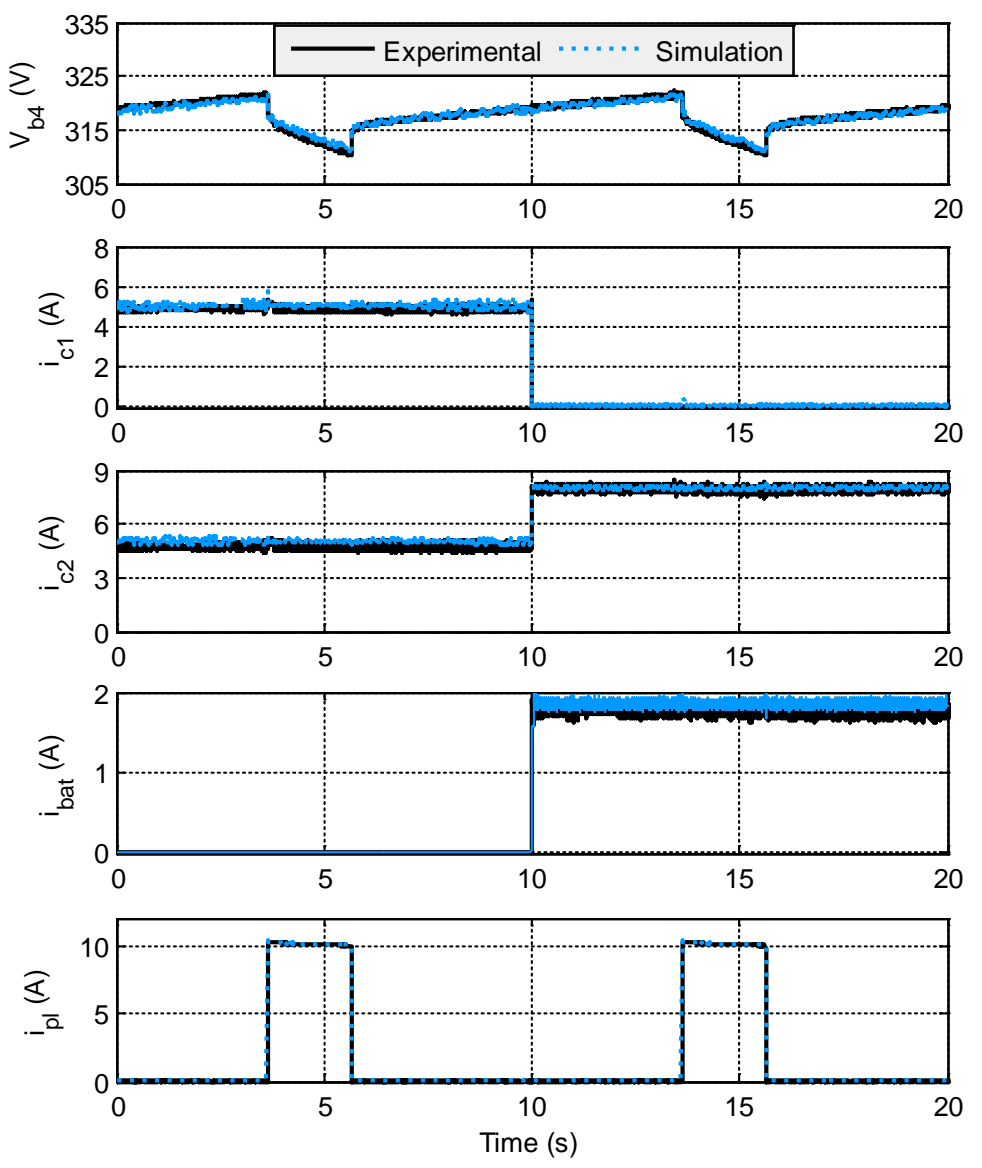

Figure 7.5: Performance of the hybrid DC microgrid during transient condition when the boost converter at bus 2 fails at $\mathrm{t}=10 \mathrm{~s}$.

\subsubsection{Transient Fault Condition}

In order to test the self reconfigurability of the system and to evaluate the simulation model during transient conditions, the grid was tested with an outage that occurred in the first converter. The test result is shown in Figure 7.5. As can be seen, when the outage occurred at $t=10$ s, the energy management system sets the second converter to its maximum power limit, which was 8-A, and the rest of the power was provided by the battery bank through the bidirectional converter. The experimental results show that the bus voltage variation was properly regulated and the voltage variation was less than the preset $5 \%$ limit. The simulation result for the same case study is also shown in Figure 7.5. 
The simulation results were consistent with the experimental test results. This agreement successfully validated both the experimental design of the system and the accuracy of the grid simulation model. 


\section{DESIGN OF DC POWER SYSTEM WITH HIGH RELIABILITY}

\subsection{Introduction}

While the advantages of the DC architecture are significant, the protection of multi-terminal and distribution DC systems has posed many challenges, such as accurate fault detection and location, proper grounding schemes, proper design of DC circuit breakers, fast isolation of faulted areas and self-reconfiguration capabilities, along with the lack of standards and guidelines. An advanced protection scheme should have selfdiagnosing and self-healing capabilities to reduce damage caused by short circuit faults and to provide continuous power to loads, even during system faults [112], [156][158]. The self-diagnosing capability of the system requires a well-designed protection scheme to be able to detect a fault very quickly and accurately locate the faulted area. Also, to realize the self-healing capability of the system, the switching devices should be properly designed and coordinated to enable fast transition from a fault condition to a normal operation. Moreover, choosing the appropriate grounding scheme is an important issue that involves many aspects of the power system, such as the reliability of the system, personal safety, transient overvoltage and insulation coordination of the system [159]-[160].

In the following, the key requirements for the protection of DC power systems are discussed. These requirements are the equipment survivability, personal safety, fault detection and location capabilities, system reconfigurability and ride-through capability. Then, different fault currents in DC systems are analyzed to provide guidelines to accurately set the protective relays and to properly design the protective devices. Also, various grounding topologies of DC power systems are explained and their advantages and 
limitations are compared. Finally, different DC circuit breaker technologies are reviewed and the performances of these breakers are compared.

\subsection{Power System Protection Requirements}

Designing a protection system is a comprehensive issue that involves many challenges. In this section, the key requirements of DC system protection are discussed and design challenges are addressed.

\subsubsection{Equipment Survivability}

DC fault currents are severe in transients, which can cause considerable damage to the equipment, such as the power electronic converters and sensitive loads. In a DC system, the power supplies are the major fault current sources, however, the capacitors at the DC bus terminal and DC filters significantly contribute to fault currents, which cause very high short circuit currents during the transient fault periods [112]. Thus, the proper design of a protection system requires careful analysis of its fault current characteristics. Section 8.3 discusses the fault current characteristics of different sources under various fault impedances.

The amount of energy absorbed by a device during a fault is proportional to the integral of $i^{2}$ over the transient fault period [161]-[162]. Thus, the maximum fault current amplitude, its shape and the fault clearing time are important factors that should be considered for a safe operation of the device. An advanced DC system should employ a protection scheme that quickly identifies a fault event and utilizes fast circuit breakers to minimize the fault clearing time and the maximum short circuit current. 


\subsubsection{Personal Safety}

Personal safety is one of the essential requirements of an electrical protection system. Particularly, arc flash can cause substantial injury to employees working on or near electrical equipment and can create further damage to the system. Thus, it is important to identify arc flash risk levels within the DC system and to adopt the necessary safety measures [163]-[165]. Moreover, the concern regarding retained charges on equipment and power lines is more significant for DC systems than it has been for AC [165]. As a result, the touch potential should be well considered when designing a DC system. This requires a proper strategy for the system grounding, which will be discussed in section 8.4.

\subsubsection{Fault Detection and Location Capabilities}

One of the most important aspects of a protection system is the fault detection and location algorithms embedded on the protection relays. These algorithms may require system current and/or voltage variables that can be locally measured and/or collected through communication networks. The key design challenges of any fault detection and location scheme are:

Speed: An advanced DC protection system should employ fast fault detection and location schemes to effectively limit the maximum fault current and minimize the system restoration time.

Accuracy: Another design aspect of the protection system is accurate setting of the protection relay that needs a detailed fault current analysis of the DC system. The analysis should also identify the maximum and minimum short circuit current when a fault occurs in different zones of the power system. 
Reliability: The implemented fault detection and location scheme need to be accurately set and well-coordinated to prevent malfunction. The system should also utilize a precise signal processing technique that avoids spurious switching on transients or noise.

Simplicity: The protection scheme should employ fewer measurement devices and require less data from communication networks to reduce the complexity and the cost of the system. Also, the protection system should use uncomplicated fault detection and location algorithms to reduce the computation burden on the protective relays.

Various fault detection and location techniques have been developed for a variety of applications and system configurations. These techniques are studied and analyzed in chapter 9 .

\subsubsection{System Reconfigurability}

A proper protection system should add reconfiguration capabilities to the grid so that the system could restore after the fault incident has been detected and the faulted area has been isolated from the grid. For this purpose, implementation of a proper number of disconnecting switches and circuit breakers, in addition to the multi-source and multiterminal capability of the grid, is essential [112], [166]-[167]. Moreover, the energy management system should be able to set the current or the voltage reference of power control devices based on the available energy sources and the required power of the loads. To this end, the energy management system may use a centralized control scheme [168][170] or distributed control approach [170]-[173]. In terms of system reconnection, load shedding would be necessary if the power consumption of the grid is higher than the generated power [171]. 


\subsubsection{Ride-Through Capability}

In advanced DC systems, the critical loads should be designed so that they can ridethrough the fault disturbances without interruption. For a system with a clearing time of typically less than 30-ms, this feature can be achieved by utilizing a shunt hold-up capacitor and clamping diodes [112], [174]. Assuming that the grid nominal voltage is EN and the minimum acceptable voltage of the load is $E_{\min }$, the required hold-up capacitor to maintain the load voltage can be found as:

$$
C_{H}=\frac{2 P_{L} t_{c l}}{\left(E_{N}^{2}-E_{\min }^{2}\right)}
$$

where $P_{L}$ is the load power and $t_{c l}$ is the fault clearing time.

It should be noted that for a high power load or for a protection system with a long fault clearing time, utilization of the hold-up capacitor may not be sufficient. In this case, implementation of a supercapacitor bank or a battery storage system as an uninterruptable power supply is necessary.

\subsection{Characteristics of DC Fault Current}

For a proper design of the protective devices and to accurately set the protective relays, detailed fault current analysis of the system is essential. In order to analyze the fault current of a DC system, consider the notional three bus MVDC network shown in Figure 8.1. This system employs AC/DC converters at bus 1 and bus 3 to interface the AC sources to the DC network. Also, a battery bank at bus 2, acting as a DC source, is connected to the grid through a DC-DC converter. In a DC system, the power supplies are the main fault current sources, however, the DC capacitor filters significantly contribute to the fault 
currents during the transient period. In order to drive the short circuit currents of the DC grid during the bus faults and the feeder faults, the equivalent circuit of the grid seen from the AC source at bus 1 is shown in Figure 8.2 (a). The AC sources connected through AC/DC converters typically provide relatively low currents that are in the order of $150 \%$ - 200\% of the converter rating currents and can be represented as a current source during a transient period [112], [175]. It should be noted that the healthy operation of a converter is assured only when the antiparallel diodes across the solid-state switches are back-biased by the DC voltages of the bus capacitors [174]. During DC grid faults, the DC bus capacitor voltage drops, so the antiparallel diodes of the converter are biased and the converter can be modeled with only its equivalent resistance and inductance [112]. Thus, the AC source 1, including its converter, is modeled as a current source in series with the $R_{S 1}$ and $L_{S 1}$ branch, representing the equivalent resistor and inductance of the AC source and the converter, respectively. Also, the $C_{B 1}, R_{C B 1}, L_{e q 1}$, and $R_{e q 1}$ shown in this model are the capacitance and resistance of the capacitor connected to the first bus, and the short circuit equivalent inductance and resistance seen from this bus, respectively.

Using the KCL law, and assuming negligible DC bus voltage during the transient fault, the short circuit current of bus1, $i_{B 1}$ can be expressed as:

$$
i_{B 1}=i_{S 1}+i_{C B 1}
$$

where $i_{S 1}$ and $i_{C B 1}$ are the current of the AC-DC converter and the DC bus capacitor, respectively. 


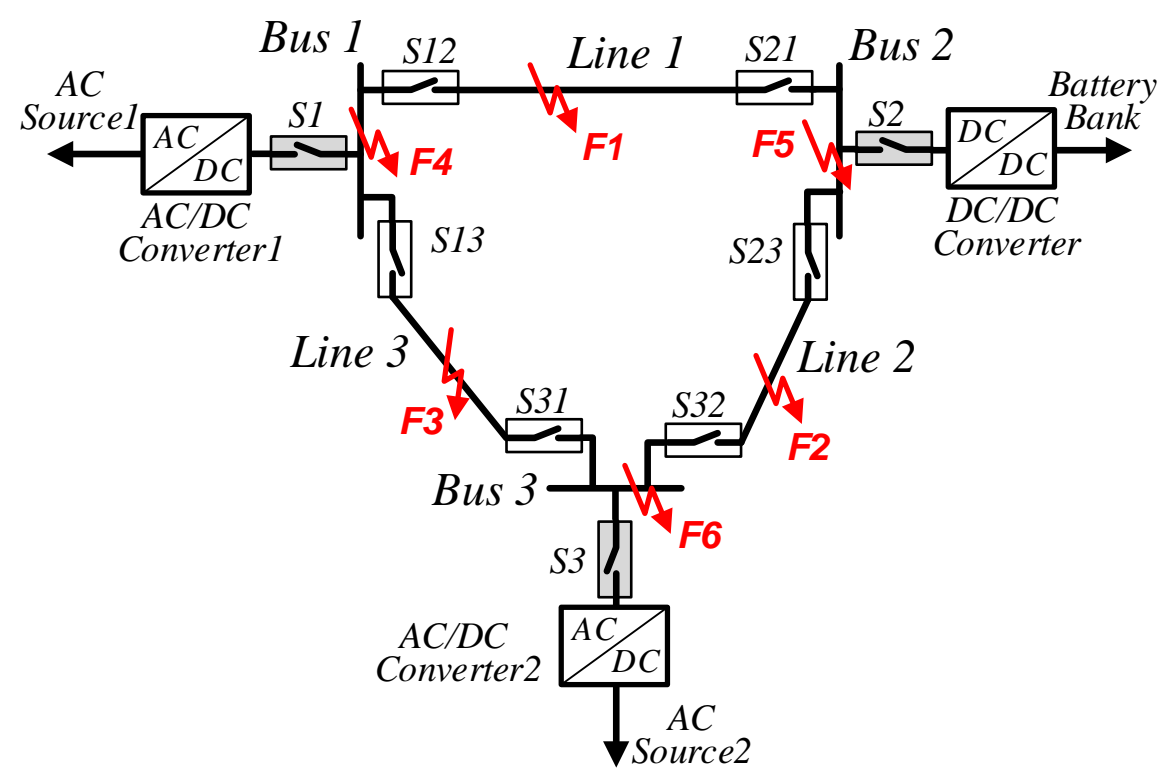

Figure 8.1: Schematic diagram of a notional three-bus MVDC.

Transient $i_{S 1}$ can be found by means of an equivalent star circuit model with six parallel branches, where the current of the $j$ th branch can be expressed as (8.3) so that $i_{S 1}$ is the envelope of the maximum values of $i_{S B j}[175]$.

$$
i_{S B j}(t)=I_{0}\left(\operatorname{Cos}\left(\omega t+\varphi_{j}-\theta_{S 1}\right)-\operatorname{Cos}\left(\varphi_{j}-\theta_{S 1}\right) e^{-t / \tau_{S 1}}\right)
$$

where $\tau_{U R}$ and $\theta_{U R}$ are the converter's short circuit time constant and its impedance phase angle, which can be found as (8.4) and (8.5). Also, $\mathrm{I}_{0}$ is the steady state short circuit current of the branches and is given by (8.6):

$$
\begin{gathered}
\tau_{S 1}=\frac{L_{S 1}}{R_{S 1}} \\
\theta_{S 1}=\tan ^{-1}\left(\frac{\omega L_{S 1}}{R_{S 1}}\right) \\
I_{0}=\frac{E_{A C}}{\sqrt{R_{S 1}^{2}+\omega^{2} L_{S 1}^{2}}}
\end{gathered}
$$


where $E_{A C}$ is the rms nominal phase voltage, $\omega$ is its angular frequency and $\varphi_{j}$ is the phase shift of the jth branch voltage with respect to a common reference.

In order to find the $i_{C B 1}$ during bus fault (F1), a KVL can be applied across the loop that includes the DC bus capacitor as:

$$
\frac{d i_{C B 1}(t)}{d t}+\frac{1}{R_{C B 1} C_{B 1}} i_{C B 1}(t)=0
$$

Solving the differential equation (8.7) yields the transient $i_{C B 1}$ during a bus fault as:

$$
i_{C B 1}(t)=\frac{E_{N}}{R_{C B 1}} e^{-t / \tau_{C B 1}}
$$

where the time constant $\tau_{C B 1}$ is given by:

$$
\tau_{C B 1}=R_{C B 1} C_{B 1}
$$

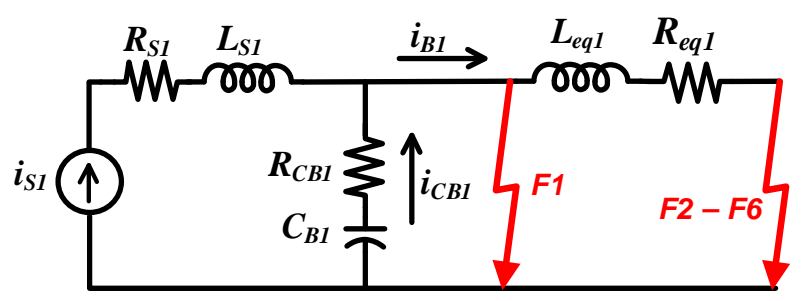

(a)

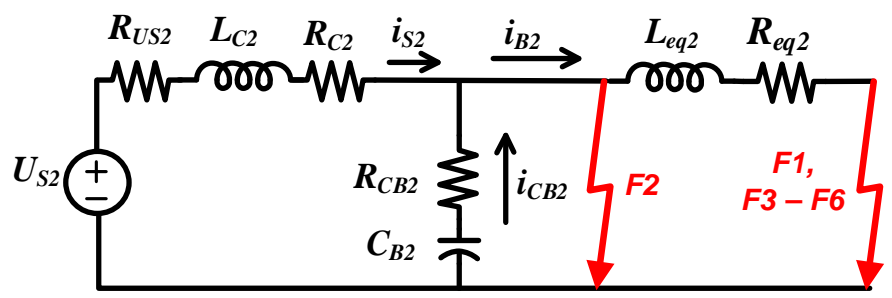

(b)

Figure 8.2: Equivalent circuit of the grid shown in Figure 8.2 during short circuit conditions: (a) seen from AC source at bus 1 , (b) seen from battery bank at bus 2 .

Also, the transient fault current of bus 1 during the remote bus faults F2 - F3 and the feeder faults F4 - F6 can be given as an algebraic sum of $i_{S 1}$ and $i_{C B 1}$. During a poleto-pole fault, since the fault often has low impedance, it can be assumed that the DC bus 
voltage during the transient fault is negligible. Thus, the $i_{S 1}$ can be found from (8.3). Also, application of KVL to the loop, including the DC bus capacitor and the short circuit equivalent inductance and resistance seen from first bus (Figure 8.2 (a)), gives the following relation for $i_{C B 1}$ during the remote bus faults and the feeder faults.

$$
\frac{d^{2} i_{C B 1}(t)}{d t^{2}}+\left(\frac{R_{C B 1}+R_{e q 1}}{L_{e q 1}}\right) \frac{d i_{C B 1}(t)}{d t}+\frac{1}{C_{B 1} L_{e q 1}}=0
$$

To solve the second-order differential equation (8.10), the damping factor $\zeta_{F}$, can be defined as (8.11). In the case that $\zeta_{F}$ is smaller than unity, the $i_{C B 1}$ is underdamped and the corresponding solution to (10) can be found as (8.12) [112],.

$$
\begin{gathered}
\zeta_{F}=\left(\frac{R_{C F 1}+R_{e q 1}}{2}\right) \times \sqrt{\frac{C_{F 1}}{L_{e q 1}}} \\
i_{C B 1}(t)=\left(A_{1} \cos \left(\omega_{F} t\right)+A_{2} \sin \left(\omega_{F} t\right)\right) e^{-\alpha_{F} t}
\end{gathered}
$$

where the attenuation factor, $\alpha_{D F}$ the decaying oscillation frequency, $\omega_{F}$ and the constants $A_{1}$ and $A_{2}$ are:

$$
\begin{gathered}
\omega_{F}=\sqrt{\frac{1}{L_{e q 1} C_{F 1}}\left(1-\zeta_{F}^{2}\right)} \\
\alpha_{F}=\frac{R_{C B 1}+R_{e q 1}}{2 L_{e q 1}} \\
A_{1}=i_{C B 0} \\
A_{2}=\left(\frac{E_{N}-\left(R_{C B 1}+R_{e q 1}\right) i_{C B 0}}{L_{e q 1}}+\alpha_{F} i_{C B 0}\right) \omega_{F}
\end{gathered}
$$

where $i_{C B O}$ is the $i_{C B 1}$ before the fault occurs. 
In order to analyze the fault current of the battery bank connected at bus 2, the equivalent model shown in Figure 8.2 (b) can be utilized. The parameters $U_{S 2}, R_{S U 2}, L_{C 2}$ and $R_{C 2}$ shown in this model are the internal voltage of the battery bank, the battery bank internal resistance, the DC-DC converter inductance and its resistance, respectively. Also, the $C_{B 2}, R_{C B 2}, L_{e q 2}$, and $R_{e q 2}$ are the capacitance and resistance of the capacitor connected to the second bus and the short circuit equivalent inductance and resistance seen from this bus, respectively. It should be noted that the internal voltage of the battery $U_{S 2}$ is a function of the charge drawn from the battery bank. However, during the transient fault time, the change in the internal voltage is small and it can be assumed to be constant. In the case of a short circuit fault at bus 2 (F2), the transient current of this bus is given by:

$$
i_{B 2}=i_{S 2}+i_{C B 2}
$$

where the $i_{C B 2}$ can be similarly found as (8.8) and the $i_{S 2}$ is the DC_DC converter current. Application of KVL to the loop, including the converter impedance (Figure 8.2 (b)), gives the following differential equation for the $i_{S 2}$ :

$$
\frac{d i_{S 2}(t)}{d t}+\frac{R_{U S 2}+R_{C 2}}{L_{C 2}} i_{S 2}(t)-\frac{1}{L_{C 2}} U_{S 2}(q)=0
$$

Solving the differential equation (8.18), the transient $i_{S 2}$ can be found as:

$$
i_{S 2}=\frac{U_{S 2}(q)}{R_{U S 2}+R_{C 2}}\left(1-e^{t / \tau_{C 2}}\right)
$$

where the time constant $\tau_{C 2}$, is given by:

$$
\tau_{C 2}=\frac{L_{B D}}{R_{\text {Batt }}+R_{L B D}}
$$


The short circuit current of bus 2 in the case of feeder faults and remote bus faults (F1 and F3 - F6) can be given as an algebraic sum of $i_{C B 2}$ and $i_{s 2}$. In this case, the $i_{C B 2}$ can be found similar to (8.12) and the $i_{S 2}$ can be found based on (8.19) with the consideration of the equivalent inductance and resistance of the fault.

The analytical analysis given in this section can be effectively utilized for the calculation of the fault current parameters, such as the maximum fault current, its derivative and the time it takes to reach the maximum value. These data provide essential guidelines for the accurate setting of the protection relays and the proper design of the protective devices.

\subsection{Grounding Scheme of DC Power System}

The grounding scheme in a DC power system is a very important issue that highly affect both the transient short circuit current and overvoltage of the system, and also its fault detection and protection capabilities [159], [160], [177]. The grounding configuration of DC power systems can be divided into three types, which are the ungrounded system, resistance grounded system and solidly grounded system in middle point. These grounding schemes are explained here in detail and their advantages and limitations are compared.

\subsubsection{Ungrounded DC System}

Compared with other grounding methods, the ungrounded DC power system has a better continuity of power supply, as it allows the system to operate for a while when single pole ground fault occurs. Also known as a floating system, the ungrounded DC grid considerably reduces ground leakage current, as well as the system corrosion level [178]. 
Moreover, since this grounding scheme doesn't need any device to be connected to the ground, it is technically simple in its implementation and has a low installation cost.

Although an ungrounded system is able to normally operate with a single pole ground fault, it is critical to detect and mitigate the problem. This is because of the fact that a second ground contact in another pole can cause a pole-to-pole fault and significant system damage. However, a single pole ground fault detection and location in an ungrounded system is very difficult, as it is seen similarly by different detectors in the system due to the low ground current [179]-[181]. Another disadvantage of this configuration is that, without a DC path to ground, the presence of even small leakage currents can cause an unpredictable DC offset since there is no DC reference point for the DC bus to be balanced [182]. Additionally, ungrounded components can have electrical noise problems throughout the system, e.g. in a motor's encoder wiring and circuitry, noise may cause erroneous information, such as missing encoder pulses [183]. Moreover, the equipment in the floating DC system requires an insulation level corresponding to the full DC voltage against the ground needed to withstand unbalanced voltage conditions. Hence, for high-voltage applications, there are higher cable costs, occupied space and weight when compared to the solidly grounded system [182].

\subsubsection{Resistance Grounded System}

The second possible method of the DC power system grounding is to connect a resistor with a certain value between either of the poles to the ground. Figure 8.3 (a) shows this grounding configuration for an AC/DC converter that is connected to a DC bus. Figure 8.3 (b) shows an alternative configuration in which two impedances, $Z_{p}$ in series are connected to the DC buses while the middle point is grounded. The $Z_{p}$ can be a properly 
sized resistor or a resistor and a capacitor in parallel. This configuration, which is also known as the virtually grounded system, is mostly utilized in DC power systems without a neutral point and in an application such as in flexible HVDC systems [157], [176]-[184]. As the resistor continuously dissipates DC power, there is a practical limitation on how small it may be. Also, increasing the size of the grounding resistor will increase the possibility of a voltage unbalance in the system and the time it takes to restore balance if an unbalance occurs. In the case of a DC power system with a neutral point, the resistor can be connected to the neutral point to limit the power loss during normal operations. Figure 8.3 (c) shows the configuration of a symmetric bipolar AC/DC converter with a neutral point that is grounded via a resistor. Other examples of a bipolar DC (BPDC) system that can be grounded via resistor in the neutral point to avoid power loss include a neutral-point-clamped inverter (NPCI), a twelve pulse uncontrolled rectifier and a series of two battery storage banks [112], [157], [185].

One of the advantages of the resistance grounded system is that there is a small current and voltage transient in the case of a ground fault and as a result, during a single pole ground fault, the system is able to continuously operate and supply the loads [177], [181], [182]. Also, the resistance grounded system reduces the concern for transient high overvoltage due to single pole ground faults [159]. However, in the case of small groundfault currents, it is difficult to measure and detect the fault, and as a result, the metal enclosures of the loads may be energized. Also, during a single pole ground fault, the insulation of equipment connected to a healthy pole has to withstand the full bipolar DC voltage until a fault is removed. Moreover, in the design of this grounding system, the safe 
operation of the sensitive loads during changing the pole-to-ground voltage must be ensured.

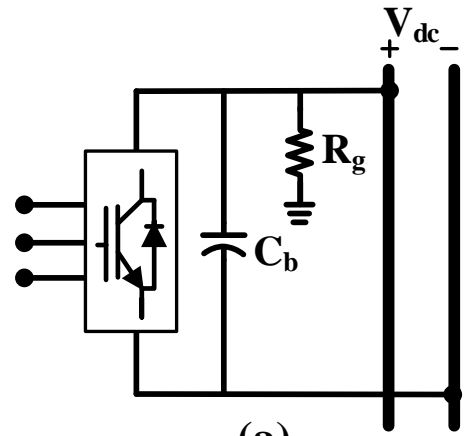

(a)

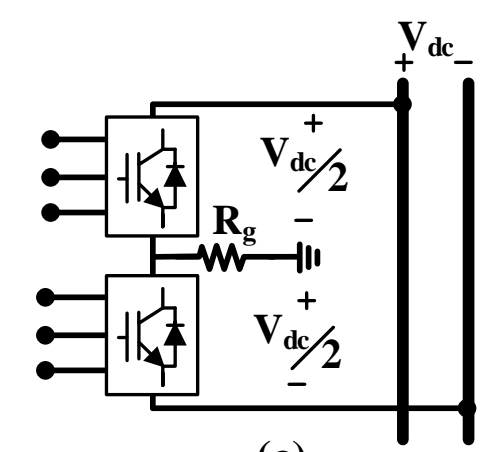

(c)

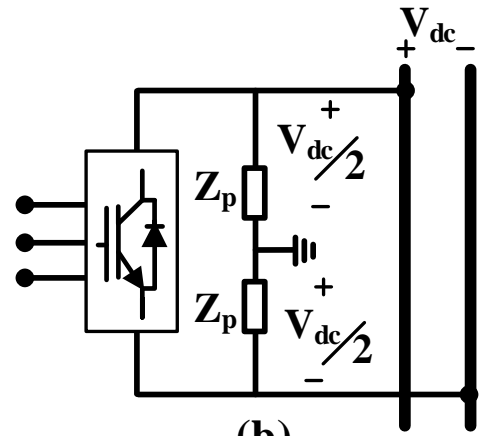

(b)

Figure 8.3: Grounding scheme of DC power system. (a), (b) and (c) are the resistance

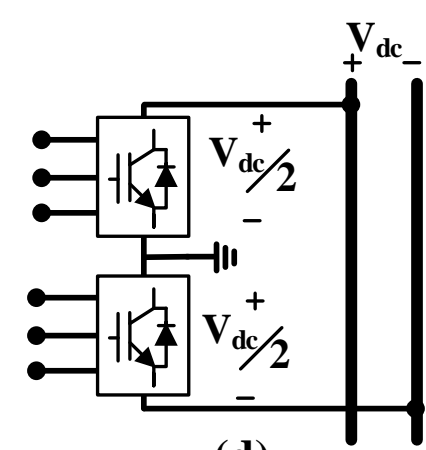

(d) grounded DC power system. (d) is a solidly grounded system in middle point.

\subsubsection{Solidly Grounded System}

In the solidly grounded BPDC power system, the ground point is electrically centered between the positive DC bus and the negative DC bus at the neutral point. Figure 8.3 (d) shows this grounding configuration for the NPCI system. Compared with other grounding methods, this configuration causes a larger ground current and a higher dc-link voltage transient. However, with a well-designed protective relay, the fault can be easily detected and it can be quickly cleared. Additionally, using proper arrangement of the disconnecting switches and circuit breakers, the system can continue to operate at half power during the time that the faulted pole is out of service [112], [182]. Another advantage 
of this configuration is that, the equipment and the cables require an insulation level of half DC pole-to-pole voltage. As a result, the system has lower cost, space and weight.

Table 8.1: Comparison of different grounding schemes for DC power system applications.

\begin{tabular}{|l|c|c|c|c|c|c|}
\hline \multirow{2}{*}{} & \multicolumn{2}{|c|}{ Ungrounded } & \multicolumn{2}{c|}{$\begin{array}{c}\text { Grounded with } \\
\text { Resistor }\end{array}$} & \multicolumn{2}{c|}{ Solidly Grounded } \\
\cline { 2 - 7 } & MPDC & BPDC & MPDC & BPDC & MPDC & BPDC \\
\hline Safety & Low & Low & High & High & High & High \\
\hline $\begin{array}{l}\text { Short Circuit } \\
\text { Current }\end{array}$ & $\begin{array}{l}\text { Very } \\
\text { Low }\end{array}$ & $\begin{array}{c}\text { Very } \\
\text { Low }\end{array}$ & Low & Moderate & Low & High \\
\hline $\begin{array}{l}\text { Transient } \\
\text { Overvoltage }\end{array}$ & High & High & Moderate & Moderate & Low & Low \\
\hline $\begin{array}{l}\text { Insulation } \\
\text { Level }\end{array}$ & $\begin{array}{l}\text { Nominal } \\
\text { Voltage }\end{array}$ & $\begin{array}{l}\text { Nominal } \\
\text { Voltage }\end{array}$ & $\begin{array}{l}\text { Nominal } \\
\text { Voltage }\end{array}$ & $\begin{array}{c}\text { Nominal } \\
\text { Voltage }\end{array}$ & $\begin{array}{c}\text { Nominal } \\
\text { Voltage }\end{array}$ & $\begin{array}{c}\text { Nominal } \\
\text { Voltage }\end{array}$ \\
\hline $\begin{array}{l}\text { Grounding } \\
\text { Power Loss }\end{array}$ & No & No & Yes & No & No & No \\
\hline $\begin{array}{l}\text { Stray Current } \\
\text { Low }\end{array}$ & $\begin{array}{l}\text { Very } \\
\text { Low }\end{array}$ & Moderate & Moderate & Low & Moderate \\
\hline $\begin{array}{l}\text { Ability to } \\
\text { Operate } \\
\text { Furing Ground }\end{array}$ & Yes & Yes & Yes & Yes & Yes & $\begin{array}{c}\text { Nominal } \\
\text { Power }\end{array}$ \\
\hline
\end{tabular}

Although a solidly grounded system in a monopole DC (MPDC) power grid is possible through direct connection of the negative pole to ground, it is not recommended for many applications due to corrosion and safety issues [182], [186]-[187]. The study presented in [187] has shown that the cumulative leakage current in the solidly grounded monopole traction system is approximately 30 times larger than the one in the ungrounded 
traction system during both acceleration and deceleration modes. In such a system, the negative pole can be connected to the ground via a diode circuit to eliminate the problems of stray-current corrosion and to keep bus potentials at a safe level [186]-[187]. Depending on the application, the conduction voltage threshold of the diode is usually adjusted in the range of $10-\mathrm{V}$ to $50-\mathrm{V}$ [187].

In order to summarize the analysis presented in this section, comparative analysis of different grounding schemes for a MPDC system without a neutral point and a BPDC arrangement with a neutral are given in Table 8.1 .

\subsection{Circuit Breakers and Disconnecting Switches}

As discussed in section 8.2, the development of an advanced protection scheme in DC power systems requires proper design and implementation of circuit breakers to effectively limit the fault current and isolate the faulty section in a DC grid. However, there are many challenges involved in designing a circuit breaker in a DC power system. Due to the absence of zero crossings in the DC current, the breakers have to interrupt the fault currents and dissipate a large amount of energy very quickly. Moreover, the inductance of a DC system is generally low, which leads to a very high rate of rise of fault current. Hence, the fast fault current interruption capabilities of the DC breaker are very important. In this section, various circuit breaker technologies developed for DC systems have been reviewed and the performances of these breakers are compared in terms of the design and control complexity, cost, operation time, and power losses. 


\subsubsection{Fuses}

Fuses are self-triggered protective devices that offer short-circuit and overcurrent protection for a wide range of DC power system applications for voltage levels up to 36kV [188]-[191]. Fuses can be ideally utilized in DC distribution networks having a low inductance and a high di/dt that keeps the time for the fuse to reach its melting point to a minimum [191]. The main disadvantage of the fuses is that they are one-time devices and must be replaced when the strip or element melts. As a result, the reconfigurability of a system that is protected by fuses is very low. Moreover, from the standpoints of safety and reliability, fuses are not a good solution, as they only trip the faulted line, leaving the unfaulted pole energized, which makes the fault detection process complicated. Thus, in an advanced DC system, they are only adopted as a backup protection for the main circuit breaker.

\subsubsection{Mechanical Circuit Breakers}

In the past decades, various mechanical circuit breaker (MCB) topologies have been developed for DC power system applications. The main concept of the MCBs is to supplement a conventional mechanical AC circuit breaker (MACCB) with a resonance circuit to create a current zero crossing for fault current interruption. In these systems, the resonance circuit can be directly connected to the main breaker, known as passive MCBs [192]-[195], or it can be actively connected through switching devices [196]-[201]. Figure 8.4 shows the basic topology of a passive MCB. In this configuration, once the MACCB is opened, a negative growing oscillation current is generated by the resonance circuit. This current ultimately exceeds the amplitude of the fault current, hence, a zero crossing in the MACCB is realized and the fault current is interrupted. In an active MCB, the capacitor of 
the resonance circuit is pre-charged and it is connected to the system once the MACCB is opened. As a result, the maximum resonance amplitude can be immediately reached that speeds up the MCB's operation. In order to further reduce the interruption time, the frequency of the oscillation current should be increased. This requires implementation of an MACCB with a high rate of rise current interruption capability [199]-[200]. Although the cost of the MCBs is lower than the other technologies, their operation speed is very low. Generally, the current interruption time of the MCBs is $30-100 \mathrm{~ms}$, which might be adequate for a system with line commutated current-source converters and a transmission system with low speed protection [202]-[205].

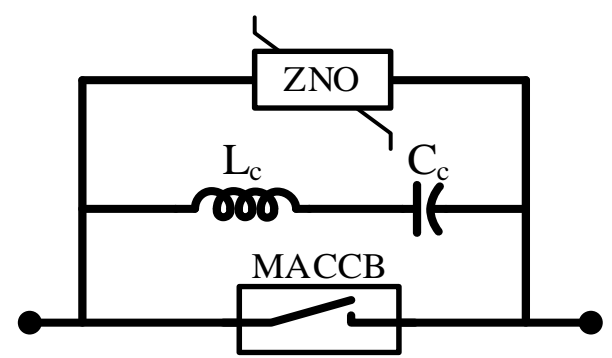

Figure 8.4: Configuration of the passive MCB.

\subsubsection{Solid-State Circuit Breakers}

A solid-state circuit breaker (SSCB) can be built using various semiconductor devices, including the integrated gate-commutated thyristor (IGCT) and insulated-gate bipolar thyristor (IGBT) [177], [205]-[207]. The topology of a unidirectional SSCB consisting of a single power semiconductor is shown in Figure 8.5. In this configuration, a surge arrester in series with a freewheeling diode is connected in parallel to the DC bus. In normal condition, the semiconductor device remains at the ON-state and leads the load current. When the semiconductor is turned off after detecting a fault, the fault current commutates to the freewheeling diode and suppresses the surge voltage. Alternatively, the 
surge arrester can be directly connected in parallel with the semiconductor device [205][207]. However, this will result in a higher rating and volume of the surge arrestor [208].

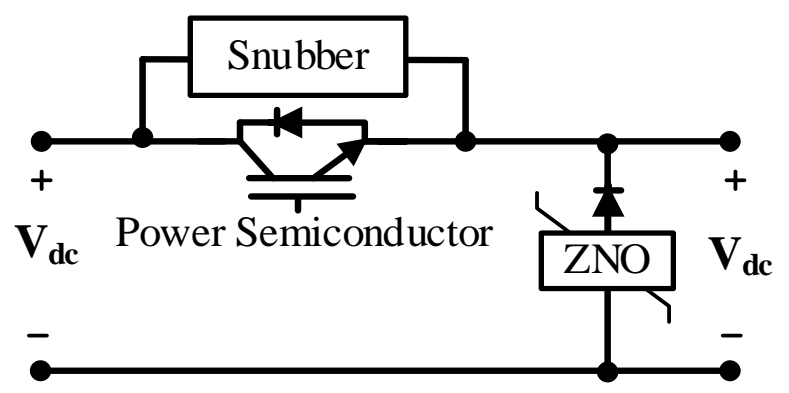

Figure 8.5: Schematic of a Unidirectional SSCB system.

Figure 8.6 also shows the circuit configuration of a bidirectional SSCB consisting of two back-to-back power semiconductors. It should be noted that the ratings of the SSCB are determined by the voltage and current ratings of the utilized semiconductor devices. Thus, for high-voltage applications, series connection of multiple semiconductor devices is necessary. In this case, a similar resistor should be connected in parallel to switching devices to maintain voltage balance in steady states. Also, in order to balance the voltage of the semiconductor device during turn-off transients, a snubber circuit can be utilized, as shown in Figure 8.6, to realize zero voltage switching [206], [208].

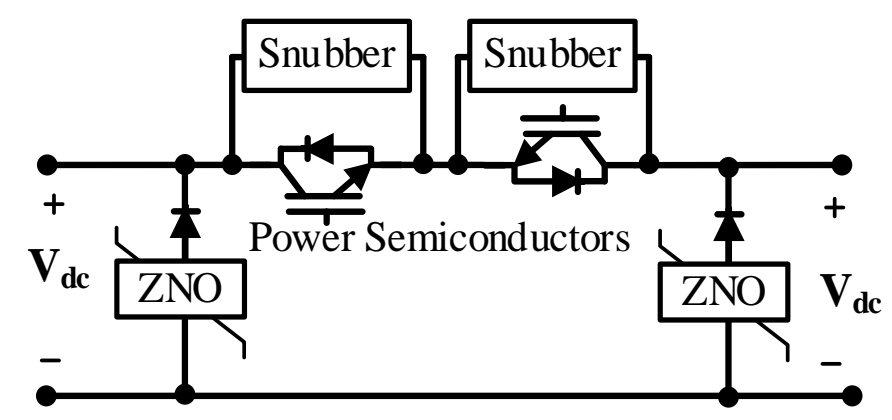

Figure 8.6: Schematic of a Bidirectional SSCB system.

The main advantage of the SSCBs is that they are very fast and are able to operate in microseconds. As a result, the utilization of the SSCBs and a protection system with fast 
fault detection capabilities enable the system to interrupt the fault current before it reaches its maximum level. Moreover, the SSCBs have a superior acoustic performance and lower maintenance costs when compared with other technologies. However, the power losses of the SSCBs in steady state operation are considerable and some mitigation, especially for high-voltage applications, is required.

\subsubsection{Hybrid Solid-State Circuit Breakers}

In order to reduce the breaker power losses during normal operation, a hybrid high voltage circuit breaker (HHVCB) consisting of a SSCB and an additional bypass branch is suggested [209]-[210]. The bypass branch is formed by a load commutation semiconductor in series with a fast mechanical disconnector. Figure 8.7 shows the topology of a bidirectional HHVCB system. During normal operation, the current will only flow through the bypass branch, and the current in the main semiconductor branch is zero. During faults, the load commutation switch immediately commutates the current to the main SSCB and the fast mechanical disconnector opens.

For proper operation of the HHVCB, the main SSCB must be able to break the peak fault current, and withstand peak DC voltage. Since the mechanical disconnector opens at zero current with low voltage stress, it can be realized as a disconnector with a lightweight contact system. Also, the load commutation switch should be rated for full transmission line current but only for conduction drop on SSCB switches, which is typically in the $\mathrm{kV}$ range for a $320 \mathrm{kV}$ HVDC breaker [209]. 


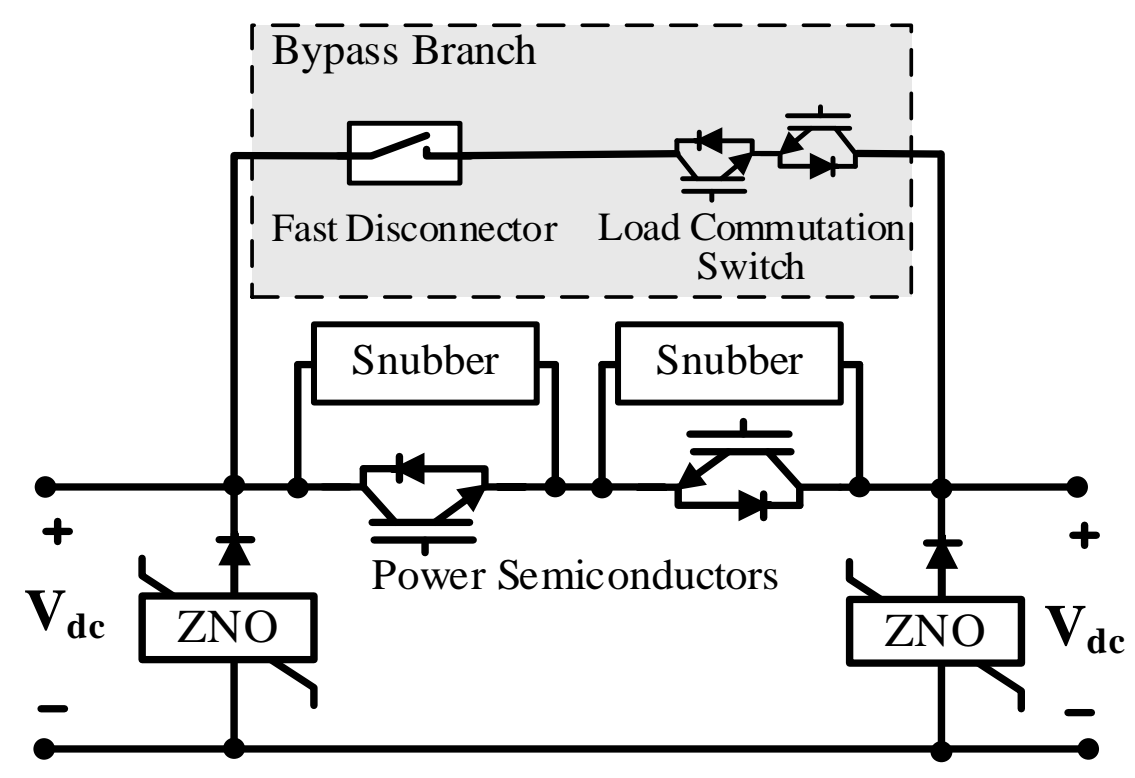

Figure 8.7: Hybrid circuit breaker for high-voltage applications.

\subsubsection{Galvanic Isolator}

One of the disadvantages of the SSCBs and HHVCBs is that they do not provide galvanic isolation. Thus, for the safety issues and in order to interrupt the leakage current after the SSCB operation, implementation of galvanic isolators is essential [177], [206], [208]. Galvanic isolators, also known as disconnecting switches, realize physical isolation through a mechanical method. Figure 8.8 shows the configuration of the circuit breaker with galvanic isolation capability. This system is composed of a main SSCB or HHVCB for high fault current interruption through semiconductor devices and two secondary galvanic isolators for isolation of the two feeders. The controller activates the galvanic isolator when the HHVCB interrupts the fault current and the leakage current is decayed to a predetermined level. 


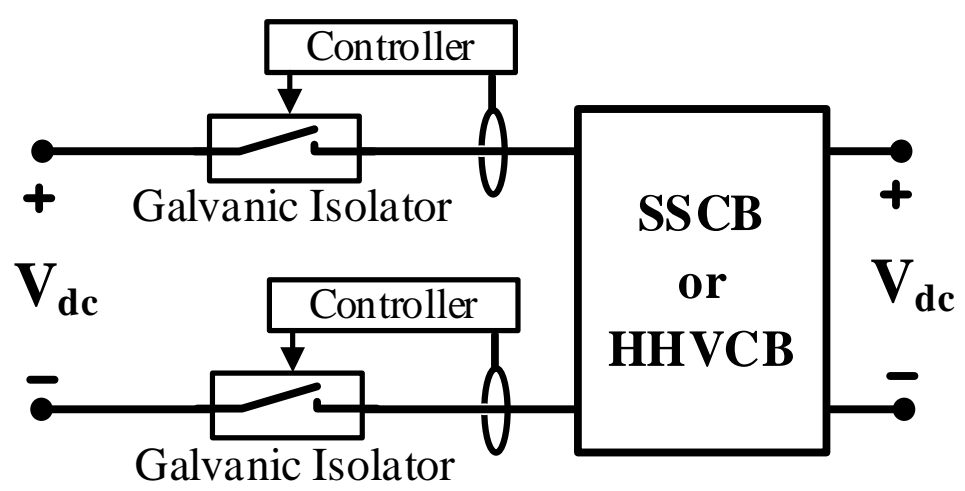

Figure 8.8: Circuit breaker with galvanic isolator.

\subsubsection{Fault-Tolerant Converters}

Utilization of the semiconductor-based, fault-tolerant DC-DC converters is an alternative approach to break DC fault current and isolate the faulty section in a DC system. Examples of such a converter are the half bridge chopper [211], full bridge chopper [212], and LCL thyristor converter [213]. In addition to fault current breaking capabilities, these converters can provide the voltage stepping and power regulation services to a DC grid. Since the fault-tolerant converters use inherent characteristics of the converter to block the current when the voltage on the DC buses drops, they can perform very fast and they do not require fast protection systems [207]. However, compared with SSCBs and HHVCBs, the fault-tolerant converters have high switching and conduction power losses, very high costs and are more complex. As a result, applications of these converters are limited to the system in which the voltage and power regulation capabilities of the converter are in demand, and also for critical loads connected to a DC system with low speed protection.

\subsubsection{Superconductive Fault Current Limiter}

The superconductive fault current limiter (SFCL) has been extensively studied for both AC and DC systems in the past decades [214]-[220]. The SFCLs make use of the physical property of the superconductors, that is they lose their zero resistance when the 
current density of the conductor reaches above a critical value [216]-[217]. Depending on the configuration and the operation principle, the SFCLs in DC power systems can be classified into resistive SFCL (RSFCL) and inductive SFCL (ISFCL). An RSFCL consists of a superconducting wire that is directly connected in series with the DC feeder [214][214]-[215].

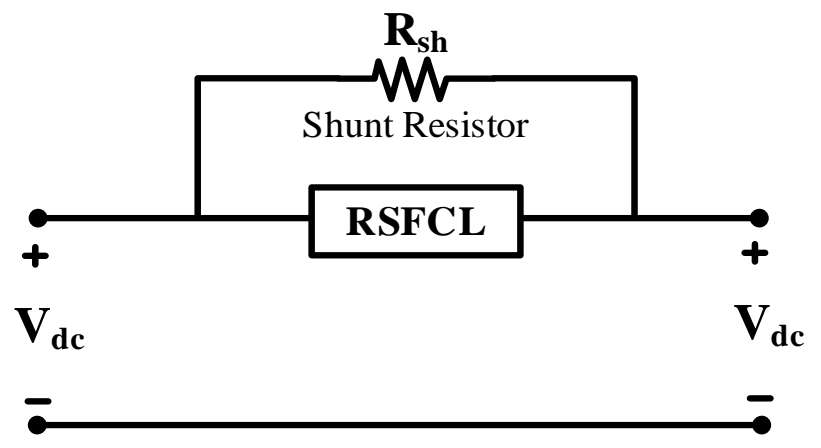

Figure 8.9: RSFCL configuration.

The main advantages of the RSFCL are the simplicity and compactness of the system. However, due to the heating of the superconductor during the fault, the cooling and recovery time of the RSFCL are of concern [217]. The ISFCLs are composed of the superconductive coils wound on an iron core that can be directly connected in series with a feeder [218], or actively connected through power electronic converters [219]-[220]. The ISFCLs are able to provide large current limiting impedance, while utilizing less superconductive wire.

Figure 8.9 shows the configuration of an RSFCL utilized for DC feeder protection. It should be noted that, in high-voltage applications, the fault current level is very high and the direct connection of RSFCL leads to a significant increase of superconductor temperature. Consequently, it takes a long time for the superconducting coil to be cooled enough and return to its superconducting zone after the fault has been cleared. Thus, the 
implementation of a shunt resistor in such a system is necessary. The shunt resistor should be designed so that the RSFCL limits the fault current effectively, while the cooling process and system restoration after fault clearance is expedited.

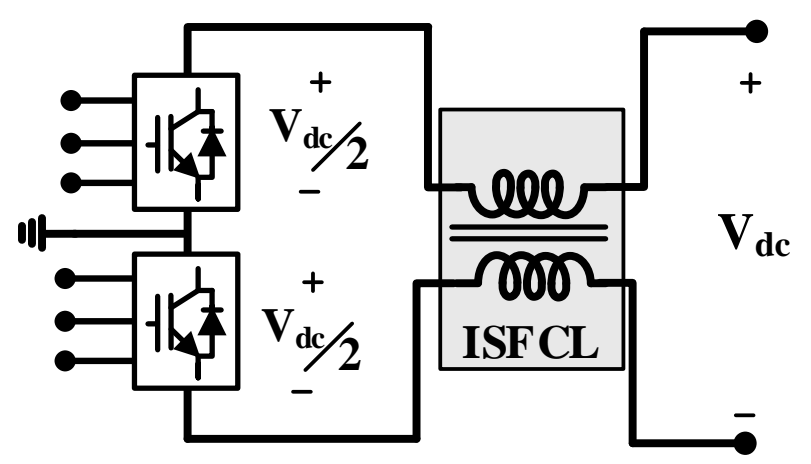

Figure 8.10: Passive ISFCL configuration.

In a DC system, an inductor can suppress the sudden increase of the fault current and it can effectively limit the changing rate of the current. However, the stored magnetic energy in an inductor in normal operations is very large and its transient voltage at the instance of the circuit breaking is considerably high. Thus, in [218], the authors have developed an ISFCL, as shown in Figure 8.10. In this configuration, the magnetic flux in the iron core of the inductor in normal operation is zero since the magnetic motive forces induced by the positive and the negative current cancel each other. On the other hand, in the case of a single pole-to-ground fault, the ISFCL suppresses the sudden increase of the short-circuit current as it generates a large counter voltage. Also, in the case of a pole-topole fault, the fault current is limited by the high, normal conducting resistance of the superconducting coil after the quench. 


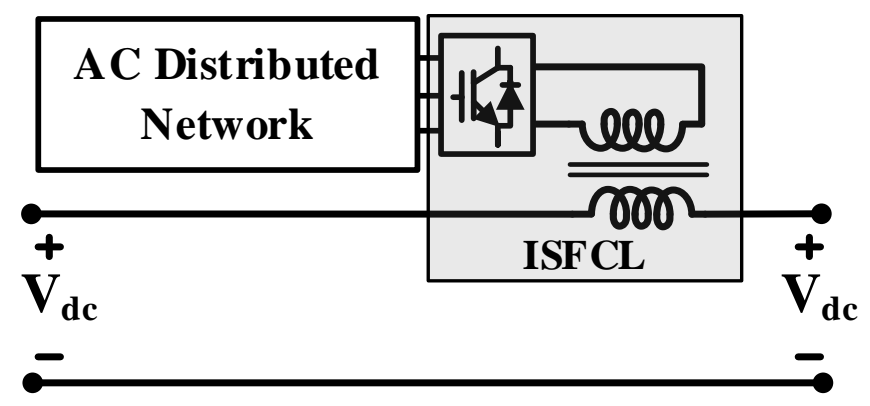

Figure 8.11: Active ISFCL configuration.

The circuit structure of an active ISFCL with the hybrid AC-DC power system is shown in Figure 8.11. The system is composed of two superconducting coils wound on an iron core. The first coil is connected with the AC power system through the PWM converter and the second coil is in series with the DC power system. During normal operation, the ISFCL presents very low power losses as a superconducting transformer’s primary leakage impendence, which has little influence on the DC system. In the case of a fault, the current of the DC system will rise rapidly, and the magnetic energy in the superconducting coil will increase. During this time, the increased energy is transferred to the AC system through the PWM converter to suppress the fault current of the DC system. In addition to its fault current limiting capability, an active ISFCL can act as a power filter for the AC distributed network through active and reactive power exchange between the DC and AC systems [219]-[220].

Since the SFCLs are not able to completely interrupt the fault current and usually cannot withstand high voltages, disconnectors have to be placed in series. Although the SFCLs with significant improvements in design were built and successfully tested, especially in the AC distribution network [221]-[222], they are still immature and their costs are very high. 
Table 8.2: Comparison of different circuit breaker technologies for DC power system applications.

\begin{tabular}{|l|c|c|c|c|c|}
\hline & MCB & SSCB & HHVCB & $\begin{array}{c}\text { Fault- } \\
\text { Tolerant } \\
\text { Converter }\end{array}$ & SFCL \\
\hline Cost & Low & Moderate & Moderate & High & $\begin{array}{c}\text { Very } \\
\text { High }\end{array}$ \\
\hline Operation Time & $\begin{array}{c}30-100 \\
\text { ms }\end{array}$ & $100-200 \mu \mathrm{s}$ & $\begin{array}{c}500 \mu \mathrm{s}-1 \\
\mathrm{~ms}\end{array}$ & $\begin{array}{c}100-200 \\
\mu \mathrm{s}\end{array}$ & $<2 \mathrm{~ms}$ \\
\hline Complexity & Low & Low & Moderate & High & Immature \\
\hline Power Loss & $\begin{array}{c}\text { p.001 } \\
\text { p.u. }\end{array}$ & $\begin{array}{c}0.001-0.02 \\
\text { p.u. }\end{array}$ & $\begin{array}{c}>0.001 \\
\text { p.u. }\end{array}$ & $\begin{array}{c}0.02-0.05 \\
\text { p.u. }\end{array}$ & $\begin{array}{c}>0.001 \\
\text { p.u. }\end{array}$ \\
\hline $\begin{array}{l}\text { High Speed } \\
\text { Protection System } \\
\text { Requirements }\end{array}$ & No & Yes & Yes & No & No \\
\hline $\begin{array}{l}\text { Inherent Galvanic } \\
\text { Isolation } \\
\text { Capability }\end{array}$ & Yes & No & No & No & No \\
\hline
\end{tabular}

In order to summarize the analysis presented in this section and better compare DC circuit breaker technologies, the characteristics and performances of various circuit breaker technologies are summarized in Table 8.2.

\subsection{Conclusion}

This chapter presented a comprehensive study on the design challenges for the protection of multi-terminal and distributed DC systems. The key requirements for the protection of these systems in terms of equipment survivability, personal safety, fault detection and location capabilities, system reconfigurability and ride-through capability 
were discussed. This study also presented a detailed fault analysis for multi-terminal DC lines and distributed system. The presented analysis provide essential guidelines for the proper design of the protective devices and relays and the accurate calculation of the fault current parameters such as the maximum fault current, its derivative and the time it takes to reach to the maximum value.

Various grounding topologies for DC power system were explained and their advantages and limitations for both monopole DC and bipolar DC systems were compared. It has been shown that the selection of the grounding topology not only effects the personal safety and insulation coordination of the system but also has significant impacts on the transient short circuit current behavior and the fault detection and protection capabilities of the system. Thus, through a comprehensive analysis, the grounding system should be designed based on its practical limitations and its impacts. More detailed studies need to be done on using advanced grounding schemes, using a mixed configuration or active components at the grounding locations to achieve a better performance while the cost and complexity of the system are minimized.

In this study, various circuit breaker technologies, as important tolls for fault isolation and system restoration, have been studied. The operation principle and configuration of these devices were explained and their performances have been compared in the terms of cost, operation time, complexity, power loss, protection speed requirements and galvanic isolation capabilities. Currently, much progress is being made in increasing the voltage capability of the silicon power semiconductors. Use of new wide bandgap power semiconductor devices, such as silicon-carbide ( $\mathrm{SiC}$ ) and gallium-nitride (GaN) and their future improvement are expected to highly increase the voltage and power rating of 
solid-state circuit breakers (SSCBs) and hybrid high voltage circuit breakers (HHVCBs). Furthermore, developing a new topology and optimizing the existing mechanical circuit breakers (MCBs) and HHVCBs are of interests as a future research subject with the goals to improve the power and voltage rating of the circuit breaker and reduce the interruption time and the cost of the system. 


\section{FAULT IDENTIFICATION AND LOCATION TECHNIQUES IN DC SYSTEM}

\subsection{Introduction}

In the last decade, multi-terminal and distributed DC power systems have been widely researched and developed, having numerous advantages over the existing AC network, such as higher energy efficiency, improved reliability, higher power quality, better energy systems integration and eco-friendliness [111]-[112]. High voltage DC (HVDC) transmission lines have been increasingly used to provide a cost-effective solution for transferring power over long distances with enhanced power flow controllability [156]. Also, multi-terminal HVDC grids offer a better integration of renewable energy resources like offshore wind farms and photovoltaic generations [157]. At lower voltage and power levels, medium voltage DC (MVDC) and low voltage DC (LVDC) distribution systems can be effectively utilized in various systems, such as electric traction systems, telecommunication and data centers, and for shipboard and aircraft systems [112], [156]. Moreover, DC distribution systems have the potential to better support future smart grids in realizing a high penetration of distributed renewables and electric vehicles [156]. In order to protect DC systems from short circuit faults, various protection schemes have been developed for different applications and voltage levels. The key design challenges of a fault detection and location scheme have been discussed in chapter 8. In this chapter, the operation principle and applications of various fault detection and location techniques suggested in the literature are presented and their advantages and limitations are discussed. 


\subsection{Differential Protection Scheme}

Differential protection is a common unit protection scheme, which operates by comparing the current magnitudes and the relative directions at the boundaries of a specified element within a network [223]-[228]. Figure 9.1 shows the configuration of this protection strategy for feeder protection. Assuming that $i_{\text {in }}$ and $i_{\text {out }}$ are the current at each end of the protected element, the difference of the two reading currents is:

$$
\Delta i=i_{\text {in }}-i_{\text {out }}
$$

When $\Delta i$ exceeds a predefined threshold, a fault inside the protected element is identified and then appropriate commands will be sent to the protective switches to separate the element from the network.

Since the differential protecting scheme monitors only the relative difference of the input and the output current of an element, it can detect the fault regardless of the fault impedance and the fault current amplitude, facilitating effective protection coordination in the network. However, technical and economic barriers exist in the deployment of the differential protection scheme in multi-terminal and distributed DC systems. In this protection scheme, current measurement sensors have to be installed in each of the protected element terminals and the system requires communication links between terminals. The main challenge in the implementation of the differential protection scheme is the requirement for fast and accurate data synchronization, especially under high di/dt conditions. Based on (9.2), for any fault external to a protected element, the $\Delta i$ should be almost zero. However, where the time delay between the $i_{\text {in }}$ and $i_{\text {out }}$ measurements equal to $\Delta t$ exist, a nonzero differential sum can result in relay mal-operation. The study in [228] has shown that a $10-\mu$ s time delay can cause $\Delta i$ calculation errors of up to $75 \%$ of the fault 
current when the fault occurs external to a protected feeder in a 400-V DC distributed system.

$$
\Delta i=i_{\text {in }}(t)-i_{\text {out }}(t-\Delta t)
$$

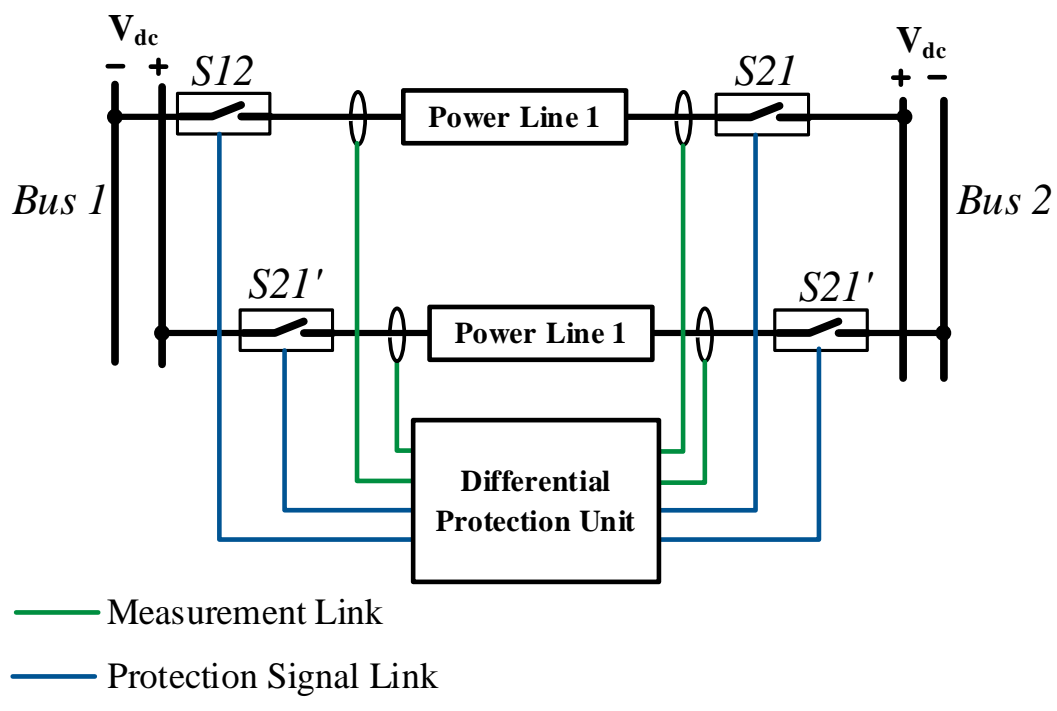

Figure 9.1: Differential protection scheme.

The second design challenge involved in the implementation of differential protection is the fault detection and isolation time in multi-terminal and DC distribution systems. The existing differential protection schemes implemented in AC systems require around 20 to $40 \mathrm{~ms}$ for proper operation, which is much higher than the operation time that is needed for DC system applications. It should be noted that the main factor that prevents the operating time reduction in an AC system is the need for individual current measurement and phasor comparison, which is around 20 ms for each cycle. However, this phasor current measurement is not the issue in DC systems, where only current magnitudes need to be compared. Moreover, as DC current is measured using a current transducer rather than via a current transformer (utilized in AC systems), the sensor output will be voltage, which facilitates easier integration with digital protection relays [228]. 
Assuming that the time it takes $\Delta i$ to reach a predefined threshold is $t_{\Delta i}$, the total operation time of a differential protection scheme toP , can be given as:

$$
t_{O P}=t_{\Delta i}+t_{C B}+t_{R}
$$

where $t_{C B}$ is the circuit breaker operation time and $t_{R}$ is the relay processing time.

In order to highlight the practical speed of this protection scheme, assume that a hybrid circuit breaker with $500 \mu$ s operation time is implemented in a distributed system. Also, the $t_{\Delta i}$ is $10 \mu$ s and the $t_{r}$ is $500 \mu$ s. Thus, the total operation time of this protection scheme will be around $1 \mathrm{~ms}$.

In addition to the necessity of accurate synchronization, the high number of measurement points and the requirement for the communication network increase the complexity and the cost of the system, and reduce its reliability due to communication and equipment failures. However, with the increasing implementation of distribution systems and smart grid concepts [229], further sensors and communication technology development are expected in the near future.

\subsection{Handshaking Protection Scheme}

The handshaking scheme is a protection method that can be utilized to detect and isolate various line-to-line faults and line-to-ground faults in a multiterminal DC system. In order to explain the principle of the handshaking scheme, assume a positive line to ground fault occurs on one of the lines of a DC bus in a multiterminal system. In this case, the current direction of the faulted line is always positive (going out from the bus to the fault point), while the current flows in the negative direction on the other healthy lines of the DC bus. For instance, for the multi-terminal DC system shown in Figure 8.1, if a fault 
F1 occurs, the protection equipment of bus 1 detects a positive fault current in line 1 while the current of line 3 is negative. Thus, the line with a positive fault current can potentially be the faulted line, whereas the line with a negative fault current is healthy.

In [166], Tang et al. developed and verified a handshaking method to locate and isolate the faults on a multi-terminal DC network. In the developed scheme, two important roles were defined for selection of the potential faulted line to disconnect its DC switches and for reclosing the DC switches of the healthy line to restore the system. The role of the potential faulted line selection in the case of a positive line to ground is that the protection unit of each bus should identify and disconnect the line carrying the largest positive DC fault current. This role ensures that the faulted line is selected if one of the lines of the protected DC bus has a fault. In the case that none of the lines of the DC bus has a fault, the line with maximum positive current can be different and the selected line doesn't matter. Once the potential faulted lines and their corresponding switches are selected, the power units of all DC buses are set off to shut down the grid. Afterward, the selected DC switches will be disconnected when the current of the line is decayed to a predefined threshold. The next step in the handshaking scheme is to identify the exact faulted line and keep its DC switches open while the other DC switches of the healthy line should be reconnected to restore the system. For this purpose, the power unit of all DC buses should be turned on and the rule for reclosing of the DC switches is that the potential faulted line is healthy and its switch can be reconnected if the DC line has been energized through the other end switch. Since the faulted line has been disconnected at both ends, it will remain disconnected and isolated from the grid and as a result, the system can restore properly. 


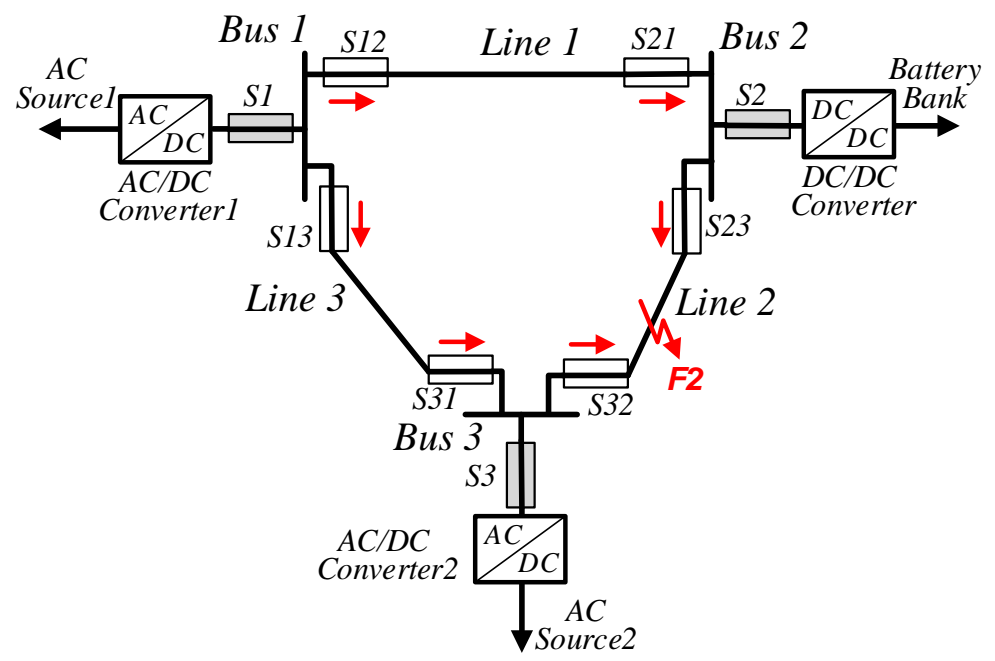

Figure 9.2: Illustration of the potential faulted line Selection in the handshaking method.

In presenting the handshaking protection scheme, consider the three bus multiterminal DC system shown in Figure 8.1. Also, assume a positive line to ground fault F2 has already been detected by a fault detection scheme, such as fault current amplitude or current derivative technique. The next step in the handshaking protection scheme is to identify the potential faulted line based on the role that is to select the line carrying the largest positive DC fault current. Figure 9.2 shows the direction of the fault current in the case of a positive line to ground fault F2. Based on the handshaking role, the protection unit of bus 2 selects line 2 as the potential faulted line and identifies switch S23 to open. For the protection unit located at bus 3 , the fault current through line 2 is positive and line 3 is negative. Thus, line 2 is identified as the potential faulted line and the switch S32 is selected for opening. Also, the protection unit of bus 1 detects positive current on line 1 and line 3. However, it does not matter which of the lines are selected as the potential faulted line. For instance, assume by applying the selection rule, the handshaking method identifies line 3 as the one through which the largest positive DC fault current flows and as a result switch S13 is selected for opening. 
The next step is to turn off the converter and de-energize the grid by opening the circuit breakers, S1, S2 and S3. Then, the selected DC switches S23, S32 and S13 should be disconnected. Figure 9.3 shows the configuration of the system after disconnecting the selected switches. As can be seen, the healthy lines have their fast DC switches closed at least at one end. This is due to the fact that the DC fault current enters one end of the nonfaulted line and exits at the other end. As a result, the healthy line will always be connected to one of the DC buses at one end through the fast DC switch which has not been selected for opening. On the contrary, the faulted line is isolated from the grid since its switches are open at both ends.

After disconnecting the selected DC switches and isolating the faulted line, all circuit breakers should be reconnected and the converters should be turned on to reenergize the DC buses. The next step is to reclose the DC switches of the un-faulted lines. Based on the handshaking scheme, a switch can be reconnected if the DC line has been energized from the other end power unit. For the given case study, because line 3 has been energized by AC/DC converter2 through bus 3, the S13 will be reconnected. In contrary, since line 2 was disconnected and de-energized from both ends, it remains disconnected form the system.

The handshaking scheme is a reliable method to locate and isolate a faulted DC line in multiterminal HVDC and MVDC systems. Moreover, since identifying the faulted line is accomplished based on local measurements without communication networks, the cost and complexity of the system can be considerably reduced. However, this method temporarily de-energizes the entire network, which can create unnecessary outages. As a result, this scheme may not be suitable for local distribution networks and LVDC 
microgrids where a large number of sources and loads are connected to the system [156], [225], [230].

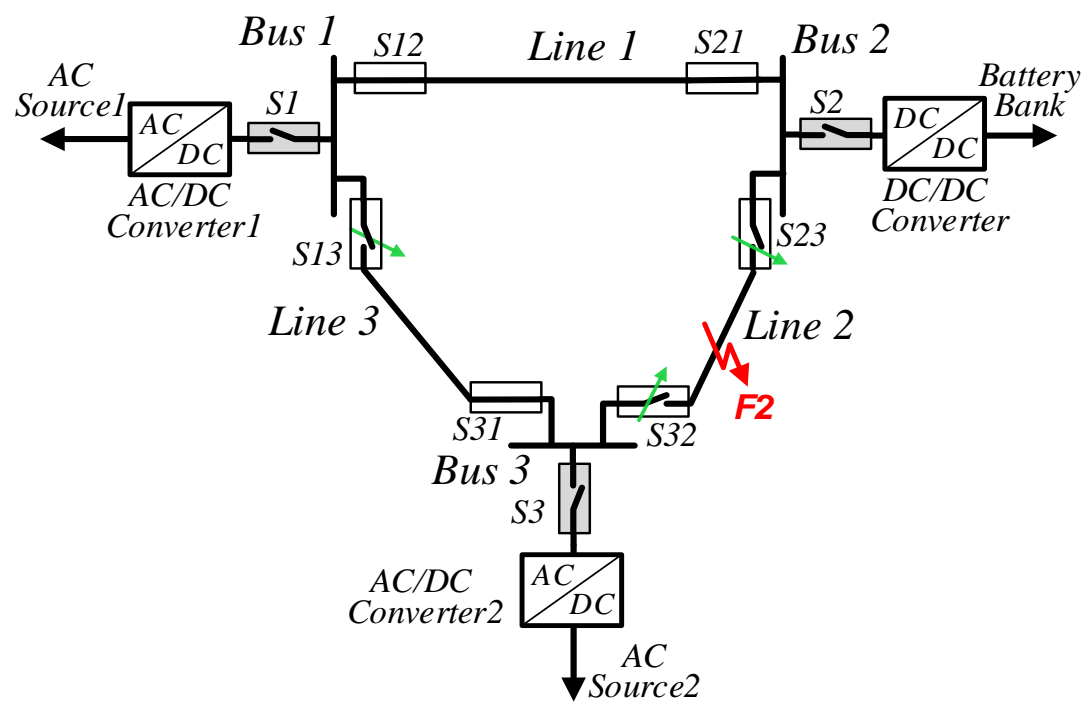

Figure 9.3: Illustration of the potential faulted line opening in the handshaking method.

\subsection{Traveling Wave Protection Techniques}

When a fault occurs in a HVDC transmission line, the voltage and current changes at the point of the fault generate electromagnetic impulse, which travels to both ends of the line. Traveling wave protection schemes utilize various signal processing techniques to identify the characteristics of the current and voltage waves reflected and received at the transmission line terminations. These protection schemes have already been applied for the protection of conventional two terminal HVDC systems [231]-[235], while various studies have been done for its application on the multiterminal HVDC systems[167], [236]-[238].

Based on the electromagnetic field theory, the current and voltage in a high voltage transmission system are related via the partial differential equations as follows:

$$
\frac{\partial E(x, t)}{\partial x}=-L_{0} \frac{\partial I(x, t)}{\partial t}
$$




$$
\begin{gathered}
\frac{\partial I(x, t)}{\partial x}=-C_{0} \frac{\partial E(x, t)}{\partial t} \\
\frac{\partial^{2} E(x, t)}{\partial x^{2}}=L_{0} C_{0} \frac{\partial^{2} E(x, t)}{\partial t^{2}} \\
\frac{\partial^{2} I(x, t)}{\partial x^{2}}=L_{0} C_{0} \frac{\partial^{2} I(x, t)}{\partial t^{2}}
\end{gathered}
$$

The general solutions to (9.6) and (9.7) are:

$$
\begin{gathered}
E(x, t)=E_{F}(x-v t)+E_{R}(x+v t) \\
I(x, t)=I_{F}(x-v t)-I_{R}(x+v t) \\
=\frac{1}{Z_{c}} E_{F}(x-v t)-\frac{1}{Z_{c}} E_{R}(x+v t)
\end{gathered}
$$

where $L_{0}$ is the inductance per unit length of the line, $C_{0}$ is the capacitance per unit length of the line, $E_{F}$ is the forward voltage wave and $I_{f}$ is the forward current wave, $E_{R}$ is backward voltage wave and $I_{R}$ is the backward current wave. Also, the $Z_{c}$ is the characteristic impedance of the line and it can be found as:

$$
Z_{c}=\sqrt{\frac{L_{0}}{C_{0}}}
$$

Also, the velocity of the traveling wave can be found as:

$$
v=\sqrt{\frac{1}{L_{0} C_{0}}}
$$




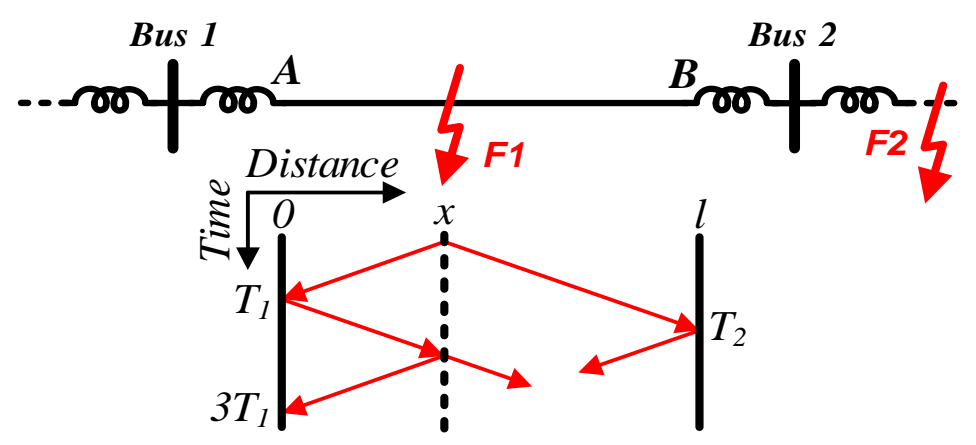

Figure 9.4: Lattice diagram corresponding to fault F1 to illustrate the travelling wave flow along the HVDC transmission line.

Figure 9.4 shows a schematic diagram of an HVDC transmission system and the fault wave propagating to terminal $A$ and $B$ where the protection relays are located. The traveling wave equation in the case of voltage at terminal $A$ can be found as [239]:

$$
\begin{aligned}
\Delta E_{A}(t)= & E_{F 1} A_{r}\left(1+\Gamma_{r}\right) u\left(t-T_{1}\right) \\
& +E_{F 1} A_{r}^{3} \Gamma_{r} \Gamma_{f}\left(1+\Gamma_{r}\right) u\left(t-3 T_{1}\right)+\ldots
\end{aligned}
$$

where $\Delta E_{\mathrm{A}}$ is the voltage change at terminal $\mathrm{A}, E_{F 1}$ is the initial voltage wave from the fault point, $\mathrm{u}(\mathrm{t})$ is the step function, $A_{r}$ is the terminal A side line attenuation coefficient and $\Gamma_{f}$ is the reflection coefficient of the fault point. Also $\Gamma_{r}$ is the terminal reflection coefficient and in the case of an inductive termination, it can be calculated as [239]:

$$
\Gamma_{r}=2 e^{-t /\left(L_{A} / Z_{C}\right)}-1
$$

in which $Z_{C}$ is the characteristic impedance of the line and $L_{A}$ is the inductor value.

It is well known that the reflection coefficient at the fault point can be expressed as:

$$
\Gamma_{f}=\frac{-Z_{c}}{2 R_{f}+Z_{c}}
$$

Also, the transmission (refraction) coefficient can be given by: 


$$
\beta_{f}=\frac{2 R_{f}}{2 R_{f}+Z_{c}}
$$

A ground fault generates backward and forward traveling voltage waves with a negative sign. Based on (9.12) and (9.13), an inductive termination can cause a steep enough voltage reflection and considerable voltage reduction, which can be utilized for inzone fault identification. However, the terminal voltage does not contain information on the fault direction. In contrast, the current derivative criterion can be applied to discriminate between forward and backward faults. A positive sign indicates a forward fault and a negative sign corresponds to a backward fault. Therefore, the overall in-zone fault criteria for the relay connected at terminal $A$ can be defined as [167], [236]:

$$
\left\{\begin{array}{c}
\frac{d E_{A}}{d t}>\sigma_{E} \\
\Delta E_{A}>\Delta_{E} \\
\frac{d i_{A}}{d t}>0
\end{array}\right.
$$

where the threshold $\Delta_{\mathrm{E}}$ discriminates between faults and normal operation and the threshold $\sigma_{\mathrm{E}}$ discriminates between fault F1 and F2.

In addition to the zonal protection capability, the traveling wave protection schemes are able to identify the accurate location of a fault based on the traveling wave propagation time. Depending on whether the measurements at the remote terminal of the transmission line are required or not, the fault location algorithms can be classified into two-end fault location schemes and one-end schemes. 
The two-end methods require detection of the first surge arrival time at the two transmission ends. The distance to the fault location from the terminal can be found by applying the traveling wave principle as [237], [239]:

$$
x_{f}=\frac{l-\left(T_{1}-T_{2}\right) v}{2}
$$

where $x_{F}$ is the distance of the fault point from terminal $A, l$ is the line distance, $T_{1}$ is the first surge arrival time at terminal $\mathrm{A}, T_{2}$ is the first surge arrival time at terminal $B$ and $v$ is the traveling wave propagation velocity.

In one-end traveling wave schemes, the fault location is estimated based on the arrival time of the first and the second reflected wave at the terminal and they do not require measurements from the other terminal. Based on (9.14) and (9.15) and since the fault is approximately a short circuit, the traveling wave reflection coefficient at the fault point is always negative and its transmitted coefficient is positive [240]. Also, based on (9.13), the reflection coefficient of an inductive termination is initially positive. Thus, a fault initially causes the terminal voltage to decrease due to the travelling wave from the fault point. If the second voltage change is positive, then it corresponds to the first reflected wave from the fault point and the fault location is less than half of the line length and the location can be found using (9.18). In the case that the second voltage change is negative, then the fault location is more than half of the line length and the voltage change corresponds to the fault wave reflected at the other termination and transmitted through the fault location. In this case, the fault distance can be calculated using (9.19) [239]-[240].

$$
x_{f_{-} I}=\frac{\left(t_{\Delta v 2}-T_{1}\right)}{2} v
$$




$$
x_{f_{-} I I}=l-\frac{\left(t_{\Delta v 2}-T_{1}\right)}{2} v
$$

in which $t_{\Delta v 2}$ is the time when the second voltage change due to traveling wave occurs.

In the case of HVDC line with a capacitive termination [239]-[240], a very steep change in the current traveling signal occurs when it reaches the terminal. Thus, the current signal can be employed to protect and locate the fault. Based on (9.9), the initial forward and backward current traveling waves have a positive and negative sign, respectively. Also, the reflection coefficient for the current traveling wave for the capacitive termination is positive since the capacitive termination initially looks like a short circuit [241]. Therefore, for a fault that occurs at less than half of the line length, the first current increase corresponds to the initial traveling wave from the fault point and the next current change is positive and it corresponds to the first reflected wave from the fault point. Thus, the fault location can be found using (9.18).

In the case that the second current change is negative, then the fault location is more than half of the line length and the current change corresponds to the fault wave reflected at the other termination that is transmitted through the fault point. Therefore, the fault location, $x_{f_{-} I I}$ for this case can be found based on (9.19).

Although the one-end schemes are superior as they do not require synchronized measurements and high bandwidth communication, the two-end methods are far more reliable if the measurement waves are properly synchronized using the global positioning system (GPS). Moreover, the accuracy of the one-end traveling based protection scheme can be highly degraded due to multiple and superimposed reflections and refractions of traveling waves [237]. In recent years, wavelets, as a powerful time-frequency signal 
processing tool have been suggested to identify the wave front arrival time especially in the presence of considerable measurement noise [241]-[242]. Nevertheless, there is a blind zone of protection for detecting close-up faults in both methods [232], [241]. Therefore, a hybrid algorithm of the traveling wave protection scheme and other techniques such as current derivative or impedance relay is necessary [232]-[233]. Moreover, the traveling wave based protection schemes require data acquisition units with very high sampling rate that increase its implementation difficulty.

\subsection{Distance Protection Techniques}

Distance protection schemes are based on the measurement of the short circuit loop impedance that is used to estimate the distance from the relay point to fault point. These methods are very common for AC system transmission line protections; however, the small value of the cable series impedance creates significant problems in using this method for DC systems. Moreover, during fault transients, the frequency of the DC system changes abruptly and no grid fundamental frequency impedance can be defined for distance protection. Furthermore, while the symmetrical component analysis is used in AC system protection to avoid the influence of fault resistance, this is not possible in DC systems [207], [243].

Some emerging techniques proposed the active distance evaluation scheme for DC systems protection. In this scheme, the impedance of the system and the fault distance are evaluated by injecting a current or voltage signal that has a known spectrum. The active distance relays are generally composed of probe units and signal processing algorithms. The probe unit can be a full bridge [180] or half bridge inverter [158] that generates voltage 
or current with arbitrary amplitude and frequency. It can also be a large capacitor that is connected to a power source via a controlled power switch to form a second-order RLC circuit through the fault path [244]. In these methods, the measured voltage and current are processed by advanced signal processing algorithms, such as fast Fourier transform (FFT) and least square curve fitting to calculate the impedance of the faulted system and estimate the distance from the relay point to fault point [158], [180], [244]. Active distance protection schemes generally do not require a complete shutdown of the microgrid rather, only the affected zone of the system is isolated and tested. For this purpose, the grid should be classified to several zones with overlapping nodes within the bus. Although the active distance protection schemes gives very detailed information about the state of the system, each node requires a probe system with a signal injection unit and high bandwidth measurements that can increase the cost and complexity of the protection system.

Some other research studies also suggested a time-impedance evaluation technique that is based on the time evolution of the apparent resistance, which does not require any signal injection units. The main principle of this technique is to estimate the impedance of the faulted system and wait for its corresponding time delay to realize selection. In order to calculate the impedance of the system, the voltage-current ratio can be calculated at the terminals of converters and contactors to determine the resistance of the cable between the device and the faulted point [245]. Alternatively, the fault distance can be evaluated by using two voltage dividers instead of a pair of voltage and current measurements [243]. This can reduce calculation problems of voltage by current division due to power switching noise and abrupt change of current during fault. The time-impedance evaluation technique requires much less computing capability than the active distance method and is faster for 
short circuit (or low impedance) fault protection. However, for high resistance faults, which are more common in ground faults, accurate distance evaluation is difficult. Usually, this kind of fault should be properly fulfilled by a backup protection such as the overcurrent protection [243].

\subsection{Artificial Intelligence Protection Techniques}

Artificial intelligence techniques, such as expert system, neural network and fuzzy logic have advanced significantly in recent years and have been widely utilized in various power system control design and protection systems [247]-[248]. Among various artificial intelligence techniques, artificial neural network (ANN) methodology shown to be better suited for DC power system protection since this technique is less complex and expensive in its development and has relatively fast calculation time and as a result is able to be utilized for real-time protections [249]-[252]. In these schemes, the transient fault current and voltage waveforms can be directly sampled as the input to neural network [249], or they can be initially processed to extract the signal features and then feed to the network [250]-[252]. Although the direct utilization of the sampled data found to be effective in fault detection and location, this approach requires a very long training process and calculation time and may also require a very complex structure for accurate operation.

For signal feature extraction in ANN-based protection schemes, the FFT method and the wavelet transform are among the frequency and time frequency signal processing techniques. However, the intrinsic drawback of the FFT method, that is the lack of information in the time domain, makes it improper for ANN-based protection methods which require signal processing technique with transients and short spikes identification 
capabilities [250]. On the contrary, the wavelet is not only able to decompose a signal into its frequency components, but can also provide a non-uniform division of the frequency domain, whereby it allows the decomposition of a signal into different levels of resolution. This feature enables the wavelet transform to be effectively employed in ANN-based protection methods to detect the abrupt-changing of the transient signals and identify the sharp increase of current during short circuit fault. In this protection method, the wavelet transform extracts a feature vector from the current waveform and feeds it to a selforganizing neural network. Then, the neural network determines whether the feature vector belongs to a normal or a fault current surge.

Generally, the wavelet transform technique can be divided in to continuous wavelet transform (CWT) and discrete wavelet transform (DWT). The CWT for a given signal x(t) can be defined as [250]:

$$
C W T(a, b)=\frac{1}{\sqrt{a}} \int_{-\infty}^{\infty} x(t) \psi\left(\frac{t-b}{a}\right) d t
$$

where $\psi(t)$ is the mother wavelet, $a$ is the scale factor and $b$ is the translation factor. The parameters $a, b$ and $t$ in CWT are continuous.

The DWT for a given signal $x$ also can defined as [250][250]:

$$
D W T(m, n)=\frac{1}{\sqrt{a_{0}^{m}}} \sum_{k} x(k) \psi\left(\frac{k-n b_{0} a_{0}^{m}}{a_{0}^{m}}\right)
$$

where $k$ is an integer variable and it refers to a sample number of the input signal and the parameters $n$ and $m$ are integer form of the parameters $a$ and $b$ in (9.20).

Since most of the protection relays are now performed using digital processors, the DWT are commonly utilized. The DWT process the fault signal at different frequency 
bands with different resolutions by decomposing the signal into a rough approximation and detail information. For this purpose, two sets of functions are utilized by DWT named Scaling functions and Wavelet functions which are associated with high pass filter (HPF) and low pass filters (LPF), respectively.

In [251] and [252], DWT have been applied to detect surges in DC third rail current waveform of DC transit systems. Also, the utilization of the WT-based multiresolution analysis (MRA) have been suggested in [250] to improve the speed of the training process.

The MRA methodology aims to represent the sophisticated signal, $x$ in terms of wavelet and scaling functions and the objective is to produce good frequency resolution and poor time resolution at low frequencies and good time resolution and poor frequency resolution at high frequencies. The philosophy of this idea is that most signals in practical applications have a low frequency components for long durations and high frequency components for short durations. Figure 9.5 shows the schematic diagram of WT-based MRA decomposition of a signal with three levels of decomposition. 


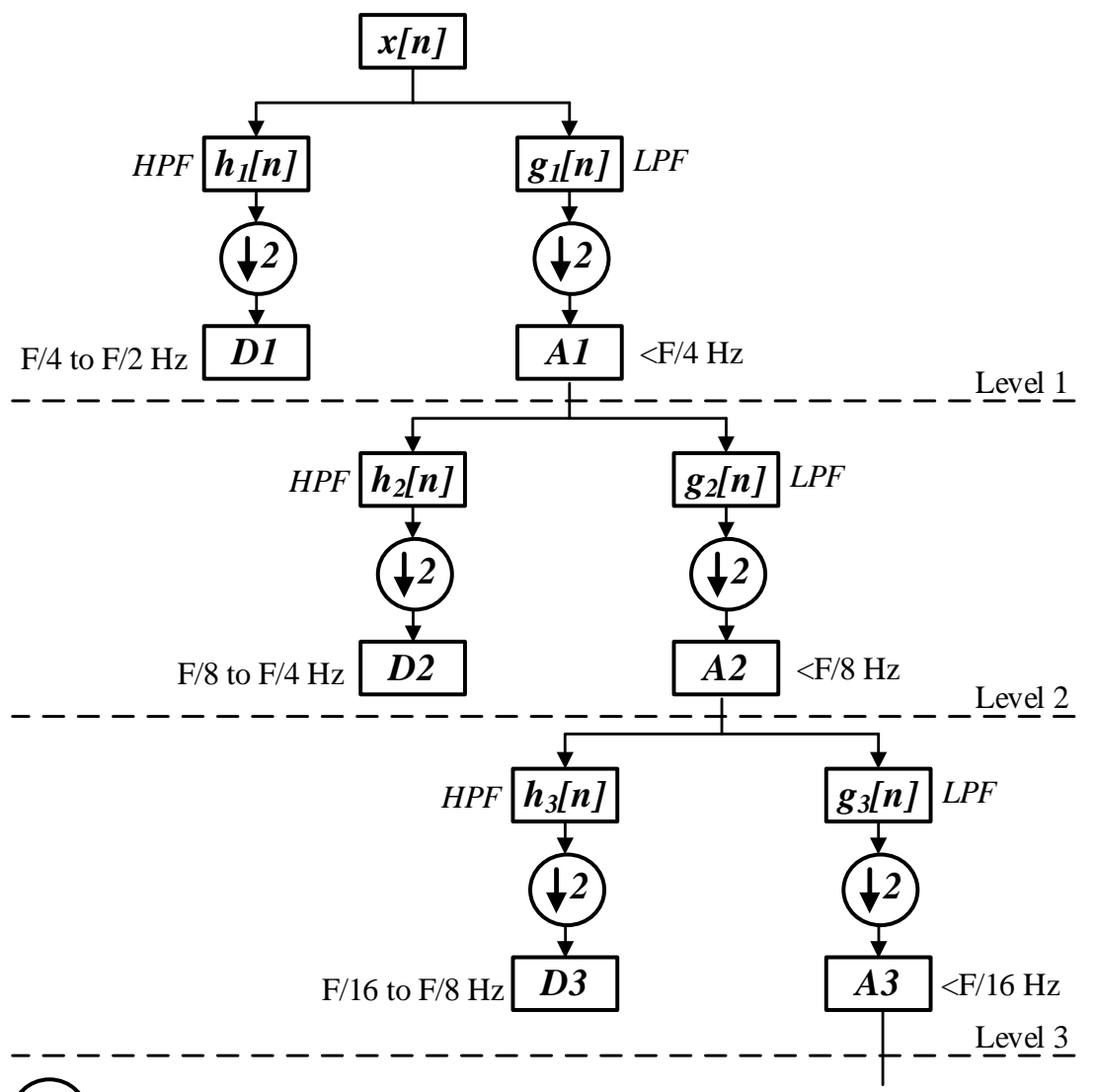

(72): Down Sampling by 2

$A_{i}$ : Approximation in Level $i$

$D_{i}$ : Details in Level $i$
HPF : High Pass Filter

LPF : Low Pass Filter

Figure 9.5: Schematic diagram WT-based MRA decomposition.

The scaling coefficients, known as approximation, are a low-resolution representation of the original signal that is the low frequency, high-scale components of the signal. This resolution is obtained by passing the original signal through a LPF. On the other hand, the wavelet coefficients, known as details, are the low-scale, high frequency components of the signal that is obtained by passing the original signal through a HPF. Shown $D 1$ in Figure 9.5 is the shortest mother wavelet and contains the highest frequency information present in the input signal, while the A1 is the approximation of the signal in the first level of decomposition. 


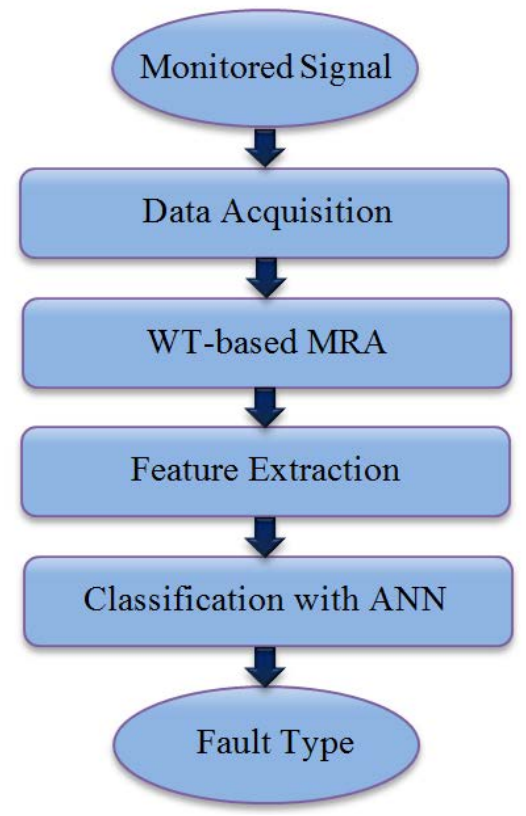

Figure 9.6: WT-based MRA protection flowchart.

Figure 9.6 shows the flowchart of the developed WT-based MRA technique in [250], for the protection of a MVDC naval shipboard power system. This technique is based on the signature analysis of the monitored signals that is performed with WT-based MRA methods. As Figure 9.6 shows, the monitored signal is initially sampled and then, the WT-based MRA techniques is applied for decomposing each signal into low frequency approximation and high frequency details. Accordingly, Parseval's theory is applied to extract the feature vectors of each sampled signal. In the next stage, the extracted feature vectors are utilized as the inputs of the neural network for classification. The output of the neural networks then indicates the fault types. The main shortcoming of the ANN-based protection methods is that the training of the neural network is a time consuming process and the trained network is only specific to the system considered. Currently, the design of network configurations is still a trial and error process and the design of the optimum network architecture involves a painstaking procedure. 


\subsection{Conclusion}

The key design challenges of a protection scheme include; speed, accuracy, reliability and simplicity. Various fault detection and location techniques have been developed for a variety of applications and system configurations. This chapter presented a comprehensive overview of fault detection and location techniques suggested in literature. The requirements and applications of these techniques were presented and their main advantages and limitations were discussed. The presented techniques in this chapter were differential protection, handshaking protection, traveling wave protection, distance protection and artificial intelligence protection. More progress is expected to develop new protection schemes using advanced signal processing methods and the existing protection techniques. However, the lack of standards, guidelines and practical experience are the main shortcomings of this research area. 


\section{DEVELOPMENT OF AN EVENT-BASED PROTECTION SCHEME FOR HYBRID DC POWER SYSTEM}

\subsection{Introduction}

In this section, we investigate a new scheme for a multi-terminal DC power system, which include hybrid energy resources and various loading schemes. In such a system, coordinated operation of circuit breakers (CBs) and disconnecting switches (DSs) can rapidly isolate short circuit faults and restore the system very fast. The proposed eventbased protection strategy transfers less data when compared with commonly used databased protection methods and does not require high speed communication and synchronization. Each protection unit is able to autonomously identify the type of event using the current derivative fault identification method and employing an artificial inductive line impedance (AILI). In order to accurately set the protection relays, detailed fault current analysis considering low pass RC filter effects are presented. The decision for fault isolation is made based on the unit judgment and the data received through high level data communication from the other interconnected units. The performance of the proposed protection scheme was evaluated under different DC feeder and bus faults. The results show that this scheme is able to accurately identify the type of fault, isolate the faulted area and restore the system quickly while limiting the load voltage drop to its preset limit.

\subsection{Proposed Approach for Protection of Hybrid DC Microgrid}

In this dissertation a new simple, yet efficient and reliable event-based protection technique for a multi-terminal DC power system is developed. When compared to databased protection methods, the event-based protection scheme transfers much less data and 
does not require high speed communication and synchronization. Moreover, the developed event-based protection scheme utilizes less measurement equipment since only the DC bus data is required while the traditional data-based protection methods require both the DC bus measurement and the feeder measurements.

In order to fully illustrate and evaluate the proposed protection scheme, a three-bus medium-voltage DC (MVDC) system and a four-bus hybrid DC power system are considered. Also, the virtual test-bed model of the four-bus hybrid DC power system explained in section 7 is utilized for evaluation of the protection scheme and the fault current study.

In order to accurately set the protection relays, the detailed fault current analysis is presented using analytical calculation and virtual test-bed model. Since the protection relays are microprocessor-based, the input signals to the digital relays can be highly contaminated with noises and high-frequency components as a consequence of using power converter and load drive switching [183], [252]. Moreover, the transient short circuit currents contain exponential and high-frequency damped oscillation components in which improper analog filtering can cause aliasing error and may also saturate the analog to digital converters (ADC) [254]-[255]. In this dissertation, the transient fault current and protection relay settings are evaluated while considering the effect of a low pass RC filter.

In the proposed protection scheme, each unit is able to autonomously classify the type of a fault into one of the three categories: (I) bus fault, (II) interconnected feeder fault and (III) adjacent feeder or adjacent bus fault. Since the DC bus and cable have low impedance, it is difficult to identify an interconnected feeder fault from a bus fault or an adjacent feeder fault. This study proposes an effective event identification technique using 
the current derivative method and employing an artificial inductive line impedance (AILI). The AILIs are small inductors, which are implemented in each feeder. The AILIs have a significant impact on the di/dt characteristic of fault current in transient condition that is utilized as a new technique for fault identification. The decision for fault isolation and grid restoration is made based on the unit judgment and the data that is received through high level data communication from the other interconnected units. The performance of the proposed event-based fault identification method is verified under different fault conditions using the hybrid DC microgrid simulation model.

\subsection{Developed Event-based Protection Scheme}

In presenting the event-based protection scheme, a small MVDC system consisting of three buses as illustrated using Figure 10.1 is considered. The pole-to-pole faults are the most problematic DC power system faults, which can be categorized as a bus fault and a feeder fault. These two possible faults have been indicated as F1-F6 in Figure 10.1. From the protection unit point of view, a fault can be classified to four groups as a bus fault, an interconnected feeder fault, an adjacent bus fault or an adjacent feeder fault. Considering the protection unit located at bus 1, fault F1 is a bus fault, while the faults F4 and F6 are an interconnected feeder fault, the faults F2 and F3 are an adjacent bus fault and the fault F5 is an adjacent feeder fault. 


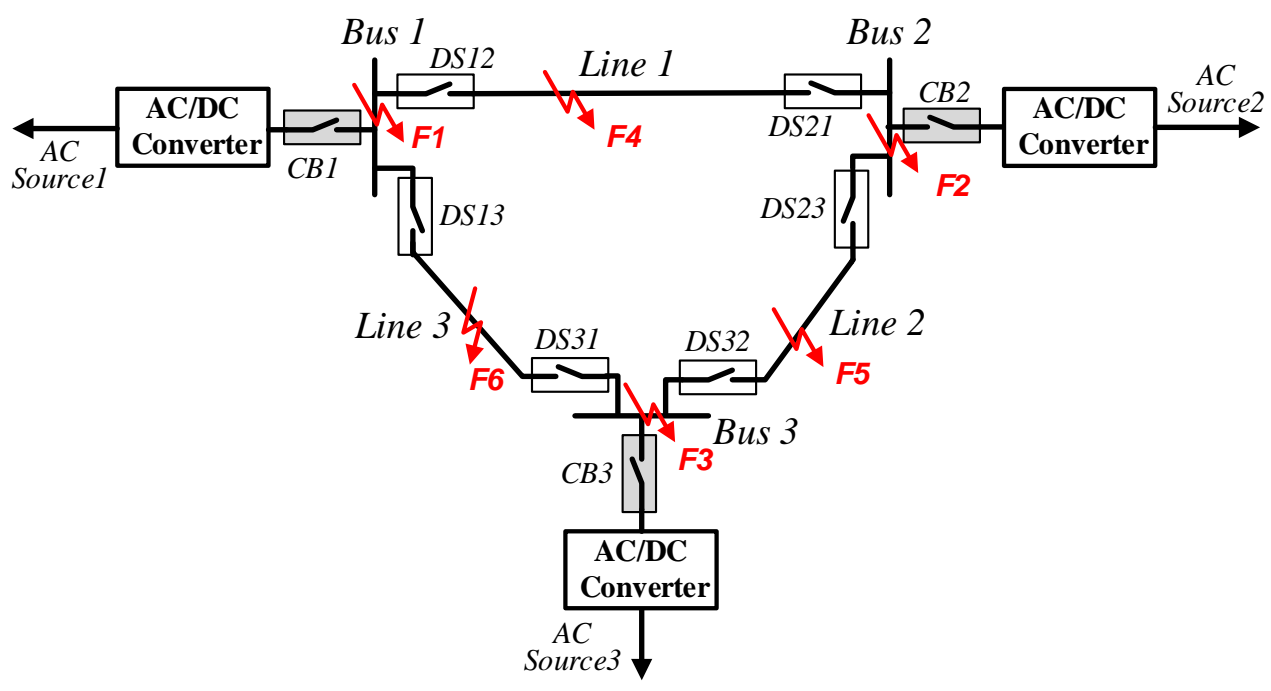

Figure 10.1: Schematic diagram of a three-bus MVDC.

As a starting point of the event-based protection scheme, it is assumed that a DC fault has already been detected and classified by fault detection schemes, which will be discussed in section 10.6. Following a short-circuit fault, a protection unit must go through a number of operation steps:

\subsubsection{Step1: De-energizing of the DC grid}

Following the fault identification and classification, the protection units should deenergize the MVDC system. This can be achieved by turning off the AC/DC converter switches. However, in the absence of the back-biasing voltage, the AC sources feed the MVDC system through the diode rectifier and as a result, the AC/DC converters are unable to de-energize the MVDC system [166]. In such a system, a circuit breaker (CB) should be implemented next to the AC/DC converter as shown in Figure 10.1. The required time to interrupt a fault current and completely isolate the faulted area through the CBs depends on the switching technology and operating speed of the contactor. 


\subsubsection{Step 2: High Level Data Communication}

Each protection unit needs to send and receive the type of event to and from the interconnected units. The communication protocol utilized in the proposed event-based protection scheme consists of high level data communication rather than the conventional protection schemes, which send and receive the actual measured voltage and current parameters. As a result, using high level data communication, the event protection scheme does not require high speed communication and synchronization.

\subsubsection{Step 3: Identification and Isolation of the Faulted Area}

The fault classification scheme in the event protection method is able to classify the type of event into three classes: (I) bus fault, (II) interconnected feeder fault and (III) adjacent feeder or adjacent bus fault. Figure 10.2 shows the strategy of identifying the potential faulted area. In the case of a bus fault, the protection unit will disconnect the CB and DSs of the load and the feeders connected to the corresponding bus. Then, it will send this event to other units. Also, the other protection units will disconnect the DS of the interconnected feeder to the faulted bus. For instance, in the case of fault F1, the protection unit 1 will disconnect the CB1 and DS12 and DS13. Also, the DS31 and DS21 will be disconnected by the protection units at bus 3 and bus 2 respectively.

In the case that an interconnected feeder fault occurs, all the protection units execute the event classification process and distribute the analyzed data. Then, the DSs of the faulted feeder will be disconnected by the protection units at its two ends. For instance, assume fault F4 occurred. The protection units in bus 1 and bus 2 will identify the fault as an interconnected feeder fault, while the protection unit at bus 3 will see an adjacent feeder fault. Following the distribution of the analyzed data, a fault in line 1 will be detected. 
Consequently, DS12 and DS21 will be disconnected by the protection units in bus 1 and bus 2, respectively.

If the protection unit identifies a fault as an adjacent feeder fault or an adjacent bus fault (third class), two cases arise. In the case that the protection unit receives a bus fault event from an adjacent bus, the unit should disconnect the corresponding feeder. Otherwise, if it receives an adjacent feeder fault, no action for fault isolation is required by that protection unit.

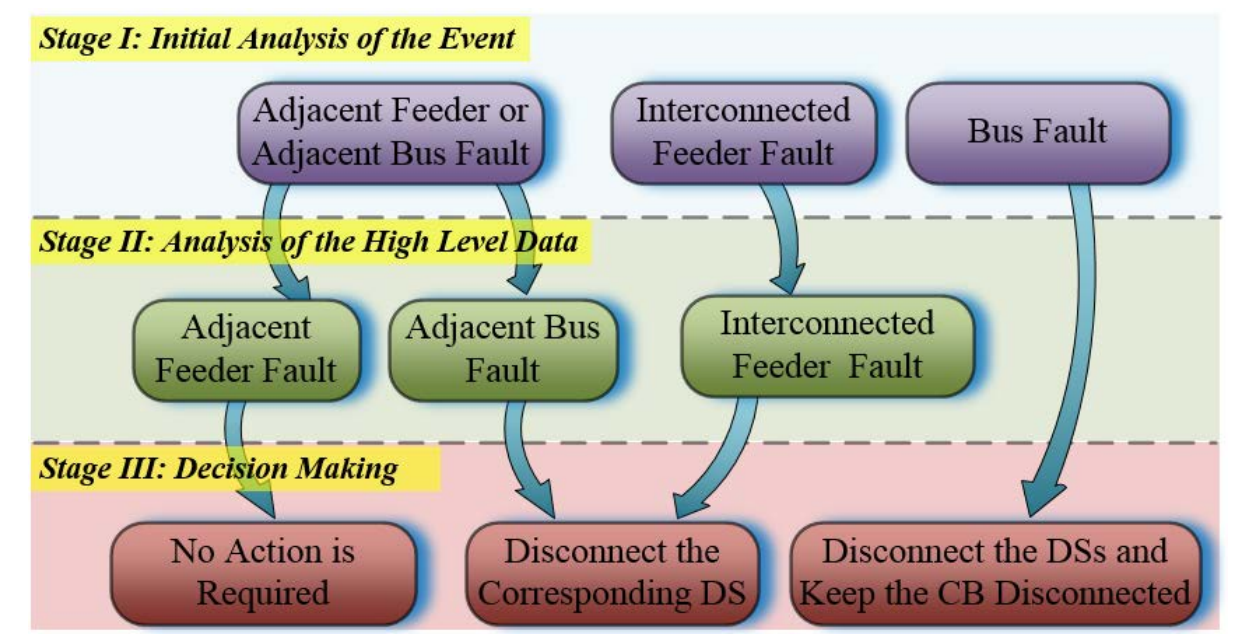

Figure 10.2: Event-based protection strategy.

\subsubsection{Step 4: Reenergizing the DC Grid}

Once the faulted area is identified and completely isolated, the grid should be reenergized. Initially, the CBs of the non-faulted buses will be turned on to recharge the DC capacitors by the 3-phase AC voltages through the diodes of the AC to DC converters. Then, the converter will be turned on to control the voltage of the DC bus and the power that flows to the DC grid. If a fault had occurred on a DC bus, the power (or current) reference of the converter will be set to a new operating point to compensate for the loss 
of the faulted bus and the power converter. Load shedding would be necessary if the power consumption of the grid is higher than the converter's capability.

\subsection{Hybrid DC microgrid Power System Design for Advanced Protection Scheme}

Figure 10.4 shows the configuration of the notional hybrid DC microgrid under study and its components. Figure 10.4 also shows some representative locations where faults could occur in the system at F1-F9. The circuit breakers (CBs) and disconnecting switches (DSs) are utilized to interrupt the fault current from the power units and to isolate the faulted area. In order to allow the load to ride-through the fault event uninterrupted, clamping diodes and a shunt hold-up capacitor are implemented. Figure 10.3 shows the configuration of the loads implemented in hybrid DC microgrid. Assuming that the nominal voltage of the grid is $E_{d c}$ and the minimum acceptable voltage of the load is $E_{\min }$ , the required hold-up capacitor to maintain the load voltage can be found using (10.1) [22].

$$
C_{H}=\frac{2 P_{L} t_{c l}}{\left(E_{d c}^{2}-E_{\min }^{2}\right)}
$$

where $t_{c l}$ is the fault clearing time and $P_{L}$ is the load power.

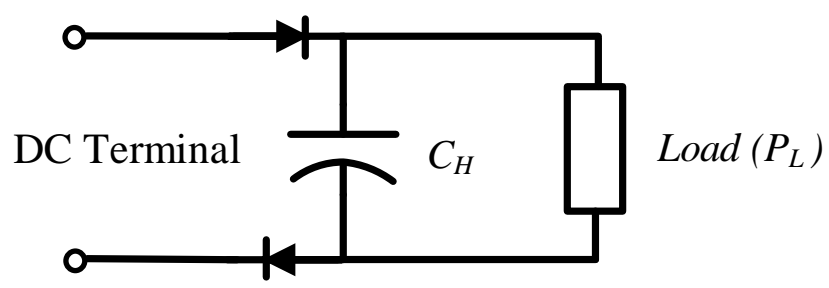

Figure 10.3: Load and its hold-up capacitor configuration. 


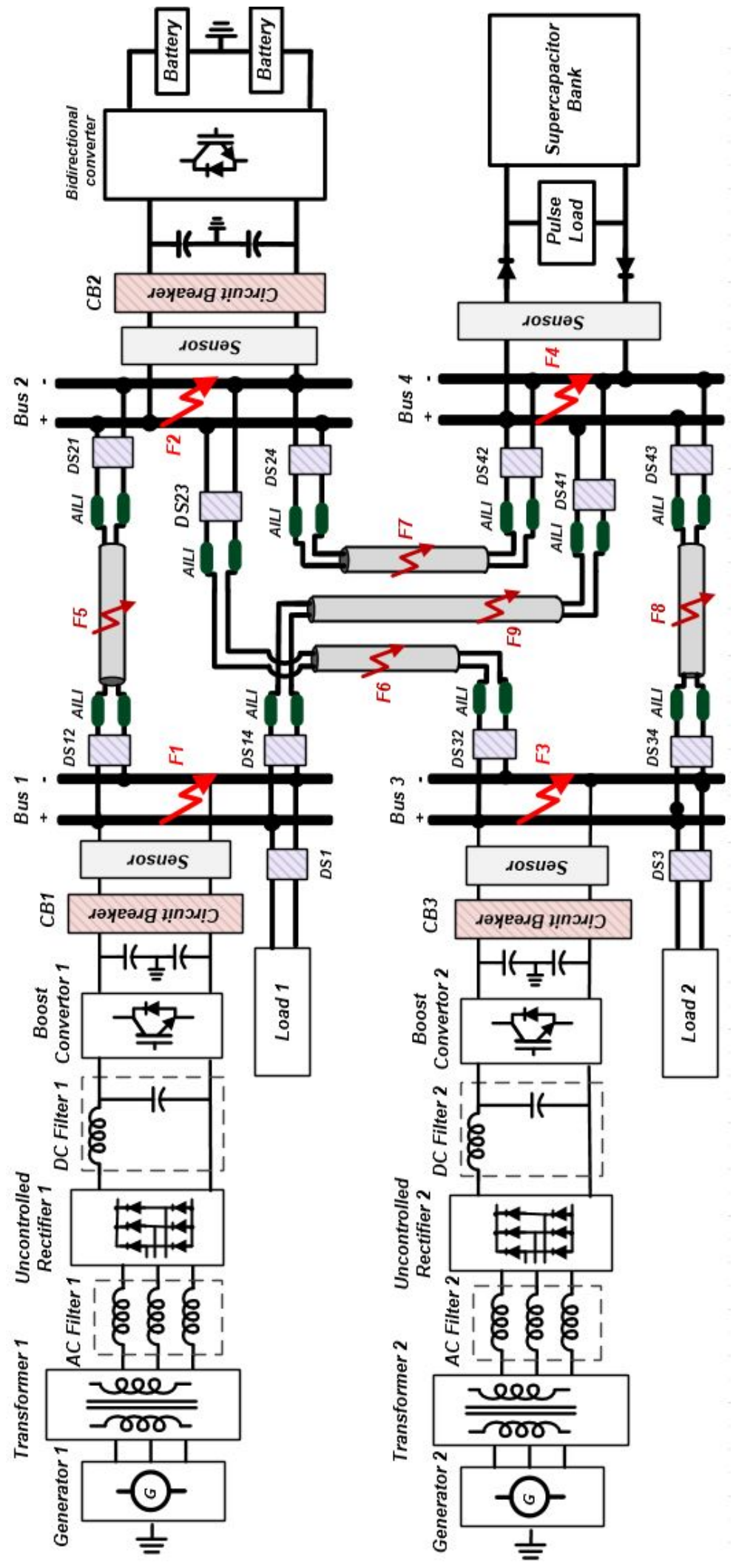

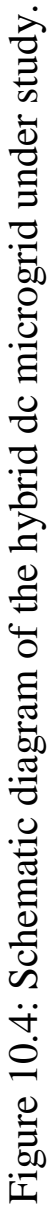


Based on the protection scheme presented in the following sections, the hold-up capacitors of each load are selected so that the load is able to ride through faults and has a limited voltage drop of $5 \%$ when the maximum $t_{c l}$ is $10 \mathrm{~ms}$.

\subsection{Short Circuit Current Calculations}

In order to evaluate our simulation model during transient fault conditions and accurately set the protection relays, the detailed analytical fault current analyses considering the effect of a low pass RC filter are presented in this section.

\subsubsection{Bus Faults}

The power sources are the major fault current contributor, but the capacitors at the DC bus terminal and DC filters also contribute to fault currents during the transient short circuit time. Figure 10.5 shows the equivalent circuit of the grid seen from the uncontrolled rectifier in the short circuit conditions. It should be noted that since the feed forward diode of the boost converter is biased during the short circuit condition, the converter is only modeled with its equivalent resistance and inductance. Applying the KCL law, the transient short circuit current of bus $1 i_{B 1}$, during fault $\mathrm{F} 1$ can be written as:

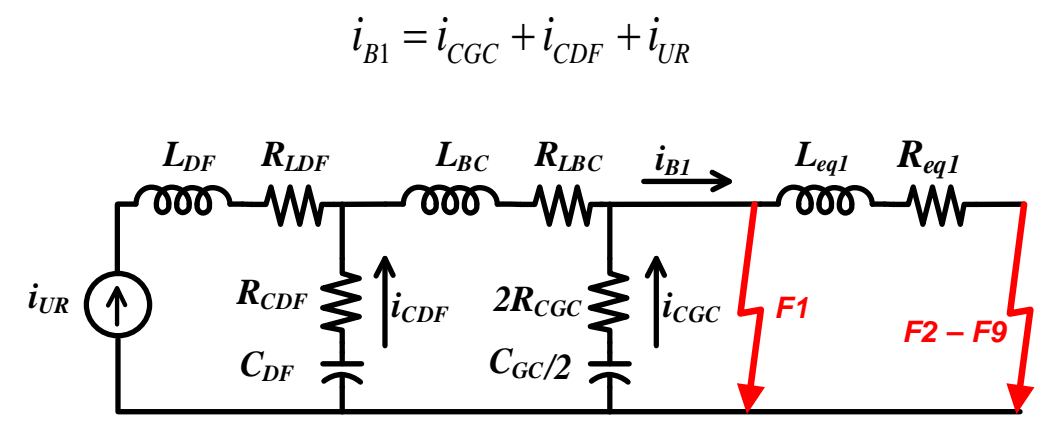

Figure 10.5: Equivalent circuit of the grid in the short circuit conditions seen from uncontrolled rectifier at bus 1 . 
where $i_{C G C}, i_{C D F}$ and $i_{U R}$ are the bus capacitor coupling current, the DC filter capacitor current and the uncontrolled rectifier current, respectively. Also, by applying KVL across the loop that includes the DC bus capacitor, it can be shown that the $i_{C G C}$ is related via:

$$
\frac{d i_{C G C}(t)}{d t}+\frac{1}{R_{C G C} C_{G C}} i_{C G C}(t)=0
$$

where $C_{G C}$ and $R_{C G C}$ are the capacitance of the DC bus capacitor and the resistance of the DC bus capacitor, respectively. Solving the differential equation (10.3) yields the transient $i_{C G C}$ as [256]:

$$
i_{C G C}(t)=\frac{E_{d c}}{2 R_{C G C}} e^{-t / \tau_{C G C}}
$$

where the time constant $\tau_{C G C}$ is given by:

$$
\tau_{C G C}=R_{C G C} C_{G C}
$$

For the sake of simplicity in the $i_{C D F}$ calculation, the voltage drop due to $i_{U R}$ on the $L_{B C}$ may be neglected. By applying KVL across the loop that includes the DC filter, it can be shown that the $i_{C D F}$ is related via a second-order differential equation as:

$$
\frac{d^{2} i_{C D F}(t)}{d t^{2}}+\left(\frac{R_{C D F}+R_{L B C}}{L_{B C}}\right) \frac{d i_{C D F}(t)}{d t}+\frac{1}{C_{D F} L_{B C}}=0
$$

where $L_{B C}$ and $C_{D F}$ are the boost converter inductance and the DC filter capacitance respectively. Also, $R_{C D F}$ and $R_{L B C}$ are the resistance of the DC filter capacitor and the resistance of the boost converter's inductor respectively.

In most practical applications, the resistance of the convertor's inductor $R_{L B C}$, and the DC filter capacitor resistance $R_{C D F}$, are relatively small, therefore the $C_{D F}\left(R_{C D F}+\right.$ 
$\left.R_{L B C}\right)^{2} / 4 L_{B C}$ is smaller than unity. Thus, the circuit is underdamped and based on differential equation (10.6), the $i_{C D F}$ can be expressed as [257]:

$$
i_{C D F}(t)=\left(A_{1} \cos \left(\omega_{D F} t\right)+A_{2} \sin \left(\omega_{D F} t\right)\right) e^{-\alpha_{D F} t}
$$

where $\omega_{D F}$ and $\alpha_{D F}$ are the decaying oscillation frequency and attenuation factor of $i_{C D F}$, respectively that can be calculated as:

$$
\begin{gathered}
\omega_{D F}=\sqrt{\frac{1}{L_{B C} C_{D F}}-\frac{\left(R_{C D F}+R_{L B C}\right)^{2}}{4 L_{B C}^{2}}} \\
\alpha_{D F}=\frac{R_{C D F}+R_{L B C}}{2 L_{B C}}
\end{gathered}
$$

Also, the $A_{1}$ and $A_{2}$ are the constants that are given by:

$$
\begin{gathered}
A_{1}=i_{L B C 0} \\
A_{2}=\left(\frac{E_{D C}-\left(R_{C D F}+R_{L B C}\right) i_{L B C 0}}{L_{B C}}+\alpha_{D F} i_{L B C 0}\right) \omega_{D F}
\end{gathered}
$$

where $i_{L B C O}$ is the boost converter input current before a fault occurs.

Assuming negligible voltage at the DC terminal of the uncontrolled rectifier during the transient fault, $i_{U R}$ can be evaluated by means of an equivalent star circuit modeling with six parallel branches. In this method, each of the branches can be modeled as a series voltage source $v_{j}$, equivalent resistor $R_{U R}$, equivalent inductance $L_{U R}$, and a diode. These are given by [175]:

$$
\begin{gathered}
v_{j}(t)=\sqrt{2} E_{A C} \operatorname{Cos}\left(\omega t+\varphi_{j}\right) \\
R_{U R}=R_{S}+R_{T R}+R_{A F}
\end{gathered}
$$




$$
L_{U R}=L_{S}+L_{T R}+L_{A F}
$$

where $E_{A C}$ is the RMS nominal AC phase voltage and $\varphi_{j}$ is the phase shift of the voltage $v_{j}$ with respect to a common reference. The Parameters $R_{S}, R_{T R}$ and $R_{A F}$ are the AC source equivalent resistance, the transformer windings resistance and the inductive AC filter resistance. Also, $L_{S}, L_{T R}$ and $L_{A F}$ are the AC source equivalent inductance, the transformer leakage inductance and AC filter inductance. The transient short circuit current of the $j$ th branch in the star equivalent circuit can be calculated as in equation (10.15) so that $i_{U R}$ is the envelope of the maximum values of $i_{B j}[175]$.

$$
i_{B j}(t)=I_{0}\left(\operatorname{Cos}\left(\omega t+\varphi_{j}-\theta_{U R}\right)-\operatorname{Cos}\left(\varphi_{j}-\theta_{U R}\right) e^{-t / t_{U R}}\right)
$$

where $I_{0}$ is the steady state short circuit current of the equivalent branches on a zero impedance fault and it can be calculated as:

$$
I_{0}=\frac{E_{A C}}{\sqrt{R_{U R}^{2}+\omega^{2} L_{U R}^{2}}}
$$

Also, $\tau_{U R}$ and $\theta_{U R}$ are the uncontrolled rectifier short circuit time constant and its impedance phase angle, which are given by (10.17) and (10.18).

$$
\begin{gathered}
\tau_{U R}=\frac{L_{U R}}{R_{U R}} \\
\theta_{U R}=\tan ^{-1}\left(\frac{\omega L_{U R}}{R_{U R}}\right)
\end{gathered}
$$

Figure 10.6 shows the equivalent circuit of the grid seen from the battery bank. In the case of a short circuit fault at bus 2 (F2), the transient current of this bus can be calculated as: 


$$
i_{B 2}=i_{C G C}+i_{B D}
$$

where the $i_{C G C}$ can be similarly found using (10.4) and the $i_{B D}$ is the bidirectional converter current. It should be noted that the internal voltage of the battery $U(q)$, is a function of charge drawn from the battery. However, during a short transient fault period, the change in the internal voltage is small and it can be assumed to be constant. Thus, application of KVL to the loop including the bidirectional converter impedance (Figure 10.6) gives the $i_{B D}$ as:

$$
\frac{d i_{B D}(t)}{d t}+\frac{R_{B a t t}+R_{L B D}}{L_{B D}} i_{B D}(t)-\frac{1}{L_{B D}} U(q)=0
$$

where $L_{B D}$ is the bidirectional converter inductance. Also, the parameters $R_{\text {batt }}$ and $R_{L B D}$ are the battery internal resistance and the resistance of the bidirectional converter's inductor respectively.

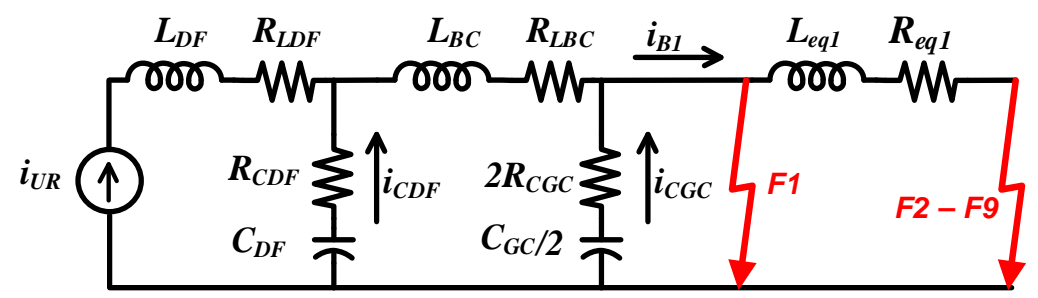

Figure 10.6: Equivalent circuit of the grid in the short circuit conditions seen from battery bank at bus 2 .

Solving the differential equation (10.20) yields the transient $i_{B D}$ as:

$$
i_{B D}=\frac{U(q)}{R_{\text {Batt }}+R_{L B D}}\left(1-e^{t / \tau_{B D}}\right)
$$

where $\tau_{\mathrm{BD}}$ is time constant of the bidirectional convertor, which is given by:

$$
\tau_{B D}=\frac{L_{B D}}{R_{\text {Batt }}+R_{L B D}}
$$




\subsubsection{Feeder Fault and Remote Bus Fault}

The transient short circuit current of bus 1 during the feeder faults F5 - F9 and the remote bus faults F2 - F4 can be given as an algebraic sum of $i_{C G C}, i_{C D F}$ and $i_{U R}$ (Figure 10.5). Since the pole-to-pole faults often have low fault impedance, the $L_{e q 1}$ and $R_{e q 1}$ can be neglected with respect to $L_{B C}$ and $R_{L B C}$, respectively. Thus, the $i_{C D F}$ and $i_{U R}$ can be found from (10.7) and (10.15). Also, application of KVL to the loop including the DC bus capacitor, the $L_{e q 1}$ and $R_{e q 1}$ (Figure 10.5), gives the following relation for $i_{C G C}$ during the feeder faults and the remote bus faults:

$$
\frac{d^{2} i_{C G C}(t)}{d t^{2}}+\left(\frac{2 R_{C G C}+R_{e q 1}}{L_{e q 1}}\right) \frac{d i_{C G C}(t)}{d t}+\frac{2}{C_{G C} L_{e q 1}}=0
$$

To solve the second-order differential equation (10.23), the damping factor $\zeta_{G C}$, is defined as (10.24). Depending on the $\zeta_{G C}$, the $i_{C G C}$ can be over-damped, critically damped or under damped. In the case that the $\zeta$ is greater than one, the $i_{C G C}$ is over-damped and it can be found as (10.25).

$$
\begin{gathered}
\zeta_{G C}=\left(\frac{2 R_{c g c}+R_{e q 1}}{2}\right) \times \sqrt{\frac{C_{G C}}{2 L_{e q 1}}} \\
i_{C G C}(t)=B_{1} e^{-\omega_{0}\left(\zeta-\sqrt{\zeta^{2}-1}\right) t}+B_{2} e^{-\omega_{0}\left(\zeta+\sqrt{\zeta^{2}-1}\right) t}
\end{gathered}
$$

where $\omega_{0}$ is the angular frequency, and $B_{1}$ and $B_{2}$ are the constants, which are given by following equations.

$$
\omega_{0}=\sqrt{\frac{2}{L_{e q 1} C_{G C}}}
$$




$$
\begin{gathered}
B_{1}=\frac{1}{2 \omega_{0} \sqrt{\zeta^{2}-1}}\left(\frac{E_{D C}-\left(2 R_{C G C}+R_{e q 1}\right) i_{C G C 0}}{L_{e q 1}}+\omega_{0} i_{C G C 0}\left(\zeta_{G C}+\sqrt{\zeta_{G C}^{2}-1}\right)\right) \\
B_{2}=i_{C G C 0}-B_{1}
\end{gathered}
$$

In the case that the $\zeta$ is one, the $i_{C G C}$ is critically damped and the fault current can be defined by the following expression based on the differential equation (10.23) [257]:

$$
i_{C G C}(t)=D_{1} e^{-\alpha C t}+D_{2} t e^{-\alpha C_{G C} t}
$$

where the attenuation factor $\alpha_{G C}$, and the constants D1 and D2 can be found as:

$$
\begin{gathered}
\alpha_{G C}=\frac{2 R_{C G C}+R_{e q 1}}{2 L_{e q 1}} \\
D_{1}=i_{C G C 0} \\
D_{2}=i_{C G C 0} \alpha_{G C}+\frac{E_{D C}-\left(2 R_{C G C}+R_{e q 1}\right)}{L_{e q 1}}
\end{gathered}
$$

The length of the cable strongly influences the fault current behavior. The linear increase of $L_{\text {eqi }}$ with cable length causes the $\zeta_{\mathrm{GC}}$ to decrease with cable length. In the case where $\zeta_{\mathrm{GC}}$ is smaller than unity, the $i_{C G C}$ is underdamped and the corresponding solution to (10.23) can be expressed as [257]:

$$
i_{C G C}(t)=\left(F_{1} \cos \left(\omega_{G C} t\right)+F_{2} \sin \left(\omega_{G C} t\right)\right) e^{-\alpha_{G C} t}
$$

where the decaying oscillation frequency $\omega_{G C}$, and the constants $F_{1}$ and $F_{2}$ can be found as:

$$
\begin{gathered}
\omega_{G C}=\omega_{0} \sqrt{1-\zeta_{G C}^{2}} \\
F_{1}=i_{C G C 0}
\end{gathered}
$$




$$
F_{2}=\left(\frac{E_{D C}-\left(2 R_{C G C}+R_{e q 1}\right) i_{C G C 0}}{L_{e q 1}}+\alpha_{G C} i_{C G C 0}\right) \omega_{G C}
$$

Similarly, in the case of the feeder faults and the remote bus faults, the short circuit current of bus 2 can be given as an algebraic sum of $i_{C G C}$ and $i_{B D}$ based on (19). However, depending on the $\zeta_{G C}$, the $i_{C G C}$ can be found from (10.25), (10.29) or (10.33) in the case of over-damped, critically damped or under damped condition, respectively.

\subsubsection{Low Pass Filter Effects}

Due to the converter switching and load drive system, the measured signals can be highly contaminated with noises and high-frequency components. Moreover, based on (10.2) and (10.19), the $i_{b i}$ contains excessive exponential components during bus faults, which can saturate the ADC. Therefore, the measured signals must be band limited by an analog low pass filter prior to sampling.

If an RC low pass filter is used, the 10 - 90\% rise time $t_{r}$ of the filter to a step input can be given by (10.37) [258]:

$$
t_{r}=\frac{0.35}{f_{c}}
$$

where $f_{c}$ is the cut off frequency of the filter.

In the case of a bus fault, since the time constant of the RC low pass filter is much smaller than the time constants of the $i_{C D F}$ and $i_{U R}$, it can be shown that the $i_{C D F}$ and $i_{U R}$ are unaffected by the filter while the measured $i_{C G C}$ can be calculated based on (10.4), as (10.38).

$$
i_{C G C}^{m}(t)=\frac{E_{d c} C_{G C}}{2\left(R_{C G C} C_{G C}-R_{f} C_{f}\right)}\left(e^{-\alpha_{G C} t}-e^{-\alpha_{f} t}\right)
$$


where $R_{f}$ and $C_{f}$ are the resistance and the capacitance of the RC low pass filter. Also, the $\tau_{C G C}$ is $1 / \alpha_{G C}$ using (10.5) and $\alpha_{f}$ is the attenuation factor of the RC low pass filter, which is given by:

$$
\alpha_{f}=\frac{1}{R_{f} C_{f}}
$$

Also, by taking the derivative from (10.38), the time in which the $i_{C G C}^{m}$ reaches its maximum $t_{i C G C}^{M a x}$, can be found as:

$$
t_{i C G C}^{M a x}=\frac{R_{f} C_{f} R_{C G C} C_{G C}}{R_{f} C_{f}-R_{C G C} C_{G C}} \ln \left(\frac{R_{f} C_{f}}{R_{C G C} C_{G C}}\right)
$$

Considering the low pass filter effect, the $i_{C G C}^{m}$ in the case of a feeder or remote bus fault and in underdamped conditions is given by (10.41) using (10.33). It should be noted that since the $\omega_{G C}$ is relatively low, only the filtering effect on the exponential component is considered. However, for the sake of simplicity and given that $t_{i C G C}^{\operatorname{Max}} \gg>1 / \alpha_{f}$, the $t_{i C G C}^{\operatorname{Max}}$ is calculated using the derivative of the $i_{C G C}$ based on (10.29) as (10.42). Thus, the maximum $i_{C G C}^{m}$ can be found by substituting (10.42) to (10.41).

$$
\begin{gathered}
i_{C G C}^{m}(t)=\frac{\alpha_{f}}{\alpha_{f}-\alpha_{G C}}\left[F_{1} \cos \left(\omega_{G C} t\right)+F_{2} \sin \left(\omega_{G C} t\right)\right]\left(e^{-\alpha_{G C} t}-e^{-\alpha_{f} t}\right) \\
t_{i_{C G C}}^{M a x} \cong \frac{1}{\omega_{G C}} \tan ^{-1}\left(\frac{F_{2} \omega_{G C}-F_{1} \alpha_{G C}}{F_{1} \omega_{G C}+F_{2} \alpha_{G C}}\right)
\end{gathered}
$$

In this dissertation, the $f_{c}$ of the filter is adjusted to $2 \mathrm{kHz}$ which leads to the signal rise time specification of $0.175 \mathrm{~ms}$. Also, the high frequency component of the 
measurements at the power converter, with a switching frequency of $5 \mathrm{kHz}$, will be attenuated by more than $8.5 \mathrm{db}$.

\subsection{Fault Event Classification Approach}

In this section, the proposed current derivative event classification method is explained in detail using the simulation results and analytical transient fault current calculations. Figure 10.7 shows the transient fault current $i_{b 1}$, and its components $i_{C G C}, i_{C D F}$

and $i_{U R}$ during fault F1. Using (10.38) and (10.40) the $t_{i C G C}^{M a x}$ and maximum $i_{C G C}^{m}$ are found to be 80.8 - $\mu$ s and 240.18-A, respectively which is consistent with the simulation results. Based on (10.7), the $i_{C D F}$ has exponentially damped oscillations at $494.2 \mathrm{rad} / \mathrm{s}$. Also, the initial current of $i_{U R}$ and its steady state fault current are nonzero, oscillating with low frequency.

As was mentioned earlier, each unit in the proposed event-based protection method should be able to distinguish between bus fault, interconnected feeder fault and adjacent bus or feeder fault. Since the DC bus and cable have a low impedance with respect to the converter impedance, using the sub-transient fault current is the most effective way for fault identification. Figure 10.8 shows sub-transient fault current $i_{b 1}$, during bus fault F1 and feeder fault F5 when a fault occurs $5 \mathrm{~m}$ from bus 1 . As can be seen, it is difficult to identify the close interconnected feeder fault from the bus fault. Also, Figure 10.8 shows the fault F5 when it occurs $5 \mathrm{~m}$ from bus 2 and fault F7 when it occurs $5 \mathrm{~m}$ from bus 2 . Due to small impedance differences, the far interconnected feeder fault and the close adjacent feeder fault have very similar characteristics. As a result, even with a more 
advanced fault identification method, such as the current derivate, it is difficult to differentiate them from each other.

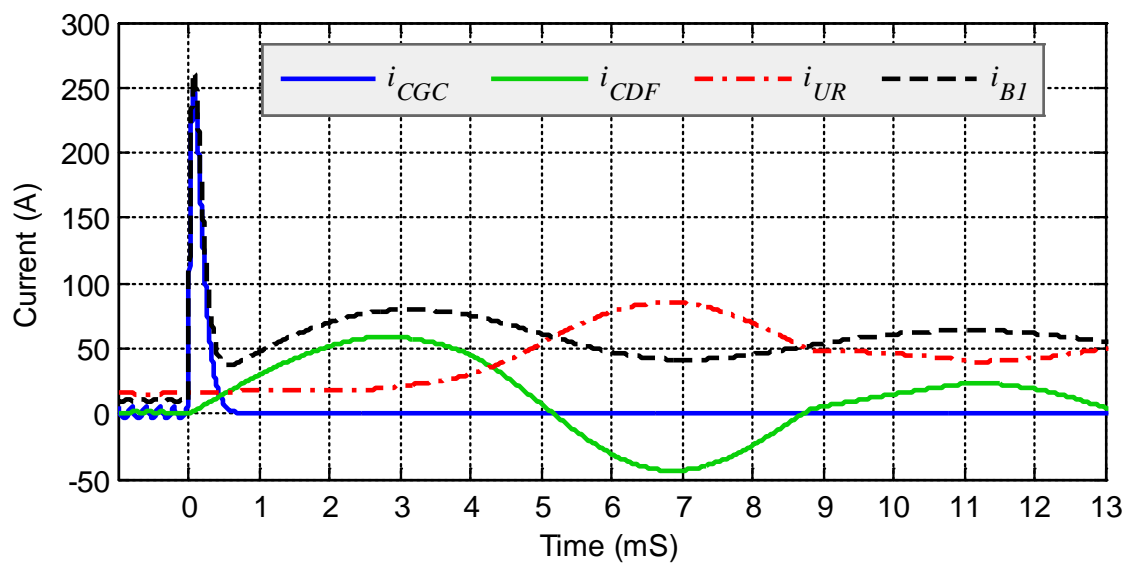

Figure 10.7: Simulation result during fault $\mathrm{F} 1$, showing $i_{C G C}, i_{C D F}$ and $i_{U R}$ contributions to the $i_{b 1}$.

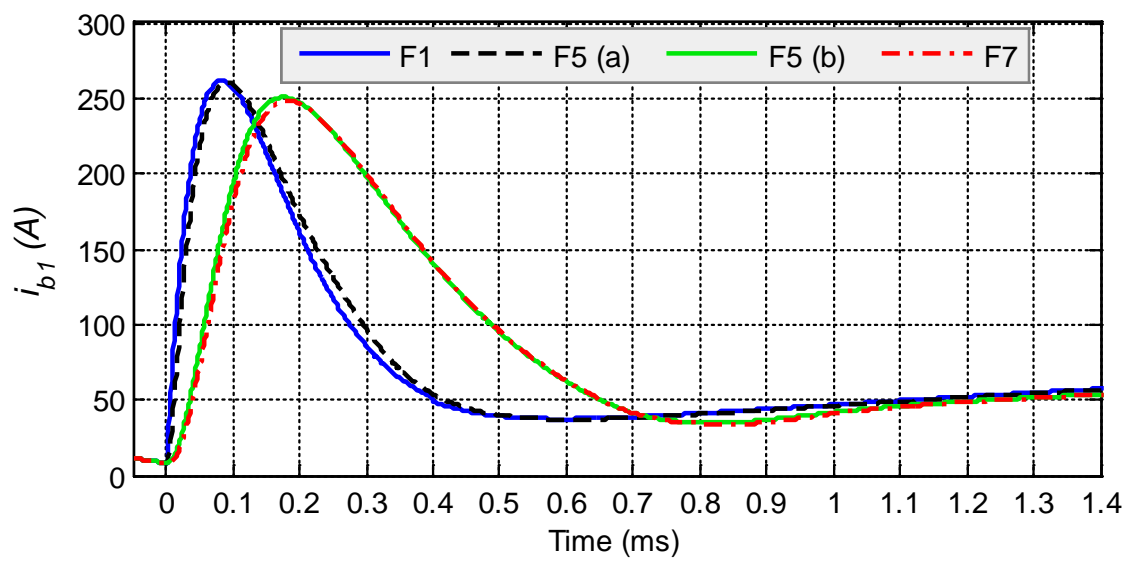

Figure 10.8: Sub-transient short circuit current $i_{b 1}$ during faults F1, F5 (a) when occurs 5 $\mathrm{m}$ from bus 1 , F5 (b) when occurs $5 \mathrm{~m}$ from bus 2 , and fault $\mathrm{F} 7$ when occurs $5 \mathrm{~m}$ from bus 2.

\subsubsection{Artificial Inductive Line Impedance Technique}

In the proposed protection scheme, small inductors as AILIs are implemented next to the DSs of each feeder (Figure 10.4). Since the $d i / d t$ in steady state operation of the DC power grid is low, the AILIs do not create considerable voltage drop in normal operation. However, in transient conditions, the AILIs have a significant impact on $d i / d t$ 
characteristics of fault currents, which can be utilized for fault identification. In fact, the AILIs are utilized to artificially add inductive impedance to the lines so that there will be enough di/dt margins for the protection unit to successfully classify a fault event. Figure 10.9 shows the sub-transient fault current $i_{b 1}$, during bus fault F1 and close feeder fault F5 occurring $5 \mathrm{~m}$ from bus 1 when three different AILIs were employed. The parameters of each AILI are listed in Table 10.1.

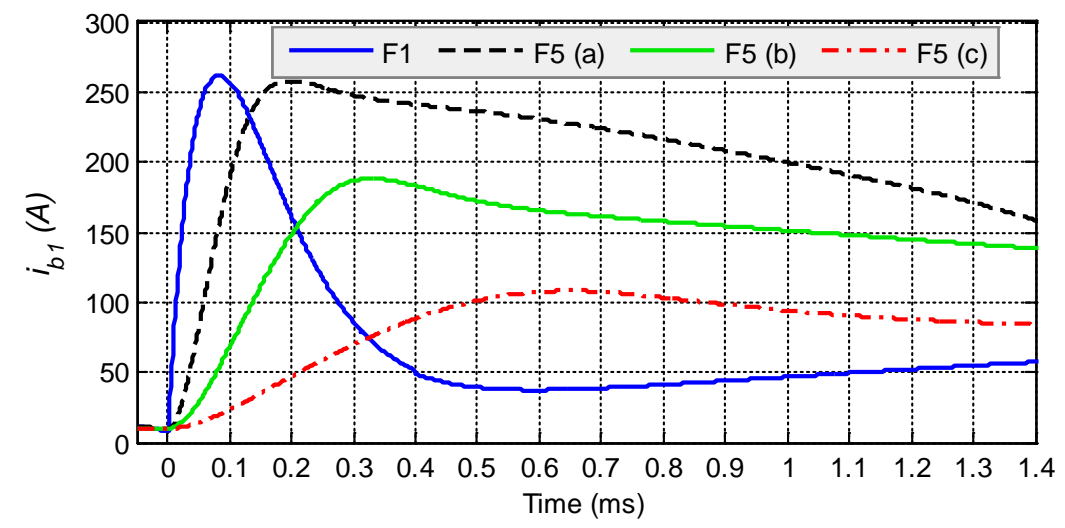

Figure 10.9: Sub-transient short circuit current $i_{b 1}$ during faults F1, F5 (a) when AILI = 0.02-mH were employed, F5 (b) when AILI $=0.1-\mathrm{mH}$ were employed and F5 (c) when AILI $=0.5-\mathrm{mH}$ were employed.

Table 10.1: Artificial inductive line impedance parameters

\begin{tabular}{|c|c|c|}
\hline Component & $\mathrm{R}(\Omega)$ & $\mathrm{L}(\mathrm{mH})$ \\
\hline AILI-1 & 0.0037 & 0.02 \\
\hline AILI-2 & 0.0195 & 0.1 \\
\hline AILI-3 & 0.112 & 0.5 \\
\hline
\end{tabular}

As can be seen in Figure 10.9, the $0.02 \mathrm{mH}$ AILI only decreased the slope of the sub-transient fault current while the $0.1 \mathrm{mH}$ and $0.5 \mathrm{mH}$ AILIs reduced the maximum value of the fault current and also its slope. However, it should be noted that the larger the AILI, the greater power losses will be in the AILI and it can also decrease the stability region of 
the system in normal operation. In this study, the $0.1 \mathrm{mH}$ AILI is implemented, which results in less than $0.25 \%$ reduction of the system efficiency at full load operation.

\subsubsection{Fault Current Derivative Method}

When a fault occurs, depending on its location, the derivatives of the current will change significantly. Using (10.38), the derivative of the fault current in the case of a bus fault can be found as (10.43). Also, the maximum derivative of the bus fault current occurs at the fault incidence and its magnitude is given by (10.44). This can be applied to set the minimum level of the fault current derivative for bus fault identification.

$$
\begin{gathered}
\frac{d i_{C G C}^{m}(t)}{d t}=\frac{E_{d c} C_{G C}}{2\left(R_{C G C} C_{G C}-R_{f} C_{f}\right)}\left(\frac{1}{R_{f} C_{f}} e^{-\alpha_{f} t}-\frac{1}{R_{C G C} C_{G C}} e^{-\alpha_{G C} t}\right) \\
\left(\frac{d i_{C G C}^{m}(t)}{d t}\right)^{M a x}=\frac{E_{d c} C_{G C}}{2 R_{C G C} C_{G C} R_{f} C_{f}}
\end{gathered}
$$

Also, in the case of a feeder fault or remote bus fault, the derivative of the fault current can be calculated as in (10.45) based on (10.41). Assuming that the variations of the sine wave component during initial fault current are negligible, the time in which the maximum of the fault current derivative occurs $t_{d i_{C G C}}^{M a x}$, can be approximated as (10.46).

$$
\begin{aligned}
\frac{d i_{C G C}^{m}(t)}{d t}=\frac{\alpha_{f}}{\alpha_{f}-\alpha_{G C}}\{ & {\left[\left(F_{2} \omega_{G C}-F_{1} \alpha_{G C}\right) \cos \left(\omega_{G C} t\right)\right.} \\
& \left.-\left(F_{1} \omega_{G C}+F_{2} \alpha_{G C}\right) \sin \left(\omega_{G C} t\right)\right] e^{-\alpha_{G C} t} \\
+ & {\left[\left(F_{1} \alpha_{f}-F_{2} \omega_{G C}\right) \cos \left(\omega_{G C} t\right)+\right.} \\
+ & \left.\left.\left(F_{1} \omega_{G C}+F_{2} \alpha_{f}\right) \sin \left(\omega_{G C} t\right)\right] e^{-\alpha_{f} t}\right\} \\
t_{d i_{C G C}}^{M a x} \cong \frac{1}{\alpha_{f}-\alpha_{G C}} & \ln \left(\frac{F_{1} \alpha_{f}^{2}-2 F_{2} \omega_{G C} \alpha_{f}-F_{1} \omega_{G C}^{2}}{F_{1} \alpha_{G C}^{2}-2 F_{2} \omega_{G C} \alpha_{G C}-F_{1} \omega_{G C}^{2}}\right)
\end{aligned}
$$


Figure 10.10 shows the derivative of the fault current (previously shown in Figure 10.9) during the bus fault and the close feeder fault when the AILI was $0.1 \mathrm{mH}$. Using (10.44), the maximum value of the fault current derivative in the case of bus fault was 8074 $A / m s$. Also, based on (10.46) and (10.45), the $t_{d^{\prime} i_{C C}}^{M a x}$ and maximum of the fault current derivative during a close feeder fault were $0.157 \mathrm{~ms}$ and $813 \mathrm{~A} / \mathrm{ms}$, respectively. This is consistent with the simulation results.

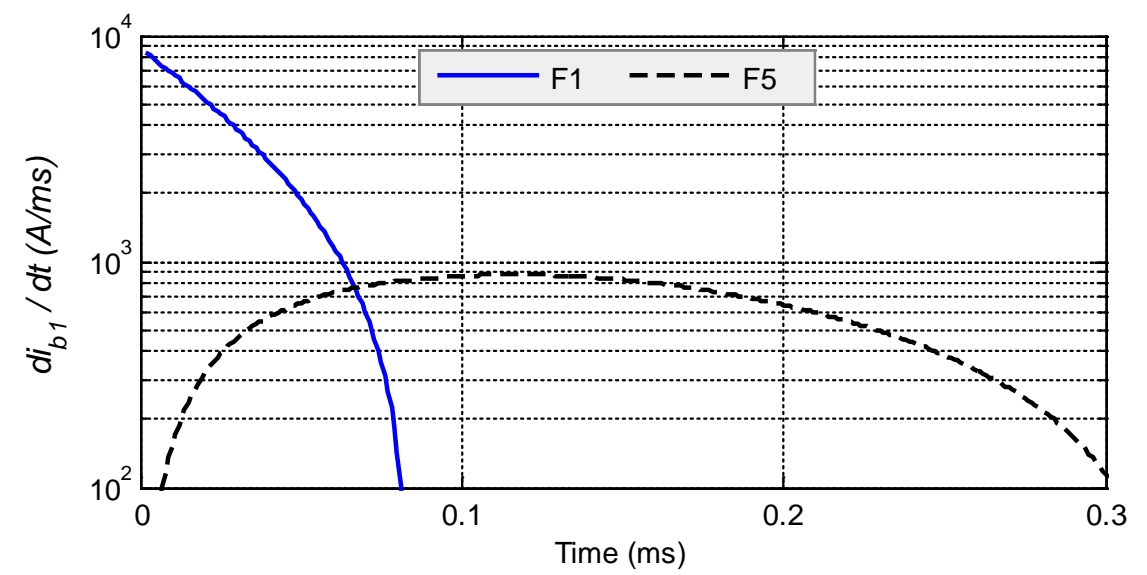

Figure 10.10: Derivative of short circuit current $i_{b 1}$ during fault F1 and fault F5 $5 \mathrm{~m}$ from bus 1 with AILI $=0.1 \mathrm{mH}$.

Compared with the current magnitude method, the current derivative method not only has better resolution for fault identification, but also it is much faster. As a result, the fault current can be interrupted at a lower level before it reaches its maximum value.

The protection of each power unit should be able to distinguish between the interconnected feeder fault and adjacent feeder fault. However, the multi-path characteristic of the hybrid DC microgrid reduces the adjacent feeder fault impedance and its value will be minimum if the fault occurs in the middle of the feeder. Figure 10.11 shows the fault current $i_{b 1}$ during far interconnected feeder faults F5 and F9 and also during adjacent feeder faults F6, F7 and F8 when it occurs in the middle of the corresponding 
feeders. As can be seen, even with the implementation of AILI, it is difficult to identify the interconnected feeder fault and adjacent feeder fault using fault current amplitude. However, when employing the derivative fault identification method (Figure 10.12), the adjacent feeder faults can be recognized as they have a lower peak with respect to the interconnected feeder fault.

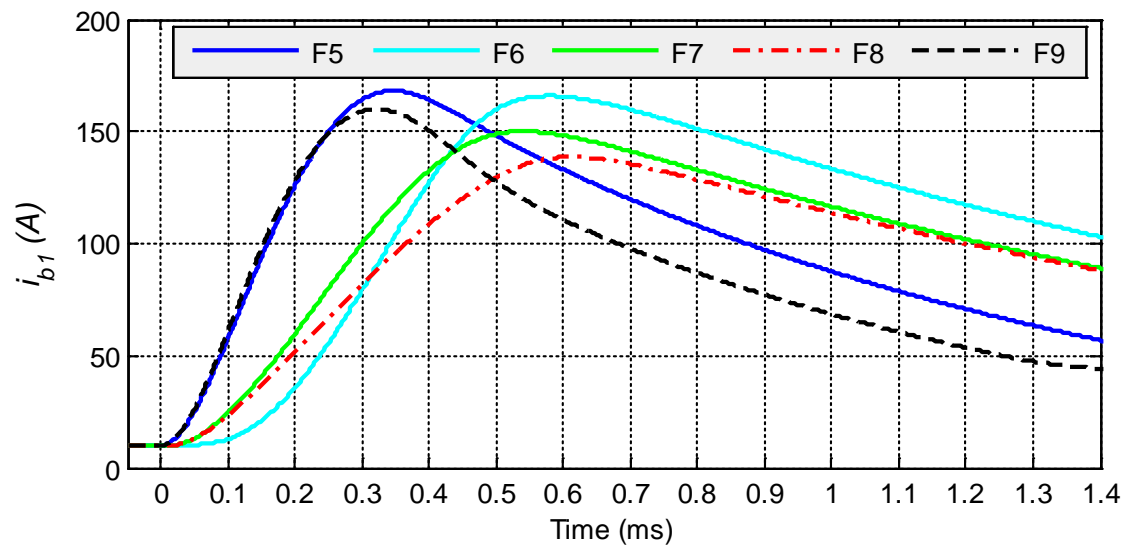

Figure 10.11: Sub-transient short circuit current $i_{b 1}$ with AILI $=0.1 \mathrm{mH}$ during faults F5 5 $\mathrm{m}$ from bus 2, F6 middle of the feeder, F7 middle of the feeder, F8 middle of the feeder and F9 $5 \mathrm{~m}$ from bus 4 .

In order to accurately set the protection relay, the minimum $d t_{i C G C}^{M}(t) / d t$ in the case of the interconnected feeder fault and maximum $d t_{i C G C}^{M}(t) / d t$ in the case of the adjacent feeder fault should be calculated. In the case of bus 1 , using the cable and AILI parameters, the maximum of $L_{e q 1}$ and $R_{e q 1}$ are $0.219 \mathrm{mH}$ and $0.1633 \Omega$, respectively. This gives rise to a $788 \mathrm{~A} / \mathrm{ms}$ maximum derivative fault current based on (10.46) and (10.45). Similarly, the minimum of the $L_{\text {eq } 1}$ and $R_{\text {eq } 1}$ in the case of an adjacent feeder fault are calculated as 0.413 $\mathrm{mH}$ and $0.202 \Omega$, respectively. This resulted in $528 \mathrm{~A} / \mathrm{ms}$ maximum derivative fault current. 


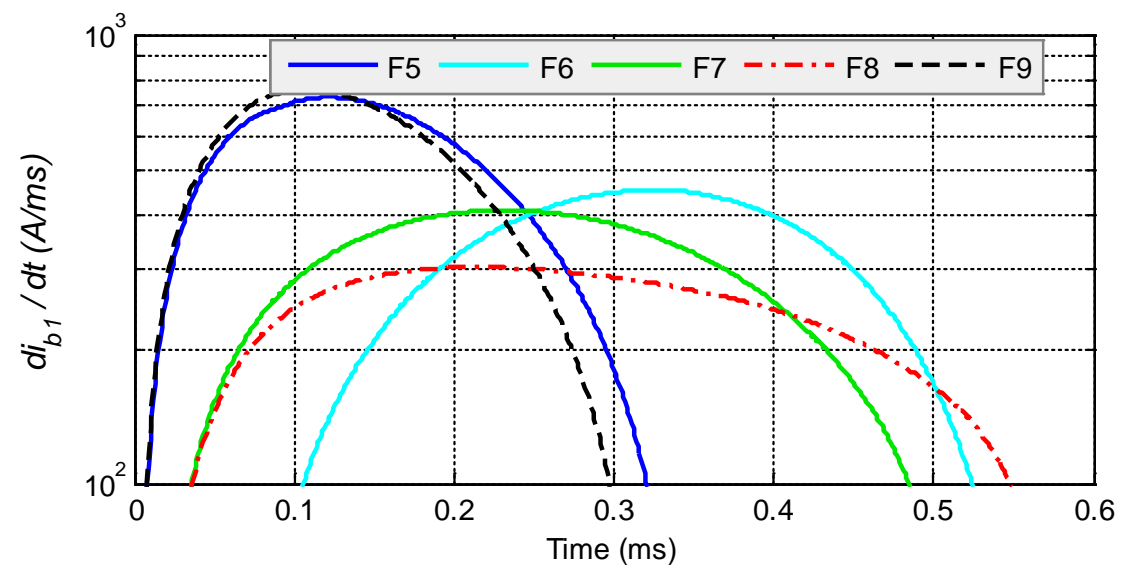

Figure 10.12: Derivative of short circuit current $i_{b 1}$ with AILI $=0.1 \mathrm{mH}$ during faults F5 5 $\mathrm{m}$ from bus 2, F6 middle of the feeder, F7 middle of the feeder, F8 middle of the feeder and F9 $5 \mathrm{~m}$ from bus 4 .

\subsubsection{Analysis of the Effects of the Cable Length}

In order to analyze the impacts of the cable length and to further validate the effectiveness of the proposed protection scheme, two additional case studies are defined in this subsection. In case 1 , the length of the cable $1-2$ was changed from $70-\mathrm{m}$ to $40-\mathrm{m}$, while in case 2, the length of this cable increased to $150-\mathrm{m}$.

Analysis of the previous subsection was shown that the most difficult fault identification was between far interconnected feeder faults F5 and adjacent feeder faults F6. Figure 10.13 shows the sub-transient short circuit current $i_{b 1}$, during faults F5 and F6 for case study 1 and case study 2. Also, Figure 10.14 shows the derivative of the fault current $i_{b 1}$, during faults F5 and F6 for the two case studies. As the results show, by employing the AILI and utilizing the derivative fault identification method, the interconnected feeder fault and the adjacent feeder fault could be clearly identified in both case studies. In case study 1 , the maximum $d t_{i c c}^{M}(t) / d t$ during faults F5 and F6 were 800.5$\mathrm{A} / \mathrm{ms}$ and $462.3-\mathrm{A} / \mathrm{ms}$, respectively. Also, in case study 2 the maximum $d t_{i c C C}^{M}(t) / d t$ during faults F5 and F6 were 601.2-A/ms and 434.8-A/ms, respectively. 


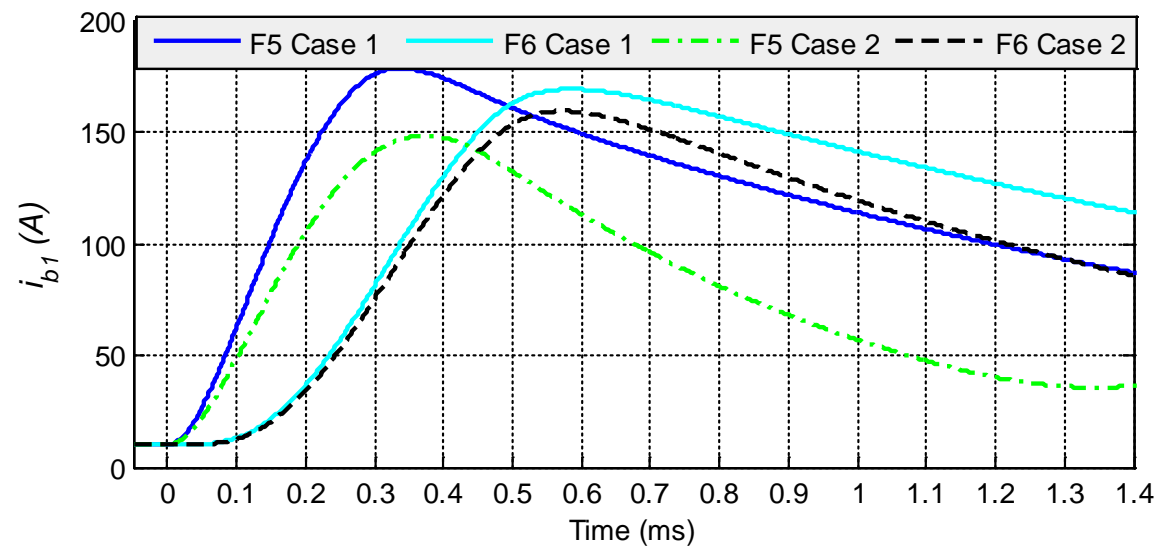

Figure 10.13: Sub-transient short circuit current $i_{b 1}$ with AILI $=0.1 \mathrm{mH}$ during faults F5 5 $\mathrm{m}$ from bus 2 and F6 middle of the feeder. Case 1: length of the cable 1-2 was $40 \mathrm{~m}$. Case 2: length of the cable $1-2$ was $150 \mathrm{~m}$.

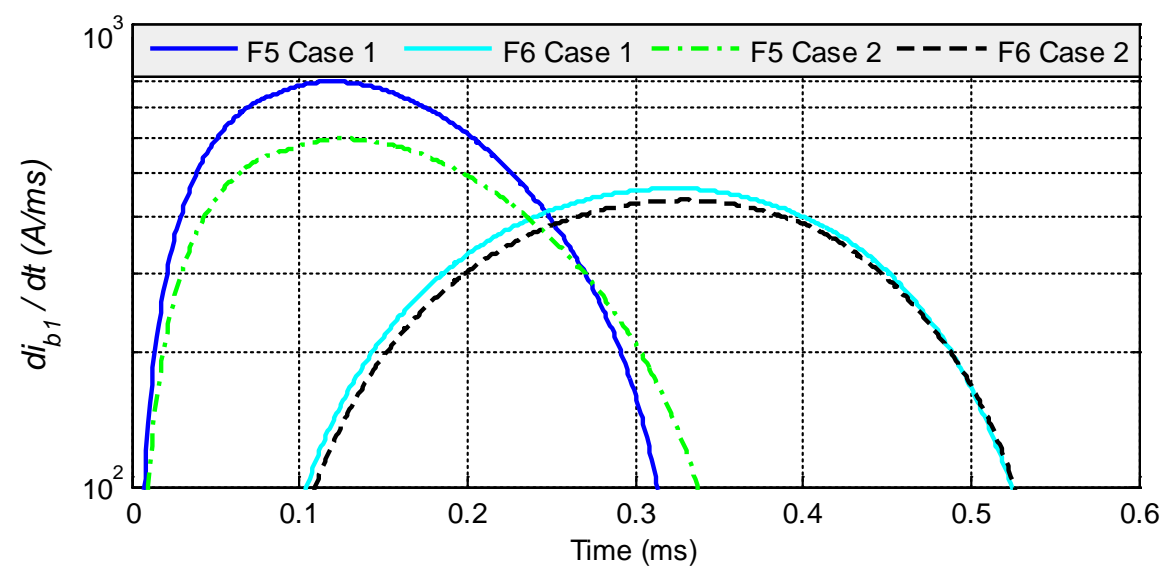

Figure 10.14: Derivative of short circuit current $i_{b 1}$ with AILI $=0.1 \mathrm{mH}$ during faults F5 5 $\mathrm{m}$ from bus 2 and F6 middle of the feeder. Case 1: length of the cable 1-2 was $40 \mathrm{~m}$. Case 2: length of the cable $1-2$ was $150 \mathrm{~m}$.

It should be noted that for a larger system and by increasing the length of the cable, the equivalent inductance of the feeder will increase. Thus, in order to reduce the fault current front slop and increase fault current derivative resolution, implementation of bigger AILIs is more feasible, which is discussed in Figure 10.9. 


\subsection{Performance Evaluation of the proposed Protection Scheme}

Using the simulation model of the hybrid DC microgrid, the performance of the proposed event-based fault identification method is verified in terms of fault isolation, load support during a fault, and system restoration.

The required time to interrupt a fault current and completely isolate the fault area through the CB and DS depends on the switching technology and operating speed of the contactor. In this study, a $150 \mu$ s and 3 ms operating time for the CB and DS are considered, respectively. This also includes communication delay. Figure 10.15 shows the performance of the protection scheme when the fault F5 was simulated. The fault occurred at $\mathrm{t}=5 \mathrm{~ms}$, 5 meters from bus 2. It should be noted that the output current of the DC bus $i_{b i}$, is measured for system protection. However, in order to better demonstrate the performance of the converter during the fault and after system restoration, the converter output current $i_{c i}$, is shown instead.

As was shown in Figure 10.12, the protection system of the first unit was able to identify an interconnected feeder fault $0.12 \mathrm{~ms}$ after the fault incident. Then, CB1 and converter 1 at t $=5.27$ ms were blocked. Similarly, the second protection unit identified an interconnected feeder fault and opened the CB2 at $t=5.27 \mathrm{~ms}$. The third protection unit also detected an adjacent feeder fault when the maximum derivative of the fault current reached to $480 \mathrm{~A} / \mathrm{ms}$ at $\mathrm{t}=5.30 \mathrm{~ms}$. Consequently, the CB3 was opened at $\mathrm{t}=5.45 \mathrm{~ms}$ and converter 3 was stopped. 

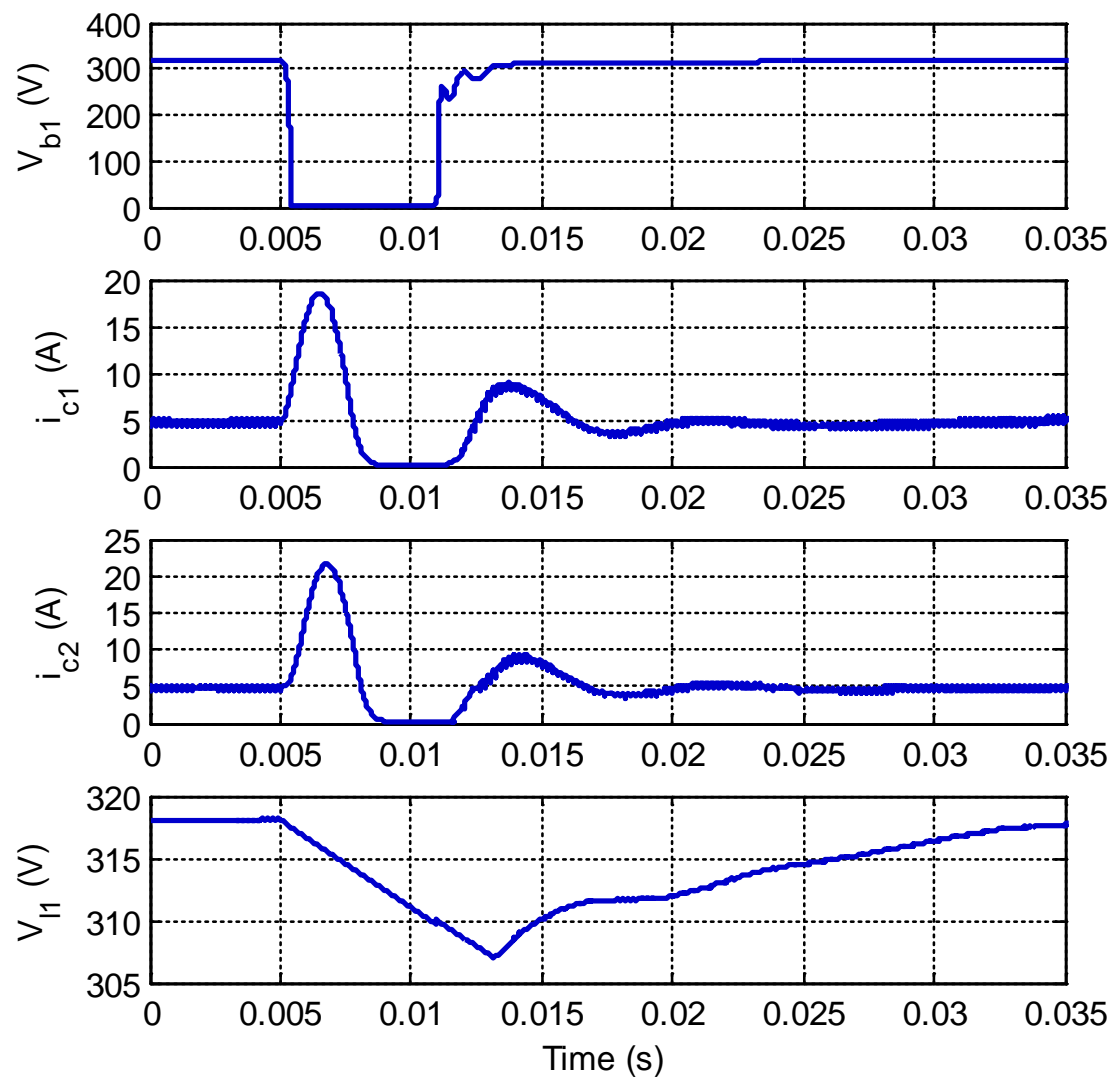

Figure 10.15: Performance of the event-based protection method during fault F5.

Figure 10.16 shows the simulation test results in the case of bus fault F1. The grid was supplying load 1 with $250 \mathrm{~W}$, load 2 with $2500 \mathrm{~W}$, and load 3, a pulse load, with 3.25 $\mathrm{kW}$ before the fault occurred. Following the fault incident, the protection of the first unit detected a bus fault since the derivative of the fault current exceeded the $2000 \mathrm{~A} / \mathrm{ms}$ threshold. Then, CB1 was completely opened at $\mathrm{t}=5.15 \mathrm{~ms}$. Also, the protection of the second and third power unit detected a $493 \mathrm{~A} / \mathrm{ms}$ and $376 \mathrm{~A} / \mathrm{ms}$ maximum fault current derivative, respectively and classified the fault as an adjacent bus or feeder fault initially. Then, upon receiving the bus fault event from the first unit, an adjacent bus fault was concluded. The results show that the maximum fault current of the first and second unit are limited to $26.17 \mathrm{~A}$ and 19.86A, respectively. Following fault isolation, the energy management algorithm explained in 5, set the reference current of the second converter to 
its maximum power capacity of $8 \mathrm{~A}$, and adjust the rest of the required power to the bidirectional converter. The results show that the developed protection scheme and the energy management system were able to accurately identify the type of fault, isolate the faulted area and restore the system very quickly while the load voltage drop was limited to $2.88 \%$.
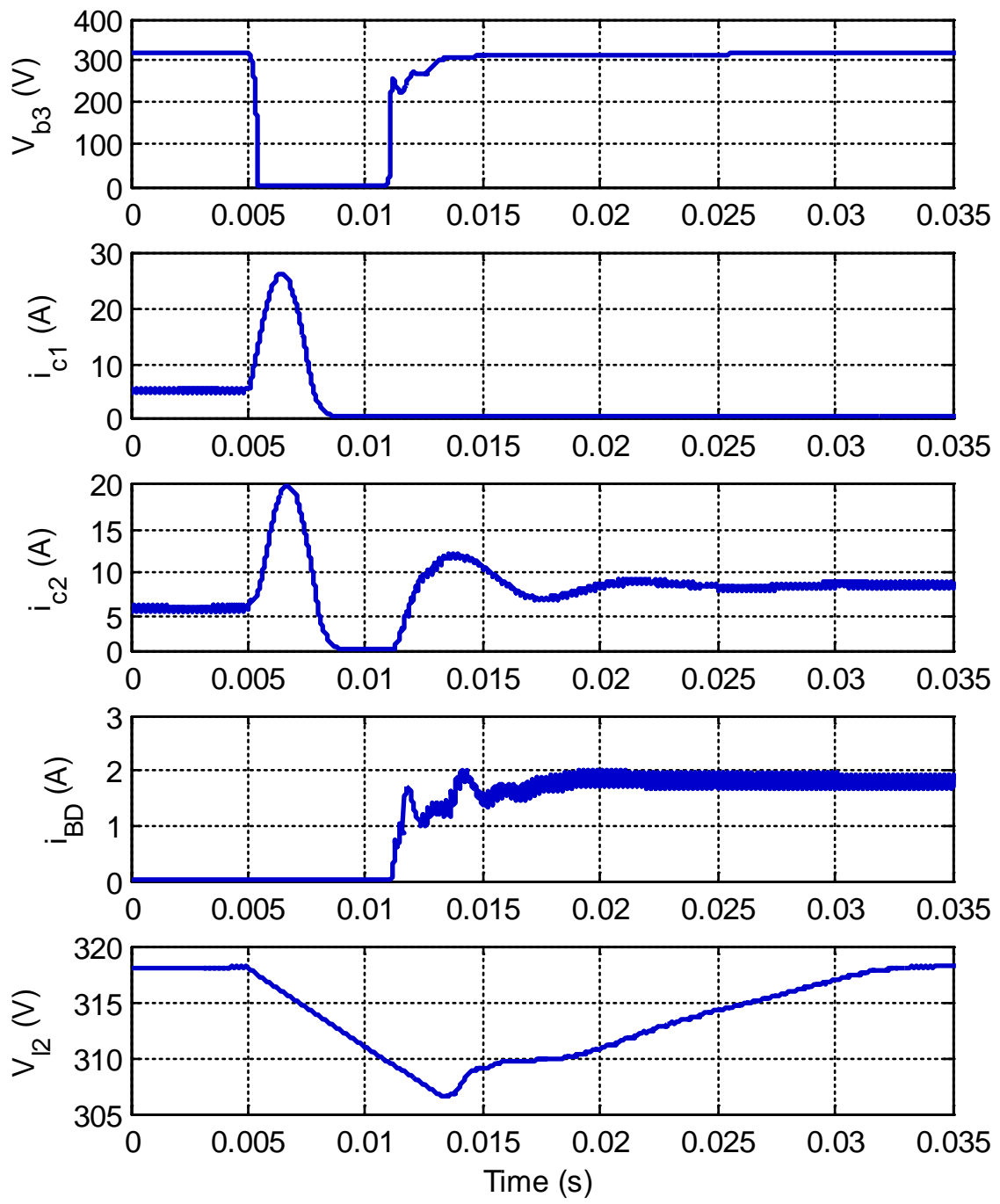

Figure 10.16: Performance of the event-based protection scheme during fault F1.

The test results have shown that the derivative fault classification technique together with the artificial inductive line impedance implementation were able to classify the type of a fault accurately, isolate the faulted area very fast, and restore system very 
quickly while the loads were able to ride-through the fault uninterrupted. Thus, it can be concluded that the developed event-based protection strategy can be utilized in multiterminal DC microgrid, such as renewable energy distributed generation microgrids, data centers, or shipboard power systems where self-diagnosing and self-reconfiguring capability is in high demand. 


\section{CONCLUSIONS AND RECOMMENDATIONS FOR FUTURE WORK}

\subsection{Conclusions}

This dissertation presented new techniques for the control, energy management and protection of the hybrid battery-supercapacitor storage system to improve the performance of the system in terms of efficiency, power quality and reliability. A laboratory-scale hardware/software-based hybrid DC power system was developed to experimentally evaluate contributions of this dissertation. This platform was equipped with a hybrid supercapacitor-battery energy storage system and was able to supply the loads with power up to $10-\mathrm{kW}$ in a steady state condition and $18-\mathrm{kW}$ in transient conditions. The implemented hybrid DC microgrid included three different types of fully programmable loads, which were the steady state load, dynamic load and pulse load. The energy storage devices, power electronic converters, programmable loads and main energy resources were completely reconfigurable for various studies. The developed setup had a real-time control and monitoring capabilities, which made the system as a unique platform for various power system studies and performance analysis, such as efficiency, voltage profile, current harmonics, power fluctuation and frequency variation, as well as system reliability.

The effects of pulse loads on AC and DC power systems were analyzed to investigate the power and energy requirements of the load in terms of dynamic and average power and energy. Various supercapacitor-battery hybrid configuration were studied, and advantages and limitations of each configuration were discussed. Also, different aspects involved in the design, control and operation of a hybrid power system that incorporates

pulse loads were investigated. Also, the design requirements of the hybrid DC power 
system used to prevent even-order harmonics and DC current circulation were examined and experimentally tested. Two protection systems for safe operation of the supercapacitor bank were designed and implemented. These included a digital voltage protection, which was embedded to the real-time control system, and an analog hysteresis protection, which was designed to monitor different supercapacitor arrays. The experimental result shown that the developed protection system ensure safe operation of the supercapacitor bank and prevent over voltage due to uneven charge distribution, especially during very fast charges.

In this dissertation, a new configuration for a direct connection of the supercapacitor bank and pulse load to a DC bus was proposed. The developed configuration improved the redundancy of the system as well as it reliability. In addition to supplying the pulse load, the supercapacitor was able to support the grid during the transient startup of the dynamic loads and reduce the stresses on power converters and storage devices. However, the energy management and control of such a redundant system were concerns since the heavy pulse load could cause high power pulsation and voltage drop on the entire grid. To reduce the adverse impacts of the pulse load, a novel, real-time energy management system and adaptive energy calculator were developed and presented in this dissertation. The developed energy management system utilized an adaptive energy calculator to dynamically calculate the required power of the grid. The performance of the energy management system were experimentally evaluated and were compared with other approaches suggested in the literature. The results showed that the developed technique caused about $0.84 \%$ more DC bus voltage variation than the direct voltage control. However, it effectively prevented high current pulsations of the converters. Moreover, the developed technique eliminated the power and frequency fluctuation of the generators 
supplying the grid. Comparison of the performance of the developed technique with continuous current averaging method showed that the developed technique adaptively adjusted the total reference current during transient time, and the bus voltage was maintained within the limit even if the measurement had a considerable error.

Grid connected DC microgrid power systems that incorporate heavy pulse load faces many challenges. Hybrid energy storage devices are of significant interest in such a system. High power and high energy capability of the hybrid storage devices improve the efficiency and reliability of the system. However, existence of the pulse loads causes various disturbances on the DC microgrid and the interconnected AC grid. In this dissertation, a new three-layer control scheme was developed to actively control a grid connected hybrid DC microgrid. Unlike conventional control methods, the developed control approach had the advantage of controlling both the voltage and the current of the system. The performance of the developed technique was experimentally evaluated. The results were shown that the developed scheme highly improves the dynamic performance of the hybrid DC microgrid and mitigated the adverse effects of the pulse load on the interconnected grid. The experimental test results for different pulse duty ratios were analyzed in terms of DC bus voltage drop, system efficiency, AC grid voltage drop and the frequency fluctuations. The results shown that the developed technique caused slightly more bus voltage variation than the conventional method; however, significant improvements achieved in terms of reducing the AC grid voltage drop, enhancing the system efficiency and mitigating Ac grid frequency fluctuation.

The DC power system is an effective architecture that leads to a more reliable power with higher efficiency, achieving a better integration of energy sources with fewer power 
electronic converters. However, protection of DC power systems, especially multi-source distribution systems and multi-terminal dc lines, involves many challenges. Through extensive study, this dissertation presented the key requirements for the protection of these systems, such as personal safety, fault detection and location capabilities, equipment survivability and ride-through capability. Various grounding topologies utilized in DC systems were detailed and their advantages and limitations were compared in terms of personal safety, transient short circuit current behavior, fault detection and protection capabilities, and insulation requirements. In multi-source systems, the proper design and coordination of circuit breakers and disconnecting switches are very important to rapidly isolate the faulted area and restore the system. Different circuit breaker technologies and configurations were studied and their performances were compared. Moreover, a comprehensive overview of various fault detection and location techniques suggested in the literature were presented. The requirements and applications of these techniques were discussed and areas for future researches were indicated.

This dissertation also presented a detailed model of a hybrid DC microgrid for transient and dynamic study. Different techniques for modeling of supercapacitor and battery energy storage devices were explained. Based on the explained techniques, an accurate virtual test-bed platform of the actual system was developed. The virtual test-bed model was implemented in the Matlab/Simulink environment. In order to evaluate the accuracy of the developed model, the normal and transient performances of the virtual testbed were compared with the experimental results. The simulation model results were consistent with the experimental test results which successfully validated the accuracy of the model. 
A novel protection scheme for a multi-terminal DC power system was developed in this dissertation. The developed protection scheme was an event-based protection strategy, which transferred less data when compared with commonly used data-based protection methods, and did not require synchronization and high speed communication. The protection relays were able to autonomously identify the type of event by employing artificial inductive line impedance and utilizing a current derivative fault identification technique. Detailed fault current analysis considering low pass RC filter effects were investigated. This analysis can be effectively utilized to accurately set the protection relays. The performance of the developed protection scheme was evaluated using the virtual testbed platform. The results shown that the developed protection scheme was accurate and fast, and the grid were able to ride-through the fault uninterrupted.

\subsection{Future Work}

To enable large scale grid integration of sustainable resources without compromising security of the operation of the power systems, important technical requirements such as Low Voltage Ride-Through (LVRT) capability is imposed to distributed stations. Advanced control of the photovoltaic station with the maximum power tracking capability for normal operation and LVRT capability during a fault condition can be studied. The proposed method can be experimentally tested by connecting the PV emulator and hybrid storage to the AC grid.

In the power train of future movable vessels, regenerative braking systems is a requirement for better system efficiency and lower exhaust emissions. The regenerative braking system should be designed so that the energy is effectively stored and reused while 
the bus voltage is maintained within the limit. A test setup composed of a generator-motor and an adjustable load can be implemented. Control and energy management enhancement in the hybrid DC power system with regenerative braking capability can be studied. Also, the effect of fast start and fast stop on the bus voltage and stability of the test-bed system can be analyzed.

Also, due to the increasing number of electronic and other non-linear loads, the harmonics and power quality of the system become more important issues. Implementation of ultracapacitor in the UPQC structure with an advanced control method is a new idea that can be experimentally tested and verified. 


\section{REFERENCES}

[1] B. Roberts, "Capturing grid power," IEEE Power Energy Mag., vol. 7, no. 4, pp.32 -41 2009.

[2] M. S. Whittingham, "History, evolution, and future status of energy storage", Proc. IEEE, vol. 100, pp.1518 -1534 2012.

[3] “DOE Global Energy Storage Database” available online at: www.energystorageexchange.org.

[4] M.A. Hozouri, A. Abbaspour, M. Fotuhi-Firuzabad and M. Moeini-Aghtaie, "On the Use of Pumped Storage for Wind Energy Maximization in Transmission-Constrained Power Systems," IEEE Transactions on Power Systems, vol.30, no.2, pp.1017,1025, March 2015.

[5] F.A. Bhuiyan and A. Yazdani, "Energy storage technologies for grid-connected and offgrid power system applications," IEEE, Electrical Power and Energy Conference (EPEC 2012), pp.303,310, 10-12 Oct. 2012.

[6] F. S. Barnes and J. G. Levine, Eds., "Large Energy Storage Systems Handbook. Boca Raton,” FL: CRC Press, 2011.

[7] D. J. Swider "Compressed air energy storage in an electricity system with significant wind power generation", IEEE Trans. Energy Convers., vol. 22, no. 1, pp.95 -102 2007.

[8] M. S. Whittingham "History, evolution, and future status of energy storage", Proc. IEEE, vol. 100, pp.1518 -1534 2012.

[9] “Grid energy storage,” U.S. Department of Energy, Technical Report December 2013.

[10] F. Zhang, M. Tokombayev, Y. Song and G. Gross, "Effective flywheel energy storage (FES) offer strategies for frequency regulation service provision,” Power Systems Computation Conference, pp.1,7, 18-22 Aug. 2014.

[11] H. Vasconcelos, C. Moreira, A. Madureira, J. Lopes and V. Miranda, “Advanced Control Solutions for Operating Isolated Power Systems: Examining the Portuguese islands,” Electrification Magazine, IEEE, vol.3, no.1, pp.25-35, March 2015.

[12] G. Cimuca, S. Breban, M. M. Radulescu, C. Saudemont and B. Robyns, "Design and control strategies of an induction-machine-based flywheel energy storage system associated toa variable-speed wind generator,” IEEE Trans. Energy Convers., vol. 25, no. 2, pp.526 -534 2010.

[13] L. Ran, D. Xiang and J. Kirtley, "Analysis of electromechanical interactions in a flywheel system with a doubly fed induction machine,” IEEE Trans. Ind. Appl., vol. 47, no. 3, pp. 1498-1506, 2011. 
[14] I.A. Gowaid, A.A. Elserougi, A.S. Abdel-Khalik, A.M. Massoud and S. Ahmed, “A series flywheel architecture for power levelling and mitigation of DC voltage transients in multi-terminal HVDC grids," Generation, Transmission \& Distribution, IET, vol.8, no.12, pp.1951,1959, 122014.

[15] R. Arghandeh, M. Pipattanasomporn, S. Rahman "Flywheel energy storage systems for ride-through applications in a facility microgrid” IEEE Trans Smart Grid, 3 (4) (2012), pp. 1955-1962.

[16] A. Joseph and M. Shahidehpour "Battery storage systems in electric power systems", Proc. IEEE Power EnergySoc. Gen. Meet., 2006.

[17] A.R. Sparacino, G.F. Reed, R.J. Kerestes, B.M. Grainger, Z.T. Smith, "Survey of Battery Energy Storage Systems and Modeling Techniques", IEEE Power and Energy Society General Meeting, pp. 1-8, 2012.

[18] B. Roberts "Capturing grid power", IEEE Power Energy Mag., vol. 7, no. 4, pp.32 -41 2009.

[19] A. Oudalov, D. Chartouni and C. Ohler "Optimizing a battery energy storage system for primary frequency control”, IEEE Trans. Power Syst., vol. 22, no. 3, pp.1259 -1266 2007.

[20] C. R. Vergara, "Parametric interface for Battery Energy Storage Systems providing ancillary services,” Proc. IEEE PES International Conference and Exhibition on Innovative Smart Grid Technologies (ISGT Europe), 2012, pp. 1-7.

[21] U. Koehler, F. J. Kruger, J. Kuempers, M. Maul, E. Niggemann, and H. H. Schoenfelder, "High performance nickel-metal hydride and lithium-ion batteries," Proc. IECEC',97, vol. 1, pp.93 -98 1997.

[22] J. Cao and A. Emadi "A new battery/ultracapacitor hybrid energy storage system for electric, hybrid, and plug-in hybrid electric vehicles", IEEE Trans. Power Electron., vol. 27, no. 1, pp.122 -132, 2012.

[23] S. M. Lukic, C. Jian, R. C. Bansal, F. Rodriguez and A. Emadi, "Energy storage systems for automotive applications,” IEEE Trans. Ind. Electron., vol. 55, no. 6, pp.2258 -2267 2008.

[24] M. A. Casacca, M. R. Capobianco, and Z. M. Salameh, "Lead-acid battery storage configurations for improved available capacity," IEEE Trans. Energy Conversion, vol. 11, pp. 139-145, Mar. 1996.

[25] J. Moreno, M. E. Ortuzar and J. W. Dixon "Energy-management system for a hybrid electric vehicle, using ultracapacitors and neural networks", IEEE Trans. Ind. Electron., vol. 53, no. 2, pp.614 -623 2006. 
[26] A. Green, "The Characteristics of the Nickel-Cadmium Battery for Energy Storage," Power Engineering Journal, Vol.13, Issue 3, June 1999, pp. 117-121.

[27] Y. Bo , Y. Makarov , J. Desteese , V. Viswanathan , P. Nyeng , B. McManus and J. Pease "On the use of energy storage technologies for regulation services in electric power systems with significant penetration of wind energy", Proc. 5th Int. Conf. EEM, pp.1 -6 2008.

[28] S. S. Williamson "Electric drive train efficiency analysis based on varied energy storage system usage for plug-in hybrid electric vehicle applications", Proc. IEEE Power Electronics Specialists Conf. (PESC), pp.1515 -1520 2007.

[29] E. Kodama and Y. Kurashima "Development of a compact sodium sulphur battery", Power Eng. J., vol. 13, no. 3, pp.136 -141 1999.

[30] W. Zhaoyin "Study on energy storage technology of sodium sulfur battery and its application in power system", Proc. Int. Conf. PowerCon, pp.1 -4 2006.

[31] David Connolly, "A review of energy storage technologies for the integration of fluctuating renewable energy," University of Limerick, October 2010.

[32] B. P. Roberts "Sodium-sulfur (NaS) batteries for utility energy storage applications", IEEE PES General Meeting, 2008.

[33] W. Hu, Z. Chen and B. Bak-Jensen "Optimal operation strategy of battery energy storage system to real-time electricity price in Denmark", Proc. IEEE Power Energy Soc. Gen. Meet., 2010.

[34] D. H. Swan, B. Dickinson and M. Arikara "Demonstration of a zinc bromine battery in an electric vehicle", IEEE Aerosp. Electron. Syst. Mag., pp.20 -23 1994.

[35] S. Nguyen, M.J. Kushner and A. Gallimore, "A study of reaction kinetics of a water plasma using a 0-D global kinetic model," IEEE International Conference on Plasma Science (ICOPS 2009) pp.1,1, 1-5 June 2009.

[36] Z. Yong-hui and Z. Ning "Research and development of hydrogen energy materials," International Conference on Electric Information and Control Engineering (ICEICE 2011), pp.2484, 2486, 15-17 April 2011.

[37] M.W. Ellis, M.R. von Spakovsky and D.J. Nelson, "Fuel cell systems: efficient, flexible energy conversion for the 21st century," IEEE Proceedings, vol.89, no.12, pp.18081818, Dec 2001.

[38] N. Bertrand, J. Sabatier, O. Briat and J. Vinassa, "Embedded fractional nonlinear supercapacitor model and its parametric estimation method," IEEE Trans. Ind. Electron., vol. 57, no. 12, pp.3991 -4000 2010. 
[39] V. Musolino, L. Piegari and E. Tironi, "New full-frequency-range supercapacitor model with easy identification procedure," IEEE Trans. Ind. Electron., vol. 60, no. 1, pp.112 1202013.

[40] V. Weidner, J.W. Srinivasan, "Mathematical modeling of electrochemical capacitors," Journal of the Electrochemical Society, 146 (1999), pp. 1650-1658.

[41] R. Shah and N. Mithulananthan, "A comparison of ultracapacitor, BESS and shunt capacitor on oscillation damping of power system with large-scale PV plants,” Proc. Universities Power Engineering Conference 2011, pp.1, 6, 25-28 Sept. 2011.

[42] J. Liang and C. Feng, "Stability improvement of micro-grids with coordinate control of fuel cell and ultracapacitor,” Proc. Power Electronics Specialists Conference, 2007. IEEE, pp. 2472-2477, 2007.

[43] C. Abbey and G. Joos "Supercapacitor energy storage for wind energy applications" IEEE Trans. Ind. Appl., vol. 43, no. 3, pp. 769-776, 2007.

[44] S.I. Gkavanoudis and C.S. Demoulias, "A new Fault Ride-Through control method for full-converter wind turbines employing Supercapacitor Energy Storage System," Universities Power Engineering Conference (UPEC), pp.1, 6, 4-7 Sept. 2012.

[45] M. Farhadi, and O. Mohammed, "Real-time operation and harmonic analysis of isolated and non-isolated hybrid DC microgrid,” Industry Applications Society Annual Meeting, 2013 IEEE, vol., no., pp.1, 6, 6-11 Oct. 2013.

[46] M. Sudhoff, S.D., "Reducing Impact of Pulsed Power Loads on Microgrid Power Systems,” Smart Grid, IEEE Transactions on, vol.1, no.3, pp.270-277, Dec. 2010.

[47] M. H. Ali, B. Wu and R. A. Dougal "An overview of SMES applications in power and energy systems”, IEEE Trans. Sustainable Energy, vol. 1, no. 1, pp.38 -47 2010.

[48] A. H. Moghadasi , H. Heydari and M. Salehifar, "Reduction in VA rating of the unified power quality conditioner with superconducting fault current limiters," Proc. 1st Power Electron. Drive Syst., pp.382 -387 2010.

[49] A. H. Moghadasi, H. Heydari and M. Farhadi, "Pareto optimality for the design of SMES solenoid coils verified by magnetic field analysis," IEEE Trans. Appl. Supercond., vol. 21, no. 1, pp.13 -20 2011.

[50] D. Ketata and L. Krichen, "Control and power management of a PEMFC-supercapacitor as a distributed generator,” Proc. 8th Int. Multi-Conf. Syst., Signals Devices, pp.1 -7 2011.

[51] A. Arsoy, Y. Liu, P. F. Ribeiro and W. Xu, "The impact of energy storage on the dynamic performance of a static synchronous compensator," Proc. of 3rd International 
Conference on Power Electronics and Motion Control, 15-18 Aug. 2000, vol.2, pp. 519524.

[52] A. Bidadfar, M. Abedi, M. Karari and C.-C. Chu, "Power swings damping improvement by control of UPFC and SMES based on direct Lyapunov method application,” Proc. 2008 IEEE Power \& Energy Soc. General Meeting, 2008.

[53] A. B. Arsoy, Y. Liu, P. F. Ribeiro, and F. Wang, “StatCom—SMES,” IEEE Ind. Appl. Mag., vol. 9, no. 2, pp. 21-28, Mar./Apr. 2003.

[54] H. Heydari and A. H. Moghadasi, "Optimization scheme in combinatorial UPQC and SFCL using normalized simulated annealing," IEEE Trans. Power Del., vol. 26, no. 3, pp.1489 -1498 2011.

[55] M. Swierczynski , R. Teodorescu , C. N. Rasmussen , P. Rodriguez and H. Vikelgaard "Overview of the energy storage systems for wind power integration enhancement", Proc.2010 IEEE Int. Symp. Ind. Electronics (ISIE), pp.3749 -3756 2010.

[56] H. Chen, T. N. Cong, W. Yang, C. Tan , Y. Li and Y. Ding "Progress in electrical energy storage system: A critical review", Prog. Natural Sci., vol. 19, no. 3, pp.291 -312 2009.

[57] F. Da $\neg$ az-Gonzalez, A. Sumper , O. Gomis-Bellmunt and V. Robles "A review of energy storage technologies for wind power applications", Elsevier Renewable Sustain. Energy Rev., vol. 16, no. 4, pp.2154 -2171 2012.

[58] H. Ibrahim , A. Ilinca and J. Perron "Energy storage systems-characteristics and comparisons", Renewable Sustainable Energy Rev., vol. 12, no. 5, pp.1221 -1250 2008.

[59] J. M. Eyer, J. J. Iannucci and G. P. Corey, "Energy Storage Benefits and Market Analysis Handbook,” Sandia National Laboratories, Dec 2004.

[60] R. J. Kerestes, G. F. Reed, A. R. Sparacino, "Economic analysis of grid level energy storage for the application of load leveling," IEEE Power and Energy Society General Meeting, 22-26 July 2012, pp. 1-9.

[61] I. Papic "Simulation model for discharging a lead-acid battery energy storage system for load leveling", IEEE Trans. Energy Conv., vol. 21, no. 2, pp.608 -615 2006.

[62] S. Li, K. Tomsovic and T. Hiyama "Load following functions using distributed energy resources", Proc. IEEE/PES Summer Meeting, pp.1756 -1761 2000.

[63] "EPRI-DOE Handbook of Energy Storage for Transmission and Distribution Applications,” EPRI, 2003.

[64] P.F. Frack, M. Martinez, M.G. Molina and P.E. Mercado "Emulation of Synchronous Generator for Frequency Control of Smart Microgrids,” Latin America Transactions, IEEE, vol.11, no.1, pp.486-491, Feb. 2013. 
[65] Y. Cho, J. W. Shim, S. Kim, S. W. Min and K. Hur, "Enhanced frequency regulation service using Hybrid Energy Storage System against increasing power-load variability,” Power and Energy Society General Meeting (PES), pp.1,5, 21-25 July 2013.

[66] L. Zhang and Y. Liu, "Bulk power system low frequency oscillation suppression by FACTS/ESS,” Proc. IEEE PES Power Syst. Conf. Exp., pp.219 -226 2004.

[67] B. Kirby and E. Hirst, “Ancillary service details: Voltage control,” Oak Ridge National Laboratory, Rep. ONRL/CON-453, 1997.

[68] J. H. Chow, R. W. De Mello and K. W. Cheung, "Electricity market design: An integrated approach to reliability assurance,” Proc. IEEE, vol. 93, no. 11, pp. 1956-1969, 2005.

[69] G. Graditi, M. G. Ippolito, E. Telaretti, and G. Zizzo, “An innovative conversion device to the grid interface of combined RES-based generators and electric storage systems," IEEE Trans. Ind. Electron., vol. 62, no. 4, pp. 2540-2550, Apr. 2015.

[70] M. Yilmaz and P. T. Krein, "Review of the impact of vehicle-to-grid technologies on distribution systems and utility interfaces," IEEE Trans. Power Electron., vol. 28, no. 12, pp.5673 -5689 2013.

[71] K. Christakou, D.-C. Tomozei, M. Bahramipanah, J.-Y. Le Boudec, and M. Paolone, "Primary voltage control in active distribution networks via broadcast signals: The case of distributed storage,” IEEE Trans. on Smart Grid, vol.5, no.5, pp.2314,2325, Sept. 2014.

[72] 26M.khaterchi, J.elhadj, M.elleuch, "DPC for three-phase inverter to improve the integration of wind turbine associated to flywheel energy storage system into the grid," The 6th international Muli-Conference on systéms,Signals and Devices, (SSD) 2009.

[73] J. Rocabert, A. Luna, F. Blaabjerg and P. Rodriguez, "Control of power converters in AC microgrids,” IEEE Trans. Power Electron., vol. 27, no. 11, pp.4734 -4749 2012.

[74] G. Graditi, M.G. Ippolito, E. Telaretti and G. Zizzo, “An Innovative Conversion Device to the Grid Interface of Combined RES-Based Generators and Electric Storage Systems,” IEEE Trans. Ind. Appl., vol. 62, no.4, pp.2540,2550, April 2015.

[75] M. Glavic, "Design of a resistive brake controller for power system stability enhancement using reinforcement learning” IEEE Trans. Control Syst. Technol., vol. 13, no. 5, pp. 743-751, 2005.

[76] A. H. M. A. Rahim, D. A. H. Alamgir, “A closed-loop quasi-optimal dynamic braking resistor and shunt reactor control strategy for transient stability”, IEEE Trans. on Power Syst., Vol. 3, No. 3, pp. 879-886, 1988. 
[77] Da Wang, M. Glavic and L. Wehenkel, “Trajectory-Based Supplementary Damping Control for Power System Electromechanical Oscillations,” Power Systems, IEEE Transactions on, vol.29, no.6, pp.2835, 2845, Nov. 2014.

[78] C. Sihler and A. M. Miri, “A stabilizer for oscillating torques in synchronous machines,” IEEE Trans. Ind. Appl., vol. 41, no. 3, pp. 748-755, 2005.

[79] J.C. Neely, R.H. Byrne, R.T. Elliott, C.A. Silva-Monroy, D.A. Schoenwald, D.J. Trudnowski and M.K. Donnelly, "Damping of inter-area oscillations using energy storage,” Power and Energy Society General Meeting (PES), 2013 IEEE, vol., no., pp.1,5, 21-25 July 2013

[80] M. H. Ali, M. Park, I. K Yu, T. Murata, and J. Tamura, “Effect of fuzzy logic-controlled SMES on damping turbine generator shaft torsional oscillations during unsuccessful reclosing," Transmission and Distribution Conference and Exposition, 2008, T\&D IEEE/PES, 21-24 April 2008 Page(s):1-8.

[81] L. Wen-Tsan, W. Yuan-Kang, L. Ching-Yin and C. Chao-Rong "Effect of low-voltageride-through technologies on the first Taiwan offshore wind farm planning” IEEE Trans. Sustain. Energy, vol. 2, no. 1, pp. 78-86, 2011.

[82] M. Molinas, J. A. Suul and T. Undeland "Low voltage ride through of wind farms with cage generators: STATCOM versus SVC” IEEE Trans. Power Electron., vol. 23, no. 3, pp. 1104-1117, 2008.

[83] S. Muyeen "A variable speed wind turbine control strategy to meet wind farm grid code requirements” IEEE Trans. Power Syst., vol. 25, no. 1, pp. 331-340, Feb., 2010.

[84] A. Mullane, G. Lightbody and R. Yacamini "Wind-turbine fault ride-through enhancement” IEEE Trans. Power Syst., vol. 20, no. 4, pp. 1929-1937, 2005.

[85] M.I. Daoud, A. Massoud, S. Ahmed, A.S. Abdel-Khalik and A. Elserougi, "Ridethrough capability enhancement of VSC-HVDC based wind farms using low speed flywheel energy storage system,” Applied Power Electronics Conference and Exposition (APEC), pp. 2706,2712, March 2014.

[86] S. Gayathri Nair and N. Senroy, "Wind turbine with flywheel for improved power smoothening and LVRT,” Power and Energy Society General Meeting (PES),1,5, 21-25 July 2013.

[87] M. A. Alam, A. H. M. A. Rahim and M. A. Abido "Supercapacitor based energy storage system for effective fault ride through of wind generation system” Proc. IEEE ISIE, pp. 2481-2486, 2010.

[88] A. Affanni, A. Bellini, G. Franceschini, P. Guglielmi and C. Tassoni, "Battery choice and management for new-generation electric vehicles,” IEEE Trans. Ind. Electron., vol. 52, no. 5, pp.1343 -1349 2005. 
[89] D. Feroldi, M. Serra and J. Riera, "Design and analysis of fuel-cell hybrid systems oriented to automotive applications,” IEEE Trans. Veh. Technol., vol. 58, no. 9, pp.4720 $-47292009$.

[90] P. P. Acarnley, B. C. Mecrow, J. S. Burdess, J. N. Fawcett, J. G. Kelly and P. G. Dickinson, "Design principles for a flywheel energy store for road vehicles," IEEE Trans. Ind. Appl., vol. 32, no. 6, pp.1402 -1408 1996.

[91] K. van Berkel, W. Klemm, T. Hofman, B. Vroemen and M. Steinbuch, "Optimal control of a mechanical hybrid powertrain with cold-start conditions,” IEEE Trans. Veh. Technol., vol. 63, no. 4, pp. 1555-1566, May, 2014.

[92] S. Shen and F. E. Veldpaus, "Analysis and control of a flywheel hybrid vehicular powertrain,” IEEE Transaction on control systems technology, vol.12, no.5, pp.645-660, Sept. 2004.

[93] P. Thounthong, V. Chunkag, P. Sethakul, B. Davat and M. Hinaje, "Comparative study of fuel-cell vehicle hybridization with battery or supercapacitor storage device,” IEEE Trans. Veh. Technol., vol. 58, no. 8, pp.3892 -3904 2009.

[94] R. de Castro, R. E. Araujo, J. P. F. Trovao, P. G. Pereirinha, P. Melo and D. Freitas, "Robust DC-link control in EVs with multiple energy storage systems," IEEE Trans. Veh. Technol., vol. 61, no. 8, pp.3553 -3565 2012.

[95] Y. Wu and H. Gao, "Optimization of fuel cell and supercapacitor for fuel-cell electric vehicles,” IEEE Trans. Veh. Technol., vol. 55, no. 6, pp.1748 -1755 2006.

[96] A. L. Allegre, A. Bouscayrol, P. Delarue, P. Barrade, E. Chattot and S. El-Fassi, “Energy storage system with supercapacitor for an innovative subway," IEEE Trans. Ind. Electron., vol. 57, no. 12, pp.4001 -4012 2010.

[97] Musolino, L. Piegari and E. Tironi, "Storage systems for transportation, land handling and naval applications,” Proc. Elect. Syst. Aircraft, Railway Ship Propulsion (ESARS), pp.1 -9 2012.

[98] X. Roboam, O. Langlois, H. Piquet, B. Morin, C. Turpin, "Hybrid power generation system for aircraft electrical emergency network," IET Trans on Electrical Systems in Transportation, Vol. 1, Issue 4, 2011, pp. 148-155.

[99] H. Tao, J. L. Duarte and M. A. M. Hendrix "Line-interactive UPS using a fuel cell as the primary source”, IEEE Trans. Ind. Electron., vol. 55, no. 8, pp.3012 -3021 2008.

[100] R. G. Lawrence, K. L. Craven and G. D. Nichols, “Flywheel UPS,” IEEE Ind. Appicat. Mag., pp.44 -50 2003. 
[101] R. S. Weissbach, G. G. Karady and R. G. Farmer, "A combined uninterruptible power supply and dynamic voltage compensator using a flywheel energy storage system," IEEE Trans. Power Del., vol. 16, no. 2, pp.265 -270 2001.

[102] J. D. Park, C. Kalev and H. F. Hofmann, “Control of high-speed solid rotor synchronous reluctance motor/generator for flywheel-based uninterruptible power supplies,” IEEE Trans. Ind. Electron., vol. 55, no. 8, pp.3038 -3046 2008.

[103] A. Lahyani, P. Venet, A. Guermazi and A. Troudi "Battery/Supercapacitors combination in uninterruptible power supply (UPS)”, IEEE Trans. Power. Electron, vol. 28, no. 4, pp.1509 -1522 2013.

[104] J. Jia, Y. Wang, S. Li and G. Wang, “A dynamic discharge structure for ultracapacitor application in the fuel cell UPS,” Proc. 10th Int. Conf. Control, Autom., Robot. Vision, pp.2102 -2107 2008.

[105] B. Roberts and J. McDowall, “Commercial successes in power storage,” IEEE Power Energy Mag., vol. 3, no. 2, pp.24 -30 2005.

[106] P. J. Binduhewa, "Uninterruptible power supply for short-time power back-up using ultracapacitors", in Proc. of IEEE International Conference on Industrial and Information Systems (ICIIS), 2011, pp. 551 - 556.

[107] M. Falahi, B K.L. utler-Purry and M. Ehsani, "Reactive Power Coordination of Shipboard Power Systems in Presence of Pulsed Loads,” Power Systems, IEEE Transactions on, vol.28, no.4, pp.3675-3682, Nov. 2013.

[108] Mustafa Farhadi, Ali Mazloomzadeh and Osama Mohammed, "Comparative Analysis of Energy Control Techniques for DC Microgrid and Pulsed Power Load Applications” 40th Annual Conference of the IEEE Industrial Electronics Society, IECON'2014, Dallas, TX - USA October 28 - November 1, 2014.

[109] Lijun Gao, R.A. Dougal, Shengyi Liu, "Power enhancement of an actively controlled battery/ultracapacitor hybrid,” Power Electronics, IEEE Transactions on, vol.20, no.1, pp.236-243, Jan. 2005.

[110] M. Farhadi, O. Mohammed, "Performance enhancement of actively controlled hybrid DC microgrid with pulsed power load,” IEEE Industry Applications Society Annual Meeting, October 2014.

[111] M. Farhadi, O. Mohammed, “Adaptive energy management in redundant hybrid DC microgrid for pulse load mitigation” IEEE Trans. Smart Grid, vol.6, no.1, pp.54,62, Jan. 2015.

[112] M. Farhadi, O. Mohammed, "Event Based Protection Scheme for a Multi-Terminal Hybrid DC Power System,” accepted for publication in the IEEE Trans. Smart Grid, 2015. 
[113] D. Shin, Y. Kim, J. Seo, N. Chang, Y. Wang, M. Pedram,, “Battery-supercapacitor hybrid system for high-rate pulsed load applications," Design, Automation \& Test in Europe Conference \& Exhibition (DATE), 2011, vol., no., pp.1,4, 14-18 March 2011.

[114] T.T. Leung, “Concept of a modified flywheel for megajoule storage and pulse conditioning,” IEEE Transactions on Magnetics, vol.27, no.1, pp.403,408, Jan 1991.

[115] S.K. Nayak and D.N. Gaonkar, "Modeling and performance analysis of hybrid fuel cell and microturbine based distributed generation system, "A review"," International Conference on Power, Energy and Control (ICPEC), pp.760,766, 6-8 Feb. 2013.

[116] F. Jurado and J. R. Saenz "Adaptive control of a fuel cell-microturbine hybrid power plant", IEEE Trans. Energy Convers., vol. 18, no. 2, pp.342 -347 2003.

[117] K. Rajashekara "Hybrid fuel-cell strategies for clean power generation", IEEE Trans. Ind. Appl., vol. 41, no. 3, pp.682 -689 2005.

[118] M. H. Nehrir , C. Wang , K. Strunz , H. Aki , R. Ramakumar, J. Bing, Z. Miao and Z. Salameh "A review of hybrid renewable/alternativeenergy systems for electric power generation: Configurations, control, andapplications", IEEE Trans. Sustain. Energy, vol. 2, no. 4, pp.392 -403 2011.

[119] A. Ipakchi and F. Albuyeh "Grid of the future", IEEE Power \& Energy Mag., vol. 7, no. 2, pp.52 -62 2009.

[120] A. H. Mohsenian-Rad , V. Wong , J. Jatskevich and R. Schober "Optimal and autonomous incentive-based energy consumption scheduling algorithm for smart grid", IEEE PES Conf. Innov. Smart Grid Technol., 2010.

[121] U. Abdulwahid , J. F. Manwell and J. G. Mcgowan "Development of a dynamic control communication system for hybrid power systems", IET Renewable Power Gener., vol. 1, no. 1, pp.70 -80 2007.

[122] X. Yu, Y. Xue, S. Sirouspour, and A. Emadi, "Microgrid and transportation electrification: A review," Transportation Electrification Conference and Expo (ITEC), 2012 IEEE, pp. 1-6, 2012.

[123] F. A. Farret and M. G. Simões, “Integration of Alternative Sources of Energy,” Wiley, 2006.

[124] C. K. Sao and P. W. Lehn, "A transformer-less energy storage system based on a cascade multilevel PWM converter with star configuration," IEEE Trans. Ind. Appl., vol. 44, no. 5, pp. 1621-1630, 2008.

[125] A. A. Mohamed "Hybrid Power System Intelligent Operation and Protection Involving Distributed Architectures and Pulsed Loads,” Ph.D. thesis, School of Electrical and Computer Engineering, Florida International University, 2013. 
[126] V. Salehi "Development and Verification of Control and Protection Strategies in Hybrid AC/DC Power Systems for Smart Grid Applications,” Ph.D. thesis, School of Electrical and Computer Engineering, Florida International University, 2012.

[127] P. Jain and H. Pinheiro, "Hybrid high frequency AC power distribution architecture for telecommunication systems", IEEE Trans. Aerosp. Electron. Syst., vol. 35, no. 1, pp.138 $-1471999$.

[128] M. Qiu , P. K. Jain and H. Zhang "Dynamic performance of an APWM resonant inverter for high frequency AC power distribution system", IEEE Trans. Power Electron., vol. 21, no. 6, pp.1556 -1563 2006.

[129] H. J. Cha and P. N. Enjeti, “A three-phase AC/AC high-frequency link matrix converter for VSCF applications,” Proc. IEEE 34th Annu. Power Electronics Specialist Conf. pp. 1971-1976, 2003.

[130] Bose, B.K.; Min-Huei Kin; Kankam, M.D. "High frequency AC vs. DC distribution system for next generation hybrid electric vehicle", Industrial Electronics, Control, and Instrumentation, vol.2 pp. 706 -712, 1996.

[131] P. Wang, J. Xiao and L. Setyawan, "Hierarchical Control of Hybrid Energy Storage System in DC Microgrids," IEEE Transactions on Industrial Electronics, , vol.99, pp.1,1.

[132] A.A.A. Radwan and Y.A. Mohamed, "Networked Control and Power Management of AC/DC Hybrid Microgrids," IEEE Systems Journal, vol. 99, pp.1-12.

[133] A. S. Hintz, U. R. Prasanna and K. Rajashekara, "Hybrid multi-agent based resilient control for EV connected micro grid system," IEEE Transportation Electrification Conference and Expo pp.1-6, 2014.

[134] H. M. Kelash, H. M. Faheem and M. Amoon, "It takes a multiagent system to manage distributed systems," IEEE Potentials, vol. 26, no. 2, pp. 39-45, 2007.

[135] V. Salehi, A. Mohamed, A. Mazloomzadeh, and O. A. Mohammed, "Laboratory-Based Smart Power System, Part I: Design and System Development," IEEE Transactions on Smart Grid, vol. 3, pp. 1-11, 2012.

[136] V. Salehi, A. Mohamed, A. Mazloomzadeh, and O. A. Mohammed, "Laboratory-Based Smart Power System, Part II: Control, Monitoring, and Protection,” IEEE Transactions on Smart Grid, vol. 3, pp. 1-13, 2012.

[137] H. Smolleck, S. J. Ranade, N. R. Prasad, and R. O. Velasco, "Effects of pulsed-power loads upon an electric power grid,” IEEE Trans. Power Del., vol. 6, no. 4, pp. 13051314, 1991. 
[138] S. Kulkarni and S. Santoso, "Impact of pulse loads on electric ship power system: With and without flywheel energy storage systems,” in Proc. IEEE Electric Ship Technol. Symp. (ESTS 2009), pp. 568-573.

[139] A. Mohamed, V. Salehi, O. Mohammed, "Real-Time Energy Management Algorithm for Mitigation of Pulse Loads in Hybrid Microgrids," Smart Grid, IEEE Transactions on , vol.3, no.4, pp.1911,1922, Dec. 2012.

[140] T. Ma, B. Serrano, O. Mohammed, "Fuzzy logic based power and thermal management system design for multi-cell lithium-ion battery bank protection and operation," Power Systems Conference (PSC), 2014 Clemson University, pp.1,5, 11-14 March 2014.

[141] T. Christen and M.W. Carlen, "Theory of Ragone plots," Journal of Power Sources, September, 2000, vol. 91, Issue 2, pp. 210-216, Dec. 2000.

[142] R.A.Dougal, Shengyi Liu, R.E. White, "Power and life extension of batteryultracapacitor hybrids," Components and Packaging Technologies, IEEE Transactions on, vol.25, no.1, pp.120-131, Mar 2002.

[143] M. Rashid, “DC Choppers,” in Power Electronics. Circuits, Devices and Applications, 2nd ed. Englewood Cliffs, NJ: Prentice-Hall, 1993, ch. 7, pp. 180-225.

[144] P. Karlsson and J. Svensson, "DC bus voltage control for a distributed power system," IEEE Trans. Power Electronics, vol. 18, no. 6, pp.1405-1412, November 2003.

[145] M. Ortuzar, J. Moreno, and J. Dixon, "Ultracapacitor-based auxiliary energy system for an electric vehicle: Implementation and evaluation,” IEEE Trans. Ind. Electron., vol. 54, no. 4, pp. 2147-2156, Aug. 2007.

[146] P. Bajpai and V. Dash, "Hybrid renewable energy systems for power generation in standalone applications: A review,” Renewable and Sustainable Energy Reviews, vol. 16, no. 5, pp. 2926-2939, Jun. 2012.

[147] Guide to the Expression of Uncertainty in Measurement, BIPM, IEC, IFCC, ISO, IUPAC, OIML, 1993.

[148] M. E. Baran and N. R. Mahajan, "DC Distribution for industrial systems: opportunities and challenges,” IEEE Trans. on industrial applications, vol. 39, no. 6, pp. 1596-1601, November/December 2003.

[149] M. Farhadi, A. Mohamed and O. Mohammed, "Connectivity and Bidirectional Energy Transfer in DC Microgrid Featuring Different Voltage Characteristics," Green Technologies Conference, 2013 IEEE, vol., no., pp.244-249, 4-5 April 2013.

[150] D. Salomonsson, L.Soder, A. Sannino, "An Adaptive Control System for a DC Microgrid for Data Centers," Industry Applications Conference, 2007. 42nd IAS Annual 
Meeting. Conference Record of the 2007 IEEE, vol., no., pp.2414,2421, 23-27 Sept. 2007.

[151] Simulink Response Optimization User's Guide. Natick, MA, USA: Math-Works Inc., 2004.

[152] R. A. Dougal, L. Gao, and S. Liu, "Ultracapacitor model with automatic order selection and capacity scaling for dynamic system simulation,” J. Power Sources, vol. 126, pp. 250-257, Feb. 2004.

[153] M. Uzunoglu, O.C. Onar, M.S. Alam, "Modeling, control and simulation of a PV/FC/UC based hybrid power generation system for stand-alone applications”, Renewable Energy, Vol. 34, Issue 3, pp. 509-520, Mar. 2009.

[154] Z. M. Salameh, M. A. Casacca, and W. A. Lynch, “A mathematical model for lead-acid batteries,” IEEE Trans. Energy Convers., vol. 7, no. 1, pp. 93-98, Mar. 1992.

[155] Shepherd, C. M., "Design of primary and secondary cells - Part 2. An equation describing battery discharge,” Journal of Electrochemical Society, Vol. 112, pp 657-664, July 1965.

[156] A. A. S. Emhemed and G. M. Burt, "An Advanced Protection Scheme for Enabling an LVDC Last Mile Distribution Network,” IEEE Trans. Smart Grid, vol. 5, no. 5, pp. 2602-2609, 2014.

[157] W. Leterme, P. Tielens, S. De Boeck, and D. Van Hertem, “Overview of Grounding and Configuration Options for Meshed HVDC Grids,” IEEE Trans. Power Del., vol. 29, no. 6, pp. 2467-2475, Dec 2014.

[158] K. Jia, E. Christopher, D. Thomas, M. Sumner and T. Bi, "Advanced DC zonal marine power system protection," IET Gener. Trans. Distrib. Vol. 8, Iss. 2, pp. 301-309, Feb. 2014.

[159] Y. Wang, Z. Yu, J. He, S. Chen, R. Zeng and B. Zhang, "Performance of Shipboard Medium-Voltage DC System of Various Grounding Modes Under Monopole Ground Fault,” IEEE Trans. Ind. Appl., vol. 51, no.6, pp.5002-5009, Nov.-Dec. 2015.

[160] S.-L. Chen, S.-C. Hsu, C.-T. Tseng, K.-H. Yan, H.-Y. Chou, and T.-M. Too, “Analysis of rail potential and stray current for Taipei metro," IEEE Transactions on Vehicular Technology, vol. 55, no. 1, pp. 67-75, 2006.

[161] S. Fletcher, P. Norman, S. Galloway and G. Burt "Determination of protection system requirements for DC unmanned aerial vehicle electrical power networks for enhanced capability and survivability", IET Electr. Syst. Transp., vol. 1, no. 4, pp.137 -147 2011.

[162] IEEE Std. 1662, "IEEE Guide for the Design and Application of Power Electronics in Electrical Power Systems on Ships", IEEE Industry Applications Society, 2009. 
[163] R. F. Ammerman and R. K. Sen, "DC-arc models and incident-energy calculations," IEEE Trans. Ind. Appl., vol. 46, no. 5, pp. 1810-1819, 2010.

[164] J. Yuventi, "DC Electric Arc-Flash Hazard-Risk Evaluations for Photovoltaic Systems," IEEE Transactions on Power Delivery, vol.29, no.1, pp.161,167, Feb. 2014.

[165] Hesla, E., "DC Task Team Report," Industry Applications, IEEE Transactions on , vol.50, no.5, pp.2996,3003, Sept.-Oct. 2014.

[166] L. Tang and B. T. Ooi, "Locating and isolating DC faults in multi-terminal DC system,” IEEE Trans. Power Del., vol. 22, no. 3, pp. 1877-1884, Jul. 2007.

[167] J. Cheng, M. Guan, L. Tang, H. Huang, X. Chen and J. Xie, "Paralleled multi-terminal DC transmission line fault locating method based on travelling wave," Generation, Transmission \& Distribution, IET , vol.8, no.12, pp.2092,2101, 122014.

[168] W. Dalbon , M. Roscia and D. Zaninelli "Hybrid photovoltaic system control for enhancing sustainable energy" Proc. IEEE Power Eng. Soc. Summer Meeting, vol. 1, pp. 134-139, 2002.

[169] M. K. Zadeh, B. Zahedi, M. Molinas, and L. E. Norum, “Centralized stabilizer for marine DC microgrid,” in Proc. IECON 2013, pp. 3359-3363, 2013.

[170] J. Curk, I. Kobal and G. Parkelj , "How to combine the benefits of distributed and centralized systems for substation control and protection in a single solution," IEE International Conference on Developments in Power System Protection, vol.2, pp.709713 Vol.2, April 2004.

[171] J. Schonberger, R. Duke and S. Round, "DC-bus signaling: A distributed control strategy for a hybrid renewable nanogrid", IEEE Trans. Ind. Electron., vol. 53, no. 5, pp.1453 $-14602006$.

[172] A. Khorsandi, M. Ashourloo and H. Mokhtari, "A decentralized control method for a low-voltage DC microgrid", IEEE Trans. Energy Convers, vol.29, no.4, pp.793-801, Dec. 2014.

[173] C. Gavriluta, J.I. Candela, J. Rocabert, A. Luna and P. Rodriguez, "Adaptive Droop for Control of Multiterminal DC Bus Integrating Energy Storage," IEEE Transactions on Power Delivery, vol.30, no.1, pp.16-24, Feb. 2015.

[174] P. Cairoli, I. Kondratiev, and R. A. Dougal, "Coordinated control of the bus tie switches and power supply converters for fault protection in DC microgrids,” IEEE Trans. Power Electron., vol. 28, no. 4, pp. 2037-2047, Apr. 2013.

[175] P. Pozzobon, "Transient and steady-state short-circuit currents in rectifiers for DC traction supply,” IEEE Trans. Veh. Technol., vol. 47, no. 4, pp. 1390-1404, Nov. 1998. 
[176] D. Paul, "DC traction power system grounding," IEEE Transactions on Industry Applications, vol. 38, no. 3, pp. 818-824, 2002.

[177] D. Salomonsson, L. Soder, and A. Sannino, "Protection of low-voltage DC microgrids," IEEE Trans. Power Del., vol. 24, no. 3, pp. 1045-1053, July 2009.

[178] I. Cotton, C. Charalambous, P. Ernst and P. Aylott, "Stray current control in DC mass transit systems," IEEE Trans. Veh. Technol., vol. 54, no. 2, pp.722 -730, 2005.

[179] D. Love and N. Hashemi, “Considerations for ground fault protection in medium-voltage industrial and cogeneration systems," IEEE Transactions on Industry Applications, vol. 24, no. 4, pp. 548-553, 1988.

[180] J.D. Park, "Ground Fault Detection and Location for Ungrounded DC Traction Power Systems," IEEE Transactions on in Vehicular Technology, vol.64, no.12, pp.5667-5676, Dec. 2015.

[181] J. Roberts, H. Altuve, and D. Hou, "Review of ground fault protection methods for grounded, ungrounded and compensated distribution systems," in 2008 IEEE Power and Energy Society General Meeting - Conversion and Delivery of Electrical Energy in the 21st Century, 2001, pp. 1-8.

[182] IEEE Std 1709-2010, "IEEE Recommended Practice for $1 \mathrm{kV}$ to $35 \mathrm{kV}$ Medium-Voltage DC Power Systems on Ships," IEEE Std 1709-2010, , pp.1-54, Nov. 2010.

[183] A. Nejadpak, A. Sarikhani, O.A. Mohammed, "Analysis of radiated EMI and noise propagation in three-phase inverter system operating under different switching patterns," IEEE Trans. Magn., vol. 49, no.5, pp 2213-2216, May 2013.

[184] J. Yu, "The effects of earthing strategies on rail potential and stray currents in DC transit railways," in International Conference on Developments in Mass Transit Systems (Conf. Pub. No. 453), pp. 303-309, 1998.

[185] J. Rodriguez , J. S. Lai and F. Z. Peng "Multilevel inverters: a survey of topologies, controls and applications", IEEE Transactions on Industrial Electronics, vol. 49, no. 4, pp.724 -738, Aug. 2002.

[186] C.-H. Lee and C.-J. Lu, “Assessment of grounding schemes on rail potential and stray currents in a DC transit system,” IEEE Transactions on Power Delivery, vol. 21, no. 4, pp. 1941-1947, 2006.

[187] C.-H. Lee and H.-M. Wang, "Effects of grounding schemes on rail potential and stray currents in Taipei rail transit systems,” IEE Proceedings- Electric Power Applications, vol. 148, no. 2, pp. 148-154, March 2001.

[188] A. Wright and P. Newbery, “Electric Fuses,” 3rd ed. London, U.K.: Inst. Elect. Eng., 2004. 
[189] M. Fang, L. J. Fu, R. T. Wang, and Z. H. Ye, "Coordination protection for DC distribution network in DC zonal shipboard power system", International Conference on Advanced Power System Automation and Protection (APAP), pp. 418-421, October 2011.

[190] M. Saqib and A. Stokes, “Arc behavior and confinement in a high voltage, high breaking capacity fuse filler,” IEEE Trans. Power Del., vol. 25, no. 1, pp. 212-220, Jan. 2010.

[191] J. P. Brozek, “DC Overcurrent Protection-Where We Stand”, IEEE Transactions on Industry Applications, vol. 29, No. 5, pp. 1029-1032, September/October 1993.

[192] Pauli, B.; Mauthe, G.; Ruoss, E.; Ecklin, G.; Porter, J.; Vithayathil, J., "Development of a high current HVDC circuit breaker with fast fault clearing capability," IEEE Transactions on Power Delivery, vol.3, no.4, pp.2072-2080, Oct. 1988.

[193] S.Tokuyama, K. Arimatsu, Y. Yoshioka, Y. Kato, and K. Hirata, "Development and Interrupting Tests on 250KV 8KA HVDC Circuit Breaker”, IEEE Transactions on Power Apparatus and Systems, vol. PAS-104, no. 9, pp. 2453-2459, Sep. 1985.

[194] Greenwood, A.N.; Lee, T.H., "Theory and Application of the Commutation Principle for HVDC Circuit Breakers, "Power Apparatus and Systems, IEEE Transactions on, vol.PAS-91, no.4, pp.1570-1574, July 1972.

[195] T. Eriksson, M. Backman and S. Halén, “A low loss mechanical HVDC breaker for HVDC Grid applications” in Proc. 2014 CIGRE Paris Conf., pp.1-8, Aug. 2014.

[196] Senda, T.; Tamagawa, T.; Higuchi, K.; Horiuchi, T.; Yanabu, S., "Development of HVDC Circuit Breaker Based on Hybrid Interruption Scheme," IEEE Transactions on Power Apparatus and Systems, vol.PAS-103, no.3, pp. 545-552, March 1984.

[197] Y. Niwa, T. Funahashi, K. Yokokura, J. Matsuzaki, M.Homma, E.Kaneko, "Basic investigation of a high speed vacuum circuit breaker and its vacuum arc characteristics”, IEE Proc.-Gnener. Transm. Distrib., Vol.153, No1, pp11-15, 2006.

[198] L. Liu, J. Zhuang; C. Wang, Z. Jiang, J. Wu and B. Chen, “A Hybrid DC Vacuum Circuit Breaker for Medium Voltage: Principle and First Measurements,” IEEE Transactions on Power Delivery, vol.30, no.5, pp.2096-2101, Oct. 2015.

[199] R. Tamura , R. Shimada , Y. Kito , Y. Kanai , H. Koike , H. Ikeda and S. Yanabu , "Parallel Interruption of Heavy Direct Current by Vacuum Circuit Breaker" , IEEE Trans. PAS , vol. 99 , pp. $1119-1127$, 1980.

[200] R. Shimada, K. Tani, H. Kishimoto and S. Tamura, "Development of High Current DC Circuit Breaker for Large Tokamak Fusion Device", IEEE PES Summer Meeting, 1978.

[201] B. Bachmann et al., "Development of a $500 \mathrm{kV}$ air-blast HVDC circuit breaker,” IEEE Trans. Power App. Syst., vol. PAS-104, no. 9, pp. 2460- 2466, Sep. 1985. 
[202] D. Jovcic and B. Wu, "Fast fault current interruption on high-power DC networks," in Proc. IEEE Power Energy Soc. Gen. Meet., pp.1-6, Jul. 2010.

[203] C. Meyer, M. Kowal, and R. W. De Doncker, "Circuit breaker concepts for future highpower dc-applications,” in Conf. Rec. IEEE IAS Annu. Meeting, vol. 2, pp. 860-866, Oct. 2005.

[204] M. Hajian, L. Zhang and D. Jovcic, "DC Transmission Grid With Low-Speed Protection Using Mechanical DC Circuit Breakers," IEEE Transactions on Power Delivery, vol. 30 , no. 3 , July 2015.

[205] C. M. Franck "HVDC circuit breakers: A review identifying future research needs", IEEE Trans. Power Del., vol. 26, no. 2, pp.998 -1007, April 2011.

[206] R. Schmerda, R. Cuzner, R. Clark, D. Nowak and S. Bunzel "Shipboard solid-state protection: Overview and applications,", IEEE Electrific. Mag., vol. 1, no. 1, pp.3239, Sept. 2013.

[207] M. Hajian , D. Jovcic and W. Bin "Evaluation of semiconductor based methods for fault isolation on high voltage DC grids", IEEE Trans. Smart Grid, vol. 4, no. 2, pp.1171 1179, June 2013.

[208] K. Sano and M. Takasaki "A surgeless solid-state DC circuit breaker for voltage-sourceconverter-based HVDC systems", IEEE Trans. Ind. Appl., vol. 50, no. 4, pp.2690 2699, July-Aug. 2013.

[209] M. Callavik, A. Blomberg, J. Häfner and B. Jacobson, “The hybrid HVDC breaker, an innovation breakthrough enabling reliable HVDC grids” ABB Grid Systems's technichal paper, Nov. 2012.

[210] J. Hafner and B. Jacobson, "Proactive hybrid HVDC breakers-A key innovation for reliable HVDC grids,” in Proc. CIGRE Bologna Conf., pp. 1-8, 2011.

[211] C. D. Barker, C. C. Davidson, D. R. Trainer, and R. S. Whitehouse, "Requirements of DC-DC converters to facilitate large DC Grids,” in Proc. CIGRE Symp., Paris, France, Aug. 2012.

[212] C. Zhan, C. Smith, A. Crane, A. Bullock, and D. Grieve, "DC transmission and distribution system for a large offshore wind farm," in Proc. 2010 IET AC and DC Power Transm., pp. 1-5.

[213] D. Jovcic, “Bi-directional, high-power DC transformer,” IEEE Trans. Power Del., vol. 24, pp. 2276-2283, Sep. 2009.

[214] Y. Chen, X. Liu , J. Sheng, L. Cai , Z. Jin , J. Gu , Z. An, X. Yang and Z. Hong "Design and application of superconducting fault current limiter in DC system", IEEE Trans. Appl. Supercond. vol.24, no.3, pp.1-5, June 2014. 
[215] H. Kraemer "Test of a 1 kA superconducting fault current limiter for DC applications", IEEE Trans. Appl. Supercond., vol. 15, no. 2, pp.1986 -1989, June 2005.

[216] M. Farhadi and H. Heydari "Rational approach for self-limiting current injection transformers confirmed by coupled electromagnetic Thermal FEM simulation", Supercond. Sci. Technol., vol. 24, no. 7, pp. 075021, 2011.

[217] A. Morandi, "State of the art of superconducting fault current limiters and their application to the electric power system,” Physica C: Supercond., vol. 484, pp. 242-247, 2013.

[218] T. Ishigohka and N. Sasaki "Fundamental test of new DC superconducting fault current limiter", IEEE Trans. Magn., vol. 27, pp.2341 -2344, 1991

[219] J. Shi, "Active superconducting DC fault current limiter based on flux compensation", Physica C: Superconductivity and Its Applications, vol. 442, no. 2, 2006.

[220] J. Shi, Y. Tang, L. Chen, J. Wang, L. Ren, J. Li. L. Li T. Peng and S. Cheng, "The application of active superconducting DC fault current limiter in hybrid AC/DC power supply system", IEEE Trans. Appl. Supercond., vol. 18, no. 2, pp.672 -675, 2008.

[221] Y. Xin et all "Manufacturing and test of a $35 \mathrm{kV} / 90$ MVA saturated iron-coretype superconductive fault current limiter for live-grid operation", IEEE Trans. Appl. Supercond., vol. 19, no. 3, pp.1934 -1937, 2009

[222] Y. Xin "Factory and field tests of a $220 \mathrm{kV} / 300$ MVA statured iron-core superconducting fault current limiter", IEEE Trans. Appl. Supercond., vol. 23, no. 3, 2013.

[223] S.D.A. Fletcher, P.J. Norman, S.J. Galloway, P. Crolla, and G.M. Burt, “Optimizing the roles of unit and non-unit protection methods within DC microgrids,” IEEE Trans. Smart Grid, vol. 3, no. 4, pp. 2079-2087, Dec. 2012.

[224] C. Yuan; M.A. Haj-ahmed, and M.S. Illindala, "An MVDC microgrid for a remote area mine site: Protection, operation and control," Industry Applications Society Annual Meeting, pp.1-9, Oct. 2014.

[225] J. Park, J. Candelaria, "Fault detection and isolation in low-voltage DC Bus microgrid system" IEEE Trans. Power Del., vol. 28, no. 2, pp. 779-787, Apr. 2013.

[226] G. Patil, and M.F.A.R. Satarkar, "Autonomous protection of low voltage DC microgrid," International Conference on Power, Automation and Communication (INPAC), pp.2326, Oct. 2014.

[227] Monadi, M.; Koch-Ciobotaru, C.; Luna, A.; Candela, J.I.; Rodriguez, P., "A protection strategy for fault detection and location for multi-terminal MVDC distribution systems 
with renewable energy systems," International Conference on Renewable Energy Research and Application (ICRERA), 2014, pp.496-501, Oct. 2014.

[228] S.D.A. Fletcher, P.J. Norman, K. Fong, S.J. Galloway, and G.M. Burt, "High-Speed Differential Protection for Smart DC Distribution Systems," Smart Grid, IEEE Transactions on, vol.5, no.5, pp.2610-2617, Sept. 2014.

[229] H. Farhangi “The path of the smart grid” IEEE Power Energy Mag., vol. 8, no. 1, pp. 18-28, Jan.-Feb 2010.

[230] R. M. Cuzner and G. Venkataramanan "The status of DC micro-grid protection", Proc. IEEE Ind. Appl. Soc. Annu. Meeting, pp. 1 -8 Oct. 2008.

[231] Z. Galijasevic and A. Abur, "Fault area estimation via intelligent processing of faultinduced transients,” IEEE Trans. Power Syst., vol. 18,no. 4, pp. 1241-1247, Nov. 2003.

[232] L. Xiaqing, G. Shuyou, Z. Yiming, L. Yunhua and F. Shengtao, "Research on Fault Diagnosis and Protect Model for DC Traction Power Supply System Based on Traveling Wave Theory," IEEE Conference on Robotics, Automation and Mechatronics, pp.1,5, Dec. 2006.

[233] V. Pathirana, E. Dirks, and P. G. McLaren, "Hardware and software implementation of a travelling wave based protection relay,” in Proc. IEEE Power Eng. Soc. General Meeting, vol. 1, pp. 701-706, Jun. 2005.

[234] A. Elhaffar and M. Lehtonen, "Multi-end traveling wave fault location based on current traveling waves,” presented at the Power Syst.Comput. Conf., Glasgow, U.K., 2008.

[235] Y. Zhang , N. Tai and B. Xu "Fault analysis and traveling-wave protection scheme for bipolar HVDC lines", IEEE Trans. Power Del., vol. 27, no. 3, pp.1583 -1591, July 2012.

[236] W. Leterme, J. Beerten and D. Van Hertem "Non-unit protection of HVDC grids with inductive DC cable termination," to be published in IEEE Trans. Power Del., 2015.

[237] O. Nanayakkara , A.D. Rajapakse and R. Wachal "Traveling-wave-based line fault location in star-connected multiterminal HVDC systems", IEEE Trans. Power Del., vol. 27, no. 4, pp.2286 -2294, Oct. 2012.

[238] O. M. K. K. Nanayakkara, A. D. Rajapakse, and R. Wachal, "Location of DC line faults in conventional HVDC systems with segments of cables and overhead lines using terminal measurements,” IEEE Trans. Power Del., vol. 27, no. 1, pp. 279-288, Jan. 2012.

[239] E. W. Kimbark, "Transient over voltages caused by monopolar ground fault on bipolar DC line: Theory and simulation,” IEEE Trans. Power App. Syst., vol. PAS-89, no. 4, pp. 584-592, Apr. 1970. 
[240] L. Jie, S. Elangovan and J. B. X. Devotta "Adaptive travelling wave protection algorithm using two correlation functions", IEEE Trans. Power Del., vol. 14, no. 1, pp.126 -131, 1999.

[241] X. Liu , A. H. Osman and O. P. Malik "Hybrid traveling wave/boundary protection for monopolar HVDC line", IEEE Trans. Power Del., vol. 24, no. 2, pp.569 -578, April 2009.

[242] P. Murthy, J. Amarnath, S. Kamakshiah, and B. Singh, "Wavelet transform approach for detection and location of faults in HVDC system,” inProc. Conf. Ind. Inf. Syst., pp. 16, 2008.

[243] J. Yang , J. E. Fletcher and J. O'Reilly "Multiterminal DC wind farm collection grid internal fault analysis and protection design", IEEE Trans. Power Del., vol. 25, no. 4, pp.2903 -2912, Oct. 2010.

[244] J.-D. Park, J. Candelaria, L. Ma and K. Dunn "DC ring-bus microgrid fault protection and identification of fault location", IEEE Trans. Power Del., vol. 28, no. 4, pp.2574 -2584, Oct. 2013.

[245] P. Cairoli and R.A. Dougal,"New Horizons in DC Shipboard Power Systems: New fault protection strategies are essential to the adoption of DC power systems," IEEE Electrification Magazine, vol.1, no.2, pp.38-45, Dec. 2013.

[246] Mustafa Farhadi and Osama Mohammed, “A New Protection Scheme for Multi-Bus DC Power Systems Using an Event Classification Approach " 50th IEEE IAS Annual Meeting, Dallas, Texas 18-22 October 2015.

[247] B. K. Bose "Expert system, fuzzy logic, and neural network applications in power electronics and motion control", Proc. IEEE, vol. 82, pp.1303 -1323, 1994.

[248] F. Filippetti , G. Franceschini , C. Tassoni and P. Vas "Recent developments of induction motor drives fault diagnosis using AI techniques", IEEE Trans. Power Electron., vol. 19, no. 4, pp.1108 -1116, 2004.

[249] N. K. Chanda and Y. Fu, "ANN-based fault classification and location in MVDC shipboard power systems,” in Proc. NAPS, pp. 1-7 Aug. 2011.

[250] W. Li, A. Monti and F. Ponci "Fault detection and classification in medium voltage DC shipboard power systems with wavelets and artificial neural networks", IEEE Trans. Instrum. Meas., vol. 63, no. 11, pp.2651 -2665, 2014.

[251] C. S. Chang, Z. Xu, and A. Khambadkone, "Enhancement and laboratory implementation of neural network detection of short circuit faults in DC transit system," IEE Electric Power Appl. Proc., vol. 150, no. 3, pp. 344-350, May 2003. 
[252] C. S. Chang, S. Kumar, B. Liu, and A. Khambadkone, "Real-time detection using wavelet transform and neural network of short-circuit faults within a train in DC transit systems,” IEE Electric Power Appl. Proc., vol. 148, no. 3, pp. 251-256, May 2001.

[253] A. Nejadpak and O. A. Mohammed, "Physics-based modeling of power converters from finite element electromagnetic field computations,” IEEE Trans. Magn., vol. 49, no. 1, pp. 567-576, Jan. 2013.

[254] S. R. Velazquez, T. Q. Nguyen, and S. R. Broadstone, "Design of hybrid filter banks for analog/digital conversion,” IEEE Trans. Signal Processing, vol. 46, no. 4, pp. 956-967, Apr. 1998.

[255] IEEE Standard for Terminology and Test Methods for Analog-to-Digital Converters, IEEE Std. 1241-2010, Jan. 2011.

[256] A. Agarwal and J. H. Lang, "Foundations of Analog and Digital Circuits," Morgan Kaufman, 2005.

[257] A. Greenwood, “Electrical Transients in Power Systems,” New York: Wiley, 1991.

[258] Carr, J.; Jackson, R. V., "Frequency domain analysis applied to digital transmission line protection," IEEE Transactions on Power Apparatus and Systems, vol.94, no.4, pp.11571166, July 1975. 
VITA

MUSTAFA FARHADI

1984

2003-2007

2008-2011

$2011-2016$

2015

2015
Born, Ghemshahr, Iran

B.S., Electrical Engineering

University of Mazandaran, Mazandaran, Iran

M.S., Electrical Engineering

Iran University of Technology, Tehran, Iran

Doctoral Candidate, Electrical Engineering

Florida International University, Miami, Florida

Award, Dissertation Year Fellowship

Florida International University, Miami, Florida

Myron Zucker Travel Award

50th IEEE IAS Annual Meeting

\section{SELECTED PUBLICATIONS AND PRESENTATIONS}

[J-1] Mustafa Farhadi and Osama Mohammed, "A New Protection Scheme for Multi-Bus DC Power Systems Using an Event Classification Approach,” Accepted for publication in IEEE Transactions on Industry Applications.

[J-2] Mustafa Farhadi and Osama Mohammed, “Energy Storage Technologies for High Power Applications,” Accepted for publication in IEEE Transactions on Industry Applications.

[J-3] Mustafa Farhadi and Osama Mohammed, "Performance enhancement of actively controlled hybrid DC microgrid and pulsed power load,” IEEE Trans. Ind. Appl., vol.51, no.5, pp.3570-3578, Sept.-Oct. 2015.

[J-4] Mustafa Farhadi and Osama Mohammed, "Event Based Protection Scheme for a MultiTerminal Hybrid DC Power System,” IEEE Trans. Smart Grid, vol.6, no.4, pp.1658-1669, July 2015.

[J-5] Mustafa Farhadi and Osama Mohammed, “Adaptive Energy Management in Redundant Hybrid DC Microgrid for Pulse Load Mitigation,” IEEE Trans. Smart Grid, vol.6, no.1, pp.5462, Jan. 2015.

[J-6] Mustafa Farhadi and Osama Mohammed, "Real-time operation and harmonic analysis of isolated and non-isolated hybrid DC microgrid,” IEEE Trans. Ind. Appl., vol.50, no.4, pp.2900-2909, Jul./Aug. 2014. 
[C-1] Mustafa Farhadi and Osama Mohammed, "Energy Storage Systems for High Power Applications,” 50th IEEE IAS Annual Meeting, Dallas, Texas, pp. 1-7, 18-22 October 2015.

[C-2] Mustafa Farhadi and Osama Mohammed, "Hybrid Energy Storage Systems for Transportation Electrification,” International Aegean Conference on Electrical machines and Power Electronics, Turkey 2-4 September 2015.

[C-3] Mustafa Farhadi and Osama Mohammed, "A New Protection Scheme for Multi-Bus DC Power Systems Using an Event Classification Approach,” 50th IEEE IAS Annual Meeting, Dallas, Texas, pp. 1-7 18-22 October 2015.

[C-4] Mustafa Farhadi and Osama Mohammed, "Design and Hardware Implementation of Laboratory-Scale Hybrid DC power System for Educational Purpose" $122^{\text {th }}$ American Society for Engineering Education Annual Conference, ASEE, Seattle, WA, June 14 - 17, 2015.

[C-5] Mustafa Farhadi and Osama Mohammed, "Hybrid DC Power System for Pulse Load Application: Design, Modeling and Operation” IEEE Green Technologies Conference, New Orleans, LA, pp.152-157, 15- 17 April, 2015.

[C-6] Mustafa Farhadi and Osama Mohammed, "Experimental Study on the Effects of the Pulse Load Characteristics on the Performance of the Slack Bus Generator," 17th International Conference on Electrical Machines and Systems (ICEMS2014), Hangzhou, China on, pp.2802-2807 October 22-25, 2014.

[C-7] Mustafa Farhadi, Ali Mazloomzadeh and Osama Mohammed, "Comparative Analysis of Energy Control Techniques for DC Microgrid and Pulsed Power Load Applications,” 40th Annual Conference of the IEEE Industrial Electronics Society, IECON'2014, Dallas, TX USA, pp. 4204-4210, October 28 - November 1, 2014.

[C-8] Mustafa Farhadi and Osama Mohammed, "Performance Enhancement of Actively Controlled Hybrid DC Microgrid and Pulsed Power Load,” 49th IEEE IAS Annual Meeting, Vancouver, BC Canada, p.p 1-8, 5-9 October 2014.

[C-9] Mustafa Farhadi and Osama Mohammed, "System Operation and Harmonic Analysis for Isolated and Non-Isolated Hybrid DC Microgrid,” Industry Applications Society Annual Meeting, 2013 IEEE , pp.1,6, 6-11 Oct. 2013.

[C-10] Mustafa Farhadi and Osama Mohammed, “Adaptive Energy Management of Hybrid DC Microgrids Featuring Ultracapacitors and Voltage Protection Scheme with Hardware Implementation,” International Smart Grid Conference \& Exhibition , ISGC\&E, July, 2013. 